\title{
Analytical Data from Phases I and II of the Willamette River Basin Water Quality Study, Oregon, 1992-94
}

\section{U.S. GEOLOGICAL SURVEY}

Open-File Report 95-373

Prepared in cooperation with the

OREGON DEPARTMENT OF ENVIRONMENTAL QUALITY

WILLAMETTE RIVER TECHNICAL ADVISORY STEERING COMMITTEE, and the

NATIONAL WATER-QUALITY ASSESSMENT PROGRAM

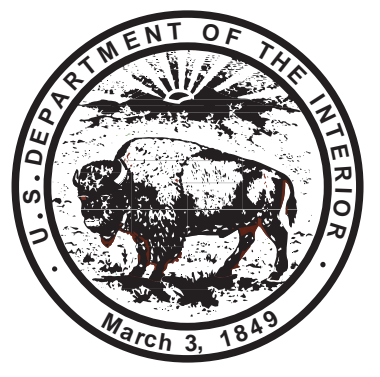


Cover photograph. Looking upstream at the Willamette River with Mt. Hood and Portland Harbor in the background, February 7, 1993. (Photograph by Dennis A. Wentz, U.S. Geological Survey.) 


\section{Analytical Data from Phases I and II of the Willamette River Basin Water Quality Study, Oregon, 1992-94}

By Howard E. Harrison, Chauncey W. Anderson, Frank A. Rinella, Timothy M. Gasser, and Ted R. Pogue Jr.

U.S. GEOLOGICAL SURVEY Open-File Report 95-373

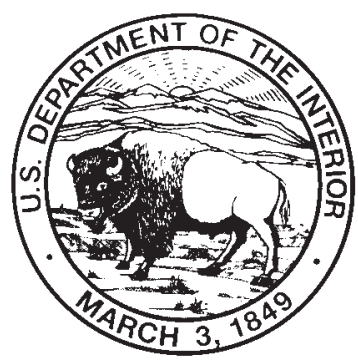

Prepared in cooperation with the OREGON DEPARTMENT OF ENVIRONMENTAL QUALITY WILLAMETTE RIVER TECHNICAL ADVISORY STEERING COMMITTEE, and the NATIONAL WATER-QUALITY ASSESSMENT PROGRAM

Portland, Oregon 1995

(Revised and Reprinted 1997) 


\title{
U.S. DEPARTMENT OF THE INTERIOR BRUCE BABBITT, Secretary
}

\author{
U.S. GEOLOGICAL SURVEY \\ GORDON P. EATON, Director
}

Any use of trade, product or firm names in this publication is for descriptive

purposes only and does not imply endorsement by the U.S. Government.

For additional information write to:

District Chief

U.S. Geological Survey, WRD

10615 S.E. Cherry Blossom Drive

Portland, OR 97216

E-mail: info-or@usgs.gov

Internet: <http://oregon.usgs.gov>
Copies of this report can be purchased from:

U.S. Geological Survey Branch of Information Services Box 25286, Federal Center Denver, CO 80225 


\section{CONTENTS}

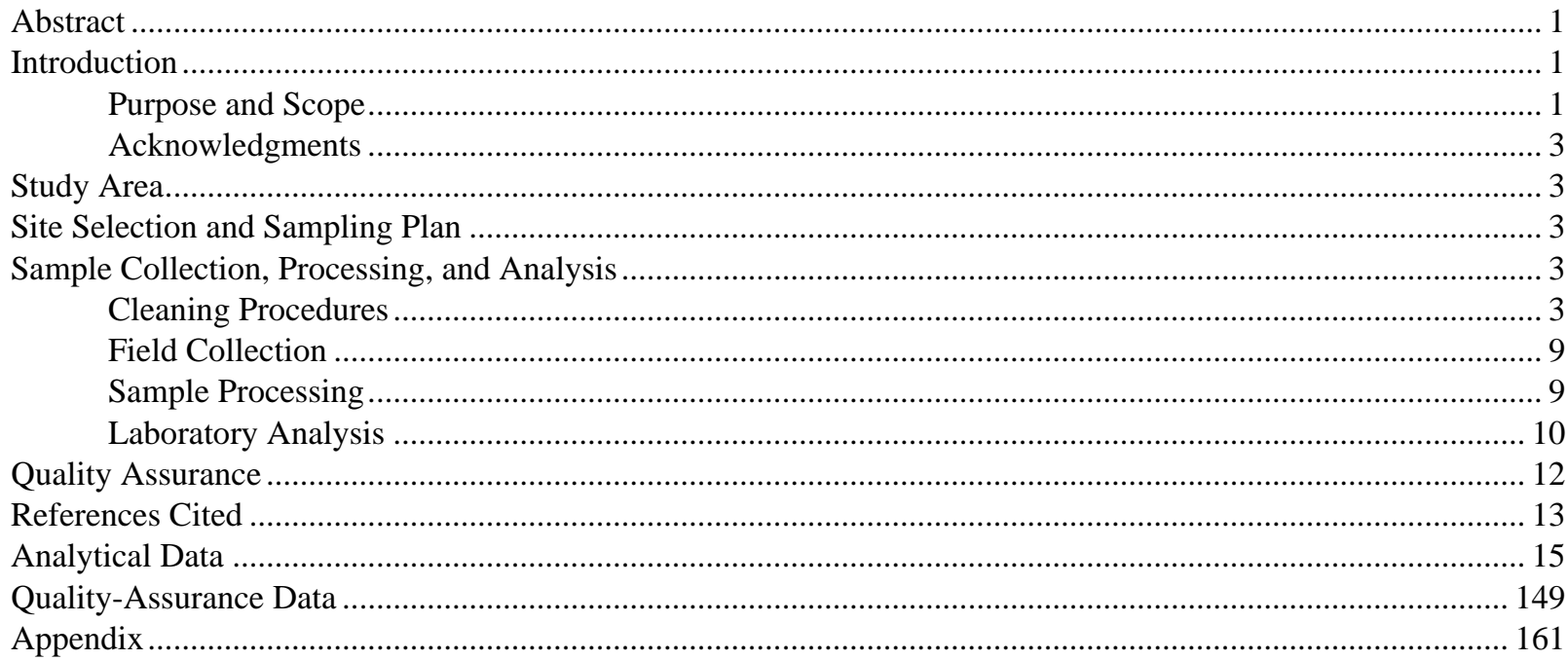

FIGURES

1. Map showing the Willamette and Sandy River Basins, Oregon ......

2. Map showing sampling sites from Phases I and II of the Willamette River Basin Water Quality Study, Oregon, 1992-94

\section{TABLES}

1. Land use in the Willamette and Sandy River Basins, Oregon ......

2. Water-quality sampling sites from Phases I and II of the Willamette River Basin Water Quality Study, Oregon, 1992-94....

3. Sampling plan for Phase I of the Willamette River Basin Water Quality Study, Oregon 1992-93 ................. 6

4. Sampling plan for Phase II of the Willamette River Basin Water Quality Study, Oregon, Spring 1994 ......... 7

5. Sampling plan for Phase II of the Willamette River Basin Water Quality Study, Oregon, Fall 1994 ............. 8

6. Summary of water-quality analyses conducted during Phases I and II of the Willamette River Basin Water Quality Study, Oregon, 1992-94 11

7. Ancillary data from Phase I of the Willamette River Basin Water Quality Study, Oregon, 1992-93 ............ 17

8. Ancillary data from Phase II of the Willamette River Basin Water Quality Study, Oregon, 1994 ............... 20

9. Concentrations of nutrients in whole and filtered water from Phase I of the Willamette River Basin Water Quality Study, Oregon, 1992-93.

10. Concentrations of nutrients in whole and filtered water from Phase II of the Willamette River Basin Water Quality Study, Oregon, 1994.

11. Concentrations of trace elements and major metals in whole water from Phase I of the Willamette River Basin Water Quality Study, Oregon, 1992-93

12. Concentrations of trace elements in whole water from Phase II of the Willamette River Basin Water Quality Study, Oregon, 1994

13. Concentrations of trace elements and major metals in filtered water from Phase I of the Willamette River Basin Water Quality Study, Oregon, 1992-93.

14. Concentrations of trace elements in filtered water from Phase II of the Willamette River Basin Water Quality Study, Oregon, 1994

15. Concentrations of trace elements associated with suspended sediment from Phase I of the Willamette River Basin Water Quality Study, Oregon 1992-93 
16. Concentrations of trace elements associated with bed sediment from Phase I of the Willamette River Basin Water Quality Study, Oregon, 1992.

17. Concentrations of organochlorine and organophosphorus compounds in whole water from Phase I of the Willamette River Basin Water Quality Study, Oregon, 1993

18. Concentrations of organochlorine compounds in whole water from Phase II of the Willamette River Basin Water Quality Study, Oregon, 1994.

19. Concentrations of organochlorine and organophosphorus compounds in filtered water from Phase I of the Willamette River Basin Water Quality Study, Oregon, 1992-93.....

20. Concentrations of organochlorine compounds in filtered water from Phase II of the Willamette River Basin Water Quality Study, Oregon, 1994.

21. Concentrations of organochlorine and organophosphorus compounds associated with suspended sediment from Phase I of the Willamette River Basin Water Quality Study, Oregon, 1992-93

22. Concentrations of organochlorine compounds associated with suspended sediment from Phase II of the Willamette River Basin Water Quality Study, Oregon, 1994

23. Concentrations of organochlorine compounds associated with bed sediment from Phase I of the Willamette River Basin Water Quality Study, Oregon, 1992

24. Concentrations of organic acids in whole water from Phase I of the Willamette River Basin Water Quality Study, Oregon, 1992.

25. Concentrations of semi-volatile compounds in whole water from Phase I of the Willamette River Basin Water Quality Study, Oregon, 1993.

26. Concentrations of semi-volatile compounds in filtered water from Phase I of the Willamette River Basin Water Quality Study, Oregon, 1992-93

27. Concentrations of semi-volatile compounds associated with suspended sediment from Phase I of the Willamette River Basin Water Quality Study, Oregon, 1992-93.....

28. Concentrations of semi-volatile compounds associated with bed sediment from Phase I of the Willamette River Basin Water Quality Study, Oregon, 1992

29. Concentrations of volatile organic compounds in whole water from Phase I of the Willamette River Basin Water Quality Study, Oregon, 1992-93

30. Concentrations of volatile organic compounds in whole water from Phase II of the Willamette River Basin Water Quality Study, Oregon, 1994.

31. Concentrations of dioxin associated with suspended sediment from Phase I of the Willamette River Basin Water Quality Study, Oregon, 1992.

32. Concentrations of dioxin associated with bed sediment from Phase I of the Willamette River Basin Water Quality Study, Oregon, 1992.

33. Concentrations of furans associated with suspended sediment from Phase I of the Willamette River Basin Water Quality Study, Oregon, 1992.

34. Concentrations of furans associated with bed sediment from Phase I of the Willamette River Basin Water Quality Study, Oregon, 1992

35. Concentrations of pesticides in filtered water analyzed by gas chromatography/mass spectroscopy from Phase I of the Willamette River Basin Water Quality Study, Oregon, 1992-93.

36. Concentrations of pesticides in filtered water analyzed by gas chromatography/mass spectroscopy from Phase II of the Willamette River Basin Water Quality Study, Oregon, 1994

37. Concentrations of pesticides in filtered water analyzed by high performance liquid chromatography from Phase I of the Willamette River Basin Water Quality Study, Oregon, 1993.

38. Concentrations of pesticides in filtered water analyzed by high performance liquid chromatography from Phase II of the Willamette River Basin Water Quality Study, Oregon, 1994

39. Concentrations of triazine herbicides in filtered water from Phase II of the Willamette River Basin Water Quality Study, Oregon, 1994

40. Concentrations of trace elements in splits of whole water samples from Phase II of the Willamette River Basin Water Quality Study, 1994, Oregon .

41. Concentrations of trace elements in splits of filtered water samples from Phase II of the Willamette River Basin Water Quality Study, Oregon, 1994 
42. Concentrations of organochlorine compounds in splits and field matrix spikes of whole water samples from Phase II of the Willamette River Basin Water Quality Study, Oregon, 1994

43. Concentrations of pesticides analyzed by gas chromatography/mass spectroscopy in splits and field matrix spikes of filtered water samples from Phase II of the Willamette River Basin Water Quality Study, Oregon, 1994.

44. Concentrations of pesticides analyzed by high performance liquid gas chromatography in splits and field matrix spikes of filtered water samples from Phase II of the Willamette River Basin Water Quality Study, Oregon, 1994

45. Concentrations of pesticides analyzed by high performance liquid gas chromatography in field matrix spikes of filtered water samples from Phase II of the Willamette River Basin Water Quality Study, Oregon, 1994.

46. Concentrations of pesticides analyzed by high performance liquid gas chromatography in field matrix spikes of filtered water samples from Phase II of the Willamette River Basin Water Quality Study, Oregon, 1994

47. Concentrations of triazine compounds in split samples from Phase II of the Willamette River Basin Water Quality Study, Oregon, 1994

48. Concentrations of triazine compounds in field matrix spikes of filtered water samples from Phase II of the Willamette River Basin Water

Quality Study, Oregon, 1994.

\section{CONVERSION FACTORS}

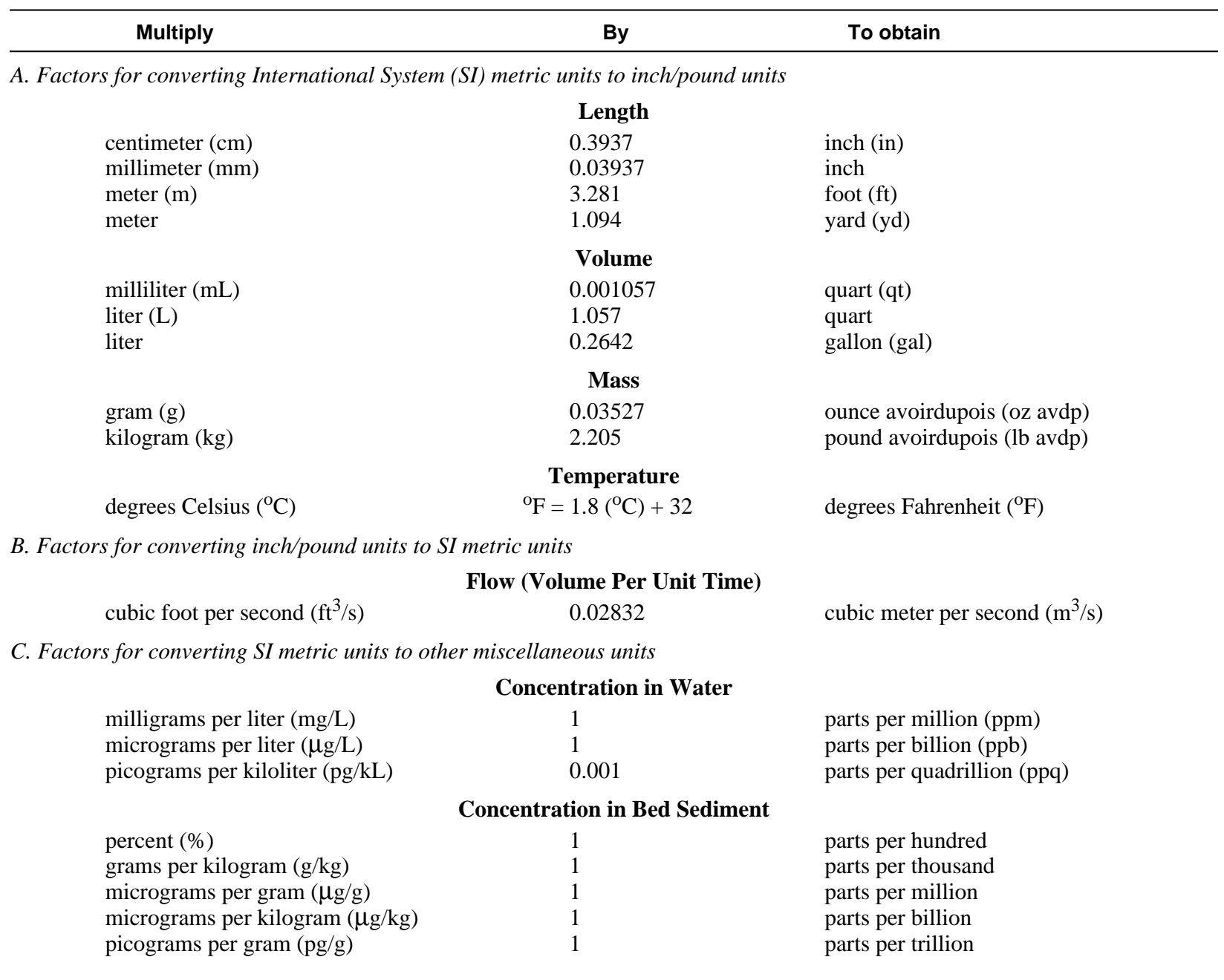


Page Intentionally Blank 


\title{
Analytical Data from Phases I and II of the Willamette River Basin Water Quality Study, Oregon, 1992-94
}

\author{
By Howard E. Harrison, Chauncey W. Anderson, Frank A. Rinella, Timothy M. Gasser \\ and Ted R. Pogue, Jr.
}

\section{Abstract}

This report presents trace-element, organic-compound (pesticides, volatile and semi-volatile organic, and dioxin and furan compounds), and nutrient concentration data from the analyses of water column, suspendedsediment, and bed-sediment samples collected by the U.S. Geological Survey as part of Phases I and II of the comprehensive Willamette River Basin Water Quality Study in western Oregon. The overall study was designed by the Oregon Department of Environmental Quality to acquire the technical and regulatory knowledge necessary to protect and enhance water quality in the Willamette River Basin.

These data were collected at 50 sites representing runoff from agricultural, forested, and urbanized subbasins. In Phase I, water samples were collected during high and low flows in 1992 and 1993 to represent a wide range of hydrologic conditions. Bed-sediment samples were collected during low flows in 1993. In Phase II, water samples were collected in the spring of 1994 after the first high-flow event following the application of agricultural fertilizers and pesticides and in the fall during the first high-flow events following the conclusion of the agricultural season.

\section{INTRODUCTION}

In 1990, the Oregon Department of Environmental Quality (ODEQ) identified the protection and enhancement of water quality in the
Willamette River Basin (fig. 1) as one of its critical long-range resource-management goals. In response to a joint State Legislative Emergency Board mandate, ODEQ formed the Willamette River Technical Advisory Steering Committee (WRTASC) and charged the committee to design a comprehensive study to develop the technical and regulatory knowledge required to protect and enhance water quality in the basin. The committee is composed of representatives of Federal, State, and local agencies, as well as from industry, environmental groups, and the public. In 1992, WRTASC designed the Willamette River Basin Water Quality Study to collect critical information necessary to direct future management efforts. As part of this study the U.S. Geological Survey (USGS) investigated (1) hydrology, (2) sediment transport, and (3) major sources of pesticides and trace elements within the basin.

\section{Purpose and Scope}

This report presents trace-element, organiccompound (pesticides, volatile and semi-volatile organic, and dioxin and furan compounds), and nutrient concentration data from the analyses of water column, suspended-sediment, and bedsediment samples collected from the Willamette River Basin (objective 3 listed above). These data were collected in two phases. Phase I was a reconnaissance of hydrophilic- and hydrophobiccontaminant concentrations in water and bed sediment spanning an 18 month period (April 1992 through September 1993). Phase II consisted of spring (March through June 1994) and fall (October through December 1994) high-flow samplings to identify and (or) quantify major sources of pesticides and trace-elements. 


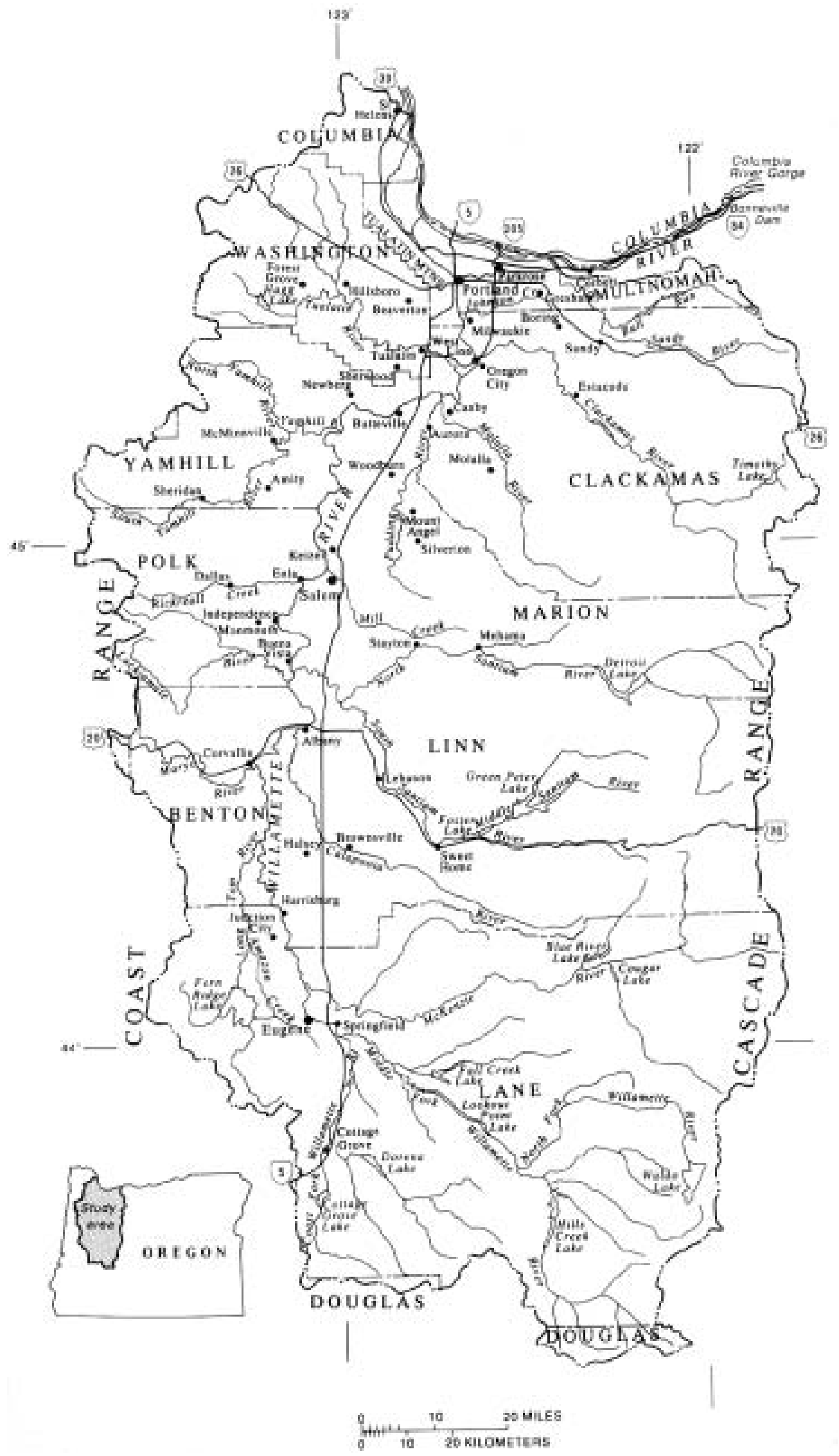

Figure 1. The Willamette and Sandy River Basins, Oregon. 


\section{Acknowledgments}

The authors wish to thank the USGS Willamette Basin National Water-Quality Assessment (NAWQA) Program for its contribution of personnel and equipment to the data-collection effort. In addition, we are grateful to the members of the WRTASC for their guidance and support in this project.

\section{STUDY AREA}

The Willamette River Basin, including the Sandy River Basin, encompasses nearly 12,000 square miles (fig. 1). The Willamette River is located in western Oregon between the Coast and Cascade Ranges and is the $13^{\text {th }}$ largest river basin in the conterminous United States (Kammerer, 1990). Fed by 13 major tributaries, the Willamette River flows north over 270 miles from its headwaters to its confluence with the Columbia River near Portland, Oregon. The basin is home to more than 1.8 million people (69 percent of the State's population). Land use in the basin is varied (table 1); forested lands are predominant on the slopes of the Cascade and Coast Ranges, and agricultural lands are predominant in the lower elevations. Although urban regions account for only a small percentage of the land area, they are concentrated along the main stem of the Willamette River in the metropolitan areas of Portland, Salem, and Eugene-Springfield.

Table 1. Land use in the Willamette and Sandy River Basins, Oregon

[Source: adapted from Fegeas and others, 1983]

\begin{tabular}{lcc}
\hline \multicolumn{1}{c}{ Category } & $\begin{array}{c}\text { Area } \\
\text { (Square miles) }\end{array}$ & $\begin{array}{c}\text { Percent of } \\
\text { basin area }\end{array}$ \\
\hline Urban & 631.7 & 5.27 \\
Agriculture & 2691.1 & 22.46 \\
Rangeland & 53.1 & 0.44 \\
Forest & 8411.1 & 70.18 \\
Open water & 112.6 & 0.94 \\
Wetland & 41.4 & 0.35 \\
Barren land & 20.8 & 0.17 \\
Tundra & 16.8 & 0.14 \\
Glacial & 6.1 & 0.05 \\
Total & $11,984.7$ & 100.00 \\
\hline
\end{tabular}

\section{SITE SELECTION AND SAMPLING PLAN}

Available data on point- and nonpointsource discharges, pesticide usage, and land-use practices were used in conjunction with current and historical water-quality records to select sampling sites. These sites were categorized according to the dominant land use within the basin as either forest, agriculture, urban-industrial or, when no other category exceeded 50 percent of the upstream drainage, as integrator. Some exceptions in this classification scheme occurred at sites where local land use dominated the water quality. Forested sites were sampled to verify the assumption that nonpoint runoff from these lands was only a minor source of trace-elements or pesticides.

Water-quality sampling locations are described in table 2 and shown in figure 2 . These 50 sites were distributed throughout the basin on the main stem of the Willamette River, as well as on major and minor tributaries. Twenty-one sites were visited in Phase I and 33 sites in Phase II; four sites were visited in both phases. Tables 3, 4, and 5 provide the sampling plans for Phase I, Phase II (spring), and Phase II (fall), respectively.

In Phase I, water samples were collected during high and low flows in 1992 and 1993 to represent a wide range of hydrologic conditions. Bed-sediment samples were collected during low flows in 1993, when sediment transport is minimal. In Phase II, water samples were collected in the spring of 1994 after the first high-flow event following the application of agricultural fertilizers and pesticides and in the fall during the first highflow events following the conclusion of the agricultural season.

\section{SAMPLE COLLECTION, PROCESSING, AND ANALYSIS \\ Cleaning Procedures}

In preparation for water-quality sampling, all sampling and processing equipment was thoroughly cleaned according to the following procedures: Trace-element-sampling and processing equipment was washed in dilute (0.1 percent) low-phosphate soap and tap water 
Table 2. Water-quality sampling sites from Phases I and II of the Willamette River Basin Water Quality Study, Oregon, 1992-94

\begin{tabular}{|c|c|c|c|c|}
\hline $\begin{array}{l}\text { Map index } \\
\text { number }\end{array}$ & Site name & Site number & $\begin{array}{l}\text { Site } \\
\text { type }\end{array}$ & $\begin{array}{c}\text { Sampled in } \\
\text { study } \\
\text { Phase }\end{array}$ \\
\hline 1 & CF Willamette R bl Big R nr London, OR & 433548123040600 & Forest & II \\
\hline 2 & CF Willamette R at Seavy Loop Rd nr Eugene, OR & 440045122585600 & Integrator & II \\
\hline 3 & A-3 Channel at Wallis and 5th St Eugene, OR & 440313123091100 & Urban-industrial & I \\
\hline 4 & $\begin{array}{l}\text { Urban outfall at Polk St Park at Eugene, OR } \\
\text { (Urban outfall at Greenway Bicycle Bridge, Eugene, OR) }\end{array}$ & 440402123063900 & Urban & II \\
\hline 5 & Mack Cr nr Blue River, OR & 441310122095801 & Forest & II \\
\hline 6 & McKenzie R nr Coburg, OR & 14165500 & Integrator & I \\
\hline 7 & McKenzie R nr Eugene, OR & 440707123041300 & Integrator & II \\
\hline 8 & Long Tom R at Bundy Bridge nr Monroe, OR & 442223123153703 & Agriculture & II \\
\hline 9 & Lake Camous Cr at Pine Grove Dr nr Harrisburg, OR & 442413123122500 & Agriculture & II \\
\hline 10 & Willamette R nr Corvallis, OR & 443207123145500 & Integrator & I \\
\hline 11 & Muddy Cr nr Peoria, OR & 443138123120901 & Agriculture & both \\
\hline 12 & Rock Cr ab Griffith Cr nr Philomath, OR & 443045123273000 & Forest & II \\
\hline 13 & Marys $\mathrm{R}$ at Corvallis, OR & 443321123155201 & Integrator & II \\
\hline 14 & Calapooia R at Albany, OR & 14173500 & Agriculture & II \\
\hline 15 & Calapooia R at mouth nr Albany, OR & 443819123064000 & Agriculture & I \\
\hline 16 & Middle Fourth Lk nr Albany, OR & 444032123050600 & Urban-industrial & I \\
\hline 17 & Luckiamute R at Buena Vista Rd nr Buena Vista, OR & 444349123094000 & Integrator & II \\
\hline 18 & Thomas Cr at Kelly Rd nr Jefferson, OR & 444123122562200 & Agriculture & II \\
\hline 19 & Santiam R at Jefferson, OR & 14189000 & Integrator & II \\
\hline 20 & Santiam R nr Jefferson, OR & 444416123030800 & Integrator & I \\
\hline 21 & Rickreall Cr nr Rickerall, OR & 445543123084400 & Agriculture & I \\
\hline 22 & Rickreall Cr nr mouth nr Salem, OR & 445547123065400 & Agriculture & II \\
\hline 23 & Pringle $\mathrm{Cr}$ at Bush Park at Salem, OR & 14190970 & Urban & II \\
\hline 24 & Mill Cr at Delaney Rd nr Turner, OR & 445037122573800 & Agriculture & II \\
\hline 25 & South Yamhill R at McMinnville, OR & 14194150 & Agriculture & II \\
\hline 26 & North Yamhill R at Hwy 99W nr McMinnville, OR & 451355123093600 & Agriculture & II \\
\hline 27 & Palmer Cr at Dayton, OR & 451309123041501 & Agriculture & $\mathrm{I}$ \\
\hline 28 & Yamhill R at Dayton, OR & 451320123041100 & Agriculture & I \\
\hline 29 & Willamette R nr Newberg, OR & 451705122575100 & Integrator & I \\
\hline 30 & Willamette R at Hwy 219 nr Newberg, OR & 451602122564400 & Integrator & II \\
\hline 31 & Champoeg Cr bl Mission Cr nr Butteville, OR & 451502122524700 & Agriculture & II \\
\hline 32 & Zollner Cr nr Mt Angel, OR & 14201300 & Agriculture & both \\
\hline 33 & Pudding R at Aurora, OR & 14202000 & Agriculture & both \\
\hline 34 & Molalla R at Knights Bridge nr Canby, OR & 451603122423301 & Integrator & II \\
\hline 35 & Dairy $\mathrm{Cr}$ at Rte $8 \mathrm{nr}$ Hillsboro, OR & 14206200 & Agriculture & II \\
\hline 36 & Beaverton $\mathrm{Cr}$ at Beaverton, $\mathrm{OR}$ & 452950122492900 & Urban & I \\
\hline 37 & Bronson $\mathrm{Cr}$ at 185 th Ave $\mathrm{nr}$ Aloha, OR & 14206298 & Urban & II \\
\hline 38 & Beaverton Cr nr Orenco, OR & 453115122535500 & Urban-industrial & I \\
\hline 39 & Fanno $\mathrm{Cr}$ at Durham, OR & 14206950 & Urban & both \\
\hline 40 & Tualatin R at West Linn, OR & 14207500 & Integrator & II \\
\hline 41 & Clackamas R at Oregon City, OR & 452221122362400 & Integrator & I \\
\hline 42 & Johnson $\mathrm{Cr}$ at Palmblad Rd nr Gresham, OR & 452823122240900 & Agriculture & II \\
\hline 43 & Johnson Cr at Hogan Rd nr Gresham, OR & 452847122244500 & Agriculture & I \\
\hline 44 & Johnson $\mathrm{Cr}$ at Milwaukie, OR & 14211550 & Urban & I \\
\hline 45 & $\begin{array}{l}\text { Commercial-Residential runoff at Harbor Way } \\
\text { at Portland, OR }\end{array}$ & 453043122402200 & Urban & II \\
\hline 46 & Willamette R at Portland Harbor, OR & 14211720 & Integrator & II \\
\hline 47 & Interstate 84 Trans Corridor at SE 3rd at Portland, OR & 453154122394200 & Urban & II \\
\hline 48 & Willamette R at St Johns Bridge at Portland, OR & 14211805 & Integrator & II \\
\hline 49 & Willamette $\mathrm{R}$ at Linnton, OR & 453547122463000 & Integrator & I \\
\hline 50 & Beaver Cr nr Troutdale, OR (Sandy River Basin) & 453205122223701 & Integrator & $\mathrm{I}$ \\
\hline
\end{tabular}




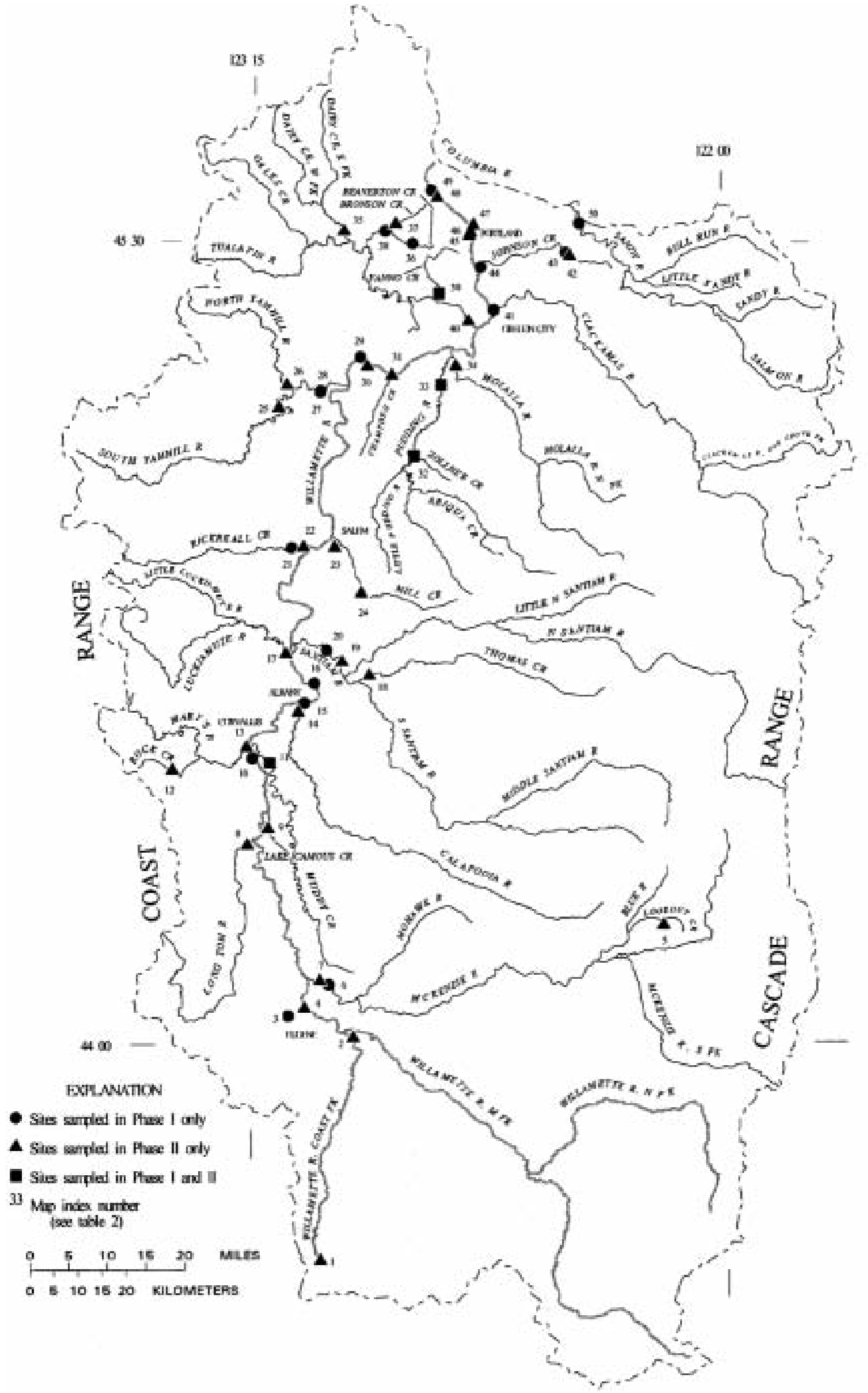

Figure 2. Sampling sites from Phases I and II of the Willamette River Basin Water Quality Study, Oregon, 1992-94 
Table 3. Sampling plan for Phase I of the Willamette River Basin Water Quality Study, Oregon 1992-93

[Sites are listed in downstream order by land use TYPE (A, agricultural, I, integrator, U, urban-industrial) and can be located in figure 2 by Map index number; Misc, miscellaneous analyses, including flow, water temperature, specific conductance, $\mathrm{pH}$, dissolved oxygen, suspended sediment, and dissolved and suspended organic carbon; WW, whole water; FW, filtered water; SS, suspended sediment; BED, bed sediment; Acids, organic acids; VOC, volatile organic compounds; GCMS, analysis of filtered water by gas chromatography and mass spectroscopy; HPLC, analysis of filtered water by high performance liquid chromatography; Nutr, nutrients in whole and filtered water; OC/OP, organochlorine and organophosphorus compounds; PCDD/PCDF, dioxin and furan compounds; refer to Appendix for list of specific constituents]

\begin{tabular}{|c|c|c|c|c|c|c|c|c|c|c|c|c|c|c|c|c|c|c|c|c|c|c|c|c|}
\hline \multirow{3}{*}{$\begin{array}{c}\text { Map } \\
\text { index } \\
\text { number }\end{array}$} & \multirow{3}{*}{ Station name } & \multirow{3}{*}{ Station number } & \multirow{3}{*}{$\begin{array}{l}\text { Site } \\
\text { type }\end{array}$} & \multirow{3}{*}{ Date } & \multirow{3}{*}{ Misc } & \multirow{3}{*}{ Nutr } & \multirow{2}{*}{\multicolumn{4}{|c|}{ Trace elements }} & \multicolumn{14}{|c|}{ Organic compounds } \\
\hline & & & & & & & & & & & \multicolumn{4}{|c|}{$\mathrm{OC} / \mathrm{OP}$} & \multicolumn{4}{|c|}{ SEMI-VOLATILE } & \multicolumn{2}{|c|}{ PESTICIDES } & \multirow{2}{*}{$\frac{\text { VOC }}{\text { WW }}$} & \multirow{2}{*}{$\frac{\text { Acids }}{\text { WW }}$} & \multicolumn{2}{|c|}{ PCDD/PCDF } \\
\hline & & & & & & & WW & FW & SS & BED & wW & FW & SS & BED & WW & FW & SS & BED & GCMS & HPLC & & & SS & BED \\
\hline \multirow[t]{4}{*}{3} & A-3 Channel at Wallis and $5^{\text {th }} \mathrm{St}$ & 440313123091100 & $\mathrm{U}$ & 920416 & $\mathrm{X}$ & & & & & & & $\mathrm{X}$ & $\mathrm{X}$ & & & $\mathrm{X}$ & $\mathrm{X}$ & & $\mathrm{X}$ & & $\mathrm{X}$ & $\mathrm{X}$ & $\mathrm{X}$ & \\
\hline & at Eugene, OR & & & 920825 & & & & & & $\mathrm{X}$ & & & & $\mathrm{X}$ & & & & $\mathrm{X}$ & & & & & & $\mathrm{X}$ \\
\hline & & & & 921016 & $\mathrm{X}$ & & $\mathrm{X}$ & $\mathrm{X}$ & & & & $\mathrm{X}$ & $\mathrm{X}$ & & & $\mathrm{x}$ & $\mathrm{x}$ & & $\mathrm{x}$ & & $\mathrm{x}$ & $\mathrm{X}$ & & \\
\hline & & & & 921029 & $\mathrm{X}$ & & $\mathrm{X}$ & $\mathrm{X}$ & $\mathrm{x}$ & & & & & & & & & & $\mathrm{x}$ & & & & & \\
\hline 16 & Middle Fourth Lake nr Albany, OR & 444032123050600 & $\mathrm{U}$ & 920901 & & & & & & $\mathrm{X}$ & & & & $\mathrm{X}$ & & & & $\mathrm{X}$ & & & & & & $\mathrm{X}$ \\
\hline 36 & Beaverton $\mathrm{Cr}$ at Beaverton, OR & 452950122492900 & $\mathrm{U}$ & 920823 & & & & & $\mathrm{x}$ & $\mathrm{x}$ & & & & $\mathrm{x}$ & & & & $\mathrm{x}$ & & & & & & $\mathrm{X}$ \\
\hline 38 & Beaverton $\mathrm{Cr}$ nr Orenco, OR & 453115122535500 & $\mathrm{U}$ & 930331 & $\mathrm{X}$ & & $\mathrm{X}$ & $\mathrm{X}$ & & & & $\mathrm{X}$ & $\mathrm{X}$ & & $\mathrm{X}$ & $\mathrm{X}$ & $\mathrm{X}$ & & $\mathrm{X}$ & $\mathrm{X}$ & $\mathrm{x}$ & & & \\
\hline \multirow[t]{2}{*}{39} & Fanno $\mathrm{Cr}$ at Durham, OR & 14206950 & $\mathrm{U}$ & 930301 & $\mathrm{X}$ & & $\mathrm{X}$ & $\mathrm{x}$ & $\mathrm{X}$ & & & $\mathrm{x}$ & $\mathrm{X}$ & & & $\mathrm{x}$ & $\mathrm{x}$ & & $\mathrm{x}$ & & $\mathrm{x}$ & & & \\
\hline & & & & 930722 & $\mathrm{X}$ & $\mathrm{X}$ & $\mathrm{X}$ & $\mathrm{X}$ & & & $\mathrm{X}$ & & & & $\mathrm{X}$ & & & & $\mathrm{x}$ & $\mathrm{X}$ & $\mathrm{x}$ & & & \\
\hline 44 & Johnson $\mathrm{Cr}$ at Milwaukie, OR & 14211550 & $\mathrm{U}$ & 930314 & $\mathrm{X}$ & & $\mathrm{X}$ & $\mathrm{X}$ & $\mathrm{X}$ & & & $\mathrm{X}$ & $\mathrm{X}$ & & & $\mathrm{x}$ & $\mathrm{X}$ & & $\mathrm{x}$ & & $\mathrm{x}$ & & & \\
\hline 6 & McKenzie R nr Coburg, OR & 14165500 & I & 920825 & & & & & & $\mathrm{X}$ & & & & $\mathrm{x}$ & & & & $\mathrm{X}$ & & & & & & \\
\hline \multirow[t]{2}{*}{10} & Willamette $\mathrm{R}$ nr Corvallis, OR & 443207123145500 & I & 930824 & $\mathrm{X}$ & $\mathrm{x}$ & $\mathrm{X}$ & $\mathrm{X}$ & & & $\mathrm{X}$ & & & & $\mathrm{X}$ & & & & $\mathrm{x}$ & $\mathrm{x}$ & $\mathrm{x}$ & & & \\
\hline & & & & 920826 & & & & & & $\mathrm{x}$ & & & & $\mathrm{X}$ & & & & $\mathrm{x}$ & & & & & & $\mathrm{X}$ \\
\hline 20 & Santiam R nr Jefferson, OR & 444416123030800 & I & 920829 & & & & & & $\mathrm{x}$ & & & & $\mathrm{X}$ & & & & $\mathrm{X}$ & & & & & & \\
\hline 29 & Willamette $R$ at Newberg, OR & 451705122575100 & I & 920901 & & & & & & $\mathrm{x}$ & & & & $\mathrm{X}$ & & & & $\mathrm{x}$ & & & & & & $\mathrm{X}$ \\
\hline 41 & Clackamas R nr Oregon City, OR & 452221122362400 & I & 920824 & & & & & & $\mathrm{X}$ & & & & $\mathrm{x}$ & & & & $\mathrm{X}$ & & & & & & \\
\hline \multirow[t]{3}{*}{49} & Willamette $\mathrm{R}$ at Linnton, OR & 453547122463000 & I & 920418 & $\mathrm{x}$ & & $\mathrm{X}$ & $\mathrm{X}$ & $\mathrm{x}$ & & & & & & & & & & & & & & & \\
\hline & & & & 920909 & & & & & & $\mathrm{x}$ & & & & $\mathrm{x}$ & & & & $\mathrm{x}$ & & & & & & $\mathrm{x}$ \\
\hline & & & & 921026 & $\mathrm{X}$ & & $\mathrm{x}$ & $\mathrm{x}$ & & & & & $\mathrm{x}$ & & & $\mathrm{x}$ & $\mathrm{x}$ & & $\mathrm{x}$ & & $\mathrm{x}$ & $\mathrm{x}$ & & \\
\hline 50 & Beaver $\mathrm{Cr}$ nr Troutdale, OR & 453225122223701 & I & 920822 & & & & & & $\mathrm{x}$ & & & & $\mathrm{x}$ & & & & $\mathrm{x}$ & & & & & & $\mathrm{X}$ \\
\hline 11 & Muddy Cr nr Peoria, OR & 443138123120901 & A & 930824 & $\mathrm{X}$ & $\mathrm{x}$ & $\mathrm{X}$ & $\mathrm{X}$ & & & $\mathrm{X}$ & & & & $\mathrm{X}$ & & & & $\mathrm{x}$ & $\mathrm{X}$ & $\mathrm{x}$ & & & \\
\hline 15 & Calapooia $\mathrm{R}$ at mouth nr Albany, OR & 443819123064000 & A & 920826 & & & & & & $\mathrm{X}$ & & & & $\mathrm{X}$ & & & & $\mathrm{X}$ & & & & & & \\
\hline 21 & Rickreall Cr nr Rickerall, OR & 445543123084400 & A & 920828 & & & & & & $\mathrm{x}$ & & & & $\mathrm{x}$ & & & & $\mathrm{x}$ & & & & & & \\
\hline 27 & Palmer $\mathrm{Cr}$ at Dayton, OR & 451309123041501 & A & 930907 & $\mathrm{X}$ & $\mathrm{x}$ & $\mathrm{X}$ & $\mathrm{x}$ & & & $\mathrm{X}$ & & & & $\mathrm{X}$ & & & & $\mathrm{x}$ & $\mathrm{x}$ & $\mathrm{x}$ & & & \\
\hline \multirow[t]{2}{*}{28} & Yamhill $\mathrm{R}$ at Dayton, OR & 451320123041100 & A & 920827 & & & & & & $\mathrm{X}$ & & & & $\mathrm{X}$ & & & & $\mathrm{X}$ & & & & & & $\mathrm{X}$ \\
\hline & & & & 930907 & $\mathrm{X}$ & $\mathrm{X}$ & & & & & $\mathrm{X}$ & & & & & & & & $\mathrm{X}$ & $\mathrm{X}$ & & & & \\
\hline 32 & Zollner Cr nr Mt Angel, OR & 14201300 & A & 930601 & $\mathrm{x}$ & & $\mathrm{X}$ & $\mathrm{X}$ & $\mathrm{X}$ & & & $\mathrm{X}$ & $\mathrm{X}$ & & & & $\mathrm{X}$ & & $\mathrm{x}$ & $\mathrm{x}$ & $\mathrm{x}$ & & & \\
\hline & & & & 930727 & $\mathrm{x}$ & $\mathrm{X}$ & $\mathrm{x}$ & $\mathrm{x}$ & & & $\mathrm{X}$ & & & & $\mathrm{X}$ & & & & $\mathrm{x}$ & $\mathrm{x}$ & $\mathrm{x}$ & & & \\
\hline 33 & Pudding $\mathrm{R}$ at Aurora, OR & 14202000 & A & 930427 & $\mathrm{X}$ & & $\mathrm{X}$ & $\mathrm{X}$ & $\mathrm{x}$ & & & $\mathrm{X}$ & $\mathrm{X}$ & & & $\mathrm{x}$ & $\mathrm{x}$ & & $\mathrm{x}$ & $\mathrm{X}$ & $\mathrm{x}$ & & & \\
\hline 43 & Johnson $\mathrm{Cr}$ at Hogan Rd nr Gresham, OR & 452847122244500 & A & 920821 & & & & & & $\mathrm{X}$ & & & & $\mathrm{X}$ & & & & $\mathrm{X}$ & & & & & & \\
\hline
\end{tabular}


Table 4. Sampling plan for Phase II of the Willamette River Basin Water Quality Study, Oregon, Spring 1994

[Sites are listed in downstream order by land use TYPE (A, agricultural, F, forested, I, integrator, U, urban-industrial) and can be located in figure 2 by Map index number; Misc, miscellaneous analyses, including flow, water temperature, specific conductance, $\mathrm{pH}$, dissolved oxygen, suspended sediment, and dissolved and suspended organic carbon; Nutr includes minimum of nitrite plus nitrate in filtered water and total phosphorus; Organics, pesticides in filtered water by solid phase extraction and gas chromatography/mass spectroscopy or high performance liquid chromatography; Triazines, low-level triazine herbicides in filtered water; OC-WW, organochlorine compounds in whole water; VOC, volatile organic compounds; TE-WW, total recoverable trace elements in whole water and hardness; TE-FW, trace elements in filtered water; refer to Appendix for list of specific constituents]

\begin{tabular}{|c|c|c|c|c|c|c|c|c|c|c|c|c|}
\hline $\begin{array}{c}\text { Map } \\
\text { index } \\
\text { number }\end{array}$ & Station name & Station number & $\begin{array}{l}\text { Site } \\
\text { type }\end{array}$ & Date & Misc & Nutr & TE-WW & TE-FW & Organics & Triazines & OC-WW & VOC \\
\hline 4 & Urban Outfall at Polk St Park at Eugene, OR & 440402123063900 & $\mathrm{U}$ & 940613 & $\mathrm{X}$ & $\mathrm{X}$ & & $\mathrm{X}$ & $\mathrm{X}$ & $\mathrm{X}$ & $\mathrm{X}$ & $\mathrm{X}$ \\
\hline 37 & Bronson $\mathrm{Cr}$ at $185^{\text {th }}$ Ave nr Aloha, OR & 14206298 & $\mathrm{U}$ & 940527 & $\mathrm{X}$ & $\mathrm{X}$ & $\mathrm{X}$ & & $\mathrm{X}$ & $\mathrm{X}$ & $\mathrm{X}$ & \\
\hline 45 & Urban runoff at Harbor Way at Portland, OR & 453043122402200 & $\mathrm{U}$ & 940617 & $\mathrm{X}$ & $\mathrm{X}$ & $\mathrm{X}$ & $\mathrm{X}$ & $\mathrm{X}$ & $\mathrm{X}$ & $X$ & \\
\hline 47 & Interstate 84 runoff at Portland, OR & 453154122394200 & $\mathrm{U}$ & 940531 & $\mathrm{X}$ & $\mathrm{X}$ & $\mathrm{X}$ & $x$ & $\mathrm{X}$ & $\mathrm{X}$ & $\mathrm{X}$ & $\mathrm{X}$ \\
\hline 1 & CF Willamette R bl Big R nr London, OR & 433548123040600 & $\mathrm{~F}$ & 940520 & $\mathrm{X}$ & $\mathrm{X}$ & $\mathrm{X}$ & & $\mathrm{X}$ & $\mathrm{X}$ & $\mathrm{X}$ & \\
\hline 5 & Mack Cr nr Blue River, OR & 441310122095801 & $\mathrm{~F}$ & 940527 & $\mathrm{X}$ & $\mathrm{X}$ & & & $\mathrm{X}$ & $\mathrm{X}$ & $\mathrm{X}$ & \\
\hline 12 & Rock Cr ab Grifffith Cr nr Philomath, OR & 443045123273000 & $\mathrm{~F}$ & 940518 & $\mathrm{X}$ & $\mathrm{X}$ & & & $\mathrm{X}$ & $\mathrm{X}$ & $\mathrm{X}$ & \\
\hline 2 & CF Willamette R at Seavey Loop Rd nr Eugene, OR & 440045122585600 & I & 940519 & $\mathrm{X}$ & $\mathrm{X}$ & $\mathrm{X}$ & & $\mathrm{X}$ & $\mathrm{X}$ & $\mathrm{X}$ & \\
\hline 7 & McKenzie R nr Eugene, OR & 440707123041300 & I & 940519 & $\mathrm{X}$ & $X$ & & & $\mathrm{X}$ & $\mathrm{X}$ & $\mathrm{X}$ & \\
\hline 13 & Marys $\mathrm{R}$ at Corvallis, OR & 443321123155201 & I & 940518 & $\mathrm{X}$ & $\mathrm{X}$ & $\mathrm{X}$ & & $\mathrm{X}$ & $\mathrm{X}$ & $\mathrm{X}$ & \\
\hline 17 & Luckiamute R nr Buena Vista, OR & 444349123094000 & I & 940516 & $\mathrm{X}$ & $\mathrm{X}$ & & & $\mathrm{X}$ & $\mathrm{X}$ & $\mathrm{X}$ & \\
\hline 19 & Santiam R at Jefferson, OR & 14189000 & I & 940517 & $\mathrm{X}$ & $\mathrm{X}$ & & & $\mathrm{X}$ & $\mathrm{X}$ & $\mathrm{X}$ & \\
\hline 30 & Willamette R at Hwy $219 \mathrm{nr}$ Newberg, OR & 451602122564400 & I & 940531 & $\mathrm{X}$ & $\mathrm{X}$ & $\mathrm{X}$ & $\mathrm{X}$ & $\mathrm{X}$ & $\mathrm{X}$ & $\mathrm{X}$ & \\
\hline 34 & Molalla R nr Canby, OR & 451603122423301 & I & 940525 & $\mathrm{X}$ & $\mathrm{X}$ & & & $\mathrm{X}$ & $\mathrm{X}$ & $\mathrm{X}$ & \\
\hline 40 & Tualatin R at West Linn, OR & 14207500 & I & 940525 & $\mathrm{X}$ & $\mathrm{X}$ & $\mathrm{X}$ & & $\mathrm{X}$ & $\mathrm{X}$ & $\mathrm{X}$ & \\
\hline 48 & Willamette R ab St. John's Bridge at Portland, OR & 14211805 & I & 940523 & $\mathrm{X}$ & $\mathrm{X}$ & $\mathrm{X}$ & & $\mathrm{X}$ & $\mathrm{X}$ & $\mathrm{X}$ & \\
\hline 9 & Lake Camous Cr at Pine Grove Dr nr Harrisburg, OR & 442413123122500 & A & 940519 & $\mathrm{X}$ & $\mathrm{X}$ & & & $\mathrm{X}$ & & $\mathrm{X}$ & \\
\hline 18 & Thomas Cr at Kelly Rd nr Jefferson, OR & 444123122562200 & A & 940516 & $\mathrm{X}$ & $\mathrm{X}$ & & & $\mathrm{X}$ & $\mathrm{X}$ & $\mathrm{X}$ & \\
\hline 22 & Rickreall Cr nr mouth nr Salem, OR & 445547123065400 & A & 940526 & $\mathrm{X}$ & $\mathrm{X}$ & & & $\mathrm{X}$ & $\mathrm{X}$ & $\mathrm{X}$ & \\
\hline 24 & Mill $\mathrm{Cr}$ at Delaney Rd nr Turner, OR & 445037122573800 & A & 940614 & $\mathrm{X}$ & $\mathrm{X}$ & $\mathrm{X}$ & & $\mathrm{X}$ & $\mathrm{X}$ & $\mathrm{X}$ & \\
\hline 25 & S. Yamhill R at McMinneville, OR & 14194150 & A & 940517 & $\mathrm{X}$ & $X$ & $\mathrm{X}$ & & $X$ & $\mathrm{X}$ & $X$ & \\
\hline 26 & N. Yamhill R nr McMinneville, OR & 451355123093600 & A & 940517 & $\mathrm{X}$ & $X$ & & & $X$ & $\mathrm{X}$ & $X$ & \\
\hline 31 & Champoeg Cr bl Mission Cr nr Butteville, OR & 451502122524701 & A & 940526 & $\mathrm{X}$ & $\mathrm{X}$ & & & $\mathrm{X}$ & $\mathrm{X}$ & $\mathrm{X}$ & \\
\hline 33 & Pudding R at Aurora, OR & 14202000 & A & 940412 & $\mathrm{X}$ & $\mathrm{X}$ & & & $\mathrm{X}$ & & & \\
\hline 35 & Dairy $\mathrm{Cr}$ at Rte $8 \mathrm{nr}$ Hillsboro, OR & 14206200 & A & 940527 & $\mathrm{X}$ & $\mathrm{X}$ & & & $\mathrm{X}$ & $\mathrm{X}$ & $\mathrm{X}$ & \\
\hline 42 & Johnson $\mathrm{Cr}$ at Palmblad Rd nr Gresham, OR & 452823122240900 & A & 940524 & $\mathrm{X}$ & $\mathrm{X}$ & & & $\mathrm{X}$ & $\mathrm{X}$ & $X$ & \\
\hline
\end{tabular}


Table 5. Sampling plan for Phase II of the Willamette River Basin Water Quality Study, Oregon, Fall 1994

[Sites are listed in downstream order by land use TYPE (A, agricultural, I, integrator, U, urban-industrial) and can be located in figure 2 by Map index number; Misc, miscellaneous analyses, including flow, water temperature, specific conductance, $\mathrm{pH}$, dissolved oxygen, suspended sediment, and dissolved- and suspended-organic carbon; Nutr includes minimum of nitrite plus nitrate in filtered water and total phosphorus; Organics, pesticides in filtered water by solid phase extraction and gas chromatography/mass spectroscopy or high performance liquid chromatography; Triazines, low-level triazine herbicides in filtered water; OC-WW, organochlorine compounds in whole water; OC-FW, organochlorine compounds in filtered water; OC-SS, organochlorine compounds in suspended sediment; VOC, volatile organic compounds; TE-WW, total recoverable trace elements in whole water and hardness; TE-FW, trace elements in filtered water; refer to Appendix for list of specific constituents]

\begin{tabular}{|c|c|c|c|c|c|c|c|c|c|c|c|c|c|c|}
\hline $\begin{array}{c}\text { Map } \\
\text { index } \\
\text { number }\end{array}$ & Station name & Station number & $\begin{array}{l}\text { Site } \\
\text { type }\end{array}$ & Date & Misc & Nutr & TE-WW & TE-FW & Organics & Triazines & OC-WW & OC-FW & OC-SS & VOC \\
\hline \multirow{3}{*}{23} & Pringle $\mathrm{Cr}$ at Bush Park at Salem, OR & 14190970 & $\mathrm{U}$ & 941123 & $\mathrm{X}$ & $\mathrm{X}$ & $\mathrm{X}$ & $\mathrm{X}$ & $\mathrm{X}$ & $\mathrm{X}$ & $\mathrm{X}$ & & & \\
\hline & & & & 941130 & $\mathrm{X}$ & $\mathrm{X}$ & $\mathrm{X}$ & & $\mathrm{x}$ & $\mathrm{X}$ & $\mathrm{x}$ & & & \\
\hline & & & & 941130 & $\mathrm{X}$ & $\mathrm{X}$ & $\mathrm{X}$ & & $\mathrm{X}$ & $\mathrm{X}$ & $\mathrm{X}$ & & & \\
\hline 37 & Bronson $\mathrm{Cr}$ at $185^{\text {th }}$ Ave nr Aloha, OR & 14206298 & $\mathrm{U}$ & 941123 & $\mathrm{x}$ & $\mathrm{x}$ & $\mathrm{X}$ & $\mathrm{x}$ & $\mathrm{x}$ & $\mathrm{X}$ & $\mathrm{x}$ & & & $\mathrm{X}$ \\
\hline 39 & Fanno Cr at Durham, OR. & 14206950 & $\mathrm{U}$ & 941027 & $\mathrm{X}$ & $\mathrm{X}$ & $\mathrm{X}$ & & $\mathrm{X}$ & $\mathrm{X}$ & $\mathrm{X}$ & & & \\
\hline \multirow[t]{5}{*}{47} & Interstate 84 runoff at Portland, OR & 453154122394200 & $\mathrm{U}$ & 941108 & $\mathrm{x}$ & $\mathrm{x}$ & $\mathrm{X}$ & $\mathrm{X}$ & $\mathrm{X}$ & $\mathrm{X}$ & $\mathrm{x}$ & $\mathrm{X}$ & $\mathrm{X}$ & $\mathrm{X}$ \\
\hline & & & & 941108 & $\mathrm{x}$ & $\mathrm{x}$ & $\mathrm{x}$ & & $\mathrm{x}$ & $\mathrm{X}$ & $\mathrm{X}$ & & & \\
\hline & & & & 941130 & $\mathrm{x}$ & $\mathrm{x}$ & $\mathrm{X}$ & & $\mathrm{x}$ & $\mathrm{X}$ & $\mathrm{X}$ & & & $\mathrm{X}$ \\
\hline & & & & 941130 & $\mathrm{X}$ & $\mathrm{X}$ & $\mathrm{X}$ & & $\mathrm{X}$ & $\mathrm{X}$ & $\mathrm{X}$ & & & \\
\hline & & & & 941202 & $\mathrm{X}$ & $\mathrm{X}$ & $\mathrm{X}$ & & $\mathrm{X}$ & $\mathrm{X}$ & $\mathrm{X}$ & & & \\
\hline 40 & Tualatin $\mathrm{R}$ at West Linn, OR & 14207500 & I & 941028 & $\mathrm{X}$ & $\mathrm{X}$ & $\mathrm{X}$ & & $\mathrm{X}$ & $\mathrm{X}$ & $\mathrm{X}$ & & & \\
\hline \multirow[t]{3}{*}{46} & Willamette $\mathrm{R}$ at Portland harbor, OR & 14211720 & I & 941025 & $\mathrm{x}$ & $\mathrm{x}$ & & $\mathrm{x}$ & $\mathrm{x}$ & & & & & \\
\hline & & & & 941029 & $\mathrm{X}$ & $\mathrm{X}$ & $\mathrm{X}$ & $\mathrm{X}$ & $\mathrm{X}$ & $\mathrm{X}$ & $\mathrm{X}$ & $\mathrm{X}$ & $\mathrm{X}$ & \\
\hline & & & & 941103 & $\mathrm{x}$ & $\mathrm{x}$ & & $\mathrm{x}$ & $\mathrm{x}$ & $\mathrm{X}$ & $\mathrm{X}$ & & & \\
\hline 8 & Long Tom $\mathrm{R}$ at Bundy Bridge $\mathrm{nr}$ Monroe, OR & 442223123153703 & A & 941101 & $\mathrm{X}$ & $\mathrm{X}$ & & & $\mathrm{x}$ & $\mathrm{X}$ & $\mathrm{X}$ & & & \\
\hline \multirow[t]{3}{*}{11} & Muddy Cr nr Peoria, OR & 443138123120901 & A & 941102 & $\mathrm{x}$ & $\mathrm{x}$ & & & $\mathrm{x}$ & $\mathrm{X}$ & $\mathrm{X}$ & $\mathrm{X}$ & $\mathrm{x}$ & \\
\hline & & & & 941106 & $\mathrm{x}$ & $\mathrm{x}$ & & & $\mathrm{x}$ & $\mathrm{X}$ & $\mathrm{X}$ & $\mathrm{X}$ & $\mathrm{X}$ & \\
\hline & & & & 941109 & $\mathrm{x}$ & $\mathrm{X}$ & & & $\mathrm{X}$ & $\mathrm{X}$ & $\mathrm{X}$ & & & \\
\hline 14 & Calapooia $\mathrm{R}$ at Albany, $\mathrm{OR}$ & 14173500 & A & 941101 & $\mathrm{X}$ & $\mathrm{X}$ & & & $\mathrm{X}$ & $\mathrm{X}$ & $\mathrm{X}$ & & & \\
\hline \multirow[t]{3}{*}{24} & Mill Cr at Delaney Rd nr Turner, OR & 445037122573800 & A & 941031 & $\mathrm{x}$ & $\mathrm{x}$ & & & $\mathrm{x}$ & $\mathrm{X}$ & $\mathrm{x}$ & & & \\
\hline & & & & 941104 & $\mathrm{X}$ & $\mathrm{x}$ & & & $\mathrm{x}$ & $\mathrm{X}$ & $\mathrm{X}$ & $\mathrm{X}$ & $\mathrm{X}$ & \\
\hline & & & & 941104 & $\mathrm{X}$ & $\mathrm{x}$ & & & $\mathrm{X}$ & $\mathrm{X}$ & $\mathrm{X}$ & & & \\
\hline 25 & S. Yamhill R at McMinneville, OR & 14194150 & A & 941102 & $\mathrm{X}$ & $\mathrm{x}$ & & & $\mathrm{x}$ & $\mathrm{X}$ & $\mathrm{X}$ & & & \\
\hline 31 & Champoeg Cr bl Mission Cr nr Butteville, OR & 451502122524700 & A & 941031 & $\mathrm{X}$ & $\mathrm{x}$ & & & $\mathrm{X}$ & $\mathrm{X}$ & $\mathrm{X}$ & & & \\
\hline 32 & Zollner Cr nr Mount Angel, OR & 14201300 & A & 941028 & $\mathrm{X}$ & $\mathrm{x}$ & & & $\mathrm{X}$ & $\mathrm{X}$ & $\mathrm{X}$ & & & \\
\hline \multirow[t]{3}{*}{33} & Pudding R at Aurora, OR & 14202000 & A & 941028 & $\mathrm{X}$ & $\mathrm{x}$ & & & $\mathrm{X}$ & $\mathrm{X}$ & $\mathrm{X}$ & $\mathrm{X}$ & $\mathrm{X}$ & \\
\hline & & & & 941029 & $\mathrm{X}$ & $\mathrm{X}$ & & & $\mathrm{X}$ & $\mathrm{X}$ & $\mathrm{X}$ & & & \\
\hline & & & & 941109 & $\mathrm{X}$ & $\mathrm{X}$ & & & $\mathrm{X}$ & $\mathrm{X}$ & $\mathrm{X}$ & & & \\
\hline 35 & Dairy $\mathrm{Cr}$ at Rte $8 \mathrm{nr}$ Hillsboro, OR & 14206200 & A & 941201 & $\mathrm{X}$ & $\mathrm{X}$ & $\mathrm{X}$ & & $\mathrm{X}$ & $\mathrm{X}$ & $\mathrm{X}$ & & & \\
\hline \multirow[t]{5}{*}{42} & Johnson $\mathrm{Cr}$ at Palmblad $\mathrm{Rd} \mathrm{nr}$ Gresham, OR & 452823122240900 & A & 941027 & $\mathrm{X}$ & & & & $\mathrm{X}$ & $\mathrm{X}$ & $\mathrm{X}$ & $\mathrm{X}$ & $\mathrm{X}$ & \\
\hline & & & & 941028 & $\mathrm{X}$ & $\mathrm{X}$ & & & $\mathrm{X}$ & $\mathrm{X}$ & $\mathrm{X}$ & $\mathrm{X}$ & $\mathrm{X}$ & \\
\hline & & & & 941101 & $\mathrm{X}$ & $\mathrm{x}$ & & & $\mathrm{x}$ & $\mathrm{X}$ & $\mathrm{X}$ & & & \\
\hline & & & & 941103 & $\mathrm{X}$ & $\mathrm{x}$ & $\mathrm{X}$ & & $\mathrm{X}$ & $\mathrm{X}$ & $\mathrm{X}$ & & & \\
\hline & & & & 941123 & $\mathrm{X}$ & $\mathrm{X}$ & & & $X$ & $X$ & $X$ & & & \\
\hline
\end{tabular}


then rinsed with copious amounts of hot tap water, dilute (5 percent) nitric (Phase I) or (5 percent) hydrochloric (Phase II) acid solution, and distilleddeionized water. Organic-sampling and processing equipment was washed in the same manner with the addition of a final reagent-grade methanol rinse. Organic-carbon-sample processing equipment was washed in the low-phosphate soap and tap water then rinsed with hot tap water, distilled-deionized water, and finally with organicfree blank water. Glass collection bottles were washed in low-phosphate soap and tap water, rinsed with hot tap water followed by distilleddeionized water, and baked for 8 hours at $325^{\circ} \mathrm{C}$. One-time-use polyethylene shipment bottles were rinsed with native water; bottles designated for trace-element samples were acid rinsed.

\section{Field Collection}

Field measurements of water temperature, $\mathrm{pH}$, specific conductance, and dissolved oxygen were made using Hydrolab multi-parameter probes. Data were collected near the center of flow at one-meter depth or less below the water surface (Phase I) or were averaged from a minimum of five verticals in the sampling cross section (Phase II). Hydrolabs were calibrated each day before and after sampling. Discharge measurements were made in accordance with standard USGS procedures (Rantz and others, 1982) or determined from established stage-discharge relationships. For urban outflows sampled through manholes, discharge was estimated.

With the exception of dissolved organic carbon (DOC), and volatile organic compounds (VOC), water samples were collected using a D-77 or a DH-81 depth-integrating sampler specially fitted with a Teflon 3-liter bottle, nozzle, and head assembly to minimize contamination. Water was collected from 5 equal-discharge interval (EDI) verticals in the cross section (Phase I) or from 10 verticals using the equal-width increment (EWI) method (Phase II). Both the EDI and EWI methods are described by Edwards and Glysson (1988).

Water for DOC analysis was collected in a baked glass bottle by grab sample at the centroid of flow. To minimize aeration, water for VOC analysis was collected at the centroid of flow by hand dipping 40-milliliter amber-glass vials (fitted with septum caps) below the water surface. VOC samples were unpreserved (Phase I) or preserved in the field with 2 drops of concentrated VOC-free hydrochloric acid (Phase II).

Sampling for trace elements was conducted using clean procedures as outlined by Horowitz and others (1994), and these samples were collected concurrently with organic samples. Bedsediment samples were collected with an Ekman dredge or Teflon scoop at 10 depositional areas at each site. Protocols for bed sampling are described by Shelton and Capel (1994).

During Phase I, water for analysis of organic compounds was composited into 10-gallon stainless steel milk containers, and water for analysis of suspended sediment was composited into polyethylene churn splitters. During Phase II, water for organic compounds, suspended organic carbon (SOC), and sediment analyses was split from the 3-liter Teflon collection bottles into 1-liter baked amber-glass bottles using a Teflon cone splitter encased in a clean chamber. Water samples for trace element and nutrient analyses were composited and transported in 10-liter polycarbonate carboys (Phase I) or into a churn splitter (Phase II) previously soaked in high-purity hydrochloric acid (5 percent by volume) as described by Horowitz and others (1994).

\section{Sample Processing}

Except during processing, all samples were kept chilled from the time of collection until analysis. Processing of field samples, including filtration and preservation, was accomplished at the USGS Oregon District laboratory in Portland.

Samples for analysis of organic constituents in filtered water were filtered through 0.7 micrometer pore size baked, glass-fiber filters. Samples for analysis of pesticides by gas chromatography/mass spectroscopy (GC/MS) or high performance liquid chromatography (HPLC) (USGS schedules 2010 and 2051, respectively) were further processed using solid-phase extraction (SPE) techniques (Sandstrom, 1989; Werner, S.L., Burkhardt, M.R., and deRusseau, S.N., USGS National Water Quality Laboratory, unpub. data, 1994). Samples for analysis of triazine compounds 
in filtered water were shipped in 1-liter baked amber-glass bottles.

During Phase I, organic compounds associated with suspended sediment were dewatered by filtration through a baked 1micrometer pore size, 293-millimeter diameter, glass-fiber filter and analyzed on the filter media as the suspended phase; the resulting filtrate was analyzed as the dissolved phase. A representative sample of the whole water was analyzed as the total recoverable sample. One hundred- to 200-liter water samples were generally required to obtain a sufficient sediment mass for analysis. Over 350 liters of water were required to analyze polychlorinated dibenzo-p-dioxin (PCDD) and polychlorinated dibenzofuran (PCDF) in suspended sediment.

Samples for organochlorine (OC) compounds in whole water were shipped in 1-liter baked amber-glass bottles without filtration.

Samples for analysis of OC associated with filtered water were filtered through baked 0.7-micrometer pore size, glass-fiber filters and shipped in 1-liter baked amber-glass bottles. Samples for analysis of OC in suspended sediment were collected on a baked 0.7-micrometer pore size, glass-fiber filters. The volume of water filtered was noted and the filters were wrapped in baked aluminum foil for shipment.

SOC samples were filtered through a 0.45 micrometer pore size, silver filter in a stainless steel column pressurized with water-pumped nitrogen gas, and the volume of sample, usually 10 to 25 milliliters, was noted. DOC samples were collected from the same column into 125-milliliter, baked amber glass bottles.

During Phase I, trace elements associated with suspended sediment were dewatered by centrifugation; centrifugation time was based on Stokes' Law and computed as the time under centrifugal acceleration to settle all suspended particles greater than 0.45 micrometers in diameter. Fifty- to 100-liter water samples generally were required to obtain sufficient sediment mass for analysis. The resulting supernatant was preserved with 0.5 milliliters of high-purity nitric acid per 250 milliliters of sample, and analyzed as the dissolved phase. A representative portion of the whole water sample was preserved with high-purity nitric acid and analyzed as the total recoverable sample.

In Phase II, trace elements and nutrients in filtered water were filtered through 0.45 micrometer pore size, capsule filters into polyethylene bottles using procedures outlined by Horowitz and others (1994). Both whole- and filtered-water trace-element samples were preserved with 0.5 milliliters of high-purity nitric acid per 250 milliliters of sample; nutrient samples were not preserved.

\section{Laboratory Analysis}

All chemical analyses were conducted at the USGS National Water Quality Laboratory (NWQL) in Arvada, Colorado, with the exception of PCDD and PCDF compounds associated with bed and suspended sediment and trace elements associated with suspended sediment. PCDD and PCDF compounds were analyzed by a USGSContract Laboratory, Quanterra, located in West Sacramento, California, using high resolution GC/ MS. Trace elements associated with suspended sediment were analyzed by the USGS Geologic Division Laboratory located in Denver, Colorado. Suspended-sediment-concentration analyses and the computation of percent finer than sand (less than 62-micrometer sieve diameter) were made by the USGS Sediment laboratory in Vancouver, Washington, as outlined by Guy (1969). Table 6 summarizes the analyses conducted and references the appropriate table(s) in this report where data are presented. Refer to Appendix for constituents analyzed, their U.S. Environmental Protection Agency STOrage and RETrieval (STORET) codes (if one exists), applicable minimum reporting level (MRL) or method detection limits (MDL), and reporting units.

The MRL is the smallest measured concentration of a constituent that may be reliably reported using a given analytical method (U.S. Environmental Protection Agency, 1992). The MDL is the minimum concentration of a substance that can be identified, measured, and reported in pure water with a 99-percent confidence that the analyte concentration is greater than zero (U.S. Environmental Protection Agency, 1992). The MDL is based on replicate spikes, above which 
Table 6. Summary of water-quality analyses conducted during Phases I and II of the Willamette River Basin Water Quality Study, Oregon, 1992-94

[USGS, U.S. Geological Survey; NWQL, USGS National Water Quality Laboratory, Arvada CO; GD, USGS Geologic Division laboratory, Denver, CO; CL, contract laboratory, Quanterra, West Sacramento, CA; OR, Oregon district laboratory, Portland, OR; WA, USGS sediment laboratory, Vancouver, WA; SPE, solid phase extraction, GC/MS, gas chromatography/mass spectroscopy; HPLC, high performance liquid chromatography;

EPA, U.S. Environmental Protection Agency]

\begin{tabular}{|c|c|c|c|c|c|}
\hline Constituent group & $\begin{array}{l}\text { Analysis } \\
\text { location }\end{array}$ & $\begin{array}{l}\text { Laboratory } \\
\text { schedule }\end{array}$ & $\begin{array}{l}\text { Phase } \\
\text { sampled }\end{array}$ & Reference or analytical method & $\begin{array}{l}\text { Table }(s) \text { in } \\
\text { this report }\end{array}$ \\
\hline Ancillary data & $\begin{array}{l}\text { NWQL, } \\
\text { OR, WA }\end{array}$ & -- & both & -- & 7,8 \\
\hline Nutrients in whole and filtered water & NWQL & 2702 & both & Fishman (1993) & 9,10 \\
\hline Trace elements in whole water & NWQL & -- & both & Fishman (1993) & 11,12 \\
\hline Trace elements in filtered water & NWQL & -- & both & Fishman (1993) & 13,14 \\
\hline Trace elements associated with suspended sediment & GD & -- & I & Arbogast (1990) & 15 \\
\hline Trace elements associated with bed sediment & NWQL & 2400 & I & Arbogast (1990) & 16 \\
\hline $\begin{array}{l}\text { Organochlorine and organophosphorus compounds in } \\
\text { whole water }\end{array}$ & NWQL & 1399 & I & Wershaw and others (1987) & 17 \\
\hline $\begin{array}{l}\text { Organochlorine and organophosphorus compounds in } \\
\text { filtered water }\end{array}$ & NWQL & 8307 & I & Wershaw and others (1987) & 19 \\
\hline $\begin{array}{l}\text { Organochlorine and organophosphorus compounds } \\
\text { associated with suspended sediment }\end{array}$ & NWQL & 8308 & I & Wershaw and others (1987) & 21 \\
\hline Organochlorine compounds associated with bed sediment & NWQL & 2501 & I & Foreman and others (1995) & 23 \\
\hline Organochlorine compounds in whole water & NWQL & 1398 & II & Wershaw and others (1987) & 18 \\
\hline Organochlorine compounds in filtered water & NWQL & 8369 & II & Wershaw and others (1987) & 20 \\
\hline $\begin{array}{l}\text { Organochlorine compounds associated with suspended } \\
\text { sediment }\end{array}$ & NWQL & 8370 & II & Wershaw and others (1987) & 22 \\
\hline Organic acids in whole water & NWQL & 8331 & I & Wershaw and others (1987) & 24 \\
\hline Semi-volatile compounds in whole water & NWQL & 1383 & I & Wershaw and others (1987) & 25 \\
\hline Semi-volatile compounds in filtered water & NWQL & 8005 & I & Wershaw and others (1987) & 26 \\
\hline $\begin{array}{l}\text { Semi-volatile compounds associated with suspended } \\
\text { sediment }\end{array}$ & NWQL & 8006 & I & Wershaw and others (1987) & 27 \\
\hline Semi-volatile compounds associated with bed sediment & NWQL & 2502 & I & $\begin{array}{l}\text { Furlong and others } \\
\text { (USGS, unpub. data, 1994) }\end{array}$ & 28 \\
\hline Volatile organic compounds in whole water & NWQL & 1392,1401 & I & Rose and Schroeder (1995) & 29 \\
\hline Volatile organic compounds in whole water & NWQL & 2090 & II & Rose and Schroeder (1995) & 30 \\
\hline $\begin{array}{l}\text { Polychlorinated dibenzo-p-dioxin associated with } \\
\text { suspended sediment }\end{array}$ & $\mathrm{CL}$ & -- & I & EPA method 8290 & 31 \\
\hline $\begin{array}{l}\text { Polychlorinated dibenzo-p-dioxin associated with bed } \\
\text { sediment }\end{array}$ & $\mathrm{CL}$ & -- & I & EPA method 8290 & 32 \\
\hline $\begin{array}{l}\text { Polychlorinated dibenzo-furans associated with } \\
\text { suspended sediment }\end{array}$ & $\mathrm{CL}$ & -- & I & EPA method 8290 & 33 \\
\hline $\begin{array}{l}\text { Polychlorinated dibenzo-furans associated with bed } \\
\text { sediment }\end{array}$ & $\mathrm{CL}$ & -- & I & EPA Method 8290 & 34 \\
\hline Pesticides in filtered water analyzed by SPE and GC/MS & NWQL & 2010 & both & Zaugg and others (in press) & 35,36 \\
\hline Pesticides in filtered water analyzed by SPE and HPLC & NWQL & 2051 & both & $\begin{array}{l}\text { Werner and others } \\
\text { (USGS, unpub. data, 1994) }\end{array}$ & 37,38 \\
\hline Triazines herbicides in filtered water & NWQL & 8015 & II & Sandstrom and others (1994) & 39 \\
\hline
\end{tabular}


reported detections for the particular constituent represent actual detections. Detections may be reported below the MDL; however, they may have a greater than 1-percent chance of representing false positive detections, that is, indicating that the analyte is present when it actually is not. The MRL is generally higher than the MDL because of unpredictable matrix effects for different waters. For PCDD and PCDF analyses the MRL varied with each sample and, in some cases, is reported as a range. For some bed sediment samples and all suspended sediment samples, the MRL was determined from the quality assurance method blanks.

At the NWQL, pesticide samples (USGS schedules 2010 and 2051) were extracted from solid-phase extraction cartridges with a solvent and analyzed by GC/MS (Zaugg and others, 1995) or HPLC (Werner, S.L.,Burkhardt, M.R., and deRusseau, S.N., USGS National Water Quality Laboratory, unpub. data, 1994). Triazine samples in filtered water were analyzed by GC/MS according to the method of Sandstrom and others (1994), modified to achieve lower MDL by the extraction of more water than originally specified for the method.

OC samples in whole water (USGS schedule 1398) were analyzed by GC according to method 0-3104-83 (Wershaw and others, 1987). OC samples in filtered water (USGS schedule 8369) were analyzed by GC according to method 0-110483, modified to achieve lower MDL by concentrating the final extract by a factor of 10 . OC samples associated with suspended-sediment (USGS schedule 8370) were analyzed by GC according to method 0-7104-83, modified to achieve lower MDL by filtering up to 4 liters of water to obtain sediment.

VOC samples were analyzed by purge and trap followed by GC/MS as described by Rose and Schroeder (1995). DOC and SOC samples were analyzed as described by Wershaw and others (1987). Analysis of OC and semi-volatile organic compounds in bed sediment were analyzed using GC/MS as described by Foreman and others (1995) and Furlong, E.T., Vaught, D.G., Merten, L.M., Foreman, W.T., and Gates, P.M. (USGS, unpub. data, 1994), respectively.

Analysis of trace elements associated with suspended and bed sediment were performed according to procedures described by Arbogast (1990). Filtered-water trace-element samples were analyzed by inductively coupled plasma and mass spectroscopy (ICP/MS) in a clean room designed to minimize metallic contamination. Samples for nutrients, hardness, and trace elements in whole water were analyzed according to Fishman (1993).

\section{QUALITY ASSURANCE}

To ensure the collection of representative samples from the water column, all water samples in this study were depth- and width- integrated using procedures outlined by Edwards and Glysson (1988). In addition, about 15 percent of the water samples were quality-control samples used to assess the accuracy and precision of the waterquality data collected in this study. Since Phase I sites and the constituents sampled were generally coincident with the ongoing Willamette Basin NAWQA study, which had its own quality-control sampling plan, no additional quality-control samples were taken. Phase I quality assurance data are not presented in this report. A combination of the following quality-control samples were collected:

(1) Blank-Contaminant-free water sent to the laboratory as a routine sample or contaminant free water analyzed at the laboratory as part of their internal quality-control procedures. These samples do not come in contact with sampling and processing equipment. They indicate laboratory accuracy of baseline concentrations.

(2) Field blank-Requires passing a volume of contaminant-free water through all sampling and processing equipment that the water samples contact. These samples are preserved and analyzed along with regular water samples. Results are used to assess equipment cleaning procedures, and identify contamination introduced by sampling protocols and (or) sample handling and transport.

(3) Split -A large volume of native water sample is divided into two or more samples and analyzed individually. Results are used to estimate the combined effects of processing and laboratory precision and (or) bias. 
(4) Standard reference-Contaminant-free water spiked with known concentrations of analytes. These samples do not contact sampling or processing equipment and provide estimates of analytical accuracy and bias.

(5) Field matrix spikes-A standard spike solution added to a split of the regular water sample. These samples are processed and analyzed in the same manner as the regular water samples and are used to assess the analytical precision and recovery rates of analytes from matrices of native water.

(6) Surrogate spikes-Addition of compounds that behave similar to target analytes to each sample to asses the analytical precision and percent recovery from the sample matrix. Percent recovery of surrogates are expected to be greater than or equal to the recovery rates of target analytes.

Quality-control results for Phase II replicate, split, and spike samples are summarized in tables 40 through 48 . Blank and field blank qualitycontrol sample results indicated possible wholewater trace-element contamination in Phase II spring sampling for copper, nickel, and zinc; this contamination makes low-level ( 1 to 2 micrograms per liter) concentrations for these elements questionable. The contamination might be attributable to metal springs in the spigots of churn splitters. Spigots made entirely of plastic were installed for the fall sampling. No detection of organic compounds occurred in any of the blank or field blank samples.

\section{REFERENCES CITED}

Arbogast, B.F. (ed), 1990, Quality assurance manual for the Branch of Geochemistry: U.S. Geological Survey Open-File Report 90-668, 184 p.

Edwards, T.K. and Glysson, D. G.,1988, Field methods for measurement of fluvial sediment: U.S. Geological Survey Open-File Report 86-531, 118 p.

Fegeas, R.G., Claire, R.W., Guptill, S.C., Anderson, K.E., and Hallam, C.A., 1983, U.S. Geological Survey digital cartographic data standards-land use and land cover digital data: U.S. Geological Survey Circular 895-E, 21p.
Fishman, M.J., (ed), 1993, Methods of analysis by the U.S. Geological Survey National Water-Quality Laboratory-Determination of inorganic and organic constituents in water and fluvial sediments: U.S. Geological Survey Open-File Report 93-125, 217 p.

Foreman, W.T., Connor, B.F., Furlong, E.T., Vaught, D.G., and Merten, L.M., 1995, Methods of analysis by the U.S. Geological Survey National Water Quality Laboratory-Determination of organochlorine pesticides and polychlorinated biphenyls in bottom sediment by dual capillarycolumn gas chromatography with electron-capture detection: U.S. Geological Survey Open-File Report 95-140, 78 p.

Guy, H.P., 1969, Laboratory theory and methods of sediment analysis: U.S. Geological Survey Techniques of Water-Resources Investigations, book 5 , chapter $\mathrm{C} 1,58 \mathrm{p}$.

Horowitz, A.J., Demas, C.R., Fitzgerald, K.K., Miller, T.M., and Rickert, D.A., 1994, U.S. Geological Survey protocol for the collection and processing of surface-water samples for the subsequent determination of inorganic constituents in filtered water: U.S. Geological Survey Open-File Report 94-539, 57 p.

Kammerer, J.C., 1990, Largest rivers in the United States: U.S. Geological Survey Open-File Report 87-242, 2 p.

Rantz, S.E., and others, 1982, Measurement and Computation of Streamflow: U.S. Geological Survey Water Supply Paper 2175, 2 vol., 631p.

Rose D.L., and Schroeder, M.P., 1995, Methods of analysis by the U.S. Geological Survey National Water-Quality Laboratory-Determination of volatile organic compounds in water by purge and trap capillary gas chromatography/mass spectrometry: U.S. Geological Survey Open-File Report 94-708, 26 p.

Sandstrom, M.W., Wydoski, D.S., Schroeder, M.P., Zamboni, J.L., and Foreman, W.T., 1994, Methods of analysis by the U.S. Geological Survey National Water-Quality Laboratory -Determination of organonitrogen herbicides in water by solid-phase extraction and capillary column gas chromatography/mass spectrometry with selectedion monitoring: U.S. Geological Survey Open-File Report 91-519, 26 p. 
Sandstrom, M.W., 1989, Field method for isolation of herbicides in surface and ground water using solidphase extraction, in Pederson, G.L., and Smith, M.M., eds., U.S. Geological Survey second national symposium on water quality-Abstracts of the technical sessions, Orlando, Florida, November 12-17, 1989: U.S. Geological Survey Open-File Report 89-409, p. 82.

Shelton, L.R. and Capel, P.D., 1994, Guidelines for collecting and processing samples of stream bed sediment for analysis of trace elements and organic contaminants for the National WaterQuality Assessment Program: U.S. Geological Survey Open-File Report 94-458, 20 p.

Timme, P.J., 1994, National Water Quality Laboratory 1994 Services catolog: U.S. Geological Survey Open-File report 94-304, 103 p.
U.S. Environmental Protection Agency, 1992, Guidelines establishing test procedures for the analysis of pollutants: U.S. Code of Federal Regulations, Title 40, p. 565-567.

Wershaw, R.L., Fishman, M.J., Grabbe, R.R., and Lowe, L.E., eds., 1987, Methods for the determination of organic substances in water and fluvial sediments: U.S. Geological Survey Techniques of Water-Resources Investigations, book 5 , chapter A3, $80 \mathrm{p}$.

Zaugg, S.D., M.W. Sandstrom, S.G. Smith, and K.M. Fehlberg, 1995, Methods of analysis by the U.S. Geological Survey National Water-Quality Laboratory-Determination of pesticides in water by $\mathrm{C}-18$ solid-phase extraction and capillarycolumn gas chromatography/mass spectrometry with selected-ion monitoring: U.S. Geological Survey Open-File Report 95-181, 49 p. 


\section{ANALYTICAL DATA}

Data in the following tables were not aligned on decimal points and trailing zeros to the right of the decimal points were not removed. Method detection limits and minimum reporting levels are listed in Appendix, and quality-assurance data are listed in tables 40 through 48. Additional Willamette Basin NAWQA samples are included in the following tables which were not listed in tables 3, 4, and 5 . 
Page Intentionally Blank 
Table 7.Ancillary data from Phase I of the Willamette River Basin Water Quality Study, Oregon, 1992-93

\begin{tabular}{|c|c|c|c|}
\hline STATION NUMBER & STATION NAME & DATE & TIME \\
\hline 440313123091100 & $\begin{array}{llllllll}\text { A-3 } & \text { CHANNEL AT WALLIS AND } & 5 \mathrm{TH} & \mathrm{ST} \text { AT EUGENE, OR } \\
\mathrm{A}-3 & \text { CHANNEL AT } & \text { WALLIS AND } & 5 \mathrm{TH} & \mathrm{ST} & \mathrm{AT} & \text { EUGENE, OR } \\
\mathrm{A}-3 & \text { CHANNEL AT } & \text { WALLIS AND } & 5 \mathrm{TH} & \mathrm{ST} & \mathrm{AT} & \text { EUGENE, OR }\end{array}$ & $\begin{array}{l}04-16-92 \\
10-16-92 \\
10-29-92\end{array}$ & $\begin{array}{l}1410 \\
1345 \\
1000\end{array}$ \\
\hline 443207123145500 & WILLAMETTE RIVER NEAR CORVALLIS, OR & $08-24-93$ & 1615 \\
\hline 443138123120901 & MUDDY CREEK NEAR PEORIA, OR & $08-24-93$ & 1300 \\
\hline 451309123041501 & PALMER C AT DAYTON, OR & $09-07-93$ & 1045 \\
\hline 451320123041100 & YAMHILL RIVER AT DAYTON, OR & $09-07-93$ & 1420 \\
\hline 14201300 & $\begin{array}{llll}\text { ZOLLNER CREEK NEAR MT ANGEL, OR } \\
\text { ZOLLNER CREEK NEAR MT ANGEL, OR }\end{array}$ & $\begin{array}{l}06-01-93 \\
07-27-93\end{array}$ & $\begin{array}{l}1115 \\
1015\end{array}$ \\
\hline 14202000 & PUDDING RIVER AT AURORA, OREG. & $04-27-93$ & 1445 \\
\hline 453115122535500 & BEAVERTON C AT 216TH AVE NEAR ORENCO, OR & $03-31-93$ & 1810 \\
\hline 14206950 & $\begin{array}{lllll}\text { FANNO CREEK AT DURHAM, OR } \\
\text { FANNO CREEK AT DURHAM, OR }\end{array}$ & $\begin{array}{l}03-01-93 \\
07-22-93\end{array}$ & $\begin{array}{l}1830 \\
1000\end{array}$ \\
\hline 14211550 & JOHNSON CREEK AT MILWAUKIE, OREG. & $03-14-93$ & 1745 \\
\hline 453547122463000 & $\begin{array}{llll}\text { WILLAMETTE RIVER AT LINNTON, OR } \\
\text { WILLAMETTE RIVER AT LINNTON, OR }\end{array}$ & $\begin{array}{l}04-18-92 \\
10-26-92\end{array}$ & $\begin{array}{l}0900 \\
1000\end{array}$ \\
\hline
\end{tabular}


Table 7. Ancillary data from Phase I of the Willamette River Basin Water Quality Study, Oregon, 1992-93-Continued

\begin{tabular}{|c|c|c|c|c|c|c|c|c|}
\hline STATION NUMBER & DATE & $\begin{array}{c}\text { TEMPER- } \\
\text { ATURE } \\
\text { WATER } \\
(\text { DEG C) }\end{array}$ & $\begin{array}{l}\text { BARO- } \\
\text { METRIC } \\
\text { PRES- } \\
\text { SURE } \\
\text { (MM } \\
\text { OF } \\
\text { HG) }\end{array}$ & $\begin{array}{c}\text { DIS- } \\
\text { CHARGE, } \\
\text { INST. } \\
\text { CUBIC } \\
\text { FEET } \\
\text { PER } \\
\text { SECOND }\end{array}$ & $\begin{array}{l}\text { SEDI- } \\
\text { MENT, } \\
\text { SUS- } \\
\text { PENDED } \\
\text { (MG/L) }\end{array}$ & $\begin{array}{l}\text { SED. } \\
\text { SUSP. } \\
\text { SIEVE } \\
\text { DIAM. } \\
\circ \text { FINER } \\
\text { THAN } \\
.062 \text { MM }\end{array}$ & $\begin{array}{l}\text { SPE- } \\
\text { CIFIC } \\
\text { CON- } \\
\text { DUCT- } \\
\text { ANCE } \\
\text { (US/CM) }\end{array}$ & $\begin{array}{c}\text { OXYGEN, } \\
\text { DIS- } \\
\text { SOLVED } \\
(\text { MG/L) }\end{array}$ \\
\hline 440313123091100 & $\begin{array}{l}04-16-92 \\
10-16-92 \\
10-29-92\end{array}$ & $\begin{array}{l}17.5 \\
13.0 \\
12.0\end{array}$ & $\begin{array}{l}-- \\
759 \\
741\end{array}$ & $\begin{array}{l}12 \\
0.17 \\
17\end{array}$ & $\begin{array}{r}63 \\
6 \\
90\end{array}$ & $\begin{array}{l}98 \\
98 \\
97\end{array}$ & $\begin{array}{r}124 \\
284 \\
35\end{array}$ & $\begin{array}{l}-- \\
11.8 \\
--\end{array}$ \\
\hline 443207123145500 & $08-24-93$ & 17.0 & 765 & 6640 & 4 & 90 & 59 & 10.2 \\
\hline 443138123120901 & $08-24-93$ & 19.0 & 766 & 38 & 14 & 95 & 159 & 7.5 \\
\hline 451309123041501 & $09-07-93$ & 18.0 & 766 & 15 & 4 & 90 & 116 & 8.0 \\
\hline 451320123041100 & $09-07-93$ & 20.5 & 766 & 40 & 8 & 91 & 183 & 8.7 \\
\hline 14201300 & $\begin{array}{l}06-01-93 \\
07-27-93\end{array}$ & $\begin{array}{l}15.5 \\
16.5\end{array}$ & $\begin{array}{l}759 \\
760\end{array}$ & $\begin{array}{l}12 \\
1.0\end{array}$ & $\begin{array}{l}33 \\
13\end{array}$ & $\begin{array}{l}98 \\
96\end{array}$ & $\begin{array}{l}328 \\
378\end{array}$ & $\begin{array}{l}7.7 \\
7.2\end{array}$ \\
\hline 14202000 & $04-27-93$ & 10.0 & 770 & 3100 & 27 & -- & 65 & 10.4 \\
\hline 453115122535500 & $03-31-93$ & 11.0 & 754 & 37 & 10 & 99 & 221 & 9.8 \\
\hline 14206950 & $\begin{array}{l}03-01-93 \\
07-22-93\end{array}$ & $\begin{array}{l}-- \\
17.0\end{array}$ & $\begin{array}{l}766 \\
759\end{array}$ & $\begin{array}{r}164 \\
15\end{array}$ & $\begin{array}{r}203 \\
6\end{array}$ & $\begin{array}{l}92 \\
92\end{array}$ & $\begin{array}{l}134 \\
196\end{array}$ & $--\frac{}{7.5}$ \\
\hline 14211550 & $03-14-93$ & 10.5 & 757 & 42 & 24 & 81 & 130 & 10.7 \\
\hline 453547122463000 & $\begin{array}{l}04-18-92 \\
10-26-92\end{array}$ & $\begin{array}{l}12.0 \\
14.5\end{array}$ & $-\frac{-}{770}$ & $\begin{array}{r}45100 \\
9720\end{array}$ & $\begin{array}{r}32 \\
4\end{array}$ & $\begin{array}{r}90 \\
100\end{array}$ & $\begin{array}{l}72 \\
90\end{array}$ & $-\overline{9} .0$ \\
\hline
\end{tabular}


Table 7. Ancillary data from Phase I of the Willamette River Basin Water Quality Study, Oregon, 1992-93-Continued

\begin{tabular}{|c|c|c|c|c|c|c|c|c|}
\hline STATION NUMBER & DATE & $\begin{array}{c}\text { OXYGEN, } \\
\text { DIS- } \\
\text { SOLVED } \\
\text { (PER- } \\
\text { CENT } \\
\text { SATUR- } \\
\text { ATION) }\end{array}$ & $\begin{array}{c}\text { PH } \\
\text { WATER } \\
\text { WHOLE } \\
\text { FIELD } \\
\text { (STAND- } \\
\text { ARD } \\
\text { UNITS) }\end{array}$ & $\begin{array}{l}\text { CARBON, } \\
\text { ORGANIC } \\
\text { DIS- } \\
\text { SOLVED } \\
(\text { MG / L } \\
\text { AS C) }\end{array}$ & $\begin{array}{l}\text { CARBON, } \\
\text { ORGANIC } \\
\text { SUS- } \\
\text { PENDED } \\
(\mathrm{MG} / \mathrm{L} \\
\text { AS C) }\end{array}$ & $\begin{array}{l}\text { CALCIUM, } \\
\text { DIS- } \\
\text { SOLVED } \\
\text { (MG/L } \\
\text { AS CA) }\end{array}$ & $\begin{array}{l}\text { MAGNE- } \\
\text { SIUM, } \\
\text { DIS- } \\
\text { SOLVED } \\
\text { (MG/L } \\
\text { AS MG) }\end{array}$ & $\begin{array}{l}\text { HARD- } \\
\text { NESS } \\
\text { TOTAL } \\
\text { (MG/L } \\
\text { AS } \\
\text { CACO3) }\end{array}$ \\
\hline 440313123091100 & $\begin{array}{l}04-16-92 \\
10-16-92 \\
10-29-92\end{array}$ & $\begin{array}{l}-- \\
112 \\
--\end{array}$ & $\begin{array}{l}7.3 \\
7.6 \\
7.1\end{array}$ & $\begin{array}{l}7.7 \\
3.7 \\
5.7\end{array}$ & $\begin{array}{l}4.0 \\
1.0 \\
0.2\end{array}$ & $\begin{array}{l}-- \\
33 \\
2.8\end{array}$ & $\begin{array}{l}-- \\
12 \\
1.0\end{array}$ & $\begin{array}{r}-- \\
130 \\
11\end{array}$ \\
\hline 443207123145500 & $08-24-93$ & 106 & 7.6 & 1.2 & 0.3 & 5.0 & 1.9 & 20 \\
\hline 443138123120901 & $08-24-93$ & 80 & 7.4 & 1.9 & 0.7 & 13 & 6.0 & 57 \\
\hline 451309123041501 & $09-07-93$ & 84 & 7.6 & 2.6 & 0.3 & 11 & 3.9 & 44 \\
\hline 451320123041100 & $09-07-93$ & 97 & 9.4 & 3.0 & 3.1 & -- & -- & -- \\
\hline 14201300 & $\begin{array}{l}06-01-93 \\
07-27-93\end{array}$ & $\begin{array}{l}78 \\
74\end{array}$ & $\begin{array}{l}7.1 \\
7.4\end{array}$ & $\begin{array}{l}6.5 \\
4.3\end{array}$ & $\begin{array}{l}1.1 \\
0.7\end{array}$ & $\begin{array}{l}29 \\
37\end{array}$ & $\begin{array}{c}9.6 \\
12\end{array}$ & $\begin{array}{l}110 \\
140\end{array}$ \\
\hline 14202000 & $04-27-93$ & 91 & 6.9 & 2.3 & 1.2 & 6.0 & 2.1 & 24 \\
\hline 453115122535500 & $03-31-93$ & 89 & 7.4 & 4.2 & 1.2 & 22 & 7.6 & 86 \\
\hline 14206950 & $\begin{array}{l}03-01-93 \\
07-22-93\end{array}$ & -- & $-\frac{-}{7.4}$ & $\begin{array}{l}4.1 \\
5.5\end{array}$ & $\begin{array}{l}5.8 \\
--\end{array}$ & $\begin{array}{l}13 \\
22\end{array}$ & $\begin{array}{l}4.2 \\
7.0\end{array}$ & $\begin{array}{l}50 \\
84\end{array}$ \\
\hline 14211550 & $03-14-93$ & 96 & 7.7 & 2.0 & 1.8 & 11 & 4.9 & 48 \\
\hline 453547122463000 & $\begin{array}{l}04-18-92 \\
10-26-92\end{array}$ & --87 & $\begin{array}{l}7.3 \\
7.1\end{array}$ & $\begin{array}{l}2.5 \\
2.0\end{array}$ & $\begin{array}{l}1.6 \\
1.7\end{array}$ & $\begin{array}{l}5.9 \\
6.9\end{array}$ & $\begin{array}{l}2.0 \\
2.4\end{array}$ & $\begin{array}{l}23 \\
27\end{array}$ \\
\hline
\end{tabular}


Table 8. Ancillary data from Phase II of the Willamette River Basin Water Quality Study, Oregon, 1994

\begin{tabular}{|c|c|c|c|}
\hline STATION NUMBER & STATION NAME & DATE & TIME \\
\hline 433548123040600 & COAST FORK WILLAMETTE R BL BIG RIVER NR LONDON, OR & $05-20-94$ & 1040 \\
\hline 440045122585600 & COAST FORK WILLAM AT SEAVY LOOP RD NR EUGENE, OR & $05-19-94$ & 1020 \\
\hline 440402123063900 & URBAN OUTFALL AT POLK ST. PARK AT EUGENE, OR & $06-13-94$ & 1210 \\
\hline 441310122095801 & MACK CREEK NEAR BLUE RIVER, OR & $05-27-94$ & 1030 \\
\hline 440707123041300 & MCKENZIE RIVER NR EUGENE, OR & $05-19-94$ & 1450 \\
\hline 442223123153703 & $\begin{array}{lllllll}\text { LONG TOM } R & \text { AT } & \text { BUNDY } & \text { BRIDGE } & \text { NR MONROE, OR } \\
\text { LONG TOM } R \text { AT } & \text { BUNDY } & \text { BRIDGE } & \text { NR MONROE, OR }\end{array}$ & $\begin{array}{l}05-19-94 \\
11-01-94\end{array}$ & $\begin{array}{l}0950 \\
1720\end{array}$ \\
\hline 442413123122500 & LAKE CAMOUS CR AT PINE GROVE DR NR HARRISBURG, OR & $05-19-94$ & 1720 \\
\hline 443138123120901 & $\begin{array}{llll}\text { MUDDY } & \text { CREEK } & \text { NEAR } & \text { PEORIA, OR } \\
\text { MUDDY } & \text { CREEK } & \text { NEAR } & \text { PEORIA, OR } \\
\text { MUDDY } & \text { CREEK } & \text { NEAR } & \text { PEORIA, OR } \\
\text { MUDDY } & \text { CREEK } & \text { NEAR } & \text { PEORIA, OR } \\
\text { MUDDY } & \text { CREEK } & \text { NEAR } & \text { PEORIA, OR } \\
\text { MUDDY } & \text { CREEK } & \text { NEAR } & \text { PEORIA, OR }\end{array}$ & $\begin{array}{l}05-26-94 \\
06-03-94 \\
06-20-94 \\
11-02-94 \\
11-06-94 \\
11-09-94\end{array}$ & $\begin{array}{l}1730 \\
0930 \\
1050 \\
1010 \\
1200 \\
1430\end{array}$ \\
\hline 443045123273000 & ROCK CREEK ABOVE GRIFFITH CREEK NR PHILOMATH, OR & $05-18-94$ & 1820 \\
\hline 443321123155201 & MARYS RIVER AT CORVALLIS, OR & $05-18-94$ & 1350 \\
\hline 14173500 & $\begin{array}{llll}\text { CALAPOOIA } & \text { RIVER AT ALBANY, OR } \\
\text { CALAPOOIA } & \text { RIVER AT ALBANY, OR }\end{array}$ & $\begin{array}{l}05-26-94 \\
11-01-94\end{array}$ & $\begin{array}{l}1220 \\
1230\end{array}$ \\
\hline 444349123094000 & LUCKIAMUTE R AT BUENA VISTA RD NR BUENA VISTA, OR & $05-16-94$ & 1720 \\
\hline 444123122562200 & THOMAS CREEK AT KELLY RD NR JEFFERSON, OR & $05-16-94$ & 1900 \\
\hline 14189000 & SANTIAM RIVER AT JEFFERSON, OR & $05-17-94$ & 1620 \\
\hline
\end{tabular}


Table 8. Ancillary data from Phase II of the Willamette River Basin Water Quality Study, Oregon, 1994-Continued

\begin{tabular}{|c|c|c|c|c|c|c|c|c|c|}
\hline STATION NUMBER & $\mathrm{DATE}$ & $\begin{array}{c}\text { TEMPER- } \\
\text { ATURE } \\
\text { WATER } \\
\left(\begin{array}{ll}\text { DEG } & \text { C }\end{array}\right)\end{array}$ & $\begin{array}{l}\text { BARO- } \\
\text { METRIC } \\
\text { PRES- } \\
\text { SURE } \\
\text { (MM } \\
\text { OF } \\
\text { HG) }\end{array}$ & $\begin{array}{c}\text { DIS- } \\
\text { CHARGE, } \\
\text { INST. } \\
\text { CUBIC } \\
\text { FEET } \\
\text { PER } \\
\text { SECOND }\end{array}$ & $\begin{array}{l}\text { SEDI- } \\
\text { MENT, } \\
\text { SUS- } \\
\text { PENDED } \\
\text { (MG/L) }\end{array}$ & $\begin{array}{r}\text { SED } \\
\text { SUSH } \\
\text { SIEVH } \\
\text { DIAI } \\
\text { FIN } \\
\text { THAI } \\
.062\end{array}$ & & $\begin{array}{l}\text { SPE- } \\
\text { CIFIC } \\
\text { CON- } \\
\text { DUCT- } \\
\text { ANCE } \\
\text { (US/CM) }\end{array}$ & $\begin{array}{c}\text { OXYGEN, } \\
\text { DIS- } \\
\text { SOLVED } \\
\text { (MG/L) }\end{array}$ \\
\hline 433548123040600 & $05-20-94$ & 10.0 & 738 & 21 & 5 & & 84 & 60 & 10.6 \\
\hline 440045122585600 & $05-19-94$ & 14.5 & 751 & 276 & 6 & & 90 & 65 & 9.4 \\
\hline 440402123063900 & $06-13-94$ & -- & 750 & 1.5 & 4 & & 76 & 193 & -- \\
\hline 441310122095801 & $05-27-94$ & 8.0 & 699 & 4.7 & $<1$ & -- & & 24 & 10.9 \\
\hline 440707123041300 & $05-19-94$ & 12.0 & 756 & 1580 & 2 & & 70 & 62 & 11.3 \\
\hline 442223123153703 & $\begin{array}{l}05-19-94 \\
11-01-94\end{array}$ & $\begin{array}{l}17.5 \\
12.0\end{array}$ & $\begin{array}{l}758 \\
756\end{array}$ & $\begin{array}{r}110 \\
1780\end{array}$ & $\begin{array}{l}24 \\
76\end{array}$ & & $\begin{array}{l}91 \\
91\end{array}$ & $\begin{array}{r}105 \\
84\end{array}$ & $\begin{array}{l}10.4 \\
10.5\end{array}$ \\
\hline 442413123122500 & $05-19-94$ & 16.5 & 756 & 7.7 & 25 & & 96 & 153 & 7.3 \\
\hline 443138123120901 & $\begin{array}{l}05-26-94 \\
06-03-94 \\
06-20-94 \\
11-02-94 \\
11-06-94 \\
11-09-94\end{array}$ & $\begin{array}{r}19.0 \\
16.0 \\
18.5 \\
10.0 \\
8.0 \\
8.5\end{array}$ & $\begin{array}{l}759 \\
758 \\
760 \\
754 \\
754 \\
746\end{array}$ & $\begin{array}{r}21 \\
29 \\
38 \\
899 \\
524 \\
230\end{array}$ & $\begin{array}{r}8 \\
9 \\
--\quad \\
158 \\
33 \\
20\end{array}$ & -- & $\begin{array}{l}90 \\
99 \\
98 \\
99 \\
94\end{array}$ & $\begin{array}{l}242 \\
251 \\
184 \\
109 \\
129 \\
140\end{array}$ & $\begin{array}{r}9.2 \\
7.9 \\
8.2 \\
8.5 \\
9.4 \\
10.1\end{array}$ \\
\hline 443045123273000 & $05-18-94$ & 13.0 & 750 & 11 & 3 & & 85 & 107 & 9.8 \\
\hline 443321123155201 & $05-18-94$ & 15.0 & 758 & 159 & 8 & & 88 & 94 & -- \\
\hline 14173500 & $\begin{array}{l}05-26-94 \\
11-01-94\end{array}$ & $\begin{array}{l}19.5 \\
10.5\end{array}$ & $\begin{array}{l}762 \\
756\end{array}$ & $\begin{array}{r}152 \\
2750\end{array}$ & $\begin{array}{r}13 \\
307\end{array}$ & & $\begin{array}{l}83 \\
86\end{array}$ & $\begin{array}{r}164 \\
73\end{array}$ & $\begin{array}{l}9.6 \\
9.2\end{array}$ \\
\hline 444349123094000 & $05-16-94$ & 13.5 & 754 & 236 & 7 & & 96 & 91 & 8.9 \\
\hline 444123122562200 & $05-16-94$ & 15.0 & 754 & 204 & 5 & & 91 & 43 & 10.4 \\
\hline 14189000 & $05-17-94$ & 12.5 & 756 & 3050 & 5 & & 73 & 44 & 10.9 \\
\hline
\end{tabular}


Table 8. Ancillary data from Phase II of the Willamette River Basin Water Quality Study, Oregon, 1994-Continued

\begin{tabular}{|c|c|c|c|c|c|c|c|c|}
\hline STATION NUMBER & DATE & $\begin{array}{c}\text { OXYGEN, } \\
\text { DIS- } \\
\text { SOLVED } \\
\text { (PER- } \\
\text { CENT } \\
\text { SATUR- } \\
\text { ATION) }\end{array}$ & $\begin{array}{c}\text { PH } \\
\text { WATER } \\
\text { WHOLE } \\
\text { FIELD } \\
\text { (STAND- } \\
\text { ARD } \\
\text { UNITS) }\end{array}$ & $\begin{array}{c}\text { CARBON, } \\
\text { ORGANIC } \\
\text { DIS- } \\
\text { SOLVED } \\
(\mathrm{MG} / \mathrm{L} \\
\text { AS C) }\end{array}$ & $\begin{array}{l}\text { CARBON, } \\
\text { ORGANIC } \\
\text { SUS- } \\
\text { PEND } \\
(\mathrm{MG} / \mathrm{L} \\
\text { AS C) }\end{array}$ & $\begin{array}{l}\text { CALCIUM } \\
\text { DIS- } \\
\text { SOLVED } \\
\text { (MG/L } \\
\text { AS CA) }\end{array}$ & $\begin{array}{l}\text { MAGNE- } \\
\text { SIUM, } \\
\text { DIS- } \\
\text { SOLVED } \\
\text { (MG/L } \\
\text { AS MG) }\end{array}$ & $\begin{array}{c}\text { HARD- } \\
\text { NESS } \\
\text { TOTAL } \\
\text { (MG/L } \\
\text { AS } \\
\text { CACO3) }\end{array}$ \\
\hline 433548123040600 & $05-20-94$ & 97 & 7.6 & 3.1 & 0.9 & 7.5 & 1.7 & 26 \\
\hline 440045122585600 & $05-19-94$ & 93 & 7.4 & 2.1 & 0.4 & 7.2 & 1.8 & 25 \\
\hline 440402123063900 & $06-13-94$ & -- & 7.2 & 4.4 & 0.9 & 18 & 6.8 & 73 \\
\hline 441310122095801 & $05-27-94$ & 100 & 6.0 & 0.6 & 0.1 & -- & -- & -- \\
\hline 440707123041300 & $05-19-94$ & 106 & 7.8 & 0.8 & 0.4 & -- & -- & -- \\
\hline 442223123153703 & $\begin{array}{l}05-19-94 \\
11-01-94\end{array}$ & $\begin{array}{r}109 \\
98\end{array}$ & $\begin{array}{l}7.5 \\
7.2\end{array}$ & $\begin{array}{l}2.5 \\
4.1\end{array}$ & $\begin{array}{l}0.6 \\
3.3\end{array}$ & $\begin{array}{l}-- \\
--\end{array}$ & $\begin{array}{l}-- \\
--\end{array}$ & $\begin{array}{l}-- \\
--\end{array}$ \\
\hline 442413123122500 & $05-19-94$ & 75 & 7.2 & 2.8 & 0.7 & -- & -- & -- \\
\hline 443138123120901 & $\begin{array}{l}05-26-94 \\
06-03-94 \\
06-20-94 \\
11-02-94 \\
11-06-94 \\
11-09-94\end{array}$ & $\begin{array}{l}99 \\
80 \\
88 \\
76 \\
80 \\
88\end{array}$ & $\begin{array}{l}7.6 \\
7.4 \\
7.5 \\
6.9 \\
6.8 \\
7.0\end{array}$ & $\begin{array}{l}2.8 \\
3.1 \\
2.5 \\
6.1 \\
7.3 \\
8.2\end{array}$ & $\begin{array}{l}0.4 \\
0.3 \\
1.6 \\
1.4 \\
1.1 \\
0.8\end{array}$ & $\begin{array}{c}-- \\
-- \\
14 \\
-- \\
-- \\
--\end{array}$ & $\begin{array}{l}-- \\
-- \\
7.0 \\
-- \\
-- \\
--\end{array}$ & $\begin{array}{l}-- \\
-- \\
-- \\
-- \\
-- \\
--\end{array}$ \\
\hline 443045123273000 & $05-18-94$ & 95 & 8.0 & 0.8 & 0.2 & -- & -- & -- \\
\hline 443321123155201 & $05-18-94$ & -- & 7.6 & 1.9 & 0.4 & 8.5 & 3.3 & 35 \\
\hline 14173500 & $\begin{array}{l}05-26-94 \\
11-01-94\end{array}$ & $\begin{array}{r}105 \\
83\end{array}$ & $\begin{array}{l}7.9 \\
6.9\end{array}$ & $\begin{array}{l}1.4 \\
4.2\end{array}$ & $\begin{array}{l}0.5 \\
4.7\end{array}$ & $\begin{array}{l}-- \\
--\end{array}$ & $\begin{array}{l}-- \\
--\end{array}$ & $\begin{array}{l}-- \\
--\end{array}$ \\
\hline 444349123094000 & $05-16-94$ & 87 & 7.1 & 1.9 & 0.3 & -- & -- & -- \\
\hline 444123122562200 & $05-16-94$ & 105 & 7.4 & 1.3 & 0.4 & -- & -- & -- \\
\hline 14189000 & $05-17-94$ & 102 & 7.4 & 1.3 & 0.4 & -- & -- & -- \\
\hline
\end{tabular}


Table 8. Ancillary data from Phase II of the Willamette River Basin Water Quality Study, Oregon, 1994-Continued

\begin{tabular}{|c|c|c|c|}
\hline STATION NUMBER & STATION NAME & DATE & TIME \\
\hline 445547123065400 & RICKREAL CREEK NR MOUTH NR SALEM, OR & $05-26-94$ & 1540 \\
\hline 14190970 & $\begin{array}{llllllll}\text { PRINGLE } & \text { C } & \text { AT } & \text { BUSH } & \text { PARK } & \text { AT } & \text { SALEM, } & \text { OR } \\
\text { PRINGLE } & \text { C } & \text { AT } & \text { BUSH } & \text { PARK } & \text { AT } & \text { SALEM, } & \text { OR } \\
\text { PRINGLE } & \text { C } & \text { AT } & \text { BUSH } & \text { PARK } & \text { AT } & \text { SALEM, } & \text { OR }\end{array}$ & $\begin{array}{l}11-23-94 \\
11-30-94 \\
11-30-94\end{array}$ & $\begin{array}{l}1200 \\
1530 \\
1710\end{array}$ \\
\hline 445037122573800 & $\begin{array}{llllllll}\text { MILL } & \text { CREEK } & \text { AT } & \text { DELANEY } & \text { ROAD } & \text { NR } & \text { TURNER, } & \text { OR } \\
\text { MILL } & \text { CREEK } & \text { AT } & \text { DELANEY } & \text { ROAD } & \text { NR } & \text { TURNER, } & \text { OR } \\
\text { MILL } & \text { CREEK } & \text { AT } & \text { DELANEY } & \text { ROAD } & \text { NR } & \text { TURNER, OR } \\
\text { MILL } & \text { CREEK } & \text { AT } & \text { DELANEY } & \text { ROAD } & \text { NR } & \text { TURNER, } & \text { OR }\end{array}$ & $\begin{array}{l}06-14-94 \\
10-31-94 \\
11-04-94 \\
11-04-94\end{array}$ & $\begin{array}{l}1140 \\
1410 \\
1120 \\
1420\end{array}$ \\
\hline 14194150 & $\begin{array}{lllll}\text { SOUTH YAMHILL RIVER AT MCMINNVILLE, } & \text { OR } \\
\text { SOUTH YAMHILL RIVER AT } & \text { MCMINNVILLE, } & \text { OR }\end{array}$ & $\begin{array}{l}05-17-94 \\
11-02-94\end{array}$ & $\begin{array}{l}1720 \\
1210\end{array}$ \\
\hline 451355123093600 & NORTH YAMHILL RIVER AT HWY. 99E NR MCMINNVILLE, OR & $05-17-94$ & 1220 \\
\hline 451602122564400 & WILLAMETTE RIVER NR NEWBERG, OR & $05-31-94$ & 1400 \\
\hline 451502122524700 & $\begin{array}{lllllll}\text { CHAMPOEG CREEK } & \text { BELOW MISSION CR NR BUTTEVILLE, OR } \\
\text { CHAMPOEG CREEK } & \text { BELOW MISSION CR NR BUTTEVILLE, OR }\end{array}$ & $\begin{array}{l}05-26-94 \\
10-31-94\end{array}$ & $\begin{array}{l}1030 \\
1300\end{array}$ \\
\hline 14201300 & $\begin{array}{llllll}\text { ZOLLNER } & \text { CREEK } & \text { NEAR } & \text { MT } & \text { ANGEL, OR } \\
\text { ZOLLNER } & \text { CREEK } & \text { NEAR } & \text { MT } & \text { ANGEL, } \\
\text { ZOLLNER } & \text { CREEK } & \text { NEAR } & \text { MT ANGEL, } & \text { OR } \\
\text { ZOLLNER } & \text { CREEK } & \text { NEAR } & \text { MT ANGEL, } & \text { OR }\end{array}$ & $\begin{array}{l}05-25-94 \\
06-13-94 \\
10-28-94 \\
11-28-94\end{array}$ & $\begin{array}{l}1450 \\
1120 \\
1320 \\
1400\end{array}$ \\
\hline 14202000 & $\begin{array}{lllll}\text { PUDDING } & \text { RIVER AT AURORA, OREG. } \\
\text { PUDDING } & \text { RIVER AT AURORA, OREG. } \\
\text { PUDDING } & \text { RIVER AT AURORA, OREG. } \\
\text { PUDDING } & \text { RIVER AT AURORA, OREG. } \\
\text { PUDDING } & \text { RIVER AT AURORA, OREG. } \\
\text { PUDDING RIVER AT AURORA, OREG. } & \text { AT } \\
\text { PUDDING RIVER AT AURORA, OREG. }\end{array}$ & $\begin{array}{l}04-12-94 \\
05-25-94 \\
06-15-94 \\
10-28-94 \\
10-29-94 \\
11-09-94 \\
11-29-94\end{array}$ & $\begin{array}{l}1110 \\
1100 \\
1145 \\
1710 \\
1230 \\
1300 \\
1110\end{array}$ \\
\hline 451603122423301 & MOLALLA R AT KNIGHTS BRIDGE NR CANBY, OR & $05-25-94$ & 1020 \\
\hline 14206200 & $\begin{array}{lllllll}\text { DAIRY CREEK AT RTE } & 8 & \text { NEAR HILLSBORO, OR } \\
\text { DAIRY CREEK AT RTE } & 8 \text { NEAR HILLSBORO, OR }\end{array}$ & $\begin{array}{l}05-27-94 \\
12-01-94\end{array}$ & $\begin{array}{l}1640 \\
1140\end{array}$ \\
\hline
\end{tabular}


Table 8. Ancillary data from Phase II of the Willamette River Basin Water Quality Study, Oregon, 1994-Continued

\begin{tabular}{|c|c|c|c|c|c|c|c|c|}
\hline STATION NUMBER & DATE & 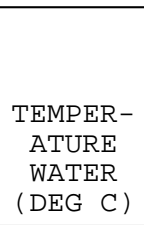 & $\begin{array}{l}\text { BARO- } \\
\text { METRIC } \\
\text { PRES- } \\
\text { SURE } \\
\text { (MM } \\
\text { OF } \\
\text { HG) }\end{array}$ & $\begin{array}{c}\text { DIS- } \\
\text { CHARGE, } \\
\text { INST. } \\
\text { CUBIC } \\
\text { FEET } \\
\text { PER } \\
\text { SECOND }\end{array}$ & $\begin{array}{l}\text { SEDI- } \\
\text { MENT, } \\
\text { SUS- } \\
\text { PENDED } \\
\text { (MG/L) }\end{array}$ & $\begin{array}{c}\text { SED. } \\
\text { SUSP. } \\
\text { SIEVE } \\
\text { DIAM. } \\
\% \text { FINER } \\
\text { THAN } \\
.062 \mathrm{MM}\end{array}$ & $\begin{array}{l}\text { SPE- } \\
\text { CIFIC } \\
\text { CON- } \\
\text { DUCT- } \\
\text { ANCE } \\
\text { (US/CM) }\end{array}$ & $\begin{array}{c}\text { OXYGEN, } \\
\text { DIS- } \\
\text { SOLVED } \\
\text { (MG/L) }\end{array}$ \\
\hline 445547123065400 & $05-26-94$ & 17.5 & 760 & 26 & 3 & -- & 168 & 9.5 \\
\hline 14190970 & $\begin{array}{l}11-23-94 \\
11-30-94 \\
11-30-94\end{array}$ & $\begin{array}{r}8.5 \\
10.5 \\
10.5\end{array}$ & $\begin{array}{r}-- \\
752 \\
753\end{array}$ & $\begin{array}{r}49 \\
500 \\
650\end{array}$ & $\begin{array}{r}10 \\
164 \\
266\end{array}$ & $\begin{array}{r}100 \\
89 \\
86\end{array}$ & $\begin{array}{l}80 \\
37 \\
40\end{array}$ & $\begin{array}{l}11.2 \\
10.3 \\
10\end{array}$ \\
\hline 445037122573800 & $\begin{array}{l}06-14-94 \\
10-31-94 \\
11-04-94 \\
11-04-94\end{array}$ & $\begin{array}{r}12.5 \\
11.5 \\
9.5 \\
9.5\end{array}$ & 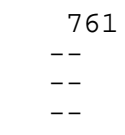 & $\begin{array}{l}187 \\
169 \\
790 \\
875\end{array}$ & $\begin{array}{l}20 \\
35 \\
34 \\
20\end{array}$ & $\begin{array}{l}43 \\
95 \\
75 \\
91\end{array}$ & $\begin{array}{r}45 \\
130 \\
112 \\
109\end{array}$ & $\begin{array}{r}11.1 \\
9.3 \\
10.1 \\
10.0\end{array}$ \\
\hline 14194150 & $\begin{array}{l}05-17-94 \\
11-02-94\end{array}$ & $\begin{array}{r}15.0 \\
9.5\end{array}$ & $\begin{array}{l}758 \\
755\end{array}$ & $\begin{array}{r}700 \\
4840\end{array}$ & $\begin{array}{l}11 \\
60\end{array}$ & $\begin{array}{l}95 \\
87\end{array}$ & $\begin{array}{r}104 \\
63\end{array}$ & $\begin{array}{l}9.6 \\
9.7\end{array}$ \\
\hline 451355123093600 & $05-17-94$ & 13.0 & 759 & 124 & 8 & 93 & 119 & 10.1 \\
\hline 451602122564400 & $05-31-94$ & 17.0 & -- & 7260 & 5 & -- & 90 & 9.5 \\
\hline 451502122524700 & $\begin{array}{l}05-26-94 \\
10-31-94\end{array}$ & $\begin{array}{l}16.0 \\
--\end{array}$ & $\begin{array}{l}761 \\
751\end{array}$ & $\begin{array}{l}8.6 \\
32\end{array}$ & $\begin{array}{r}7 \\
31\end{array}$ & -- & $\begin{array}{l}300 \\
251\end{array}$ & $\begin{array}{l}8.4 \\
--\end{array}$ \\
\hline 14201300 & $\begin{array}{l}05-25-94 \\
06-13-94 \\
10-28-94 \\
11-28-94\end{array}$ & $\begin{array}{r}17.5 \\
16.5 \\
12.5 \\
8.5\end{array}$ & $\begin{array}{l}761 \\
757 \\
764 \\
767\end{array}$ & $\begin{array}{l}0.32 \\
2.5 \\
82 \\
41\end{array}$ & $\begin{array}{r}6 \\
7 \\
72 \\
11\end{array}$ & $\begin{array}{r}98 \\
98 \\
98 \\
100\end{array}$ & $\begin{array}{l}386 \\
407 \\
433 \\
389\end{array}$ & $\begin{array}{r}7.2 \\
7.0 \\
5.8 \\
10.3\end{array}$ \\
\hline 14202000 & $\begin{array}{l}04-12-94 \\
05-25-94 \\
06-15-94 \\
10-28-94 \\
10-29-94 \\
11-09-94 \\
11-29-94\end{array}$ & $\begin{array}{r}10.0 \\
18.5 \\
15.0 \\
12.0 \\
11.0 \\
8.0 \\
6.5\end{array}$ & $\begin{array}{c}766 \\
764 \\
763 \\
764 \\
-- \\
-- \\
762\end{array}$ & $\begin{array}{r}2450 \\
300 \\
482 \\
3650 \\
3100 \\
2220 \\
2390\end{array}$ & $\begin{array}{r}39 \\
<1 \\
9 \\
162 \\
68 \\
45 \\
19\end{array}$ & $\begin{array}{r}73 \\
100 \\
94 \\
89 \\
88 \\
90 \\
86\end{array}$ & $\begin{array}{r}63 \\
95 \\
77 \\
100 \\
88 \\
93 \\
77\end{array}$ & $\begin{array}{r}9.8 \\
7.7 \\
9.4 \\
8.4 \\
9.1 \\
10.4 \\
11.1\end{array}$ \\
\hline 451603122423301 & $05-25-94$ & 18.5 & 764 & 123 & -- & -- & 53 & 9.8 \\
\hline 14206200 & $\begin{array}{l}05-27-94 \\
12-01-94\end{array}$ & $\begin{array}{r}14.0 \\
8.5\end{array}$ & $\begin{array}{l}761 \\
755\end{array}$ & $\begin{array}{r}64 \\
1350\end{array}$ & $\begin{array}{l}13 \\
90\end{array}$ & $\begin{array}{l}97 \\
95\end{array}$ & $\begin{array}{l}92 \\
95\end{array}$ & $\begin{array}{l}8.4 \\
9.2\end{array}$ \\
\hline
\end{tabular}


Table 8. Ancillary data from Phase II of the Willamette River Basin Water Quality Study, Oregon, 1994-Continued

\begin{tabular}{|c|c|c|c|c|c|c|c|c|}
\hline STATION NUMBER & DATE & $\begin{array}{c}\text { OXYGEN, } \\
\text { DIS- } \\
\text { SOLVED } \\
\text { (PER- } \\
\text { CENT } \\
\text { SATUR- } \\
\text { ATION) }\end{array}$ & $\begin{array}{l}\text { PH } \\
\text { WATER } \\
\text { WHOLE } \\
\text { FIELD } \\
\text { (STAND- } \\
\text { ARD } \\
\text { UNITS) }\end{array}$ & $\begin{array}{l}\text { CARBON, } \\
\text { ORGANIC } \\
\text { DIS- } \\
\text { SOLVED } \\
(\mathrm{MG} / \mathrm{L} \\
\text { AS C) }\end{array}$ & $\begin{array}{l}\text { CARBON, } \\
\text { ORGANIC } \\
\text { SUS- } \\
\text { PENDED } \\
(\mathrm{MG} / \mathrm{L} \\
\text { AS C) }\end{array}$ & $\begin{array}{l}\text { CALCIUM } \\
\text { DIS- } \\
\text { SOLVED } \\
\text { (MG/L } \\
\text { AS CA) }\end{array}$ & $\begin{array}{l}\text { MAGNE- } \\
\text { SIUM, } \\
\text { DIS- } \\
\text { SOLVED } \\
\text { (MG/L } \\
\text { AS MG) }\end{array}$ & $\begin{array}{l}\text { HARD- } \\
\text { NESS } \\
\text { TOTAL } \\
\text { (MG/L } \\
\text { AS } \\
\text { CACO3) }\end{array}$ \\
\hline 445547123065400 & $05-26-94$ & 99 & 7.2 & 1.9 & 0.4 & -- & -- & -- \\
\hline 14190970 & $\begin{array}{l}11-23-94 \\
11-30-94 \\
11-30-94\end{array}$ & $\begin{array}{r}-- \\
93 \\
90\end{array}$ & $\begin{array}{l}7.1 \\
6.7 \\
6.5\end{array}$ & $\begin{array}{l}2.1 \\
2.5 \\
2.4\end{array}$ & $\begin{array}{l}0.6 \\
7.4 \\
8.1\end{array}$ & $\begin{array}{l}6.4 \\
3.6 \\
3.2\end{array}$ & $\begin{array}{l}2.8 \\
1.2 \\
1.0\end{array}$ & $\begin{array}{l}28 \\
14 \\
12\end{array}$ \\
\hline 445037122573800 & $\begin{array}{l}06-14-94 \\
10-31-94 \\
11-04-94 \\
11-04-94\end{array}$ & $\begin{array}{l}104 \\
-- \\
-- \\
--\end{array}$ & $\begin{array}{l}7.3 \\
7.1 \\
6.7 \\
6.7\end{array}$ & $\begin{array}{l}1.2 \\
3.1 \\
4.3 \\
4.5\end{array}$ & $\begin{array}{l}0.7 \\
1.4 \\
1.4 \\
1.0\end{array}$ & $\begin{array}{l}-- \\
-- \\
-- \\
--\end{array}$ & $\begin{array}{l}-- \\
-- \\
-- \\
--\end{array}$ & $\begin{array}{l}-- \\
-- \\
-- \\
--\end{array}$ \\
\hline 14194150 & $\begin{array}{l}05-17-94 \\
11-02-94\end{array}$ & $\begin{array}{l}95 \\
86\end{array}$ & $\begin{array}{l}7.5 \\
6.9\end{array}$ & $\begin{array}{l}1.4 \\
3.9\end{array}$ & $\begin{array}{l}0.3 \\
1.4\end{array}$ & $\begin{array}{l}9.4 \\
--\end{array}$ & $\begin{array}{l}3.0 \\
--\end{array}$ & $-{ }_{-}^{36}$ \\
\hline 451355123093600 & $05-17-94$ & 96 & 7.6 & 2.1 & 0.3 & -- & -- & -- \\
\hline 451602122564400 & $05-31-94$ & -- & 7.5 & 1.7 & 0.4 & 7.0 & 2.4 & 27 \\
\hline 451502122524700 & $\begin{array}{l}05-26-94 \\
10-31-94\end{array}$ & --85 & $\begin{array}{l}7.8 \\
--\end{array}$ & $\begin{array}{l}4.0 \\
7.8\end{array}$ & $\begin{array}{l}0.6 \\
2.0\end{array}$ & $\begin{array}{l}-- \\
--\end{array}$ & $\begin{array}{l}-- \\
--\end{array}$ & $\begin{array}{l}-- \\
--\end{array}$ \\
\hline 14201300 & $\begin{array}{l}05-25-94 \\
06-13-94 \\
10-28-94 \\
11-28-94\end{array}$ & $\begin{array}{l}75 \\
72 \\
54 \\
87\end{array}$ & $\begin{array}{l}7.4 \\
7.4 \\
6.7 \\
7.1\end{array}$ & $\begin{array}{l}3.5 \\
4.2 \\
7.8 \\
3.3\end{array}$ & $\begin{array}{l}0.4 \\
0.6 \\
-- \\
0.7\end{array}$ & $\begin{array}{l}-- \\
37 \\
38 \\
35\end{array}$ & $\begin{array}{l}-- \\
12 \\
12 \\
12\end{array}$ & $\begin{array}{l}-- \\
140 \\
140 \\
140\end{array}$ \\
\hline 14202000 & $\begin{array}{l}04-12-94 \\
05-25-94 \\
06-15-94 \\
10-28-94 \\
10-29-94 \\
11-09-94 \\
11-29-94\end{array}$ & $\begin{array}{r}87 \\
82 \\
93 \\
-78 \\
-- \\
-- \\
-91\end{array}$ & $\begin{array}{l}7.0 \\
7.3 \\
7.3 \\
6.7 \\
6.8 \\
6.8 \\
7.0\end{array}$ & $\begin{array}{l}1.6 \\
1.7 \\
1.6 \\
5.5 \\
5.5 \\
3.4 \\
2.0\end{array}$ & $\begin{array}{l}1.5 \\
0.4 \\
0.6 \\
1.6 \\
2.4 \\
1.2 \\
0.6\end{array}$ & $\begin{array}{l}5.5 \\
-- \\
6.2 \\
8.0 \\
-- \\
-- \\
7.1\end{array}$ & $\begin{array}{l}2.0 \\
-- \\
2.5 \\
2.7 \\
-- \\
-- \\
2.6\end{array}$ & $\begin{array}{l}-22 \\
-- \\
26 \\
-- \\
-- \\
-\end{array}$ \\
\hline 451603122423301 & $05-25-94$ & 104 & 7.5 & 1.1 & 0.3 & -- & -- & -- \\
\hline 14206200 & $\begin{array}{l}05-27-94 \\
12-01-94\end{array}$ & $\begin{array}{l}82 \\
80\end{array}$ & $\begin{array}{l}7.2 \\
6.6\end{array}$ & $\begin{array}{l}5.7 \\
4.1\end{array}$ & $\begin{array}{l}0.4 \\
3.1\end{array}$ & $\begin{array}{l}-- \\
7.6\end{array}$ & $\begin{array}{l}-- \\
2.8\end{array}$ & ${ }^{--} 31$ \\
\hline
\end{tabular}


Table 8. Ancillary data from Phase II of the Willamette River Basin Water Quality Study, Oregon, 1994-Continued

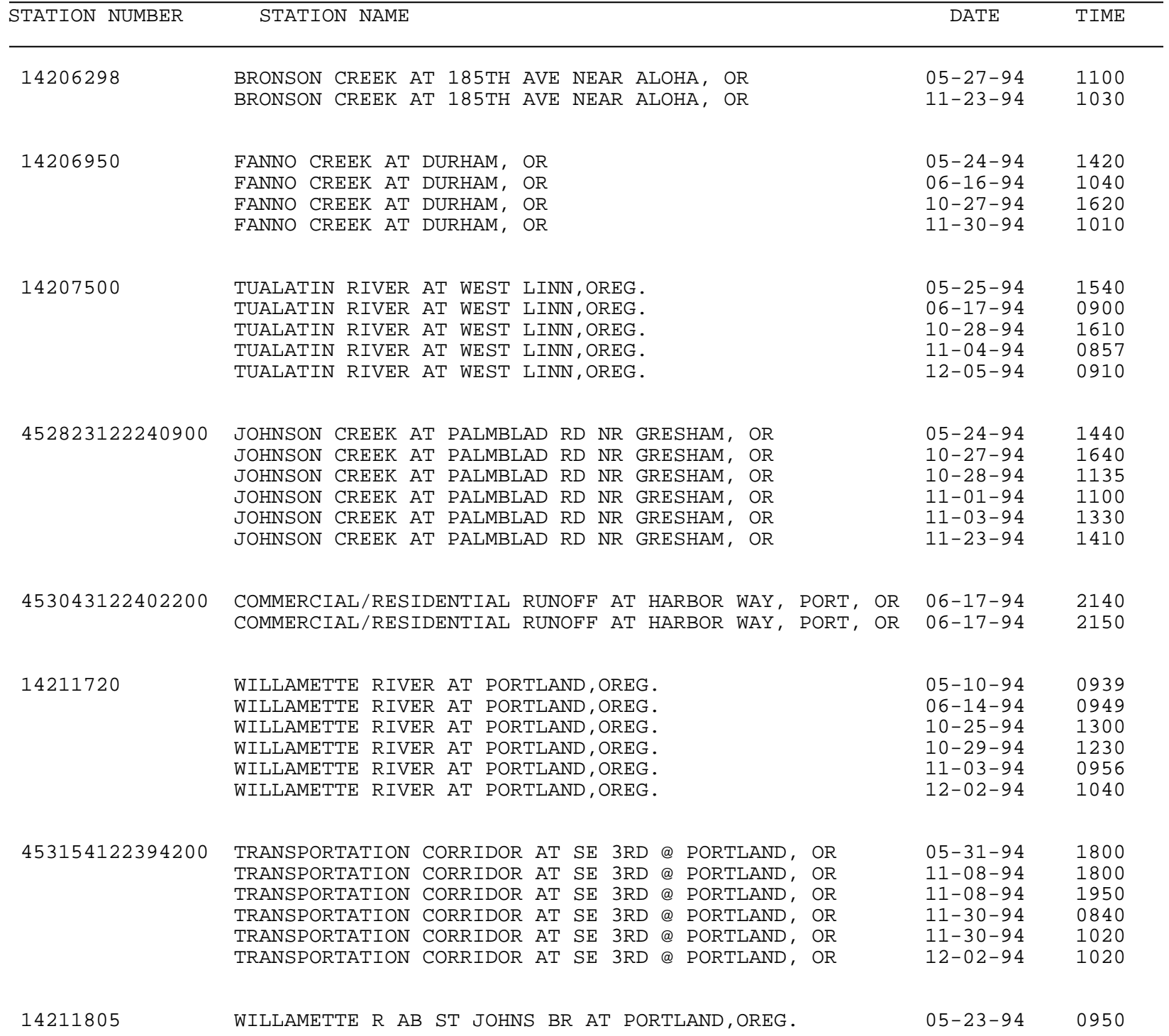


Table 8. Ancillary data from Phase II of the Willamette River Basin Water Quality Study, Oregon, 1994-Continued

\begin{tabular}{|c|c|c|c|c|c|c|c|c|}
\hline STATION NUMBER & DATE & 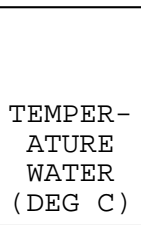 & $\begin{array}{l}\text { BARO- } \\
\text { METRIC } \\
\text { PRES- } \\
\text { SURE } \\
\text { (MM } \\
\text { OF } \\
\text { HG) }\end{array}$ & $\begin{array}{c}\text { DIS- } \\
\text { CHARGE, } \\
\text { INST. } \\
\text { CUBIC } \\
\text { FEET } \\
\text { PER } \\
\text { SECOND }\end{array}$ & $\begin{array}{l}\text { SEDI- } \\
\text { MENT, } \\
\text { SUS- } \\
\text { PENDED } \\
\text { (MG/L) }\end{array}$ & $\begin{array}{c}\text { SED. } \\
\text { SUSP. } \\
\text { SIEVE } \\
\text { DIAM. } \\
\text { FINER } \\
\text { THAN } \\
.062 \text { MM }\end{array}$ & $\begin{array}{l}\text { SPE- } \\
\text { CIFIC } \\
\text { CON- } \\
\text { DUCT- } \\
\text { ANCE } \\
\text { (US/CM) }\end{array}$ & $\begin{array}{c}\text { OXYGEN, } \\
\text { DIS- } \\
\text { SOLVED } \\
\text { (MG/L) }\end{array}$ \\
\hline 14206298 & $\begin{array}{l}05-27-94 \\
11-23-94\end{array}$ & $\begin{array}{r}20.0 \\
5.0\end{array}$ & $\begin{array}{l}762 \\
760\end{array}$ & $\begin{array}{l}0.51 \\
6.2\end{array}$ & $\begin{array}{r}7 \\
18\end{array}$ & $\begin{array}{r}71 \\
100\end{array}$ & $\begin{array}{l}237 \\
168\end{array}$ & $\begin{array}{r}6.9 \\
11.3\end{array}$ \\
\hline 14206950 & $\begin{array}{l}05-24-94 \\
06-16-94 \\
10-27-94 \\
11-30-94\end{array}$ & $\begin{array}{r}18.5 \\
15.0 \\
13.0 \\
9.5\end{array}$ & $\begin{array}{l}759 \\
765 \\
755 \\
757\end{array}$ & $\begin{array}{r}7.6 \\
9.1 \\
946 \\
179\end{array}$ & $\begin{array}{r}8 \\
7 \\
770 \\
166\end{array}$ & $\begin{array}{r}100 \\
92 \\
93 \\
95\end{array}$ & $\begin{array}{r}235 \\
194 \\
67 \\
122\end{array}$ & $\begin{array}{r}10.4 \\
8.2 \\
7.6 \\
10.4\end{array}$ \\
\hline 14207500 & $\begin{array}{l}05-25-94 \\
06-17-94 \\
10-28-94 \\
11-04-94 \\
12-05-94\end{array}$ & $\begin{array}{r}19.0 \\
17.5 \\
12.5 \\
9.0 \\
6.0\end{array}$ & $\begin{array}{r}761 \\
766 \\
-- \\
749 \\
758\end{array}$ & $\begin{array}{r}233 \\
230 \\
1640 \\
2020 \\
4470\end{array}$ & $\begin{array}{r}12 \\
9 \\
48 \\
31 \\
51\end{array}$ & $\begin{array}{l}-- \\
-- \\
95 \\
96 \\
97\end{array}$ & $\begin{array}{r}213 \\
254 \\
163 \\
112 \\
82\end{array}$ & $\begin{array}{r}10.5 \\
9.7 \\
7.9 \\
8.9 \\
10.0\end{array}$ \\
\hline 452823122240900 & $\begin{array}{l}05-24-94 \\
10-27-94 \\
10-28-94 \\
11-01-94 \\
11-03-94 \\
11-23-94\end{array}$ & $\begin{array}{r}17.5 \\
12.5 \\
12.0 \\
10.0 \\
9.0 \\
7.0\end{array}$ & $\begin{array}{r}752 \\
754 \\
762 \\
-- \\
755 \\
755\end{array}$ & $\begin{array}{l}1.8 \\
246 \\
68 \\
283 \\
67 \\
50\end{array}$ & $\begin{array}{r}5 \\
266 \\
48 \\
91 \\
14 \\
18\end{array}$ & $\begin{array}{r}96 \\
92 \\
96 \\
95 \\
94 \\
100\end{array}$ & $\begin{array}{r}102 \\
108 \\
116 \\
95 \\
96 \\
85\end{array}$ & $\begin{array}{r}9.5 \\
9.8 \\
10.4 \\
9.9 \\
10.7 \\
11.8\end{array}$ \\
\hline 453043122402200 & $\begin{array}{l}06-17-94 \\
06-17-94\end{array}$ & $\begin{array}{l}18.0 \\
18.0\end{array}$ & $\begin{array}{l}761 \\
761\end{array}$ & $\begin{array}{l}3.0 \\
3.0\end{array}$ & $\begin{array}{l}42 \\
--\end{array}$ & $\begin{array}{l}-- \\
--\end{array}$ & $\begin{array}{l}195 \\
195\end{array}$ & $\begin{array}{l}9.3 \\
9.3\end{array}$ \\
\hline 14211720 & $\begin{array}{l}05-10-94 \\
06-14-94 \\
10-25-94 \\
10-29-94 \\
11-03-94 \\
12-02-94\end{array}$ & $\begin{array}{r}16.5 \\
17.5 \\
13.0 \\
12.0 \\
9.5 \\
8.0\end{array}$ & $\begin{array}{l}766 \\
764 \\
757 \\
765 \\
766 \\
758\end{array}$ & $\begin{array}{r}13000 \\
10800 \\
9740 \\
47300 \\
81800 \\
108000\end{array}$ & $\begin{array}{r}6 \\
10 \\
7 \\
30 \\
81 \\
120\end{array}$ & $\begin{array}{r}\mathrm{E} 93 \\
94 \\
100 \\
100 \\
96 \\
88\end{array}$ & $\begin{array}{l}90 \\
79 \\
88 \\
79 \\
59 \\
57\end{array}$ & $\begin{array}{r}9.8 \\
8.8 \\
9.6 \\
11.4 \\
12.6 \\
13.5\end{array}$ \\
\hline 453154122394200 & $\begin{array}{l}05-31-94 \\
11-08-94 \\
11-08-94 \\
11-30-94 \\
11-30-94 \\
12-02-94\end{array}$ & $\begin{array}{r}17.0 \\
9.0 \\
9.0 \\
11.0 \\
11.5 \\
12.0\end{array}$ & $\begin{array}{r}763 \\
750 \\
750 \\
758 \\
758 \\
--\end{array}$ & $\begin{array}{l}0.20 \\
-- \\
-- \\
-- \\
-- \\
--\end{array}$ & $\begin{array}{r}97 \\
127 \\
59 \\
233 \\
215 \\
17\end{array}$ & $\begin{array}{l}-- \\
96 \\
97 \\
96 \\
93 \\
78\end{array}$ & $\begin{array}{r}227 \\
89 \\
99 \\
76 \\
90 \\
274\end{array}$ & $\begin{array}{r}8.4 \\
10.7 \\
10.3 \\
10.3 \\
10.0 \\
--\end{array}$ \\
\hline 14211805 & $05-23-94$ & 16.5 & 768 & 17800 & 14 & 97 & 120 & 9.3 \\
\hline
\end{tabular}


Table 8. Ancillary data from Phase II of the Willamette River Basin Water Quality Study, Oregon, 1994-Continued

\begin{tabular}{|c|c|c|c|c|c|c|c|c|}
\hline STATION NUMBER & DATE & $\begin{array}{c}\text { OXYGEN, } \\
\text { DIS- } \\
\text { SOLVED } \\
\text { (PER- } \\
\text { CENT } \\
\text { SATUR- } \\
\text { ATION) }\end{array}$ & $\begin{array}{l}\text { PH } \\
\text { WATER } \\
\text { WHOLE } \\
\text { FIELD } \\
\text { (STAND- } \\
\text { ARD } \\
\text { UNITS) }\end{array}$ & $\begin{array}{l}\text { CARBON, } \\
\text { ORGANIC } \\
\text { DIS- } \\
\text { SOLVED } \\
(\mathrm{MG} / \mathrm{L} \\
\text { AS C) }\end{array}$ & $\begin{array}{l}\text { CARBON, } \\
\text { ORGANIC } \\
\text { SUS- } \\
\text { PENDED } \\
(\text { MG / L } \\
\text { AS C) }\end{array}$ & $\begin{array}{l}\text { CALCIUM, } \\
\text { DIS- } \\
\text { SOLVED } \\
\text { (MG/L } \\
\text { AS CA) }\end{array}$ & $\begin{array}{l}\text { MAGNE- } \\
\text { SIUM, } \\
\text { DIS- } \\
\text { SOLVED } \\
\text { (MG/L } \\
\text { AS MG) }\end{array}$ & $\begin{array}{l}\text { HARD- } \\
\text { NESS } \\
\text { TOTAL } \\
\text { (MG/L } \\
\text { AS } \\
\text { CACO3) }\end{array}$ \\
\hline 14206298 & $\begin{array}{l}05-27-94 \\
11-23-94\end{array}$ & $\begin{array}{l}76 \\
88\end{array}$ & $\begin{array}{l}7.7 \\
7.2\end{array}$ & $\begin{array}{l}2.1 \\
5.1\end{array}$ & $\begin{array}{l}0.4 \\
1.1\end{array}$ & $\begin{array}{l}21 \\
14\end{array}$ & $\begin{array}{l}8.5 \\
5.0\end{array}$ & $\begin{array}{l}87 \\
56\end{array}$ \\
\hline 14206950 & $\begin{array}{l}05-24-94 \\
06-16-94 \\
10-27-94 \\
11-30-94\end{array}$ & $\begin{array}{r}112 \\
80 \\
72 \\
92\end{array}$ & $\begin{array}{l}7.7 \\
7.3 \\
6.7 \\
7.3\end{array}$ & $\begin{array}{l}3.6 \\
4.4 \\
6.5 \\
4.4\end{array}$ & $\begin{array}{l}0.6 \\
0.3 \\
-- \\
4.6\end{array}$ & $\begin{array}{c}-- \\
21 \\
5.3 \\
11\end{array}$ & $\begin{array}{l}-- \\
7.4 \\
1.8 \\
3.5\end{array}$ & $\begin{array}{r}-- \\
83 \\
21 \\
42\end{array}$ \\
\hline 14207500 & $\begin{array}{l}05-25-94 \\
06-17-94 \\
10-28-94 \\
11-04-94 \\
12-05-94\end{array}$ & $\begin{array}{r}114 \\
101 \\
-- \\
79 \\
80\end{array}$ & $\begin{array}{l}7.9 \\
7.6 \\
6.9 \\
6.8 \\
7.0\end{array}$ & $\begin{array}{l}2.8 \\
3.3 \\
5.1 \\
-- \\
3.4\end{array}$ & $\begin{array}{l}1.7 \\
1.9 \\
-- \\
-- \\
2.0\end{array}$ & $\begin{array}{l}17 \\
20 \\
14 \\
9.7 \\
7.2\end{array}$ & $\begin{array}{l}4.8 \\
5.2 \\
3.7 \\
2.9 \\
2.4\end{array}$ & $\begin{array}{l}62 \\
71 \\
50 \\
36 \\
28\end{array}$ \\
\hline 452823122240900 & $\begin{array}{l}05-24-94 \\
10-27-94 \\
10-28-94 \\
11-01-94 \\
11-03-94 \\
11-23-94\end{array}$ & $\begin{array}{r}100 \\
93 \\
96 \\
--\quad \\
94 \\
99\end{array}$ & $\begin{array}{l}7.4 \\
6.6 \\
6.8 \\
6.5 \\
6.8 \\
7.1\end{array}$ & $\begin{array}{l}3.1 \\
6.6 \\
3.9 \\
3.4 \\
2.2 \\
2.9\end{array}$ & $\begin{array}{l}0.5 \\
5.7 \\
1.8 \\
2.2 \\
0.5 \\
0.7\end{array}$ & $\begin{array}{l}-- \\
-- \\
-- \\
-- \\
-- \\
--\end{array}$ & $\begin{array}{l}-- \\
-- \\
-- \\
-- \\
-- \\
--\end{array}$ & $\begin{array}{l}-- \\
-- \\
-- \\
-- \\
-- \\
--\end{array}$ \\
\hline 453043122402200 & $\begin{array}{l}06-17-94 \\
06-17-94\end{array}$ & $\begin{array}{l}98 \\
98\end{array}$ & $\begin{array}{l}7.3 \\
7.3\end{array}$ & $\begin{array}{l}26 \\
4.8\end{array}$ & $\begin{array}{l}8.4 \\
0.3\end{array}$ & $\stackrel{12}{--}$ & $\begin{array}{l}3.4 \\
--\end{array}$ & $-{ }_{-}^{44}$ \\
\hline 14211720 & $\begin{array}{l}05-10-94 \\
06-14-94 \\
10-25-94 \\
10-29-94 \\
11-03-94 \\
12-02-94\end{array}$ & $\begin{array}{r}100 \\
92 \\
93 \\
106 \\
109 \\
115\end{array}$ & $\begin{array}{l}7.2 \\
7.2 \\
7.2 \\
7.2 \\
7.0 \\
7.0\end{array}$ & $\begin{array}{l}1.7 \\
1.6 \\
1.8 \\
3.4 \\
3.8 \\
2.5\end{array}$ & $\begin{array}{l}0.5 \\
0.7 \\
-- \\
1.1 \\
2.4 \\
3.3\end{array}$ & $\begin{array}{l}7.0 \\
6.2 \\
6.8 \\
6.5 \\
5.2 \\
5.0\end{array}$ & $\begin{array}{l}2.4 \\
2.1 \\
2.1 \\
2.1 \\
1.6 \\
1.6\end{array}$ & $\begin{array}{l}27 \\
24 \\
26 \\
25 \\
20 \\
19\end{array}$ \\
\hline 453154122394200 & $\begin{array}{l}05-31-94 \\
11-08-94 \\
11-08-94 \\
11-30-94 \\
11-30-94 \\
12-02-94\end{array}$ & $\begin{array}{r}87 \\
93 \\
91 \\
94 \\
92\end{array}$ & $\begin{array}{l}7.7 \\
7.2 \\
7.3 \\
7.8 \\
7.9 \\
8.0\end{array}$ & $\begin{array}{r}28 \\
6.9 \\
7.8 \\
4.8 \\
5.0 \\
3.4\end{array}$ & $\begin{array}{c}6.3 \\
7.4 \\
3.9 \\
16 \\
13 \\
1.1\end{array}$ & $\begin{array}{l}29 \\
12 \\
15 \\
12 \\
15 \\
32\end{array}$ & $\begin{array}{l}2.9 \\
1.0 \\
1.6 \\
0.70 \\
1.0 \\
7.8\end{array}$ & $\begin{array}{r}84 \\
34 \\
44 \\
33 \\
42 \\
110\end{array}$ \\
\hline 14211805 & $05-23-94$ & 95 & 7.2 & 1.5 & 0.6 & 8.1 & 2.9 & 32 \\
\hline
\end{tabular}


Table 9. Concentrations of nutrients in whole and filtered water from Phase I of the Willamette River Basin Water Quality Study, Oregon, 1993

[USGS laboratory schedule 2702]

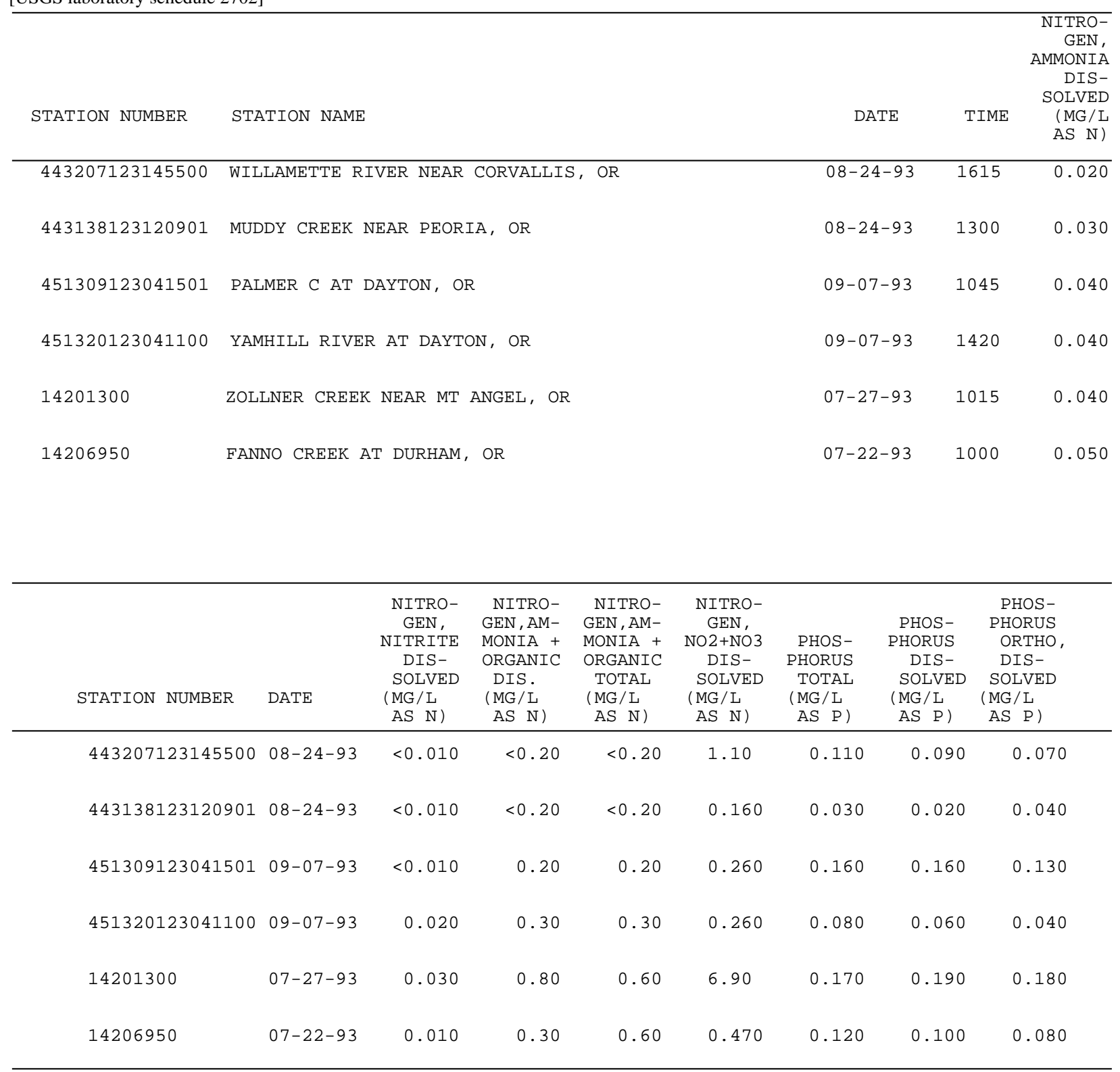


Table 10. Concentrations of nutrients in whole and filtered water from Phase II of the Willamette River Basin Water Quality Study, Oregon, 1994

USGS laboratory schedule 2702]

\begin{tabular}{|c|c|c|c|c|}
\hline STATION NUMBER & STATION NAME & DATE & TIME & $\begin{array}{l}\text { NITRO- } \\
\text { GEN, } \\
\text { AMMONIA } \\
\text { DIS- } \\
\text { SOLVED } \\
\text { (MG/L } \\
\text { AS N) }\end{array}$ \\
\hline 433548123040600 & COAST FORK WILLAMETTE R BL BIG RIVER NR LONDON, OR & $05-20-94$ & 1040 & 0.030 \\
\hline 440045122585600 & COAST FORK WILLAM AT SEAVY LOOP RD NR EUGENE, OR & $05-19-94$ & 1020 & 0.040 \\
\hline 440402123063900 & URBAN OUTFALL AT POLK ST. PARK AT EUGENE, OR & $06-13-94$ & 1210 & 0.020 \\
\hline 441310122095801 & MACK CREEK NEAR BLUE RIVER, OR & $05-27-94$ & 1030 & 0.020 \\
\hline 440707123041300 & MCKENZIE RIVER NR EUGENE, OR & $05-19-94$ & 1450 & 0.020 \\
\hline 442223123153703 & $\begin{array}{llllllll}\text { LONG } & \text { TOM } & R & \text { AT } & \text { BUNDY } & \text { BRIDGE } & \text { NR MONROE, } & \text { OR } \\
\text { LONG } & \text { TOM } & R & \text { AT } & \text { BUNDY } & \text { BRIDGE } & \text { NR MONROE, } & \text { OR }\end{array}$ & $\begin{array}{l}05-19-94 \\
11-01-94\end{array}$ & $\begin{array}{l}0950 \\
1720\end{array}$ & $\begin{array}{l}0.020 \\
0.040\end{array}$ \\
\hline 442413123122500 & LAKE CAMOUS CR AT PINE GROVE DR NR HARRISBURG, OR & $05-19-94$ & 1720 & 0.050 \\
\hline 443138123120901 & $\begin{array}{llll}\text { MUDDY } & \text { CREEK } & \text { NEAR } & \text { PEORIA, OR } \\
\text { MUDDY } & \text { CREEK } & \text { NEAR } & \text { PEORIA, OR } \\
\text { MUDDY } & \text { CREEK } & \text { NEAR PEORIA, OR } \\
\text { MUDDY } & \text { CREEK } & \text { NEAR PEORIA, OR } \\
\text { MUDDY } & \text { CREEK } & \text { NEAR PEORIA, OR } \\
\text { MUDDY } & \text { CREEK } & \text { NEAR } & \text { PEORIA, OR }\end{array}$ & $\begin{array}{l}05-26-94 \\
06-03-94 \\
06-20-94 \\
11-02-94 \\
11-06-94 \\
11-09-94\end{array}$ & $\begin{array}{l}1730 \\
0930 \\
1050 \\
1010 \\
1200 \\
1430\end{array}$ & $\begin{array}{l}0.030 \\
0.030 \\
0.030 \\
0.050 \\
0.080 \\
--\end{array}$ \\
\hline 443045123273000 & ROCK CREEK ABOVE GRIFFITH CREEK NR PHILOMATH, OR & $05-18-94$ & 1820 & 0.040 \\
\hline 443321123155201 & MARYS RIVER AT CORVALLIS, OR & $05-18-94$ & 1350 & $<0.010$ \\
\hline 14173500 & $\begin{array}{llll}\text { CALAPOOIA RIVER AT ALBANY, OR } \\
\text { CALAPOOIA RIVER AT ALBANY, OR }\end{array}$ & $\begin{array}{l}05-26-94 \\
11-01-94\end{array}$ & $\begin{array}{l}1220 \\
1230\end{array}$ & $\begin{array}{l}0.020 \\
0.080\end{array}$ \\
\hline 444349123094000 & LUCKIAMUTE R AT BUENA VISTA RD NR BUENA VISTA, OR & $05-16-94$ & 1720 & $<0.010$ \\
\hline 444123122562200 & THOMAS CREEK AT KELLY RD NR JEFFERSON, OR & $05-16-94$ & 1900 & $<0.010$ \\
\hline 14189000 & SANTIAM RIVER AT JEFFERSON, OR & $05-17-94$ & 1620 & $<0.010$ \\
\hline
\end{tabular}


Table 10. Concentrations of nutrients in whole and filtered water from Phase II of the Willamette River Basin Water Quality Study, Oregon, 1994-Continued

\begin{tabular}{|c|c|c|c|c|c|c|c|c|}
\hline STATION NUMBER & DATE & $\begin{array}{c}\text { NITRO- } \\
\text { GEN, } \\
\text { NITRITE } \\
\text { DIS- } \\
\text { SOLVED } \\
\text { (MG/L } \\
\text { AS N) }\end{array}$ & $\begin{array}{l}\text { NITRO- } \\
\text { GEN, AM- } \\
\text { MONIA + } \\
\text { ORGANIC } \\
\text { DIS. } \\
\text { (MG/L } \\
\text { AS N) }\end{array}$ & $\begin{array}{c}\text { NITRO- } \\
\text { GEN, AM- } \\
\text { MONIA + } \\
\text { ORGANIC } \\
\text { TOTAL } \\
\text { (MG/L } \\
\text { AS N) }\end{array}$ & $\begin{array}{c}\text { NITRO- } \\
\text { GEN, } \\
\text { NO2+NO3 } \\
\text { DIS- } \\
\text { SOLVED } \\
\text { (MG/L } \\
\text { AS N) }\end{array}$ & $\begin{array}{c}\text { PHOS- } \\
\text { PHORUS } \\
\text { TOTAL } \\
\text { (MG/L } \\
\text { AS P) }\end{array}$ & $\begin{array}{c}\text { PHOS- } \\
\text { PHORUS } \\
\text { DIS- } \\
\text { SOLVED } \\
\text { (MG/L } \\
\text { AS P) }\end{array}$ & $\begin{array}{l}\text { PHOS- } \\
\text { PHORUS } \\
\text { ORTHO, } \\
\text { DIS- } \\
\text { SOLVED } \\
\text { (MG/L } \\
\text { AS P) }\end{array}$ \\
\hline 433548123040600 & $05-20-94$ & $<0.010$ & $<0.20$ & $<0.20$ & 0.088 & 0.020 & $<0.010$ & 0.010 \\
\hline 440045122585600 & $05-19-94$ & 0.010 & $<0.20$ & 0.40 & 0.130 & 0.140 & 0.020 & 0.010 \\
\hline 440402123063900 & $06-13-94$ & 0.010 & 0.30 & 0.50 & 1.20 & 0.140 & 0.110 & 0.090 \\
\hline 441310122095801 & $05-27-94$ & $<0.010$ & $<0.20$ & $<0.20$ & 0.051 & $<0.010$ & $<0.010$ & 0.010 \\
\hline 440707123041300 & $05-19-94$ & $<0.010$ & $<0.20$ & $<0.20$ & $<0.050$ & 0.010 & 0.020 & 0.030 \\
\hline 442223123153703 & $\begin{array}{l}05-19-94 \\
11-01-94\end{array}$ & $\begin{array}{l}<0.010 \\
<0.010\end{array}$ & $\begin{array}{r}<0.20 \\
0.20\end{array}$ & $\begin{array}{l}0.30 \\
0.90\end{array}$ & $\begin{array}{l}0.880 \\
0.640\end{array}$ & $\begin{array}{l}0.030 \\
0.110\end{array}$ & $\begin{array}{l}0.020 \\
0.040\end{array}$ & $\begin{array}{l}0.010 \\
0.030\end{array}$ \\
\hline 442413123122500 & $05-19-94$ & 0.030 & 0.30 & 0.40 & 0.690 & 0.120 & 0.080 & 0.080 \\
\hline 443138123120901 & $\begin{array}{l}05-26-94 \\
06-03-94 \\
06-20-94 \\
11-02-94 \\
11-06-94 \\
11-09-94\end{array}$ & $\begin{array}{r}<0.010 \\
0.010 \\
<0.010 \\
0.020 \\
0.040 \\
--\end{array}$ & $\begin{aligned} 0.20 \\
<0.20 \\
<0.20 \\
0.40 \\
0.60 \\
--\end{aligned}$ & $\begin{array}{l}0.30 \\
0.30 \\
0.30 \\
1.3 \\
0.80 \\
--\end{array}$ & $\begin{array}{l}1.20 \\
1.60 \\
1.20 \\
2.50 \\
4.10 \\
3.20\end{array}$ & $\begin{array}{l}0.090 \\
0.080 \\
0.110 \\
0.230 \\
0.140 \\
0.080\end{array}$ & $\begin{array}{l}0.080 \\
0.070 \\
0.100 \\
0.070 \\
0.080 \\
--\end{array}$ & $\begin{array}{l}0.090 \\
0.070 \\
0.090 \\
0.080 \\
0.070 \\
--\end{array}$ \\
\hline 443045123273000 & $05-18-94$ & $<0.010$ & $<0.20$ & $<0.20$ & $<0.050$ & $<0.010$ & $<0.010$ & $<0.010$ \\
\hline 443321123155201 & $05-18-94$ & $<0.010$ & $<0.20$ & $<0.20$ & 0.120 & 0.020 & 0.020 & 0.020 \\
\hline 14173500 & $\begin{array}{l}05-26-94 \\
11-01-94\end{array}$ & $\begin{array}{r}<0.010 \\
0.020\end{array}$ & $\begin{array}{r}<0.20 \\
0.30\end{array}$ & $\begin{array}{r}<0.20 \\
0.80\end{array}$ & $\begin{array}{l}0.710 \\
1.70\end{array}$ & $\begin{array}{l}0.020 \\
0.260\end{array}$ & $\begin{array}{r}<0.010 \\
0.080\end{array}$ & $\begin{array}{l}0.020 \\
0.090\end{array}$ \\
\hline 444349123094000 & $05-16-94$ & $<0.010$ & $<0.20$ & $<0.20$ & 0.200 & 0.010 & $<0.010$ & 0.010 \\
\hline 444123122562200 & $05-16-94$ & $<0.010$ & $<0.20$ & $<0.20$ & 0.120 & $<0.010$ & $<0.010$ & $<0.010$ \\
\hline 14189000 & $05-17-94$ & $<0.010$ & $<0.20$ & 0.30 & 0.150 & 0.020 & $<0.010$ & $<0.010$ \\
\hline
\end{tabular}


Table 10. Concentrations of nutrients in whole and filtered water from Phase II of the Willamette River Basin Water Quality Study, Oregon, 1994-Continued

\begin{tabular}{|c|c|c|c|c|}
\hline STATION NUMBER & STATION NAME & DATE & TIME & $\begin{array}{l}\text { NITRO- } \\
\text { GEN, } \\
\text { AMMONIA } \\
\text { DIS- } \\
\text { SOLVED } \\
\text { (MG/L } \\
\text { AS N) }\end{array}$ \\
\hline 445547123065400 & RICKREAL CREEK NR MOUTH NR SALEM, OR & $05-26-94$ & 1540 & 0.020 \\
\hline 14190970 & $\begin{array}{lllllll}\text { PRINGLE } & \text { AT } & \text { BUSH PARK AT } & \text { SALEM, OR } \\
\text { PRINGLE } & \text { C } & \text { AT } & \text { BUSH PARK } & \text { AT } & \text { SALEM, OR } \\
\text { PRINGLE } & \text { C } & \text { AT } & \text { BUSH PARK } & \text { AT } & \text { SALEM, OR }\end{array}$ & $\begin{array}{l}11-23-94 \\
11-30-94 \\
11-30-94\end{array}$ & $\begin{array}{l}1200 \\
1530 \\
1710\end{array}$ & $\begin{array}{l}-- \\
-- \\
--\end{array}$ \\
\hline 445037122573800 & $\begin{array}{llllll}\text { MILL CREEK AT DELANEY ROAD } & \text { NR } & \text { TURNER, OR } \\
\text { MILL CREEK AT } & \text { DELANEY ROAD NR TURNER, OR } \\
\text { MILL CREEK AT } & \text { DELANEY ROAD NR TURNER, OR } \\
\text { MILL CREEK AT } & \text { DELANEY ROAD NR TURNER, OR }\end{array}$ & $\begin{array}{l}06-14-94 \\
10-31-94 \\
11-04-94 \\
11-04-94\end{array}$ & $\begin{array}{l}1140 \\
1410 \\
1120 \\
1420\end{array}$ & $\begin{array}{l}0.030 \\
-- \\
-- \\
--\end{array}$ \\
\hline 14194150 & $\begin{array}{llll}\text { SOUTH YAMHILL RIVER AT MCMINNVILLE, OR } \\
\text { SOUTH YAMHILL RIVER AT MCMINNVILLE, OR }\end{array}$ & $\begin{array}{l}05-17-94 \\
11-02-94\end{array}$ & $\begin{array}{l}1720 \\
1210\end{array}$ & $\begin{array}{c}<0.010 \\
--\end{array}$ \\
\hline 451355123093600 & NORTH YAMHILL RIVER AT HWY. 99E NR MCMINNVILLE, OR & $05-17-94$ & 1220 & $<0.010$ \\
\hline 451602122564400 & WILLAMETTE RIVER NR NEWBERG, OR & $05-31-94$ & 1400 & 0.060 \\
\hline 451502122524700 & $\begin{array}{l}\text { CHAMPOEG CREEK BELOW MISSION CR NR BUTTEVILLE, OR } \\
\text { CHAMPOEG CREEK BELOW MISSION CR NR BUTTEVILLE, OR }\end{array}$ & $\begin{array}{l}05-26-94 \\
10-31-94\end{array}$ & $\begin{array}{l}1030 \\
1300\end{array}$ & $\begin{array}{l}0.030 \\
--\end{array}$ \\
\hline 14201300 & $\begin{array}{llll}\text { ZOLLNER CREEK NEAR MT ANGEL, OR } \\
\text { ZOLLNER CREEK NEAR MT ANGEL, OR } \\
\text { ZOLLNER CREEK NEAR MT ANGEL, OR } \\
\text { ZOLLNER CREEK NEAR MT ANGEL, OR }\end{array}$ & $\begin{array}{l}05-25-94 \\
06-13-94 \\
10-28-94 \\
11-28-94\end{array}$ & $\begin{array}{l}1450 \\
1120 \\
1320 \\
1400\end{array}$ & $\begin{array}{l}0.070 \\
0.050 \\
0.270 \\
0.360\end{array}$ \\
\hline 14202000 & $\begin{array}{lll}\text { PUDDING RIVER AT AURORA, OREG. } \\
\text { PUDDING RIVER AT AURORA, OREG. } \\
\text { PUDDING RIVER AT AURORA, OREG. } \\
\text { PUDDING RIVER AT AURORA, OREG. } \\
\text { PUDDING RIVER AT AURORA, OREG. } \\
\text { PUDDING RIVER AT AURORA, OREG. } \\
\text { PUDDING RIVER AT AURORA, OREG. }\end{array}$ & $\begin{array}{l}04-12-94 \\
05-25-94 \\
06-15-94 \\
10-28-94 \\
10-29-94 \\
11-09-94 \\
11-29-94\end{array}$ & $\begin{array}{l}1110 \\
1100 \\
1145 \\
1710 \\
1230 \\
1300 \\
1110\end{array}$ & $\begin{array}{l}0.020 \\
0.060 \\
0.060 \\
0.050 \\
0.040 \\
-- \\
0.040\end{array}$ \\
\hline 451603122423301 & MOLALLA R AT KNIGHTS BRIDGE NR CANBY, OR & $05-25-94$ & 1020 & 0.050 \\
\hline 14206200 & $\begin{array}{llllll}\text { DAIRY CREEK AT RTE } 8 \text { NEAR HILLSBORO, OR } \\
\text { DAIRY CREEK AT RTE } 8 \text { NEAR HILLSBORO, OR }\end{array}$ & $\begin{array}{l}05-27-94 \\
12-01-94\end{array}$ & $\begin{array}{l}1640 \\
1140\end{array}$ & $\begin{array}{l}0.030 \\
--\end{array}$ \\
\hline
\end{tabular}


Table 10. Concentrations of nutrients in whole and filtered water from Phase II of the Willamette River Basin Water Quality Study, Oregon, 1994-Continued

\begin{tabular}{|c|c|c|c|c|c|c|c|c|}
\hline STATION NUMBER & DATE & $\begin{array}{c}\text { NITRO- } \\
\text { GEN, } \\
\text { NITRITE } \\
\text { DIS- } \\
\text { SOLVED } \\
\text { (MG/L } \\
\text { AS N) }\end{array}$ & $\begin{array}{l}\text { NITRO- } \\
\text { GEN, AM- } \\
\text { MONIA + } \\
\text { ORGANIC } \\
\text { DIS. } \\
\text { (MG/L } \\
\text { AS N) }\end{array}$ & $\begin{array}{c}\text { NITRO- } \\
\text { GEN, AM- } \\
\text { MONIA + } \\
\text { ORGANIC } \\
\text { TOTAL } \\
\text { (MG/L } \\
\text { AS N) }\end{array}$ & $\begin{array}{c}\text { NITRO- } \\
\text { GEN, } \\
\text { NO2+NO3 } \\
\text { DIS- } \\
\text { SOLVED } \\
\text { (MG/L } \\
\text { AS N) }\end{array}$ & $\begin{array}{l}\text { PHOS- } \\
\text { PHORUS } \\
\text { TOTAL } \\
(\text { MG / L } \\
\text { AS P) }\end{array}$ & $\begin{array}{c}\text { PHOS- } \\
\text { PHORUS } \\
\text { DIS- } \\
\text { SOLVED } \\
\text { (MG/L } \\
\text { AS P) }\end{array}$ & $\begin{array}{l}\text { PHOS- } \\
\text { PHORUS } \\
\text { ORTHO, } \\
\text { DIS- } \\
\text { SOLVED } \\
\text { (MG/L } \\
\text { AS P) }\end{array}$ \\
\hline 445547123065400 & $05-26-94$ & 0.010 & 0.20 & 0.20 & 1.70 & 0.100 & 0.090 & 0.090 \\
\hline 14190970 & $\begin{array}{l}11-23-94 \\
11-30-94 \\
11-30-94\end{array}$ & $\begin{array}{l}-- \\
-- \\
--\end{array}$ & $\begin{array}{l}-- \\
-- \\
--\end{array}$ & $\begin{array}{l}-- \\
-- \\
--\end{array}$ & $\begin{array}{l}1.80 \\
0.810 \\
0.710\end{array}$ & $\begin{array}{l}0.030 \\
0.070 \\
0.320\end{array}$ & $\begin{array}{l}-- \\
-- \\
--\end{array}$ & $\begin{array}{l}-- \\
-- \\
--\end{array}$ \\
\hline 445037122573800 & $\begin{array}{l}06-14-94 \\
10-31-94 \\
11-04-94 \\
11-04-94\end{array}$ & $\begin{array}{c}<0.010 \\
-- \\
-- \\
--\end{array}$ & $\begin{array}{l}<0.20 \\
-- \\
-- \\
--\end{array}$ & $\begin{array}{l}<0.20 \\
-- \\
-- \\
--\end{array}$ & $\begin{array}{l}0.460 \\
6.70 \\
5.70 \\
5.50\end{array}$ & $\begin{array}{l}0.020 \\
0.110 \\
0.140 \\
0.160\end{array}$ & $\begin{array}{l}0.020 \\
-- \\
-- \\
--\end{array}$ & $\begin{array}{c}<0.010 \\
-- \\
-- \\
--\end{array}$ \\
\hline 14194150 & $\begin{array}{l}05-17-94 \\
11-02-94\end{array}$ & $\begin{array}{c}<0.010 \\
--\end{array}$ & $\begin{array}{c}<0.20 \\
--\end{array}$ & $\begin{array}{c}<0.20 \\
--\end{array}$ & $\begin{array}{l}0.220 \\
1.00\end{array}$ & $\begin{array}{l}0.010 \\
0.040\end{array}$ & $\begin{array}{c}<0.010 \\
--\end{array}$ & $\begin{array}{c}<0.010 \\
--\end{array}$ \\
\hline 451355123093600 & $05-17-94$ & $<0.010$ & $<0.20$ & $<0.20$ & 0.190 & 0.020 & $<0.010$ & 0.010 \\
\hline 451602122564400 & $05-31-94$ & 0.020 & 0.20 & $<0.20$ & 0.410 & 0.060 & 0.050 & 0.050 \\
\hline 451502122524700 & $\begin{array}{l}05-26-94 \\
10-31-94\end{array}$ & $\begin{array}{c}0.010 \\
--\end{array}$ & $\begin{array}{l}0.40 \\
--\end{array}$ & $\begin{array}{l}0.50 \\
--\end{array}$ & $\begin{array}{l}1.40 \\
1.90\end{array}$ & $\begin{array}{c}0.260 \\
--\end{array}$ & $\begin{array}{l}0.240 \\
--\end{array}$ & $\begin{array}{c}0.230 \\
--\end{array}$ \\
\hline 14201300 & $\begin{array}{l}05-25-94 \\
06-13-94 \\
10-28-94 \\
11-28-94\end{array}$ & $\begin{array}{l}0.050 \\
0.030 \\
0.220 \\
0.120\end{array}$ & $\begin{array}{l}0.40 \\
0.60 \\
1.3 \\
0.80\end{array}$ & $\begin{array}{l}0.50 \\
0.70 \\
2.0 \\
0.80\end{array}$ & $\begin{array}{r}9.00 \\
6.60 \\
22.0 \\
19.0\end{array}$ & $\begin{array}{l}0.220 \\
0.260 \\
0.630 \\
0.170\end{array}$ & $\begin{array}{l}0.220 \\
0.240 \\
0.350 \\
0.140\end{array}$ & $\begin{array}{l}0.220 \\
0.230 \\
0.350 \\
0.150\end{array}$ \\
\hline 14202000 & $\begin{array}{l}04-12-94 \\
05-25-94 \\
06-15-94 \\
10-28-94 \\
10-29-94\end{array}$ & $\begin{array}{r}<0.010 \\
0.030 \\
0.010 \\
0.020 \\
0.030\end{array}$ & $\begin{array}{r}<0.20 \\
<0.20 \\
<0.20 \\
0.40 \\
0.30\end{array}$ & $\begin{aligned} 0.20 \\
0.20 \\
<0.20 \\
1.1 \\
0.70\end{aligned}$ & $\begin{array}{l}1.60 \\
1.10 \\
0.720 \\
4.10 \\
3.50\end{array}$ & $\begin{array}{l}0.080 \\
0.070 \\
0.070 \\
0.380 \\
0.220\end{array}$ & $\begin{array}{l}0.030 \\
0.050 \\
0.060 \\
0.100 \\
0.090\end{array}$ & $\begin{array}{l}0.020 \\
0.060 \\
0.070 \\
0.070 \\
0.080\end{array}$ \\
\hline & $\begin{array}{l}11-09-94 \\
11-29-94\end{array}$ & 0.020 & $\begin{array}{l}-- \\
0.20\end{array}$ & $\begin{array}{l}-- \\
0.20\end{array}$ & $\begin{array}{l}3.10 \\
2.80\end{array}$ & $\begin{array}{l}0.080 \\
0.070\end{array}$ & 0.040 & 0.040 \\
\hline 451603122423301 & $05-25-94$ & 0.030 & $<0.20$ & $<0.20$ & 0.260 & 0.010 & 0.020 & $<0.010$ \\
\hline 14206200 & $\begin{array}{l}05-27-94 \\
12-01-94\end{array}$ & $\begin{array}{c}<0.010 \\
--\end{array}$ & $\begin{array}{c}<0.20 \\
--\end{array}$ & $\begin{array}{l}0.30 \\
--\end{array}$ & $\begin{array}{l}0.510 \\
3.80\end{array}$ & $\begin{array}{l}0.060 \\
0.120\end{array}$ & $\begin{array}{c}0.040 \\
--\end{array}$ & $\begin{array}{c}0.040 \\
--\end{array}$ \\
\hline
\end{tabular}


Table 10. Concentrations of nutrients in whole and filtered water from Phase II of the Willamette River Basin Water Quality Study, Oregon, 1994-Continued

\begin{tabular}{|c|c|c|c|c|}
\hline STATION NUMBER & STATION NAME & DATE & TIME & $\begin{array}{l}\text { NITRO- } \\
\text { GEN, } \\
\text { AMMONIA } \\
\text { DIS- } \\
\text { SOLVED } \\
\text { (MG/L } \\
\text { AS N) }\end{array}$ \\
\hline 14206298 & $\begin{array}{lllll}\text { BRONSON CREEK AT } & 185 \mathrm{TH} \text { AVE NEAR ALOHA, OR } \\
\text { BRONSON CREEK AT } 185 \mathrm{TH} \text { AVE NEAR ALOHA, OR }\end{array}$ & $\begin{array}{l}05-27-94 \\
11-23-94\end{array}$ & $\begin{array}{l}1100 \\
1030\end{array}$ & $\begin{array}{l}0.030 \\
--\end{array}$ \\
\hline 14206950 & $\begin{array}{llll}\text { FANNO } & \text { CREEK AT DURHAM, OR } \\
\text { FANNO } & \text { CREEK AT DURHAM, OR } \\
\text { FANNO } & \text { CREEK AT } & \text { DURHAM, OR } \\
\text { FANNO } & \text { CREEK AT } & \text { DURHAM, OR }\end{array}$ & $\begin{array}{l}05-24-94 \\
06-16-94 \\
10-27-94 \\
11-30-94\end{array}$ & $\begin{array}{l}1420 \\
1040 \\
1620 \\
1010\end{array}$ & $\begin{array}{l}0.080 \\
0.090 \\
0.020 \\
0.030\end{array}$ \\
\hline 14207500 & $\begin{array}{llll}\text { TUALATIN RIVER AT WEST LINN, OREG. } \\
\text { TUALATIN RIVER AT WEST LINN, OREG. } \\
\text { TUALATIN RIVER AT WEST LINN, OREG. } \\
\text { TUALATIN RIVER AT WEST LINN, OREG. } \\
\text { TUALATIN RIVER AT } & \text { WEST LINN, OREG. }\end{array}$ & $\begin{array}{l}05-25-94 \\
06-17-94 \\
10-28-94 \\
11-04-94 \\
12-05-94\end{array}$ & $\begin{array}{l}1540 \\
0900 \\
1610 \\
0857 \\
0910\end{array}$ & $\begin{array}{l}0.040 \\
0.010 \\
0.140 \\
0.080 \\
0.240\end{array}$ \\
\hline 452823122240900 & $\begin{array}{lllllll}\text { JOHNSON } & \text { CREEK } & \text { AT } & \text { PALMBLAD } & \text { RD } & \text { NR } & \text { GRESHAM, OR } \\
\text { JOHNSON } & \text { CREEK } & \text { AT } & \text { PALMBLAD } & \text { RD } & \text { NR } & \text { GRESHAM, OR } \\
\text { JOHNSON } & \text { CREEK } & \text { AT } & \text { PALMBLAD } & \text { RD } & \text { NR } & \text { GRESHAM, OR } \\
\text { JOHNSON } & \text { CREEK AT } & \text { PALMBLAD } & \text { RD } & \text { NR } & \text { GRESHAM, OR } \\
\text { JOHNSON } & \text { CREEK } & \text { AT } & \text { PALMBLAD } & \text { RD } & \text { NR } & \text { GRESHAM, OR } \\
\text { JOHNSON } & \text { CREEK } & \text { AT } & \text { PALMBLAD } & \text { RD } & \text { NR } & \text { GRESHAM, OR }\end{array}$ & $\begin{array}{l}05-24-94 \\
10-27-94 \\
10-28-94 \\
11-01-94 \\
11-03-94 \\
11-23-94\end{array}$ & $\begin{array}{l}1440 \\
1640 \\
1135 \\
1100 \\
1330 \\
1410\end{array}$ & $\begin{array}{l}0.050 \\
-- \\
-- \\
0.050 \\
-- \\
--\end{array}$ \\
\hline 453043122402200 & $\begin{array}{l}\text { COMMERCIAL/RESIDENTIAL RUNOFF AT HARBOR WAY, PORT, OR } \\
\text { COMMERCIAL/RESIDENTIAL RUNOFF AT HARBOR WAY, PORT, OR }\end{array}$ & $\begin{array}{l}06-17-94 \\
06-17-94\end{array}$ & $\begin{array}{l}2140 \\
2150\end{array}$ & $\begin{array}{l}0.440 \\
--\end{array}$ \\
\hline 14211720 & $\begin{array}{lll}\text { WILLAMETTE } & \text { RIVER AT PORTLAND, OREG. } \\
\text { WILLAMETTE } & \text { RIVER AT PORTLAND, OREG. } \\
\text { WILLAMETTE } & \text { RIVER AT PORTLAND, OREG. } \\
\text { WILLAMETTE } & \text { RIVER AT PORTLAND, OREG. } \\
\text { WILLAMETTE } & \text { RIVER AT PORTLAND, OREG. } \\
\text { WILLAMETTE } & \text { RIVER AT PORTLAND, OREG. }\end{array}$ & $\begin{array}{l}05-10-94 \\
06-14-94 \\
10-25-94 \\
10-29-94 \\
11-03-94 \\
12-02-94\end{array}$ & $\begin{array}{l}0939 \\
0949 \\
1300 \\
1230 \\
0956 \\
1040\end{array}$ & $\begin{array}{l}0.050 \\
0.120 \\
0.080 \\
0.060 \\
0.040 \\
0.040\end{array}$ \\
\hline 453154122394200 & 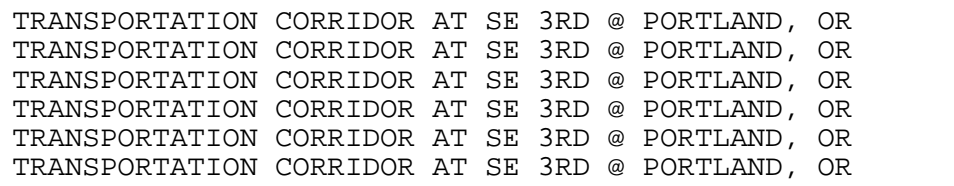 & $\begin{array}{l}05-31-94 \\
11-08-94 \\
11-08-94 \\
11-30-94 \\
11-30-94 \\
12-02-94\end{array}$ & $\begin{array}{l}1800 \\
1800 \\
1950 \\
0840 \\
1020 \\
1020\end{array}$ & $\begin{array}{l}1.50 \\
-- \\
-- \\
-- \\
-- \\
--\end{array}$ \\
\hline 14211805 & WILLAMETTE R AB ST JOHNS BR AT PORTLAND, OREG. & $05-23-94$ & 0950 & 0.050 \\
\hline
\end{tabular}


Table 10. Concentrations of nutrients in whole and filtered water from Phase II of the Willamette River Basin Water Quality Study, Oregon, 1994-Continued

\begin{tabular}{|c|c|c|c|c|c|c|c|c|}
\hline STATION NUMBER & DATE & $\begin{array}{c}\text { NITRO- } \\
\text { GEN, } \\
\text { NITRITE } \\
\text { DIS- } \\
\text { SOLVED } \\
\text { (MG/L } \\
\text { AS N) }\end{array}$ & $\begin{array}{l}\text { NITRO- } \\
\text { GEN, AM- } \\
\text { MONIA + } \\
\text { ORGANIC } \\
\text { DIS. } \\
\text { (MG/L } \\
\text { AS N) }\end{array}$ & $\begin{array}{c}\text { NITRO- } \\
\text { GEN, AM- } \\
\text { MONIA + } \\
\text { ORGANIC } \\
\text { TOTAL } \\
\text { (MG/L } \\
\text { AS N) }\end{array}$ & $\begin{array}{c}\text { NITRO- } \\
\text { GEN, } \\
\text { NO2+NO3 } \\
\text { DIS- } \\
\text { SOLVED } \\
\text { (MG/L } \\
\text { AS N) }\end{array}$ & $\begin{array}{c}\text { PHOS- } \\
\text { PHORUS } \\
\text { TOTAL } \\
(\text { MG / L } \\
\text { AS P) }\end{array}$ & $\begin{array}{c}\text { PHOS- } \\
\text { PHORUS } \\
\text { DIS- } \\
\text { SOLVED } \\
\text { (MG/L } \\
\text { AS P) }\end{array}$ & $\begin{array}{l}\text { PHOS- } \\
\text { PHORUS } \\
\text { ORTHO, } \\
\text { DIS- } \\
\text { SOLVED } \\
\text { (MG/L } \\
\text { AS P) }\end{array}$ \\
\hline 14206298 & $\begin{array}{l}05-27-94 \\
11-23-94\end{array}$ & $\begin{array}{c}<0.010 \\
--\end{array}$ & $\begin{array}{l}0.40 \\
--\end{array}$ & $\begin{array}{l}0.50 \\
--\end{array}$ & $\begin{array}{c}<0.050 \\
1.20\end{array}$ & $\begin{array}{l}0.080 \\
0.150\end{array}$ & $\begin{array}{l}0.030 \\
--\end{array}$ & $\begin{array}{c}0.040 \\
--\end{array}$ \\
\hline 14206950 & $\begin{array}{l}05-24-94 \\
06-16-94 \\
10-27-94 \\
11-30-94\end{array}$ & $\begin{array}{r}0.020 \\
<0.010 \\
0.020 \\
0.010\end{array}$ & $\begin{array}{r}<0.20 \\
0.30 \\
0.30 \\
0.20\end{array}$ & $\begin{array}{l}0.30 \\
0.40 \\
0.80 \\
0.40\end{array}$ & $\begin{array}{l}0.480 \\
0.420 \\
0.790 \\
0.560\end{array}$ & $\begin{array}{l}0.100 \\
0.120 \\
0.210 \\
0.160\end{array}$ & $\begin{array}{l}0.040 \\
0.070 \\
0.130 \\
0.050\end{array}$ & $\begin{array}{l}0.040 \\
0.060 \\
0.110 \\
0.060\end{array}$ \\
\hline 14207500 & $\begin{array}{l}05-25-94 \\
06-17-94 \\
10-28-94 \\
11-04-94 \\
12-05-94\end{array}$ & $\begin{array}{l}0.030 \\
0.010 \\
0.020 \\
0.020 \\
0.020\end{array}$ & $\begin{array}{l}0.20 \\
0.30 \\
0.40 \\
-- \\
0.40\end{array}$ & $\begin{array}{l}0.50 \\
0.30 \\
0.70 \\
0.60 \\
0.60\end{array}$ & $\begin{array}{l}1.90 \\
1.40 \\
1.20 \\
2.10 \\
2.00\end{array}$ & $\begin{array}{l}0.060 \\
0.040 \\
0.200 \\
0.160 \\
0.140\end{array}$ & $\begin{array}{r}0.030 \\
<0.010 \\
0.090 \\
0.070 \\
0.090\end{array}$ & $\begin{array}{r}0.020 \\
<0.010 \\
0.070 \\
0.050 \\
0.060\end{array}$ \\
\hline 452823122240900 & $\begin{array}{l}05-24-94 \\
10-27-94 \\
10-28-94 \\
11-01-94 \\
11-03-94 \\
11-23-94\end{array}$ & $\begin{array}{c}0.020 \\
-- \\
-- \\
<0.010 \\
-- \\
--\end{array}$ & $\begin{array}{l}0.30 \\
-- \\
-- \\
0.30 \\
-- \\
--\end{array}$ & $\begin{array}{l}0.40 \\
-- \\
-- \\
0.40 \\
-- \\
--\end{array}$ & $\begin{array}{l}2.00 \\
-- \\
-- \\
5.60 \\
0.440 \\
4.30\end{array}$ & $\begin{array}{l}0.070 \\
-- \\
-- \\
0.070 \\
0.040 \\
0.050\end{array}$ & $\begin{array}{l}0.060 \\
-- \\
-- \\
0.040 \\
-- \\
--\end{array}$ & $\begin{array}{l}0.040 \\
-- \\
-- \\
0.030 \\
-- \\
--\end{array}$ \\
\hline 453043122402200 & $\begin{array}{l}06-17-94 \\
06-17-94\end{array}$ & $\begin{array}{l}0.040 \\
--\end{array}$ & $\begin{array}{l}1.0 \\
--\end{array}$ & $\begin{array}{l}1.4 \\
--\end{array}$ & $\begin{array}{l}0.820 \\
--\end{array}$ & $\begin{array}{l}0.180 \\
--\end{array}$ & $\begin{array}{l}0.120 \\
--\end{array}$ & $\begin{array}{c}0.080 \\
--\end{array}$ \\
\hline 14211720 & $\begin{array}{l}05-10-94 \\
06-14-94 \\
10-25-94 \\
10-29-94 \\
11-03-94 \\
12-02-94\end{array}$ & $\begin{array}{l}0.010 \\
0.010 \\
0.010 \\
0.010 \\
0.010 \\
0.010\end{array}$ & $\begin{array}{r}<0.20 \\
<0.20 \\
<0.20 \\
0.20 \\
<0.20 \\
<0.20\end{array}$ & $\begin{array}{r}0.30 \\
0.30 \\
<0.20 \\
0.40 \\
0.50 \\
0.50\end{array}$ & $\begin{array}{l}0.540 \\
0.330 \\
0.280 \\
0.740 \\
1.10 \\
0.940\end{array}$ & $\begin{array}{l}0.050 \\
0.070 \\
0.070 \\
0.140 \\
0.140 \\
0.170\end{array}$ & $\begin{array}{l}0.030 \\
0.050 \\
0.050 \\
0.070 \\
0.050 \\
0.030\end{array}$ & $\begin{array}{l}0.040 \\
0.040 \\
0.062 \\
0.060 \\
0.034 \\
0.034\end{array}$ \\
\hline 453154122394200 & $\begin{array}{l}05-31-94 \\
11-08-94 \\
11-08-94 \\
11-30-94 \\
11-30-94 \\
12-02-94\end{array}$ & $\begin{array}{l}0.350 \\
-- \\
-- \\
-- \\
-- \\
--\end{array}$ & $\begin{array}{l}2.5 \\
-- \\
-- \\
-- \\
-- \\
--\end{array}$ & $\begin{array}{l}3.4 \\
-- \\
-- \\
-- \\
-- \\
--\end{array}$ & $\begin{array}{l}2.20 \\
0.510 \\
0.770 \\
0.270 \\
0.370 \\
3.40\end{array}$ & $\begin{array}{l}0.260 \\
0.250 \\
0.120 \\
0.060 \\
0.050 \\
0.090\end{array}$ & $\begin{array}{l}0.110 \\
-- \\
-- \\
-- \\
-- \\
--\end{array}$ & $\begin{array}{l}0.090 \\
-- \\
-- \\
-- \\
-- \\
--\end{array}$ \\
\hline 14211805 & $05-23-94$ & 0.020 & 0.30 & 0.20 & 0.530 & 0.060 & 0.070 & 0.070 \\
\hline
\end{tabular}


Table 11. Concentrations of trace elements and major metals in whole water from Phase I of the Willamette River Basin Water Quality Study, Oregon, 1992-93

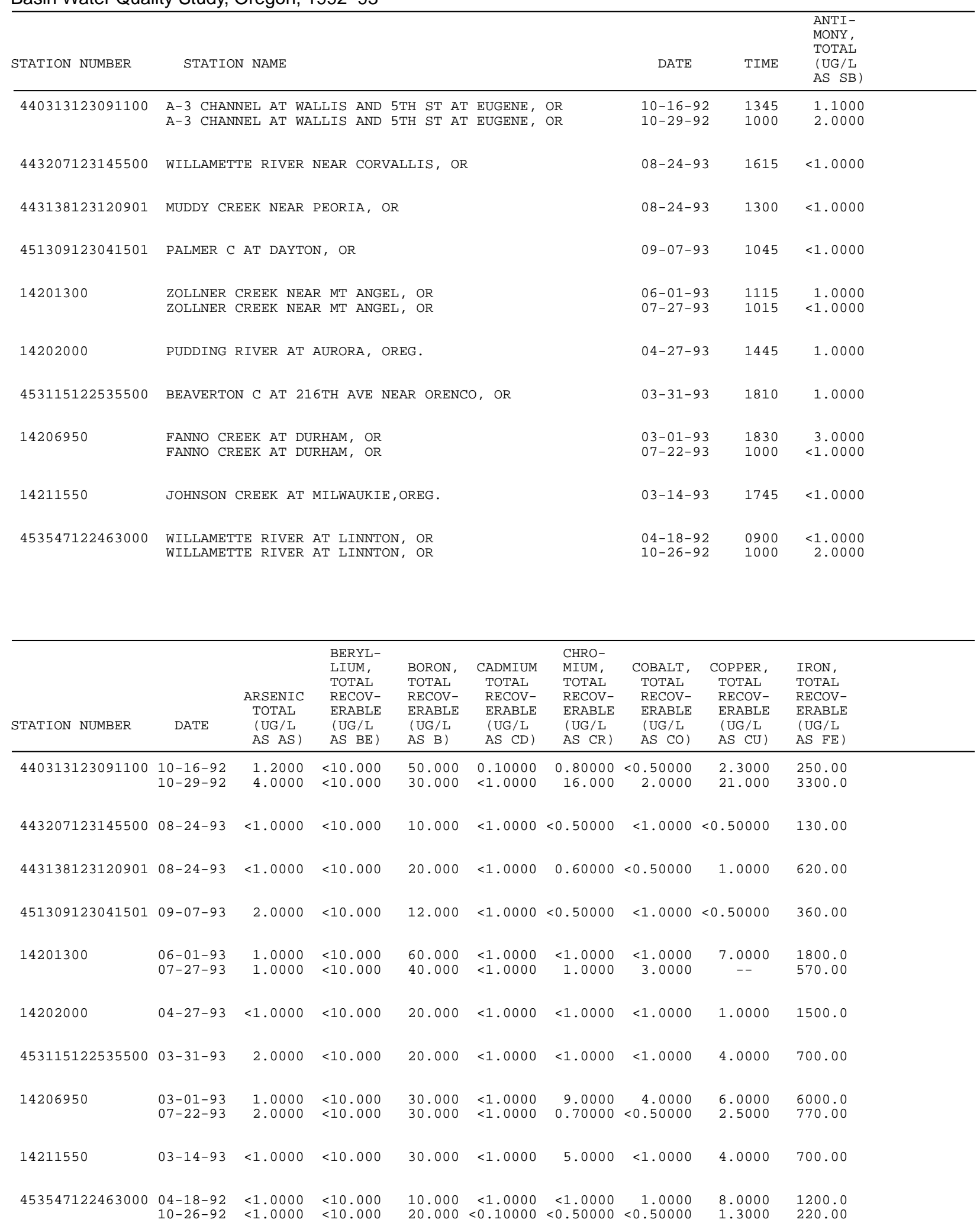


Table 11. Concentrations of trace elements and major metals in whole water from Phase I of the Willamette River Basin Water Quality Study, Oregon, 1992-93—Continued

\begin{tabular}{|c|c|c|c|c|c|c|c|c|}
\hline STATION NUMBER & DATE & $\begin{array}{l}\text { LEAD, } \\
\text { TOTAL } \\
\text { RECOV- } \\
\text { ERABLE } \\
\text { (UG/L } \\
\text { AS PB) }\end{array}$ & $\begin{array}{l}\text { MANGA- } \\
\text { NESE, } \\
\text { TOTAL } \\
\text { RECOV- } \\
\text { ERABLE } \\
\text { (UG/L } \\
\text { AS MN) }\end{array}$ & $\begin{array}{c}\text { MERCURY } \\
\text { TOTAL } \\
\text { RECOV- } \\
\text { ERABLE } \\
\text { (UG/L } \\
\text { AS HG) }\end{array}$ & $\begin{array}{c}\text { NICKEL, } \\
\text { TOTAL } \\
\text { RECOV- } \\
\text { ERABLE } \\
\text { (UG/L } \\
\text { AS NI) }\end{array}$ & $\begin{array}{l}\text { SELE- } \\
\text { NIUM, } \\
\text { TOTAL } \\
\text { (UG/L } \\
\text { AS SE) }\end{array}$ & $\begin{array}{c}\text { SILVER, } \\
\text { TOTAL } \\
\text { RECOV- } \\
\text { ERABLE } \\
\text { (UG/L } \\
\text { AS AG) }\end{array}$ & $\begin{array}{l}\text { ZINC, } \\
\text { TOTAL } \\
\text { RECOV- } \\
\text { ERABLE } \\
\text { (UG/L } \\
\text { AS ZN) }\end{array}$ \\
\hline 440313123091100 & $\begin{array}{l}10-16-92 \\
10-29-92\end{array}$ & $\begin{array}{l}1.4000 \\
30.000\end{array}$ & $\begin{array}{l}16.000 \\
160.00\end{array}$ & $\begin{array}{r}<0.10000 \\
0.10000\end{array}$ & $\begin{array}{r}<1.0000 \\
8.0000\end{array}$ & $\begin{array}{l}<1.0000 \\
<1.0000\end{array}$ & $\begin{array}{l}<1.0000 \\
<1.0000\end{array}$ & $\begin{array}{l}11.000 \\
130.00\end{array}$ \\
\hline 443207123145500 & $08-24-93$ & $<0.50000$ & 8.7000 & $<0.10000$ & $<1.0000$ & $<1.0000$ & $<1.0000$ & $<10.000$ \\
\hline 443138123120901 & $08-24-93$ & $<0.50000$ & 67.000 & $<0.10000$ & $<1.0000$ & $<1.0000$ & $<1.0000$ & 1.3000 \\
\hline 451309123041501 & $09-07-93$ & 0.60000 & 124.00 & $<0.10000$ & $<1.0000$ & $<1.0000$ & $<1.0000$ & 0.60000 \\
\hline 14201300 & $\begin{array}{l}06-01-93 \\
07-27-93\end{array}$ & $\begin{array}{r}2.0000 \\
<0.50000\end{array}$ & $\begin{array}{l}170.00 \\
170.00\end{array}$ & $\begin{array}{l}<0.10000 \\
<0.10000\end{array}$ & $\begin{array}{r}2.0000 \\
0.80000\end{array}$ & $\begin{array}{l}<1.0000 \\
<1.0000\end{array}$ & $\begin{array}{l}<1.0000 \\
<1.0000\end{array}$ & $\begin{array}{l}20.000 \\
13.000\end{array}$ \\
\hline 14202000 & $04-27-93$ & $<1.0000$ & 50.000 & $<0.10000$ & $<1.0000$ & $<1.0000$ & $<1.0000$ & $<10.000$ \\
\hline 453115122535500 & $03-31-93$ & $<1.0000$ & 110.00 & $<0.10000$ & 9.0000 & $<1.0000$ & $<1.0000$ & 20.000 \\
\hline 14206950 & $\begin{array}{l}03-01-93 \\
07-22-93\end{array}$ & $\begin{array}{r}16.000 \\
0.50000\end{array}$ & $\begin{array}{l}420.00 \\
180.00\end{array}$ & $\begin{array}{l}<0.10000 \\
<0.10000\end{array}$ & $\begin{array}{r}7.0000 \\
<1.0000\end{array}$ & $\begin{array}{l}<1.0000 \\
<1.0000\end{array}$ & $\begin{array}{l}<1.0000 \\
<1.0000\end{array}$ & $\begin{array}{l}80.000 \\
11.000\end{array}$ \\
\hline 14211550 & $03-14-93$ & 3.0000 & 30.000 & $<0.10000$ & 6.0000 & $<1.0000$ & $<1.0000$ & 20.000 \\
\hline 453547122463000 & $\begin{array}{l}04-18-92 \\
10-26-92\end{array}$ & $\begin{array}{r}1.0000 \\
<0.50000\end{array}$ & $\begin{array}{l}40.000 \\
19.000\end{array}$ & $\begin{array}{l}<0.10000 \\
<0.10000\end{array}$ & $\begin{array}{l}1.0000 \\
3.0000\end{array}$ & $\begin{array}{l}<1.0000 \\
<1.0000\end{array}$ & $\begin{array}{l}<1.0000 \\
<1.0000\end{array}$ & $\begin{array}{l}10.000 \\
1.1000\end{array}$ \\
\hline
\end{tabular}


Table 12. Concentrations of trace elements in whole water from Phase II of the Willamette River Basin Water Quality Study, Oregon, 1994

\begin{tabular}{|c|c|c|c|c|}
\hline STATION NUMBER & STATION NAME & DATE & TIME & $\begin{array}{c}\text { ARSENIC } \\
\text { TOTAL } \\
\text { (UG/L } \\
\text { AS AS) }\end{array}$ \\
\hline 433548123040600 & COAST FORK WILLAMETTE R BL BIG RIVER NR LONDON, OR & $05-20-94$ & 1040 & -- \\
\hline 440045122585600 & COAST FORK WILLAM AT SEAVY LOOP RD NR EUGENE, OR & $05-19-94$ & 1020 & -- \\
\hline 443321123155201 & MARYS RIVER AT CORVALLIS, OR & $05-18-94$ & 1350 & -- \\
\hline 14190970 & $\begin{array}{lllllll}\text { PRINGLE } & \text { AT } & \text { BUSH } & \text { PARK AT SALEM, OR } \\
\text { PRINGLE } & \text { C AT } & \text { BUSH PARK AT SALEM, OR } \\
\text { PRINGLE } & \text { C AT } & \text { BUSH PARK AT } & \text { SALEM, OR }\end{array}$ & $\begin{array}{l}11-23-94 \\
11-30-94 \\
11-30-94\end{array}$ & $\begin{array}{l}1200 \\
1530 \\
1710\end{array}$ & $\begin{array}{l}<1.0000 \\
<1.0000 \\
<1.0000\end{array}$ \\
\hline 445037122573800 & MILL CREEK AT DELANEY ROAD NR TURNER, OR & $06-14-94$ & 1140 & -- \\
\hline 14194150 & SOUTH YAMHILL RIVER AT MCMINNVILLE, OR & $05-17-94$ & 1720 & -- \\
\hline 451602122564400 & WILLAMETTE RIVER NR NEWBERG, OR & $05-31-94$ & 1400 & -- \\
\hline 14206200 & DAIRY CREEK AT RTE 8 NEAR HILLSBORO, OR & $12-01-94$ & 1140 & 1.0000 \\
\hline 14206298 & $\begin{array}{lllll}\text { BRONSON CREEK AT } & 185 \mathrm{TH} \text { AVE NEAR ALOHA, OR } \\
\text { BRONSON CREEK AT } & 185 \mathrm{TH} \text { AVE NEAR ALOHA, OR }\end{array}$ & $\begin{array}{l}05-27-94 \\
11-23-94\end{array}$ & $\begin{array}{l}1100 \\
1030\end{array}$ & $<1 . \overline{0} 000$ \\
\hline 14206950 & FANNO CREEK AT DURHAM, OR & $10-27-94$ & 1620 & -- \\
\hline 14207500 & $\begin{array}{l}\text { TUALATIN RIVER AT WEST LINN, OREG. } \\
\text { TUALATIN RIVER AT WEST LINN, OREG. }\end{array}$ & $\begin{array}{l}05-25-94 \\
10-28-94\end{array}$ & $\begin{array}{l}1540 \\
1610\end{array}$ & $\begin{array}{l}-- \\
--\end{array}$ \\
\hline 452823122240900 & JOHNSON CREEK AT PALMBLAD RD NR GRESHAM, OR & $11-03-94$ & 1330 & -- \\
\hline 453043122402200 & COMMERCIAL/RESIDENTIAL RUNOFF AT HARBOR WAY, PORT, OR & $06-17-94$ & 2140 & -- \\
\hline 14211720 & WILLAMETTE RIVER AT PORTLAND, OREG. & $10-29-94$ & 1230 & -- \\
\hline 453154122394200 & 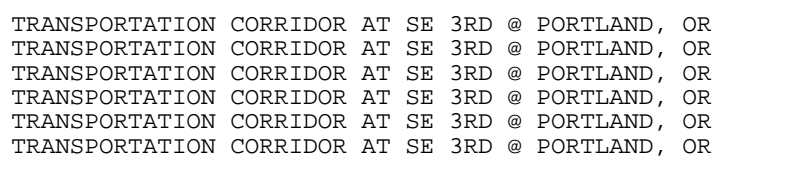 & $\begin{array}{l}05-31-94 \\
11-08-94 \\
11-08-94 \\
11-30-94 \\
11-30-94 \\
12-02-94\end{array}$ & $\begin{array}{l}1800 \\
1800 \\
1950 \\
0840 \\
1020 \\
1020\end{array}$ & $\begin{array}{l}1.0000 \\
1.0000 \\
1.0000 \\
1.0000 \\
2.0000\end{array}$ \\
\hline 14211805 & WILLAMETTE R AB ST JOHNS BR AT PORTLAND, OREG. & $05-23-94$ & 0950 & -- \\
\hline
\end{tabular}


Table 12. Concentrations of trace elements in whole water from Phase II of the Willamette River Basin Water Quality Study, Oregon, 1994-Continued

\begin{tabular}{|c|c|c|c|c|c|c|c|c|}
\hline STATION NUMBER & DATE & $\begin{array}{l}\text { CADMIUM } \\
\text { TOTAL } \\
\text { RECOV- } \\
\text { ERABLE } \\
\text { (UG/L } \\
\text { AS CD) }\end{array}$ & $\begin{array}{l}\text { CHRO- } \\
\text { MIUM, } \\
\text { TOTAL } \\
\text { RECOV- } \\
\text { ERABLE } \\
\text { (UG/L } \\
\text { AS CR) }\end{array}$ & $\begin{array}{c}\text { COPPER, } \\
\text { TOTAL } \\
\text { RECOV- } \\
\text { ERABLE } \\
\text { (UG/L } \\
\text { AS CU) }\end{array}$ & $\begin{array}{l}\text { LEAD, } \\
\text { TOTAL } \\
\text { RECOV- } \\
\text { ERABLE } \\
\text { (UG/L } \\
\text { AS PB) }\end{array}$ & $\begin{array}{c}\text { MERCURY } \\
\text { TOTAL } \\
\text { RECOV- } \\
\text { ERABLE } \\
\text { (UG/L } \\
\text { AS HG) }\end{array}$ & $\begin{array}{l}\text { NICKEL, } \\
\text { TOTAL } \\
\text { RECOV- } \\
\text { ERABLE } \\
\text { (UG/L } \\
\text { AS NI) }\end{array}$ & $\begin{array}{l}\text { ZINC, } \\
\text { TOTAL } \\
\text { RECOV- } \\
\text { ERABLE } \\
\text { (UG/L } \\
\text { AS ZN) }\end{array}$ \\
\hline 433548123040600 & $05-20-94$ & $<1.0000$ & $<1.0000$ & 1.0000 & $<1.0000$ & $<0.10000$ & $<1.0000$ & $<10.000$ \\
\hline 440045122585600 & $05-19-94$ & $<1.0000$ & $<1.0000$ & 1.0000 & $<1.0000$ & $<0.10000$ & $<1.0000$ & $<10.000$ \\
\hline 443321123155201 & $05-18-94$ & $<1.0000$ & 1.9000 & 1.0000 & $<1.0000$ & -- & 3.0000 & $<10.000$ \\
\hline 14190970 & $\begin{array}{l}11-23-94 \\
11-30-94 \\
11-30-94\end{array}$ & $\begin{array}{l}<1.0000 \\
<1.0000 \\
<1.0000\end{array}$ & $\begin{array}{r}<1.0000 \\
4.8000 \\
6.6000\end{array}$ & $\begin{array}{l}2.0000 \\
8.0000 \\
11.000\end{array}$ & $\begin{array}{l}1.0000 \\
21.000 \\
44.000\end{array}$ & $\begin{array}{l}-- \\
-- \\
--\end{array}$ & $\begin{array}{r}<1.0000 \\
3.0000 \\
5.0000\end{array}$ & $\begin{array}{r}<10.000 \\
60.000 \\
90.000\end{array}$ \\
\hline 445037122573800 & $06-14-94$ & $<1.0000$ & $<1.0000$ & $<1.0000$ & $<1.0000$ & -- & $<1.0000$ & $<10.000$ \\
\hline 14194150 & $05-17-94$ & $<1.0000$ & $<1.0000$ & 3.0000 & $<1.0000$ & -- & 1.0000 & $<10.000$ \\
\hline 451602122564400 & $05-31-94$ & $<1.0000$ & $<1.0000$ & 1.0000 & $<1.0000$ & -- & 1.0000 & $<10.000$ \\
\hline 14206200 & $12-01-94$ & $<1.0000$ & 3.3000 & 3.0000 & 2.0000 & -- & 2.0000 & 10.000 \\
\hline 14206298 & $\begin{array}{l}05-27-94 \\
11-23-94\end{array}$ & $\begin{array}{l}<1.0000 \\
<1.0000\end{array}$ & $\begin{array}{r}<1.0000 \\
2.5000\end{array}$ & $\begin{array}{l}1.0000 \\
3.0000\end{array}$ & $\begin{array}{l}1.0000 \\
1.0000\end{array}$ & $\begin{array}{l}-- \\
--\end{array}$ & $\begin{array}{l}2.0000 \\
2.0000\end{array}$ & $\begin{array}{l}<10.000 \\
<10.000\end{array}$ \\
\hline 14206950 & $10-27-94$ & $<1.0000$ & 3.9000 & 4.0000 & 5.0000 & -- & 3.0000 & 30.000 \\
\hline 14207500 & $\begin{array}{l}05-25-94 \\
10-28-94\end{array}$ & $\begin{array}{l}<1.0000 \\
<1.0000\end{array}$ & $\begin{array}{r}<1.0000 \\
2.2000\end{array}$ & $\begin{array}{l}3.0000 \\
5.0000\end{array}$ & $\begin{array}{r}<1.0000 \\
2.0000\end{array}$ & $\begin{array}{l}-- \\
--\end{array}$ & $\begin{array}{l}2.0000 \\
2.0000\end{array}$ & $\begin{array}{r}<10.000 \\
20.000\end{array}$ \\
\hline 452823122240900 & $11-03-94$ & $<1.0000$ & $<1.0000$ & $<1.0000$ & $<1.0000$ & -- & $<1.0000$ & $<10.000$ \\
\hline 453043122402200 & $06-17-94$ & 7.0000 & -- & 45.000 & 44.000 & -- & 6.0000 & 290.00 \\
\hline 14211720 & $10-29-94$ & $<1.0000$ & 1.1000 & 2.0000 & $<1.0000$ & -- & 1.0000 & $<10.000$ \\
\hline 453154122394200 & $\begin{array}{l}05-31-94 \\
11-08-94 \\
11-08-94 \\
11-30-94 \\
11-30-94 \\
12-02-94\end{array}$ & $\begin{array}{r}1.0000 \\
2.0000 \\
1.0000 \\
2.0000 \\
2.0000 \\
<1.0000\end{array}$ & $\begin{array}{l}7.3000 \\
8.6000 \\
5.6000 \\
14.000 \\
14.000 \\
5.0000\end{array}$ & $\begin{array}{l}36.000 \\
19.000 \\
12.000 \\
21.000 \\
21.000 \\
9.0000\end{array}$ & $\begin{array}{l}36.000 \\
39.000 \\
21.000 \\
67.000 \\
59.000 \\
14.000\end{array}$ & $\begin{array}{l}-- \\
-- \\
-- \\
-- \\
-- \\
--\end{array}$ & $\begin{array}{l}12.000 \\
8.0000 \\
1.0000 \\
9.0000 \\
9.0000 \\
3.0000\end{array}$ & $\begin{array}{l}200.00 \\
210.00 \\
150.00 \\
280.00 \\
280.00 \\
70.000\end{array}$ \\
\hline 14211805 & $05-23-94$ & $<1.0000$ & $<1.0000$ & 1.0000 & $<1.0000$ & -- & $<1.0000$ & $<10.000$ \\
\hline
\end{tabular}


Table 13. Concentrations of trace elements and major metals in filtered water from Phase I of the Willamette River Basin Water Quality Study, Oregon, 1992-93

\begin{tabular}{|c|c|c|c|c|}
\hline STATION NUMBER & STATION NAME & DATE & TIME & $\begin{array}{l}\text { ANTI- } \\
\text { MONY, } \\
\text { DIS- } \\
\text { SOLVED } \\
\text { (UG / L } \\
\text { AS SB) }\end{array}$ \\
\hline 440313123091100 & 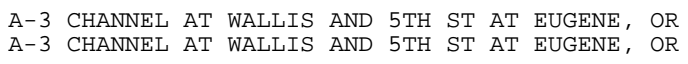 & $\begin{array}{l}10-16-92 \\
10-29-92\end{array}$ & $\begin{array}{l}1345 \\
1000\end{array}$ & $\begin{array}{l}1.0000 \\
2.0000\end{array}$ \\
\hline 443207123145500 & WILLAMETTE RIVER NEAR CORVALLIS, OR & $08-24-93$ & 1615 & $<1.0000$ \\
\hline 443138123120901 & MUDDY CREEK NEAR PEORIA, OR & $08-24-93$ & 1300 & $<1.0000$ \\
\hline 451309123041501 & PALMER C AT DAYTON, OR & $09-07-93$ & 1045 & $<1.0000$ \\
\hline 14201300 & $\begin{array}{l}\text { ZOLLNER CREEK NEAR MT ANGEL, OR } \\
\text { ZOLLNER CREEK NEAR MT ANGEL, OR }\end{array}$ & $\begin{array}{l}06-01-93 \\
07-27-93\end{array}$ & $\begin{array}{l}1115 \\
1015\end{array}$ & $\begin{array}{l}<1.0000 \\
<1.0000\end{array}$ \\
\hline 14202000 & PUDDING RIVER AT AURORA, OREG. & $04-27-93$ & 1445 & $<1.0000$ \\
\hline 453115122535500 & BEAVERTON C AT 216TH AVE NEAR ORENCO, OR & $03-31-93$ & 1810 & 1.0000 \\
\hline 14206950 & $\begin{array}{llll}\text { FANNO CREEK AT DURHAM, OR } \\
\text { FANNO CREEK AT DURHAM, OR }\end{array}$ & $\begin{array}{l}03-01-93 \\
07-22-93\end{array}$ & $\begin{array}{l}1830 \\
1000\end{array}$ & $\begin{array}{r}1.0000 \\
<1.0000\end{array}$ \\
\hline 14211550 & JOHNSON CREEK AT MILWAUKIE, OREG. & $03-14-93$ & 1745 & $<1.0000$ \\
\hline 453547122463000 & $\begin{array}{l}\text { WILLAMETTE RIVER AT LINNTON, OR } \\
\text { WILLAMETTE RIVER AT LINNTON, OR }\end{array}$ & $\begin{array}{l}04-18-92 \\
10-26-92\end{array}$ & $\begin{array}{l}0900 \\
1000\end{array}$ & $\begin{array}{l}<1.0000 \\
<1.0000\end{array}$ \\
\hline
\end{tabular}


Table 13. Concentrations of trace elements and major metals in filtered water from Phase I of the Willamette River Basin Water Quality Study, Oregon, 1992-93—Continued

\begin{tabular}{|c|c|c|c|c|c|c|c|c|c|}
\hline STATION NUMBER & DATE & $\begin{array}{c}\text { ARSENIC } \\
\text { DIS- } \\
\text { SOLVED } \\
\text { (UG/L } \\
\text { AS AS) }\end{array}$ & $\begin{array}{l}\text { BERYL- } \\
\text { LIUM, } \\
\text { DIS- } \\
\text { SOLVED } \\
\text { (UG/L } \\
\text { AS BE) }\end{array}$ & $\begin{array}{l}\text { BORON, } \\
\text { DIS- } \\
\text { SOLVED } \\
\text { (UG/L } \\
\text { AS B) }\end{array}$ & $\begin{array}{c}\text { CADMIUM } \\
\text { DIS- } \\
\text { SOLVED } \\
\text { (UG/L } \\
\text { AS CD) }\end{array}$ & $\begin{array}{l}\text { CHRO- } \\
\text { MIUM, } \\
\text { DIS- } \\
\text { SOLVED } \\
\text { (UG/L } \\
\text { AS CR) }\end{array}$ & $\begin{array}{l}\text { COBALT, } \\
\text { DIS- } \\
\text { SOLVED } \\
\text { (UG/L } \\
\text { AS CO) }\end{array}$ & $\begin{array}{l}\text { COPPER, } \\
\text { DIS- } \\
\text { SOLVED } \\
\text { (UG/L } \\
\text { AS CU) }\end{array}$ & $\begin{array}{l}\text { IRON, } \\
\text { DIS- } \\
\text { SOLVED } \\
\text { (UG/L } \\
\text { AS FE) }\end{array}$ \\
\hline 440313123091100 & $\begin{array}{l}10-16-92 \\
10-29-92\end{array}$ & $\begin{array}{l}1.0000 \\
3.0000\end{array}$ & $\begin{array}{l}<10.000 \\
<10.000\end{array}$ & 20.000 & $\begin{array}{l}<0.10000 \\
<0.10000\end{array}$ & $\begin{array}{r}<0.50000 \\
5.4000\end{array}$ & $\begin{array}{l}<0.50000 \\
<0.50000\end{array}$ & $\begin{array}{l}1.0000 \\
7.4000\end{array}$ & $\begin{array}{l}160.00 \\
330.00\end{array}$ \\
\hline 443207123145500 & $08-24-93$ & $<1.0000$ & $<0.50000$ & 10.000 & $<0.10000$ & $<0.50000$ & $<0.50000$ & $<0.50000$ & 67.000 \\
\hline 443138123120901 & $08-24-93$ & $<1.0000$ & $<0.50000$ & 20.000 & $<0.10000$ & $<0.50000$ & $<0.50000$ & 0.70000 & 300.00 \\
\hline 451309123041501 & $09-07-93$ & 2.0000 & $<0.50000$ & 14.000 & $<0.10000$ & $<0.50000$ & $<0.50000$ & $<0.50000$ & 200.00 \\
\hline 14201300 & $\begin{array}{l}06-01-93 \\
07-27-93\end{array}$ & $\begin{array}{l}1.0000 \\
1.0000\end{array}$ & $\begin{array}{r}<10.000 \\
<0.50000\end{array}$ & $\begin{array}{l}50.000 \\
40.000\end{array}$ & $\begin{array}{r}0.10000 \\
<0.10000\end{array}$ & $\begin{array}{r}<0.50000 \\
0.70000\end{array}$ & $\begin{array}{r}<0.50000 \\
3.2000\end{array}$ & $\begin{array}{c}3.5000 \\
--\end{array}$ & $\begin{array}{l}120.00 \\
290.00\end{array}$ \\
\hline 14202000 & $04-27-93$ & $<1.0000$ & $<10.000$ & 20.000 & 0.20000 & 1.0000 & $<0.50000$ & 2.2000 & 100.00 \\
\hline 453115122535500 & $03-31-93$ & $<1.0000$ & $<10.000$ & 10.000 & 0.70000 & 2.5000 & $<0.50000$ & 4.6000 & 340.00 \\
\hline 14206950 & $\begin{array}{l}03-01-93 \\
07-22-93\end{array}$ & $\begin{array}{r}<1.0000 \\
2.0000\end{array}$ & $\begin{array}{r}<10.000 \\
<0.50000\end{array}$ & $\begin{array}{l}20.000 \\
30.000\end{array}$ & $\begin{array}{l}0.20000 \\
0.10000\end{array}$ & $\begin{array}{r}1.8000 \\
0.60000\end{array}$ & $\begin{array}{l}<0.50000 \\
<0.50000\end{array}$ & 2.4000 & $\begin{array}{l}510.00 \\
590.00\end{array}$ \\
\hline 14211550 & $03-14-93$ & $<1.0000$ & $<10.000$ & 40.000 & $<0.10000$ & 0.90000 & $<0.50000$ & 1.9000 & 270.00 \\
\hline 453547122463000 & $\begin{array}{l}04-18-92 \\
10-26-92\end{array}$ & $\begin{array}{l}<1.0000 \\
<1.0000\end{array}$ & $\begin{array}{l}<10.000 \\
<10.000\end{array}$ & $\begin{array}{c}<10.000 \\
--\end{array}$ & $\begin{array}{l}<0.10000 \\
<0.10000\end{array}$ & $\begin{array}{l}<0.50000 \\
<0.50000\end{array}$ & $\begin{array}{l}<0.50000 \\
<0.50000\end{array}$ & $\begin{array}{l}1.3000 \\
1.1000\end{array}$ & $\begin{array}{l}370.00 \\
80.000\end{array}$ \\
\hline
\end{tabular}


Table 13. Concentrations of trace elements and major metals in filtered water from Phase I of the Willamette River Basin Water Quality Study, Oregon, 1992-93-Continued

\begin{tabular}{|c|c|c|c|c|c|c|c|c|}
\hline STATION NUMBER & DATE & $\begin{array}{l}\text { LEAD, } \\
\text { DIS- } \\
\text { SOLVED } \\
\text { (UG/L } \\
\text { AS PB) }\end{array}$ & $\begin{array}{l}\text { MANGA- } \\
\text { NESE, } \\
\text { DIS- } \\
\text { SOLVED } \\
\text { (UG/L } \\
\text { AS MN) }\end{array}$ & $\begin{array}{c}\text { MERCURY } \\
\text { DIS- } \\
\text { SOLVED } \\
\text { (UG/L } \\
\text { AS HG) }\end{array}$ & $\begin{array}{l}\text { NICKEL, } \\
\text { DIS- } \\
\text { SOLVED } \\
\text { (UG/L } \\
\text { AS NI) }\end{array}$ & $\begin{array}{l}\text { SELE- } \\
\text { NIUM, } \\
\text { DIS- } \\
\text { SOLVED } \\
\text { (UG/L } \\
\text { AS SE) }\end{array}$ & $\begin{array}{c}\text { SILVER, } \\
\text { DIS- } \\
\text { SOLVED } \\
\text { (UG/L } \\
\text { AS AG) }\end{array}$ & $\begin{array}{l}\text { ZINC, } \\
\text { DIS- } \\
\text { SOLVED } \\
\text { (UG/L } \\
\text { AS ZN) }\end{array}$ \\
\hline 440313123091100 & $\begin{array}{l}10-16-92 \\
10-29-92\end{array}$ & $\begin{array}{r}<0.50000 \\
2.2000\end{array}$ & $\begin{array}{l}5.1000 \\
89.000\end{array}$ & $\begin{array}{l}<0.10000 \\
<0.10000\end{array}$ & $\begin{array}{r}<1.0000 \\
1.0000\end{array}$ & $\begin{array}{l}<1.0000 \\
<1.0000\end{array}$ & $\begin{array}{l}<1.0000 \\
<1.0000\end{array}$ & $\begin{array}{r}1.4000 \\
<0.50000\end{array}$ \\
\hline 443207123145500 & $08-24-93$ & $<0.50000$ & 3.9000 & $<0.10000$ & $<1.0000$ & $<1.0000$ & $<1.0000$ & $<0.50000$ \\
\hline 443138123120901 & $08-24-93$ & $<0.50000$ & 30.000 & $<0.10000$ & $<1.0000$ & $<1.0000$ & $<1.0000$ & 0.50000 \\
\hline 451309123041501 & $09-07-93$ & 0.50000 & 82.000 & $<0.10000$ & $<1.0000$ & $<1.0000$ & $<1.0000$ & $<0.50000$ \\
\hline 14201300 & $\begin{array}{l}06-01-93 \\
07-27-93\end{array}$ & $\begin{array}{l}<0.50000 \\
<0.50000\end{array}$ & $\begin{array}{l}110.00 \\
160.00\end{array}$ & $\begin{array}{l}<0.10000 \\
<0.10000\end{array}$ & $\begin{array}{r}1.0000 \\
0.64000\end{array}$ & $\begin{array}{l}<1.0000 \\
<1.0000\end{array}$ & $\begin{array}{l}<1.0000 \\
<1.0000\end{array}$ & $\begin{array}{r}<0.50000 \\
12.000\end{array}$ \\
\hline 14202000 & $04-27-93$ & 0.70000 & 9.0000 & $<0.10000$ & $<1.0000$ & $<1.0000$ & $<1.0000$ & $<0.50000$ \\
\hline 453115122535500 & $03-31-93$ & 0.70000 & 39.000 & $<0.10000$ & $<1.0000$ & $<1.0000$ & $<1.0000$ & $<0.50000$ \\
\hline 14206950 & $\begin{array}{l}03-01-93 \\
07-22-93\end{array}$ & $\begin{array}{r}2.3000 \\
<0.50000\end{array}$ & $\begin{array}{l}54.000 \\
170.00\end{array}$ & $\begin{array}{l}<0.10000 \\
<0.10000\end{array}$ & $\begin{array}{r}2.0000 \\
<1.0000\end{array}$ & $\begin{array}{l}<1.0000 \\
<1.0000\end{array}$ & $\begin{array}{l}<1.0000 \\
<1.0000\end{array}$ & $\begin{array}{r}<0.50000 \\
10.000\end{array}$ \\
\hline 14211550 & $03-14-93$ & 0.70000 & 11.000 & $<0.10000$ & $<1.0000$ & $<1.0000$ & $<1.0000$ & 0.50000 \\
\hline 453547122463000 & $\begin{array}{l}04-18-92 \\
10-26-92\end{array}$ & $\begin{array}{l}<0.50000 \\
<0.50000\end{array}$ & $\begin{array}{l}3.2000 \\
6.0000\end{array}$ & $<\frac{--}{<0.10000}$ & $\begin{array}{r}<1.0000 \\
3.0000\end{array}$ & $\begin{array}{l}<1.0000 \\
<1.0000\end{array}$ & $\begin{array}{l}<1.0000 \\
<1.0000\end{array}$ & $\begin{array}{l}<0.50000 \\
<0.50000\end{array}$ \\
\hline
\end{tabular}


Table 14. Concentrations of trace elements in filtered water from Phase II of the Willamette River Basin Water Quality Study, Oregon, 1994

\begin{tabular}{|c|c|c|c|c|}
\hline STATION NUMBER & STATION NAME & DATE & TIME & $\begin{array}{l}\text { ARSENIC } \\
\text { DIS- } \\
\text { SOLVED } \\
\text { (UG/L } \\
\text { AS AS) }\end{array}$ \\
\hline 440402123063900 & URBAN OUTFALL AT POLK ST. PARK AT EUGENE, OR & $06-13-94$ & 1210 & 1.0000 \\
\hline 14190970 & PRINGLE C AT BUSH PARK AT SALEM, OR & $11-23-94$ & 1200 & $<1.0000$ \\
\hline 451602122564400 & WILLAMETTE RIVER NR NEWBERG, OR & $05-31-94$ & 1400 & $<1.0000$ \\
\hline 14206298 & BRONSON CREEK AT 185TH AVE NEAR ALOHA, OR & $11-23-94$ & 1030 & $<1.0000$ \\
\hline 14207500 & TUALATIN RIVER AT WEST LINN, OREG. & $11-04-94$ & 0857 & -- \\
\hline 453043122402200 & COMMERCIAL/RESIDENTIAL RUNOFF AT HARBOR WAY, PORT, OR & $06-17-94$ & 2140 & 1.0000 \\
\hline 14211720 & $\begin{array}{llll}\text { WILLAMETTE } & \text { RIVER AT PORTLAND, OREG. } \\
\text { WILLAMETTE } & \text { RIVER AT } \\
\text { WILLAMETTE } & \text { RIVER AT } & \text { PORTLAND, OREG. } \\
\text { WILLAMETTE } & \text { RIVER AT } & \text { PORTLAND, OREG. } \\
\text { WILLAMETTE } & \text { RIVER AT PORTLAND, OREG. }\end{array}$ & $\begin{array}{l}05-10-94 \\
06-14-94 \\
10-25-94 \\
10-29-94 \\
11-03-94\end{array}$ & $\begin{array}{l}0939 \\
0949 \\
1300 \\
1230 \\
0956\end{array}$ & $\begin{array}{l}<1.0000 \\
<1.0000 \\
<1.0000 \\
<1.0000\end{array}$ \\
\hline 453154122394200 & $\begin{array}{llllll}\text { TRANSPORTATION CORRIDOR AT SE 3RD @ PORTLAND, OR } \\
\text { TRANSPORTATION CORRIDOR AT SE 3RD @ PORTLAND, OR }\end{array}$ & $\begin{array}{l}05-31-94 \\
11-08-94\end{array}$ & $\begin{array}{l}1800 \\
1800\end{array}$ & $\begin{array}{l}2.0000 \\
2.0000\end{array}$ \\
\hline
\end{tabular}


Table 14. Concentrations of trace elements in filtered water from Phase II of the Willamette River Basin Water Quality Study, Oregon, 1994-Continued

\begin{tabular}{|c|c|c|c|c|c|c|c|c|c|}
\hline STATION NUMBER & DATE & $\begin{array}{l}\text { ALUM- } \\
\text { INUM, } \\
\text { DIS- } \\
\text { SOLVED } \\
\text { (UG/L } \\
\text { AS AL) }\end{array}$ & $\begin{array}{l}\text { ANTI- } \\
\text { MONY, } \\
\text { DIS- } \\
\text { SOLVED } \\
\text { (UG/L } \\
\text { AS SB) }\end{array}$ & $\begin{array}{l}\text { BARIUM, } \\
\text { DIS- } \\
\text { SOLVED } \\
\text { (UG/L } \\
\text { AS BA) }\end{array}$ & $\begin{array}{l}\text { BERYL- } \\
\text { LIUM, } \\
\text { DIS- } \\
\text { SOLVED } \\
\text { (UG/L } \\
\text { AS BE) }\end{array}$ & $\begin{array}{l}\text { CADMIUM } \\
\text { DIS- } \\
\text { SOLVED } \\
\text { (UG/L } \\
\text { AS CD) }\end{array}$ & $\begin{array}{l}\text { CHRO- } \\
\text { MIUM, } \\
\text { DIS- } \\
\text { SOLVED } \\
\text { (UG/L } \\
\text { AS CR) }\end{array}$ & $\begin{array}{l}\text { COBALT, } \\
\text { DIS- } \\
\text { SOLVED } \\
\text { (UG/L } \\
\text { AS CO) }\end{array}$ & $\begin{array}{l}\text { COPPER, } \\
\text { DIS- } \\
\text { SOLVED } \\
\text { (UG/L } \\
\text { AS CU) }\end{array}$ \\
\hline 440402123063900 & $06-13-94$ & 23.000 & $<1.0000$ & 6.0000 & $<1.0000$ & $<1.0000$ & $<1.0000$ & $<1.0000$ & 13.000 \\
\hline 14190970 & $11-23-94$ & 113.00 & $<1.0000$ & 28.000 & $<1.0000$ & $<1.0000$ & $<1.0000$ & $<1.0000$ & 1.0000 \\
\hline 451602122564400 & $05-31-94$ & 17.000 & $<1.0000$ & 5.0000 & $<1.0000$ & $<1.0000$ & $<1.0000$ & $<1.0000$ & 2.0000 \\
\hline 14206298 & $11-23-94$ & 107.00 & $<1.0000$ & 19.000 & $<1.0000$ & $<1.0000$ & $<1.0000$ & $<1.0000$ & 2.0000 \\
\hline 14207500 & $11-04-94$ & 340.00 & -- & 17.000 & -- & -- & -- & $<3.0000$ & -- \\
\hline 453043122402200 & $06-17-94$ & 49.000 & $<1.0000$ & 14.000 & $<1.0000$ & $<1.0000$ & $<1.0000$ & $<1.0000$ & 21.000 \\
\hline 14211720 & $\begin{array}{l}05-10-94 \\
06-14-94 \\
10-25-94 \\
10-29-94 \\
11-03-94\end{array}$ & $\begin{array}{r}<10.000 \\
15.000 \\
25.000 \\
93.000 \\
160.00\end{array}$ & $\begin{array}{l}\quad-- \\
<1.0000 \\
<1.0000 \\
<1.0000 \\
<1.0000\end{array}$ & $\begin{array}{l}7.0000 \\
6.0000 \\
5.0000 \\
8.0000 \\
7.0000\end{array}$ & $\begin{array}{l}\quad-- \\
<1.0000 \\
<1.0000 \\
<1.0000 \\
<1.00000\end{array}$ & $\begin{array}{l}\quad-- \\
<1.0000 \\
<1.0000 \\
<1.0000 \\
<1.0000\end{array}$ & $\begin{array}{l}<-- \\
<1.0000 \\
<1.0000 \\
<1.0000 \\
<1.00000\end{array}$ & $\begin{array}{l}<3.0000 \\
<1.0000 \\
<1.0000 \\
<1.0000 \\
<1.0000\end{array}$ & $\begin{array}{r}-- \\
<1.0000 \\
5.0000 \\
<1.0000 \\
2.0000\end{array}$ \\
\hline 453154122394200 & $\begin{array}{l}05-31-94 \\
11-08-94\end{array}$ & $\begin{array}{l}220.00 \\
203.00\end{array}$ & $\begin{array}{l}4.0000 \\
2.0000\end{array}$ & $\begin{array}{l}63.000 \\
52.000\end{array}$ & $\begin{array}{l}<1.0000 \\
<1.0000\end{array}$ & $\begin{array}{l}<1.0000 \\
<1.0000\end{array}$ & $\begin{array}{l}3.0000 \\
2.0000\end{array}$ & $\begin{array}{l}<1.0000 \\
<1.0000\end{array}$ & $\begin{array}{l}16.000 \\
9.0000\end{array}$ \\
\hline
\end{tabular}


Table 14. Concentrations of trace elements in filtered water from Phase II of the Willamette River Basin Water Quality Study, Oregon, 1994-Continued

\begin{tabular}{|c|c|c|c|c|c|c|c|c|c|}
\hline STATION NUMBER & DATE & $\begin{array}{l}\text { LEAD, } \\
\text { DIS- } \\
\text { SOLVED } \\
\text { (UG/L } \\
\text { AS PB) }\end{array}$ & $\begin{array}{l}\text { MANGA- } \\
\text { NESE, } \\
\text { DIS- } \\
\text { SOLVED } \\
\text { (UG/L } \\
\text { AS MN) }\end{array}$ & $\begin{array}{l}\text { MOLYB- } \\
\text { DENUM, } \\
\text { DIS- } \\
\text { SOLVED } \\
\text { (UG/L } \\
\text { AS MO) }\end{array}$ & $\begin{array}{l}\text { NICKEL, } \\
\text { DIS- } \\
\text { SOLVED } \\
\text { (UG/L } \\
\text { AS NI) }\end{array}$ & $\begin{array}{l}\text { SELE- } \\
\text { NIUM, } \\
\text { DIS- } \\
\text { SOLVED } \\
\text { (UG/L } \\
\text { AS SE) }\end{array}$ & $\begin{array}{l}\text { SILVER, } \\
\text { DIS- } \\
\text { SOLVED } \\
\text { (UG/L } \\
\text { AS AG) }\end{array}$ & $\begin{array}{l}\text { URANIUM } \\
\text { NATURAL } \\
\text { DIS- } \\
\text { SOLVED } \\
\text { (UG/L } \\
\text { AS U) }\end{array}$ & $\begin{array}{l}\text { ZINC, } \\
\text { DIS- } \\
\text { SOLVED } \\
\text { (UG/L } \\
\text { AS ZN) }\end{array}$ \\
\hline 440402123063900 & $06-13-94$ & $<1.0000$ & 8.0000 & 10.000 & 1.0000 & $<1.0000$ & $<1.0000$ & $<1.0000$ & 49.000 \\
\hline 14190970 & $11-23-94$ & $<1.0000$ & 33.000 & $<1.0000$ & $<1.0000$ & $<1.0000$ & $<1.0000$ & $<1.0000$ & 6.0000 \\
\hline 451602122564400 & $05-31-94$ & $<1.0000$ & 12.000 & $<1.0000$ & $<1.0000$ & $<1.0000$ & $<1.0000$ & $<1.0000$ & 2.0000 \\
\hline 14206298 & $11-23-94$ & $<1.0000$ & 30.000 & $<1.0000$ & $<1.0000$ & $<1.0000$ & $<1.0000$ & $<1.0000$ & 4.0000 \\
\hline 14207500 & $11-04-94$ & -- & 96.000 & $<10.000$ & 5.0000 & $<1.0000$ & $<1.0000$ & -- & -- \\
\hline 453043122402200 & $06-17-94$ & 2.0000 & 19.000 & 11.000 & 3.0000 & $<1.0000$ & $<1.0000$ & $<1.0000$ & 97.000 \\
\hline 14211720 & $\begin{array}{l}05-10-94 \\
06-14-94 \\
10-25-94 \\
10-29-94 \\
11-03-94\end{array}$ & $\begin{array}{l}\quad--\overline{0} \\
<1.0000 \\
<1.0000 \\
<1.0000 \\
<1.0000\end{array}$ & $\begin{array}{l}11.000 \\
6.0000 \\
10.000 \\
8.0000 \\
10.000\end{array}$ & $\begin{array}{l}<10.000 \\
<1.0000 \\
<1.0000 \\
<1.0000 \\
<1.0000\end{array}$ & $\begin{array}{r}<1.0000 \\
<1.0000 \\
1.0000 \\
<1.0000 \\
<1.0000\end{array}$ & $\begin{array}{l}<1.0000 \\
<1.0000 \\
<1.0000 \\
<1.0000 \\
<1.0000\end{array}$ & $\begin{array}{l}<1.00000 \\
<1.00000 \\
<1.00000 \\
<1.00000 \\
<1.00000\end{array}$ & $\begin{array}{l}\quad-- \\
<1.0000 \\
<1.0000 \\
<1.0000 \\
<1.0000\end{array}$ & $\begin{array}{r}--- \\
2.0000 \\
<1.0000 \\
1.0000 \\
2.0000\end{array}$ \\
\hline 453154122394200 & $\begin{array}{l}05-31-94 \\
11-08-94\end{array}$ & $\begin{array}{l}4.0000 \\
3.0000\end{array}$ & $\begin{array}{l}27.000 \\
28.000\end{array}$ & $\begin{array}{l}7.0000 \\
2.0000\end{array}$ & $\begin{array}{l}7.0000 \\
4.0000\end{array}$ & $\begin{array}{l}<1.0000 \\
<1.0000\end{array}$ & $\begin{array}{l}<1.0000 \\
<1.0000\end{array}$ & $\begin{array}{l}<1.0000 \\
<1.0000\end{array}$ & $\begin{array}{l}54.000 \\
54.000\end{array}$ \\
\hline
\end{tabular}


Table 15. Concentrations of trace elements associated with suspended sediment from Phase I of the Willamette River Basin Water Quality Study, Oregon 1992-93

[Al, Aluminum; Au, Gold; Hg, Mercury; Se, Selenium; Ca, Calcium; Ba, Barium; Ho, Holmium; Sn, Tin; Fe, Iron; Be, Beryllium;

La, Lanthanum; Sr, Strontium; K, Potassium; Bi, Bismuth; Li, Lithium; Ta, Tantalum; Mg, Magnesium; Cd, Cadmium; Mo, Molybdenum;

Th, Thorium; Na, Sodium; Ce, Cerium; Nb, Niobium; Tl, Thallium; P, Phosphorus; Co, Cobalt; Nd, Neodymium; U, Uranium; Ti, Titanium;

Cr, Chromium; Ni, Nickel; V, Vanadium; Mn, Manganese; Cu, Copper; Pb, Lead; Y, Yttrium; Ag, Silver;Eu, Europium; Sb, Antimony;

Yb, Ytterbium; As, Arsenic; Ga, Gallium; Sc, Scandium; Zn, Zinc; 。, percent, ug/g, micrograms per gram]

Total elemental analysis of the suspended sediment as done by the Geologic Division of the U.S. Geological Survey

\begin{tabular}{|c|c|c|c|c|c|c|c|c|c|c|c|c|}
\hline Station Name & Date & Time & $\begin{array}{l}\text { Al } \\
(\%)\end{array}$ & $\begin{array}{l}\mathrm{Ca} \\
(\%)\end{array}$ & $\begin{array}{l}\mathrm{Fe} \\
(\%)\end{array}$ & $\begin{array}{l}K \\
(\%)\end{array}$ & $\begin{array}{l}\mathrm{Mg} \\
(\%)\end{array}$ & $\begin{array}{l}\mathrm{Na} \\
(\%)\end{array}$ & $\begin{array}{l}P \\
(\%)\end{array}$ & $\begin{array}{l}\mathrm{Ti} \\
(\%)\end{array}$ & $\begin{array}{c}\mathrm{Mn} \\
\text { (ug/g) }\end{array}$ & $\begin{array}{c}\mathrm{Ag} \\
(\mathrm{ug} / \mathrm{g})\end{array}$ \\
\hline Fanno Cr At Durham & $03-01-93$ & 1830 & 7.3 & 1.7 & 5.9 & 1.3 & 0.87 & 1.2 & 0.18 & 0.68 & 2700 & 0.9 \\
\hline A-3 Channel at Eugene & $10-29-92$ & 1000 & 7.0 & 2.7 & 5.4 & 0.69 & 1.3 & 1.4 & 0.35 & 0.53 & 1600 & 1.5 \\
\hline Johnson Cr at Gresham & $03-14-93$ & 1745 & 6.7 & 1.7 & 5.2 & 1.1 & 0.86 & 1.2 & 0.23 & 0.57 & 1000 & 0.4 \\
\hline Beaverton $\mathrm{Cr}$ at Orenco & $03-31-93$ & 1810 & 7.6 & 1.3 & 7.3 & 1.1 & 0.91 & 0.78 & 0.38 & 0.54 & 6200 & -- \\
\hline Pudding $\mathrm{R}$ at Aurora & $04-27-93$ & 1445 & 8.4 & 1.6 & 6.4 & 0.96 & 0.83 & 1.0 & 0.16 & 0.84 & 1500 & 0.3 \\
\hline Willamette $\mathrm{R}$ at Linnton & $04-18-92$ & 0900 & 8.8 & 1.5 & 6.4 & 0.83 & 1.0 & 0.81 & 0.22 & 0.72 & 2200 & 0.7 \\
\hline Zollner Cr nr Mt Angel & $06-01-93$ & 1115 & 8.6 & 1.2 & 7.1 & 1.4 & 0.98 & 0.60 & 0.43 & 0.73 & 4300 & 0.3 \\
\hline
\end{tabular}

\begin{tabular}{|c|c|c|c|c|c|c|c|c|c|c|c|c|c|}
\hline STATION NAME & $\begin{array}{c}\text { As } \\
(\mathrm{ug} / \mathrm{g})\end{array}$ & $\begin{array}{c}\mathrm{Au} \\
(\mathrm{ug} / \mathrm{g})\end{array}$ & $\begin{array}{l}\mathrm{Ba} \\
(\mathrm{ug} / \mathrm{g})\end{array}$ & $\begin{array}{c}\mathrm{Be} \\
(\mathrm{ug} / \mathrm{g})\end{array}$ & $\begin{array}{c}\mathrm{Bi} \\
(\mathrm{ug} / \mathrm{g})\end{array}$ & $\begin{array}{c}\mathrm{Cd} \\
(\mathrm{ug} / \mathrm{g})\end{array}$ & $\begin{array}{c}\mathrm{Ce} \\
(\mathrm{ug} / \mathrm{g})\end{array}$ & $\begin{array}{c}\text { Co } \\
\text { (ug/g) }\end{array}$ & $\begin{array}{c}\mathrm{Cr} \\
(\mathrm{ug} / \mathrm{g})\end{array}$ & $\begin{array}{c}\mathrm{Cu} \\
(\mathrm{ug} / \mathrm{g})\end{array}$ & $\begin{array}{c}E u \\
(\mathrm{ug} / \mathrm{g})\end{array}$ & $\begin{array}{c}\mathrm{Ga} \\
(\mathrm{ug} / \mathrm{g})\end{array}$ & $\begin{array}{c}\mathrm{Hg} \\
(\mathrm{ug} / \mathrm{g})\end{array}$ \\
\hline Fanno $\mathrm{Cr}$ At Durham & 8.5 & $<8$ & 670 & 2 & $<10$ & 0.8 & 66 & 27 & 80 & 44 & $<2$ & 17 & 0.12 \\
\hline A-3 Channel at Eugene & 19.0 & $<8$ & 420 & $<1$ & $<10$ & 3.7 & 39 & 27 & 200 & 180 & $<2$ & 14 & 1.20 \\
\hline Johnson Cr at Gresham & 5.8 & $<8$ & 560 & 1 & $<10$ & 1.3 & 47 & 25 & 91 & 70 & $<2$ & 14 & 0.18 \\
\hline Beaverton $\mathrm{Cr}$ at Beaverton & 18.0 & $<8$ & 670 & 2 & $<10$ & $<2$ & 58 & 31 & 110 & 79 & $<2$ & 19 & 0.44 \\
\hline Pudding $\mathrm{R}$ at Aurora & 7.3 & $<8$ & 590 & 2 & $<10$ & 0.3 & 55 & 27 & 55 & 34 & $<2$ & 18 & 0.06 \\
\hline Willamette $\mathrm{R}$ at Linnton & 7.8 & $<8$ & 440 & 1 & $<10$ & 0.9 & 56 & 29 & 85 & 68 & $<2$ & 20 & 0.14 \\
\hline Zollner Cr nr Mt Angel & 13.0 & $<8$ & 790 & 2 & $<10$ & 1.6 & 68 & 29 & 93 & 52 & $<2$ & 22 & 0.31 \\
\hline STATION NAME & $\begin{array}{c}\text { Ho } \\
(\mathrm{ug} / \mathrm{g})\end{array}$ & $\begin{array}{c}\mathrm{La} \\
(\mathrm{ug} / \mathrm{g})\end{array}$ & $\begin{array}{c}\mathrm{Li} \\
(\mathrm{ug} / \mathrm{g})\end{array}$ & $\begin{array}{c}\text { Mo } \\
(\mathrm{ug} / \mathrm{g})\end{array}$ & $\begin{array}{c}\mathrm{Nb} \\
(\mathrm{ug} / \mathrm{g})\end{array}$ & $\begin{array}{c}\mathrm{Nd} \\
(\mathrm{ug} / \mathrm{g})\end{array}$ & $\begin{array}{c}\mathrm{Ni} \\
(\mathrm{ug} / \mathrm{g})\end{array}$ & $\begin{array}{c}\mathrm{Pb} \\
\text { (ug/g) }\end{array}$ & $\begin{array}{c}\mathrm{Sb} \\
(\mathrm{ug} / \mathrm{g})\end{array}$ & $\begin{array}{c}\mathrm{Sc} \\
(\mathrm{ug} / \mathrm{g})\end{array}$ & $\begin{array}{c}\mathrm{Se} \\
(\mathrm{ug} / \mathrm{g})\end{array}$ & $\begin{array}{c}\mathrm{Sn} \\
(\mathrm{ug} / \mathrm{g})\end{array}$ & $\begin{array}{c}\mathrm{Sr} \\
(\mathrm{ug} / \mathrm{g})\end{array}$ \\
\hline Fanno Cr At Durham & $<4$ & 33 & 23 & 1.8 & 14 & 30 & 33 & 88.4 & 2.3 & 19 & 0.3 & $<5$ & 240 \\
\hline A-3 Channel at Eugene & $<4$ & 18 & 15 & 7.0 & 11 & 20 & 64 & 242.0 & 5.7 & 18 & 0.3 & $<5$ & 310 \\
\hline Johnson Cr at Gresham & $<4$ & 24 & 19 & 5.4 & 14 & 23 & 53 & 115.0 & 1.8 & 15 & 0.4 & $<5$ & 230 \\
\hline Beaverton $\mathrm{Cr}$ at Beaverton & $<4$ & 30 & 31 & $<2$ & 12 & 27 & 42 & 74.0 & -- & 20 & 0.4 & $<5$ & 170 \\
\hline Pudding $\mathrm{R}$ at Aurora & $<4$ & 27 & 23 & 0.9 & 15 & 27 & 22 & 16.0 & 0.6 & 24 & 0.3 & $<5$ & 230 \\
\hline Willamette $\mathrm{R}$ at Linnton & $<4$ & 26 & 27 & 1.1 & 15 & 26 & 38 & 28.0 & 0.8 & 25 & 0.6 & 7 & 200 \\
\hline Zollner Cr nr Mt Angel & $<4$ & 36 & 35 & 1.1 & 16 & 34 & 35 & 40.0 & 1.3 & 23 & 0.4 & $<5$ & 160 \\
\hline STATION NAME & $\begin{array}{c}\mathrm{Ta} \\
(\mathrm{ug} / \mathrm{g})\end{array}$ & $\begin{array}{c}\text { Th } \\
(\mathrm{ug} / \mathrm{g})\end{array}$ & $\begin{array}{c}\mathrm{Tl} \\
(\mathrm{ug} / \mathrm{g})\end{array}$ & $\begin{array}{c}\mathrm{U} \\
(\mathrm{ug} / \mathrm{g})\end{array}$ & $\begin{array}{c}\mathrm{V} \\
(\mathrm{ug} / \mathrm{g})\end{array}$ & $\begin{array}{c}Y \\
(u g / g)\end{array}$ & $\begin{array}{c}Y b \\
(u g / g)\end{array}$ & $\begin{array}{l}\mathrm{Zn} \\
(\mathrm{ug} / \mathrm{g})\end{array}$ & $\begin{array}{l}\text { Suspended } \\
\text { sediment } \\
(\mathrm{mg} / \mathrm{L})\end{array}$ & & & & \\
\hline Fanno Cr At Durham & $<40$ & 8.1 & 0.46 & 2.1 & 170 & 26 & 2 & 370 & 203 & & & & \\
\hline A-3 Channel at Eugene & $<40$ & 2.7 & 0.20 & 1.4 & 140 & 21 & 2 & 1200 & 90 & & & & \\
\hline Johnson Cr at Gresham & $<40$ & 6.5 & 0.30 & 1.8 & 150 & 20 & 2 & 470 & 24 & & & & \\
\hline Beaverton $\mathrm{Cr}$ at Beaverton & $<40$ & 8.0 & -- & -- & 170 & 27 & 2 & 440 & 10 & & & & \\
\hline Pudding $\mathrm{R}$ at Aurora & $<40$ & 6.0 & 0.30 & 1.9 & 200 & 28 & 3 & 120 & 27 & & & & \\
\hline Willamette $\mathrm{R}$ at Linnton & $<40$ & 5.7 & 0.20 & 1.8 & 180 & 27 & 2 & 200 & 32 & & & & \\
\hline Zollner Cr nr Mt Angel & $<40$ & 10.3 & 0.52 & 2.3 & 180 & 32 & 3 & 350 & 33 & & & & \\
\hline
\end{tabular}

Conversion Factors

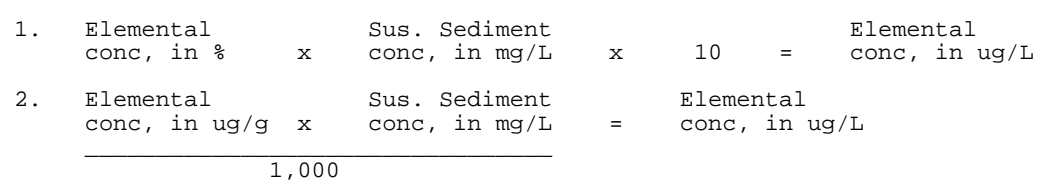


Table 16. Concentrations of trace elements associated with bed sediment from Phase I of the Willamette River Basin Water Quality Study, Oregon, 1992

[USGS laboratory schedule 2400; WS, wet sieved; DW, dry weight; BM, bed material; UG/G, micrograms per gram]

\begin{tabular}{|c|c|c|c|c|c|}
\hline STATION NUMBER & STATION NAME & DATE & TIME & $\begin{array}{c}\text { CARBON, } \\
\text { INORG, } \\
\text { SED, BM } \\
\text { WS, <63U } \\
\text { DW, REC } \\
\text { (PERCENT) }\end{array}$ & $\begin{array}{l}\text { CARBON, } \\
\text { ORGANIC } \\
\text { SED, BM } \\
\text { WS, }<63 U \\
\text { DW, REC } \\
\text { (PERCENT) }\end{array}$ \\
\hline 440313123091100 & A-3 CHANNEL AT WALLIS AND 5TH ST AT EUGENE, OR & $08-25-92$ & 1230 & 0.04000 & 8.6500 \\
\hline 14165500 & MCKENZIE RIVER NEAR COBURG, OREG. & $08-25-92$ & 1645 & $<0.01000$ & 6.5100 \\
\hline 443207123145500 & WILLAMETTE RIVER NEAR CORVALLIS, OR & $08-26-92$ & 1030 & $<0.01000$ & 4.7600 \\
\hline 443819123064000 & CALAPOOIA RIVER AT MOUTH AT ALBANY, OR & $08-26-92$ & 1500 & 0.01000 & 1.9900 \\
\hline 444032123050600 & MIDDLE FOURTH LAKE NEAR ALBANY, OR & $09-01-92$ & 1100 & $<0.01000$ & 5.2100 \\
\hline 444416123030800 & SANTIAM RIVER NEAR JEFFERSON, OR & $08-29-92$ & 1000 & 0.01000 & 3.5400 \\
\hline 445543123084400 & RICKREALL CREEK NEAR RICKREALL, OR & $08-28-92$ & 1345 & 0.02000 & 2.5200 \\
\hline 451320123041100 & YAMHILL RIVER AT DAYTON, OR & $08-27-92$ & 1400 & $<0.01000$ & 1.7500 \\
\hline 451705122575100 & WILLAMETTE RIVER AT NEWBERG, OR & $09-01-92$ & 1600 & $<0.01000$ & 2.4800 \\
\hline 452950122492900 & BEAVERTON CREEK AT BEAVERTON, OR & $08-23-92$ & 1100 & $<0.01000$ & 4.9400 \\
\hline 452221122362400 & CLACKAMAS RIVER AT OREGON CITY, OR & $08-24-92$ & 1130 & 0.02000 & 3.5900 \\
\hline 452847122244500 & JOHNSON CREEK AT GRESHAM, OR & $08-21-92$ & 1330 & $<0.01000$ & 2.0900 \\
\hline 453547122463000 & WILLAMETTE RIVER AT LINNTON, OR & $09-09-92$ & 1130 & 0.01000 & 2.7300 \\
\hline 453205122223701 & BEAVER CREEK NEAR TROUTDALE, & $08-22-92$ & 0945 & 0.02000 & 2.8700 \\
\hline
\end{tabular}


Table 16. Concentrations of trace elements associated with bed sediment from Phase I of the Willamette River Basin Water Quality Study, Oregon, 1992-Continued

\begin{tabular}{|c|c|c|c|c|c|c|c|c|c|c|}
\hline STATION NUMBER & DATE & $\begin{array}{c}\text { CARBON, } \\
\text { ORG + } \\
\text { INORG, } \\
\text { SED, BM } \\
\text { WS, <63U } \\
\text { DW, REC } \\
\text { (percent) }\end{array}$ & $\begin{array}{l}\text { ALUM- } \\
\text { INUM } \\
\text { BOT MAT } \\
<63 \text { WS } \\
\text { FIELD } \\
\text { (percent) }\end{array}$ & $\begin{array}{l}\text { ANTI- } \\
\text { MONY } \\
\text { BOT MAT } \\
<63 \text { W WS } \\
\text { FIELD } \\
\text { (UG/G) }\end{array}$ & $\begin{array}{c}\text { ARSENIC } \\
\text { BOT MAT } \\
<63 \text { W WS } \\
\text { FIELD } \\
(\mathrm{UG} / \mathrm{G})\end{array}$ & $\begin{array}{l}\text { BARIUM } \\
\text { BOT MAT } \\
<63 \text { WS } \\
\text { FIELD } \\
\text { (UG/G) }\end{array}$ & $\begin{array}{l}\text { BERYL- } \\
\text { LIUM } \\
\text { BOT MAT } \\
<63 U \text { WS } \\
\text { FIELD } \\
\text { (UG/G) }\end{array}$ & $\begin{array}{l}\text { BISMUTH } \\
\text { BOT MAT } \\
<180 \text { UWS } \\
\text { FIELD } \\
(U G / G)\end{array}$ & $\begin{array}{l}\text { CADMIUM } \\
\text { BOT MAT } \\
<63 \text { WS } \\
\text { FIELD } \\
(U G / G)\end{array}$ & $\begin{array}{c}\text { CALCIUM } \\
\text { BOT MAT } \\
<63 \mathrm{U} \text { WS } \\
\text { FIELD } \\
\text { (percent) }\end{array}$ \\
\hline 440313123091100 & $08-25-92$ & 8.6900 & 7.3000 & 4.0000 & 12.000 & 360.00 & 1.0000 & $<10.000$ & 2.2000 & 2.9000 \\
\hline 14165500 & $08-25-92$ & 6.5100 & 5.9000 & 1.0000 & 3.7000 & 240.00 & $<1.0000$ & $<10.000$ & 0.30000 & 1.5000 \\
\hline 443207123145500 & $08-26-92$ & 4.7600 & 6.8000 & 1.0000 & 4.1000 & 300.00 & 1.0000 & $<10.000$ & 0.20000 & 1.9000 \\
\hline 443819123064000 & $08-26-92$ & 2.0000 & 8.1000 & 1.0000 & 6.8000 & 480.00 & 1.0000 & $<10.000$ & 0.20000 & 1.8000 \\
\hline 444032123050600 & $09-01-92$ & 5.2100 & 7.6000 & 2.0000 & 5.7000 & 420.00 & 1.0000 & $<10.000$ & 0.50000 & 2.0000 \\
\hline 444416123030800 & $08-29-92$ & 3.5500 & 8.0000 & 1.0000 & 7.5000 & 350.00 & 1.0000 & $<10.000$ & 0.30000 & 2.0000 \\
\hline 445543123084400 & $08-28-92$ & 2.5400 & 7.7000 & 2.0000 & 5.2000 & 400.00 & 2.0000 & $<10.000$ & 0.30000 & 2.1000 \\
\hline 451320123041100 & $08-27-92$ & 1.7500 & 7.9000 & 2.0000 & 6.6000 & 540.00 & 2.0000 & $<10.000$ & 0.30000 & 1.6000 \\
\hline 451705122575100 & $09-01-92$ & 2.4800 & 8.3000 & 1.0000 & 4.6000 & 420.00 & 1.0000 & $<10.000$ & 0.20000 & 2.3000 \\
\hline 452950122492900 & $08-23-92$ & 4.9400 & 6.8000 & 4.0000 & 10.000 & 620.00 & 2.0000 & $<10.000$ & 3.1000 & 1.7000 \\
\hline 452221122362400 & $08-24-92$ & 3.6100 & 7.3000 & 1.0000 & 7.3000 & 450.00 & 1.0000 & $<10.000$ & 0.30000 & 1.9000 \\
\hline 452847122244500 & $08-21-92$ & 2.0900 & 6.7000 & 0.80000 & 3.5000 & 670.00 & 2.0000 & $<10.000$ & 0.30000 & 1.1000 \\
\hline 453547122463000 & $09-09-92$ & 2.7400 & 8.5000 & 1.0000 & 6.6000 & 490.00 & 1.0000 & $<10.000$ & 0.40000 & 2.0000 \\
\hline 453205122223701 & $08-22-92$ & 2.8900 & 7.3000 & 1.0000 & 4.2000 & 680.00 & 2.0000 & $<10.000$ & 0.50000 & 1.7000 \\
\hline
\end{tabular}


Table 16. Concentrations of trace elements associated with bed sediment from Phase I of the Willamette River Basin Water Quality Study, Oregon, 1992-Continued

\begin{tabular}{|c|c|c|c|c|c|c|c|c|c|c|}
\hline STATION NUMBER & DATE & $\begin{array}{l}\text { CERIUM } \\
\text { BOT MAT } \\
<63 \text { WS } \\
\text { FIELD } \\
(U G / G)\end{array}$ & $\begin{array}{l}\text { CHRO- } \\
\text { MIUM } \\
\text { BOT MAT } \\
<63 \text { WS } \\
\text { FIELD } \\
(\text { UG/G) }\end{array}$ & $\begin{array}{l}\text { COBALT } \\
\text { BOT MAT } \\
<63 \text { WS } \\
\text { FIELD } \\
(\text { UG/G) }\end{array}$ & $\begin{array}{l}\text { COPPER } \\
\text { BOT MAT } \\
<63 \text { WS } \\
\text { FIELD } \\
(\text { UG/G) }\end{array}$ & $\begin{array}{l}\text { EURO- } \\
\text { PIUM } \\
\text { BOT MAT } \\
<63 \text { W WS } \\
\text { FIELD } \\
(\text { UG/G) }\end{array}$ & $\begin{array}{l}\text { GALLIUM } \\
\text { BOT MAT } \\
<63 \text { WS } \\
\text { FIELD } \\
(\text { UG/G) }\end{array}$ & $\begin{array}{l}\text { GOLD } \\
\text { BOT MAT } \\
<63 \text { WS } \\
\text { FIELD } \\
(\mathrm{UG} / \mathrm{G})\end{array}$ & $\begin{array}{l}\text { HOLMIUM } \\
\text { BOT MAT } \\
<63 \text { WS } \\
\text { FIELD } \\
(\text { UG/G) }\end{array}$ & $\begin{array}{l}\text { IRON } \\
\text { BOT MAT } \\
<63 \mathrm{U} \text { WS } \\
\text { FIELD } \\
\text { (percent) }\end{array}$ \\
\hline 440313123091100 & $08-25-92$ & 31.000 & 140.00 & 30.000 & 140.00 & $<2.000$ & 21.000 & $<8.0000$ & $<4.0000$ & 5.6000 \\
\hline 14165500 & $08-25-92$ & 23.000 & 70.000 & 19.000 & 43.000 & $<2.000$ & 14.000 & $<8.0000$ & $<4.0000$ & 4.5000 \\
\hline 443207123145500 & $08-26-92$ & 28.000 & 88.000 & 21.000 & 36.000 & $<2.000$ & 16.000 & $<8.0000$ & $<4.0000$ & 4.5000 \\
\hline 443819123064000 & $08-26-92$ & 43.000 & 90.000 & 26.000 & 44.000 & $<2.000$ & 22.000 & $<8.0000$ & $<4.0000$ & 5.3000 \\
\hline 444032123050600 & $09-01-92$ & 8.0000 & 96.000 & 17.000 & 72.000 & $<2.000$ & 18.000 & $<8.0000$ & 5.0000 & 4.9000 \\
\hline 444416123030800 & $08-29-92$ & 36.000 & 94.000 & 36.000 & 52.000 & $<2.000$ & 21.000 & $<8.0000$ & $<4.0000$ & 5.9000 \\
\hline 445543123084400 & $08-28-92$ & 42.000 & 150.00 & 34.000 & 120.00 & $<2.000$ & 22.000 & $<8.0000$ & $<4.0000$ & 7.7000 \\
\hline 451320123041100 & $08-27-92$ & 49.000 & 120.00 & 31.000 & 73.000 & $<2.000$ & 21.000 & $<8.0000$ & $<4.0000$ & 6.9000 \\
\hline 451705122575100 & $09-01-92$ & 38.000 & 110.00 & 24.000 & 48.000 & $<2.000$ & 20.000 & $<8.0000$ & $<4.0000$ & 5.4000 \\
\hline 452950122492900 & $08-23-92$ & 56.000 & 110.00 & 29.000 & 150.00 & $<2.000$ & 20.000 & $<8.0000$ & $<4.0000$ & 5.2000 \\
\hline 452221122362400 & $08-24-92$ & 44.000 & 89.000 & 25.000 & 36.000 & $<2.000$ & 19.000 & $<8.0000$ & $<4.0000$ & 5.3000 \\
\hline 452847122244500 & $08-21-92$ & 62.000 & 74.000 & 19.000 & 23.000 & $<2.000$ & 17.000 & $<8.0000$ & $<4.0000$ & 3.7000 \\
\hline 453547122463000 & $09-09-92$ & 46.000 & 83.000 & 26.000 & 65.000 & $<2.000$ & 20.000 & $<8.0000$ & $<4.0000$ & 5.8000 \\
\hline 453205122223701 & $08-22-92$ & 53.000 & 73.000 & 23.000 & 38.000 & $<2.000$ & 19.000 & $<8.0000$ & $<4.0000$ & 4.5000 \\
\hline
\end{tabular}


Table 16. Concentrations of trace elements associated with bed sediment from Phase I of the Willamette River Basin Water Quality Study, Oregon, 1992-Continued

\begin{tabular}{|c|c|c|c|c|c|c|c|c|c|c|}
\hline STATION NUMBER & DATE & $\begin{array}{c}\text { LANTHA- } \\
\text { NUM } \\
\text { BOT MAT } \\
<63 \text { W WS } \\
\text { FIELD } \\
\text { (UG/G) } \\
\end{array}$ & $\begin{array}{l}\text { LEAD } \\
\text { BOT MAT } \\
<63 \text { WS } \\
\text { FIELD } \\
(\mathrm{UG} / \mathrm{G}) \\
\end{array}$ & $\begin{array}{c}\text { LITHIUM } \\
\text { BOT MAT } \\
<63 \text { W WS } \\
\text { FIELD } \\
(U G / G) \\
\end{array}$ & $\begin{array}{l}\text { MAGNE- } \\
\text { SIUM } \\
\text { BOT MAT } \\
<63 \text { W WS } \\
\text { FIELD } \\
\text { (percent) }\end{array}$ & $\begin{array}{l}\text { MANGA- } \\
\text { NESE } \\
\text { BOT MAT } \\
<63 \text { U WS } \\
\text { FIELD } \\
\text { (UG/G) }\end{array}$ & $\begin{array}{c}\text { MERCURY } \\
\text { BOT MAT } \\
<63 \text { WS } \\
\text { FIELD } \\
\text { (UG/G) }\end{array}$ & $\begin{array}{c}\text { MOLYB- } \\
\text { DENUM } \\
\text { BOT MAT } \\
<63 \text { WS } \\
\text { FIELD } \\
(\text { UG/G) }\end{array}$ & $\begin{array}{c}\text { NEODYM- } \\
\text { IUM } \\
\text { BOT MAT } \\
<63 \text { W WS } \\
\text { FIELD } \\
\text { (UG/G) }\end{array}$ & $\begin{array}{l}\text { NICKEL } \\
\text { BOT MAT } \\
<63 \text { WS } \\
\text { FIELD } \\
(\mathrm{UG} / \mathrm{G}) \\
\end{array}$ \\
\hline 440313123091100 & $08-25-92$ & 18.000 & 140.00 & 20.000 & 1.4000 & 2600.0 & 1.2000 & $<2.0000$ & 24.000 & 56.000 \\
\hline 14165500 & $08-25-92$ & 14.000 & 19.000 & 20.000 & 0.90000 & 1100.0 & 0.08000 & $<2.0000$ & 16.000 & 26.000 \\
\hline 443207123145500 & $08-26-92$ & 17.000 & 13.000 & 20.000 & 1.1000 & 1300.0 & 0.07000 & $<2.0000$ & 19.000 & 33.000 \\
\hline 443819123064000 & $08-26-92$ & 26.000 & 9.0000 & 20.000 & 1.0000 & 2300.0 & $<0.02000$ & $<2.0000$ & 27.000 & 25.000 \\
\hline 444032123050600 & $09-01-92$ & 25.000 & 28.000 & 20.000 & 0.84000 & 450.00 & 0.25000 & $<2.0000$ & 29.000 & 63.000 \\
\hline 444416123030800 & $08-29-92$ & 20.000 & 14.000 & 20.000 & 1.2000 & 2000.0 & 0.05000 & $<2.0000$ & 22.000 & 35.000 \\
\hline 445543123084400 & $08-28-92$ & 29.000 & 10.000 & 30.000 & 1.7000 & 960.00 & 0.05000 & $<2.0000$ & 34.000 & 59.000 \\
\hline 451320123041100 & $08-27-92$ & 30.000 & 11.000 & 40.000 & 1.3000 & 1100.0 & 0.05000 & $<2.0000$ & 31.000 & 42.000 \\
\hline 451705122575100 & $09-01-92$ & 23.000 & 9.0000 & 20.000 & 1.3000 & 920.00 & 0.06000 & $<2.0000$ & 25.000 & 37.000 \\
\hline 452950122492900 & $08-23-92$ & 30.000 & 240.00 & 20.000 & 0.86000 & 1700.0 & 0.45000 & $<2.0000$ & 28.000 & 41.000 \\
\hline 452221122362400 & $08-24-92$ & 27.000 & 23.000 & 20.000 & 1.1000 & 1200.0 & 0.08000 & $<2.0000$ & 27.000 & 34.000 \\
\hline 452847122244500 & $08-21-92$ & 37.000 & 27.000 & 20.000 & 0.58000 & 1000.0 & 0.05000 & $<2.0000$ & 31.000 & 25.000 \\
\hline 453547122463000 & $09-09-92$ & 27.000 & 27.000 & 30.000 & 1.2000 & 1200.0 & 0.11000 & $<2.0000$ & 29.000 & 35.000 \\
\hline 453205122223701 & $08-22-92$ & 32.000 & 29.000 & 20.000 & 0.81000 & 1600.0 & 0.10000 & $<2.0000$ & 29.000 & 30.000 \\
\hline
\end{tabular}


Table 16. Concentrations of trace elements associated with bed sediment from Phase I of the Willamette River Basin Water Quality Study, Oregon, 1992-Continued

\begin{tabular}{|c|c|c|c|c|c|c|c|c|c|c|}
\hline STATION NUMBER & DATE & $\begin{array}{c}\text { NIOBIUM } \\
\text { BOT MAT } \\
<63 \text { WS } \\
\text { FIELD } \\
\text { (UG/G) }\end{array}$ & $\begin{array}{c}\text { PHOS- } \\
\text { PHORUS } \\
\text { BOT MAT } \\
<63 \text { WS } \\
\text { FIELD } \\
\text { (percent) } \\
\end{array}$ & $\begin{array}{c}\text { POTAS- } \\
\text { SIUM } \\
\text { BOT MAT } \\
<63 U \text { WS } \\
\text { FIELD } \\
\text { (percent) }\end{array}$ & $\begin{array}{l}\text { SCAN- } \\
\text { DIUM } \\
\text { BOT MAT } \\
<63 \text { W WS } \\
\text { FIELD } \\
\text { (UG/G) } \\
\end{array}$ & $\begin{array}{c}\text { SELE- } \\
\text { NIUM } \\
\text { BOT MAT } \\
<63 U \text { WS } \\
\text { FIELD } \\
\text { (UG/G) }\end{array}$ & $\begin{array}{l}\text { SILVER } \\
\text { BOT MAT } \\
<63 \text { WS } \\
\text { FIELD } \\
\text { (UG/G) } \\
\end{array}$ & $\begin{array}{l}\text { SODIUM } \\
\text { BOT MAT } \\
<63 \text { WS } \\
\text { FIELD } \\
\text { (percent) }\end{array}$ & $\begin{array}{c}\text { STRON- } \\
\text { TIUM } \\
\text { BOT MAT } \\
<63 \text { U WS } \\
\text { FIELD } \\
\text { (UG/G) } \\
\end{array}$ & $\begin{array}{c}\text { TANTA- } \\
\text { LUM } \\
\text { BOT MAT } \\
<63 \text { W WS } \\
\text { FIELD } \\
\text { (UG/G) } \\
\end{array}$ \\
\hline 440313123091100 & $08-25-92$ & 8.0000 & 0.35000 & 0.68000 & 20.000 & 0.20000 & 1.4000 & 1.4000 & 330.00 & $<40.000$ \\
\hline 14165500 & $08-25-92$ & 6.0000 & 0.21000 & 0.42000 & 16.000 & 0.50000 & 0.20000 & 0.76000 & 190.00 & $<40.000$ \\
\hline 443207123145500 & $08-26-92$ & 7.0000 & 0.17000 & 0.56000 & 18.000 & 0.20000 & 0.30000 & 0.99000 & 250.00 & $<40.000$ \\
\hline 443819123064000 & $08-26-92$ & 11.000 & 0.11000 & 0.98000 & 21.000 & 0.10000 & 0.20000 & 1.4000 & 270.00 & $<40.000$ \\
\hline 444032123050600 & $09-01-92$ & 150.00 & 0.20000 & 0.97000 & 21.000 & 0.20000 & 0.90000 & 1.3000 & 240.00 & $<40.000$ \\
\hline 444416123030800 & $08-29-92$ & 9.0000 & 0.13000 & 0.64000 & 23.000 & 0.20000 & 0.10000 & 1.1000 & 260.00 & $<40.000$ \\
\hline 445543123084400 & $08-28-92$ & 17.000 & 0.15000 & 1.0000 & 30.000 & 0.50000 & 0.30000 & 0.93000 & 180.00 & $<40.000$ \\
\hline 451320123041100 & $08-27-92$ & 16.000 & 0.14000 & 1.2000 & 26.000 & 0.80000 & 0.30000 & 1.0000 & 190.00 & $<40.000$ \\
\hline 451705122575100 & $09-01-92$ & 9.0000 & 0.13000 & 0.85000 & 22.000 & 0.30000 & 0.50000 & 1.5000 & 320.00 & $<40.000$ \\
\hline 452950122492900 & $08-23-92$ & 11.000 & 0.18000 & 1.3000 & 18.000 & 0.30000 & 1.8000 & 1.4000 & 230.00 & $<40.000$ \\
\hline 452221122362400 & $08-24-92$ & 10.000 & 0.15000 & 0.94000 & 18.000 & 0.20000 & 0.10000 & 1.1000 & 230.00 & $<40.000$ \\
\hline 452847122244500 & $08-21-92$ & 12.000 & 0.11000 & 1.4000 & 12.000 & 0.20000 & 0.10000 & 1.3000 & 240.00 & $<40.000$ \\
\hline 453547122463000 & $09-09-92$ & 11.000 & 0.17000 & 0.98000 & 22.000 & 0.40000 & 0.60000 & 1.3000 & 280.00 & $<40.000$ \\
\hline 453205122223701 & $08-22-92$ & 12.000 & 0.14000 & 1.3000 & 16.000 & 0.30000 & 0.20000 & 1.6000 & 300.00 & $<40.000$ \\
\hline
\end{tabular}


Table 16. Concentrations of trace elements associated with bed sediment from Phase I of the Willamette River Basin Water Quality Study, Oregon, 1992-Continued

\begin{tabular}{|c|c|c|c|c|c|c|c|c|c|}
\hline STATION NUMBER & DATE & $\begin{array}{c}\text { THORIUM } \\
\text { BOT MAT } \\
<63 \text { WS } \\
\text { FIELD } \\
(\text { UG } / G)\end{array}$ & $\begin{array}{c}\text { TIN } \\
\text { BOT MAT } \\
<63 \text { U WS } \\
\text { FIELD } \\
(\mathrm{UG} / \mathrm{G})\end{array}$ & $\begin{array}{c}\text { TITA- } \\
\text { NIUM, } \\
\text { BOT MAT } \\
<63 U \text { WS } \\
\text { FIELD } \\
\text { (PERCENT) }\end{array}$ & $\begin{array}{c}\text { URANIUM } \\
\text { BOT MAT } \\
<63 \text { WS } \\
\text { FIELD } \\
\text { (UG/G) }\end{array}$ & $\begin{array}{l}\text { VANA- } \\
\text { DIUM } \\
\text { BOT MAT } \\
<63 \text { W WS } \\
\text { FIELD } \\
\text { (UG/G) }\end{array}$ & $\begin{array}{l}\text { YTTER- } \\
\text { BIUM } \\
\text { BOT MAT } \\
<63 \text { W WS } \\
\text { FIELD } \\
\text { (UG/G) }\end{array}$ & $\begin{array}{l}\text { YTTRIUM } \\
\text { BOT MAT } \\
<63 \text { W WS } \\
\text { FIELD } \\
\text { (UG/G) }\end{array}$ & $\begin{array}{l}\text { ZINC } \\
\text { BOT MAT } \\
<63 \text { WS } \\
\text { FIELD } \\
\text { (UG/G) }\end{array}$ \\
\hline 440313123091100 & $08-25-92$ & 4.0000 & $<10.000$ & 0.65000 & 1.5000 & 150.00 & 2.0000 & 23.000 & 840.00 \\
\hline 14165500 & $08-25-92$ & $<4.0000$ & $<10.000$ & 0.54000 & 1.6000 & 160.00 & 2.0000 & 18.000 & 97.000 \\
\hline 443207123145500 & $08-26-92$ & 4.0000 & $<10.000$ & 0.59000 & 1.1000 & 140.00 & 2.0000 & 19.000 & 100.00 \\
\hline 443819123064000 & $08-26-92$ & 6.0000 & $<10.000$ & 0.95000 & 2.0000 & 170.00 & 2.0000 & 22.000 & 110.00 \\
\hline 444032123050600 & $09-01-92$ & 11.000 & 10.000 & 0.71000 & 14.000 & 130.00 & 6.0000 & 45.000 & 160.00 \\
\hline 444416123030800 & $08-29-92$ & 5.0000 & $<10.000$ & 0.79000 & 1.5000 & 180.00 & 2.0000 & 24.000 & 110.00 \\
\hline 445543123084400 & $08-28-92$ & 7.0000 & $<10.000$ & 1.4000 & 2.2000 & 290.00 & 3.0000 & 29.000 & 140.00 \\
\hline 451320123041100 & $08-27-92$ & 7.0000 & $<10.000$ & 1.2000 & 2.5000 & 250.00 & 3.0000 & 27.000 & 130.00 \\
\hline 451705122575100 & $09-01-92$ & 5.0000 & $<10.000$ & 0.81000 & 2.1000 & 170.00 & 3.0000 & 24.000 & 120.00 \\
\hline 452950122492900 & $08-23-92$ & 8.0000 & $<10.000$ & 0.71000 & 2.7000 & 160.00 & 2.0000 & 35.000 & 670.00 \\
\hline 452221122362400 & $08-24-92$ & 8.0000 & $<10.000$ & 0.80000 & 2.9000 & 170.00 & 2.0000 & 22.000 & 110.00 \\
\hline 452847122244500 & $08-21-92$ & 10.000 & $<10.000$ & 0.65000 & 4.0000 & 100.00 & 2.0000 & 17.000 & 130.00 \\
\hline 453547122463000 & $09-09-92$ & 6.0000 & $<10.000$ & 0.79000 & 2.5000 & 160.00 & 2.0000 & 26.000 & 190.00 \\
\hline 453205122223701 & $08-22-92$ & 9.0000 & $<10.000$ & 0.68000 & 2.9000 & 130.00 & 2.0000 & 21.000 & 190.00 \\
\hline
\end{tabular}


Table 17. Concentrations of organochlorine and organophosphorus compounds in whole water from Phase I of the Willamette River Basin Water Quality Study, Oregon, 1993

[USGS laboratory schedule 1399]

\begin{tabular}{|c|c|c|c|c|c|c|}
\hline STATION NUMBER & STATION NAME & DATE & TIME & $\begin{array}{c}\text { ALDRIN } \\
\text { TOTAL } \\
\text { (UG/L) }\end{array}$ & $\begin{array}{c}\text { CHLOR- } \\
\text { N, DANE, } \\
\text { TOTAL } \\
(\mathrm{UG} / \mathrm{L}) \\
\end{array}$ & $\begin{array}{r}\mathrm{p}, \mathrm{p}^{\prime}- \\
\mathrm{DDD} \\
\text { TOTAL } \\
(\mathrm{UG} / \mathrm{L}) \\
\end{array}$ \\
\hline 443207123145500 & WILLAMETTE RIVER NEAR CORVALLIS, OR & $08-24-93$ & 1615 & $<0.00100$ & $<0.10000$ & $<0.00100$ \\
\hline 443138123120901 & MUDDY CREEK NEAR PEORIA, OR & $08-24-93$ & 1300 & $<0.00100$ & $<0.10000$ & $<0.00100$ \\
\hline 451309123041501 & PALMER C AT DAYTON, OR & $09-07-93$ & 1045 & $<0.00100$ & $<0.10000$ & $<0.00100$ \\
\hline 451320123041100 & YAMHILL RIVER AT DAYTON, OR & $09-07-93$ & 1420 & $<0.00100$ & $<0.10000$ & $<0.00100$ \\
\hline 14201300 & ZOLLNER CREEK NEAR MT ANGEL, OR & $07-27-93$ & 1015 & $<0.00100$ & $<0.10000$ & $<0.00100$ \\
\hline 14206950 & FANNO CREEK AT DURHAM, OR & $07-22-93$ & 1000 & $<0.00100$ & $<0.10000$ & $<0.00100$ \\
\hline
\end{tabular}

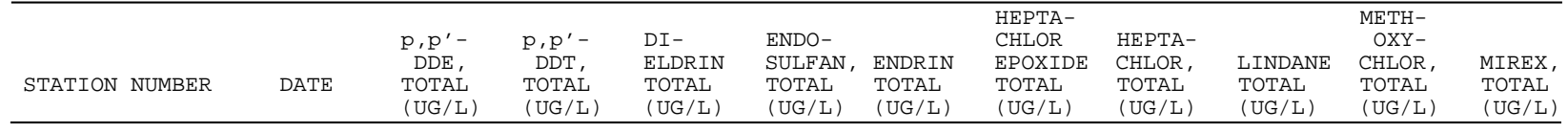

$44320712314550008-24-93<0.00100<0.00100<0.00100<0.00100<0.00100<0.00100<0.00100<0.00100<0.01000<0.01000$

$44313812312090108-24-93<0.00100<0.00100<0.00100<0.00100<0.00100<0.00100<0.00100<0.00100<0.01000<0.01000$

$45130912304150109-07-93<0.00100<0.00100<0.00100<0.00100<0.00100<0.00100<0.00100<0.00100<0.01000<0.01000$

$45132012304110009-07-93<0.00100<0.00100<0.00100<0.00100<0.00100<0.00100<0.00100<0.00100<0.01000<0.01000$

14201300

$07-27-93<0.00100<0.00100<0.00100<0.00100<0.00100<0.00100<0.001000 .02000<0.01000<0.01000$

14206950

$07-22-93<0.00100<0.00100<0.00100<0.00100<0.00100<0.00100<0.00100<0.00100<0.01000<0.01000$

\begin{tabular}{|c|c|c|c|c|c|c|c|c|c|c|c|}
\hline STATION NUMBER & DATE & $\begin{array}{c}\text { GROSS } \\
\text { PCB, } \\
\text { TOTAL } \\
(\mathrm{UG} / \mathrm{L})\end{array}$ & $\begin{array}{c}\text { GROSS } \\
\text { PCN< } \\
\text { TOTAL } \\
(\mathrm{UG} / \mathrm{L})\end{array}$ & $\begin{array}{c}\text { PER- } \\
\text { THANE } \\
\text { TOTAL } \\
(\mathrm{UG} / \mathrm{L})\end{array}$ & $\begin{array}{c}\text { TOX- } \\
\text { APHENE, } \\
\text { TOTAL } \\
\text { (UG/L) }\end{array}$ & $\begin{array}{l}\text { DI- } \\
\text { AZINON, } \\
\text { TOTAL } \\
\text { (UG/L) }\end{array}$ & $\begin{array}{c}\text { ETHION, } \\
\text { TOTAL } \\
\text { (UG/L) }\end{array}$ & $\begin{array}{l}\text { MALA- } \\
\text { THION, } \\
\text { TOTAL } \\
\text { (UG/L) }\end{array}$ & $\begin{array}{l}\text { METHYL } \\
\text { PARA- } \\
\text { THION, } \\
\text { TOTAL } \\
\text { (UG/L) }\end{array}$ & $\begin{array}{l}\text { PARA- } \\
\text { THION, } \\
\text { TOTAL } \\
\text { (UG/L) }\end{array}$ & $\begin{array}{l}\text { TRI- } \\
\text { THION } \\
\text { TOTAL } \\
\text { (UG/L) }\end{array}$ \\
\hline 443207123145500 & $08-24-93$ & $<0.10000$ & $<0.10000$ & $<0.10000$ & $<1.0000$ & $<0.01000$ & $<0.01000$ & 0.03000 & $<0.01000$ & $<0.01000$ & $<0.01000$ \\
\hline 443138123120901 & $08-24-93$ & $<0.10000$ & $<0.10000$ & $<0.10000$ & $<1.0000$ & $<0.01000$ & $<0.01000$ & $<0.01000$ & $<0.01000$ & $<0.01000$ & $<0.01000$ \\
\hline 451309123041501 & $09-07-93$ & $<0.10000$ & $<0.10000$ & $<0.10000$ & $<1.0000$ & $<0.01000$ & $<0.01000$ & $<0.01000$ & $<0.01000$ & $<0.01000$ & $<0.01000$ \\
\hline 451320123041100 & $09-07-93$ & $<0.10000$ & $<0.10000$ & $<0.10000$ & $<1.0000$ & $<0.01000$ & $<0.01000$ & $<0.01000$ & $<0.01000$ & $<0.01000$ & $<0.01000$ \\
\hline 14201300 & $07-27-93$ & $<0.10000$ & $<0.10000$ & $<0.10000$ & $<1.0000$ & 1.0000 & $<0.01000$ & $<0.01000$ & $<0.01000$ & $<0.01000$ & $<0.01000$ \\
\hline 14206950 & $07-22-93$ & $<0.10000$ & $<0.10000$ & $<0.10000$ & $<1.0000$ & 0.06000 & $<0.01000$ & $<0.01000$ & $<0.01000$ & $<0.01000$ & $<0.01000$ \\
\hline
\end{tabular}


Table 18. Concentrations of organochlorine compounds in whole water from Phase II of the Willamette River Basin Water Quality Study, Oregon, 1994

[USGS laboratory schedule 1398]

\begin{tabular}{|c|c|c|c|c|}
\hline STATION NUMBER & STATION NAME & DATE & TIME & $\begin{array}{c}\text { ALDRIN, } \\
\text { TOTAL } \\
\text { (UG/L) }\end{array}$ \\
\hline 433548123040600 & COAST FORK WILLAMETTE R BL BIG RIVER NR LONDON, OR & $05-20-94$ & 1040 & $<0.00100$ \\
\hline 440045122585600 & COAST FORK WILLAM AT SEAVY LOOP RD NR EUGENE, OR & $05-19-94$ & 1020 & $<0.00100$ \\
\hline 440402123063900 & URBAN OUTFALL AT POLK ST. PARK AT EUGENE, OR & $06-13-94$ & 1210 & $<0.00100$ \\
\hline 441310122095801 & MACK CREEK NEAR BLUE RIVER, OR & $05-27-94$ & 1030 & $<0.00100$ \\
\hline 440707123041300 & MCKENZIE RIVER NR EUGENE, OR & $05-19-94$ & 1450 & $<0.00100$ \\
\hline 442223123153703 & $\begin{array}{llllllll}\text { LONG TOM } & R & \text { AT } & \text { BUNDY } & \text { BRIDGE } & \text { NR MONROE, OR } \\
\text { LONG } & \text { TOM } & R & A T & \text { BUNDY } & \text { BRIDGE } & \text { NR MONROE, OR }\end{array}$ & $\begin{array}{l}05-19-94 \\
11-01-94\end{array}$ & $\begin{array}{l}0950 \\
1720\end{array}$ & $\begin{array}{l}<0.00100 \\
<0.00100\end{array}$ \\
\hline 442413123122500 & LAKE CAMOUS CR AT PINE GROVE DR NR HARRISBURG, OR & $05-19-94$ & 1720 & $<0.00100$ \\
\hline 443138123120901 & $\begin{array}{llll}\text { MUDDY } & \text { CREEK NEAR PEORIA, OR } \\
\text { MUDDY } & \text { CREEK NEAR PEORIA, OR } \\
\text { MUDDY } & \text { CREEK NEAR PEORIA, OR } \\
\text { MUDDY } & \text { CREEK NEAR PEORIA, OR } \\
\text { MUDDY CREEK NEAR PEORIA, OR }\end{array}$ & $\begin{array}{l}05-26-94 \\
06-03-94 \\
11-02-94 \\
11-06-94 \\
11-09-94\end{array}$ & $\begin{array}{l}1730 \\
0930 \\
1010 \\
1200 \\
1430\end{array}$ & $\begin{array}{l}<0.00100 \\
<0.00100 \\
<0.00100 \\
<0.00100 \\
<0.00100\end{array}$ \\
\hline 443045123273000 & ROCK CREEK ABOVE GRIFFITH CREEK NR PHILOMATH, OR & $05-18-94$ & 1820 & $<0.00100$ \\
\hline 443321123155201 & MARYS RIVER AT CORVALLIS, OR & $05-18-94$ & 1350 & $<0.00100$ \\
\hline 14173500 & $\begin{array}{lll}\text { CALAPOOIA RIVER AT ALBANY, OR } \\
\text { CALAPOOIA RIVER AT ALBANY, OR }\end{array}$ & $\begin{array}{l}05-26-94 \\
11-01-94\end{array}$ & $\begin{array}{l}1220 \\
1230\end{array}$ & $\begin{array}{l}<0.00100 \\
<0.00100\end{array}$ \\
\hline 444349123094000 & LUCKIAMUTE R AT BUENA VISTA RD NR BUENA VISTA, OR & $05-16-94$ & 1720 & $<0.00100$ \\
\hline 444123122562200 & THOMAS CREEK AT KELLY RD NR JEFFERSON, OR & $05-16-94$ & 1900 & $<0.00100$ \\
\hline 14189000 & SANTIAM RIVER AT JEFFERSON, OR & $05-17-94$ & 1620 & $<0.00100$ \\
\hline
\end{tabular}


Table 18. Concentrations of organochlorine compounds in whole water from Phase II of the Willamette River Basin Water Quality Study, Oregon, 1994-Continued

\begin{tabular}{|c|c|c|c|c|c|c|c|c|c|}
\hline STATION NUMBER & DATE & $\begin{array}{l}\text { CHLOR- } \\
\text { DANE, } \\
\text { TOTAL } \\
\text { (UG/L) }\end{array}$ & $\begin{array}{l}\text { DI- } \\
\text { ELDRIN } \\
\text { TOTAL } \\
\text { (UG/L) }\end{array}$ & $\begin{array}{c}\mathrm{p}_{,} \mathrm{p}^{\prime}- \\
\text { DDD, } \\
\text { TOTAL } \\
(\mathrm{UG} / \mathrm{L})\end{array}$ & $\begin{array}{c}\mathrm{P}_{,} \mathrm{p}^{\prime}- \\
\text { DDE, } \\
\text { TOTAL } \\
\text { (UG/L) }\end{array}$ & $\begin{array}{c}\mathrm{p}_{,} \mathrm{p}^{\prime}- \\
\mathrm{DDT}, \\
\mathrm{TOTAL} \\
(\mathrm{UG} / \mathrm{L})\end{array}$ & $\begin{array}{l}\text { ENDRIN } \\
\text { TOTAL } \\
\text { (UG/L) }\end{array}$ & $\begin{array}{l}\text { ENDO- } \\
\text { SULFAN, } \\
\text { TOTAL } \\
\text { (UG/L) }\end{array}$ & $\begin{array}{l}\text { HEPTA- } \\
\text { CHLOR, } \\
\text { TOTAL } \\
\text { (UG/L) }\end{array}$ \\
\hline 433548123040600 & $05-20-94$ & $<0.10000$ & $<0.00100$ & $<0.00100$ & $<0.00100$ & $<0.00100$ & $<0.00100$ & $<0.00100$ & $<0.00100$ \\
\hline 440045122585600 & $05-19-94$ & $<0.10000$ & $<0.00100$ & $<0.00100$ & $<0.00100$ & $<0.00100$ & $<0.00100$ & $<0.00100$ & $<0.00100$ \\
\hline 440402123063900 & $06-13-94$ & $<0.10000$ & $<0.00100$ & $<0.00100$ & $<0.00100$ & $<0.00100$ & $<0.00100$ & $<0.00100$ & $<0.00100$ \\
\hline 441310122095801 & $05-27-94$ & $<0.10000$ & $<0.00100$ & $<0.00100$ & $<0.00100$ & $<0.00100$ & $<0.00100$ & $<0.00100$ & $<0.00100$ \\
\hline 440707123041300 & $05-19-94$ & $<0.10000$ & $<0.00100$ & $<0.00100$ & $<0.00100$ & $<0.00100$ & $<0.00100$ & $<0.00100$ & $<0.00100$ \\
\hline 442223123153703 & $\begin{array}{l}05-19-94 \\
11-01-94\end{array}$ & $\begin{array}{l}<0.10000 \\
<0.10000\end{array}$ & $\begin{array}{l}<0.00100 \\
<0.00100\end{array}$ & $\begin{array}{l}<0.00100 \\
<0.00100\end{array}$ & $\begin{array}{l}<0.00100 \\
<0.00100\end{array}$ & $\begin{array}{l}<0.00100 \\
<0.00100\end{array}$ & $\begin{array}{l}<0.00100 \\
<0.00100\end{array}$ & $\begin{array}{l}<0.00100 \\
<0.00100\end{array}$ & $\begin{array}{l}<0.00100 \\
<0.00100\end{array}$ \\
\hline 442413123122500 & $05-19-94$ & $<0.10000$ & $<0.00100$ & $<0.00100$ & $<0.00100$ & $<0.00100$ & $<0.00100$ & $<0.00100$ & $<0.00100$ \\
\hline 443138123120901 & $\begin{array}{l}05-26-94 \\
06-03-94 \\
11-02-94 \\
11-06-94 \\
11-09-94\end{array}$ & $\begin{array}{l}<0.10000 \\
<0.10000 \\
<0.10000 \\
<0.10000 \\
<0.10000\end{array}$ & $\begin{array}{l}<0.00100 \\
<0.00100 \\
0.001000 \\
<0.00100 \\
<0.00100\end{array}$ & $\begin{array}{l}<0.00100 \\
<0.00100 \\
<0.00100 \\
<0.00100 \\
<0.00100\end{array}$ & $\begin{array}{l}<0.00100 \\
<0.00100 \\
<0.00100 \\
<0.00100 \\
<0.00100\end{array}$ & $\begin{array}{l}<0.00100 \\
<0.00100 \\
<0.00100 \\
<0.00100 \\
<0.00100\end{array}$ & $\begin{array}{l}<0.00100 \\
<0.00100 \\
<0.00100 \\
<0.00100 \\
<0.00100\end{array}$ & $\begin{array}{l}<0.00100 \\
<0.00100 \\
<0.00100 \\
<0.00100 \\
<0.00100\end{array}$ & $\begin{array}{l}<0.00100 \\
<0.00100 \\
<0.00100 \\
<0.00100 \\
<0.00100\end{array}$ \\
\hline 443045123273000 & $05-18-94$ & $<0.10000$ & $<0.00100$ & $<0.00100$ & $<0.00100$ & $<0.00100$ & $<0.00100$ & $<0.00100$ & $<0.00100$ \\
\hline 443321123155201 & $05-18-94$ & $<0.10000$ & $<0.00100$ & $<0.00100$ & $<0.00100$ & 0.002000 & $<0.00100$ & $<0.00100$ & $<0.00100$ \\
\hline 14173500 & $\begin{array}{l}05-26-94 \\
11-01-94\end{array}$ & $\begin{array}{l}<0.10000 \\
<0.10000\end{array}$ & $\begin{array}{l}<0.00100 \\
<0.00100\end{array}$ & $\begin{array}{l}<0.00100 \\
<0.00100\end{array}$ & $\begin{array}{l}<0.00100 \\
<0.00100\end{array}$ & $\begin{array}{l}<0.00100 \\
<0.00100\end{array}$ & $\begin{array}{l}<0.00100 \\
<0.00100\end{array}$ & $\begin{array}{l}<0.00100 \\
<0.00100\end{array}$ & $\begin{array}{l}<0.00100 \\
<0.00100\end{array}$ \\
\hline 444349123094000 & $05-16-94$ & $<0.10000$ & $<0.00100$ & $<0.00100$ & $<0.00100$ & $<0.00100$ & $<0.00100$ & $<0.00100$ & $<0.00100$ \\
\hline 444123122562200 & $05-16-94$ & $<0.10000$ & $<0.00100$ & $<0.00100$ & $<0.00100$ & $<0.00100$ & $<0.00100$ & $<0.00100$ & $<0.00100$ \\
\hline 14189000 & $05-17-94$ & $<0.10000$ & $<0.00100$ & $<0.00100$ & $<0.00100$ & $<0.00100$ & $<0.00100$ & $<0.00100$ & $<0.00100$ \\
\hline
\end{tabular}


Table 18. Concentrations of organochlorine compounds in whole water from Phase II of the Willamette River Basin Water Quality Study, Oregon, 1994-Continued

\begin{tabular}{|c|c|c|c|c|c|c|c|c|c|}
\hline STATION NUMBER & DATE & $\begin{array}{c}\text { HEPTA- } \\
\text { CHLOR } \\
\text { EPOXIDE } \\
\text { TOTAL } \\
\text { (UG/L) }\end{array}$ & $\begin{array}{c}\text { LINDANE } \\
\text { TOTAL } \\
\text { (UG/L) }\end{array}$ & $\begin{array}{c}\text { METH- } \\
\text { OXY- } \\
\text { CHLOR, } \\
\text { TOTAL } \\
\text { (UG/L) }\end{array}$ & $\begin{array}{c}\text { MIREX, } \\
\text { TOTAL } \\
(\mathrm{UG} / \mathrm{L})\end{array}$ & $\begin{array}{l}\text { GROSS } \\
\text { PCB, } \\
\text { TOTAL } \\
\text { (UG/L) }\end{array}$ & $\begin{array}{l}\text { GROSS } \\
\text { PCN, } \\
\text { TOTAL } \\
\text { (UG/L) }\end{array}$ & $\begin{array}{c}\text { PER- } \\
\text { THANE } \\
\text { TOTAL } \\
(U G / L)\end{array}$ & $\begin{array}{c}\text { TOX- } \\
\text { APHENE, } \\
\text { TOTAL } \\
\text { (UG/L) }\end{array}$ \\
\hline 433548123040600 & $05-20-94$ & $<0.00100$ & $<0.00100$ & $<0.01000$ & $<0.01000$ & $<0.10000$ & $<0.10000$ & $<0.10000$ & $<1.0000$ \\
\hline 440045122585600 & $05-19-94$ & $<0.00100$ & $<0.00100$ & $<0.01000$ & $<0.01000$ & $<0.10000$ & $<0.10000$ & $<0.10000$ & $<1.0000$ \\
\hline 440402123063900 & $06-13-94$ & $<0.00100$ & $<0.00100$ & $<0.01000$ & $<0.01000$ & $<0.10000$ & $<0.10000$ & $<0.10000$ & $<1.0000$ \\
\hline 441310122095801 & $05-27-94$ & $<0.00100$ & $<0.00100$ & $<0.01000$ & $<0.01000$ & $<0.10000$ & $<0.10000$ & $<0.10000$ & $<1.0000$ \\
\hline 440707123041300 & $05-19-94$ & $<0.00100$ & $<0.00100$ & $<0.01000$ & $<0.01000$ & $<0.10000$ & $<0.10000$ & $<0.10000$ & $<1.0000$ \\
\hline 442223123153703 & $\begin{array}{l}05-19-94 \\
11-01-94\end{array}$ & $\begin{array}{l}<0.00100 \\
<0.00100\end{array}$ & $\begin{array}{l}<0.00100 \\
<0.00100\end{array}$ & $\begin{array}{l}<0.01000 \\
<0.01000\end{array}$ & $\begin{array}{l}<0.01000 \\
<0.01000\end{array}$ & $\begin{array}{l}<0.10000 \\
<0.10000\end{array}$ & $\begin{array}{l}<0.10000 \\
<0.10000\end{array}$ & $\begin{array}{l}<0.10000 \\
<0.10000\end{array}$ & $\begin{array}{l}<1.0000 \\
<1.0000\end{array}$ \\
\hline 442413123122500 & $05-19-94$ & $<0.00100$ & $<0.00100$ & $<0.01000$ & $<0.01000$ & $<0.10000$ & $<0.10000$ & $<0.10000$ & $<1.0000$ \\
\hline 443138123120901 & $\begin{array}{l}05-26-94 \\
06-03-94 \\
11-02-94 \\
11-06-94 \\
11-09-94\end{array}$ & $\begin{array}{l}<0.00100 \\
<0.00100 \\
<0.00100 \\
<0.00100 \\
<0.00100\end{array}$ & $\begin{array}{l}<0.00100 \\
<0.00100 \\
0.001000 \\
<0.00100 \\
<0.00100\end{array}$ & $\begin{array}{l}<0.01000 \\
<0.01000 \\
<0.01000 \\
<0.01000 \\
<0.01000\end{array}$ & $\begin{array}{l}<0.01000 \\
<0.01000 \\
<0.01000 \\
<0.01000 \\
<0.01000\end{array}$ & $\begin{array}{l}<0.10000 \\
<0.10000 \\
<0.10000 \\
<0.10000 \\
<0.10000\end{array}$ & $\begin{array}{l}<0.10000 \\
<0.10000 \\
<0.10000 \\
<0.10000 \\
<0.10000\end{array}$ & $\begin{array}{l}<0.10000 \\
<0.10000 \\
<0.10000 \\
<0.10000 \\
<0.10000\end{array}$ & $\begin{array}{l}<1.0000 \\
<1.0000 \\
<1.0000 \\
<1.0000 \\
<1.0000\end{array}$ \\
\hline 443045123273000 & $05-18-94$ & $<0.00100$ & $<0.00100$ & $<0.01000$ & $<0.01000$ & $<0.10000$ & $<0.10000$ & $<0.10000$ & $<1.0000$ \\
\hline 443321123155201 & $05-18-94$ & $<0.00100$ & $<0.00100$ & $<0.01000$ & $<0.01000$ & $<0.10000$ & $<0.10000$ & $<0.10000$ & $<1.0000$ \\
\hline 14173500 & $\begin{array}{l}05-26-94 \\
11-01-94\end{array}$ & $\begin{array}{l}<0.00100 \\
<0.00100\end{array}$ & $\begin{array}{l}<0.00100 \\
0.001000\end{array}$ & $\begin{array}{l}<0.01000 \\
<0.01000\end{array}$ & $\begin{array}{l}<0.01000 \\
<0.01000\end{array}$ & $\begin{array}{l}<0.10000 \\
<0.10000\end{array}$ & $\begin{array}{l}<0.10000 \\
<0.10000\end{array}$ & $\begin{array}{l}<0.10000 \\
<0.10000\end{array}$ & $\begin{array}{l}<1.0000 \\
<1.0000\end{array}$ \\
\hline 444349123094000 & $05-16-94$ & $<0.00100$ & $<0.00100$ & $<0.01000$ & $<0.01000$ & $<0.10000$ & $<0.10000$ & $<0.10000$ & $<1.0000$ \\
\hline 444123122562200 & $05-16-94$ & $<0.00100$ & $<0.00100$ & $<0.01000$ & $<0.01000$ & $<0.10000$ & $<0.10000$ & $<0.10000$ & $<1.0000$ \\
\hline 14189000 & $05-17-94$ & $<0.00100$ & $<0.00100$ & $<0.01000$ & $<0.01000$ & $<0.10000$ & $<0.10000$ & $<0.10000$ & $<1.0000$ \\
\hline
\end{tabular}


Table 18. Concentrations of organochlorine compounds in whole water from Phase II of the Willamette River Basin Water Quality Study, Oregon, 1994-Continued

\begin{tabular}{|c|c|c|c|c|}
\hline STATION NUMBER & STATION NAME & DATE & TIME & $\begin{array}{l}\text { ALDRIN, } \\
\text { TOTAL } \\
\text { (UG/L) }\end{array}$ \\
\hline 445547123065400 & RICKREAL CREEK NR MOUTH NR SALEM, OR & $05-26-94$ & 1540 & $<0.00100$ \\
\hline 14190970 & $\begin{array}{lllllll}\text { PRINGLE } & \text { AT } & \text { BUSH PARK AT SALEM, OR } \\
\text { PRINGLE C AT } & \text { BUSH PARK AT SALEM, OR } \\
\text { PRINGLE } & \text { C AT } & \text { BUSH PARK AT } & \text { SALEM, OR }\end{array}$ & $\begin{array}{l}11-23-94 \\
11-30-94 \\
11-30-94\end{array}$ & $\begin{array}{l}1200 \\
1530 \\
1710\end{array}$ & $\begin{array}{l}<0.00100 \\
<0.00100 \\
<0.00100\end{array}$ \\
\hline 445037122573800 & $\begin{array}{lllllll}\text { MILL } & \text { CREEK } & \text { AT } & \text { DELANEY } & \text { ROAD } & \text { NR } & \text { TURNER, OR } \\
\text { MILL } & \text { CREEK } & \text { AT } & \text { DELANEY } & \text { ROAD } & \text { NR } & \text { TURNER, OR } \\
\text { MILL } & \text { CREEK } & \text { AT } & \text { DELANEY } & \text { ROAD } & \text { NR } & \text { TURNER, OR } \\
\text { MILL } & \text { CREEK } & \text { AT } & \text { DELANEY } & \text { ROAD } & \text { NR } & \text { TURNER, OR }\end{array}$ & $\begin{array}{l}06-14-94 \\
10-31-94 \\
11-04-94 \\
11-04-94\end{array}$ & $\begin{array}{l}1140 \\
1410 \\
1120 \\
1420\end{array}$ & $\begin{array}{l}<0.00100 \\
<0.00100 \\
<0.00100 \\
<0.00100\end{array}$ \\
\hline 14194150 & $\begin{array}{llll}\text { SOUTH YAMHILL RIVER AT MCMINNVILLE, OR } \\
\text { SOUTH YAMHILL RIVER AT MCMINNVILLE, OR }\end{array}$ & $\begin{array}{l}05-17-94 \\
11-02-94\end{array}$ & $\begin{array}{l}1720 \\
1210\end{array}$ & $\begin{array}{l}<0.00100 \\
<0.00100\end{array}$ \\
\hline 451355123093600 & NORTH YAMHILL RIVER AT HWY. 99E NR MCMINNVILLE, OR & $05-17-94$ & 1220 & $<0.00100$ \\
\hline 451602122564400 & WILLAMETTE RIVER NR NEWBERG, OR & $05-31-94$ & 1400 & $<0.00100$ \\
\hline 451502122524700 & $\begin{array}{l}\text { CHAMPOEG CREEK BELOW MISSION CR NR BUTTEVILLE, OR } \\
\text { CHAMPOEG CREEK BELOW MISSION CR NR BUTTEVILLE, OR }\end{array}$ & $\begin{array}{l}05-26-94 \\
10-31-94\end{array}$ & $\begin{array}{l}1030 \\
1300\end{array}$ & $\begin{array}{l}<0.00100 \\
<0.00100\end{array}$ \\
\hline 14201300 & $\begin{array}{llll}\text { ZOLLNER CREEK NEAR MT ANGEL, OR } \\
\text { ZOLLNER CREEK NEAR MT ANGEL, OR }\end{array}$ & $\begin{array}{l}05-25-94 \\
10-28-94\end{array}$ & $\begin{array}{l}1450 \\
1320\end{array}$ & $\begin{array}{l}<0.00100 \\
<0.00100\end{array}$ \\
\hline 14202000 & $\begin{array}{llll}\text { PUDDING RIVER AT } & \text { AURORA, OREG. } \\
\text { PUDDING RIVER AT AURORA, OREG. } \\
\text { PUDDING RIVER AT AURORA, OREG. } \\
\text { PUDDING RIVER AT AURORA, OREG. }\end{array}$ & $\begin{array}{l}05-25-94 \\
10-28-94 \\
10-29-94 \\
11-09-94\end{array}$ & $\begin{array}{l}1100 \\
1710 \\
1230 \\
1300\end{array}$ & $\begin{array}{l}<0.00100 \\
<0.00100 \\
<0.00100 \\
<0.00100\end{array}$ \\
\hline 451603122423301 & MOLALLA R AT KNIGHTS BRIDGE NR CANBY, OR & $05-25-94$ & 1020 & $<0.00100$ \\
\hline 14206200 & $\begin{array}{llllllll}\text { DAIRY CREEK AT RTE } 8 & \text { NEAR HILLSBORO, OR } \\
\text { DAIRY CREEK AT } & \text { RTE } 8 & \text { NEAR HILLSBORO, OR }\end{array}$ & $\begin{array}{l}05-27-94 \\
12-01-94\end{array}$ & $\begin{array}{l}1640 \\
1140\end{array}$ & $\begin{array}{l}<0.00100 \\
<0.00100\end{array}$ \\
\hline
\end{tabular}


Table 18. Concentrations of organochlorine compounds in whole water from Phase II of the Willamette River Basin Water Quality Study, Oregon, 1994-Continued

\begin{tabular}{|c|c|c|c|c|c|c|c|c|c|}
\hline STATION NUMBER & DATE & $\begin{array}{l}\text { CHLOR- } \\
\text { DANE, } \\
\text { TOTAL } \\
\text { (UG/L) }\end{array}$ & \begin{tabular}{l}
\multicolumn{1}{c}{ DI- } \\
ELDRIN \\
TOTAL \\
(UG/L)
\end{tabular} & $\begin{array}{c}\mathrm{p}, \mathrm{p}^{\prime}- \\
\text { DDD, } \\
\text { TOTAL } \\
(\mathrm{UG} / \mathrm{L})\end{array}$ & $\begin{array}{l}\mathrm{P}, \mathrm{p}^{\prime}- \\
\text { DDE, } \\
\text { TOTAL } \\
(\mathrm{UG} / \mathrm{L})\end{array}$ & $\begin{array}{l}\mathrm{p}, \mathrm{p}^{\prime}- \\
\mathrm{DDT}, \\
\mathrm{TOTAL} \\
(\mathrm{UG} / \mathrm{L})\end{array}$ & $\begin{array}{l}\text { ENDRIN } \\
\text { TOTAL } \\
\text { (UG/L) }\end{array}$ & $\begin{array}{l}\text { ENDO- } \\
\text { SULFAN, } \\
\text { TOTAL } \\
\text { (UG/L) }\end{array}$ & $\begin{array}{l}\text { HEPTA- } \\
\text { CHLOR, } \\
\text { TOTAL } \\
\text { (UG/L) }\end{array}$ \\
\hline 445547123065400 & $05-26-94$ & $<0.10000$ & $<0.00100$ & $<0.00100$ & $<0.00100$ & $<0.00100$ & $<0.00100$ & $<0.00100$ & $<0.00100$ \\
\hline 14190970 & $\begin{array}{l}11-23-94 \\
11-30-94 \\
11-30-94\end{array}$ & $\begin{array}{l}<0.10000 \\
<0.10000 \\
<0.10000\end{array}$ & $\begin{array}{l}<0.00100 \\
0.002000 \\
0.003000\end{array}$ & $\begin{array}{l}<0.00100 \\
0.001000 \\
0.001000\end{array}$ & $\begin{array}{l}<0.00100 \\
0.002000 \\
0.002000\end{array}$ & $\begin{array}{l}<0.00100 \\
<0.00200 \\
<0.00200\end{array}$ & $\begin{array}{l}<0.00100 \\
<0.00100 \\
<0.00100\end{array}$ & $\begin{array}{l}<0.00100 \\
<0.00100 \\
<0.00100\end{array}$ & $\begin{array}{l}<0.00100 \\
<0.00100 \\
<0.00100\end{array}$ \\
\hline 445037122573800 & $\begin{array}{l}06-14-94 \\
10-31-94 \\
11-04-94 \\
11-04-94\end{array}$ & $\begin{array}{l}<0.10000 \\
<0.10000 \\
<0.10000 \\
<0.10000\end{array}$ & $\begin{array}{l}<0.00100 \\
<0.00100 \\
0.001000 \\
0.001000\end{array}$ & $\begin{array}{l}<0.00100 \\
<0.00100 \\
<0.00100 \\
<0.00100\end{array}$ & $\begin{array}{l}<0.00100 \\
<0.00100 \\
<0.00100 \\
<0.00100\end{array}$ & $\begin{array}{l}<0.00100 \\
<0.00100 \\
<0.00100 \\
<0.00100\end{array}$ & $\begin{array}{l}<0.00100 \\
<0.00100 \\
<0.00100 \\
<0.00100\end{array}$ & $\begin{array}{l}<0.00100 \\
<0.00100 \\
<0.00100 \\
<0.00100\end{array}$ & $\begin{array}{l}<0.00100 \\
<0.00100 \\
<0.00100 \\
<0.00100\end{array}$ \\
\hline 14194150 & $\begin{array}{l}05-17-94 \\
11-02-94\end{array}$ & $\begin{array}{l}<0.10000 \\
<0.10000\end{array}$ & $\begin{array}{l}<0.00100 \\
<0.00100\end{array}$ & $\begin{array}{l}<0.00100 \\
<0.00100\end{array}$ & $\begin{array}{l}<0.00100 \\
<0.00100\end{array}$ & $\begin{array}{l}<0.00100 \\
<0.00100\end{array}$ & $\begin{array}{l}<0.00100 \\
<0.00100\end{array}$ & $\begin{array}{l}<0.00100 \\
<0.00100\end{array}$ & $\begin{array}{l}<0.00100 \\
<0.00100\end{array}$ \\
\hline 451355123093600 & $05-17-94$ & $<0.10000$ & $<0.00100$ & $<0.00100$ & $<0.00100$ & $<0.00100$ & $<0.00100$ & $<0.00100$ & $<0.00100$ \\
\hline 451602122564400 & $05-31-94$ & $<0.10000$ & $<0.00100$ & $<0.00100$ & $<0.00100$ & $<0.00100$ & $<0.00100$ & $<0.00100$ & $<0.00100$ \\
\hline 451502122524700 & $\begin{array}{l}05-26-94 \\
10-31-94\end{array}$ & $\begin{array}{l}<0.10000 \\
<0.10000\end{array}$ & $\begin{array}{l}0.001000 \\
0.005000\end{array}$ & $\begin{array}{l}<0.00100 \\
0.001000\end{array}$ & $\begin{array}{l}<0.00100 \\
0.003000\end{array}$ & $\begin{array}{l}<0.00100 \\
0.002000\end{array}$ & $\begin{array}{l}<0.00100 \\
<0.00100\end{array}$ & $\begin{array}{l}<0.00100 \\
<0.00100\end{array}$ & $\begin{array}{l}<0.00100 \\
<0.00100\end{array}$ \\
\hline 14201300 & $\begin{array}{l}05-25-94 \\
10-28-94\end{array}$ & $\begin{array}{l}<0.10000 \\
<0.10000\end{array}$ & $\begin{array}{l}0.001000 \\
0.002000\end{array}$ & $\begin{array}{l}<0.00100 \\
<0.00100\end{array}$ & $\begin{array}{l}<0.00100 \\
0.002000\end{array}$ & $\begin{array}{l}<0.00100 \\
0.002000\end{array}$ & $\begin{array}{l}<0.00100 \\
<0.00100\end{array}$ & $\begin{array}{l}0.006000 \\
0.001000\end{array}$ & $\begin{array}{l}<0.00100 \\
<0.00100\end{array}$ \\
\hline 14202000 & $\begin{array}{l}05-25-94 \\
10-28-94 \\
10-29-94 \\
11-09-94\end{array}$ & $\begin{array}{l}<0.10000 \\
<0.10000 \\
<0.10000 \\
<0.10000\end{array}$ & $\begin{array}{l}<0.00100 \\
0.001000 \\
0.001000 \\
<0.00100\end{array}$ & $\begin{array}{l}<0.00100 \\
<0.00100 \\
<0.00100 \\
<0.00100\end{array}$ & $\begin{array}{l}<0.00100 \\
0.001000 \\
0.001000 \\
<0.00100\end{array}$ & $\begin{array}{l}<0.00100 \\
0.002000 \\
0.001000 \\
<0.00100\end{array}$ & $\begin{array}{l}<0.00100 \\
<0.00100 \\
<0.00100 \\
<0.00100\end{array}$ & $\begin{array}{l}<0.00100 \\
<0.00100 \\
<0.00100 \\
<0.00100\end{array}$ & $\begin{array}{l}<0.00100 \\
<0.00100 \\
<0.00100 \\
<0.00100\end{array}$ \\
\hline 451603122423301 & $05-25-94$ & $<0.10000$ & $<0.00100$ & $<0.00100$ & $<0.00100$ & $<0.00100$ & $<0.00100$ & $<0.00100$ & $<0.00100$ \\
\hline 14206200 & $\begin{array}{l}05-27-94 \\
12-01-94\end{array}$ & $\begin{array}{l}<0.10000 \\
<0.10000\end{array}$ & $\begin{array}{l}<0.00100 \\
0.001000\end{array}$ & $\begin{array}{l}<0.00100 \\
<0.00100\end{array}$ & $\begin{array}{l}<0.00100 \\
<0.00100\end{array}$ & $\begin{array}{l}<0.00100 \\
<0.00100\end{array}$ & $\begin{array}{l}<0.00100 \\
<0.00100\end{array}$ & $\begin{array}{l}<0.00100 \\
<0.00100\end{array}$ & $\begin{array}{l}<0.00100 \\
<0.00100\end{array}$ \\
\hline
\end{tabular}


Table 18. Concentrations of organochlorine compounds in whole water from Phase II of the Willamette River Basin Water Quality Study, Oregon, 1994-Continued

\begin{tabular}{|c|c|c|c|c|c|c|c|c|c|}
\hline STATION NUMBER & DATE & $\begin{array}{c}\text { HEPTA- } \\
\text { CHLOR } \\
\text { EPOXIDE } \\
\text { TOTAL } \\
(\mathrm{UG} / \mathrm{L}) \\
\end{array}$ & $\begin{array}{c}\text { LINDANE } \\
\text { TOTAL } \\
(\mathrm{UG} / \mathrm{L})\end{array}$ & $\begin{array}{c}\text { METH- } \\
\text { OXY- } \\
\text { CHLOR, } \\
\text { TOTAL } \\
\text { (UG/L) } \\
\end{array}$ & $\begin{array}{c}\text { MIREX, } \\
\text { TOTAL } \\
(\mathrm{UG} / \mathrm{L})\end{array}$ & $\begin{array}{c}\text { GROSS } \\
\text { PCB, } \\
\text { TOTAL } \\
\text { (UG/L) }\end{array}$ & $\begin{array}{c}\text { GROSS } \\
\text { PCN, } \\
\text { TOTAL } \\
\text { (UG/L) }\end{array}$ & $\begin{array}{c}\text { PER- } \\
\text { THANE, } \\
\text { TOTAL } \\
(\mathrm{UG} / \mathrm{L}) \\
\end{array}$ & $\begin{array}{c}\text { TOX- } \\
\text { APHENE, } \\
\text { TOTAL } \\
(\mathrm{UG} / \mathrm{L}) \\
\end{array}$ \\
\hline 445547123065400 & $05-26-94$ & $<0.00100$ & $<0.00100$ & $<0.01000$ & $<0.01000$ & $<0.10000$ & $<0.10000$ & $<0.10000$ & $<1.0000$ \\
\hline 14190970 & $\begin{array}{l}11-23-94 \\
11-30-94 \\
11-30-94\end{array}$ & $\begin{array}{l}<0.00100 \\
<0.00100 \\
<0.00100\end{array}$ & $\begin{array}{l}<0.00100 \\
0.001000 \\
0.001000\end{array}$ & $\begin{array}{l}<0.01000 \\
<0.01000 \\
<0.01000\end{array}$ & $\begin{array}{l}<0.01000 \\
<0.01000 \\
<0.01000\end{array}$ & $\begin{array}{r}<0.10000 \\
<0.10000 \\
0.10000\end{array}$ & $\begin{array}{l}<0.10000 \\
<0.10000 \\
<0.10000\end{array}$ & $\begin{array}{l}<0.10000 \\
<0.10000 \\
<0.10000\end{array}$ & $\begin{array}{l}<1.0000 \\
<1.0000 \\
<1.0000\end{array}$ \\
\hline 445037122573800 & $\begin{array}{l}06-14-94 \\
10-31-94 \\
11-04-94 \\
11-04-94\end{array}$ & $\begin{array}{l}<0.00100 \\
<0.00100 \\
<0.00100 \\
<0.00100\end{array}$ & $\begin{array}{l}<0.00100 \\
<0.00100 \\
<0.00100 \\
<0.00100\end{array}$ & $\begin{array}{l}<0.01000 \\
<0.01000 \\
<0.01000 \\
<0.01000\end{array}$ & $\begin{array}{l}<0.01000 \\
<0.01000 \\
<0.01000 \\
<0.01000\end{array}$ & $\begin{array}{l}<0.10000 \\
<0.10000 \\
<0.10000 \\
<0.10000\end{array}$ & $\begin{array}{l}<0.10000 \\
<0.10000 \\
<0.10000 \\
<0.10000\end{array}$ & $\begin{array}{l}<0.10000 \\
<0.10000 \\
<0.10000 \\
<0.10000\end{array}$ & $\begin{array}{l}<1.0000 \\
<1.0000 \\
<1.0000 \\
<1.0000\end{array}$ \\
\hline 14194150 & $\begin{array}{l}05-17-94 \\
11-02-94\end{array}$ & $\begin{array}{l}<0.00100 \\
<0.00100\end{array}$ & $\begin{array}{l}<0.00100 \\
<0.00100\end{array}$ & $\begin{array}{l}<0.01000 \\
<0.01000\end{array}$ & $\begin{array}{l}<0.01000 \\
<0.01000\end{array}$ & $\begin{array}{l}<0.10000 \\
<0.10000\end{array}$ & $\begin{array}{l}<0.10000 \\
<0.10000\end{array}$ & $\begin{array}{l}<0.10000 \\
<0.10000\end{array}$ & $\begin{array}{l}<1.0000 \\
<1.0000\end{array}$ \\
\hline 451355123093600 & $05-17-94$ & $<0.00100$ & $<0.00100$ & $<0.01000$ & $<0.01000$ & $<0.10000$ & $<0.10000$ & $<0.10000$ & $<1.0000$ \\
\hline 451602122564400 & $05-31-94$ & $<0.00100$ & $<0.00100$ & $<0.01000$ & $<0.01000$ & $<0.10000$ & $<0.10000$ & $<0.10000$ & $<1.0000$ \\
\hline 451502122524700 & $\begin{array}{l}05-26-94 \\
10-31-94\end{array}$ & $\begin{array}{l}<0.00100 \\
<0.00100\end{array}$ & $\begin{array}{l}0.03200 \\
0.03000\end{array}$ & $\begin{array}{l}<0.01000 \\
<0.01000\end{array}$ & $\begin{array}{l}<0.01000 \\
<0.01000\end{array}$ & $\begin{array}{l}<0.10000 \\
<0.10000\end{array}$ & $\begin{array}{l}<0.10000 \\
<0.10000\end{array}$ & $\begin{array}{l}<0.10000 \\
<0.10000\end{array}$ & $\begin{array}{l}<1.0000 \\
<1.0000\end{array}$ \\
\hline 14201300 & $\begin{array}{l}05-25-94 \\
10-28-94\end{array}$ & $\begin{array}{l}<0.00100 \\
<0.00100\end{array}$ & $\begin{array}{l}0.002000 \\
0.001000\end{array}$ & $\begin{array}{l}<0.01000 \\
<0.01000\end{array}$ & $\begin{array}{l}<0.01000 \\
<0.01000\end{array}$ & $\begin{array}{l}<0.10000 \\
<0.10000\end{array}$ & $\begin{array}{l}<0.10000 \\
<0.10000\end{array}$ & $\begin{array}{l}<0.10000 \\
<0.10000\end{array}$ & $\begin{array}{l}<1.0000 \\
<1.0000\end{array}$ \\
\hline 14202000 & $\begin{array}{l}05-25-94 \\
10-28-94 \\
10-29-94 \\
11-09-94\end{array}$ & $\begin{array}{l}<0.00100 \\
<0.00100 \\
<0.00100 \\
<0.00100\end{array}$ & $\begin{array}{l}<0.00100 \\
0.006000 \\
<0.00100 \\
<0.00100\end{array}$ & $\begin{array}{l}<0.01000 \\
<0.01000 \\
<0.01000 \\
<0.01000\end{array}$ & $\begin{array}{l}<0.01000 \\
<0.01000 \\
<0.01000 \\
<0.01000\end{array}$ & $\begin{array}{l}<0.10000 \\
<0.10000 \\
<0.10000 \\
<0.10000\end{array}$ & $\begin{array}{l}<0.10000 \\
<0.10000 \\
<0.10000 \\
<0.10000\end{array}$ & $\begin{array}{l}<0.10000 \\
<0.10000 \\
<0.10000 \\
<0.10000\end{array}$ & $\begin{array}{l}<1.0000 \\
<1.0000 \\
<1.0000 \\
<1.00000\end{array}$ \\
\hline 451603122423301 & $05-25-94$ & $<0.00100$ & $<0.00100$ & $<0.01000$ & $<0.01000$ & $<0.10000$ & $<0.10000$ & $<0.10000$ & $<1.0000$ \\
\hline 14206200 & $\begin{array}{l}05-27-94 \\
12-01-94\end{array}$ & $\begin{array}{l}<0.00100 \\
<0.00100\end{array}$ & $\begin{array}{l}<0.00100 \\
<0.00100\end{array}$ & $\begin{array}{l}<0.01000 \\
<0.01000\end{array}$ & $\begin{array}{l}<0.01000 \\
<0.01000\end{array}$ & $\begin{array}{l}<0.10000 \\
<0.10000\end{array}$ & $\begin{array}{l}<0.10000 \\
<0.10000\end{array}$ & $\begin{array}{l}<0.10000 \\
<0.10000\end{array}$ & $\begin{array}{l}<1.0000 \\
<1.0000\end{array}$ \\
\hline
\end{tabular}


Table 18. Concentrations of organochlorine compounds in whole water from Phase II of the Willamette River Basin Water Quality Study, Oregon, 1994-Continued

\begin{tabular}{|c|c|c|c|c|}
\hline STATION NUMBER & STATION NAME & DATE & TIME & $\begin{array}{c}\text { ALDRIN, } \\
\text { TOTAL } \\
\text { (UG/L) }\end{array}$ \\
\hline 14206298 & $\begin{array}{lllll}\text { BRONSON CREEK AT } 185 \mathrm{TH} \text { AVE NEAR ALOHA, OR } \\
\text { BRONSON CREEK AT } 185 \mathrm{TH} \text { AVE NEAR ALOHA, OR }\end{array}$ & $\begin{array}{l}05-27-94 \\
11-23-94\end{array}$ & $\begin{array}{l}1100 \\
1030\end{array}$ & $\begin{array}{l}<0.00100 \\
<0.00100\end{array}$ \\
\hline 14206950 & $\begin{array}{llll}\text { FANNO CREEK AT DURHAM, OR } \\
\text { FANNO CREEK AT DURHAM, OR }\end{array}$ & $\begin{array}{l}05-24-94 \\
10-27-94\end{array}$ & $\begin{array}{l}1420 \\
1620\end{array}$ & $\begin{array}{l}<0.00100 \\
<0.00100\end{array}$ \\
\hline 14207500 & $\begin{array}{l}\text { TUALATIN RIVER AT WEST LINN, OREG. } \\
\text { TUALATIN RIVER AT WEST LINN, OREG. }\end{array}$ & $\begin{array}{l}05-25-94 \\
10-28-94\end{array}$ & $\begin{array}{l}1540 \\
1610\end{array}$ & $\begin{array}{l}<0.00100 \\
<0.00100\end{array}$ \\
\hline 452823122240900 & $\begin{array}{llllll}\text { JOHNSON } & \text { CREEK AT PALMBLAD } & \text { RD } & \text { NR } & \text { GRESHAM, OR } \\
\text { JOHNSON } & \text { CREEK AT } & \text { PALMBLAD } & \text { RD } & \text { NR } & \text { GRESHAM, OR } \\
\text { JOHNSON } & \text { CREEK AT } & \text { PALMBLAD } & \text { RD } & \text { NR } & \text { GRESHAM, OR } \\
\text { JOHNSON } & \text { CREEK AT } & \text { PALMBLAD } & \text { RD } & \text { NR } & \text { GRESHAM, OR } \\
\text { JOHNSON } & \text { CREEK AT } & \text { PALMBLAD } & \text { RD } & \text { NR } & \text { GRESHAM, OR } \\
\text { JOHNSON } & \text { CREEK AT } & \text { PALMBLAD } & \text { RD } & \text { NR } & \text { GRESHAM, OR }\end{array}$ & $\begin{array}{l}05-24-94 \\
10-27-94 \\
10-28-94 \\
11-01-94 \\
11-03-94 \\
11-23-94\end{array}$ & $\begin{array}{l}1440 \\
1640 \\
1135 \\
1100 \\
1330 \\
1410\end{array}$ & $\begin{array}{l}<0.00100 \\
<0.00100 \\
<0.00100 \\
<0.00100 \\
<0.00100 \\
<0.00100\end{array}$ \\
\hline 453043122402200 & COMMERCIAL/RESIDENTIAL RUNOFF AT HARBOR WAY, PORT, OR & $06-17-94$ & 2150 & $<0.00100$ \\
\hline 14211720 & $\begin{array}{llll}\text { WILLAMETTE } & \text { RIVER AT PORTLAND, OREG. } \\
\text { WILLAMETTE } & \text { RIVER AT PORTLAND, OREG. } \\
\text { WILLAMETTE } & \text { RIVER AT PORTLAND, OREG. } \\
\text { WILLAMETTE } & \text { RIVER AT PORTLAND, OREG. }\end{array}$ & $\begin{array}{l}05-10-94 \\
10-29-94 \\
11-03-94 \\
12-02-94\end{array}$ & $\begin{array}{l}0939 \\
1230 \\
0956 \\
1040\end{array}$ & $\begin{array}{l}<0.00100 \\
<0.00100 \\
<0.00100 \\
<0.00100\end{array}$ \\
\hline 453154122394200 & 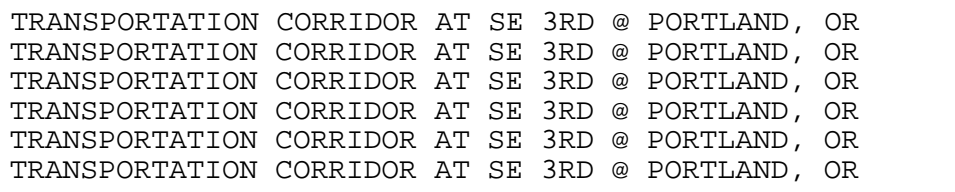 & $\begin{array}{l}05-31-94 \\
11-08-94 \\
11-08-94 \\
11-30-94 \\
11-30-94 \\
12-02-94\end{array}$ & $\begin{array}{l}1800 \\
1800 \\
1950 \\
0840 \\
1020 \\
1020\end{array}$ & $\begin{array}{l}<0.01000 \\
<0.00100 \\
<0.00100 \\
<0.00100 \\
<0.00100 \\
<0.00100\end{array}$ \\
\hline 14211805 & WILLAMETTE R AB ST JOHNS BR AT PORTLAND, OREG. & $05-23-94$ & 0950 & $<0.00100$ \\
\hline
\end{tabular}


Table 18. Concentrations of organochlorine compounds in whole water from Phase II of the Willamette River Basin Water Quality Study, Oregon, 1994-Continued

\begin{tabular}{|c|c|c|c|c|c|c|c|c|c|}
\hline STATION NUMBER & DATE & $\begin{array}{l}\text { CHLOR- } \\
\text { DANE, } \\
\text { TOTAL } \\
\text { (UG/L) }\end{array}$ & $\begin{array}{l}\text { DI- } \\
\text { ELDRIN } \\
\text { TOTAL } \\
\text { (UG/L) }\end{array}$ & $\begin{array}{c}\mathrm{p}^{\prime} \mathrm{p}^{\prime}- \\
\text { DDD, } \\
\text { TOTAL } \\
(\mathrm{UG} / \mathrm{L}) \\
\end{array}$ & $\begin{array}{l}\mathrm{p}^{\prime} \mathrm{p}^{\prime}- \\
\text { DDE, } \\
\text { TOTAL } \\
\text { (UG/L) }\end{array}$ & $\begin{array}{c}\mathrm{p}^{\prime} \mathrm{p}^{\prime}- \\
\mathrm{DDT}, \\
\text { TOTAL } \\
(\mathrm{UG} / \mathrm{L})\end{array}$ & $\begin{array}{l}\text { ENDRIN } \\
\text { TOTAL } \\
\text { (UG/L) }\end{array}$ & $\begin{array}{l}\text { ENDO- } \\
\text { SULFAN, } \\
\text { TOTAL } \\
\text { (UG/L) } \\
\end{array}$ & $\begin{array}{l}\text { HEPTA- } \\
\text { CHLOR, } \\
\text { TOTAL } \\
(\mathrm{UG} / \mathrm{L}) \\
\end{array}$ \\
\hline 14206298 & $\begin{array}{l}05-27-94 \\
11-23-94\end{array}$ & $\begin{array}{l}<0.10000 \\
<0.10000\end{array}$ & $\begin{array}{l}<0.00100 \\
<0.00100\end{array}$ & $\begin{array}{l}<0.00100 \\
<0.00100\end{array}$ & $\begin{array}{l}<0.00100 \\
<0.00100\end{array}$ & $\begin{array}{l}<0.00100 \\
<0.00100\end{array}$ & $\begin{array}{l}<0.00100 \\
<0.00100\end{array}$ & $\begin{array}{l}<0.00100 \\
<0.00100\end{array}$ & $\begin{array}{l}<0.00100 \\
<0.00100\end{array}$ \\
\hline 14206950 & $\begin{array}{l}05-24-94 \\
10-27-94\end{array}$ & $\begin{array}{l}<0.10000 \\
<0.10000\end{array}$ & $\begin{array}{l}<0.00100 \\
0.002000\end{array}$ & $\begin{array}{l}<0.00100 \\
<0.00100\end{array}$ & $\begin{array}{l}<0.00100 \\
<0.00100\end{array}$ & $\begin{array}{l}<0.00100 \\
<0.00200\end{array}$ & $\begin{array}{l}<0.00100 \\
<0.00100\end{array}$ & $\begin{array}{l}<0.00100 \\
<0.00100\end{array}$ & $\begin{array}{l}<0.00100 \\
<0.00100\end{array}$ \\
\hline 14207500 & $\begin{array}{l}05-25-94 \\
10-28-94\end{array}$ & $\begin{array}{l}<0.10000 \\
<0.10000\end{array}$ & $\begin{array}{l}<0.00100 \\
0.001000\end{array}$ & $\begin{array}{l}<0.00100 \\
<0.00100\end{array}$ & $\begin{array}{l}<0.00100 \\
0.001000\end{array}$ & $\begin{array}{l}<0.00100 \\
0.002000\end{array}$ & $\begin{array}{l}<0.00100 \\
<0.00100\end{array}$ & $\begin{array}{l}0.002000 \\
<0.00100\end{array}$ & $\begin{array}{l}<0.00100 \\
<0.00100\end{array}$ \\
\hline 452823122240900 & $\begin{array}{l}05-24-94 \\
10-27-94 \\
10-28-94 \\
11-01-94 \\
11-03-94 \\
11-23-94\end{array}$ & $\begin{array}{l}<0.10000 \\
<0.10000 \\
<0.10000 \\
<0.10000 \\
<0.10000 \\
<0.10000\end{array}$ & $\begin{array}{r}0.007000 \\
0.02000 \\
0.02000 \\
0.01000 \\
0.02000 \\
0.005000\end{array}$ & $\begin{array}{l}0.001000 \\
0.002000 \\
0.002000 \\
0.001000 \\
0.001000 \\
<0.00100\end{array}$ & $\begin{array}{l}<0.00100 \\
0.009000 \\
0.006000 \\
0.003000 \\
0.002000 \\
<0.00100\end{array}$ & $\begin{array}{r}0.001000 \\
0.01000 \\
0.008000 \\
0.002000 \\
0.002000 \\
0.002000\end{array}$ & $\begin{array}{l}<0.00100 \\
<0.00100 \\
<0.00100 \\
<0.00100 \\
<0.00100 \\
<0.00100\end{array}$ & $\begin{array}{l}<0.00100 \\
<0.00100 \\
<0.00100 \\
<0.00100 \\
<0.00100 \\
<0.00100\end{array}$ & $\begin{array}{l}<0.00100 \\
<0.00100 \\
<0.00100 \\
<0.00100 \\
<0.00100 \\
<0.00100\end{array}$ \\
\hline 453043122402200 & $06-17-94$ & $<0.10000$ & $<0.00100$ & $<0.00100$ & $<0.00100$ & $<0.00100$ & $<0.00100$ & $<0.00100$ & $<0.00100$ \\
\hline 14211720 & $\begin{array}{l}05-10-94 \\
10-29-94 \\
11-03-94 \\
12-02-94\end{array}$ & $\begin{array}{l}<0.10000 \\
<0.10000 \\
<0.10000 \\
<0.10000\end{array}$ & $\begin{array}{l}<0.00100 \\
<0.00100 \\
<0.00100 \\
<0.00100\end{array}$ & $\begin{array}{l}<0.00100 \\
<0.00100 \\
<0.00100 \\
<0.00100\end{array}$ & $\begin{array}{l}<0.00100 \\
<0.00100 \\
<0.00100 \\
<0.00100\end{array}$ & $\begin{array}{l}<0.00100 \\
0.001000 \\
<0.00100 \\
<0.00100\end{array}$ & $\begin{array}{l}<0.00100 \\
<0.00100 \\
<0.00100 \\
<0.00100\end{array}$ & $\begin{array}{l}<0.00100 \\
<0.00100 \\
<0.00100 \\
<0.00100\end{array}$ & $\begin{array}{l}<0.00100 \\
<0.00100 \\
<0.00100 \\
<0.00100\end{array}$ \\
\hline 453154122394200 & $\begin{array}{l}05-31-94 \\
11-08-94 \\
11-08-94 \\
11-30-94 \\
11-30-94 \\
12-02-94\end{array}$ & $\begin{array}{l}<0.10000 \\
<0.10000 \\
<0.10000 \\
<0.10000 \\
<0.10000 \\
<0.10000\end{array}$ & $\begin{array}{l}<0.01000 \\
<0.00100 \\
0.001000 \\
0.002000 \\
0.001000 \\
<0.00100\end{array}$ & $\begin{array}{l}<0.01000 \\
<0.00100 \\
<0.00100 \\
<0.00200 \\
<0.00100 \\
<0.00100\end{array}$ & $\begin{array}{r}<0.01000 \\
0.03000 \\
0.002000 \\
0.003000 \\
0.003000 \\
<0.00100\end{array}$ & $\begin{array}{r}<0.01000 \\
0.03000 \\
<0.00100 \\
<0.00200 \\
<0.00400 \\
<0.00100\end{array}$ & $\begin{array}{l}<0.01000 \\
<0.00100 \\
<0.00100 \\
<0.00100 \\
<0.00100 \\
<0.00100\end{array}$ & $\begin{array}{l}<0.01000 \\
<0.00100 \\
<0.00100 \\
<0.00100 \\
<0.00100 \\
<0.00100\end{array}$ & $\begin{array}{l}<0.01000 \\
0.001000 \\
<0.00100 \\
<0.00100 \\
<0.00100 \\
<0.00100\end{array}$ \\
\hline 14211805 & $05-23-94$ & $<0.10000$ & $<0.00100$ & $<0.00100$ & $<0.00100$ & $<0.00100$ & $<0.00100$ & $<0.00100$ & $<0.00100$ \\
\hline
\end{tabular}


Table 18. Concentrations of organochlorine compounds in whole water from Phase II of the Willamette River Basin Water Quality Study, Oregon, 1994-Continued

\begin{tabular}{|c|c|c|c|c|c|c|c|c|c|}
\hline STATION NUMBER & DATE & $\begin{array}{c}\text { HEPTA- } \\
\text { CHLOR } \\
\text { EPOXIDE } \\
\text { TOTAL } \\
\text { (UG/L) } \\
\end{array}$ & $\begin{array}{l}\text { LINDANE } \\
\text { TOTAL } \\
\text { (UG/L) }\end{array}$ & $\begin{array}{c}\text { METH- } \\
\text { OXY- } \\
\text { CHLOR, } \\
\text { TOTAL } \\
\text { (UG/L) } \\
\end{array}$ & $\begin{array}{c}\text { MIREX, } \\
\text { TOTAL } \\
\text { (UG/L) }\end{array}$ & $\begin{array}{c}\text { GROSS } \\
\text { PCB, } \\
\text { TOTAL } \\
\text { (UG/L) }\end{array}$ & $\begin{array}{c}\text { GROSS } \\
\text { PCN, } \\
\text { TOTAL } \\
\text { (UG/L) }\end{array}$ & $\begin{array}{c}\text { PER- } \\
\text { THANE } \\
\text { TOTAL } \\
(\text { UG/L) }\end{array}$ & $\begin{array}{c}\text { TOX- } \\
\text { AP HENE, } \\
\text { TOTAL } \\
\text { (UG/L) }\end{array}$ \\
\hline 14206298 & $\begin{array}{l}05-27-94 \\
11-23-94\end{array}$ & $\begin{array}{l}<0.00100 \\
<0.00100\end{array}$ & $\begin{array}{l}<0.00100 \\
<0.00100\end{array}$ & $\begin{array}{l}<0.01000 \\
<0.01000\end{array}$ & $\begin{array}{l}<0.01000 \\
<0.01000\end{array}$ & $\begin{array}{l}<0.10000 \\
<0.10000\end{array}$ & $\begin{array}{l}<0.10000 \\
<0.10000\end{array}$ & $\begin{array}{l}<0.10000 \\
<0.10000\end{array}$ & $\begin{array}{l}<1.0000 \\
<1.0000\end{array}$ \\
\hline 14206950 & $\begin{array}{l}05-24-94 \\
10-27-94\end{array}$ & $\begin{array}{l}<0.00100 \\
<0.00100\end{array}$ & $\begin{array}{l}<0.00100 \\
0.001000\end{array}$ & $\begin{array}{l}<0.01000 \\
<0.01000\end{array}$ & $\begin{array}{l}<0.01000 \\
<0.01000\end{array}$ & $\begin{array}{l}<0.10000 \\
<0.10000\end{array}$ & $\begin{array}{l}<0.10000 \\
<0.10000\end{array}$ & $\begin{array}{l}<0.10000 \\
<0.10000\end{array}$ & $\begin{array}{l}<1.0000 \\
<1.0000\end{array}$ \\
\hline 14207500 & $\begin{array}{l}05-25-94 \\
10-28-94\end{array}$ & $\begin{array}{l}<0.00100 \\
<0.00100\end{array}$ & $\begin{array}{l}0.002000 \\
0.002000\end{array}$ & $\begin{array}{l}<0.01000 \\
<0.01000\end{array}$ & $\begin{array}{l}<0.01000 \\
<0.01000\end{array}$ & $\begin{array}{l}<0.10000 \\
<0.10000\end{array}$ & $\begin{array}{l}<0.10000 \\
<0.10000\end{array}$ & $\begin{array}{l}<0.10000 \\
<0.10000\end{array}$ & $\begin{array}{l}<1.0000 \\
<1.0000\end{array}$ \\
\hline 452823122240900 & $\begin{array}{l}05-24-94 \\
10-27-94 \\
10-28-94 \\
11-01-94 \\
11-03-94 \\
11-23-94\end{array}$ & $\begin{array}{l}<0.00100 \\
<0.00100 \\
<0.00100 \\
<0.00100 \\
<0.00100 \\
<0.00100\end{array}$ & $\begin{array}{l}0.005000 \\
0.001000 \\
0.001000 \\
0.006000 \\
0.002000 \\
<0.00100\end{array}$ & $\begin{array}{l}<0.01000 \\
<0.01000 \\
<0.01000 \\
<0.01000 \\
<0.01000 \\
<0.01000\end{array}$ & $\begin{array}{l}<0.01000 \\
<0.01000 \\
<0.01000 \\
<0.01000 \\
<0.01000 \\
<0.01000\end{array}$ & $\begin{array}{l}<0.10000 \\
<0.10000 \\
<0.10000 \\
<0.10000 \\
<0.10000 \\
<0.10000\end{array}$ & $\begin{array}{l}<0.10000 \\
<0.10000 \\
<0.10000 \\
<0.10000 \\
<0.10000 \\
<0.10000\end{array}$ & $\begin{array}{l}<0.10000 \\
<0.10000 \\
<0.10000 \\
<0.10000 \\
<0.10000 \\
<0.10000\end{array}$ & $\begin{array}{l}<1.0000 \\
<1.0000 \\
<1.0000 \\
<1.0000 \\
<1.0000 \\
<1.0000\end{array}$ \\
\hline 453043122402200 & $06-17-94$ & $<0.00100$ & $<0.00100$ & $<0.01000$ & $<0.01000$ & $<0.10000$ & $<0.10000$ & $<0.10000$ & $<1.0000$ \\
\hline 14211720 & $\begin{array}{l}05-10-94 \\
10-29-94 \\
11-03-94 \\
12-02-94\end{array}$ & $\begin{array}{l}<0.00100 \\
<0.00100 \\
<0.00100 \\
<0.00100\end{array}$ & $\begin{array}{l}<0.00100 \\
0.007000 \\
<0.00100 \\
<0.00100\end{array}$ & $\begin{array}{l}<0.01000 \\
<0.01000 \\
<0.01000 \\
<0.01000\end{array}$ & $\begin{array}{l}<0.01000 \\
<0.01000 \\
<0.01000 \\
<0.01000\end{array}$ & $\begin{array}{l}<0.10000 \\
<0.10000 \\
<0.10000 \\
<0.10000\end{array}$ & $\begin{array}{l}<0.10000 \\
<0.10000 \\
<0.10000 \\
<0.10000\end{array}$ & $\begin{array}{l}<0.10000 \\
<0.10000 \\
<0.10000 \\
<0.10000\end{array}$ & $\begin{array}{l}<1.0000 \\
<1.0000 \\
<1.0000 \\
<1.0000\end{array}$ \\
\hline 453154122394200 & $\begin{array}{l}05-31-94 \\
11-08-94 \\
11-08-94 \\
11-30-94 \\
11-30-94 \\
12-02-94\end{array}$ & $\begin{array}{l}<0.01000 \\
<0.00100 \\
<0.00100 \\
<0.00100 \\
<0.00100 \\
<0.00100\end{array}$ & $\begin{array}{l}<0.01000 \\
0.005000 \\
0.004000 \\
0.001000 \\
0.001000 \\
<0.00100\end{array}$ & $\begin{array}{l}<0.01000 \\
<0.01000 \\
<0.01000 \\
<0.01000 \\
<0.01000 \\
<0.01000\end{array}$ & $\begin{array}{l}<0.10000 \\
<0.01000 \\
<0.01000 \\
<0.01000 \\
<0.01000 \\
<0.01000\end{array}$ & $\begin{array}{r}0.10000 \\
0.20000 \\
0.10000 \\
0.10000 \\
0.20000 \\
<0.10000\end{array}$ & $\begin{array}{l}<0.10000 \\
<0.10000 \\
<0.10000 \\
<0.10000 \\
<0.10000 \\
<0.10000\end{array}$ & $\begin{array}{l}<1.0000 \\
<0.10000 \\
<0.10000 \\
<0.10000 \\
<0.10000 \\
<0.10000\end{array}$ & $\begin{array}{l}<1.0000 \\
<1.0000 \\
<1.0000 \\
<1.0000 \\
<1.0000 \\
<1.0000\end{array}$ \\
\hline 14211805 & $05-23-94$ & $<0.00100$ & $<0.00100$ & $<0.01000$ & $<0.01000$ & $<0.10000$ & $<0.10000$ & $<0.10000$ & $<1.0000$ \\
\hline
\end{tabular}


Table 19. Concentrations of organochlorine and organophosphorus compounds in filtered water from Phase I of the Willamette River Basin Water Quality Study, Oregon, 1992-93

[USGS laboratory schedule 8307]

\begin{tabular}{|c|c|c|c|c|c|c|c|c|c|}
\hline \multirow{2}{*}{$\begin{array}{l}\text { STATION NUMBER } \\
440313123091100\end{array}$} & \multicolumn{5}{|c|}{ STATION NAME } & \multicolumn{3}{|c|}{ DATE } & $\begin{array}{l}\text { ALDRIN, } \\
\text { DIS- } \\
\text { SOLVED } \\
\text { (UG/L) } \\
\end{array}$ \\
\hline & $\begin{array}{ll}A-3 & \text { CHA } \\
A-3 & \text { CHA }\end{array}$ & $\begin{array}{lll}\text { NNEL AT } & \text { W } \\
\text { NNEL AT } & \text { W }\end{array}$ & $\begin{array}{l}\text { ALLIS AND } \\
\text { ALLIS AND }\end{array}$ & $\begin{array}{lll}5 \mathrm{TH} & \mathrm{ST} & \mathrm{AT} \\
5 \mathrm{TH} & \mathrm{ST} & \mathrm{AT}\end{array}$ & $\begin{array}{l}\text { T EUGENE, } \\
\text { T EUGENE, }\end{array}$ & $\begin{array}{l}\text { OR } \\
\text { OR }\end{array}$ & $\begin{array}{l}04-16-9 \\
10-16-9\end{array}$ & $\begin{array}{l}1410 \\
1345\end{array}$ & $\begin{array}{l}<0.00100 \\
<0.00100\end{array}$ \\
\hline 14201300 & ZOLLNER & CREEK NE & AR MT ANGE & $E L, \quad O R$ & & & $06-01-9$ & 1115 & $<0.00100$ \\
\hline 14202000 & PUDDING & RIVER AT & AURORA, & OREG. & & & $04-27-9$ & 1445 & $<0.00100$ \\
\hline 453115122535500 & BEAVERT & ON C AT 2: & $16 \mathrm{TH}$ AVE $\mathrm{N}$ & NEAR ORENC & $\mathrm{CO}, \mathrm{OR}$ & & $03-31-9$ & 1810 & $<0.00100$ \\
\hline 14206950 & FANNO C & REEK AT D & URHAM, OR & & & & $03-01-9$ & 1830 & $<0.00100$ \\
\hline 14211550 & JOHNSON & CREEK AT & MILWAUKIE & E, OREG. & & & $03-14-9$ & 1745 & $<0.00100$ \\
\hline STATION NUMBER & DATE & $\begin{array}{l}\text { CHLOR- } \\
\text { DANE, } \\
\text { DIS- } \\
\text { SOLVED } \\
\text { (UG/L) } \\
\end{array}$ & $\begin{array}{l}\mathrm{p}, \mathrm{p}^{\prime}- \\
\text { DDD, } \\
\text { DIS- } \\
\text { SOLVED } \\
(\mathrm{UG} / \mathrm{L}) \\
\end{array}$ & $\begin{array}{l}\mathrm{p}, \mathrm{p}^{\prime}- \\
\text { DDE, } \\
\text { DIS- } \\
\text { SOLVED } \\
(\mathrm{UG} / \mathrm{L}) \\
\end{array}$ & $\begin{array}{l}\mathrm{p}, \mathrm{p}^{\prime}- \\
\text { DDT, } \\
\text { DIS- } \\
\text { SOLVED } \\
(\mathrm{UG} / \mathrm{L}) \\
\end{array}$ & $\begin{array}{l}\text { DI- } \\
\text { ELDRIN } \\
\text { DIS- } \\
\text { SOLVED } \\
(\text { UG / L) } \\
\end{array}$ & $\begin{array}{l}\text { ENDO- } \\
\text { SULFAN } \\
\text { DISSOLV } \\
\text { (UG/L) } \\
\end{array}$ & $\begin{array}{l}\text { ENDRIN, } \\
\text { DIS- } \\
\text { SOLVED } \\
\text { (UG/L) }\end{array}$ & $\begin{array}{l}\text { HEPTA- } \\
\text { CHLOR } \\
\text { EPOXIDE } \\
\text { DIS- } \\
\text { SOLVED } \\
(\text { UG/L) }\end{array}$ \\
\hline 440313123091100 & $\begin{array}{l}04-16-92 \\
10-16-92\end{array}$ & $\begin{array}{l}<0.10000 \\
<0.10000\end{array}$ & $\begin{array}{l}<0.00100 \\
<0.00100\end{array}$ & $\begin{array}{l}<0.00100 \\
<0.00100\end{array}$ & $\begin{array}{l}<0.00100 \\
<0.00100\end{array}$ & $\begin{array}{l}<0.00100 \\
<0.00100\end{array}$ & $\begin{array}{l}<0.00100 \\
<0.00100\end{array}$ & $\begin{array}{l}<0.00100 \\
<0.00100\end{array}$ & $\begin{array}{l}<0.00100 \\
<0.00100\end{array}$ \\
\hline 14201300 & $06-01-93$ & $<0.10000$ & $<0.00100$ & $<0.00100$ & $<0.00100$ & -- & 0.002000 & $<0.00100$ & $<0.00100$ \\
\hline 14202000 & $04-27-93$ & $<0.10000$ & $<0.00100$ & $<0.00100$ & $<0.00100$ & $<0.00100$ & $<0.00100$ & $<0.00100$ & $<0.00100$ \\
\hline 453115122535500 & $03-31-93$ & $<0.10000$ & $<0.00100$ & $<0.00100$ & $<0.00100$ & $<0.00100$ & $<0.00100$ & $<0.00100$ & $<0.00100$ \\
\hline 14206950 & $03-01-93$ & $<0.10000$ & $<0.00100$ & $<0.00100$ & $<0.00100$ & 0.001000 & $<0.00100$ & $<0.00100$ & $<0.00100$ \\
\hline 14211550 & $03-14-93$ & $<0.10000$ & 0.001000 & $<0.00100$ & $<0.00100$ & 0.002000 & $<0.00100$ & $<0.00100$ & $<0.00100$ \\
\hline STATION NUMBER & DATE & $\begin{array}{l}\text { HEPTA- } \\
\text { CHLOR, } \\
\text { DIS- } \\
\text { SOLVED } \\
\text { (UG/L) }\end{array}$ & $\begin{array}{l}\text { LINDANE } \\
\text { DIS- } \\
\text { SOLVED } \\
\text { (UG/L) }\end{array}$ & $\begin{array}{l}\text { METH- } \\
\text { OXY- } \\
\text { CHLOR } \\
\text { DISSOLV } \\
\text { (UG/L) }\end{array}$ & $\begin{array}{l}\text { MIREX, } \\
\text { DIS- } \\
\text { SOLVED } \\
(\text { UG/L) }\end{array}$ & $\begin{array}{l}\text { GROSS } \\
\text { PCB, } \\
\text { DIS- } \\
\text { SOLVED } \\
\text { (UG/L) }\end{array}$ & $\begin{array}{l}\text { GROSS } \\
\text { PCN, } \\
\text { DIS } \\
\text { SOLVED } \\
\text { (UG/L) }\end{array}$ & $\begin{array}{l}\text { PER- } \\
\text { THANE } \\
\text { DISSOLV } \\
\text { (UG/L) }\end{array}$ & $\begin{array}{l}\text { TOX- } \\
\text { APHENE, } \\
\text { DIS- } \\
\text { SOLVED } \\
\text { (UG/L) }\end{array}$ \\
\hline 440313123091100 & $\begin{array}{l}04-16-92 \\
10-16-92\end{array}$ & $\begin{array}{l}<0.00100 \\
<0.00100\end{array}$ & $\begin{array}{l}<0.00100 \\
<0.00100\end{array}$ & $\begin{array}{l}<0.01000 \\
<0.01000\end{array}$ & $\begin{array}{l}<0.01000 \\
<0.01000\end{array}$ & $\begin{array}{l}<0.10000 \\
<0.10000\end{array}$ & $\begin{array}{l}<0.10000 \\
<0.10000\end{array}$ & $\begin{array}{l}<0.10000 \\
<0.10000\end{array}$ & $\begin{array}{l}<1.0000 \\
<1.0000\end{array}$ \\
\hline 14201300 & $06-01-93$ & $<0.00100$ & -- & $<0.01000$ & $<0.01000$ & $<0.10000$ & $<0.10000$ & $<0.10000$ & $<1.0000$ \\
\hline 14202000 & $04-27-93$ & $<0.00100$ & $<0.00100$ & $<0.01000$ & $<0.01000$ & $<0.10000$ & $<0.10000$ & $<0.10000$ & $<1.0000$ \\
\hline 453115122535500 & $03-31-93$ & $<0.00100$ & $<0.00100$ & $<0.01000$ & $<0.01000$ & $<0.10000$ & $<0.10000$ & $<0.10000$ & $<1.0000$ \\
\hline 14206950 & $03-01-93$ & $<0.00100$ & $<0.00100$ & $<0.01000$ & $<0.01000$ & $<0.10000$ & $<0.10000$ & $<0.10000$ & $<1.0000$ \\
\hline 14211550 & $03-14-93$ & $<0.00100$ & 0.001000 & $<0.01000$ & $<0.01000$ & $<0.10000$ & $<0.10000$ & $<0.10000$ & $<1.0000$ \\
\hline STATION NUMBER & DATE & $\begin{array}{l}\text { CHLOR- } \\
\text { PYRIFOS } \\
\text { DIS- } \\
\text { SOLVED } \\
\text { (UG/L) }\end{array}$ & $\begin{array}{l}\text { DI- } \\
\text { AZINON, } \\
\text { DIS- } \\
\text { SOLVED } \\
\text { (UG/L) }\end{array}$ & $\begin{array}{l}\text { ETHION } \\
\text { DISSOLV } \\
\text { (UG/L) }\end{array}$ & $\begin{array}{l}\text { FONOFOS } \\
\text { DIS- } \\
\text { SOLVED } \\
\text { (UG/L) }\end{array}$ & $\begin{array}{l}\text { MALA- } \\
\text { THION, } \\
\text { DIS- } \\
\text { SOLVED } \\
\text { (UG/L) }\end{array}$ & $\begin{array}{l}\text { METHYL } \\
\text { PARA- } \\
\text { THION, } \\
\text { DIS- } \\
\text { SOLVED } \\
\text { (UG/L) }\end{array}$ & $\begin{array}{l}\text { PARA- } \\
\text { THION, } \\
\text { DIS- } \\
\text { SOLVED } \\
\text { (UG/L) }\end{array}$ & $\begin{array}{l}\text { TRI- } \\
\text { THION } \\
\text { DISSOLV } \\
\quad(\text { UG/L) }\end{array}$ \\
\hline 440313123091100 & $\begin{array}{l}04-16-92 \\
10-16-92\end{array}$ & $\begin{array}{r}<0.02000 \\
0.01000\end{array}$ & $\begin{array}{r}0.02000 \\
<0.01000\end{array}$ & $\begin{array}{l}<0.02000 \\
<0.01000\end{array}$ & $\begin{array}{r}<0.02000 \\
0.07000\end{array}$ & $\begin{array}{l}<0.02000 \\
<0.01000\end{array}$ & $\begin{array}{l}<0.02000 \\
<0.01000\end{array}$ & $\begin{array}{l}<0.02000 \\
<0.01000\end{array}$ & $\begin{array}{l}<0.02000 \\
<0.01000\end{array}$ \\
\hline 14201300 & $06-01-93$ & -- & -- & -- & -- & -- & -- & -- & -- \\
\hline 14202000 & $04-27-93$ & $<0.00500$ & 0.01000 & $<0.00500$ & $<0.00500$ & $<0.00500$ & $<0.00500$ & $<0.00500$ & $<0.00500$ \\
\hline 453115122535500 & $03-31-93$ & -- & 0.06000 & $<0.00500$ & $<0.00500$ & $<0.00500$ & $<0.00500$ & $<0.00500$ & $<0.00500$ \\
\hline 14206950 & $03-01-93$ & $<0.01000$ & 0.03000 & $<0.01000$ & $<0.01000$ & $<0.01000$ & $<0.01000$ & $<0.01000$ & $<0.01000$ \\
\hline 14211550 & $03-14-93$ & 0.01000 & 0.02000 & $<0.01000$ & $<0.01000$ & 0.02000 & $<0.01000$ & $<0.01000$ & $<0.01000$ \\
\hline
\end{tabular}


Table 20. Concentrations of organochlorine compounds in filtered water from Phase II of the Willamette River Basin Water Quality Study, Oregon, 1994 [USGS laboratory schedule 8369; ug/L, micrograms per liter]

\begin{tabular}{|c|c|c|c|c|c|c|c|c|c|c|}
\hline Station Name & Station number & Date & Time & $\begin{array}{l}\text { Aldrin } \\
\text { (ug/L) }\end{array}$ & $\begin{array}{l}\text { Chlordane } \\
\text { (ug/L) }\end{array}$ & $\begin{array}{l}\text { p, p' DDD } \\
\text { (ug/L) }\end{array}$ & $\begin{array}{l}\text { p, p' DDE } \\
\text { (ug/L) }\end{array}$ & $\begin{array}{l}\text { p, p' DDT } \\
\text { (ug/L) }\end{array}$ & $\begin{array}{l}\text { Dieldrin } \\
\text { (ug/L) }\end{array}$ & $\begin{array}{c}\text { Endosulfan I } \\
\quad(\mathrm{ug} / \mathrm{L})\end{array}$ \\
\hline \multirow[t]{2}{*}{ Muddy Cr. nr Peoria, OR } & 443138123120901 & 941102 & 1010 & $<0.001$ & $<0.1$ & $<0.001$ & $<0.001$ & $<0.001$ & $<0.001$ & $<0.001$ \\
\hline & & 941106 & 1200 & $<.001$ & $<.1$ & $<.001$ & $<.001$ & $<.001$ & $<.001$ & $<.001$ \\
\hline Mill Cr. nr Turner, OR & 445037122573800 & 941104 & 1120 & $<.001$ & $<.1$ & $<.001$ & $<.001$ & $<.001$ & .001 & $<.001$ \\
\hline Pudding R. at Aurora, OR & 14202000 & 941028 & 1710 & $<.001$ & $<.1$ & $<.001$ & $<.001$ & $<.001$ & .001 & $<.001$ \\
\hline \multirow[t]{2}{*}{ Johnson Cr. nr Gresham, OR } & 452823122240900 & 941027 & 1640 & $<.001$ & $<.1$ & .001 & .003 & .003 & .010 & $<.001$ \\
\hline & & 941028 & 1135 & $<.001$ & $<.1$ & .001 & .002 & .003 & .010 & $<.001$ \\
\hline Willamette R. at Portland, OR & 14211720 & 941029 & 1230 & $<.001$ & $<.1$ & $<.001$ & $<.001$ & $<.001$ & $<.001$ & $<.001$ \\
\hline $\begin{array}{l}\text { Interstate } 84 \text { Trans } \\
\text { Corridor at SE 3rd at } \\
\text { Portland, OR }\end{array}$ & 453154122394200 & 941108 & 1800 & $<.001$ & $<.1$ & .001 & .001 & .003 & $<.001$ & $<.001$ \\
\hline
\end{tabular}

\begin{tabular}{|c|c|c|c|c|c|c|c|c|c|c|c|}
\hline Station number & Date & Endrin & $\begin{array}{l}\text { HeptachlorE } \\
\text { poxide } \\
\text { (ug/L) }\end{array}$ & $\begin{array}{l}\text { Heptachlor } \\
\quad \text { (ug/L) }\end{array}$ & $\begin{array}{c}\text { Lindane } \\
\text { (ug/L) }\end{array}$ & $\begin{array}{c}\text { Methoxy- } \\
\text { chlor } \\
\text { (ug/L) }\end{array}$ & $\begin{array}{l}\text { Mirex } \\
\text { (ug/L) }\end{array}$ & $\begin{array}{c}\text { Gross } \\
\text { PCB } \\
\text { (ug/L) }\end{array}$ & $\begin{array}{c}\text { Gross } \\
\text { PCN } \\
\text { (ug/L) }\end{array}$ & $\begin{array}{l}\text { Perthane } \\
\text { (ug/L) }\end{array}$ & $\begin{array}{l}\text { Toxaphene } \\
\text { (ug/L) }\end{array}$ \\
\hline \multirow[t]{2}{*}{443138123120901} & 941102 & $<0.001$ & $<0.001$ & $<0.001$ & $<0.001$ & $<0.01$ & $<0.01$ & $<0.1$ & $<0.1$ & $<0.1$ & $<1.0$ \\
\hline & 941106 & $<.001$ & $<.001$ & $<.001$ & $<.001$ & $<.01$ & $<.01$ & $<.1$ & $<.1$ & $<.1$ & $<1.0$ \\
\hline 445037122573800 & 941104 & $<.001$ & $<.001$ & $<.001$ & $<.001$ & $<.01$ & $<.01$ & $<.1$ & $<.1$ & $<.1$ & $<1.0$ \\
\hline 14202000 & 941028 & $<.001$ & $<.001$ & $<.001$ & $<.001$ & $<.01$ & $<.01$ & $<.1$ & $<.1$ & $<.1$ & $<1.0$ \\
\hline \multirow[t]{2}{*}{452823122240900} & 941027 & $<.001$ & $<.001$ & $<.001$ & .001 & $<.01$ & $<.01$ & $<.1$ & $<.1$ & $<.1$ & $<1.0$ \\
\hline & 941028 & $<.001$ & $<.001$ & $<.001$ & .001 & $<.01$ & $<.01$ & $<.1$ & $<.1$ & $<.1$ & $<1.0$ \\
\hline 14211720 & 941029 & $<.001$ & $<.001$ & $<.001$ & .001 & $<.01$ & $<.01$ & $<.1$ & $<.1$ & $<.1$ & $<1.0$ \\
\hline 453154122394200 & 941108 & $<.001$ & $<.001$ & $<.001$ & .003 & $<.01$ & $<.01$ & .1 & $<.1$ & $<.1$ & $<1.0$ \\
\hline
\end{tabular}


Table 21. Concentrations of organochlorine and organophosphorus compounds associated with suspended sediment from Phase I of the Willamette River Basin Water Quality Study, Oregon, 1992-93

[USGS laboratory schedule 8308; UG/L, micrograms per liter]

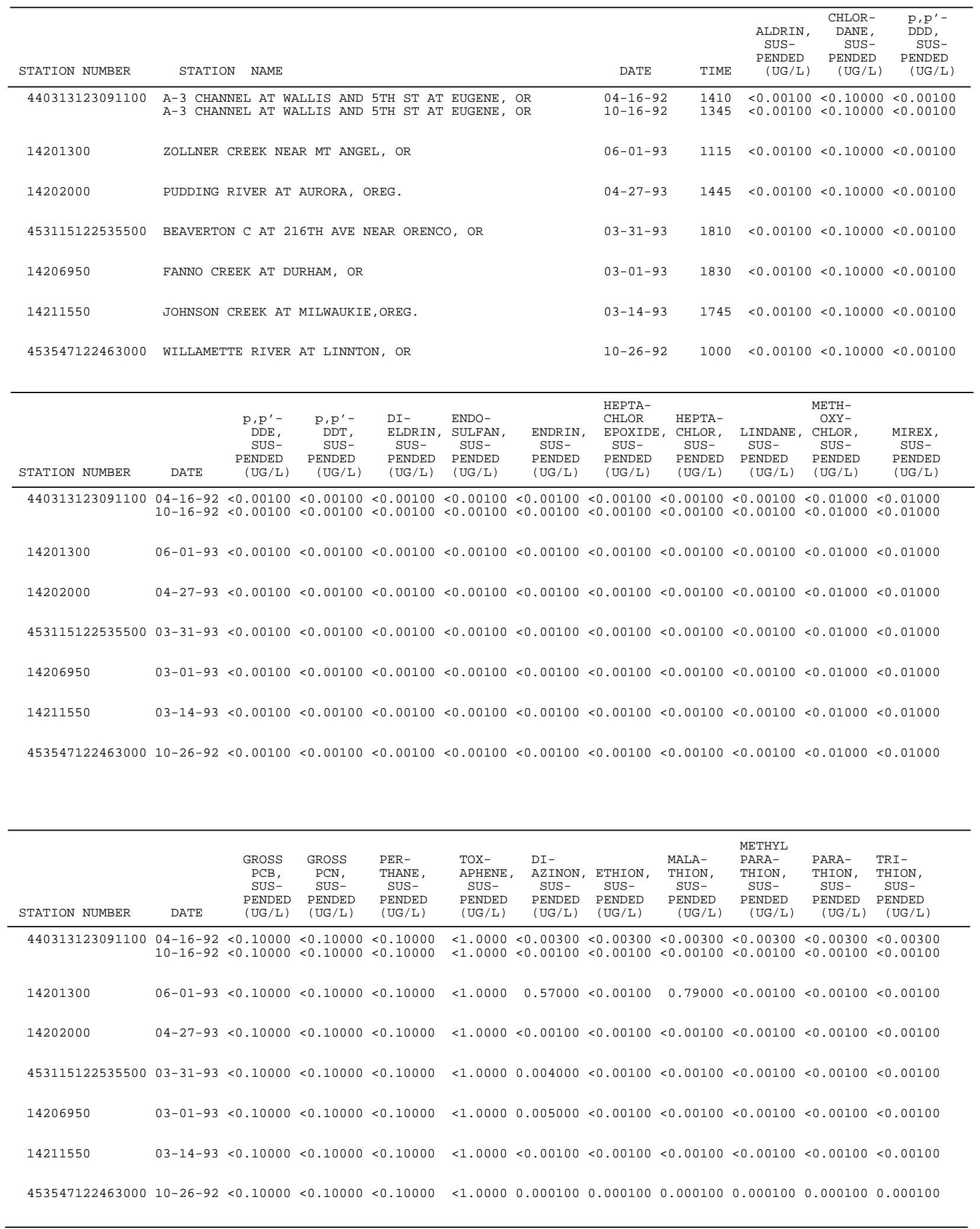


Table 22. Concentrations of organochlorine compounds associated with suspended sediment from Phase II of the Willamette River Basin Water Quality Study, Oregon, 1994

[USGS laboratory schedule 8370; ug/L, micrograms per liter]

\begin{tabular}{|c|c|c|c|c|c|c|c|c|c|c|}
\hline Station Name & Station number & Date & Time & $\begin{array}{l}\text { Aldrin } \\
\text { (ug/L) }\end{array}$ & $\begin{array}{l}\text { Chlordane } \\
\text { (ug/L) }\end{array}$ & $\begin{array}{l}\text { p, p' DDD } \\
\text { (ug/L) }\end{array}$ & $\begin{array}{l}\text { p, p' DDE } \\
\text { (ug/L) }\end{array}$ & $\begin{array}{l}\text { p, p' DDT } \\
\text { (ug/L) }\end{array}$ & $\begin{array}{c}\text { Dieldrin } \\
\text { (ug/L) }\end{array}$ & $\begin{array}{c}\text { Endosulfan I } \\
\quad(\text { ug/L) }\end{array}$ \\
\hline \multirow[t]{2}{*}{ Muddy Cr. nr Peoria, OR } & 443138123120901 & 941102 & 1010 & $<0.003$ & $<0.3$ & $<0.003$ & $<0.003$ & $<0.003$ & $<0.012$ & $<0.003$ \\
\hline & & 941106 & 1200 & $<.001$ & $<.1$ & $<.001$ & $<.001$ & $<.001$ & $<.004$ & $<.001$ \\
\hline Mill Cr. nr Turner, OR & 445037122573800 & 941104 & 1120 & $<.002$ & $<.2$ & $<.002$ & $<.002$ & $<.002$ & $<.008$ & $<.002$ \\
\hline Pudding R. at Aurora, OR & 14202000 & 941028 & 1710 & $<.002$ & $<.2$ & .002 & .003 & .004 & $<.008$ & $<.002$ \\
\hline \multirow[t]{2}{*}{ Johnson Cr. nr Gresham, OR } & 452823122240900 & 941027 & 1640 & $<.004$ & $<.4$ & .006 & .020 & .031 & $<.016$ & $<.004$ \\
\hline & & 941028 & 1135 & $<.002$ & $<.2$ & .002 & .005 & .007 & $<.008$ & $<.002$ \\
\hline Willamette R. at Portland, OR & 14211720 & 941029 & 1230 & $<.001$ & $<.1$ & $<.001$ & $<.001$ & $<.001$ & $<.004$ & $<.001$ \\
\hline $\begin{array}{l}\text { Interstate } 84 \text { Trans } \\
\text { Corridor at SE 3rd at }\end{array}$ & 453154122394200 & 941108 & 1800 & $<.001$ & $<.1$ & .002 & .021 & .018 & $<.004$ & $<.001$ \\
\hline
\end{tabular}

Corridor at SE 3rd at

Portland, OR

\begin{tabular}{|c|c|c|c|c|c|c|c|c|c|c|c|}
\hline Station number & Date & Endrin & $\begin{array}{c}\text { HeptachlorE } \\
\text { poxide } \\
(\mathrm{ug} / \mathrm{L})\end{array}$ & $\begin{array}{l}\text { Heptachlor } \\
\quad(\mathrm{ug} / \mathrm{L})\end{array}$ & $\begin{array}{c}\text { Lindane } \\
\text { (ug/L) }\end{array}$ & $\begin{array}{l}\text { Methoxy- } \\
\text { chlor } \\
\text { (ug/L) }\end{array}$ & $\begin{array}{l}\text { Mirex } \\
\text { (ug/L) }\end{array}$ & $\begin{array}{c}\text { Gross } \\
\text { PCB } \\
\text { (ug/L) }\end{array}$ & $\begin{array}{r}\text { Gross } \\
\text { PCN } \\
(\mathrm{ug} / \mathrm{L})\end{array}$ & $\begin{array}{c}\text { Perthane } \\
\text { (ug/L) }\end{array}$ & $\begin{array}{l}\text { Toxaphene } \\
\text { (ug/L) }\end{array}$ \\
\hline \multirow[t]{2}{*}{443138123120901} & 941102 & $<0.003$ & $<0.003$ & $<0.003$ & $<0.003$ & $<0.012$ & $<0.003$ & $<0.3$ & $<0.3$ & $<0.3$ & $<3.0$ \\
\hline & 941106 & $<.001$ & $<.001$ & $<.001$ & $<.001$ & $<.004$ & $<.001$ & $<.1$ & $<.1$ & $<.1$ & $<1.0$ \\
\hline 445037122573800 & 941104 & $<.002$ & $<.002$ & $<.002$ & $<.002$ & $<.008$ & $<.002$ & $<.2$ & $<.2$ & $<.2$ & $<2.0$ \\
\hline 14202000 & 941028 & $<.002$ & $<.002$ & $<.002$ & $<.002$ & $<.008$ & $<.002$ & $<.2$ & $<.2$ & $<.2$ & $<2.0$ \\
\hline \multirow[t]{2}{*}{452823122240900} & 941027 & $<.004$ & $<.004$ & $<.004$ & $<.004$ & $<.016$ & $<.004$ & $<.4$ & $<.4$ & $<.4$ & $<4.0$ \\
\hline & 941028 & $<.002$ & $<.002$ & $<.002$ & $<.002$ & $<.008$ & $<.002$ & $<.2$ & $<.2$ & $<.2$ & $<2.0$ \\
\hline 14211720 & 941029 & $<.001$ & $<.001$ & $<.001$ & $<.001$ & $<.004$ & $<.001$ & $<.1$ & $<.1$ & $<.1$ & $<1.0$ \\
\hline 453154122394200 & 941108 & $<.001$ & $<.001$ & $<.001$ & $<.001$ & $<.004$ & $<.001$ & $<.1$ & $<.1$ & $<.1$ & $<1.0$ \\
\hline
\end{tabular}


Table 23. Concentrations of organochlorine compounds associated with bed sediment from Phase I of the Willamette River Basin Water Quality Study, Oregon, 1992

[USGS laboratory schedule 2501; WS, wet sieved; DW, dry weight; BM, bed material; G/KG, grams per kilogram; UG/KG, micrograms per kilogram]

\begin{tabular}{|c|c|c|c|c|c|c|}
\hline STATION NUMBER & STATION NAME & DATE & TIME & $\begin{array}{c}\text { CARBON, } \\
\text { ORGANIC } \\
\text { SED, BM } \\
\text { WS, <2MM } \\
\text { DW, REC } \\
(\mathrm{G} / \mathrm{KG}) \\
\end{array}$ & $\begin{array}{l}\text { CARBON, } \\
\text { INORG, } \\
\text { SED, BM } \\
\text { WS, <2MM } \\
\text { DW, REC } \\
(\mathrm{G} / \mathrm{KG})\end{array}$ & $\begin{array}{r}\text { CARBON, } \\
\text { ORG + } \\
\text { INORG } \\
\text { SED, BM } \\
\text { WS, <2MM } \\
(\mathrm{G} / \mathrm{KG}) \\
\end{array}$ \\
\hline 440313123091100 & A-3 CHANNEL AT WALLIS AND 5TH ST AT EUGENE, OR & $08-25-92$ & 1230 & 18.000 & 0.40000 & 18.000 \\
\hline 14165500 & MCKENZIE RIVER NEAR COBURG, OR & $08-25-92$ & 1645 & 85.000 & 0.20000 & 85.000 \\
\hline 443207123145500 & WILLAMETTE RIVER NEAR CORVALLIS, OR & $08-26-92$ & 1030 & 18.000 & $<0.10000$ & 18.000 \\
\hline 443819123064000 & CALAPOOIA RIVER AT MOUTH AT ALBANY, OR & $08-26-92$ & 1500 & 13.000 & 1.4000 & 14.000 \\
\hline 444032123050600 & MIDDLE FOURTH LAKE NEAR ALBANY, OR & $09-01-92$ & 1100 & 77.000 & 0.20000 & 77.000 \\
\hline 444416123030800 & SANTIAM RIVER NEAR JEFFERSON, OR & $08-29-92$ & 1000 & 3.0000 & $<0.10000$ & 3.0000 \\
\hline 445543123084400 & RICKREALL CREEK NEAR RICKREALL, OR & $08-28-92$ & 1345 & 12.000 & $<0.10000$ & 12.000 \\
\hline 451320123041100 & YAMHILL RIVER AT DAYTON, OR & $08-27-92$ & 1400 & 3.7000 & $<0.10000$ & 3.7000 \\
\hline 451705122575100 & WILLAMETTE RIVER AT NEWBERG, OR & $09-01-92$ & 1600 & 11.000 & $<0.10000$ & 11.000 \\
\hline 452950122492900 & BEAVERTON CREEK AT BEAVERTON, OR & $08-23-92$ & 1100 & 46.000 & $<0.10000$ & 46.000 \\
\hline 452221122362400 & CLACKAMAS RIVER AT OREGON CITY, OR & $08-24-92$ & 1130 & 5.8000 & 0.30000 & 6.1000 \\
\hline 452847122244500 & JOHNSON CREEK AT GRESHAM, OR & $08-21-92$ & 1330 & 9.4000 & 0.60000 & 10.000 \\
\hline 453547122463000 & WILLAMETTE RIVER AT LINNTON, OR & $09-09-92$ & 1130 & 17.000 & $<0.10000$ & 17.000 \\
\hline 453205122223701 & BEAVER CREEK NEAR TROUTDALE, OR & $08-22-92$ & 0945 & 8.4000 & 0.40000 & 8.8000 \\
\hline
\end{tabular}


Table 23. Concentrations of organochlorine compounds associated with bed sediment from Phase I of the Willamette River Basin Water Quality Study, Oregon, 1992-Continued

\begin{tabular}{|c|c|c|c|c|c|c|c|c|c|c|}
\hline STATION NUMBER & DATE & $\begin{array}{c}\text { BED } \\
\text { MAT. } \\
\text { SIEVE } \\
\text { DIAM. } \\
\% \text { FINER } \\
\text { THAN } \\
.062 \text { MM }\end{array}$ & $\begin{array}{l} \\
\text { ALDRIN, } \\
\text { SED, BM } \\
\text { WS, <2MM } \\
\text { DW, REC } \\
\text { (UG/KG) }\end{array}$ & $\begin{array}{l}\text { ALPHA- } \\
\text { HCH, } \\
\text { SED, BM } \\
\text { WS, <2MM } \\
\text { DW, REC } \\
(\mathrm{UG} / \mathrm{KG})\end{array}$ & $\begin{array}{l}\text { BETA- } \\
\text { HCH, } \\
\text { SED, BM } \\
\text { WS, <2MM } \\
\text { DW, REC } \\
(\mathrm{UG} / \mathrm{KG})\end{array}$ & $\begin{array}{l}\text { TRANS- } \\
\text { CHLOR- } \\
\text { DANE, } \\
\text { SED, BM } \\
\text { WS, }<2 M M \\
\text { DW, REC } \\
(\mathrm{UG} / \mathrm{KG})\end{array}$ & $\begin{array}{l}\text { CIS- } \\
\text { CHLOR- } \\
\text { DANE, } \\
\text { SED, BM } \\
\text { WS, <2MM } \\
\text { DW, REC } \\
\text { (UG/KG) }\end{array}$ & $\begin{array}{l}\text { CHLORO- } \\
\text { NEB, } \\
\text { SED, BM } \\
\text { WS, <2MM } \\
\text { DW, REC } \\
(\mathrm{UG} / \mathrm{KG})\end{array}$ & $\begin{array}{l}\text { CIS- } \\
\text { NONA- } \\
\text { CHLOR, } \\
\text { SED, BM } \\
\text { WS, <2MM } \\
\text { DW, REC } \\
\text { (UG/KG) }\end{array}$ & $\begin{array}{l}\text { CIS- } \\
\text { PER- } \\
\text { METHRIN } \\
\text { SED, BM } \\
\text { WS, <2MM } \\
\text { DW, REC } \\
(\mathrm{UG} / \mathrm{KG})\end{array}$ \\
\hline 440313123091100 & $08-25-92$ & 23.000 & $<2.0000$ & $<2.0000$ & $<3.0000$ & $<2.0000$ & $<2.0000$ & $<10.000$ & $<2.0000$ & $<10.000$ \\
\hline 14165500 & $08-25-92$ & 84.000 & $<2.5000$ & $<2.5000$ & $<2.5000$ & $<2.5000$ & $<2.5000$ & $<12.000$ & $<2.5000$ & -- \\
\hline 443207123145500 & $08-26-92$ & 22.000 & $<1.0000$ & $<1.0000$ & $<1.0000$ & $<1.0000$ & $<1.0000$ & $<5.0000$ & $<1.0000$ & $<5.0000$ \\
\hline 443819123064000 & $08-26-92$ & 33.000 & $<1.0000$ & $<1.0000$ & $<1.0000$ & $<1.0000$ & $<1.0000$ & $<5.0000$ & $<1.0000$ & $<5.0000$ \\
\hline 444032123050600 & $09-01-92$ & 95.000 & $<2.0000$ & $<2.0000$ & $<2.0000$ & $<2.0000$ & $<2.0000$ & $<10.000$ & $<2.0000$ & $<10.000$ \\
\hline 444416123030800 & $08-29-92$ & 3.0000 & $<1.0000$ & $<1.0000$ & $<1.0000$ & $<1.0000$ & $<1.0000$ & $<5.0000$ & $<1.0000$ & $<5.0000$ \\
\hline 445543123084400 & $08-28-92$ & 17.000 & $<1.0000$ & $<1.0000$ & $<1.0000$ & $<1.0000$ & $<1.0000$ & $<5.0000$ & $<1.0000$ & $<5.0000$ \\
\hline 451320123041100 & $08-27-92$ & 9.0000 & $<1.0000$ & $<1.0000$ & $<1.0000$ & $<1.0000$ & $<1.0000$ & $<5.0000$ & $<1.0000$ & $<5.0000$ \\
\hline 451705122575100 & $09-01-92$ & 20.000 & $<1.0000$ & $<1.0000$ & $<1.0000$ & $<1.0000$ & $<1.0000$ & $<5.0000$ & $<1.0000$ & $<5.0000$ \\
\hline 452950122492900 & $08-23-92$ & 66.000 & $<2.0000$ & $<2.0000$ & $<2.0000$ & 8.0000 & 8.3000 & $<10.000$ & 3.5000 & $<10.000$ \\
\hline 452221122362400 & $08-24-92$ & 4.0000 & $<1.0000$ & $<1.0000$ & $<1.0000$ & $<1.0000$ & $<1.0000$ & $<5.0000$ & $<1.0000$ & $<5.0000$ \\
\hline 452847122244500 & $08-21-92$ & 34.000 & $<1.0000$ & $<1.0000$ & $<1.0000$ & 2.6000 & 2.4000 & $<5.0000$ & $<2.0000$ & $<5.0000$ \\
\hline 453547122463000 & $09-09-92$ & 49.000 & $<1.0000$ & $<1.0000$ & $<1.0000$ & $<1.0000$ & $<1.0000$ & $<5.0000$ & $<1.0000$ & $<5.0000$ \\
\hline 453205122223701 & $08-22-92$ & 37.000 & $<1.0000$ & $<1.0000$ & $<1.0000$ & 7.6000 & 7.2000 & $<5.0000$ & 4.6000 & $<5.0000$ \\
\hline
\end{tabular}


Table 23. Concentrations of organochlorine compounds associated with bed sediment from Phase I of the Willamette River Basin Water Quality Study, Oregon, 1992-Continued

\begin{tabular}{|c|c|c|c|c|c|c|c|c|c|c|}
\hline STATION NUMBER & DATE & $\begin{array}{l}\text { HEXA- } \\
\text { CHLORO- } \\
\text { BENZENE } \\
\text { SED, BM } \\
\text { WS, <2MM } \\
\text { DW, REC } \\
\text { (UG/KG) }\end{array}$ & $\begin{array}{l} \\
\text { DCPA, } \\
\text { SED, BM } \\
\text { WS, <2MM } \\
\text { DW, REC } \\
(\mathrm{UG} / \mathrm{KG}) \\
\end{array}$ & $\begin{array}{c}\mathrm{p}, \mathrm{p}^{\prime}- \\
\text { DDD } \\
\text { SED, BM } \\
\text { WS, <2MM } \\
\text { DW, REC } \\
\text { (UG/KG) }\end{array}$ & $\begin{array}{c}\circ, \mathrm{p}^{\prime}- \\
\text { DDD, } \\
\text { SED, BM } \\
\text { WS, <2MM } \\
\text { DW, REC } \\
(\mathrm{UG} / \mathrm{KG})\end{array}$ & $\begin{array}{c}\text { o, } \mathrm{p}^{\prime}- \\
\text { DDE, } \\
\mathrm{SED}, \mathrm{BM} \\
\mathrm{WS},<2 \mathrm{MM} \\
\mathrm{DW}, \mathrm{REC} \\
(\mathrm{UG} / \mathrm{KG}) \\
\end{array}$ & $\begin{array}{c}\mathrm{p}, \mathrm{p}^{\prime}- \\
\mathrm{DDE}, \\
\mathrm{SED}, \mathrm{BM} \\
\mathrm{WS},<2 \mathrm{MM} \\
\mathrm{DW}, \mathrm{REC} \\
(\mathrm{UG} / \mathrm{KG}) \\
\end{array}$ & $\begin{array}{c}\mathrm{p}, \mathrm{p}^{\prime}- \\
\text { DDT, } \\
\text { SED, BM } \\
\text { WS, <2MM } \\
\text { DW, REC } \\
(\mathrm{UG} / \mathrm{KG}) \\
\end{array}$ & $\begin{array}{l}\circ, \mathrm{p}^{\prime}- \\
\text { DDT, } \\
\text { SED, BM } \\
\text { WS, <2MM } \\
\text { DW, REC } \\
(\mathrm{UG} / \mathrm{KG}) \\
\end{array}$ & $\begin{array}{l}\text { DIEL- } \\
\text { DRIN, } \\
\text { SED, BM } \\
\text { WS, <2MM } \\
\text { DW, REC } \\
(\mathrm{UG} / \mathrm{KG}) \\
\end{array}$ \\
\hline 440313123091100 & $08-25-92$ & $<50.000$ & $<10.000$ & $<2.0000$ & $<2.0000$ & $<2.0000$ & $<2.0000$ & $<4.0000$ & $<4.0000$ & $<2.0000$ \\
\hline 14165500 & $08-25-92$ & $<50.000$ & $<12.000$ & -- & -- & $<2.5000$ & $<2.5000$ & -- & -- & $<2.5000$ \\
\hline 443207123145500 & $08-26-92$ & $<50.000$ & $<5.0000$ & $<1.0000$ & $<1.0000$ & $<1.0000$ & $<1.0000$ & $<2.0000$ & $<2.0000$ & $<1.0000$ \\
\hline 443819123064000 & $08-26-92$ & $<50.000$ & $<5.0000$ & -- & -- & $<1.0000$ & $<1.0000$ & -- & -- & $<1.0000$ \\
\hline 444032123050600 & $09-01-92$ & 130.00 & $<10.000$ & $<2.0000$ & $<2.0000$ & $<2.0000$ & $<2.0000$ & $<4.0000$ & $<4.0000$ & $<2.0000$ \\
\hline 444416123030800 & $08-29-92$ & $<50.000$ & $<5.0000$ & -- & -- & $<1.0000$ & $<1.0000$ & -- & -- & $<1.0000$ \\
\hline 445543123084400 & $08-28-92$ & $<50.000$ & $<5.0000$ & -- & -- & $<1.0000$ & $<1.0000$ & -- & -- & $<1.0000$ \\
\hline 451320123041100 & $08-27-92$ & $<50.000$ & $<5.0000$ & $<1.0000$ & $<1.0000$ & $<1.0000$ & $<1.0000$ & $<2.0000$ & $<2.0000$ & $<1.0000$ \\
\hline 451705122575100 & $09-01-92$ & $<50.000$ & $<5.0000$ & $<1.0000$ & $<1.0000$ & $<1.0000$ & $<1.0000$ & $<2.0000$ & $<2.0000$ & $<1.0000$ \\
\hline 452950122492900 & $08-23-92$ & $<50.000$ & $<10.000$ & 88.000 & 25.000 & $<2.0000$ & 8.3000 & 21.000 & 18.000 & 4.2000 \\
\hline 452221122362400 & $08-24-92$ & $<50.000$ & $<5.0000$ & $<1.0000$ & $<1.0000$ & $<1.0000$ & $<1.0000$ & $<2.0000$ & $<2.0000$ & $<1.0000$ \\
\hline 452847122244500 & $08-21-92$ & $<50.000$ & $<5.0000$ & 110.00 & 27.000 & 1.9000 & 120.00 & 81.000 & 44.000 & 15.000 \\
\hline 453547122463000 & $09-09-92$ & $<50.000$ & $<5.0000$ & 5.8000 & $<1.0000$ & $<1.0000$ & 3.8000 & 14.000 & $<2.0000$ & $<1.0000$ \\
\hline 453205122223701 & $08-22-92$ & $<50.000$ & $<5.0000$ & 21.000 & $<1.0000$ & $<1.0000$ & 16.000 & 17.000 & $<2.0000$ & 18.000 \\
\hline
\end{tabular}


Table 23. Concentrations of organochlorine compounds associated with bed sediment from Phase I of the Willamette River Basin Water Quality Study, Oregon, 1992-Continued

\begin{tabular}{|c|c|c|c|c|c|c|c|c|c|c|}
\hline STATION NUMBER & DATE & $\begin{array}{l}\text { ENDO- } \\
\text { SULFAN } \\
\text { I, } \\
\text { SED, BM } \\
\text { WS, <2MM } \\
\text { DW, REC } \\
\text { (UG/KG) }\end{array}$ & $\begin{array}{l}\text { ENDRIN, } \\
\text { SED, BM } \\
\text { WS, <2MM } \\
\text { DW, REC } \\
\text { (UG/KG) }\end{array}$ & $\begin{array}{l}\text { HEPTA- } \\
\text { CHLOR } \\
\text { EPOXIDE } \\
\text { SED, BM } \\
\text { WS, <2MM } \\
\text { DW, REC } \\
\text { (UG/KG) }\end{array}$ & $\begin{array}{l}\text { HEPTA- } \\
\text { CHLOR, } \\
\text { SED, BM } \\
\text { WS, <2MM } \\
\text { DW, REC } \\
\text { (UG/KG) }\end{array}$ & $\begin{array}{l}\text { ISODRIN } \\
\text { SED, BM } \\
\text { WS, <2MM } \\
\text { DW, REC } \\
\text { (UG/KG) }\end{array}$ & $\begin{array}{l}\text { LINDANE } \\
\text { SED, BM } \\
\text { WS, <2MM } \\
\text { DW, REC } \\
\text { (UG/KG) }\end{array}$ & $\begin{array}{l}\text { MIREX, } \\
\text { SED, BM } \\
\text { WS, <2MM } \\
\text { DW, REC } \\
\text { (UG/KG) }\end{array}$ & $\begin{array}{l}\text { O, } \mathrm{p}^{\prime}- \\
\text { METHOXY } \\
\text { CHLOR, } \\
\text { SED, BM } \\
\text { WS, <2MM } \\
\text { DW, REC } \\
\text { (UG/KG) }\end{array}$ & $\begin{array}{l}\text { OXY- } \\
\text { CHLOR- } \\
\text { DANE, } \\
\text { SED, BM } \\
\text { WS, <2MM } \\
\text { DW, REC } \\
\text { (UG/KG) }\end{array}$ \\
\hline 440313123091100 & $08-25-92$ & $<2.0000$ & $<4.0000$ & $<2.0000$ & $<2.0000$ & $<2.0000$ & $<2.0000$ & $<2.0000$ & $<10.000$ & $<2.0000$ \\
\hline 14165500 & $08-25-92$ & $<2.5000$ & $<5.0000$ & $<2.5000$ & $<2.5000$ & $<2.5000$ & $<2.5000$ & $<2.5000$ & $<12.000$ & $<2.5000$ \\
\hline 443207123145500 & $08-26-92$ & $<1.0000$ & $<2.0000$ & $<1.0000$ & $<1.0000$ & $<1.0000$ & $<1.0000$ & $<1.0000$ & $<5.0000$ & $<1.0000$ \\
\hline 443819123064000 & $08-26-92$ & $<1.0000$ & $<2.0000$ & $<1.0000$ & $<1.0000$ & $<1.0000$ & $<1.0000$ & $<1.0000$ & $<5.0000$ & $<1.0000$ \\
\hline 444032123050600 & $09-01-92$ & $<2.0000$ & $<4.0000$ & $<2.0000$ & $<2.0000$ & $<2.0000$ & $<2.0000$ & $<2.0000$ & $<10.000$ & $<2.0000$ \\
\hline 444416123030800 & $08-29-92$ & $<1.0000$ & $<2.0000$ & $<1.0000$ & $<1.0000$ & $<1.0000$ & $<1.0000$ & $<1.0000$ & -- & $<1.0000$ \\
\hline 445543123084400 & $08-28-92$ & $<1.0000$ & $<2.0000$ & $<1.0000$ & $<1.0000$ & $<1.0000$ & $<1.0000$ & $<1.0000$ & -- & $<1.0000$ \\
\hline 451320123041100 & $08-27-92$ & $<1.0000$ & $<2.0000$ & $<1.0000$ & $<1.0000$ & $<1.0000$ & $<1.0000$ & $<1.0000$ & $<5.0000$ & $<1.0000$ \\
\hline 451705122575100 & $09-01-92$ & $<1.0000$ & $<2.0000$ & $<1.0000$ & $<1.0000$ & $<1.0000$ & $<1.0000$ & $<1.0000$ & $<5.0000$ & $<1.0000$ \\
\hline 452950122492900 & $08-23-92$ & $<2.0000$ & $<4.0000$ & $<2.0000$ & $<2.0000$ & $<2.0000$ & $<2.0000$ & $<2.0000$ & $<10.000$ & $<2.0000$ \\
\hline 452221122362400 & $08-24-92$ & $<1.0000$ & $<2.0000$ & $<1.0000$ & $<1.0000$ & $<1.0000$ & $<1.0000$ & $<1.0000$ & $<5.0000$ & $<1.0000$ \\
\hline 452847122244500 & $08-21-92$ & $<2.0000$ & $<2.0000$ & $<1.0000$ & $<1.0000$ & $<1.0000$ & $<1.0000$ & $<1.0000$ & $<5.0000$ & $<1.0000$ \\
\hline 453547122463000 & $09-09-92$ & $<1.0000$ & $<2.0000$ & $<1.0000$ & $<1.0000$ & $<1.0000$ & $<1.0000$ & $<1.0000$ & $<5.0000$ & $<1.0000$ \\
\hline 453205122223701 & $08-22-92$ & $<1.0000$ & $<2.0000$ & 1.1000 & $<1.0000$ & $<1.0000$ & $<1.0000$ & $<1.0000$ & $<5.0000$ & $<1.0000$ \\
\hline
\end{tabular}


Table 23. Concentrations of organochlorine compounds associated with bed sediment from Phase I of the Willamette River Basin Water Quality Study, Oregon, 1992-Continued

\begin{tabular}{|c|c|c|c|c|c|c|c|c|c|c|}
\hline STATION NUMBER & DATE & $\begin{array}{l}\text { PCB, } \\
\text { SED, BM } \\
\text { WS, <2MM } \\
\text { DW, REC } \\
\text { (UG/KG) }\end{array}$ & $\begin{array}{l}\text { PENTA- } \\
\text { CHLORO- } \\
\text { ANISOLE } \\
\text { SED, BM } \\
\text { WS, }<2 \mathrm{MM} \\
\text { DW, REC } \\
(\mathrm{UG} / \mathrm{KG})\end{array}$ & $\begin{array}{c}\text { p, p'- } \\
\text { METHOXY } \\
\text { CHLOR, } \\
\text { SED, BM } \\
\text { WS, <2MM } \\
\text { DW, REC } \\
(\mathrm{UG} / \mathrm{KG})\end{array}$ & $\begin{array}{l}\text { TOXA- } \\
\text { PHENE } \\
\text { SED, BM } \\
\text { WS, }<2 M M \\
\text { DW, REC } \\
\text { (UG/KG) }\end{array}$ & $\begin{array}{l}\text { TRANS- } \\
\text { NONA- } \\
\text { CHLOR, } \\
\text { SED, BM } \\
\text { WS, }<2 M M \\
\text { DW, REC } \\
(\mathrm{UG} / \mathrm{KG})\end{array}$ & $\begin{array}{l}\text { TRANS- } \\
\text { PER- } \\
\text { METHRIN } \\
\text { SED, BM } \\
\text { WS, <2MM } \\
\text { DW, REC } \\
\text { (UG/KG) }\end{array}$ & $\begin{array}{l}\text { ALPHA- } \\
\text { HCH, D6 } \\
\text { SURROGT } \\
\text { SED, BM } \\
\text { WS, <2MM } \\
\text { DW, REC } \\
\text { (PERCENT) }\end{array}$ & $\begin{array}{l}\text { BIPHENL } \\
\text { 35DICHR } \\
\text { SURROGT } \\
\text { SED, BM } \\
\text { WS, <2MM } \\
\text { DW, REC } \\
\text { (PERCENT) }\end{array}$ & $\begin{array}{l}\text { OCTCHLR } \\
\text { BIPHENL } \\
\text { SURROGT } \\
\text { SED, BM } \\
\text { WS, <2MM } \\
\text { DW, REC } \\
\text { (PERCENT) }\end{array}$ \\
\hline 440313123091100 & $08-25-92$ & $<200.00$ & $<50.000$ & $<10.000$ & $<200.00$ & $<2.0000$ & $<10.000$ & 84.000 & 110.00 & 30.000 \\
\hline 14165500 & $08-25-92$ & $<250.00$ & $<50.000$ & $<12.000$ & $<250.00$ & $<2.5000$ & $<12.000$ & 61.000 & 34.000 & 43.000 \\
\hline 443207123145500 & $08-26-92$ & $<100.00$ & $<50.000$ & $<5.0000$ & $<200.00$ & $<1.0000$ & $<5.0000$ & 91.000 & 77.000 & 41.000 \\
\hline 443819123064000 & $08-26-92$ & $<100.00$ & $<50.000$ & $<5.0000$ & $<200.00$ & $<1.0000$ & -- & 81.000 & 50.000 & 53.000 \\
\hline 444032123050600 & $09-01-92$ & 1700.0 & $<50.000$ & $<10.000$ & $<200.00$ & $<2.0000$ & $<10.000$ & 48.000 & 110.00 & 98.000 \\
\hline 444416123030800 & $08-29-92$ & $<100.00$ & $<50.000$ & -- & $<200.00$ & $<1.0000$ & $<5.0000$ & 56.000 & 45.000 & 39.000 \\
\hline 445543123084400 & $08-28-92$ & $<100.00$ & $<50.000$ & -- & $<200.00$ & $<1.0000$ & $<5.0000$ & 74.000 & 54.000 & 55.000 \\
\hline 451320123041100 & $08-27-92$ & $<100.00$ & $<50.000$ & $<5.0000$ & $<200.00$ & $<1.0000$ & $<5.0000$ & 44.000 & 66.000 & 69.000 \\
\hline 451705122575100 & $09-01-92$ & $<100.00$ & $<50.000$ & $<5.0000$ & $<200.00$ & $<1.0000$ & $<5.0000$ & 47.000 & 69.000 & 65.000 \\
\hline 452950122492900 & $08-23-92$ & 110.00 & $<50.000$ & $<10.000$ & $<200.00$ & 7.0000 & $<10.000$ & 40.000 & 66.000 & 36.000 \\
\hline 452221122362400 & $08-24-92$ & 100.00 & $<50.000$ & $<5.0000$ & $<200.00$ & $<1.0000$ & $<5.0000$ & 43.000 & 39.000 & 42.000 \\
\hline 452847122244500 & $08-21-92$ & $<100.00$ & $<50.000$ & $<5.0000$ & 340.00 & 2.6000 & $<5.0000$ & 43.000 & 43.000 & 43.000 \\
\hline 453547122463000 & $09-09-92$ & $<100.00$ & $<50.000$ & $<5.0000$ & $<200.00$ & $<1.0000$ & $<5.0000$ & 91.000 & 110.00 & 94.000 \\
\hline 453205122223701 & $08-22-92$ & $<100.00$ & $<50.000$ & $<5.0000$ & $<200.00$ & 7.3000 & $<5.0000$ & 41.000 & 47.000 & 40.000 \\
\hline
\end{tabular}


Table 24. Concentrations of organic acids in whole water from Phase I of the Willamette River Basin Water Quality Study, Oregon, 1992

[USGS laboratory schedule 8331; UGd/L, micrograms per liter]

\begin{tabular}{|c|c|c|c|c|c|c|c|c|c|}
\hline STATION NUMBER & STATION NAME & DATE & TIME & $\begin{array}{l}2,4-D, \\
\text { TOTAL } \\
\text { (UG/L) }\end{array}$ & $\begin{array}{c}2, \quad 4-D P \\
\text { TOTAL } \\
\text { (UG/L) }\end{array}$ & $\begin{array}{l}\text { DICAMBA } \\
\text { TOTAL } \\
\text { (UG/L) }\end{array}$ & $\begin{array}{l}\text { PICLORAM } \\
\text { TOTAL } \\
\text { (UG/L) }\end{array}$ & $\begin{array}{l}\text { SILVEX, } \\
\text { TOTAL } \\
\text { (UG/L) }\end{array}$ & $\begin{array}{c}2,4,5-T \\
\text { TOTAL } \\
\text { (UG/L) }\end{array}$ \\
\hline 440313123091100 & $\begin{array}{l}\text { A-3 CHANNEL AT WALLIS AND } 5 \text { TH ST AT EUGENE, OR } \\
\text { A-3 CHANNEL AT WALLIS AND } 5 \text { TH ST AT EUGENE, OR }\end{array}$ & $\begin{array}{l}04-16-92 \\
10-16-92\end{array}$ & $\begin{array}{l}1410 \\
1345\end{array}$ & $\begin{array}{r}1.7000 \\
<0.01000\end{array}$ & $\begin{array}{l}<0.01000 \\
<0.01000\end{array}$ & $\begin{array}{r}0.02000 \\
<0.01000\end{array}$ & $\begin{array}{r}0.09000 \\
<0.01000\end{array}$ & $\begin{array}{l}<0.01000 \\
<0.01000\end{array}$ & $\begin{array}{l}<0.01000 \\
<0.01000\end{array}$ \\
\hline 453547122463000 & WILLAMETTE RIVER AT LINNTON, OR & $10-26-92$ & 1000 & $<0.01000$ & $<0.01000$ & $<0.01000$ & $<0.01000$ & $<0.01000$ & $<0.01000$ \\
\hline
\end{tabular}


Table 25. Concentrations of semi-volatile compounds in whole water from Phase I of the Willamette River Basin Water Quality Study, Oregon, 1993

[USGS laboratory schedule 1383; UG/L, micrograms per gram]

\begin{tabular}{|c|c|c|c|c|c|c|c|c|c|c|c|}
\hline STATION NUMBER & STATIC & ON NAME & & & & & DATE & TIME & $\begin{array}{c}\text { ACE- } \\
\text { NAPHTH- } \\
\text { ENE } \\
\text { TOTAL } \\
\text { (UG/L) }\end{array}$ & $\begin{array}{c}\text { ACE- } \\
\text { NAPHTH- } \\
\text { YLENE } \\
\text { TOTAL } \\
\text { (UG/L) }\end{array}$ & $\begin{array}{r}\text { ANTHRA- } \\
\text { CENE } \\
\text { TOTAL } \\
(\mathrm{UG} / \mathrm{L})\end{array}$ \\
\hline 443207123145500 & WILLAMEI & TTE RIVER & NEAR CORV & ALLIS, OR & & & $08-24-93$ & 1615 & $<5.0000$ & $<5.0000$ & $<5.0000$ \\
\hline 443138123120901 & MUDDY CF & REEK NEAR & PEORIA, O & & & & $08-24-93$ & 1300 & $<5.0000$ & $<5.0000$ & $<5.0000$ \\
\hline 451309123041501 & PALMER C & C AT DAYTOI & $\mathrm{N}, \mathrm{OR}$ & & & & $09-07-93$ & 1045 & $<5.0000$ & $<5.0000$ & $<5.0000$ \\
\hline 14201300 & ZOLLNER & CREEK NEAF & R MT ANGEI &,$\quad \mathrm{OR}$ & & & $07-27-93$ & 1015 & $<5.0000$ & $<5.0000$ & $<5.0000$ \\
\hline 14206950 & FANNO CR & REEK AT DUF & RHAM, OR & & & & $07-22-93$ & 1000 & $<5.0000$ & $<5.0000$ & $<5.0000$ \\
\hline STATION NUMBER & DATE & $\begin{array}{c}4- \\
\text { BROMO- } \\
\text { PHENYL } \\
\text { PHENYL } \\
\text { ETHER } \\
\text { TOTAL } \\
\text { (UG/L) } \\
\end{array}$ & $\begin{array}{c}\text { BENZI- } \\
\text { DINE } \\
\text { TOTAL } \\
\text { (UG/L) }\end{array}$ & $\begin{array}{l}\text { BENZO } \\
\text { (A) } \\
\text { ANTHR } \\
\text { ACENE } \\
\text { TOTAL } \\
\text { (UG/L) }\end{array}$ & $\begin{array}{l}\text { BENZO } \\
\text { (A) } \\
\text { PYRENE } \\
\text { TOTAL } \\
\text { (UG/L) }\end{array}$ & $\begin{array}{l}\text { BENZO } \\
\text { (B) } \\
\text { FLUOR- } \\
\text { ANTHENE } \\
\text { TOTAL } \\
\text { (UG/L) } \\
\end{array}$ & $\begin{array}{l}\text { BENZO } \\
\text { (GHI) } \\
\text { PERY- } \\
\text { LENE } \\
\text { TOTAL } \\
\text { (UG/L) }\end{array}$ & $\begin{array}{l}\text { BENZO } \\
(\mathrm{K}) \\
\text { FLUOR- } \\
\text { ANTHENE } \\
\text { TOTAL } \\
(\mathrm{UG} / \mathrm{L}) \\
\end{array}$ & $\begin{array}{l}\text { N-BUTYL } \\
\text { BENZYL } \\
\text { PHTHAL- } \\
\text { ATE } \\
\text { TOTAL } \\
\text { (UG/L) }\end{array}$ & $\begin{array}{c}\text { BIS } \\
(2- \\
\text { CHLORO- } \\
\text { ETHOXY) } \\
\text { METHANE } \\
\text { TOTAL } \\
\text { (UG/L) }\end{array}$ & $\begin{array}{c}\text { BIS } \\
(2- \\
\text { CHLORO- } \\
\text { ETHYL) } \\
\text { ETHER } \\
\text { TOTAL } \\
\text { (UG/L) } \\
\end{array}$ \\
\hline 443207123145500 & $08-24-93$ & $<5.0000$ & $<40.000$ & $<10.000$ & $<10.000$ & $<10.000$ & $<10.000$ & $<10.000$ & $<5.0000$ & $<5.0000$ & $<5.0000$ \\
\hline 443138123120901 & $08-24-93$ & $<5.0000$ & $<40.000$ & $<10.000$ & $<10.000$ & $<10.000$ & $<10.000$ & $<10.000$ & $<5.0000$ & $<5.0000$ & $<5.0000$ \\
\hline 451309123041501 & $09-07-93$ & $<5.0000$ & $<40.000$ & $<10.000$ & $<10.000$ & $<10.000$ & $<10.000$ & $<10.000$ & $<5.0000$ & $<5.0000$ & $<5.0000$ \\
\hline 14201300 & $07-27-93$ & $<5.0000$ & $<40.000$ & $<10.000$ & $<10.000$ & $<10.000$ & $<10.000$ & $<10.000$ & $<5.0000$ & $<5.0000$ & $<5.0000$ \\
\hline 14206950 & $07-22-93$ & $<5.0000$ & $<40.000$ & $<10.000$ & $<10.000$ & $<10.000$ & $<10.000$ & $<10.000$ & $<5.0000$ & $<5.0000$ & $<5.0000$ \\
\hline
\end{tabular}

\begin{tabular}{|c|c|c|c|c|c|c|c|c|c|c|}
\hline STATION NUMBER & DATE & $\begin{array}{c}\text { BIS (2- } \\
\text { CHLORO- } \\
\text { ISO- } \\
\text { PROPYL) } \\
\text { ETHER } \\
\text { TOTAL } \\
\text { (UG/L) }\end{array}$ & $\begin{array}{c}2- \\
\text { CHLORO- } \\
\text { NAPH- } \\
\text { THALENE } \\
\text { TOTAL } \\
\text { (UG/L) }\end{array}$ & $\begin{array}{c}2- \\
\text { CHLORO- } \\
\text { PHENOL } \\
\text { TOTAL } \\
\text { (UG/L) }\end{array}$ & $\begin{array}{c}4- \\
\text { CHLORO- } \\
\text { PHENYL } \\
\text { PHENYL } \\
\text { ETHER } \\
\text { TOTAL } \\
\text { (UG/L) }\end{array}$ & $\begin{array}{l}\quad 4- \\
\text { CHLORO } \\
\text { 3-METHYI } \\
\text { PHENOL } \\
\text { TOTAL } \\
\text { (UG/L) }\end{array}$ & $\begin{array}{ll} & \\
\text { L } & \text { CHRY- } \\
\text { SENE } \\
\text { TOTAL } \\
\text { (UG/L) }\end{array}$ & $\begin{array}{l}1,2-D I- \\
\text { PHENYL- } \\
\text { HYDRA- } \\
\text { ZINE } \\
\text { TOTAL } \\
\text { (UG/L) }\end{array}$ & $\begin{array}{l}\text { 1,2-DI- } \\
\text { CHLORO- } \\
\text { BENZENE } \\
\text { TOTAL } \\
\text { (UG/L) }\end{array}$ & $\begin{array}{l}1,3-D I- \\
\text { CHLORO- } \\
\text { BENZENE } \\
\text { TOTAL } \\
\text { (UG/L) }\end{array}$ \\
\hline 443207123145500 & $08-24-93$ & $<5.0000$ & $<5.0000$ & $<5.0000$ & $<5.0000$ & $<30.000$ & $<10.000$ & $<5.0000$ & $<5.0000$ & $<5.0000$ \\
\hline 443138123120901 & $08-24-93$ & $<5.0000$ & $<5.0000$ & $<5.0000$ & $<5.0000$ & $<30.000$ & $<10.000$ & $<5.0000$ & $<5.0000$ & $<5.0000$ \\
\hline 451309123041501 & $09-07-93$ & $<5.0000$ & $<5.0000$ & $<5.0000$ & $<5.0000$ & $<30.000$ & $<10.000$ & $<5.0000$ & $<5.0000$ & $<5.0000$ \\
\hline 14201300 & $07-27-93$ & $<5.0000$ & $<5.0000$ & $<5.0000$ & $<5.0000$ & $<30.000$ & $<10.000$ & $<5.0000<$ & $<0.20000$ & $<0.20000$ \\
\hline 14206950 & $07-22-93$ & $<5.0000$ & $<5.0000$ & $<5.0000$ & $<5.0000$ & $<30.000$ & $<10.000$ & $<5.0000$ & $<5.0000$ & $<5.0000$ \\
\hline STATION NUMBER & DATE & $\begin{array}{c}1,4-D I- \\
\text { CHLORO- } \\
\text { BENZENE } \\
\text { TOTAL } \\
\text { (UG/L) }\end{array}$ & $\begin{array}{c}2,4-D I- \\
\text { CHLORO- } \\
\text { PHENOL } \\
\text { TOTAL } \\
\text { (UG/L) }\end{array}$ & $\begin{array}{l}2,4-D I- \\
\text { METHYL- } \\
\text { PHENOL } \\
\text { TOTAL } \\
\text { (UG/L) }\end{array}$ & $\begin{array}{c}2,4,- \\
\text { DI- } \\
\text { NITRO- } \\
\text { PHENOL } \\
\text { TOTAL } \\
\text { (UG/L) }\end{array}$ & $\begin{array}{l}2,4-D I- \\
\text { NITRO- } \\
\text { TOLUENE } \\
\text { TOTAL } \\
\text { (UG/L) }\end{array}$ & $\begin{array}{l}2,6-D I- \\
\text { NITRO- } \\
\text { TOLUENE } \\
\text { TOTAL } \\
\text { (UG/L) }\end{array}$ & $\begin{array}{l}3,3^{\prime}- \\
\text { DI- } \\
\text { CHLORO- } \\
\text { BENZI- } \\
\text { DINE } \\
\text { TOTAL } \\
\text { (UG/L) }\end{array}$ & $\begin{array}{l}\text { DI-N- } \\
\text { BUTYL } \\
\text { PHTHA- } \\
\text { LATE } \\
\text { TOTAL } \\
\text { (UG/L) }\end{array}$ & $\begin{array}{l}\text { DI-N- } \\
\text { OCTYL } \\
\text { PHTHA- } \\
\text { LATE } \\
\text { TOTAL } \\
\text { (UG/L) }\end{array}$ \\
\hline 443207123145500 & $08-24-93$ & $<5.0000$ & $<5.0000$ & $<5.0000$ & $<20.000$ & $<5.0000$ & $<5.0000$ & $<20.000$ & 0.17000 & $<10.000$ \\
\hline 443138123120901 & $08-24-93$ & $<5.0000$ & $<5.0000$ & $<5.0000$ & $<20.000$ & $<5.0000$ & $<5.0000$ & $<20.000$ & 0.24900 & $<10.000$ \\
\hline 451309123041501 & $09-07-93$ & $<5.0000$ & $<5.0000$ & $<5.0000$ & $<20.000$ & $<5.0000$ & $<5.0000$ & $<20.000$ & 1.8000 & $<10.000$ \\
\hline 14201300 & $07-27-93$ & $<0.20000$ & $<5.0000$ & $<5.0000$ & $<20.000$ & $<5.0000$ & $<5.0000$ & $<20.000$ & $<5.0000$ & $<10.000$ \\
\hline 14206950 & $07-22-93$ & $<5.0000$ & $<5.0000$ & $<5.0000$ & $<20.000$ & $<5.0000$ & $<5.0000$ & $<20.000$ & $<5.0000$ & $<10.000$ \\
\hline
\end{tabular}


Table 25. Concentrations of semi-volatile compounds in whole water from Phase I of the Willamette River Basin Water Quality Study, Oregon, 1993-Continued

\begin{tabular}{|c|c|c|c|c|c|c|c|c|c|c|}
\hline STATION NUMBER & DATE & $\begin{array}{l}1,2,5,6 \\
\text { DIBENZO } \\
\text { (AH) AN- } \\
\text { THRACENE } \\
\text { TOTAL } \\
\text { (UG/L) }\end{array}$ & $\begin{array}{cl} & \text { DI- } \\
\text { ETHYL } & \\
\text { PHTHA- } \\
\text { E LATE } \\
\text { TOTAL } \\
\text { (UG/L) }\end{array}$ & $\begin{array}{l}\quad \text { DI- } \\
\text { METHYL } \\
\text { PHTHA- } \\
\text { LATE } \\
\text { TOTAL } \\
\text { (UG/L) }\end{array}$ & $\begin{array}{c}4,6- \\
\text { DINITRO } \\
\text { 2-METHYL } \\
\text { PHENOL } \\
\text { TOTAL } \\
\text { (UG/L) }\end{array}$ & $\begin{array}{l}\text { BIS (2- } \\
\text { ETHYL } \\
\text { HEXYL) } \\
\text { PHTHA- } \\
\text { LATE } \\
\text { TOTAL } \\
\text { (UG/L) }\end{array}$ & $\begin{array}{l}\text { FLUOR- } \\
\text { ANTHENE } \\
\text { TOTAL } \\
\text { (UG/L) }\end{array}$ & $\begin{array}{c}\text { FLUOR- } \\
\text { ENE } \\
\text { TOTAL } \\
\text { (UG/L) }\end{array}$ & $\begin{array}{c}\text { HEXA- } \\
\text { CHLORO- } \\
\text { BENZENE } \\
\text { TOTAL } \\
\text { (UG/L) }\end{array}$ & $\begin{array}{c}\text { HEXA- } \\
\text { CHLORO- } \\
\text { BUT- } \\
\text { ADIENE } \\
\text { TOTAL } \\
\text { (UG/L) }\end{array}$ \\
\hline 443207123145500 & $08-24-93$ & $<10.000$ & $<5.0000$ & $<5.0000$ & $<30.000$ & $<5.0000$ & $<5.0000$ & $<5.0000$ & $<5.0000$ & $<5.0000$ \\
\hline 443138123120901 & $08-24-93$ & $<10.000$ & $<5.0000$ & 1.5000 & $<30.000$ & $<5.0000$ & $<5.0000$ & $<5.0000$ & $<5.0000$ & $<5.0000$ \\
\hline 451309123041501 & $09-07-93$ & $<10.000$ & 0.80000 & $<5.0000$ & $<30.000$ & $<5.0000$ & $<5.0000$ & $<5.0000$ & $<5.0000$ & $<5.0000$ \\
\hline 14201300 & $07-27-93$ & $<10.000$ & $<5.0000$ & 1.4000 & $<30.000$ & $<5.0000$ & $<5.0000$ & $<5.0000$ & $<5.0000$ & $<0.20000$ \\
\hline 14206950 & $07-22-93$ & $<10.000$ & $<5.0000$ & $<5.0000$ & $<30.000$ & $<5.0000$ & $<5.0000$ & $<5.0000$ & $<5.0000$ & $<5.0000$ \\
\hline STATION NUMBER & DATE & $\begin{array}{c}\text { HEXA- } \\
\text { CHLORO- } \\
\text { CYCLO- } \\
\text { PENT- } \\
\text { ADIENE } \\
\text { TOTAL } \\
\text { (UG/L) }\end{array}$ & $\begin{array}{l}\text { HEXA- } \\
\text { CHLORO- } \\
\text { ETHANE } \\
\text { TOTAL } \\
\text { (UG/L) }\end{array}$ & $\begin{array}{c}\text { INDENO } \\
(1,2,3- \\
\text { CD }) \\
\text { PYRENE } \\
\text { TOTAL } \\
\text { (UG/L) }\end{array}$ & $\begin{array}{l}\text { ISO- } \\
\text { PHORONE } \\
\text { TOTAL } \\
\text { (UG/L) }\end{array}$ & $\begin{array}{c}\text { N- } \\
\text { NITRO- } \\
\text { SODI-N- } \\
\text { PROPYL- } \\
\text { AMINE } \\
\text { TOTAL } \\
\text { (UG/L) }\end{array}$ & $\begin{array}{c}\text { N-NITRO } \\
\text {-SODI- } \\
\text { PHENYL- } \\
\text { AMINE } \\
\text { TOTAL } \\
\text { (UG/L) }\end{array}$ & $\begin{array}{ll} & 2- \\
- & \text { NITRO- } \\
\text { PHENOL } \\
\text { TOTAL } \\
\text { (UG/L) }\end{array}$ & $\begin{array}{l}4- \\
\text { NITRO- } \\
\text { PHENOL } \\
\text { TOTAL } \\
\text { (UG/L) }\end{array}$ & $\begin{array}{l}\text { NAPHTH- } \\
\text { ALENE } \\
\text { TOTAL } \\
\text { (UG/L) }\end{array}$ \\
\hline 443207123145500 & $08-24-93$ & $<5.0000$ & $<5.0000$ & $<10.000$ & $<5.0000$ & $<5.0000$ & $<5.0000$ & $<5.0000$ & $<30.000$ & $<5.0000$ \\
\hline 443138123120901 & $08-24-93$ & $<5.0000$ & $<5.0000$ & $<10.000$ & $<5.0000$ & $<5.0000$ & $<5.0000$ & $<5.0000$ & $<30.000$ & $<5.0000$ \\
\hline 451309123041501 & $09-07-93$ & $<5.0000$ & $<5.0000$ & $<10.000$ & $<5.0000$ & $<5.0000$ & $<5.0000$ & $<5.0000$ & $<30.000$ & $<5.0000$ \\
\hline 14201300 & $07-27-93$ & $<5.0000$ & $<5.0000$ & $<10.000$ & $<5.0000$ & $<5.0000$ & $<5.0000$ & $<5.0000$ & $<30.000$ & $<0.20000$ \\
\hline 14206950 & $07-22-93$ & $<5.0000$ & $<5.0000$ & $<10.000$ & $<5.0000$ & $<5.0000$ & $<5.0000$ & $<5.0000$ & $<30.000$ & $<5.0000$ \\
\hline STATION NUMBER & DATE & $\begin{array}{l}\text { NITRO- } \\
\text { BENZENE } \\
\text { TOTAL } \\
\text { (UG/L) }\end{array}$ & $\begin{array}{l}\text { N-NITRO } \\
\text {-SODI- } \\
\text { METHYL- } \\
\text { AMINE } \\
\text { TOTAL } \\
\text { (UG/L) }\end{array}$ & $\begin{array}{l}\text { PENTA- } \\
\text { CHLORO- } \\
\text { PHENOL } \\
\text { TOTAL } \\
\text { (UG/L) }\end{array}$ & $\begin{array}{l}\text { PHENAN- } \\
\text { THRENE } \\
\text { TOTAL } \\
\text { (UG/L) }\end{array}$ & $\begin{array}{l}\text { PHENOL } \\
\text { TOTAL } \\
\text { (UG/L) }\end{array}$ & $\begin{array}{l}\text { PYRENE } \\
\text { TOTAL } \\
\text { (UG/L) }\end{array}$ & $\begin{array}{l}1,2,4- \\
\text { TRI- } \\
\text { CHLORO- } \\
\text { BENZENE } \\
\text { TOTAL } \\
\text { (UG/L) }\end{array}$ & $\begin{array}{l}2,4,6 \\
\text { TRI- } \\
\text { CHLORC } \\
\text { E PHENOI } \\
\text { TOTAL } \\
\text { (UG/L) }\end{array}$ & $\begin{array}{l}5- \\
20- \\
\mathrm{S}^{5}\end{array}$ \\
\hline 443207123145500 & $08-24-93$ & $<5.0000$ & $<5.0000$ & $<30.000$ & $<5.0000$ & $<5.0000$ & $<5.0000$ & $<5.0000$ & $<20.000$ & \\
\hline 443138123120901 & $08-24-93$ & $<5.0000$ & $<5.0000$ & $<30.000$ & $<5.0000$ & $<5.0000$ & $<5.0000$ & $<5.0000$ & $<20.000$ & \\
\hline 451309123041501 & $09-07-93$ & $<5.0000$ & $<5.0000$ & $<30.000$ & $<5.0000$ & $<5.0000$ & $<5.0000$ & $<5.0000$ & $<20.000$ & \\
\hline 14201300 & $07-27-93$ & $<5.0000$ & $<5.0000$ & $<30.000$ & $<5.0000$ & $<5.0000$ & $<5.0000$ & $<0.20000$ & $<20.000$ & \\
\hline 14206950 & $07-22-93$ & $<5.0000$ & $<5.0000$ & $<30.000$ & $<5.0000$ & $<5.0000$ & $<5.0000$ & $<5.0000$ & $<20.000$ & \\
\hline
\end{tabular}


Table 26. Concentrations of semi-volatile compounds in filtered water from Phase I of the Willamette River Basin Water Quality Study, Oregon, 1992-93

[USGS laboratory schedule 8005]

\begin{tabular}{|c|c|c|c|c|c|}
\hline STATION & STATION & DATE & TIME & $\begin{array}{c}\text { ACE- } \\
\text { NAPHTH- } \\
\text { ENE } \\
\text { DISSOLV } \\
\text { (UG/L) }\end{array}$ & $\begin{array}{c}\text { ACE- } \\
\text { NAPHTH- } \\
\text { YLENE } \\
\text { DISSOLV } \\
\text { (UG/L) }\end{array}$ \\
\hline 440313123091100 & $\begin{array}{llllll}\text { A-3 } & \text { CHANNEL AT WALLIS AND } & 5 \mathrm{TH} & \mathrm{ST} & \mathrm{AT} & \text { EUGENE, OR } \\
\text { A-3 } & \text { CHANNEL AT WALLIS AND } & 5 \mathrm{TH} & \mathrm{ST} & \mathrm{AT} & \text { EUGENE, OR }\end{array}$ & $\begin{array}{l}04-16-92 \\
10-16-92\end{array}$ & $\begin{array}{l}1415 \\
1205\end{array}$ & $\begin{array}{l}<5.0000 \\
<5.0000\end{array}$ & $\begin{array}{l}<5.0000 \\
<5.0000\end{array}$ \\
\hline 14202000 & PUDDING RIVER AT AURORA, OREG. & $04-27-93$ & 1450 & $<5.0000$ & $<5.0000$ \\
\hline 453115122535500 & BEAVERTON C AT 216TH AVE NEAR ORENCO, OR & $03-31-93$ & 1815 & $<5.0000$ & $<5.0000$ \\
\hline 14206950 & FANNO CREEK AT DURHAM, OR & $03-01-93$ & 1835 & $<5.0000$ & $<5.0000$ \\
\hline 14211550 & JOHNSON CREEK AT MILWAUKIE, OREG. & $03-14-93$ & 1750 & $<5.0000$ & $<5.0000$ \\
\hline 453547122463000 & WILLAMETTE RIVER AT LINNTON, OR & $10-26-92$ & 1005 & $<5.0000$ & $<5.0000$ \\
\hline
\end{tabular}

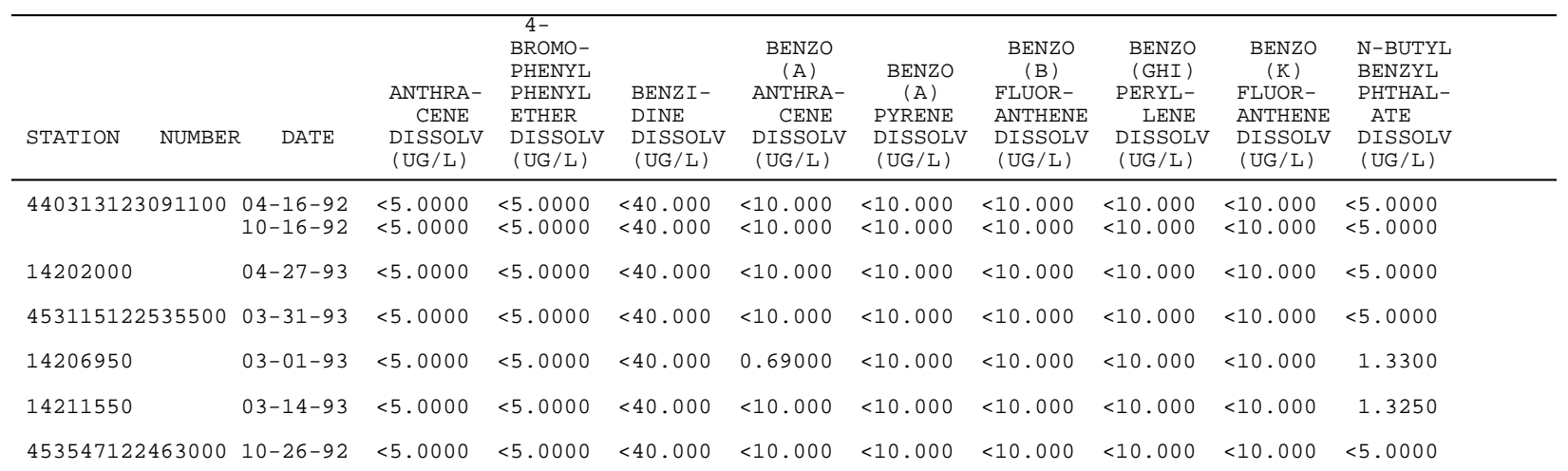

\begin{tabular}{|c|c|c|c|c|c|c|c|c|c|c|}
\hline STATION & DATE & $\begin{array}{l}\begin{array}{c}\text { BIS } \\
(2-\end{array} \\
\text { CHLORO- } \\
\text { ETHOXY) } \\
\text { METHANE } \\
\text { DISSOLV } \\
(\text { UG/L) }\end{array}$ & $\begin{array}{c}\text { BIS } \\
\text { (2- } \\
\text { CHLORO- } \\
\text { ETHYL) } \\
\text { ETHER } \\
\text { DISSOLV } \\
(\text { UG/L) }\end{array}$ & $\begin{array}{c}\text { BIS }(2- \\
\text { CHLORO- } \\
\text { ISO- } \\
\text { PROPYL) } \\
\text { ETHER } \\
\text { DISSOLV } \\
\text { (UG/L) }\end{array}$ & $\begin{array}{c}2- \\
\text { CHLORO- } \\
\text { NAPH- } \\
\text { THALENE } \\
\text { DISSOLV } \\
\text { (UG/L) }\end{array}$ & \begin{tabular}{l}
\multicolumn{1}{c}{$2-$} \\
CHLORO- \\
PHENOL \\
DISSOLV \\
(UG/L)
\end{tabular} & \begin{tabular}{l}
\multicolumn{1}{c}{$4-$} \\
CHLORO- \\
PHENYL \\
PHENYL \\
ETHER \\
DISSOLV \\
(UG/L)
\end{tabular} & $\begin{array}{l}4- \\
\text { CHLORO- } \\
3- \\
\text { METHYL- } \\
\text { PHENOL } \\
\text { DISSOLV } \\
\text { (UG/L) }\end{array}$ & $\begin{array}{l}\text { CHRY- } \\
\text { SENE } \\
\text { DISSOLV } \\
(\text { UG/L) }\end{array}$ & $\begin{array}{c}1,2- \\
\text { DI- } \\
\text { PHENYL- } \\
\text { HYDRA- } \\
\text { ZINE } \\
\text { DISSOLV } \\
(\text { UG/L) }\end{array}$ \\
\hline 440313123091100 & $\begin{array}{l}04-16-92 \\
10-16-92\end{array}$ & $\begin{array}{l}<5.0000 \\
<5.0000\end{array}$ & $\begin{array}{l}<5.0000 \\
<5.0000\end{array}$ & $\begin{array}{l}<5.0000 \\
<5.0000\end{array}$ & $\begin{array}{l}<5.0000 \\
<5.0000\end{array}$ & $\begin{array}{l}<5.0000 \\
<5.0000\end{array}$ & $\begin{array}{l}<5.0000 \\
<5.0000\end{array}$ & $\begin{array}{l}<30.000 \\
<30.000\end{array}$ & $\begin{array}{l}<10.000 \\
<10.000\end{array}$ & $\begin{array}{l}<5.0000 \\
<5.0000\end{array}$ \\
\hline 14202000 & $04-27-93$ & $<5.0000$ & $<5.0000$ & $<5.0000$ & $<5.0000$ & $<5.0000$ & $<5.0000$ & $<30.000$ & $<10.000$ & $<5.0000$ \\
\hline 453115122535500 & $03-31-93$ & $<5.0000$ & $<5.0000$ & $<5.0000$ & $<5.0000$ & $<5.0000$ & $<5.0000$ & $<30.000$ & $<10.000$ & $<5.0000$ \\
\hline 14206950 & $03-01-93$ & $<5.0000$ & $<5.0000$ & $<5.0000$ & $<5.0000$ & $<5.0000$ & $<5.0000$ & $<30.000$ & $<10.000$ & $<5.0000$ \\
\hline 14211550 & $03-14-93$ & $<5.0000$ & $<5.0000$ & $<5.0000$ & $<5.0000$ & $<5.0000$ & $<5.0000$ & $<30.000$ & $<10.000$ & $<5.0000$ \\
\hline 453547122463000 & $10-26-92$ & $<5.0000$ & $<5.0000$ & $<5.0000$ & $<5.0000$ & $<5.0000$ & $<5.0000$ & $<30.000$ & $<10.000$ & $<5.0000$ \\
\hline
\end{tabular}

\begin{tabular}{|c|c|c|c|c|c|c|c|c|c|c|}
\hline STATION & DATE & $\begin{array}{l}1,2-D I- \\
\text { CHLORO- } \\
\text { BENZENE } \\
\text { DISSOLV } \\
\text { (UG/L) }\end{array}$ & $\begin{array}{l}1,3-D I- \\
\text { CHLORO- } \\
\text { BENZENE } \\
\text { DISSOLV } \\
(\mathrm{UG} / \mathrm{L})\end{array}$ & $\begin{array}{l}\text { 1, 4-DI- } \\
\text { CHLORO- } \\
\text { BENZENE } \\
\text { DISSOLV } \\
\text { (UG/L) }\end{array}$ & $\begin{array}{l}2,4-D I- \\
\text { CHLORO- } \\
\text { PHENOL } \\
\text { DISSOLV } \\
\text { (UG/L) }\end{array}$ & $\begin{array}{l}2,4-D I- \\
\text { NITRO } \\
\text { PHENOL } \\
\text { DISSOLV } \\
\text { (UG/L) }\end{array}$ & $\begin{array}{l}2,4-D I- \\
\text { NITRO- } \\
\text { TOLUENE } \\
\text { DISSOLV } \\
\text { (UG/L) }\end{array}$ & $\begin{array}{l}2,6-D I- \\
\text { NITRO- } \\
\text { TOLUENE } \\
\text { DISSOLV } \\
\text { (UG/L) }\end{array}$ & $\begin{array}{c}3,3^{\prime}- \\
\text { DI- } \\
\text { CHLORO- } \\
\text { BENZI- } \\
\text { DINE } \\
\text { DISSOLV } \\
\text { (UG/L) }\end{array}$ & $\begin{array}{l}\text { DI-N- } \\
\text { BUTYL } \\
\text { PHTHAL- } \\
\quad \text { ATE } \\
\text { DISSOLV } \\
\text { (UG/L) }\end{array}$ \\
\hline 440313123091100 & $\begin{array}{l}04-16-92 \\
10-16-92\end{array}$ & $\begin{array}{l}<5.0000 \\
<5.0000\end{array}$ & $\begin{array}{l}<5.0000 \\
<5.0000\end{array}$ & $\begin{array}{l}<5.0000 \\
<5.0000\end{array}$ & $\begin{array}{l}<5.0000 \\
<5.0000\end{array}$ & $\begin{array}{l}<20.000 \\
<20.000\end{array}$ & $\begin{array}{l}<5.0000 \\
<5.0000\end{array}$ & $\begin{array}{l}<5.0000 \\
<5.0000\end{array}$ & $\begin{array}{l}<20.000 \\
<20.000\end{array}$ & $\begin{array}{l}<5.0000 \\
<5.0000\end{array}$ \\
\hline 14202000 & $04-27-93$ & $<5.0000$ & $<5.0000$ & $<5.0000$ & $<5.0000$ & $<20.000$ & $<5.0000$ & $<5.0000$ & $<20.000$ & $<5.0000$ \\
\hline 453115122535500 & $03-31-93$ & $<5.0000$ & $<5.0000$ & $<5.0000$ & $<5.0000$ & $<20.000$ & $<5.0000$ & $<5.0000$ & $<20.000$ & $<5.0000$ \\
\hline 14206950 & $03-01-93$ & $<5.0000$ & $<5.0000$ & $<5.0000$ & $<5.0000$ & $<20.000$ & $<5.0000$ & $<5.0000$ & $<20.000$ & 1.5000 \\
\hline 14211550 & $03-14-93$ & $<5.0000$ & $<5.0000$ & $<5.0000$ & $<5.0000$ & $<20.000$ & $<5.0000$ & $<5.0000$ & $<20.000$ & 1.3100 \\
\hline 453547122463000 & $10-26-92$ & $<5.0000$ & $<5.0000$ & $<5.0000$ & $<5.0000$ & $<20.000$ & $<5.0000$ & $<5.0000$ & $<20.000$ & $<5.0000$ \\
\hline
\end{tabular}


Table 26. Concentrations of semi-volatile compounds in filtered water from Phase I of the Willamette River Basin Water Quality Study, Oregon, 1992-93-Continued

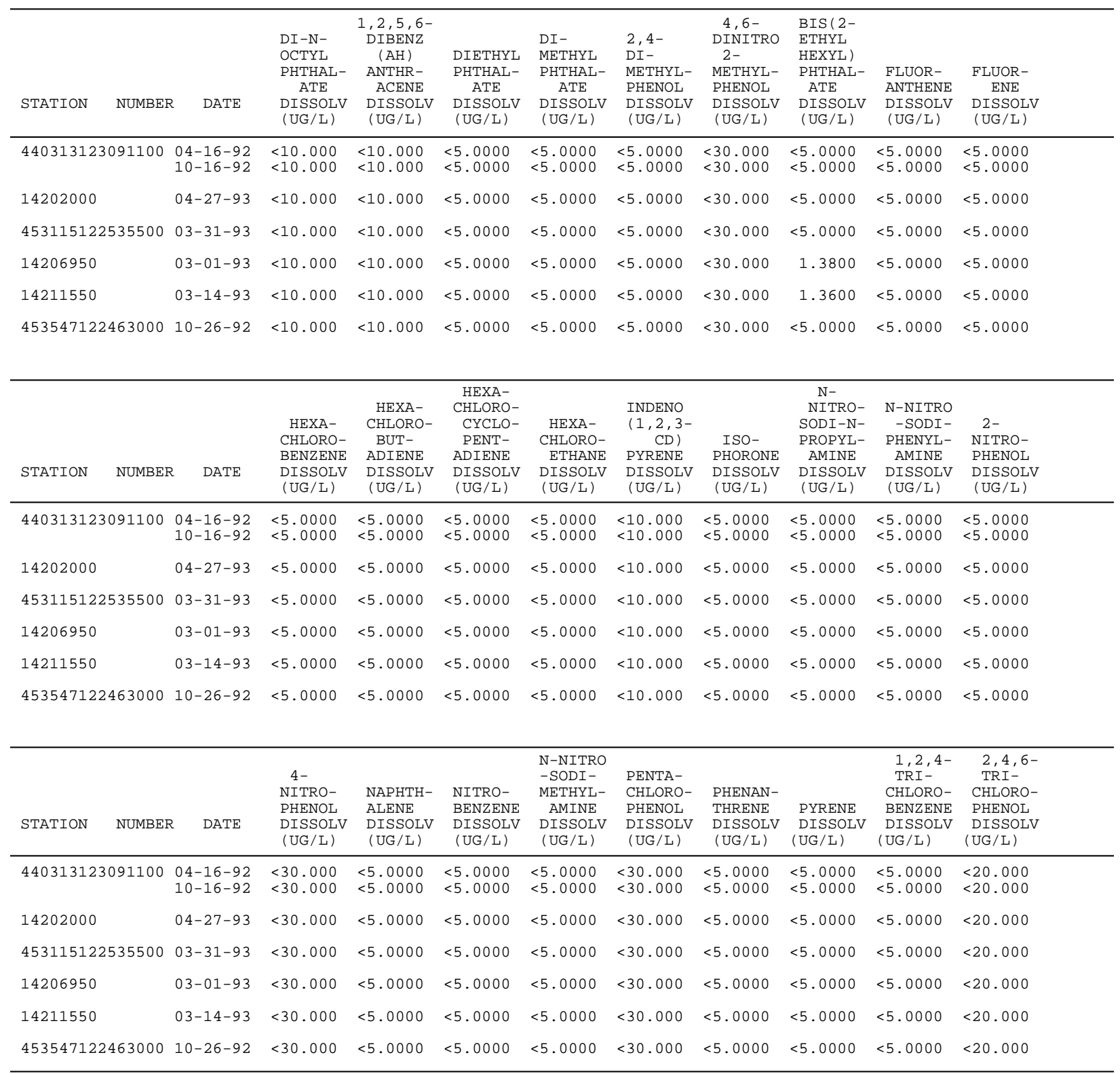


Table 27. Concentrations of semi-volatile compounds associated with suspended sediment from Phase I of the Willamette River Basin Water Quality Study, Oregon, 1992-93

[USGS laboratory schedule 8006; UG/L, micrograms per liter]

\begin{tabular}{|c|c|c|c|c|c|c|c|c|c|c|}
\hline STATION NUMBER & STATION & N NAME & & & & & DATE & TIME & $\begin{array}{l}\text { ACE- } \\
\text { NAPHTH- } \\
\text { ENE } \\
\text { SUSP. } \\
\text { (UG/L) }\end{array}$ & $\begin{array}{l}\text { ACE- } \\
\text { NAPHTH- } \\
\text { YLENE } \\
\text { SUSP } \\
\text { (UG/L) }\end{array}$ \\
\hline 440313123091100 & $\begin{array}{ll}\text { A-3 } & \text { CHAN } \\
\text { A-3 } & \text { CHAN }\end{array}$ & $\begin{array}{lll}\text { NNEL } & \text { AT } & \text { WAI } \\
\text { NNEL } & \text { AT } & \text { WAI }\end{array}$ & $\begin{array}{l}\text { LLIS AND } 5 \\
\text { LLIS AND } 5\end{array}$ & $\begin{array}{lll}5 \mathrm{TH} & \mathrm{ST} & \mathrm{AT} \\
5 \mathrm{TH} & \mathrm{ST} & \mathrm{AT}\end{array}$ & $\begin{array}{l}\text { EUGENE, } \\
\text { EUGENE, }\end{array}$ & $\begin{array}{l}\text { OR } \\
\text { OR }\end{array}$ & $\begin{array}{l}04-16-92 \\
10-16-92\end{array}$ & $\begin{array}{l}1415 \\
1350\end{array}$ & $\begin{array}{l}<5.0000 \\
<5.0000\end{array}$ & $\begin{array}{r}0.006000 \\
<5.0000\end{array}$ \\
\hline 14201300 & ZOLLNER & CREEK NEAI & R MT ANGEI & $L, \quad O R$ & & & $06-01-93$ & 1120 & $<5.0000$ & 0.47000 \\
\hline 14202000 & PUDDING & RIVER AT & AURORA, OP & REG. & & & $04-27-93$ & 1450 & $<5.0000$ & $<5.0000$ \\
\hline 453115122535500 & BEAVERTO & $\mathrm{ON}$ C AT 21 & $6 \mathrm{TH}$ AVE NE & EAR ORENCO & $O, O R$ & & $03-31-93$ & 1815 & $<5.0000$ & $<5.0000$ \\
\hline 14206950 & FANNO CR & REEK AT DUH & RHAM， OR & & & & $03-01-93$ & 1835 & $<5.0000$ & 0.97000 \\
\hline 14211550 & JOHNSON & CREEK AT & MILWAUKIE, & OREG & & & $03-14-93$ & 1750 & $<5.0000$ & 1.0340 \\
\hline 453547122463000 & WILLAMET & TTE RIVER & AT LINNTON & $N, O R$ & & & $10-26-92$ & 1005 & $<5.0000$ & $<5.0000$ \\
\hline STATION NUMBER & DATE & $\begin{array}{c}\text { ANTHRA- } \\
\text { CENE } \\
\text { SUSP . } \\
\text { (UG/L) }\end{array}$ & $\begin{array}{c}4- \\
\text { BROMO- } \\
\text { PHENYI } \\
\text { PHENYL } \\
\text { ETHER } \\
\text { SUSP } \\
\text { (UG/L) }\end{array}$ & $\begin{array}{l} \\
\\
\text { BENZI- } \\
\text { DINE } \\
\text { SUSP. } \\
\text { (UG/L) }\end{array}$ & $\begin{array}{l}\text { BENZO (A) } \\
\text { ANTHR } \\
\text { ACENE } \\
\text { SUSP } \\
\text { (UG/L) }\end{array}$ & $\begin{array}{l}\text { BENZO } \\
\text { (A) } \\
\text { PYRENE } \\
\text { SUSP. } \\
(\text { UG/L) }\end{array}$ & $\begin{array}{c}\text { BENZO (B) } \\
\text { FLUOR- } \\
\text { AN- } \\
\text { THENE } \\
\text { SUSP } \\
(\text { UG/L) }\end{array}$ & $\begin{array}{l}\text { BENZO } \\
\text { (GHI) } \\
\text { PERYLENE } \\
\text { SUSP. } \\
\text { (UG/L) }\end{array}$ & $\begin{array}{l}\text { BENZO (K) } \\
\text { FLUOR- } \\
\text { AN- } \\
\text { THENE } \\
\text { SUSP } \\
\text { (UG/L) }\end{array}$ & $\begin{array}{l}\text { N-BUTYL } \\
\text { BENZYL } \\
\text { PHTHAL- } \\
\text { ATE } \\
\text { SUSP. } \\
\text { (UG/L) }\end{array}$ \\
\hline 440313123091100 & $\begin{array}{l}04-16-92 \\
10-16-92\end{array}$ & $\begin{array}{l}0.006000 \\
0.003000\end{array}$ & $\begin{array}{l}<5.0000 \\
<5.0000\end{array}$ & $\begin{array}{l}<40.000 \\
1.94000\end{array}$ & $\begin{array}{l}0.01600 \\
0.008000\end{array}$ & $\begin{array}{l}<10.000 \\
<10.000\end{array}$ & $\begin{array}{l}<10.000 \\
<10.000\end{array}$ & $\begin{array}{l}<10.000 \\
<10.000\end{array}$ & $\begin{array}{l}<10.000 \\
<10.000\end{array}$ & $\begin{array}{l}<5.0000 \\
<5.0000\end{array}$ \\
\hline 14201300 & $06-01-93$ & 0.46000 & $<5.0000$ & $<40.000$ & $<10.000$ & 0.84000 & 0.95000 & $<10.000$ & 0.25000 & $<5.0000$ \\
\hline 14202000 & $04-27-93$ & 0.53000 & $<5.0000$ & 0.99000 & 0.69000 & $<10.000$ & $<10.000$ & $<10.000$ & $<10.000$ & 1.3600 \\
\hline 453115122535500 & $03-31-93$ & 0.54500 & $<5.0000$ & 8.2200 & 0.72200 & 1.1000 & 1.4200 & $<10.000$ & 1.6500 & $<5.0000$ \\
\hline 14206950 & $03-01-93$ & 0.54000 & $<5.0000$ & $<40.000$ & 0.75000 & 1.0300 & 1.4000 & $<10.000$ & 1.6000 & $<5.0000$ \\
\hline 14211550 & $03-14-93$ & 0.63300 & $<5.0000$ & $<40.000$ & 0.96000 & 1.2000 & 1.7110 & $<10.000$ & 1.8350 & $<5.0000$ \\
\hline 453547122463000 & $10-26-92$ & $<5.0000$ & $<5.0000$ & $<40.000$ & $<10.000$ & $<10.000$ & $<10.000$ & $<10.000$ & $<10.000$ & 3.3110 \\
\hline STATION NUMBER & DATE & $\begin{array}{c}\begin{array}{c}\text { BIS } \\
(2-\end{array} \\
\text { CHLORO- } \\
\text { ETHOXY) } \\
\text { METHANE } \\
\text { SUSP } \\
\text { (UG/L) }\end{array}$ & $\begin{array}{c}\text { BIS } \\
(2- \\
\text { CHLORO- } \\
\text { ETHYL) } \\
\text { ETHER } \\
\text { SUSP } \\
\text { (UG/L) }\end{array}$ & $\begin{array}{c}\text { BIS (2- } \\
\text { CHLORO- } \\
\text { ISO- } \\
\text { PROPYL) } \\
\text { ETHER } \\
\text { SUSP. } \\
\text { (UG/L) }\end{array}$ & $\begin{array}{l}2- \\
\text { CHLORO- } \\
\text { NAPH- } \\
\text { THALENE } \\
\text { SUSP. } \\
\text { (UG/L) }\end{array}$ & $\begin{array}{l}2- \\
\text { CHLORO- } \\
\text { PHENOL } \\
\text { SUSP. } \\
\text { (UG/L) }\end{array}$ & $\begin{array}{c}4- \\
\text { CHLORO- } \\
\text { PHENYL } \\
\text { PHENYL } \\
\text { ETHER } \\
\text { SUSP. } \\
\text { (UG/L) }\end{array}$ & $\begin{array}{l}4- \\
\text { CHLORO- } \\
\text { 3-METHYL } \\
\text { PHENOL } \\
\text { SUSP } \\
\text { (UG/L) } \\
\end{array}$ & $\begin{array}{l}\text { CHRY- } \\
\text { SENE } \\
\text { SUSP. } \\
\text { (UG/L) }\end{array}$ & $\begin{array}{c}1,2-D I- \\
\text { PHENYL- } \\
\text { HYDRA- } \\
\text { ZINE } \\
\text { SUSP. } \\
\text { (UG/L) }\end{array}$ \\
\hline 440313123091100 & $\begin{array}{l}04-16-92 \\
10-16-92\end{array}$ & $\begin{array}{l}<5.0000 \\
<5.0000\end{array}$ & $\begin{array}{l}<5.0000 \\
<5.0000\end{array}$ & $\begin{array}{l}<5.0000 \\
<5.0000\end{array}$ & $\begin{array}{l}<5.0000 \\
<5.0000\end{array}$ & $\begin{array}{l}<5.0000 \\
<5.0000\end{array}$ & $\begin{array}{l}<5.0000 \\
<5.0000\end{array}$ & $\begin{array}{l}<30.000 \\
<30.000\end{array}$ & $\begin{array}{l}0.03400 \\
0.01300\end{array}$ & $\begin{array}{l}<5.0000 \\
<5.0000\end{array}$ \\
\hline 14201300 & $06-01-93$ & $<5.0000$ & $<5.0000$ & $<5.0000$ & $<5.0000$ & $<5.0000$ & $<5.0000$ & $<30.000$ & 0.41000 & $<5.0000$ \\
\hline 14202000 & $04-27-93$ & $<5.0000$ & $<5.0000$ & $<5.0000$ & $<5.0000$ & $<5.0000$ & $<5.0000$ & $<30.000$ & 0.60000 & $<5.0000$ \\
\hline 453115122535500 & $03-31-93$ & $<5.0000$ & $<5.0000$ & $<5.0000$ & $<5.0000$ & $<5.0000$ & $<5.0000$ & $<30.000$ & 0.80000 & $<5.0000$ \\
\hline 14206950 & $03-01-93$ & $<5.0000$ & $<5.0000$ & $<5.0000$ & $<5.0000$ & $<5.0000$ & $<5.0000$ & $<30.000$ & 0.84000 & $<5.0000$ \\
\hline 14211550 & $03-14-93$ & $<5.0000$ & $<5.0000$ & $<5.0000$ & $<5.0000$ & $<5.0000$ & $<5.0000$ & $<30.000$ & 1.2900 & $<5.0000$ \\
\hline 453547122463000 & $10-26-92$ & $<5.0000$ & $<5.0000$ & $<5.0000$ & 0.45100 & $<5.0000$ & $<5.0000$ & $<30.000$ & $<10.000$ & $<5.0000$ \\
\hline
\end{tabular}


Table 27. Concentrations of semi-volatile compounds associated with suspended sediment from Phase I of the Willamette River Basin Water Quality Study, Oregon, 1992-93-Continued

\begin{tabular}{|c|c|c|c|c|c|c|c|c|c|c|}
\hline STATION NUMBER & DATE & $\begin{array}{c}1,2-D I- \\
\text { CHLORO- } \\
\text { BENZENE } \\
\text { SUSP } \\
\text { (UG/L) }\end{array}$ & $\begin{array}{c}1,3-D I- \\
\text { CHLORO- } \\
\text { BENZENE } \\
\text { SUSP } \\
\text { (UG/L) }\end{array}$ & $\begin{array}{c}\text { 1, 4-DI- } \\
\text { CHLORO- } \\
\text { BENZENE } \\
\text { SUSP } \\
\text { (UG/L) }\end{array}$ & $\begin{array}{c}2,4-D I- \\
\text { CHLORO- } \\
\text { PHENOL } \\
\text { SUSP } \\
\text { (UG/L) }\end{array}$ & $\begin{array}{c}2,4- \\
\text { DI- } \\
\text { NITRO } \\
\text { PHENOL } \\
\text { SUSP . } \\
\text { (UG/L) }\end{array}$ & $\begin{array}{l}2,4-D I- \\
\text { NITRO- } \\
\text { TOLUENE } \\
\text { SUSP. } \\
\text { (UG/L) }\end{array}$ & $\begin{array}{l}2,6-D I- \\
\text { NITRO- } \\
\text { TOLUENE } \\
\text { SUSP. } \\
\text { (UG/L) }\end{array}$ & $\begin{array}{c}3,3^{\prime}- \\
\text { DI- } \\
\text { CHLORO- } \\
\text { BENZI- } \\
\text { DINE } \\
\text { SUSP. } \\
\text { (UG/L) }\end{array}$ & $\begin{array}{c}\text { DI-N- } \\
\text { BUTYL } \\
\text { PHTHAL- } \\
\text { ATE } \\
\text { SUSP } \\
\text { (UG/L) }\end{array}$ \\
\hline 440313123091100 & $\begin{array}{l}04-16-92 \\
10-16-92\end{array}$ & $\begin{array}{l}<5.0000 \\
<5.0000\end{array}$ & $\begin{array}{l}<5.0000 \\
<5.0000\end{array}$ & $\begin{array}{l}<5.0000 \\
<5.0000\end{array}$ & $\begin{array}{l}<5.0000 \\
<5.0000\end{array}$ & $\begin{array}{l}<20.000 \\
<20.000\end{array}$ & $\begin{array}{l}<5.0000 \\
<5.0000\end{array}$ & $\begin{array}{l}<5.0000 \\
<5.0000\end{array}$ & $\begin{array}{l}<20.000 \\
<20.000\end{array}$ & $\begin{array}{l}<5.0000 \\
0.09100\end{array}$ \\
\hline 14201300 & $06-01-93$ & $<5.0000$ & $<5.0000$ & $<5.0000$ & $<5.0000$ & $<20.000$ & $<5.0000$ & $<5.0000$ & $<20.000$ & $<5.0000$ \\
\hline 14202000 & $04-27-93$ & $<5.0000$ & $<5.0000$ & $<5.0000$ & $<5.0000$ & $<20.000$ & $<5.0000$ & $<5.0000$ & $<20.000$ & 1.8000 \\
\hline 453115122535500 & $03-31-93$ & $<5.0000$ & $<5.0000$ & $<5.0000$ & $<5.0000$ & $<20.000$ & $<5.0000$ & $<5.0000$ & $<20.000$ & $<5.0000$ \\
\hline 14206950 & $03-01-93$ & $<5.0000$ & $<5.0000$ & $<5.0000$ & $<5.0000$ & $<20.000$ & $<5.0000$ & $<5.0000$ & $<20.000$ & 2.4800 \\
\hline 14211550 & $03-14-93$ & $<5.0000$ & $<5.0000$ & $<5.0000$ & $<5.0000$ & $<20.000$ & $<5.0000$ & $<5.0000$ & $<20.000$ & $<5.0000$ \\
\hline 453547122463000 & $10-26-92$ & $<5.0000$ & $<5.0000$ & $<5.0000$ & $<5.0000$ & $<20.000$ & $<5.0000$ & $<5.0000$ & $<20.000$ & 39.980 \\
\hline STATION NUMBER & DATE & $\begin{array}{l}\text { DI-N- } \\
\text { OCTYL } \\
\text { PHT } \\
\text { HALATE } \\
\text { SUSP. } \\
\text { (UG/L) }\end{array}$ & $\begin{array}{l}1,2,5,6 \\
\text { DIBENZO } \\
\text { (AH) AN } \\
\text { THRACENE } \\
\text { SUSP. } \\
\text { (UG/L) }\end{array}$ & $\begin{array}{l}\text { DIETHYL } \\
\text { PHTHAL- } \\
\text { ATE } \\
\text { SUSP. } \\
\text { (UG/L) }\end{array}$ & $\begin{array}{l}\quad \text { DI- } \\
\text { METHYL } \\
\text { PHTHAL- } \\
\text { ATE } \\
\text { SUSP. } \\
\text { (UG/L) }\end{array}$ & $\begin{array}{c}\quad \text { 4, 6- } \\
\text { DINITRO } \\
\text { 2-METHYL } \\
\text { PHENOL } \\
\text { SUSP. } \\
\text { (UG/L) }\end{array}$ & $\begin{array}{l}\text { BIS (2- } \\
\text { ETHYL } \\
\text { HEXYL) } \\
\text { PHTHAL- } \\
\text { ATE } \\
\text { SUSP. } \\
\text { (UG/L) }\end{array}$ & $\begin{array}{c}\text { FLUOR- } \\
\text { ANTHENE } \\
\text { SUSP } \\
(\text { UG/L) }\end{array}$ & $\begin{array}{l}\text { FLUOR- } \\
\text { ENE } \\
\text { SUSP . } \\
\text { (UG/L) }\end{array}$ & $\begin{array}{l}\text { HEXA- } \\
\text { CHLORO- } \\
\text { BENZENE } \\
\text { SUSP } \\
\text { (UG/L) }\end{array}$ \\
\hline 440313123091100 & $\begin{array}{l}04-16-92 \\
10-16-92\end{array}$ & $\begin{array}{l}<10.000 \\
<10.000\end{array}$ & $\begin{array}{l}<10.000 \\
<10.000\end{array}$ & $\begin{array}{l}<5.0000 \\
<5.0000\end{array}$ & $\begin{array}{l}<5.0000 \\
<5.0000\end{array}$ & $\begin{array}{l}<30.000 \\
<30.000\end{array}$ & $\begin{array}{l}<5.0000 \\
0.69000\end{array}$ & $\begin{array}{r}0.01500 \\
0.004000\end{array}$ & $\begin{array}{l}0.004000 \\
0.003000\end{array}$ & $\begin{array}{l}<5.0000 \\
<5.0000\end{array}$ \\
\hline 14201300 & $06-01-93$ & $<10.000$ & $<10.000$ & $<5.0000$ & $<5.0000$ & $<30.000$ & 2.9000 & 1.7800 & $<5.0000$ & $<5.0000$ \\
\hline 14202000 & $04-27-93$ & 1.7700 & $<10.000$ & $<5.0000$ & $<5.0000$ & $<30.000$ & 4.3700 & 0.60000 & $<5.0000$ & $<5.0000$ \\
\hline 453115122535500 & $03-31-93$ & $<10.000$ & $<10.000$ & $<5.0000$ & $<5.0000$ & $<30.000$ & $<5.0000$ & 0.82000 & $<5.0000$ & $<5.0000$ \\
\hline 14206950 & $03-01-93$ & 1.8000 & $<10.000$ & $<5.0000$ & $<5.0000$ & $<30.000$ & 4.6000 & 0.86000 & $<5.0000$ & $<5.0000$ \\
\hline 14211550 & $03-14-93$ & 2.1020 & $<10.000$ & $<5.0000$ & $<5.0000$ & $<30.000$ & 11.180 & 1.4500 & $<5.0000$ & $<5.0000$ \\
\hline 453547122463000 & $10-26-92$ & 2.2600 & $<10.000$ & 0.66000 & $<5.0000$ & $<30.000$ & 54.360 & 0.20400 & $<5.0000$ & $<5.0000$ \\
\hline STATION NUMBER & DATE & $\begin{array}{l}\text { HEXA- } \\
\text { CHLORO- } \\
\text { BUT- } \\
\text { ADIENE } \\
\text { SUSP . } \\
\text { (UG/L) }\end{array}$ & $\begin{array}{c}\text { HEXA- } \\
\text { CHLORO- } \\
\text { CYCLO- } \\
\text { PENT- } \\
\text { ADIENE } \\
\text { SUSP } \\
\text { (UG/L) }\end{array}$ & $\begin{array}{l}\text { HEXA- } \\
\text { CHLORO- } \\
\text { ETHANE } \\
\text { SUSP } \\
\text { (UG/L) }\end{array}$ & $\begin{array}{l}\text { INDENO } \\
(1,2,3- \\
\text { CD ) } \\
\text { PYRENE } \\
\text { SUSP. } \\
\text { (UG/L) }\end{array}$ & $\begin{array}{l}\text { ISO- } \\
\text { PHORONE } \\
\text { SUSP. } \\
(\mathrm{UG} / \mathrm{L})\end{array}$ & $\begin{array}{c}\text { N- } \\
\text { NITRO- } \\
\text { SODI-N- } \\
\text { PROPYL- } \\
\text { AMINE } \\
\text { SUSP } \\
\text { (UG/L) }\end{array}$ & $\begin{array}{c}\text { N-NITRO } \\
\text {-SODI- } \\
\text { PHENY- } \\
\text { LAMINE } \\
\text { SUSP . } \\
\text { (UG/L) }\end{array}$ & $\begin{array}{l}2- \\
\text { NITRO- } \\
\text { PHENOL } \\
\text { SUSP } \\
\text { (UG/L) }\end{array}$ & $\begin{array}{c}4- \\
\text { NITRO- } \\
\text { PHENOL } \\
\text { SUSP. } \\
\text { (UG/L) }\end{array}$ \\
\hline 440313123091100 & $\begin{array}{l}04-16-92 \\
10-16-92\end{array}$ & $\begin{array}{l}<5.0000 \\
<5.0000\end{array}$ & $\begin{array}{l}<5.0000 \\
<5.0000\end{array}$ & $\begin{array}{l}<5.0000 \\
<5.0000\end{array}$ & $\begin{array}{l}<10.000 \\
<10.000\end{array}$ & $\begin{array}{l}<5.0000 \\
<5.0000\end{array}$ & $\begin{array}{l}<5.0000 \\
<5.0000\end{array}$ & $\begin{array}{l}<5.0000 \\
<5.0000\end{array}$ & $\begin{array}{l}<5.0000 \\
<5.0000\end{array}$ & $\begin{array}{l}<30.000 \\
<30.000\end{array}$ \\
\hline 14201300 & $06-01-93$ & $<5.0000$ & $<5.0000$ & $<5.0000$ & $<10.000$ & $<5.0000$ & $<5.0000$ & $<5.0000$ & $<5.0000$ & $<30.000$ \\
\hline 14202000 & $04-27-93$ & $<5.0000$ & $<5.0000$ & $<5.0000$ & $<10.000$ & $<5.0000$ & $<5.0000$ & $<5.0000$ & $<5.0000$ & $<30.000$ \\
\hline 453115122535500 & $03-31-93$ & $<5.0000$ & $<5.0000$ & $<5.0000$ & $<10.000$ & $<5.0000$ & $<5.0000$ & $<5.0000$ & $<5.0000$ & $<30.000$ \\
\hline 14206950 & $03-01-93$ & $<5.0000$ & $<5.0000$ & $<5.0000$ & $<10.000$ & $<5.0000$ & $<5.0000$ & $<5.0000$ & $<5.0000$ & $<30.000$ \\
\hline 14211550 & $03-14-93$ & $<5.0000$ & $<5.0000$ & $<5.0000$ & $<10.000$ & $<5.0000$ & $<5.0000$ & $<5.0000$ & $<5.0000$ & $<30.000$ \\
\hline 453547122463000 & $10-26-92$ & $<5.0000$ & $<5.0000$ & $<5.0000$ & $<10.000$ & $<5.0000$ & $<5.0000$ & $<5.0000$ & $<5.0000$ & $<30.000$ \\
\hline
\end{tabular}


Table 27. Concentrations of semi-volatile compounds associated with suspended sediment from Phase I of the Willamette River Basin Water Quality Study, Oregon, 1992-93-Continued

\begin{tabular}{|c|c|c|c|c|c|c|c|c|c|c|}
\hline STATION NUMBER & DATE & $\begin{array}{l}\text { NAPHTH- } \\
\text { ALENE } \\
\text { SUSP } \\
\text { (UG/L) }\end{array}$ & $\begin{array}{l}\text { NITRO- } \\
\text { BENZENE } \\
\text { SUSP } \\
(U G / L)\end{array}$ & $\begin{array}{l}\text { N-NITRO } \\
\text { - SODI- } \\
\text { METHY- } \\
\text { LAMINE } \\
\text { SUSP. } \\
\text { (UG/L) }\end{array}$ & $\begin{array}{c}\text { PENTA- } \\
\text { CHLORO- } \\
\text { PHENOL } \\
\text { SUSP } \\
\text { (UG/L) }\end{array}$ & $\begin{array}{c}\text { PHENAN- } \\
\text { THRENE } \\
\text { SUSP } \\
\text { (UG/L) }\end{array}$ & $\begin{array}{l}\text { PHENOL } \\
\text { SUSP } \\
\text { (UG/L) }\end{array}$ & $\begin{array}{l}\text { PYRENE } \\
\text { SUSP } \\
\text { (UG/L) }\end{array}$ & $\begin{array}{c}1,2,4- \\
\text { TRI- } \\
\text { CHLORO- } \\
\text { BENZENE } \\
\text { SUSP } \\
(U G / L)\end{array}$ & $\begin{array}{c}2,4,6- \\
\text { TRI- } \\
\text { CHLORO- } \\
\text { PHENOL } \\
\text { SUSP } \\
\text { (UG/L) }\end{array}$ \\
\hline 440313123091100 & $\begin{array}{l}04-16-92 \\
10-16-92\end{array}$ & $\begin{array}{l}0.01300 \\
0.01000\end{array}$ & $\begin{array}{l}<5.0000 \\
<5.0000\end{array}$ & $\begin{array}{l}<5.0000 \\
<5.0000\end{array}$ & $\begin{array}{l}0.15300 \\
0.08400\end{array}$ & $\begin{array}{l}0.01200 \\
0.00900\end{array}$ & $\begin{array}{l}0.04000 \\
0.01200\end{array}$ & $\begin{array}{l}0.01800 \\
0.00500\end{array}$ & $\begin{array}{l}<5.0000 \\
<5.0000\end{array}$ & $\begin{array}{l}<20.000 \\
<20.000\end{array}$ \\
\hline 14201300 & $06-01-93$ & $<5.0000$ & $<5.0000$ & $<5.0000$ & $<30.000$ & 0.87000 & $<5.0000$ & 1.4400 & $<5.0000$ & $<20.000$ \\
\hline 14202000 & $04-27-93$ & 0.66000 & $<5.0000$ & $<5.0000$ & $<30.000$ & 0.64000 & 1.7000 & 0.56000 & $<5.0000$ & $<20.000$ \\
\hline 453115122535500 & $03-31-93$ & $<5.0000$ & $<5.0000$ & $<5.0000$ & $<30.000$ & 0.82300 & $<5.0000$ & 0.81000 & $<5.0000$ & $<20.000$ \\
\hline 14206950 & $03-01-93$ & 0.73000 & $<5.0000$ & $<5.0000$ & $<30.000$ & 0.80000 & 1.7600 & 1.0000 & $<5.0000$ & $<20.000$ \\
\hline 14211550 & $03-14-93$ & 0.72000 & $<5.0000$ & $<5.0000$ & $<30.000$ & 0.94000 & 1.7300 & 2.2300 & $<5.0000$ & $<20.000$ \\
\hline 453547122463000 & $10-26-92$ & 0.14200 & $<5.0000$ & $<5.0000$ & $<30.000$ & 0.27000 & $<5.0000$ & 0.20000 & $<5.0000$ & $<20.000$ \\
\hline
\end{tabular}


Table 28. Concentrations of semi-volatile compounds associated with bed sediment from Phase I of the Willamette River Basin Water Quality Study, Oregon, 1992

[USGS laboratory schedule 2502; WS, wet sieved; DW, dry weight; BM, bed material; G/KG, grams per kilogram; UG/KG, micrograms per kilogram]

\begin{tabular}{|c|c|c|c|c|c|c|}
\hline STATION NUMBER & STATION NAME & DATE & TIME & $\begin{array}{l}\text { CARBON, } \\
\text { ORGANIC } \\
\text { SED, BM } \\
\text { WS, <2MM } \\
\text { DW, REC } \\
(\mathrm{G} / \mathrm{KG})\end{array}$ & $\begin{array}{l}\text { CARBON, } \\
\text { INORG, } \\
\text { SED, BM } \\
\text { WS, <2MM } \\
\text { DW, REC } \\
(\mathrm{G} / \mathrm{KG})\end{array}$ & $\begin{array}{c}\text { CARBON, } \\
\text { ORG + } \\
\text { INORG } \\
\text { SED, BM } \\
\text { WS, <2MM } \\
\text { DW, REC } \\
(\mathrm{G} / \mathrm{KG})\end{array}$ \\
\hline 440313123091100 & A-3 CHANNEL AT WALLIS AND 5TH ST AT EUGENE, OR & $08-25-92$ & 1230 & 18.000 & 0.40000 & 18.000 \\
\hline 14165500 & MCKENZIE RIVER NEAR COBURG, OREG. & $08-25-92$ & 1645 & 85.000 & 0.20000 & 85.000 \\
\hline 443207123145500 & WILLAMETTE RIVER NEAR CORVALLIS, OR & $08-26-92$ & 1030 & 18.000 & $<0.10000$ & 18.000 \\
\hline 443819123064000 & CALAPOOIA RIVER AT MOUTH AT ALBANY, OR & $08-26-92$ & 1500 & 13.000 & 1.4000 & 14.000 \\
\hline 444032123050600 & MIDDLE FOURTH LAKE NEAR ALBANY, OR & $09-01-92$ & 1100 & 77.000 & 0.20000 & 77.000 \\
\hline 444416123030800 & SANTIAM RIVER NEAR JEFFERSON, OR & $08-29-92$ & 1000 & 3.0000 & $<0.10000$ & 3.0000 \\
\hline 445543123084400 & RICKREALL CREEK NEAR RICKREALL, OR & $08-28-92$ & 1345 & 12.000 & $<0.10000$ & 12.000 \\
\hline 451320123041100 & YAMHILL RIVER AT DAYTON, OR & $08-27-92$ & 1400 & 3.7000 & $<0.10000$ & 3.7000 \\
\hline 451705122575100 & WILLAMETTE RIVER AT NEWBERG, OR & $09-01-92$ & 1600 & 11.000 & $<0.10000$ & 11.000 \\
\hline 452950122492900 & BEAVERTON CREEK AT BEAVERTON, OR & $08-23-92$ & 1100 & 46.000 & $<0.10000$ & 46.000 \\
\hline 452221122362400 & CLACKAMAS RIVER AT OREGON CITY, OR & $08-24-92$ & 1130 & 5.8000 & 0.30000 & 6.1000 \\
\hline 452847122244500 & JOHNSON CREEK AT GRESHAM, OR & $08-21-92$ & 1330 & 9.4000 & 0.60000 & 10.000 \\
\hline 453547122463000 & WILLAMETTE RIVER AT LINNTON, OR & $09-09-92$ & 1130 & 17.000 & $<0.10000$ & 17.000 \\
\hline 453205122223701 & BEAVER CREEK NEAR TROUTDALE, OR & $08-22-92$ & 0945 & 8.4000 & 0.40000 & 8.8000 \\
\hline
\end{tabular}


Table 28. Concentrations of semi-volatile compounds associated with bed sediment from Phase I of the Willamette River Basin Water Quality Study, Oregon, 1992-Continued

\begin{tabular}{|c|c|c|c|c|c|c|c|c|c|c|}
\hline STATION NUMBER & DATE & $\begin{array}{c}\text { BED } \\
\text { MAT. } \\
\text { SIEVE } \\
\text { DIAM. } \\
\text { O FINER } \\
\text { THAN } \\
.062 \text { MM }\end{array}$ & $\begin{array}{l}\text { ACENAPH } \\
\text { THENE } \\
\text { SED, BM } \\
\text { WS, <2MM } \\
\text { DW, REC } \\
\text { (UG/KG) }\end{array}$ & $\begin{array}{l}\text { ACENAPH } \\
\text { THYLENE } \\
\text { SED, BM } \\
\text { WS, <2MM } \\
\text { DW, REC } \\
(\mathrm{UG} / \mathrm{KG})\end{array}$ & $\begin{array}{l}\text { ACRI- } \\
\text { DINE } \\
\text { SED, BM } \\
\text { WS, <2MM } \\
\text { DW, REC } \\
\text { (UG/KG) }\end{array}$ & $\begin{array}{c}\text { C8- } \\
\text { ALKYL- } \\
\text { PHENOL } \\
\text { SED, BM } \\
\text { WS, <2MM } \\
\text { DW, REC } \\
\text { (UG/KG) }\end{array}$ & $\begin{array}{l}\text { ANTHRA- } \\
\text { CENE } \\
\text { SED, BM } \\
\text { WS, <2MM } \\
\text { DW, REC } \\
\text { (UG/KG) }\end{array}$ & $\begin{array}{l}\text { ANTHRA- } \\
\text { QUINONE } \\
\text { SED, BM } \\
\text { WS, <2MM } \\
\text { DW, REC } \\
(U G / K G)\end{array}$ & $\begin{array}{l}\text { AZO- } \\
\text { BENZENE } \\
\text { SED, BM } \\
\text { WS, <2MM } \\
\text { DW, REC } \\
\text { (UG/KG) }\end{array}$ & $\begin{array}{c}2,2^{\prime}-\mathrm{BI} \\
\text { QUINO- } \\
\text { LINE } \\
\text { SED, BM } \\
\text { WS, <2MM } \\
\text { DW, REC } \\
\text { (UG/KG) }\end{array}$ \\
\hline 440313123091100 & $08-25-92$ & 23.000 & 23.000 & 36.000 & $<50.000$ & $<50.000$ & $<50.000$ & $<50.000$ & $<50.000$ & $<50.000$ \\
\hline 14165500 & $08-25-92$ & 84.000 & $<50.000$ & $<50.000$ & $<50.000$ & $<50.000$ & $<50.000$ & $<50.000$ & $<50.000$ & $<50.000$ \\
\hline 443207123145500 & $08-26-92$ & 22.000 & $<50.000$ & $<50.000$ & $<50.000$ & $<50.000$ & $<50.000$ & $<50.000$ & $<50.000$ & $<50.000$ \\
\hline 443819123064000 & $08-26-92$ & 33.000 & $<50.000$ & $<50.000$ & $<50.000$ & $<50.000$ & $<50.000$ & $<50.000$ & $<50.000$ & $<50.000$ \\
\hline 444032123050600 & $09-01-92$ & 95.000 & 52.000 & 20.000 & $<50.000$ & $<50.000$ & 15.000 & 180.00 & $<50.000$ & $<50.000$ \\
\hline 444416123030800 & $08-29-92$ & 3.0000 & $<50.000$ & $<50.000$ & $<50.000$ & $<50.000$ & $<50.000$ & $<50.000$ & $<50.000$ & $<50.000$ \\
\hline 445543123084400 & $08-28-92$ & 17.000 & $<50.000$ & $<50.000$ & $<50.000$ & $<50.000$ & 32.000 & $<50.000$ & 30.000 & $<50.000$ \\
\hline 451320123041100 & $08-27-92$ & 9.0000 & $<50.000$ & $<50.000$ & $<50.000$ & $<50.000$ & $<50.000$ & $<50.000$ & $<50.000$ & $<50.000$ \\
\hline 451705122575100 & $09-01-92$ & 20.000 & $<50.000$ & $<50.000$ & $<50.000$ & $<50.000$ & $<50.000$ & $<50.000$ & $<50.000$ & $<50.000$ \\
\hline 452950122492900 & $08-23-92$ & 66.000 & 27.000 & $<50.000$ & 83.000 & $<50.000$ & 150.00 & 350.00 & $<50.000$ & $<50.000$ \\
\hline 452221122362400 & $08-24-92$ & 4.0000 & $<50.000$ & $<50.000$ & $<50.000$ & $<50.000$ & $<50.000$ & $<50.000$ & $<50.000$ & $<50.000$ \\
\hline 452847122244500 & $08-21-92$ & 34.000 & 14.000 & 47.000 & $<50.000$ & $<50.000$ & 61.000 & 42.000 & $<50.000$ & $<50.000$ \\
\hline 453547122463000 & $09-09-92$ & 49.000 & 190.00 & 48.000 & 34.000 & $<50.000$ & 300.00 & 60.000 & $<50.000$ & $<50.000$ \\
\hline 453205122223701 & $08-22-92$ & 37.000 & $<50.000$ & $<50.000$ & $<50.000$ & $<50.000$ & $<50.000$ & $<50.000$ & $<50.000$ & $<50.000$ \\
\hline
\end{tabular}


Table 28. Concentrations of semi-volatile compounds associated with bed sediment from Phase I of the Willamette River Basin Water Quality Study, Oregon, 1992-Continued

\begin{tabular}{|c|c|c|c|c|c|c|c|c|c|}
\hline STATION NUMBER & DATE & $\begin{array}{l}\text { 2-CHLORO } \\
\text { ETHOXY- } \\
\text { METHANE } \\
\text { SED, BM } \\
\text { WS, <2MM } \\
\text { DW, REC } \\
\text { (UG/KG) }\end{array}$ & $\begin{array}{c}\text { 4-BROMO- } \\
\text { PHENYL } \\
\text { PHNYLETHER } \\
\text { SED, BM } \\
\text { WS, <2MM } \\
\text { DW, REC } \\
\text { (UG/KG) }\end{array}$ & $\begin{array}{l}\text { BENZO (A) } \\
\text { ANTHRA- } \\
\text { R CENE } \\
\text { SED, BM } \\
\text { WS, }<2 \text { MM } \\
\text { DW, REC } \\
\text { (UG/KG) }\end{array}$ & $\begin{array}{c}\text { BENZO (B) } \\
\text { FLUOR- } \\
\text { ANTHENE } \\
\text { SED, BM } \\
\text { WS, <2MM } \\
\text { DW, REC } \\
\text { (UG/KG) }\end{array}$ & $\begin{array}{c}\text { BENZO (G } \\
\text { HI) PERY } \\
\text { LENE } \\
\text { SED, BM } \\
\text { WS, <2MM } \\
\text { DW, REC } \\
\text { (UG/KG) }\end{array}$ & $\begin{array}{l}\text { BENZO (K) } \\
\text { FLUOR- } \\
\text { ANTHENE } \\
\text { SED, BM } \\
\text { WS, }<2 M M \\
\text { DW, REC } \\
\text { (UG/KG) }\end{array}$ & $\begin{array}{l}\text { BENZO (C) } \\
\text { QUINOLINE } \\
\text { BED MAT } \\
\text { WS <2MM } \\
\text { DRY WGT } \\
\text { REC } \\
\text { (UG/KG) }\end{array}$ & $\begin{array}{c}\text { BENZO } \\
\text { (A) } \\
\text { PYRENE } \\
\text { SED, BM } \\
\text { WS, <2MM } \\
\text { DW, REC } \\
\text { (UG/KG) }\end{array}$ \\
\hline 440313123091100 & $08-25-92$ & $<50.000$ & $<50.000$ & 100.00 & 73.000 & 110.00 & 70.000 & $<50.000$ & 110.00 \\
\hline 14165500 & $08-25-92$ & $<50.000$ & $<50.000$ & $<50.000$ & $<50.000$ & $<50.000$ & $<50.000$ & $<50.000$ & $<50.000$ \\
\hline 443207123145500 & $08-26-92$ & $<50.000$ & $<50.000$ & $<50.000$ & $<50.000$ & $<50.000$ & $<50.000$ & $<50.000$ & $<50.000$ \\
\hline 443819123064000 & $08-26-92$ & $<50.000$ & $<50.000$ & $<50.000$ & 39.000 & $<50.000$ & 39.000 & $<50.000$ & 33.000 \\
\hline 444032123050600 & $09-01-92$ & $<50.000$ & $<50.000$ & 26.000 & $<50.000$ & $<50.000$ & $<50.000$ & $<50.000$ & $<50.000$ \\
\hline 444416123030800 & $08-29-92$ & $<50.000$ & $<50.000$ & $<50.000$ & $<50.000$ & $<50.000$ & $<50.000$ & $<50.000$ & $<50.000$ \\
\hline 445543123084400 & $08-28-92$ & $<50.000$ & $<50.000$ & $<50.000$ & $<50.000$ & $<50.000$ & $<50.000$ & $<50.000$ & $<50.000$ \\
\hline 451320123041100 & $08-27-92$ & $<50.000$ & $<50.000$ & $<50.000$ & $<50.000$ & $<50.000$ & $<50.000$ & $<50.000$ & $<50.000$ \\
\hline 451705122575100 & $09-01-92$ & $<50.000$ & $<50.000$ & 46.000 & 25.000 & 14.000 & 25.000 & $<50.000$ & 26.000 \\
\hline 452950122492900 & $08-23-92$ & $<50.000$ & $<50.000$ & 880.00 & 780.00 & 590.00 & 820.00 & $<50.000$ & 630.00 \\
\hline 452221122362400 & $08-24-92$ & $<50.000$ & $<50.000$ & $<50.000$ & $<50.000$ & $<50.000$ & $<50.000$ & $<50.000$ & $<50.000$ \\
\hline 452847122244500 & $08-21-92$ & $<50.000$ & $<50.000$ & 150.00 & 180.00 & 250.00 & 160.00 & $<50.000$ & 210.00 \\
\hline 453547122463000 & $09-09-92$ & $<50.000$ & $<50.000$ & 1400.0 & 2600.0 & 1700.0 & 2500.0 & $<50.000$ & 2700.0 \\
\hline 453205122223701 & $08-22-92$ & $<50.000$ & $<50.000$ & 28.000 & 34.000 & 55.000 & 53.000 & $<50.000$ & 41.000 \\
\hline
\end{tabular}


Table 28. Concentrations of semi-volatile compounds associated with bed sediment from Phase I of the Willamette River Basin Water Quality Study, Oregon, 1992-Continued

\begin{tabular}{|c|c|c|c|c|c|c|c|c|c|}
\hline STATION NUMBER & DATE & $\begin{array}{l}\text { BIS2ETHYL } \\
\text { HEXYL-PH } \\
\text { THALATE } \\
\text { SED, BM } \\
\text { WS, <2MM } \\
\text { DW, REC } \\
\text { (UG/KG) }\end{array}$ & $\begin{array}{l}\text { L BUTYL } \\
\text { BENZYL- } \\
\text { PHTHALATE } \\
\text { SED, BM } \\
\text { WS, <2MM } \\
\text { DW, REC } \\
\text { (UG/KG) }\end{array}$ & $\begin{array}{c}124-\mathrm{TRI} \\
\text { CHLORO } \\
\text { E BENZENE } \\
\text { SED, BM } \\
\text { WS, <2MM } \\
\text { DW, REC } \\
\text { (UG/KG) }\end{array}$ & $\begin{array}{c}\text { 2CHLORO- } \\
\text { PHENOL } \\
\text { BED MAT } \\
\text { WS <2MM } \\
\text { DRY WGT } \\
\text { REC } \\
\text { (UG/KG) }\end{array}$ & $\begin{array}{l}\text { - 2CHLORO- } \\
\text { NAPTHAL } \\
\text { ENE } \\
\text { SED, BM } \\
\text { WS, <2MM } \\
\text { DW, REC } \\
\text { (UG/KG) }\end{array}$ & $\begin{array}{l}\text { - } 4 \text { CHLORO } \\
\text { PHENYL-PH } \\
\text { ENYLETHER } \\
\text { SED, BM } \\
\text { WS, <2MM } \\
\text { DW, REC } \\
\text { (UG/KG) }\end{array}$ & $\begin{array}{l}\text { H CHRY- } \\
\text { R SENE } \\
\text { SED, BM } \\
\text { WS, <2MM } \\
\text { DW, REC } \\
\text { (UG/KG) }\end{array}$ & $\begin{array}{l}\text { PENTACHL } \\
\text { ORONITRO } \\
\text { BENZENE } \\
\text { SED, BM } \\
\text { WS, <2MM } \\
\text { DW, REC } \\
\text { (UG/KG) }\end{array}$ \\
\hline 440313123091100 & $08-25-92$ & 2100.0 & 380.00 & $<50.000$ & $<50.000$ & $<50.000$ & $<50.000$ & 180.00 & $<50.000$ \\
\hline 14165500 & $08-25-92$ & 19.000 & $<50.000$ & $<50.000$ & $<50.000$ & $<50.000$ & $<50.000$ & $<50.000$ & $<50.000$ \\
\hline 443207123145500 & $08-26-92$ & 61.000 & $<50.000$ & $<50.000$ & $<50.000$ & $<50.000$ & $<50.000$ & $<50.000$ & $<50.000$ \\
\hline 443819123064000 & $08-26-92$ & 72.000 & 58.000 & $<50.000$ & $<50.000$ & $<50.000$ & $<50.000$ & $<50.000$ & $<50.000$ \\
\hline 444032123050600 & $09-01-92$ & 46.000 & 81.000 & 12.000 & $<50.000$ & $<50.000$ & $<50.000$ & 54.000 & $<50.000$ \\
\hline 444416123030800 & $08-29-92$ & 88.000 & 65.000 & $<50.000$ & $<50.000$ & $<50.000$ & $<50.000$ & $<50.000$ & $<50.000$ \\
\hline 445543123084400 & $08-28-92$ & 100.00 & 74.000 & $<50.000$ & $<50.000$ & $<50.000$ & $<50.000$ & $<50.000$ & $<50.000$ \\
\hline 451320123041100 & $08-27-92$ & $<50.000$ & $<50.000$ & $<50.000$ & $<50.000$ & $<50.000$ & $<50.000$ & $<50.000$ & $<50.000$ \\
\hline 451705122575100 & $09-01-92$ & 10.000 & $<50.000$ & $<50.000$ & $<50.000$ & $<50.000$ & $<50.000$ & 45.000 & $<50.000$ \\
\hline 452950122492900 & $08-23-92$ & 1100.0 & 780.00 & $<50.000$ & $<50.000$ & $<50.000$ & $<50.000$ & 1500.0 & $<50.000$ \\
\hline 452221122362400 & $08-24-92$ & 64.000 & 48.000 & $<50.000$ & $<50.000$ & $<50.000$ & $<50.000$ & $<50.000$ & $<50.000$ \\
\hline 452847122244500 & $08-21-92$ & 74.000 & 54.000 & $<50.000$ & $<50.000$ & $<50.000$ & $<50.000$ & 210.00 & $<50.000$ \\
\hline 453547122463000 & $09-09-92$ & 88.000 & 38.000 & $<50.000$ & $<50.000$ & $<50.000$ & $<50.000$ & 1900.0 & $<50.000$ \\
\hline 453205122223701 & $08-22-92$ & 49.000 & 30.000 & $<50.000$ & $<50.000$ & $<50.000$ & $<50.000$ & 71.000 & $<50.000$ \\
\hline
\end{tabular}


Table 28. Concentrations of semi-volatile compounds associated with bed sediment from Phase I of the Willamette River Basin Water Quality Study, Oregon, 1992-Continued

\begin{tabular}{|c|c|c|c|c|c|c|c|c|c|}
\hline STATION NUMBER & DATE & $\begin{array}{l}\text { HEXA- } \\
\text { CHLORO- } \\
\text { BENZENE } \\
\text { SED, BM } \\
\text { WS, <2MM } \\
\text { DW, REC } \\
\text { (UG/KG) }\end{array}$ & $\begin{array}{l}1,2-\mathrm{DI} \\
\text { CHLORO- } \\
\text { BENZENE } \\
\text { SED, BM } \\
\text { WS, <2MM } \\
\text { DW, REC } \\
\text { (UG/KG) }\end{array}$ & $\begin{array}{l}1,3-\mathrm{DI} \\
\text { CHLORO- } \\
\text { BENZENE } \\
\text { SED, BM } \\
\text { WS, <2MM } \\
\text { DW, REC } \\
\text { (UG/KG) }\end{array}$ & $\begin{array}{l}1,4-D I \\
\text { CHLORO- } \\
\text { BENZENE } \\
\text { SED, BM } \\
\text { WS, <2MM } \\
\text { DW, REC } \\
\text { (UG/KG) }\end{array}$ & $\begin{array}{l}2,4-D I- \\
\text { NITRO- } \\
\text { TOLUENE } \\
\text { SED, BM } \\
\text { WS, <2MM } \\
\text { DW, REC } \\
\text { (UG/KG) }\end{array}$ & $\begin{array}{l}2,6-D I- \\
\text { NITRO- } \\
\text { TOLUENE } \\
\text { SED, BM } \\
\text { WS, <2MM } \\
\text { DW, REC } \\
\text { (UG/KG) }\end{array}$ & $\begin{array}{c}3,5- \\
\text { DIMETHYL } \\
\text { PHENOL } \\
\text { SED, BM } \\
\text { WS, <2MM } \\
\text { DW, REC } \\
\text { (UG/KG) }\end{array}$ & $\begin{array}{l}\text { DIBENZO } \\
\text { THIO- } \\
\text { PHENE } \\
\text { SED, BM } \\
\text { WS, <2MM } \\
\text { DW, REC } \\
\text { (UG/KG) }\end{array}$ \\
\hline 440313123091100 & $08-25-92$ & $<50.000$ & $<50.000$ & $<50.000$ & $<50.000$ & $<50.000$ & $<500.00$ & $<50.000$ & $<50.000$ \\
\hline 14165500 & $08-25-92$ & $<50.000$ & $<50.000$ & $<50.000$ & $<50.000$ & $<50.000$ & $<500.00$ & $<50.000$ & $<50.000$ \\
\hline 443207123145500 & $08-26-92$ & $<50.000$ & $<50.000$ & $<50.000$ & $<50.000$ & $<50.000$ & $<500.00$ & $<50.000$ & $<50.000$ \\
\hline 443819123064000 & $08-26-92$ & $<50.000$ & $<50.000$ & $<50.000$ & $<50.000$ & $<50.000$ & $<500.00$ & $<50.000$ & $<50.000$ \\
\hline 444032123050600 & $09-01-92$ & 130.00 & $<50.000$ & 87.000 & 31.000 & $<50.000$ & $<500.00$ & $<50.000$ & $<50.000$ \\
\hline 444416123030800 & $08-29-92$ & $<50.000$ & $<50.000$ & $<50.000$ & $<50.000$ & $<50.000$ & $<500.00$ & $<50.000$ & $<50.000$ \\
\hline 445543123084400 & $08-28-92$ & $<50.000$ & $<50.000$ & $<50.000$ & $<50.000$ & $<50.000$ & $<500.00$ & $<50.000$ & $<50.000$ \\
\hline 451320123041100 & $08-27-92$ & $<50.000$ & $<50.000$ & $<50.000$ & $<50.000$ & $<50.000$ & $<500.00$ & $<50.000$ & $<50.000$ \\
\hline 451705122575100 & $09-01-92$ & $<50.000$ & $<50.000$ & $<50.000$ & $<50.000$ & $<50.000$ & $<500.00$ & $<50.000$ & $<50.000$ \\
\hline 452950122492900 & $08-23-92$ & $<50.000$ & $<5.0000$ & $<50.000$ & $<50.000$ & $<50.000$ & $<500.00$ & $<50.000$ & 61.000 \\
\hline 452221122362400 & $08-24-92$ & $<50.000$ & $<50.000$ & $<50.000$ & $<50.000$ & $<50.000$ & $<500.00$ & $<50.000$ & $<50.000$ \\
\hline 452847122244500 & $08-21-92$ & $<50.000$ & $<50.000$ & $<50.000$ & $<50.000$ & $<50.000$ & $<500.00$ & $<50.000$ & 33.000 \\
\hline 453547122463000 & $09-09-92$ & $<50.000$ & $<50.000$ & $<50.000$ & $<50.000$ & $<50.000$ & $<500.00$ & $<50.000$ & 120.00 \\
\hline 453205122223701 & $08-22-92$ & $<50.000$ & $<50.000$ & $<50.000$ & $<50.000$ & $<50.000$ & $<500.00$ & $<50.000$ & $<50.000$ \\
\hline
\end{tabular}


Table 28. Concentrations of semi-volatile compounds associated with bed sediment from Phase I of the Willamette River Basin Water Quality Study, Oregon, 1992-Continued

\begin{tabular}{|c|c|c|c|c|c|c|c|c|c|c|}
\hline STATION NUMBER & DATE & $\begin{array}{l}\text { DI-N- } \\
\text { BUTYL- } \\
\text { PHTHALATE } \\
\text { SED, BM } \\
\text { WS, <2MM } \\
\text { DW, REC } \\
\text { (UG/KG) }\end{array}$ & $\begin{array}{l}\text { DIBENZO } \\
\text { (AH) ANTH } \\
\text { E RACENE } \\
\text { SED, BM } \\
\text { WS, <2MM } \\
\text { DW, REC } \\
\text { (UG/KG) }\end{array}$ & $\begin{array}{l}\text { DIETHYL } \\
\text { PHTHALATE } \\
\text { SED, BM } \\
\text { WS, <2MM } \\
\text { DW, REC } \\
\text { (UG/KG) }\end{array}$ & $\begin{array}{l}\text { DIMETHYL } \\
\text { PHTHAL- } \\
\text { E ATE } \\
\text { SED, BM } \\
\text { WS, }<2 M M \\
\text { DW, REC } \\
\text { (UG/KG) }\end{array}$ & $\begin{array}{l}\text { L DI-N- } \\
\text { OCTYL- } \\
\text { PHTHALATE } \\
\text { SED, BM } \\
\text { WS, <2MM } \\
\text { DW, REC } \\
\text { (UG/KG) }\end{array}$ & $\begin{array}{l}\text { 2-ETHYL- } \\
\text { NAPTHAL } \\
\text { E ENE } \\
\text { SED BM } \\
\text { WS <2MM } \\
\text { DW REC } \\
\text { (UG/KG) }\end{array}$ & $\begin{array}{c}\text { FLUOR- } \\
\text { ANTHENE } \\
\text { BED MAT } \\
\text { WS <2MM } \\
\text { DRY WGT } \\
\text { REC } \\
(\text { UG/KG) }\end{array}$ & $\begin{array}{c}9 \mathrm{H}- \\
\text { CARBA- } \\
\text { ZOLE } \\
\text { SED, BM } \\
\text { WS, <2MM } \\
\text { DW, REC } \\
\text { (UG/KG) }\end{array}$ & $\begin{array}{l}\text { 9H- } \\
\text { FLUORENE } \\
\text { SED, BM } \\
\text { WS, <2MM } \\
\text { DW, REC } \\
\text { (UG/KG) }\end{array}$ \\
\hline 440313123091100 & $08-25-92$ & 150.00 & $<50.000$ & $<50.000$ & $<50.000$ & $<50.000$ & 15.000 & 150.00 & $<50.000$ & 40.000 \\
\hline 14165500 & $08-25-92$ & 42.000 & $<50.000$ & $<50.000$ & $<50.000$ & $<50.000$ & $<50.000$ & $<50.000$ & $<50.000$ & $<50.000$ \\
\hline 443207123145500 & $08-26-92$ & $<50.000$ & $<50.000$ & $<50.000$ & $<50.000$ & $<50.000$ & $<50.000$ & $<50.000$ & $<50.000$ & $<50.000$ \\
\hline 443819123064000 & $08-26-92$ & 54.000 & $<50.000$ & 18.000 & $<50.000$ & $<50.000$ & $<50.000$ & 37.000 & $<50.000$ & $<50.000$ \\
\hline 444032123050600 & $09-01-92$ & 60.000 & $<50.000$ & 10.000 & 9.0000 & $<50.000$ & 15.000 & 180.00 & $<50.000$ & 50.000 \\
\hline 444416123030800 & $08-29-92$ & 68.000 & $<50.000$ & 21.000 & $<50.000$ & $<50.000$ & $<50.000$ & 38.000 & $<50.000$ & $<50.000$ \\
\hline 445543123084400 & $08-28-92$ & 43.000 & $<50.000$ & 22.000 & $<50.000$ & $<50.000$ & $<50.000$ & 49.000 & $<50.000$ & $<50.000$ \\
\hline 451320123041100 & $08-27-92$ & 16.000 & $<50.000$ & $<50.000$ & $<50.000$ & $<50.000$ & $<50.000$ & $<50.000$ & $<50.000$ & $<50.000$ \\
\hline 451705122575100 & $09-01-92$ & 14.000 & $<50.000$ & $<50.000$ & $<50.000$ & $<50.000$ & $<50.000$ & 62.000 & $<50.000$ & $<50.000$ \\
\hline 452950122492900 & $08-23-92$ & 26.000 & 200.00 & $<50.000$ & 25.000 & $<50.000$ & $<50.000$ & 1700.0 & 150.00 & 55.000 \\
\hline 452221122362400 & $08-24-92$ & 42.000 & $<50.000$ & 17.000 & $<50.000$ & $<50.000$ & $<50.000$ & 31.000 & $<50.000$ & $<50.000$ \\
\hline 452847122244500 & $08-21-92$ & 54.000 & 66.000 & 17.000 & $<50.000$ & $<50.000$ & 10.000 & 240.00 & 26.000 & 25.000 \\
\hline 453547122463000 & $09-09-92$ & 31.000 & 510.00 & 11.000 & $<50.000$ & $<50.000$ & 14.000 & 2100.0 & 210.00 & 160.00 \\
\hline 453205122223701 & $08-22-92$ & 17.000 & $<50.000$ & $<50.000$ & $<50.000$ & $<50.000$ & $<50.000$ & 70.000 & $<50.000$ & $<50.000$ \\
\hline
\end{tabular}


Table 28. Concentrations of semi-volatile compounds associated with bed sediment from Phase I of the Willamette River Basin Water Quality Study, Oregon, 1992-Continued

\begin{tabular}{|c|c|c|c|c|c|c|c|c|c|c|}
\hline STATION NUMBER & DATE & $\begin{array}{l}\text { INDENO } \\
\text { 123-CD } \\
\text { PYRENE } \\
\text { SED, BM } \\
\text { WS, <2MM } \\
\text { DW, REC } \\
\text { (UG/KG) }\end{array}$ & $\begin{array}{l}\text { ISO- } \\
\text { QUINO- } \\
\text { LINE } \\
\text { SED, BM } \\
\text { WS, <2MM } \\
\text { DW, REC } \\
\text { (UG/KG) }\end{array}$ & $\begin{array}{l}\text { ISOPHOR } \\
\text { ONE } \\
\text { SED, BM } \\
\text { WS, <2MM } \\
\text { DW, REC } \\
\text { (UG/KG) }\end{array}$ & $\begin{array}{l}\text { 1,2-DI- } \\
\text { METHYLNA } \\
\text { PTHALENE } \\
\text { SED, BM } \\
\text { WS, <2MM } \\
\text { DW, REC } \\
\text { (UG/KG) }\end{array}$ & $\begin{array}{l}1,6-D I- \\
\text { METHYLNA } \\
\text { PTHALENE } \\
\text { SED, BM } \\
\text { WS, <2MM } \\
\text { DW, REC } \\
\text { (UG/KG) }\end{array}$ & $\begin{array}{l}\text { 1-METHYL } \\
\text { 9H-FLU- } \\
\text { ORENE } \\
\text { SED, BM } \\
\text { WS, <2MM } \\
\text { DW, REC } \\
\text { (UG/KG) }\end{array}$ & $\begin{array}{l}\text { 1-METHYL } \\
\text { PHENAN- } \\
\text { THRENE } \\
\text { SED, BM } \\
\text { WS, <2MM } \\
\text { DW, REC } \\
\text { (UG/KG) }\end{array}$ & $\begin{array}{l}1- \\
\text { METHYL } \\
\text { PYRENE } \\
\text { SED, BM } \\
\text { WS, <2MM } \\
\text { DW, REC } \\
\text { (UG/KG) }\end{array}$ & $\begin{array}{l}\text { 2, 3, 6-TRI } \\
\text { METHYLNA } \\
\text { PTHALENE } \\
\text { SED, BM } \\
\text { WS, <2MM } \\
\text { DW, REC } \\
\text { (UG/KG) }\end{array}$ \\
\hline 440313123091100 & $08-25-92$ & 27.000 & $<50.000$ & $<50.000$ & $<50.000$ & 58.000 & $<50.000$ & 64.000 & 31.000 & 61.000 \\
\hline 14165500 & $08-25-92$ & $<50.000$ & $<50.000$ & $<50.000$ & $<50.000$ & $<50.000$ & $<50.000$ & $<50.000$ & $<50.000$ & $<50.000$ \\
\hline 443207123145500 & $08-26-92$ & $<50.000$ & $<50.000$ & $<50.000$ & $<50.000$ & $<50.000$ & $<50.000$ & $<50.000$ & $<50.000$ & $<50.000$ \\
\hline 443819123064000 & $08-26-92$ & 45.000 & $<50.000$ & $<50.000$ & $<50.000$ & $<50.000$ & $<50.000$ & $<50.000$ & $<50.000$ & $<50.000$ \\
\hline 444032123050600 & $09-01-92$ & $<50.000$ & $<50.000$ & $<50.000$ & 6.0000 & 45.000 & $<50.000$ & 59.000 & 22.000 & 22.000 \\
\hline 444416123030800 & $08-29-92$ & $<50.000$ & $<50.000$ & $<50.000$ & $<50.000$ & $<50.000$ & $<50.000$ & $<50.000$ & $<50.000$ & $<50.000$ \\
\hline 445543123084400 & $08-28-92$ & $<50.000$ & $<50.000$ & $<50.000$ & $<50.000$ & 15.000 & $<50.000$ & $<50.000$ & $<50.000$ & $<50.000$ \\
\hline 451320123041100 & $08-27-92$ & $<50.000$ & $<50.000$ & $<50.000$ & $<50.000$ & $<50.000$ & $<50.000$ & $<50.000$ & $<50.000$ & $<50.000$ \\
\hline 451705122575100 & $09-01-92$ & 16.000 & $<50.000$ & $<50.000$ & $<50.000$ & $<50.000$ & $<50.000$ & $<50.000$ & $<50.000$ & $<50.000$ \\
\hline 452950122492900 & $08-23-92$ & 460.00 & $<50.000$ & $<50.000$ & $<50.000$ & $<50.000$ & $<50.000$ & 120.00 & 400.00 & $<50.000$ \\
\hline 452221122362400 & $08-24-92$ & $<50.000$ & $<50.000$ & $<50.000$ & $<50.000$ & $<50.000$ & $<50.000$ & $<50.000$ & $<50.000$ & $<50.000$ \\
\hline 452847122244500 & $08-21-92$ & 230.00 & $<50.000$ & $<50.000$ & 6.0000 & 16.000 & 22.000 & 44.000 & 51.000 & 24.000 \\
\hline 453547122463000 & $09-09-92$ & 1500.0 & $<50.000$ & $<50.000$ & 7.0000 & 36.000 & 25.000 & 84.000 & 150.00 & $<50.000$ \\
\hline 453205122223701 & $08-22-92$ & 36.000 & $<50.000$ & $<50.000$ & $<50.000$ & $<50.000$ & $<50.000$ & $<50.000$ & $<50.000$ & $<50.000$ \\
\hline
\end{tabular}


Table 28. Concentrations of semi-volatile compounds associated with bed sediment from Phase I of the Willamette River Basin Water Quality Study, Oregon, 1992-Continued

\begin{tabular}{|c|c|c|c|c|c|c|c|c|c|c|}
\hline STATION NUMBER & DATE & $\begin{array}{l}\text { 2, 6-DIME } \\
\text { THYLNAPH } \\
\text { THALENE } \\
\text { SED, BM } \\
\text { WS, <2MM } \\
\text { DW, REC } \\
\text { (UG/KG) }\end{array}$ & $\begin{array}{l}\text { E } 2 \text {-METHYL } \\
\text { ANTHRA- } \\
\text { CENE } \\
\text { SED, BM } \\
\text { WS, <2MM } \\
\text { DW, REC } \\
\text { (UG/KG) }\end{array}$ & $\begin{array}{l}\text { L } 45 \text {-METHY } \\
\text { ENEPHEN } \\
\text { ANTHRENE } \\
\text { SED, BM } \\
\text { WS, <2MM } \\
\text { DW, REC } \\
\text { (UG/KG) }\end{array}$ & $\begin{array}{l}\text { YL } 4-\text { CHLORC } \\
\text { 3-METHYL } \\
\text { E PHENOL } \\
\text { SED, BM } \\
\text { WS, <2MM } \\
\text { DW, REC } \\
\text { (UG/KG) }\end{array}$ & $\begin{array}{l}\text { O N-NITROS } \\
\text { DIPHENYL } \\
\text { AMINE } \\
\text { SED, BM } \\
\text { WS, <2MM } \\
\text { DW, REC } \\
\text { (UG/KG) }\end{array}$ & $\begin{array}{l}\text { SO N-NITRO } \\
\text { SODI-N-PR } \\
\text { OPYLAMINE } \\
\text { SED, BM } \\
\text { WS, <2MM } \\
\text { DW, REC } \\
\text { (UG/KG) }\end{array}$ & $\begin{array}{l}\text { NAPHTH- } \\
\text { ALENE } \\
\text { SED, BM } \\
\text { WS, <2MM } \\
\text { DW, REC } \\
\text { (UG/KG) }\end{array}$ & $\begin{array}{c}\text { NITRO- } \\
\text { BENZENE } \\
\text { SED, BM } \\
\text { WS, <2MM } \\
\text { DW, REC } \\
\text { (UG/KG) }\end{array}$ & $\begin{array}{l}\text { P- } \\
\text { CRESOL } \\
\text { SED, BM } \\
\text { WS, <2MM } \\
\text { DW, REC } \\
\text { (UG/KG) }\end{array}$ \\
\hline 440313123091100 & $08-25-92$ & 81.000 & 56.000 & $<50.000$ & $<50.000$ & 50.000 & $<50.000$ & 220.00 & $<50.000$ & 1700.0 \\
\hline 14165500 & $08-25-92$ & $<50.000$ & $<50.000$ & $<50.000$ & $<50.000$ & $<50.000$ & $<50.000$ & $<50.000$ & $<50.000$ & 2200.0 \\
\hline 443207123145500 & $08-26-92$ & 41.000 & $<50.000$ & $<50.000$ & $<50.000$ & $<50.000$ & $<50.000$ & $<50.000$ & $<50.000$ & 47.000 \\
\hline 443819123064000 & $08-26-92$ & $<50.000$ & $<50.000$ & $<50.000$ & $<50.000$ & $<50.000$ & $<50.000$ & 5.0000 & $<50.000$ & 170.00 \\
\hline 444032123050600 & $09-01-92$ & 50.000 & $<50.000$ & 40.000 & $<50.000$ & $<50.000$ & $<50.000$ & 88.000 & $<50.000$ & 97.000 \\
\hline 444416123030800 & $08-29-92$ & 12.000 & $<50.000$ & $<50.000$ & $<50.000$ & $<50.000$ & $<50.000$ & $<50.000$ & $<50.000$ & $<50.000$ \\
\hline 445543123084400 & $08-28-92$ & 19.000 & $<50.000$ & $<50.000$ & $<50.000$ & $<50.000$ & $<50.000$ & $<5.0000$ & $<50.000$ & 24.000 \\
\hline 451320123041100 & $08-27-92$ & $<50.000$ & $<50.000$ & $<50.000$ & $<50.000$ & $<50.000$ & $<50.000$ & $<50.000$ & $<50.000$ & $<50.000$ \\
\hline 451705122575100 & $09-01-92$ & $<50.000$ & $<50.000$ & $<50.000$ & $<50.000$ & $<50.000$ & $<50.000$ & 50.000 & $<50.000$ & $<50.000$ \\
\hline 452950122492900 & $08-23-92$ & $<50.000$ & $<50.000$ & 140.00 & $<50.000$ & 45.000 & $<50.000$ & 50.000 & $<50.000$ & 29.000 \\
\hline 452221122362400 & $08-24-92$ & $<50.000$ & $<50.000$ & $<50.000$ & $<50.000$ & $<50.000$ & $<50.000$ & $<50.000$ & $<50.000$ & $<50.000$ \\
\hline 452847122244500 & $08-21-92$ & 12.000 & 35.000 & 45.000 & $<50.000$ & $<50.000$ & $<50.000$ & 11.000 & $<50.000$ & 26.000 \\
\hline 453547122463000 & $09-09-92$ & 37.000 & 54.000 & 190.00 & 190.00 & $<50.000$ & $<50.000$ & 120.00 & $<50.000$ & 62.000 \\
\hline 453205122223701 & $08-22-92$ & $<50.000$ & $<50.000$ & $<50.000$ & $<50.000$ & $<50.000$ & $<50.000$ & 50.000 & $<50.000$ & 52.000 \\
\hline
\end{tabular}


Table 28. Concentrations of semi-volatile compounds associated with bed sediment from Phase I of the Willamette River Basin Water Quality Study, Oregon, 1992-Continued

\begin{tabular}{|c|c|c|c|c|c|c|c|c|c|c|}
\hline STATION NUMBER & DATE & $\begin{array}{l}\text { PENTA- } \\
\text { CHLORO- } \\
\text { ANISOLE } \\
\text { SED, BM } \\
\text { WS, <2MM } \\
\text { DW, REC } \\
\text { (UG/KG) }\end{array}$ & $\begin{array}{l}\text { PHENAN } \\
\text { THRENE } \\
\text { SED, BM } \\
\text { WS, <2MM } \\
\text { DW, REC } \\
\text { (UG/KG) }\end{array}$ & $\begin{array}{c}\text { PHENAN- } \\
\text { THRI- } \\
\text { DINE } \\
\text { SED, BM } \\
\text { WS, <2MM } \\
\text { DW, REC } \\
\text { (UG/KG) }\end{array}$ & $\begin{array}{l}\text { PHENOL } \\
\text { SED, BM } \\
\text { WS, <2MM } \\
\text { DW, REC } \\
\text { (UG/KG) }\end{array}$ & $\begin{array}{l}\text { PYRENE } \\
\text { SED, BM } \\
\text { WS, <2MM } \\
\text { DW, REC } \\
\text { (UG/KG) }\end{array}$ & $\begin{array}{l}\text { QUINO- } \\
\text { LINE } \\
\text { SED, BM } \\
\text { WS, <2MM } \\
\text { DW, REC } \\
\text { (UG/KG) }\end{array}$ & $\begin{array}{l}\text { BIP HENL } \\
\text { 2FLUORO } \\
\text { SURROGT } \\
\text { SED, BM } \\
\text { WS, <2MM } \\
\text { DW, REC } \\
\text { PERCENT }\end{array}$ & $\begin{array}{l}\text { TERPHEN } \\
\text { YL D14- } \\
\text { SURROGT } \\
\text { SED, BM } \\
\text { WS, <2MM } \\
\text { DW, REC } \\
\text { PERCENT }\end{array}$ & $\begin{array}{l}\text { BENZENE } \\
\text { NITROD } \\
\text { SURROGT } \\
\text { SED, BM } \\
\text { WS, <2MM } \\
\text { DW, REC } \\
\text { PERCENT }\end{array}$ \\
\hline 440313123091100 & $08-25-92$ & $<50.000$ & 210.00 & $<50.000$ & 110.00 & 200.00 & $<50.000$ & 56.000 & 66.000 & 49.000 \\
\hline 14165500 & $08-25-92$ & $<50.000$ & 21.000 & $<50.000$ & 310.00 & $<50.000$ & $<50.000$ & 27.000 & 35.000 & 31.000 \\
\hline 443207123145500 & $08-26-92$ & $<50.000$ & $<50.000$ & $<50.000$ & 14.000 & $<50.000$ & $<50.000$ & 69.000 & 99.000 & 66.000 \\
\hline 443819123064000 & $08-26-92$ & $<50.000$ & 18.000 & $<50.000$ & 15.000 & 42.000 & $<50.000$ & 39.000 & 53.000 & 49.000 \\
\hline 444032123050600 & $09-01-92$ & $<50.000$ & 210.00 & $<50.000$ & 36.000 & 170.00 & 20.000 & 58.000 & 74.000 & 61.000 \\
\hline 444416123030800 & $08-29-92$ & $<50.000$ & $<50.000$ & $<50.000$ & 26.000 & 44.000 & $<50.000$ & 37.000 & 51.000 & 46.000 \\
\hline 445543123084400 & $08-28-92$ & $<50.000$ & 27.000 & $<50.000$ & 5.0000 & 54.000 & $<50.000$ & 52.000 & 71.000 & 66.000 \\
\hline 451320123041100 & $08-27-92$ & $<50.000$ & $<50.000$ & $<50.000$ & $<50.000$ & $<50.000$ & $<50.000$ & 33.000 & 41.000 & 35.000 \\
\hline 451705122575100 & $09-01-92$ & $<50.000$ & $<50.000$ & $<50.000$ & $<50.000$ & 49.000 & $<50.000$ & 35.000 & 40.000 & 36.000 \\
\hline 452950122492900 & $08-23-92$ & $<50.000$ & 870.00 & 15.000 & 22.000 & 1500.0 & $<50.000$ & 28.000 & 38.000 & 29.000 \\
\hline 452221122362400 & $08-24-92$ & $<50.000$ & $<50.000$ & $<50.000$ & $<5.0000$ & 36.000 & $<50.000$ & 33.000 & 48.000 & 35.000 \\
\hline 452847122244500 & $08-21-92$ & $<50.000$ & 170.00 & $<50.000$ & 5.0000 & 300.00 & $<50.000$ & 36.000 & 52.000 & 38.000 \\
\hline 453547122463000 & $09-09-92$ & $<50.000$ & 1400.0 & 38.000 & 19.000 & 1900.0 & $<50.000$ & 68.000 & 82.000 & 74.000 \\
\hline 453205122223701 & $08-22-92$ & $<50.000$ & 37.000 & $<50.000$ & 22.000 & 90.000 & $<50.000$ & 34.000 & 40.000 & 34.000 \\
\hline
\end{tabular}


Table 29. Concentrations of volatile organic compounds in whole water from Phase I of the Willamette River Basin Water Quality Study, Oregon, 1992-93

[USGS laboratory schedules 1392 and 1401; UG/L, micrograms per liter ]

\begin{tabular}{|c|c|c|c|c|c|c|}
\hline \multirow[b]{2}{*}{ STATION NUMBER } & \multirow[b]{2}{*}{ STATION NAME } & \multirow[b]{2}{*}{ DATE } & \multirow[b]{2}{*}{ TIME } & \multicolumn{3}{|c|}{$\begin{array}{ll}\text { ACRO }- & \text { ACRYLO- } \\
\end{array}$} \\
\hline & & & & $\begin{array}{l}\text { LEIN } \\
\text { TOTAL } \\
\text { (UG/L) }\end{array}$ & $\begin{array}{c}\text { NITRILE } \\
\text { TOTAL } \\
\text { (UG/L) }\end{array}$ & $\begin{array}{l}\text { BENZENE } \\
\text { TOTAI } \\
\text { (UG/L) }\end{array}$ \\
\hline 440313123091100 & A-3 CHANNEL AT WALLIS AND 5TH ST AT EUGENE, OR & $04-16-92$ & 1410 & $<20.0$ & $<20$. & 000 \\
\hline & A-3 CHANNEL AT WALLIS AND 5TH ST AT EUGENE, OR & $10-16-92$ & 1345 & $<20.000$ & $<20.000$ & $<0.20000$ \\
\hline 443207123145500 & WILLAMETTE RIVER NEAR CORVALLIS, OR & $08-24-93$ & 1615 & $<20.000$ & $<20.000$ & $<0.20000$ \\
\hline 443138123120901 & MUDDY CREEK NEAR PEORIA, OR & $08-24-93$ & 1300 & $<20.000$ & $<20.000$ & $<0.20000$ \\
\hline 451309123041501 & PALMER C AT DAYTON, OR & $09-07-93$ & 1045 & $<20.000$ & $<20.000$ & $<0.20000$ \\
\hline 14201300 & $\begin{array}{l}\text { ZOLLNER CREEK NEAR MT ANGEL, OR } \\
\text { ZOLLNER CREEK NEAR MT ANGEL, OR }\end{array}$ & $\begin{array}{l}06-01-93 \\
07-27-93\end{array}$ & $\begin{array}{l}1115 \\
1015\end{array}$ & $\begin{array}{l}<20.000 \\
<20.000\end{array}$ & $\begin{array}{l}<20.000 \\
<20.000\end{array}$ & $\begin{array}{l}<0.20000 \\
<0.20000\end{array}$ \\
\hline 14202000 & PUDDING RIVER AT AURORA, OREG. & $04-27-93$ & 1445 & $<20.000$ & $<20.000$ & $<0.20000$ \\
\hline 453115122535500 & BEAVERTON C AT 216TH AVE NEAR ORENCO, OR & $03-31-93$ & 1810 & $<20.000$ & $<20.000$ & $<0.20000$ \\
\hline 14206950 & $\begin{array}{l}\text { FANNO CREEK AT DURHAM, OR } \\
\text { FANNO CREEK AT DURHAM, OR }\end{array}$ & $\begin{array}{l}03-01-93 \\
07-22-93\end{array}$ & $\begin{array}{l}1830 \\
1000\end{array}$ & $\begin{array}{l}<20.000 \\
<20.000\end{array}$ & $\begin{array}{l}<20.000 \\
<20.000\end{array}$ & $\begin{array}{l}<0.20000 \\
<0.20000\end{array}$ \\
\hline 14211550 & JOHNSON CREEK AT MILWAUKIE, OREG. & $03-14-93$ & 1745 & $<20.000$ & $<20.000$ & $<0.20000$ \\
\hline 453547122463000 & WILLAMETTE RIVER AT LINNTON, OR & $10-26-92$ & 1000 & $<20.000$ & $<20.000$ & $<0.20000$ \\
\hline
\end{tabular}

\begin{tabular}{|c|c|c|c|c|c|c|c|c|c|c|c|}
\hline STATION NUMBER & DATE & $\begin{array}{l}\text { BROMO- } \\
\text { BENZENE } \\
\text { TOTAL } \\
\text { (UG/L) }\end{array}$ & $\begin{array}{c}\text { BROMO- } \\
\text { CHLORO- } \\
\text { METHANE } \\
\text { TOTAL } \\
\text { (UG/L) }\end{array}$ & $\begin{array}{l} \\
\text { TRI- } \\
\text { BROMO- } \\
\text { METHANE } \\
\text { TOTAL } \\
\text { (UG/L) }\end{array}$ & $\begin{array}{l}1- \\
\text { CHLORO- } \\
2- \\
\text { METHYL- } \\
\text { E BENZENE } \\
\text { TOTAL } \\
\text { (UG/L) }\end{array}$ & $\begin{array}{c}1- \\
\text { CHLORO- } \\
4- \\
\text { METHYL- } \\
\text { BENZENE } \\
\text { TOTAL } \\
\text { (UG/L) }\end{array}$ & $\begin{array}{l}2- \\
\text { CHLORO- } \\
\text { ETHYL- } \\
\text { VINYL- } \\
\text { ETHER } \\
\text { TOTAL } \\
\text { (UG/L) }\end{array}$ & $\begin{array}{l}\text { CARBON- } \\
\text { TETRA- } \\
\text { CHLO- } \\
\text { RIDE } \\
\text { TOTAL } \\
\text { (UG/L) }\end{array}$ & $\begin{array}{l}- \\
\text { CHLORO- } \\
\text { BENZENE } \\
\text { TOTAL } \\
\text { (UG/L) }\end{array}$ & $\begin{array}{l}\text { DI- } \\
\text { BROMO- } \\
\text { CHLORO- } \\
\text { METHANE } \\
\text { TOTAL } \\
\text { (UG/L) }\end{array}$ & $\begin{array}{c} \\
\text { CHLORO- } \\
\text { ETHANE } \\
\text { TOTAL } \\
\text { (UG/L) }\end{array}$ \\
\hline 440313123091100 & $\begin{array}{l}04-16-92 \\
10-16-92\end{array}$ & $\begin{array}{l}<0.20000 \\
<0.20000\end{array}$ & $\begin{array}{l}<0.20000 \\
<0.20000\end{array}$ & $\begin{array}{l}<0.20000 \\
<0.20000<\end{array}$ & $\begin{array}{l}<0.20000 \\
<0.20000\end{array}$ & $\begin{array}{l}<0.20000 \\
<0.20000\end{array}$ & $\begin{array}{l}<1.0000 \\
<1.0000\end{array}$ & $\begin{array}{l}<0.20000 \\
<0.20000\end{array}$ & $\begin{array}{l}<0.20000 \\
<0.20000\end{array}$ & $\begin{array}{l}<0.20000 \\
<0.20000\end{array}$ & $\begin{array}{l}<0.20000 \\
<0.20000\end{array}$ \\
\hline 443207123145500 & $08-24-93$ & $<0.20000$ & $<0.20000$ & $<0.20000$ & $<0.20000$ & $<0.20000$ & $<1.0000$ & $<0.20000$ & $<0.20000$ & $<0.20000$ & $<0.20000$ \\
\hline 443138123120901 & $08-24-93$ & $<0.20000$ & $<0.20000$ & $<0.20000$ & $<0.20000$ & $<0.20000$ & $<1.0000$ & $<0.20000$ & $<0.20000$ & $<0.20000$ & $<0.20000$ \\
\hline 451309123041501 & $09-07-93$ & $<0.20000$ & $<0.20000$ & $<0.20000$ & $<0.20000$ & $<0.20000$ & $<1.0000$ & $<0.20000$ & $<0.20000$ & $<0.20000$ & $<0.20000$ \\
\hline 14201300 & $\begin{array}{l}06-01-93 \\
07-27-93\end{array}$ & $\begin{array}{l}<0.20000 \\
<0.20000\end{array}$ & $\begin{array}{l}<0.20000 \\
<0.20000\end{array}$ & $\begin{array}{l}<0.20000< \\
<0.20000<\end{array}$ & $\begin{array}{l}<0.20000 \\
<0.20000\end{array}$ & $\begin{array}{l}<0.20000 \\
<0.20000\end{array}$ & $\begin{array}{l}<1.0000 \\
<1.0000\end{array}$ & $\begin{array}{l}<0.20000 \\
<0.20000\end{array}$ & $\begin{array}{l}<0.20000 \\
<0.20000\end{array}$ & $\begin{array}{l}<0.20000 \\
<0.20000\end{array}$ & $\begin{array}{l}<0.20000 \\
<0.20000\end{array}$ \\
\hline 14202000 & $04-27-93$ & $<0.20000$ & $<0.20000$ & $<0.20000<$ & $<0.20000$ & $<0.20000$ & $<1.0000$ & $<0.20000$ & $<0.20000$ & $<0.20000$ & $<0.20000$ \\
\hline 453115122535500 & $03-31-93$ & $<0.20000$ & $<0.20000$ & $<0.20000$ & $<0.20000$ & $<0.20000$ & $<1.0000$ & $<0.20000$ & $<0.20000$ & $<0.20000$ & $<0.20000$ \\
\hline 14206950 & $\begin{array}{l}03-01-93 \\
07-22-93\end{array}$ & $\begin{array}{l}<0.20000 \\
<0.20000\end{array}$ & $\begin{array}{l}<0.20000 \\
<0.20000\end{array}$ & $\begin{array}{l}<0.20000< \\
<0.20000<\end{array}$ & $\begin{array}{l}<0.20000 \\
<0.20000\end{array}$ & $\begin{array}{l}<0.20000 \\
<0.20000\end{array}$ & $\begin{array}{l}<1.0000 \\
<1.0000\end{array}$ & $\begin{array}{l}<0.20000 \\
<0.20000\end{array}$ & $\begin{array}{l}<0.20000 \\
<0.20000\end{array}$ & $\begin{array}{l}<0.20000 \\
<0.20000\end{array}$ & $\begin{array}{l}<0.20000 \\
<0.20000\end{array}$ \\
\hline 14211550 & $03-14-93$ & $<0.20000$ & $<0.20000$ & $<0.20000<$ & $<0.20000$ & $<0.20000$ & $<1.0000$ & $<0.20000$ & $<0.20000$ & $<0.20000$ & $<0.20000$ \\
\hline 453547122463000 & $10-26-92$ & $<0.20000$ & $<0.20000$ & $<0.20000$ & $<0.20000$ & $<0.20000$ & $<1.0000$ & $<0.20000$ & $<0.20000$ & $<0.20000$ & $<0.20000$ \\
\hline
\end{tabular}


Table 29. Concentrations of volatile organic compounds in whole water from Phase I of the Willamette River Basin Water Quality Study, Oregon, 1992-93-Continued

\begin{tabular}{|c|c|c|c|c|c|c|c|c|c|c|c|}
\hline STATION NUMBER & DATE & $\begin{array}{l}\text { TRI- } \\
\text { CHLORO- } \\
\text { METHANE } \\
\text { TOTAL } \\
\text { (UG/L) }\end{array}$ & $\begin{array}{l}\text { METHYL- } \\
\text { CHLO- } \\
\text { RIDE } \\
\text { TOTAL } \\
\text { (UG/L) }\end{array}$ & $\begin{array}{c}\text { CIS-1, } \\
\text {-DI- } \\
\text { CHLORO- } \\
\text { ETHENE } \\
\text { TOTAL } \\
\text { (UG/L) }\end{array}$ & $\begin{array}{c}\text { CIS-1, } 3 \\
\text {-DI- } \\
\text { CHLORO- } \\
\text { PROPENE } \\
\text { TOTAL } \\
\text { (UG/L) }\end{array}$ & $\begin{array}{c}1,1- \\
\text { DI- } \\
\text { CHLORO- } \\
\text { ETHYLENE } \\
\text { TOTAL } \\
\text { (UG/L) }\end{array}$ & $\begin{array}{c}1,1- \\
\text { DI- } \\
\text { CHLORO- } \\
\text { ETHANE } \\
\text { TOTAL } \\
\text { (UG/L) }\end{array}$ & $\begin{array}{c}1,2- \\
\text { DIBROMO } \\
\text { ETHANE } \\
\text { TOTAL } \\
\text { (UG/L) }\end{array}$ & $\begin{array}{c}1,2- \\
\text { DI- } \\
\text { CHLORO- } \\
\text { BENZENE } \\
\text { TOTAL } \\
\text { UG/L) }\end{array}$ & $\begin{array}{c}1,2- \\
\text { DI- } \\
\text { CHLORO- } \\
\text { ETHANE } \\
\text { TOTAL } \\
\text { (UG/L) }\end{array}$ & $\begin{array}{c}1,2- \\
\text { DI- } \\
- \text { CHLORO- } \\
\text { PROPANE } \\
\text { TOTAL } \\
(\mathrm{UG} / \mathrm{L})\end{array}$ \\
\hline 440313123091100 & $\begin{array}{c}04-16-92 \\
10-16-92\end{array}$ & $\begin{array}{l}0.20000 \\
0.20000\end{array}$ & $\begin{array}{l}<0.20000 \\
<0.20000\end{array}$ & $\begin{array}{l}0.40000 \\
0.90000\end{array}$ & $\begin{array}{l}<0.20000 \\
<0.20000\end{array}$ & $\begin{array}{l}0.20000 \\
0.30000\end{array}$ & $\begin{array}{l}0.20000 \\
0.40000\end{array}$ & $\begin{array}{l}<0.20000 \\
<0.20000\end{array}$ & $\begin{array}{l}<0.20000 \\
<0.20000\end{array}$ & $\begin{array}{l}<0.20000 \\
<0.20000\end{array}$ & $\begin{array}{l}<0.20000 \\
<0.20000\end{array}$ \\
\hline 443207123145500 & $08-24-93$ & $<0.20000$ & $<0.20000$ & $<0.20000$ & $<0.20000$ & $<0.20000$ & $<0.20000$ & $<0.20000$ & $<5.0000$ & $<0.20000$ & $<0.20000$ \\
\hline 443138123120901 & $08-24-93$ & $<0.20000$ & $<0.20000$ & $<0.20000$ & $<0.20000$ & $<0.20000$ & $<0.20000$ & $<0.20000$ & $<5.0000$ & $<0.20000$ & $<0.20000$ \\
\hline 451309123041501 & $09-07-93$ & $<0.20000$ & $<0.20000$ & $<0.20000$ & $<0.20000$ & $<0.20000$ & $<0.20000$ & $<0.20000$ & $<5.0000$ & $<0.20000$ & $<0.20000$ \\
\hline 14201300 & $\begin{array}{l}06-01-93 \\
07-27-93\end{array}$ & $\begin{array}{l}<0.20000 \\
<0.20000\end{array}$ & $\begin{array}{l}<0.20000 \\
<0.20000\end{array}$ & $\begin{array}{l}<0.20000 \\
<0.20000\end{array}$ & $\begin{array}{l}<0.20000 \\
<0.20000\end{array}$ & $\begin{array}{l}<0.20000 \\
<0.20000\end{array}$ & $\begin{array}{l}<0.20000 \\
<0.20000\end{array}$ & $\begin{array}{l}<0.20000 \\
<0.20000\end{array}$ & $\begin{array}{l}<0.20000 \\
<0.20000\end{array}$ & $\begin{array}{l}<0.20000 \\
<0.20000\end{array}$ & $\begin{array}{l}<0.20000 \\
<0.20000\end{array}$ \\
\hline 14202000 & $04-27-93$ & $<0.20000$ & $<0.20000$ & $<0.20000$ & $<0.20000$ & $<0.20000$ & $<0.20000$ & $<0.20000$ & $<0.20000$ & $<0.20000$ & $<0.20000$ \\
\hline 453115122535500 & $03-31-93$ & $<0.20000$ & $<0.20000$ & $<0.20000$ & $<0.20000$ & $<0.20000$ & $<0.20000$ & $<0.20000$ & $<0.20000$ & $<0.20000$ & $<0.20000$ \\
\hline 14206950 & $\begin{array}{l}03-01-93 \\
07-22-93\end{array}$ & $\begin{array}{l}<0.20000 \\
<0.20000\end{array}$ & $\begin{array}{l}<0.20000 \\
<0.20000\end{array}$ & $\begin{array}{l}0.60000 \\
0.30000\end{array}$ & $\begin{array}{l}<0.20000 \\
<0.20000\end{array}$ & $\begin{array}{l}<0.20000 \\
<0.20000\end{array}$ & $\begin{array}{l}<0.20000 \\
<0.20000\end{array}$ & $\begin{array}{l}<0.20000 \\
<0.20000\end{array}$ & $\begin{array}{r}<0.20000 \\
<5.0000\end{array}$ & $\begin{array}{l}<0.20000 \\
<0.20000\end{array}$ & $\begin{array}{l}<0.20000 \\
<0.20000\end{array}$ \\
\hline 14211550 & $03-14-93$ & $<0.20000$ & $<0.20000$ & $<0.20000$ & $<0.20000$ & $<0.20000$ & $<0.20000$ & $<0.20000$ & $<0.20000$ & $<0.20000$ & $<0.20000$ \\
\hline 453547122463000 & $10-26-92$ & $<0.20000$ & $<0.20000$ & $<0.20000$ & $<0.20000$ & $<0.20000$ & $<0.20000$ & $<0.20000$ & $<0.20000$ & $<0.20000$ & $<0.20000$ \\
\hline STATION NUMBER & DATE & $\begin{array}{l}\text { 1,3-DI- } \\
\text { CHLORO- } \\
\text { BENZENE } \\
\text { TOTAL } \\
\text { (UG/L) }\end{array}$ & $\begin{array}{c}1,4-D I- \\
\text { CHLORO- } \\
\text { BENZENE } \\
\text { TOTAL } \\
\text { (UG/L) }\end{array}$ & $\begin{array}{c}1,1-\mathrm{DI}- \\
\text { CHLORO- } \\
\text { PROPENE } \\
\text { TOTAL } \\
\text { (UG/L) }\end{array}$ & $\begin{array}{c}1,3-D I- \\
\text { CHLORO- } \\
\text { PROPANE } \\
\text { TOTAL } \\
\text { (UG/L) }\end{array}$ & $\begin{array}{c}2,2-\mathrm{DI}- \\
\text { CHLORO- } \\
\text { PROPANE } \\
\text { TOTAL } \\
\text { (UG/L) }\end{array}$ & $\begin{array}{c}1,2- \\
\text { DIBROMO } \\
3- \\
\text { CHLORO- } \\
\text { PROPANE } \\
\text { TOTAL } \\
\text { (UG/L) }\end{array}$ & $\begin{array}{l} \\
\text { DI- } \\
\text { BROMO- } \\
\text { METHANE } \\
\text { TOTAL } \\
\text { (UG/L) }\end{array}$ & $\begin{array}{c}\text { DI- } \\
\text { CHLORO } \\
\text { BROMO- } \\
\text { METHAN } \\
\text { TOTAL } \\
\text { (UG/L) }\end{array}$ & $\begin{array}{c}\text { DI- } \\
\text { CHLORO- } \\
\text { O- DI- } \\
\text { FLUORO- } \\
\text { NE } \text { METHANE } \\
\text { TOTAL } \\
(\text { UG } / L)\end{array}$ & $\begin{array}{c}\text { TRI- } \\
\text { CHLORO- } \\
\text { TRI- } \\
\text { FLUORO- } \\
\text { ETHANE } \\
\text { TOTAL } \\
\text { (UG/L) } \\
\end{array}$ \\
\hline 440313123091100 & $\begin{array}{c}04-16-92 \\
10-16-92\end{array}$ & $\begin{array}{l}<0.20000 \\
<0.20000\end{array}$ & $\begin{array}{l}<0.20000 \\
<0.20000\end{array}$ & $\begin{array}{l}<0.20000 \\
<0.20000\end{array}$ & $\begin{array}{l}<0.20000 \\
<0.20000\end{array}$ & $\begin{array}{l}<0.20000 \\
<0.20000\end{array}$ & $\begin{array}{l}<1.0000 \\
<1.0000\end{array}$ & $\begin{array}{l}<0.20000 \\
<0.20000\end{array}$ & $\begin{array}{l}<0.20000 \\
<0.20000\end{array}$ & $\begin{array}{l}<0.20000 \\
<0.20000\end{array}$ & $\begin{array}{l}<0.50000 \\
<0.50000\end{array}$ \\
\hline 443207123145500 & $08-24-93$ & $<5.0000$ & $<5.0000$ & $<0.20000$ & $<0.20000$ & $<0.20000$ & $<1.0000$ & $<0.20000$ & $<0.20000$ & $<0.20000$ & $<0.50000$ \\
\hline 443138123120901 & $08-24-93$ & $<5.0000$ & $<5.0000$ & $<0.20000$ & $<0.20000$ & $<0.20000$ & $<1.0000$ & $<0.20000$ & $<0.20000$ & $<0.20000$ & $<0.50000$ \\
\hline 451309123041501 & $09-07-93$ & $<5.0000$ & $<5.0000$ & $<0.20000$ & $<0.20000$ & $<0.20000$ & $<1.0000$ & $<0.20000$ & $<0.20000$ & $<0.20000$ & $<0.50000$ \\
\hline 14201300 & $\begin{array}{l}06-01-93 \\
07-27-93\end{array}$ & $\begin{array}{l}<0.20000 \\
<0.20000\end{array}$ & $\begin{array}{l}<0.20000 \\
<0.20000\end{array}$ & $\begin{array}{l}<0.20000 \\
<0.20000\end{array}$ & $\begin{array}{l}<0.20000 \\
<0.20000\end{array}$ & $\begin{array}{l}<0.20000 \\
<0.20000\end{array}$ & $\begin{array}{l}<1.0000 \\
<1.0000\end{array}$ & $\begin{array}{l}<0.20000 \\
<0.20000\end{array}$ & $\begin{array}{l}<0.20000 \\
<0.20000\end{array}$ & $\begin{array}{l}<0.20000 \\
<0.20000\end{array}$ & $\begin{array}{l}<0.50000 \\
<0.50000\end{array}$ \\
\hline 14202000 & $04-27-93$ & $<0.20000$ & $<0.20000$ & $<0.20000$ & $<0.20000$ & $<0.20000$ & $<1.0000$ & $<0.20000$ & $<0.20000$ & $<0.20000$ & $<0.50000$ \\
\hline 453115122535500 & $03-31-93$ & $<0.20000$ & $<0.20000$ & $<0.20000$ & $<0.20000$ & $<0.20000$ & $<1.0000$ & $<0.20000$ & $<0.20000$ & $<0.20000$ & $<0.50000$ \\
\hline 14206950 & $\begin{array}{l}03-01-93 \\
07-22-93\end{array}$ & $\begin{array}{r}<0.20000 \\
<5.0000\end{array}$ & $\begin{array}{r}<0.20000 \\
<5.0000\end{array}$ & $\begin{array}{l}<0.20000 \\
<0.20000\end{array}$ & $\begin{array}{l}<0.20000 \\
<0.20000\end{array}$ & $\begin{array}{l}<0.20000 \\
<0.20000\end{array}$ & $\begin{array}{l}<1.0000 \\
<1.0000\end{array}$ & $\begin{array}{l}<0.20000 \\
<0.20000\end{array}$ & $\begin{array}{l}<0.20000 \\
<0.20000\end{array}$ & $\begin{array}{l}<0.20000 \\
<0.20000\end{array}$ & $\begin{array}{l}<0.50000 \\
<0.50000\end{array}$ \\
\hline 14211550 & $03-14-93$ & $<0.20000$ & $<0.20000$ & $<0.20000$ & $<0.20000$ & $<0.20000$ & $<1.0000$ & $<0.20000$ & $<0.20000$ & $<0.20000$ & $<0.50000$ \\
\hline 453547122463000 & $10-26-92$ & $<0.20000$ & $<0.20000$ & $<0.20000$ & $<0.20000$ & $<0.20000$ & $<1.0000$ & $<0.20000$ & $<0.20000$ & $<0.20000$ & $<0.50000$ \\
\hline
\end{tabular}


Table 29. Concentrations of volatile organic compounds in whole water from Phase I of the Willamette River Basin Water Quality Study, Oregon, 1992-93-Continued

\begin{tabular}{|c|c|c|c|c|c|c|c|c|c|c|c|}
\hline STATION NUMBER & DATE & $\begin{array}{l}\text { ETHYL- } \\
\text { BENZENE } \\
\text { TOTAL } \\
(\text { UG/L) }\end{array}$ & $\begin{array}{l}\text { HEXA- } \\
\text { CHLORO- } \\
\text { BUT- } \\
\text { ADIENE } \\
\text { TOTAL } \\
\text { (UG/L) }\end{array}$ & $\begin{array}{l}\text { ISO- } \\
\text { PROPYL- } \\
\text { BENZENE } \\
\text { TOTAL } \\
\text { (UG/L) }\end{array}$ & $\begin{array}{l}\text { METHYL- } \\
\text { BROMIDE } \\
\text { TOTAL } \\
(\mathrm{UG} / \mathrm{L})\end{array}$ & $\begin{array}{l}\text { METHYL- } \\
\text { ENE } \\
\text { CHLO- } \\
\text { RIDE } \\
\text { TOTAL } \\
\text { (UG/L) }\end{array}$ & $\begin{array}{c}2- \\
\text { METHOXY } \\
2- \\
\text { METHYL } \\
\text { PROPENE } \\
\text { TOTAL } \\
\text { (UG/L) }\end{array}$ & $\begin{array}{l}\mathrm{N}- \\
\text { BUTYL } \\
\text { BENZENE } \\
\text { TOTAL } \\
\text { (UG/L) }\end{array}$ & $\begin{array}{l}\mathrm{N}- \\
\text { PROPYL- } \\
\text { BENZENE } \\
\text { TOTAL } \\
\text { (UG/L) }\end{array}$ & $\begin{array}{l}\text { NAPHTH- } \\
\text { ALENE } \\
\text { TOTAL } \\
\text { (UG/L) }\end{array}$ & $\begin{array}{c}1- \\
\text { METHYL- } \\
4-I S O- \\
\text { PROPYL- } \\
\text { BENZENE } \\
\text { TOTAL } \\
\text { (UG/L) }\end{array}$ \\
\hline 440313123091100 & $\begin{array}{c}04-16-92 \\
10-16-92\end{array}$ & $\begin{array}{l}<0.20000 \\
<0.20000\end{array}$ & $\begin{array}{l}<0.20000 \\
<0.20000\end{array}$ & $\begin{array}{l}<0.20000 \\
<0.20000\end{array}$ & $\begin{array}{l}<0.20000 \\
<0.20000\end{array}$ & $\begin{array}{r}0.80000 \\
<0.20000\end{array}$ & $\begin{array}{l}<1.0000 \\
<1.0000\end{array}$ & $\begin{array}{l}<0.20000 \\
<0.20000\end{array}$ & $\begin{array}{l}<0.20000 \\
<0.20000\end{array}$ & $\begin{array}{l}<0.20000 \\
<0.20000\end{array}$ & $\begin{array}{l}<0.20000 \\
<0.20000\end{array}$ \\
\hline 443207123145500 & $08-24-93$ & $3<0.20000$ & $<5.0000$ & $<0.20000$ & $<0.20000$ & $<0.20000$ & $<1.0000$ & $<0.20000$ & $<0.20000$ & $<5.0000$ & $<0.20000$ \\
\hline 443138123120901 & $08-24-93$ & $3<0.20000$ & $<5.0000$ & $<0.20000$ & $<0.20000$ & $<0.20000$ & $<1.0000$ & $<0.20000$ & $<0.20000$ & $<5.0000$ & $<0.20000$ \\
\hline 451309123041501 & - $09-07-93$ & $3<0.20000$ & $<5.0000$ & $<0.20000$ & $<0.20000$ & $<0.20000$ & $<1.0000$ & $<0.20000$ & $<0.20000$ & $<5.0000$ & $<0.20000$ \\
\hline 14201300 & $\begin{array}{l}06-01-93 \\
07-27-93\end{array}$ & $\begin{array}{l}<0.20000 \\
<0.20000\end{array}$ & $\begin{array}{l}<0.20000 \\
<0.20000\end{array}$ & $\begin{array}{l}<0.20000 \\
<0.20000\end{array}$ & $\begin{array}{l}<0.20000 \\
<0.20000\end{array}$ & $\begin{array}{l}<0.20000 \\
<0.20000\end{array}$ & $\begin{array}{l}<1.0000 \\
<1.0000\end{array}$ & $\begin{array}{l}<0.20000 \\
<0.20000\end{array}$ & $\begin{array}{l}<0.20000 \\
<0.20000\end{array}$ & $\begin{array}{l}<0.20000 \\
<0.20000\end{array}$ & $\begin{array}{l}<0.20000 \\
<0.20000\end{array}$ \\
\hline 14202000 & $04-27-93$ & $<0.20000$ & $<0.20000$ & $<0.20000$ & $<0.20000$ & $<0.20000$ & $<1.0000$ & $<0.20000$ & $<0.20000$ & $<0.20000$ & $<0.20000$ \\
\hline 453115122535500 & $03-31-93$ & $<0.20000$ & $<0.20000$ & $<0.20000$ & $<0.20000$ & $<0.20000$ & $<1.0000$ & $<0.20000$ & $<0.20000$ & $<0.20000$ & $<0.20000$ \\
\hline 14206950 & $\begin{array}{l}03-01-93 \\
07-22-93\end{array}$ & $\begin{array}{l}<0.20000 \\
<0.20000\end{array}$ & $\begin{array}{c}<0.20000 \\
<5.0000\end{array}$ & $\begin{array}{l}<0.20000 \\
<0.20000\end{array}$ & $\begin{array}{l}<0.20000 \\
<0.20000\end{array}$ & $\begin{array}{r}0.30000 \\
<0.20000\end{array}$ & $\begin{array}{l}<1.0000 \\
<1.0000\end{array}$ & $\begin{array}{l}<0.20000 \\
<0.20000\end{array}$ & $\begin{array}{l}<0.20000 \\
<0.20000\end{array}$ & $\begin{array}{r}<0.20000 \\
<5.0000\end{array}$ & $\begin{array}{l}<0.20000 \\
<0.20000\end{array}$ \\
\hline 14211550 & $03-14-93$ & $<0.20000$ & $<0.20000$ & $<0.20000$ & $<0.20000$ & $<0.20000$ & $<1.0000$ & $<0.20000$ & $<0.20000$ & $<0.20000$ & $<0.20000$ \\
\hline 453547122463000 & $10-26-92$ & $<0.20000$ & $<0.20000$ & $<0.20000$ & $<0.20000$ & $<0.20000$ & $<1.0000$ & $<0.20000$ & $<0.20000$ & $<0.20000$ & $<0.20000$ \\
\hline
\end{tabular}

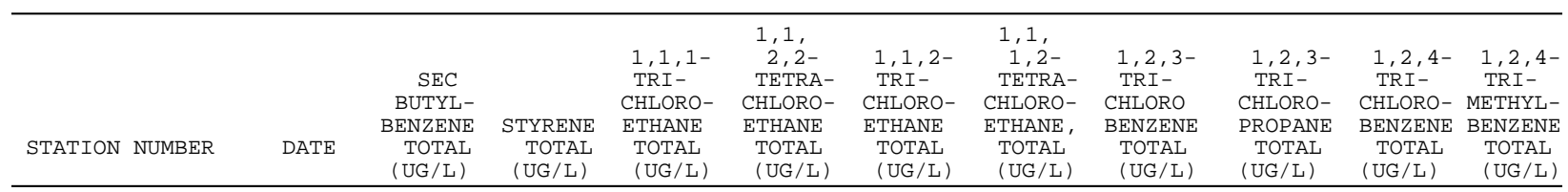

$44031312309110004-16-92<0.20000<0.20000 \quad 0.90000<0.20000<0.20000<0.20000<0.20000<0.20000<0.20000<0.20000$ $10-16-92<0.20000<0.20000 \quad 0.70000<0.20000<0.20000<0.20000<0.20000<0.20000<0.20000<0.20000$

$44320712314550008-24-93<0.20000<0.20000<0.20000<0.20000<0.20000<0.20000<0.20000<0.20000<5.0000<0.20000$

$44313812312090108-24-93<0.20000<0.20000<0.20000<0.20000<0.20000<0.20000<0.20000<0.20000<5.0000<0.20000$

$45130912304150109-07-93<0.20000<0.20000<0.20000<0.20000<0.20000<0.20000<0.20000<0.20000<5.0000<0.20000$

$14201300 \quad 06-01-93<0.20000<0.20000<0.20000<0.20000<0.20000<0.20000<0.20000<0.20000<0.20000<0.20000$ $07-27-93<0.20000<0.20000<0.20000<0.20000<0.20000<0.20000<0.20000<0.20000<0.20000<0.20000$

$14202000 \quad 04-27-93<0.20000<0.20000<0.20000<0.20000<0.20000<0.20000<0.20000<0.20000<0.20000<0.20000$

$453115122535500 \quad 03-31-93<0.20000<0.20000<0.20000<0.20000<0.20000<0.20000<0.20000<0.20000<0.20000<0.20000$

$14206950 \quad 03-01-93<0.20000<0.20000<0.20000<0.20000<0.20000<0.20000<0.20000<0.20000<0.20000<0.20000$ $07-22-93<0.20000<0.20000<0.20000<0.20000<0.20000<0.20000<0.20000<0.20000<5.0000<0.20000$

$14211550 \quad 03-14-93<0.20000<0.20000<0.20000<0.20000<0.20000<0.20000<0.20000<0.20000<0.20000<0.20000$

$45354712246300010-26-92<0.20000<0.20000<0.20000<0.20000<0.20000<0.20000<0.20000<0.20000<0.20000<0.20000$ 
Table 29. Concentrations of volatile organic compounds in whole water from Phase I of the Willamette River Basin Water Quality Study, Oregon, 1992-93-Continued

\begin{tabular}{|c|c|c|c|c|c|c|c|c|c|c|c|}
\hline STATION NUMBER & DATE & $\begin{array}{l}\text { TRANS- } \\
1,2- \\
\text { DI- } \\
\text { CHLORO- } \\
\text { ETHYLENE } \\
\text { TOTAL } \\
\text { (UG/L) }\end{array}$ & $\begin{array}{l}\text { 1, 3,5- } \\
\text { TRI- } \\
\text { METHYL- } \\
\text { BENZENE } \\
\text { TOTAL } \\
\text { (UG/L) }\end{array}$ & $\begin{array}{l}\text { TERT- } \\
\text { - BUTYL- } \\
\text { BENZENE } \\
\text { TOTAL } \\
(\mathrm{UG} / \mathrm{L})\end{array}$ & $\begin{array}{l}\text { TETRA- } \\
\text { CHLORO- } \\
\text { ETHYL- } \\
\text { ENE } \\
\text { TOTAL } \\
\text { (UG/L) }\end{array}$ & $\begin{array}{l}\text { TOLUENE } \\
\text { TOTAL } \\
\text { (UG/L) }\end{array}$ & $\begin{array}{l}\text { TRANS- } \\
1,3-D I- \\
\text { CHLORO- } \\
\text { PROPENE } \\
\text { TOTAL } \\
\text { (UG/L) }\end{array}$ & $\begin{array}{ll} & \text { TRI- } \\
\text { CHLORO- } \\
\text { ETHYL- } \\
\text { ENE } \\
\text { TOTAL } \\
(\text { UG/L) }\end{array}$ & $\begin{array}{l}\text { TRI- } \\
\text { CHLORO- } \\
\text { FLUORO- } \\
\text { METHANE } \\
\text { TOTAL } \\
(\text { UG } / \text { L) }\end{array}$ & $\begin{array}{cc}- & \text { VINYL } \\
- & \text { CHLO- } \\
\text { RIDE } \\
\text { TOTAL } \\
\text { (UG/L) }\end{array}$ & $\begin{array}{l}\text { XYLENE } \\
\text { TOTAL } \\
\text { (UG/L) }\end{array}$ \\
\hline 440313123091100 & $\begin{array}{l}04-16-92 \\
10-16-92\end{array}$ & $\begin{array}{l}<0.20000 \\
<0.20000\end{array}$ & $\begin{array}{l}<0.20000 \\
<0.20000<\end{array}$ & $\begin{array}{l}<0.20000 \\
<0.20000\end{array}$ & $\begin{array}{l}1.8000 \\
1.1000\end{array}$ & $\begin{array}{l}<0.20000 \\
<0.20000\end{array}$ & $\begin{array}{l}<0.20000 \\
<0.20000\end{array}$ & $\begin{array}{r}0.90000 \\
1.3000\end{array}$ & $\begin{array}{l}<0.20000 \\
<0.20000\end{array}$ & $\begin{array}{l}<0.20000 \\
<0.20000\end{array}$ & $\begin{array}{l}<0.20000 \\
<0.20000\end{array}$ \\
\hline 443207123145500 & $08-24-93$ & $<0.20000$ & $<0.20000$ & $<0.20000$ & $<0.20000$ & $<0.20000$ & $<0.20000$ & $<0.20000$ & $<0.20000$ & $<0.20000$ & $<0.20000$ \\
\hline 443138123120901 & $08-24-93$ & $<0.20000$ & $<0.20000$ & $<0.20000$ & $<0.20000$ & $<0.20000$ & $<0.20000$ & $<0.20000$ & $<0.20000$ & $<0.20000$ & $<0.20000$ \\
\hline 451309123041501 & $09-07-93$ & $<0.20000$ & $<0.20000$ & $<0.20000$ & $<0.20000$ & $<0.20000$ & $<0.20000$ & $<0.20000$ & $<0.20000$ & $<0.20000$ & $<0.20000$ \\
\hline 14201300 & $\begin{array}{l}06-01-93 \\
07-27-93\end{array}$ & $\begin{array}{l}<0.20000 \\
<0.20000\end{array}$ & $\begin{array}{l}<0.20000< \\
<0.20000<\end{array}$ & $\begin{array}{l}<0.20000 \\
<0.20000\end{array}$ & $\begin{array}{l}<0.20000 \\
<0.20000\end{array}$ & $\begin{array}{l}<0.20000 \\
<0.20000\end{array}$ & $\begin{array}{l}<0.20000 \\
<0.20000\end{array}$ & $\begin{array}{l}<0.20000 \\
<0.20000\end{array}$ & $\begin{array}{l}<0.20000 \\
<0.20000\end{array}$ & $\begin{array}{l}<0.20000 \\
<0.20000\end{array}$ & $\begin{array}{l}<0.20000 \\
<0.20000\end{array}$ \\
\hline 14202000 & $04-27-93$ & $<0.20000$ & $<0.20000<$ & $<0.20000$ & $<0.20000$ & $<0.20000$ & $<0.20000$ & $<0.20000$ & $<0.20000$ & $<0.20000$ & $<0.20000$ \\
\hline 453115122535500 & $03-31-93$ & $<0.20000$ & $<0.20000<$ & $<0.20000$ & $<0.20000$ & $<0.20000$ & $<0.20000$ & $<0.20000$ & $<0.20000$ & $<0.20000$ & $<0.20000$ \\
\hline 14206950 & $\begin{array}{l}03-01-93 \\
07-22-93\end{array}$ & $\begin{array}{l}<0.20000 \\
<0.20000\end{array}$ & $\begin{array}{l}<0.20000< \\
<0.20000<\end{array}$ & $\begin{array}{l}<0.20000 \\
<0.20000\end{array}$ & $\begin{array}{l}0.30000 \\
0.40000\end{array}$ & $\begin{array}{l}<0.20000 \\
<0.20000\end{array}$ & $\begin{array}{l}<0.20000 \\
<0.20000\end{array}$ & $\begin{array}{l}0.60000 \\
0.20000\end{array}$ & $\begin{array}{l}<0.20000 \\
<0.20000\end{array}$ & $\begin{array}{l}<0.20000 \\
<0.20000\end{array}$ & $\begin{array}{l}<0.20000 \\
<0.20000\end{array}$ \\
\hline 14211550 & $03-14-93$ & $<0.20000$ & $<0.20000<$ & $<0.20000$ & $<0.20000$ & $<0.20000$ & $<0.20000$ & $<0.20000$ & $<0.20000$ & $<0.20000$ & $<0.20000$ \\
\hline 453547122463000 & $10-26-92$ & $<0.20000$ & $<0.20000$ & $<0.20000$ & $<0.20000$ & $<0.20000$ & $<0.20000$ & $<0.20000$ & $<0.20000$ & $<0.20000$ & $<0.20000$ \\
\hline
\end{tabular}


Table 30. Concentrations of volatile organic compounds in whole water from Phase II of the Willamette River Basin Water Quality Study, Oregon, 1994 [USGS laboratory schedule 2090; UG/L, micrograms per liter]

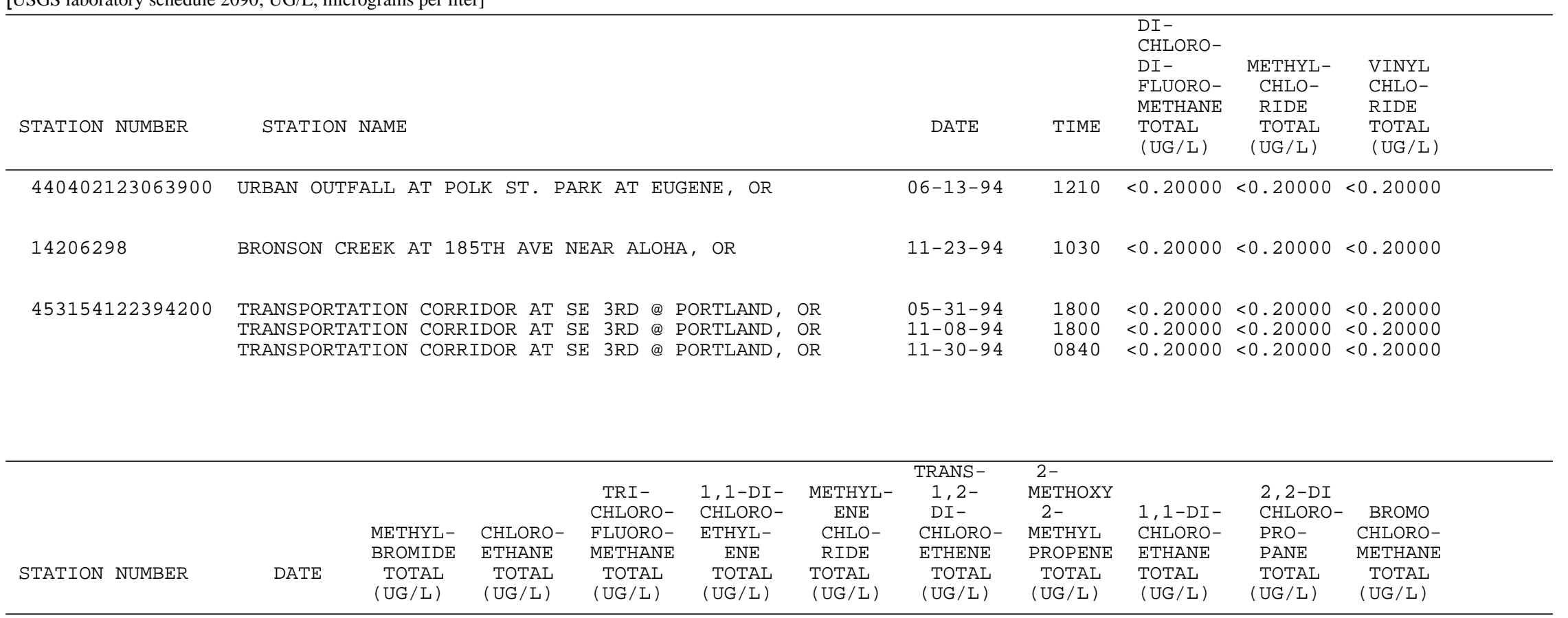

\begin{tabular}{|c|c|c|c|c|c|c|c|c|c|c|c|}
\hline 440402123063900 & $06-13-94$ & $<0.20000$ & $<0.20000$ & $<0.20000$ & 0.30000 & $<0.20000$ & $<0.20000$ & $<0.20000$ & $<0.20000$ & $<0.20000$ & $<0.20000$ \\
\hline 14206298 & $11-23-94$ & $<0.20000$ & $<0.20000$ & $<0.20000$ & $<0.20000$ & $<0.20000$ & $<0.20000$ & $<0.20000$ & $<0.20000$ & $<0.20000$ & $<0.20000$ \\
\hline 453154122394200 & $\begin{array}{l}05-31-94 \\
11-08-94 \\
11-30-94\end{array}$ & $\begin{array}{l}<0.20000 \\
<0.20000 \\
<0.20000\end{array}$ & $\begin{array}{l}<0.20000 \\
<0.20000 \\
<0.20000\end{array}$ & $\begin{array}{l}<0.20000 \\
<0.20000 \\
<0.20000\end{array}$ & $\begin{array}{l}<0.20000 \\
<0.20000 \\
<0.20000\end{array}$ & $\begin{array}{r}0.30000 \\
<0.20000 \\
<0.20000\end{array}$ & $\begin{array}{l}<0.20000 \\
<0.20000 \\
<0.20000\end{array}$ & $\begin{array}{l}<0.20000 \\
<0.20000 \\
<0.20000\end{array}$ & $\begin{array}{l}<0.20000 \\
<0.20000 \\
<0.20000\end{array}$ & $\begin{array}{l}<0.20000 \\
<0.20000 \\
<0.20000\end{array}$ & $\begin{array}{l}<0.20000 \\
<0.20000 \\
<0.20000\end{array}$ \\
\hline
\end{tabular}


Table 30. Concentrations of volatile organic compounds in whole water from Phase II of the Willamette River Basin Water Quality Study, Oregon, 1994-Continued

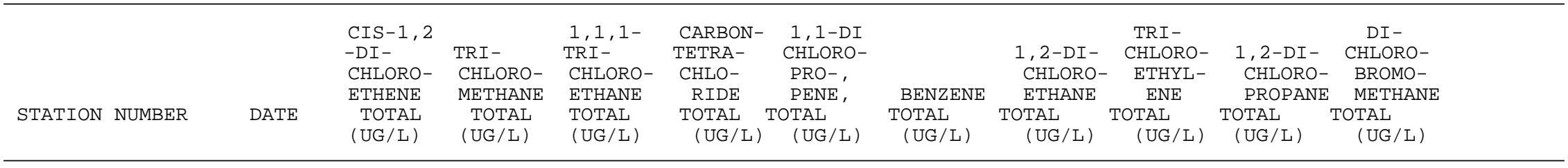

\begin{tabular}{|c|c|c|c|c|c|c|c|c|c|c|c|}
\hline 440402123063900 & $06-13-94$ & 0.80000 & 0.80000 & 0.80000 & $<0.20000$ & $<0.20000$ & $<0.20000$ & $<0.20000$ & $<0.20000$ & $<0.20000$ & $<0.20000$ \\
\hline 14206298 & $11-23-94$ & $<0.20000$ & $<0.20000$ & $<0.20000$ & $<0.20000$ & $<0.20000$ & $<0.20000$ & $<0.20000$ & $<0.20000$ & $<0.20000$ & $<0.20000$ \\
\hline 453154122394200 & $\begin{array}{l}05-31-94 \\
11-08-94 \\
11-30-94\end{array}$ & $\begin{array}{l}<0.20000 \\
<0.20000 \\
<0.20000\end{array}$ & $\begin{array}{l}<0.20000 \\
<0.20000 \\
<0.20000\end{array}$ & $\begin{array}{l}<0.20000 \\
<0.20000 \\
<0.20000\end{array}$ & $\begin{array}{l}<0.20000 \\
<0.20000 \\
<0.20000\end{array}$ & $\begin{array}{l}<0.20000 \\
<0.20000 \\
<0.20000\end{array}$ & $\begin{array}{r}1.5000 \\
1.0000 \\
0.30000\end{array}$ & $\begin{array}{l}<0.20000 \\
<0.20000 \\
<0.20000\end{array}$ & $\begin{array}{l}<0.20000 \\
<0.20000 \\
<0.20000\end{array}$ & $\begin{array}{l}<0.20000 \\
<0.20000 \\
<0.20000\end{array}$ & $\begin{array}{l}<0.20000 \\
<0.20000 \\
<0.20000\end{array}$ \\
\hline
\end{tabular}

$11-30-94<0.20000<0.20000<0.20000<0.20000<0.20000 \quad 0.30000<0.20000<0.20000<0.20000<0.20000$

\begin{tabular}{|c|c|c|c|c|c|c|c|c|c|c|c|}
\hline & & $\begin{array}{l}\text { DI- } \\
\text { BROMO- } \\
\text { METHANE }\end{array}$ & $\begin{array}{l}\text { CIS } \\
1,3-D I- \\
\text { CHLORO- } \\
\text { PROPENE }\end{array}$ & TOLUENE & $\begin{array}{l}\text { TRANS- } \\
1,3-D I- \\
\text { CHLORO- } \\
\text { PROPENE }\end{array}$ & $\begin{array}{c}1,1,2- \\
\text { TRI- } \\
\text { CHLORO- } \\
\text { ETHANE }\end{array}$ & $\begin{array}{l}\text { TETRA- } \\
\text { CHLORO- } \\
\text { ETHYL- } \\
\text { ENE }\end{array}$ & $\begin{array}{l}1,3-D I- \\
\text { CHLORO- } \\
\text { PROPANE }\end{array}$ & $\begin{array}{l}\text { DI- } \\
\text { BROMO- } \\
\text { CHLORO- } \\
\text { METHANE }\end{array}$ & $\begin{array}{l}1,2- \\
\text { DIBROMO } \\
\text { ETHANE }\end{array}$ & $\begin{array}{l}\text { CHLORO- } \\
\text { BENZENE }\end{array}$ \\
\hline STATION NUMBER & DATE & $\begin{array}{l}\text { TOTAL } \\
\text { (UG/L) }\end{array}$ & $\begin{array}{l}\text { TOTAL } \\
\text { (UG/L) }\end{array}$ & $\begin{array}{l}\text { TOTAL } \\
\text { (UG/L) }\end{array}$ & $\begin{array}{l}\text { TOTAL } \\
\text { (UG/L) }\end{array}$ & $\begin{array}{l}\text { TOTAL } \\
\text { (UG/L) }\end{array}$ & $\begin{array}{l}\text { TOTAL } \\
\text { (UG/L) }\end{array}$ & $\begin{array}{l}\text { TOTAL } \\
\text { (UG/L) }\end{array}$ & $\begin{array}{l}\text { TOTAL } \\
\text { (UG/L) }\end{array}$ & $\begin{array}{l}\text { TOTAL } \\
\text { (UG/L) }\end{array}$ & $\begin{array}{l}\text { TOTAL } \\
\quad(\mathrm{UG} / \mathrm{L})\end{array}$ \\
\hline
\end{tabular}

\footnotetext{
$44040212306390006-13-94<0.20000<0.20000<0.20000<0.20000<0.200000 .40000<0.20000<0.20000<0.20000<0.20000$

14206298

$11-23-94<0.20000<0.20000<0.20000<0.20000<0.20000<0.20000<0.20000<0.20000<0.20000<0.20000$

$453154122394200 \quad 05-31-94<0.20000<0.20000 \quad 0.20000<0.20000<0.20000<0.20000<0.20000<0.20000<0.20000<0.20000$ $11-08-94<0.20000<0.20000 \quad 0.70000<0.20000<0.20000<0.20000<0.20000<0.20000<0.20000<0.20000$ $11-30-94<0.20000<0.20000<0.20000<0.20000<0.20000<0.20000<0.20000<0.20000<0.20000<0.20000$
} 
Table 30. Concentrations of volatile organic compounds in whole water from Phase II of the Willamette River Basin Water Quality Study, Oregon, 1994—Continued

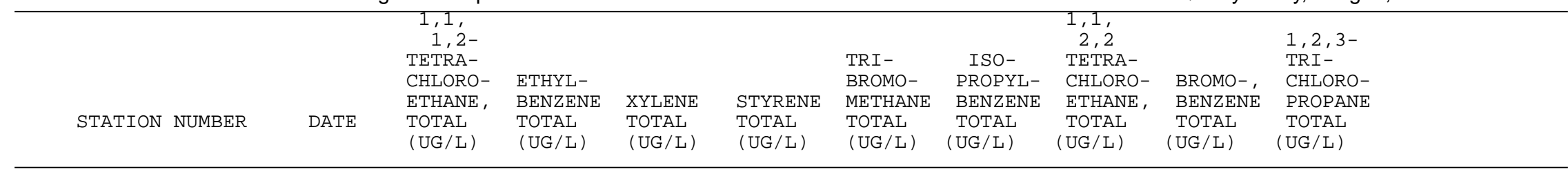

$44040212306390006-13-94<0.20000<0.20000<0.20000<0.20000<0.20000<0.20000<0.20000<0.20000<0.20000$

$14206298 \quad 11-23-94<0.20000<0.20000<0.20000<0.20000<0.20000<0.20000<0.20000<0.20000<0.20000$

$453154122394200 \quad 05-31-94<0.20000<0.20000 \quad 0.40000<0.20000<0.20000<0.20000<0.20000<0.20000<0.20000$ $\begin{array}{ll}11-08-94<0.20000<0.20000 & 0.60000<0.20000<0.20000<0.20000<0.20000<0.20000<0.20000 \\ 11-30-94<0.20000<0.20000<0.20000<0.20000<0.20000<0.20000<0.20000<0.20000<0.20000\end{array}$

\&

\begin{tabular}{|c|c|c|c|c|c|c|c|c|c|c|}
\hline & & & $1-$ & & & & $1-$ & & & \\
\hline & & & $\begin{array}{l}\text { CHLORO- } \\
2-\end{array}$ & TERT- & $\begin{array}{l}1,2,4- \\
\text { TRI- }\end{array}$ & SEC & $\begin{array}{l}\text { METHYL } \\
\text { 4-ISO- }\end{array}$ & $1,3-D I-$ & $1,4-D I-$ & \\
\hline & & $\begin{array}{l}\text { N-PROPY } \\
\text { BENZENE }\end{array}$ & $\begin{array}{l}\text { METHYL } \\
\text { BENZENE }\end{array}$ & $\begin{array}{l}\text { BUTYL- } \\
\text { BENZENE }\end{array}$ & $\begin{array}{l}\text { METHYL } \\
\text { BENZENE }\end{array}$ & $\begin{array}{l}\text { BUTYL- } \\
\text { BENZENE }\end{array}$ & $\begin{array}{l}\text { PROPYL- } \\
\text { BENZENE }\end{array}$ & $\begin{array}{l}\text { CHLORO- } \\
\text { BENZENE }\end{array}$ & $\begin{array}{l}\text { CHLORO- } \\
\text { BENZENE }\end{array}$ & $\begin{array}{l}\text { N-BUTYL } \\
\text { BENZENE }\end{array}$ \\
\hline STATION NUMBER & DATE & $\begin{array}{l}\text { TOTAL } \\
\text { (UG/L) }\end{array}$ & $\begin{array}{l}\text { TOTAL } \\
\text { (UG/L) }\end{array}$ & $\begin{array}{l}\text { TOTAL } \\
\text { (UG/L) }\end{array}$ & $\begin{array}{l}\text { TOTAL } \\
\text { (UG/L) }\end{array}$ & $\begin{array}{l}\text { TOTAL } \\
\text { (UG/L) }\end{array}$ & $\begin{array}{l}\text { TOTAL } \\
\text { (UG/L) }\end{array}$ & $\begin{array}{l}\text { TOTAL } \\
\text { (UG/L) }\end{array}$ & $\begin{array}{l}\text { TOTAL } \\
\text { (UG/L) }\end{array}$ & $\begin{array}{l}\text { TOTAL } \\
\text { (UG/L) }\end{array}$ \\
\hline
\end{tabular}

$44040212306390006-13-94<0.20000<0.20000<0.20000<0.20000<0.20000<0.20000<0.20000<0.20000<0.20000$

$14206298 \quad 11-23-94<0.20000<0.20000<0.20000<0.20000<0.20000<0.20000<0.20000<0.20000<0.20000$

$453154122394200 \quad 05-31-94<0.20000<0.20000<0.20000<0.20000<0.20000<0.20000<0.20000<0.20000<0.20000$ $11-08-94<0.20000<0.20000<0.20000 \quad 0.20000<0.20000<0.20000<0.20000<0.20000<0.20000$ $11-30-94<0.20000<0.20000<0.20000<0.20000<0.20000<0.20000<0.20000<0.20000<0.20000$ 
Table 30. Concentrations of volatile organic compounds in whole water from Phase II of the Willamette River Basin Water Quality Study, Oregon, 1994—Continued

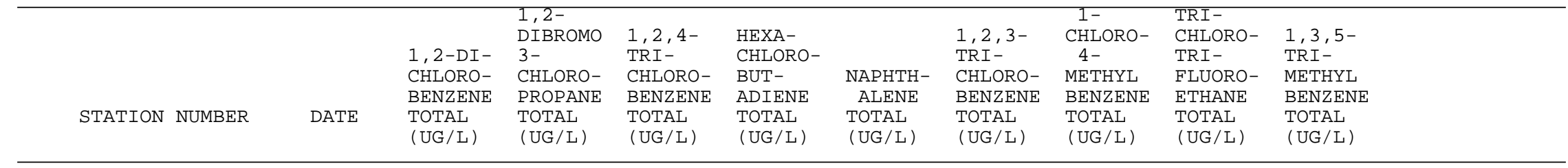

$440402123063900 \quad 06-13-94<0.20000<1.0000<0.20000<0.20000<0.20000<0.20000<0.20000<0.20000<0.20000$

$14206298 \quad 11-23-94<0.20000<1.0000<0.20000<0.20000<0.20000<0.20000<0.20000<0.20000<0.20000$

$453154122394200 \quad 05-31-94<0.20000<1.0000<0.20000<0.20000<0.20000<0.20000<0.20000<0.20000 \quad 0.30000$

$11-08-94<0.20000<1.0000<0.20000<0.20000 \quad 0.50000<0.20000<0.20000<0.20000<0.20000$

$11-30-94<0.20000<1.0000<0.20000<0.20000<0.20000<0.20000<0.20000<0.20000<0.20000$ 
Table 31. Concentrations of dioxin associated with suspended sediment from Phase I of the Willamette River Basin Water Quality Study, Oregon, 1992 [E = estimate; $\mathrm{PG} / \mathrm{KL}$, picograms per kiloliter]

STATION NUMBER STATION NAME TCDD 2,3,7,8- PeCDD 1,2,
$3,7,8-$ $1,2,3$
$4,7,8-$ $\begin{array}{llllll}1,2, & & 1,2,3, & 1,2,3, & 1,2,3, & \\ 3,7,8- & \text { HxCDD } & 4,7,8- & 6,7,8- & 7,8,9 & \text { HpCDD } \\ \text { PeCDD } & \text { (total) } & \text { HxCDD } & \text { HxCDD } & \text { HxCDD } & \text { (total) }\end{array}$

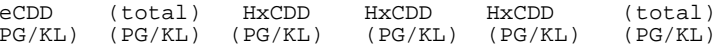


Table 32. Concentrations of dioxin associated with bed sediment from Phase I of the Willamette River Basin Water Quality Study, Oregon, 1992 [PG/G, picograms per gram]

\begin{tabular}{|c|c|c|c|c|c|c|c|}
\hline STATION NUMBER & STATION NAME & DATE & TIME & $\begin{array}{l}\text { TCDDs } \\
\text { (TOTAL) } \\
\text { (PG/G) }\end{array}$ & $\begin{array}{l}2,3,7,8- \\
\text { TCDD } \\
(\mathrm{PG} / \mathrm{G})\end{array}$ & $\begin{array}{l}\text { PeCDDs } \\
\text { (TOTAL) } \\
\text { (PG/G) }\end{array}$ & $\begin{array}{l}1,2, \\
3,7,8- \\
\text { PeCDD } \\
\text { (PG/G) }\end{array}$ \\
\hline $\begin{array}{l}440313123091100 \\
443207123145500 \\
444032123050600 \\
451320123041100 \\
451705122575100\end{array}$ & $\begin{array}{l}\text { A-3 CHANNEL AT WALLIS AND } 5 \text { TH ST AT EUGENE, OR } \\
\text { WILLAMETTE RIVER NEAR CORVALLIS, OR } \\
\text { MIDDLE FOURTH LAKE NEAR ALBANY, OR } \\
\text { YAMHILL RIVER AT DAYTON, OR } \\
\text { WILLAMETTE RIVER AT NEWBERG, OR }\end{array}$ & $\begin{array}{l}08-25-92 \\
08-26-92 \\
09-01-92 \\
08-27-92 \\
09-01-92\end{array}$ & $\begin{array}{l}1230 \\
1030 \\
1100 \\
1400 \\
1600\end{array}$ & $\begin{array}{l}55 \\
<2.4 \\
16 \\
<0.67 \\
<1.5\end{array}$ & $\begin{array}{l}3.0 \\
<0.90 \\
<2.5 \\
<0.49 \\
<0.55\end{array}$ & $\begin{array}{l}210 \\
<2.2 \\
27 \\
<1.5 \\
<2.2\end{array}$ & $\begin{array}{l}20 \\
<1.1 \\
<4.2 \\
<0.98 \\
<0.64\end{array}$ \\
\hline $\begin{array}{l}452950122492900 \\
453547122463000 \\
453205122223700\end{array}$ & $\begin{array}{l}\text { BEAVERTON CREEK AT BEAVERTON, OR } \\
\text { WILLAMETTE RIVER AT LINNTON, OR } \\
\text { BEAVER C NEAR TROUTDALE, OR }\end{array}$ & $\begin{array}{l}08-23-92 \\
09-09-92 \\
08-22-92\end{array}$ & $\begin{array}{l}1100 \\
1130 \\
0945\end{array}$ & $\begin{array}{l}9.3 \\
<0.78 \\
<0.91\end{array}$ & $\begin{array}{l}<2.2 \\
<0.78 \\
<0.91\end{array}$ & $\begin{array}{l}26 \\
<6.4 \\
<2.7\end{array}$ & $\begin{array}{l}<5.8 \\
<1.2 \\
<1.2\end{array}$ \\
\hline
\end{tabular}

\begin{tabular}{|c|c|c|c|c|c|c|c|c|}
\hline STATION NUMBER & DATE & $\begin{array}{l}\text { HXCDDs } \\
\text { (TOTAL) } \\
\text { (PG/G) }\end{array}$ & $\begin{array}{l}1,2,3, \\
4,7,8- \\
\text { HxCDD } \\
(\mathrm{PG} / \mathrm{G})\end{array}$ & $\begin{array}{l}1,2,3, \\
6,7,8- \\
\text { HXCDD } \\
\text { (PG/G) }\end{array}$ & $\begin{array}{l}1,2,3, \\
7,8,9 \\
\text { HxCDD } \\
\text { (PG/G) }\end{array}$ & $\begin{array}{l}\text { HpCDDs } \\
\text { (TOTAL) } \\
\text { (PG/G) }\end{array}$ & $\begin{array}{l}1,2,3,4, \\
6,7,8- \\
\text { HpCDD } \\
\text { (PG/G) }\end{array}$ & $\begin{array}{c}\mathrm{OCDD} \\
(\mathrm{PG} / \mathrm{G})\end{array}$ \\
\hline $\begin{array}{l}440313123091100 \\
443207123145500 \\
444032123050600 \\
451320123041100 \\
451705122575100\end{array}$ & $\begin{array}{l}08-25-92 \\
08-26-92 \\
09-01-92 \\
08-27-92 \\
09-01-92\end{array}$ & $\begin{aligned} 1000 \\
<11 \\
280 \\
<3.0 \\
<6.1\end{aligned}$ & $\begin{array}{l}30 \\
<1.2 \\
<7.1 \\
<0.27 \\
<0.34\end{array}$ & $\begin{array}{l}200 \\
<1.9 \\
47 \\
<0.93 \\
<1.0\end{array}$ & $\begin{array}{l}65 \\
<0.84 \\
19 \\
<0.26 \\
<0.58\end{array}$ & $\begin{array}{r}5200 \\
33 \\
1700 \\
26 \\
25\end{array}$ & $\begin{array}{r}3200 \\
16 \\
880 \\
14 \\
13\end{array}$ & $\begin{array}{r}32000 \\
120 \\
8500 \\
110 \\
98\end{array}$ \\
\hline $\begin{array}{l}452950122492900 \\
453547122463000 \\
453205122223700\end{array}$ & $\begin{array}{l}08-23-92 \\
09-09-92 \\
08-22-92\end{array}$ & $\begin{array}{l}190 \\
38 \\
35\end{array}$ & $\begin{array}{l}<8.9 \\
<1.6 \\
<2.1\end{array}$ & $\begin{array}{l}38 \\
<7.3 \\
8.2\end{array}$ & $\begin{array}{l}17 \\
<2.7 \\
<3.9\end{array}$ & $\begin{array}{r}1500 \\
280 \\
260\end{array}$ & $\begin{array}{l}800 \\
130 \\
160\end{array}$ & $\begin{array}{l}6100 \\
1300 \\
1400\end{array}$ \\
\hline
\end{tabular}


Table 33. Concentrations of furans associated with suspended sediment from Phase I of the Willamette River Basin Water Quality Study, Oregon, 1992 [E = estimate; $\mathrm{PG} / \mathrm{KL}$, picograms per kiloliter]

\begin{tabular}{|c|c|c|c|c|c|c|c|c|c|c|c|c|}
\hline STATION NUMBER & STATION NAME & DATE & TIME & $\begin{array}{l}\text { TCDF } \\
\text { (TOTAL) } \\
\text { (PG/KL) }\end{array}$ & $\begin{array}{l}2,3,7 \\
8-T C D F \\
\text { (PG/KL) }\end{array}$ & $\begin{array}{l}\text { PeCDF } \\
\text { (TOTAL) } \\
\text { (PG/KL) }\end{array}$ & $\begin{array}{l}1,2,3,7, \\
\text { 8-PeCDF } \\
\text { (PG/KL) }\end{array}$ & $\begin{array}{l}2,3,4,7, \\
8-\mathrm{PeCDF} \\
\text { (PG/KL) }\end{array}$ & $\begin{array}{l}\text { HXCDF } \\
\text { (TOTAL) } \\
\text { (PG/KL) }\end{array}$ & $\begin{array}{l}1,2, \\
3,4,7 \\
8-\mathrm{HXCDF} \\
(\mathrm{PG} / \mathrm{KL})\end{array}$ & $\begin{array}{l}1,2, \\
3,6,7, \\
8-\mathrm{H} \times \mathrm{CDF} \\
(\mathrm{PG} / \mathrm{KL})\end{array}$ & $\begin{array}{l}2,3, \\
4,6,7 \\
8-\mathrm{H} \times \mathrm{CDF} \\
\text { (PG/KL) }\end{array}$ \\
\hline 440313123091100 & A-3 CHANNEL AT & $04-16-92$ & 1410 & 47000 & 2500 & 58000 & 1900 & 5500 & 260000 & 6900 & 7700 & 18000 \\
\hline
\end{tabular}

WALLIS AND 5TH
ST AT EUGENE, OR

\begin{tabular}{|c|c|c|c|c|c|c|}
\hline \multirow[t]{2}{*}{ STATION NUMBER } & \multirow{2}{*}{$\begin{array}{l}\text { DATE } \\
04-16-92\end{array}$} & \multirow{2}{*}{$\begin{array}{l}1,2, \\
3,7,8, \\
9-\mathrm{HXCDF} \\
\text { (PG/KL) } \\
\end{array}$} & \multirow{2}{*}{$\begin{array}{l}\text { HPCDF } \\
\text { (TOTAL) } \\
\text { (PG/KL) }\end{array}$} & $\begin{array}{l}1,2,3 \\
4,6,7 \\
8-\mathrm{HpCDF} \\
\text { (PG/KL) } \\
\end{array}$ & $\begin{array}{l}1,2,3 \\
4,7,8, \\
9-\mathrm{H} P \mathrm{CDF} \\
\text { (PG/KL) } \\
\end{array}$ & $\begin{array}{l}\mathrm{OCDF} \\
(\mathrm{PG} / \mathrm{KL})\end{array}$ \\
\hline & & & & 9600 & E410000 & \\
\hline
\end{tabular}


Table 34. Concentrations of furans associated with bed sediment from Phase I of the Willamette River Basin Water Quality Study, Oregon, 1992 PG/G, picograms per gram

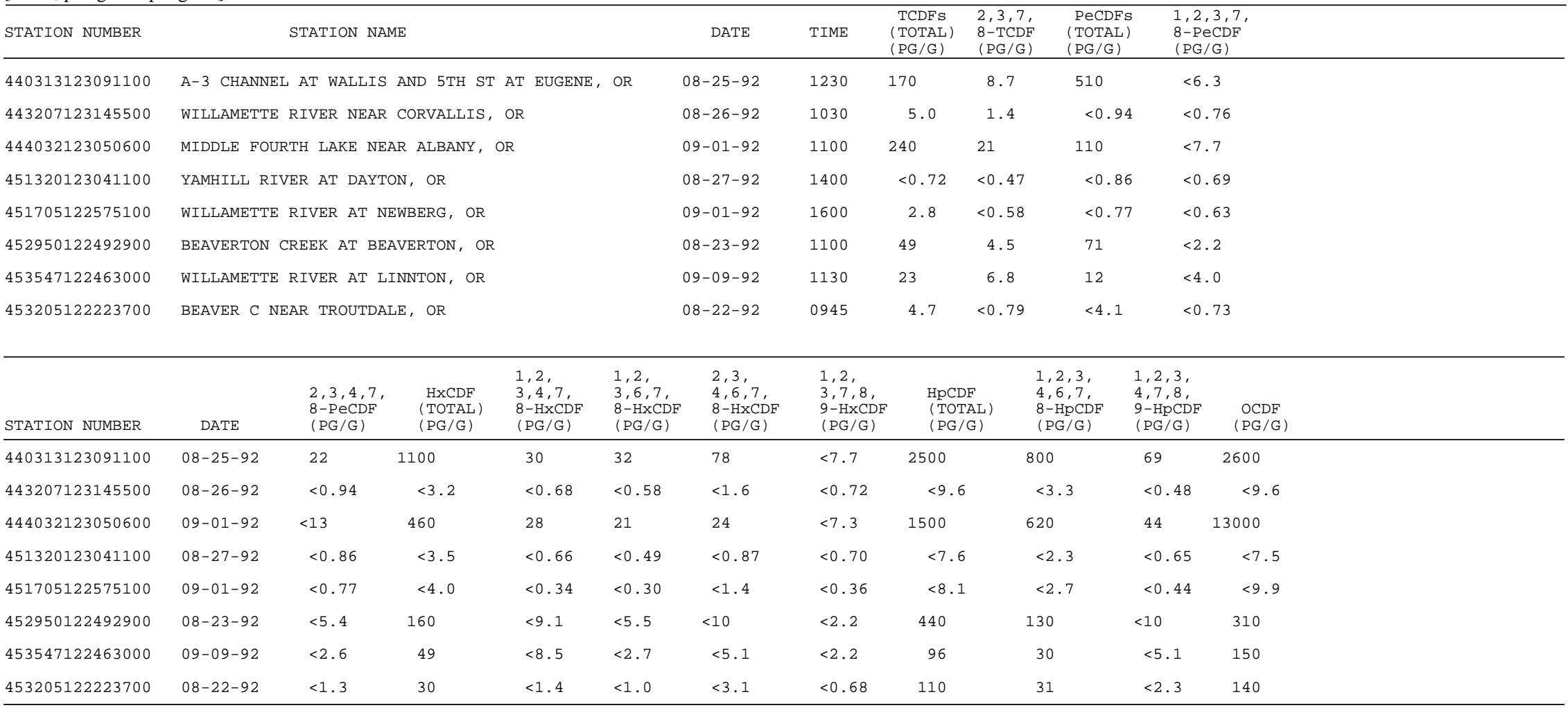


Table 35. Concentrations of pesticides in filtered water analyzed by gas chromatography/mass spectroscopy from Phase I of the Willamette River Basin Water Quality Study, Oregon, 1992-93

[USGS laboratory schedule 2010; < , less than; E, estimate; UG/L, micrograms per liter]




Table 35. Concentrations of pesticides in filtered water analyzed by gas chromatography/mass spectroscopy from Phase I of the Willamette River Basin Water Quality Study, Oregon, 1992-93-Continued

\begin{tabular}{|c|c|c|c|c|c|c|c|c|c|c|}
\hline STATION NUMBER & DATE & $\begin{array}{l}\text { TRIAL- } \\
\text { LATE } \\
\text { WATER } \\
\text { FLTRD } \\
0.7 \text { U } \\
\text { GF, REC } \\
\text { (UG/L) }\end{array}$ & $\begin{array}{l}\text { BEN- } \\
\text { FLUR- } \\
\text { ALIN } \\
\text { WAT FLD } \\
0.7 \text { U } \\
\text { GF, REC } \\
\text { (UG/L) }\end{array}$ & $\begin{array}{l}\text { ETHAL- } \\
\text { FLUR- } \\
\text { ALIN } \\
\text { WAT FLT } \\
0.7 \mathrm{U} \\
\text { GF' REC } \\
\text { (UG/L) }\end{array}$ & $\begin{array}{l}\text { PENDI- } \\
\text { METH- } \\
\text { ALIN } \\
\text { WAT FLT } \\
0.7 \mathrm{U} \\
\text { GF, REC } \\
\text { (UG/L) }\end{array}$ & $\begin{array}{l}\text { TRI- } \\
\text { FLUR- } \\
\text { ALIN } \\
\text { WAT FLT } \\
0.7 \mathrm{U} \\
\text { GF, REC } \\
\text { (UG/L) }\end{array}$ & $\begin{array}{l}2,6-D I- \\
\text { ETHYL } \\
\text { ANILINE } \\
\text { WAT FLT } \\
0.7 \text { U } \\
\text { GF, REC } \\
\text { (UG/L) }\end{array}$ & $\begin{array}{l}\text { PRO- } \\
\text { PARGITE } \\
\text { WATER } \\
\text { FLTRD } \\
0.7 \text { U } \\
\text { GF, REC } \\
\text { (UG/L) }\end{array}$ & 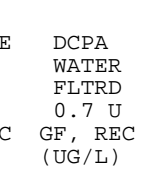 & $\begin{array}{l}\text { DI- } \\
\text { ELDRIN } \\
\text { DIS- } \\
\text { SOLVED } \\
\text { (UG/L) }\end{array}$ \\
\hline 440313123091100 & $\begin{array}{l}04-16-92 \\
10-16-92 \\
10-29-92\end{array}$ & $\begin{array}{l}<0.00100 \\
<0.00100 \\
<0.00100\end{array}$ & $\begin{array}{r}<0.00200 \\
<0.00200 \\
0.01200\end{array}$ & $\begin{array}{l}<0.00400 \\
<0.00400 \\
<0.00400\end{array}$ & $\begin{array}{l}<0.00400 \\
<0.00400 \\
<0.00400\end{array}$ & $\begin{array}{l}<0.00200 \\
<0.00200 \\
<0.00200\end{array}$ & $\begin{array}{l}<0.00300 \\
<0.00300 \\
<0.00300\end{array}$ & $\begin{array}{l}<0.01300 \\
<0.01300 \\
<0.01300\end{array}$ & $\begin{array}{l}<0.05000 \\
<0.00200 \\
0.004000\end{array}$ & $\begin{array}{l}<0.05000 \\
<0.00100 \\
<0.00100\end{array}$ \\
\hline 443207123145500 & $08-24-93$ & $<0.00100$ & $<0.00200$ & $<0.00400$ & $<0.00400$ & $<0.00200$ & $<0.00300$ & $<0.01300$ & $<0.00200$ & $<0.00100$ \\
\hline 443138123120901 & $08-24-93$ & $<0.00100$ & $<0.00200$ & $<0.00400$ & $<0.00400$ & $<0.00200$ & $<0.00300$ & $<0.01300$ & $<0.00200$ & $<0.00100$ \\
\hline 451309123041501 & $09-07-93$ & $<0.00100$ & $<0.00200$ & $<0.00400$ & $<0.00400$ & $<0.00200$ & $<0.00300$ & $<0.01300$ & $<0.00200$ & $<0.00100$ \\
\hline 14201300 & $\begin{array}{l}06-01-93 \\
07-27-93\end{array}$ & $\begin{array}{l}<0.00100 \\
<0.00100\end{array}$ & $\begin{array}{l}<0.00200 \\
<0.00200\end{array}$ & $\begin{array}{l}<0.00400 \\
<0.00400\end{array}$ & $\begin{array}{l}<0.00400 \\
<0.00400\end{array}$ & $\begin{array}{r}0.01200 \\
<0.00200\end{array}$ & $\begin{array}{l}<0.00300 \\
<0.00300\end{array}$ & $\begin{array}{l}<0.01300 \\
<0.01300\end{array}$ & $\begin{array}{r}0.01500 \\
<0.00200\end{array}$ & $\begin{array}{l}<0.00100 \\
<0.00100\end{array}$ \\
\hline 14202000 & $04-27-93$ & $<0.00100$ & $<0.00200$ & $<0.00400$ & $<0.00400$ & $<0.00200$ & $<0.00300$ & $<0.01300$ & 0.002000 & $<0.00100$ \\
\hline 453115122535500 & $03-31-93$ & $<0.00100$ & $<0.00200$ & $<0.00400$ & $<0.00400$ & $<0.00200$ & $<0.00300$ & $<0.01300$ & $<0.00200$ & $<0.00100$ \\
\hline 14206950 & $\begin{array}{l}03-01-93 \\
07-22-93\end{array}$ & $\begin{array}{l}<0.00100 \\
<0.00100\end{array}$ & $\begin{array}{l}<0.00200 \\
<0.00200\end{array}$ & $\begin{array}{l}<0.00400 \\
<0.00400\end{array}$ & $\begin{array}{l}<0.00400 \\
<0.00400\end{array}$ & $\begin{array}{l}<0.00200 \\
<0.00200\end{array}$ & $\begin{array}{l}<0.00300 \\
<0.00300\end{array}$ & $\begin{array}{l}<0.01300 \\
<0.01300\end{array}$ & $\begin{array}{r}<0.00200 \\
0.01000\end{array}$ & $\begin{array}{l}<0.00100 \\
<0.00100\end{array}$ \\
\hline 14211550 & $03-14-93$ & $<0.00100$ & $<0.00200$ & $<0.00400$ & $<0.00400$ & $<0.00200$ & $<0.00300$ & $<0.01300$ & $<0.00200$ & $<0.00100$ \\
\hline 453547122463000 & $10-26-92$ & 0.002000 & 0.006000 & $<0.00400$ & $<0.00400$ & 0.007000 & $<0.00300$ & $<0.01300$ & 0.002000 & $<0.00100$ \\
\hline STATION NUMBER & DATE & $\begin{array}{c}\text { P, } \mathrm{P}^{\prime} \\
\text { DDE } \\
\text { DISSOLV } \\
(\mathrm{UG} / \mathrm{L}) \\
\end{array}$ & $\begin{array}{c}\text { ALPHA } \\
\text { HCH } \\
\text { DIS- } \\
\text { SOLVED } \\
\text { (UG/L) } \\
\end{array}$ & $\begin{array}{l}\text { LINDANE } \\
\text { DIS- } \\
\text { SOLVED } \\
\text { (UG/L) }\end{array}$ & $\begin{array}{c}\text { CHLOR- } \\
\text { PYRIFOS } \\
\text { DIS- } \\
\text { SOLVED } \\
\text { (UG/L) } \\
\end{array}$ & $\begin{array}{c}\text { DI- } \\
\text { AZINON, } \\
\text { DIS- } \\
\text { SOLVED } \\
\text { (UG/L) } \\
\end{array}$ & $\begin{array}{l}\text { DISUL- } \\
\text { FOTON } \\
\text { WATER } \\
\text { FLTRD } \\
0.7 \text { U } \\
\text { GE' REC } \\
\text { (UG/L) }\end{array}$ & $\begin{array}{cc}\text { ETHO- } \\
\text { PROP } \\
\text { WATER } \\
\text { FLTRD } \\
0.7 \mathrm{U} \\
\text { C GF, REC } \\
\text { (UG/L) } \\
\end{array}$ & $\begin{array}{c}\text { FONOFOS } \\
\text { WATER } \\
\text { DISS } \\
\text { REC } \\
\text { (UG/L) } \\
\end{array}$ & $\begin{array}{l}\text { MALA- } \\
\text { THION, } \\
\text { DIS- } \\
\text { SOLVED } \\
\text { (UG/L) } \\
\end{array}$ \\
\hline 440313123091100 & $\begin{array}{l}04-16-92 \\
10-16-92 \\
10-29-92\end{array}$ & $\begin{array}{l}<0.00600 \\
0.002000 \\
<0.00600\end{array}$ & $\begin{array}{l}<0.00200 \\
<0.00200 \\
<0.00200\end{array}$ & $\begin{array}{l}<0.00400 \\
<0.00400 \\
<0.00400\end{array}$ & $\begin{array}{r}<0.00400 \\
0.02200 \\
0.08600\end{array}$ & $\begin{array}{r}<0.00200 \\
<0.00200 \\
0.04400\end{array}$ & $\begin{array}{l}<0.05000 \\
<0.10000 \\
<0.10000\end{array}$ & $\begin{array}{l}<0.00300 \\
<0.00300 \\
<0.00300\end{array}$ & $\begin{array}{r}<0.00300 \\
0.03000 \\
<0.00300\end{array}$ & $\begin{array}{l}<0.00500 \\
<0.00500 \\
<0.00500\end{array}$ \\
\hline 443207123145500 & $08-24-93$ & $<0.00600$ & $<0.00200$ & $<0.00400$ & $<0.00400$ & $<0.00200$ & $<0.01700$ & $<0.00300$ & $<0.00300$ & 0.01600 \\
\hline 443138123120901 & $08-24-93$ & $<0.00600$ & $<0.00200$ & $<0.00400$ & $<0.00400$ & $<0.00200$ & $<0.01700$ & $<0.00300$ & $<0.00300$ & $<0.00500$ \\
\hline 451309123041501 & $09-07-93$ & $<0.00600$ & $<0.00200$ & $<0.00400$ & $<0.00400$ & $<0.00200$ & $<0.01700$ & $<0.00300$ & $<0.00300$ & $<0.00500$ \\
\hline 14201300 & $\begin{array}{l}06-01-93 \\
07-27-93\end{array}$ & $\begin{array}{l}<0.00600 \\
<0.00600\end{array}$ & $\begin{array}{l}<0.00200 \\
<0.00200\end{array}$ & $\begin{array}{r}<0.00400 \\
0.02100\end{array}$ & $\begin{array}{l}0.01000 \\
0.03200\end{array}$ & $\begin{array}{r}0.03000 \\
1.2000\end{array}$ & $\begin{array}{l}<0.01700 \\
<0.01700\end{array}$ & $\begin{array}{l}0.28000 \\
0.06600\end{array}$ & $\begin{array}{l}0.10000 \\
0.01900\end{array}$ & $\begin{array}{l}<0.00500 \\
<0.00500\end{array}$ \\
\hline 14202000 & $04-27-93$ & $<0.00600$ & $<0.00200$ & $<0.00400$ & $<0.00400$ & 0.002000 & $<0.01700$ & $<0.00300$ & -- & 0.01000 \\
\hline 453115122535500 & $03-31-93$ & $<0.00600$ & $<0.00200$ & $<0.00400$ & $<0.00400$ & 0.06300 & $<0.01700$ & $<0.00300$ & $<0.00300$ & $<0.00500$ \\
\hline 14206950 & $\begin{array}{l}03-01-93 \\
07-22-93\end{array}$ & $\begin{array}{l}<0.00600 \\
<0.00600\end{array}$ & $\begin{array}{l}<0.00200 \\
<0.00200\end{array}$ & $\begin{array}{l}<0.00400 \\
<0.00400\end{array}$ & $\begin{array}{r}0.002000 \\
0.02100\end{array}$ & $\begin{array}{l}0.02500 \\
0.05700\end{array}$ & $\begin{array}{l}<0.01700 \\
<0.01700\end{array}$ & $\begin{array}{l}<0.00300 \\
<0.00300\end{array}$ & $\begin{array}{l}<0.00300 \\
<0.00300\end{array}$ & $\begin{array}{r}0.02000 \\
<0.00500\end{array}$ \\
\hline 14211550 & $03-14-93$ & $<0.00600$ & $<0.00200$ & $<0.00400$ & $<0.00400$ & 0.01700 & $<0.01700$ & $<0.00300$ & $<0.00300$ & $<0.00500$ \\
\hline 453547122463000 & $10-26-92$ & 0.006000 & $<0.00200$ & $<0.00400$ & $<0.00400$ & $<0.01000$ & $<0.10000$ & $<0.00300$ & $<0.00300$ & $<0.00500$ \\
\hline
\end{tabular}


Table 35. Concentrations of pesticides in filtered water analyzed by gas chromatography/mass spectroscopy from Phase I of the Willamette River Basin Water Quality Study, Oregon, 1992-93-Continued

\begin{tabular}{|c|c|c|c|c|c|c|c|c|c|c|}
\hline STATION NUMBER & DATE & $\begin{array}{l}\text { METHYL } \\
\text { AZIN- } \\
\text { PHOS } \\
\text { WAT FLT } \\
0.7 \text { U } \\
\text { GF, REC } \\
\text { (UG/L) }\end{array}$ & $\begin{array}{c}\text { METHYL } \\
\text { PARA- } \\
\text { THION } \\
\text { WAT FLT } \\
0.7 \text { U } \\
\text { GF, REC } \\
\text { (UG/L) }\end{array}$ & $\begin{array}{l}\text { PARA- } \\
\text { THION, } \\
\text { DIS- } \\
\text { SOLVED } \\
\text { (UG/L) }\end{array}$ & $\begin{array}{l}\text { PHORATE } \\
\text { WATER } \\
\text { FLTRD } \\
0.7 \text { U } \\
\text { GE, REC } \\
(\mathrm{UG} / \mathrm{L})\end{array}$ & $\begin{array}{l}\text { TER- } \\
\text { BUFOS } \\
\text { WATER } \\
\text { FLTRD } \\
0.7 \mathrm{U} \\
\text { GF, REC } \\
\text { (UG/L) }\end{array}$ & $\begin{array}{c}\text { PER- } \\
\text { METHRIN } \\
\text { CIS } \\
\text { WAT FLT } \\
0.7 \mathrm{U} \\
\text { GF, REC } \\
\text { (UG/L) }\end{array}$ & $\begin{array}{l}\text { LIN- } \\
\text { URON } \\
\text { WATER } \\
\text { FLTRD } \\
0.7 \mathrm{U} \\
\text { GF, REC } \\
\text { (UG/L) }\end{array}$ & $\begin{array}{c}\text { TEBU- } \\
\text { THIURON } \\
\text { WATER } \\
\text { FLTRD } \\
0.7 \mathrm{U} \\
\text { GF, REC } \\
\text { (UG/L) }\end{array}$ & $\begin{array}{l}\text { ATRA- } \\
\text { ZINE, } \\
\text { WATER, } \\
\text { DISS, } \\
\text { REC } \\
\text { (UG/L) }\end{array}$ \\
\hline 440313123091100 & $\begin{array}{l}04-16-92 \\
10-16-92 \\
10-29-92\end{array}$ & $\begin{array}{l}<0.00100 \\
<0.00100 \\
<0.00100\end{array}$ & $\begin{array}{l}<0.00200 \\
<0.00200 \\
<0.00200\end{array}$ & $\begin{array}{l}<0.00600 \\
<0.00600 \\
<0.00600\end{array}$ & $\begin{array}{l}<0.00200 \\
<0.00200 \\
<0.00200\end{array}$ & $\begin{array}{l}<0.05000 \\
<0.00700 \\
<0.00700\end{array}$ & $\begin{array}{l}<0.00500 \\
<0.00500 \\
<0.00500\end{array}$ & $\begin{array}{l}<0.00200 \\
<0.00200 \\
<0.00200\end{array}$ & $\begin{array}{r}<0.01000 \\
0.06300 \\
<0.01000\end{array}$ & $\begin{array}{l}0.16000 \\
0.07000 \\
0.03900\end{array}$ \\
\hline 443207123145500 & $08-24-93$ & $<0.00100$ & $<0.00200$ & $<0.00600$ & $<0.00200$ & $<0.00700$ & $<0.00500<$ & $<0.00200$ & $<0.01000<$ & $<0.00100$ \\
\hline 443138123120901 & $08-24-93$ & $<0.00100$ & $<0.00200$ & $<0.00600$ & $<0.00200$ & $<0.00700$ & $<0.00500$ & $<0.00200$ & $<0.01000$ & 0.01900 \\
\hline 451309123041501 & $09-07-93$ & $<0.00100$ & $<0.00200$ & $<0.00600$ & $<0.00200$ & $<0.00700$ & $<0.00500$ & $<0.00200$ & $<0.01000$ & 0.04300 \\
\hline 14201300 & $\begin{array}{l}06-01-93 \\
07-27-93\end{array}$ & $\begin{array}{l}\mathrm{E} 0.18000 \\
<0.00100\end{array}$ & $\begin{array}{l}<0.00200 \\
<0.00200\end{array}$ & $\begin{array}{l}<0.00600 \\
<0.00600\end{array}$ & $\begin{array}{l}<0.00200 \\
<0.00200\end{array}$ & $\begin{array}{l}<0.00700 \\
<0.00700\end{array}$ & $\begin{array}{l}<0.00500 \\
<0.00500\end{array}$ & $\begin{array}{l}<0.00200 \\
<0.00200\end{array}$ & $\begin{array}{l}<0.01000 \\
<0.01000\end{array}$ & $\begin{array}{r}2.7000 \\
0.33000\end{array}$ \\
\hline 14202000 & $04-27-93$ & $<0.00100$ & $<0.00200$ & $<0.00600$ & $<0.00200$ & $<0.00700$ & $<0.00500<$ & $<0.00200$ & $<0.01000$ & 0.18000 \\
\hline 453115122535500 & $03-31-93$ & $<0.00100$ & $<0.00200$ & $<0.00600$ & $<0.00200$ & $<0.00700$ & $<0.00500<$ & $<0.00200$ & $<0.01000$ & 0.01800 \\
\hline 14206950 & $\begin{array}{l}03-01-93 \\
07-22-93\end{array}$ & $\begin{array}{l}<0.00100 \\
<0.00100\end{array}$ & $\begin{array}{l}<0.00200 \\
<0.00200\end{array}$ & $\begin{array}{l}<0.00600 \\
<0.00600\end{array}$ & $\begin{array}{l}<0.00200 \\
<0.00200\end{array}$ & $\begin{array}{l}<0.00700 \\
<0.00700\end{array}$ & $\begin{array}{l}<0.00500 \\
<0.00500\end{array}$ & $\begin{array}{l}<0.00200 \\
<0.00200\end{array}$ & $\begin{array}{l}0.02500 \\
0.04900\end{array}$ & $\begin{array}{l}0.02200 \\
0.04000\end{array}$ \\
\hline 14211550 & $03-14-93$ & $<0.00100$ & $<0.00200$ & $<0.00600$ & $<0.00200$ & $<0.00700$ & $<0.00500<$ & $<0.00200$ & $<0.01000$ & 0.02400 \\
\hline 453547122463000 & $10-26-92$ & $<0.00100$ & $<0.00200$ & $<0.00600$ & $<0.00200$ & $<0.00700$ & $<0.00500$ & $<0.00200$ & 0.005000 & 0.01100 \\
\hline STATION NUMBER & DATE & $\begin{array}{l}\text { CYANA- } \\
\text { ZINE, } \\
\text { WATER, } \\
\text { DISS, } \\
\text { REC } \\
\text { (UG/L) }\end{array}$ & $\begin{array}{l}\text { DESETHY } \\
\text { ATRA- } \\
\text { ZINE, } \\
\text { WATER, } \\
\text { DISS, } \\
\text { REC } \\
\text { (UG/L) }\end{array}$ & $\begin{array}{cc}\text { YL } \\
\text { METRI- } \\
\text { BUZIN } \\
\text { SENCOR } \\
\text { WATER } \\
\text { DISSOLV } \\
\text { (UG/L) }\end{array}$ & $\begin{array}{l}\text { PRO- } \\
\text { METON, } \\
\text { WATER, } \\
\text { DISS, } \\
\text { REC } \\
\text { (UG/L) }\end{array}$ & $\begin{array}{l}\text { SI- } \\
\text { MAZINE, } \\
\text { WATER, } \\
\text { DISS, } \\
\text { REC } \\
\text { (UG/L) }\end{array}$ & $\begin{array}{l}\text { TER- } \\
\text { BACIL } \\
\text { WATER } \\
\text { FLTRD } \\
0.7 \text { U } \\
\text { GF, REC } \\
\text { (UG/L) }\end{array}$ & $\begin{array}{l}\text { DIAZ- } \\
\text { INON } \\
\text { D10 SRG } \\
\text { WAT FLT } \\
0.7 \text { U } \\
\text { GF, REC } \\
\text { PERCENT }\end{array}$ & $\begin{array}{c}\text { TERBUTH } \\
\text { YLAZINE } \\
\text { SURROGT } \\
\text { WAT FLT } \\
0.7 \text { U } \\
\text { GF, REC } \\
\text { PERCENT }\end{array}$ & $\begin{array}{c}\text { HCH } \\
\text { ALPHA } \\
\text { D } 6 \text { SRG } \\
\text { WAT FLT } \\
0.7 \text { U } \\
\text { GF, REC } \\
\text { PERCENT }\end{array}$ \\
\hline 440313123091100 & $\begin{array}{l}04-16-92 \\
10-16-92 \\
10-29-92\end{array}$ & $\begin{array}{r}<0.00400 \\
<0.00400 \\
0.02100\end{array}$ & $\begin{array}{l}<0.05000 \\
<0.00200 \\
<0.00200\end{array}$ & $\begin{array}{r}<0.00400 \\
0.03900 \\
0.22000\end{array}$ & $\begin{array}{r}<0.00300 \\
<0.00300 \\
0.02800\end{array}$ & $\begin{array}{r}<0.00500 \\
0.01000 \\
<0.00500\end{array}$ & $\begin{array}{l}<0.00700 \\
<0.00700 \\
<0.00700\end{array}$ & $\begin{array}{c}-- \\
111.00 \\
85.300\end{array}$ & $\begin{array}{c}-- \\
102.00 \\
96.500\end{array}$ & $\begin{array}{c}-- \\
110.00 \\
110.00\end{array}$ \\
\hline 443207123145500 & $08-24-93$ & $<0.00400$ & $<0.00200$ & $<0.00400$ & $<0.00300$ & $<0.00500$ & $<0.00700$ & 70.000 & 97.800 & 90.000 \\
\hline 443138123120901 & $08-24-93$ & $<0.00400$ & $<0.00200$ & $<0.00400$ & $<0.00300$ & 0.01500 & E0.04900 & 80.000 & 97.500 & 80.000 \\
\hline 451309123041501 & $09-07-93$ & $<0.00400$ & $<0.00200$ & $<0.00400$ & $<0.00300$ & $<0.00500$ & $<0.00700$ & 70.000 & 89.900 & 90.000 \\
\hline 14201300 & $\begin{array}{l}06-01-93 \\
07-27-93\end{array}$ & $\begin{array}{l}<0.00400 \\
<0.00400\end{array}$ & $\begin{array}{l}\mathrm{E} 0.27000 \\
\mathrm{E} 0.02800\end{array}$ & $\begin{array}{r}0.01100 \\
<0.00400\end{array}$ & $\begin{array}{l}0.005000 \\
<0.00300\end{array}$ & $\begin{array}{l}0.61000 \\
0.07600\end{array}$ & $\begin{array}{l}<0.00700 \\
<0.00700\end{array}$ & $\begin{array}{l}122.00 \\
100.00\end{array}$ & $\begin{array}{l}116.00 \\
100.00\end{array}$ & $\begin{array}{l}85.000 \\
100.00\end{array}$ \\
\hline 14202000 & $04-27-93$ & $<0.00400$ & $\mathrm{E} 0.03600$ & 0.006000 & $<0.00300$ & 0.11000 & E0.00900 & 75.600 & 99.800 & 89.000 \\
\hline 453115122535500 & $03-31-93$ & $<0.00400$ & $<0.00200$ & $<0.00400$ & 0.02700 & 0.09400 & $<0.00700$ & 102.00 & 86.500 & 97.000 \\
\hline 14206950 & $\begin{array}{l}03-01-93 \\
07-22-93\end{array}$ & $\begin{array}{l}<0.00400 \\
<0.00400\end{array}$ & $\begin{array}{l}<0.00200 \\
<0.00200\end{array}$ & $\begin{array}{l}<0.00400 \\
<0.00400\end{array}$ & $\begin{array}{l}0.03200 \\
0.04200\end{array}$ & $\begin{array}{l}0.03000 \\
0.13000\end{array}$ & $\begin{array}{l}<0.00700 \\
<0.00700\end{array}$ & $\begin{array}{l}95.600 \\
100.00\end{array}$ & $\begin{array}{l}107.00 \\
152.00\end{array}$ & $\begin{array}{l}92.000 \\
100.00\end{array}$ \\
\hline 14211550 & $03-14-93$ & $<0.00400$ & $<0.00200$ & 0.05400 & 0.02000 & 0.02500 & $<0.00700$ & 115.00 & 141.00 & 110.00 \\
\hline 453547122463000 & $10-26-92$ & $<0.00400$ & $<0.00200$ & 0.006000 & $<0.00300$ & $<0.00500$ & $<0.00700$ & 90.700 & 118.00 & 130.00 \\
\hline
\end{tabular}


Table 36. Concentrations of pesticides in filtered water analyzed by gas chromatography/mass spectroscopy from Phase II of the Willamette River Basin Water Quality Study, Oregon, 1994

[USGS laboratory schedule $2010 ;<$, less than; E, estimate; UG/L, micrograms per liter]

\begin{tabular}{|c|c|c|c|c|c|c|}
\hline STATION NUMBER & STATION NAME & DATE & TIME & $\begin{array}{l}\text { ALA- } \\
\text { CHLOR, } \\
\text { WATER } \\
\text { DISSOLV } \\
(\text { UG/L) }\end{array}$ & $\begin{array}{l}\text { METO- } \\
\text { LACHLOR } \\
\text { WATER } \\
\text { DISSOLV } \\
\text { (UG/L) }\end{array}$ & $\begin{array}{l}\text { NAPROP- } \\
\text { AMIDE } \\
\text { WATER } \\
\text { DISSOLV } \\
\text { (UG/L) }\end{array}$ \\
\hline 433548123040600 & COAST FORK WILLAMETTE R BL BIG RIVER NR LONDON, OR & $05-20-94$ & 1040 & $<0.00200$ & $<0.00400$ & $<0.00400$ \\
\hline 440045122585600 & COAST FORK WILLAM AT SEAVY LOOP RD NR EUGENE, OR & $05-19-94$ & 1020 & $<0.00200$ & $<0.00400$ & $<0.00400$ \\
\hline 440402123063900 & URBAN OUTFALL AT POLK ST. PARK AT EUGENE, OR & $06-13-94$ & 1210 & $<0.00200$ & 0.003000 & $<0.00400$ \\
\hline 441310122095801 & MACK CREEK NEAR BLUE RIVER, OR & $05-27-94$ & 1030 & $<0.00200$ & $<0.00400$ & $<0.00400$ \\
\hline 440707123041300 & MCKENZIE RIVER NR EUGENE, OR & $05-19-94$ & 1450 & $<0.00200$ & $<0.00400$ & $<0.00400$ \\
\hline 442223123153703 & $\begin{array}{lllllll}\text { LONG TOM R AT BUNDY BRIDGE } & \text { NR MONROE, OR } \\
\text { LONG TOM R AT } & \text { BUNDY } & \text { BRIDGE } & \text { NR MONROE, OR }\end{array}$ & $\begin{array}{l}05-19-94 \\
11-01-94\end{array}$ & $\begin{array}{l}0950 \\
1720\end{array}$ & $\begin{array}{l}<0.00200 \\
<0.00200\end{array}$ & $\begin{array}{r}<0.00400 \\
0.02000\end{array}$ & $\begin{array}{l}<0.00400 \\
<0.00400\end{array}$ \\
\hline 442413123122500 & LAKE CAMOUS CR AT PINE GROVE DR NR HARRISBURG, OR & $05-19-94$ & 1720 & $<0.00200$ & 0.009000 & $<0.00400$ \\
\hline 443138123120901 & $\begin{array}{llll}\text { MUDDY } & \text { CREEK NEAR PEORIA, OR } \\
\text { MUDDY } & \text { CREEK NEAR PEORIA, OR } \\
\text { MUDDY } & \text { CREEK NEAR PEORIA, OR } \\
\text { MUDDY } & \text { CREEK NEAR PEORIA, OR } \\
\text { MUDDY } & \text { CREEK NEAR PEORIA, OR } \\
\text { MUDDY CREEK NEAR PEORIA, OR }\end{array}$ & $\begin{array}{l}05-26-94 \\
06-03-94 \\
06-20-94 \\
11-02-94 \\
11-06-94 \\
11-09-94\end{array}$ & $\begin{array}{l}1730 \\
0930 \\
1050 \\
1010 \\
1200 \\
1430\end{array}$ & $\begin{array}{l}<0.00200 \\
<0.00200 \\
<0.00200 \\
<0.00200 \\
<0.00200 \\
<0.00200\end{array}$ & $\begin{array}{r}0.005000 \\
0.003000 \\
0.004000 \\
0.64000 \\
0.69000 \\
0.41000\end{array}$ & $\begin{array}{l}<0.00400 \\
<0.00400 \\
<0.00400 \\
<0.00400 \\
<0.00400 \\
<0.00400\end{array}$ \\
\hline 443045123273000 & ROCK CREEK ABOVE GRIFFITH CREEK NR PHILOMATH, OR & $05-18-94$ & 1820 & $<0.00200$ & $<0.00400$ & $<0.00400$ \\
\hline 443321123155201 & MARYS RIVER AT CORVALLIS, OR & $05-18-94$ & 1350 & $<0.00200$ & $<0.00400$ & $<0.00400$ \\
\hline 14173500 & $\begin{array}{l}\text { CALAPOOIA RIVER AT ALBANY, OR } \\
\text { CALAPOOIA RIVER AT ALBANY, OR }\end{array}$ & $\begin{array}{l}05-26-94 \\
11-01-94\end{array}$ & $\begin{array}{l}1220 \\
1230\end{array}$ & $\begin{array}{l}<0.00200 \\
<0.00200\end{array}$ & $\begin{array}{r}0.003000 \\
1.9000\end{array}$ & $\begin{array}{l}<0.00400 \\
<0.00400\end{array}$ \\
\hline 444349123094000 & LUCKIAMUTE R AT BUENA VISTA RD NR BUENA VISTA, OR & $05-16-94$ & 1720 & $<0.00200$ & $<0.00400$ & $<0.00400$ \\
\hline 444123122562200 & THOMAS CREEK AT KELLY RD NR JEFFERSON, OR & $05-16-94$ & 1900 & $<0.00200$ & $<0.00400$ & $<0.00400$ \\
\hline 14189000 & SANTIAM RIVER AT JEFFERSON, OR & $05-17-94$ & 1620 & $<0.00200$ & $<0.00400$ & $<0.00400$ \\
\hline
\end{tabular}


Table 36. Concentrations of pesticides in filtered water analyzed by gas chromatography/mass spectroscopy from Phase II of the Willamette River Basin Water Quality Study, Oregon, 1994—Continued

\begin{tabular}{|c|c|c|c|c|c|c|c|c|c|c|c|}
\hline STATION NUMBER & DATE & $\begin{array}{l}\text { PRON- } \\
\text { AMIDE } \\
\text { WATER, } \\
\text { DISSOLV } \\
(\text { UG/L) }\end{array}$ & $\begin{array}{l}\text { PROPA- } \\
\text { CHLOR } \\
\text { WATER, } \\
\text { DISSOLV } \\
(\text { UG/L) }\end{array}$ & $\begin{array}{l}\text { PRO- } \\
\text { PANIL } \\
\text { WATER, } \\
\text { DISSOLV } \\
\text { (UG/L) }\end{array}$ & $\begin{array}{l}\text { BUTYL- } \\
\text { WATER, } \\
\text { DISSOLV } \\
(U G / L)\end{array}$ & $\begin{array}{l}\text { CAR- } \\
\text { BARYL } \\
\text { WATER, } \\
\text { DISSOLV } \\
(\text { UG/L) }\end{array}$ & $\begin{array}{l}\text { CARBO- } \\
\text { FURAN } \\
\text { WATER, } \\
\text { DISSOLV } \\
\text { (UG/L) }\end{array}$ & $\begin{array}{l}\text { EPTC } \\
\text { WATER, } \\
\text { DISSOLV } \\
(\mathrm{UG} / \mathrm{L})\end{array}$ & \begin{tabular}{l}
\multicolumn{1}{c}{ MOL- } \\
INATE \\
WATER, \\
DISSOLV \\
(UG/L)
\end{tabular} & $\begin{array}{l}\text { PEB- } \\
\text { ULATE } \\
\text { WATER, } \\
\text { DISSOLV } \\
\text { (UG/L) }\end{array}$ & $\begin{array}{l}\text { THIO- } \\
\text { BENCARB } \\
\text { WATER, } \\
\text { DISSOLV } \\
\text { (UG/L) }\end{array}$ \\
\hline 433548123040600 & $05-20-94$ & $<0.01800$ & $<0.00700$ & $<0.00400$ & $<0.00200$ & $<0.00300$ & $<0.00300$ & $<0.00200$ & $<0.00300$ & $<0.00400$ & $<0.00200$ \\
\hline 440045122585600 & $05-19-94$ & $<0.01800$ & $<0.00700$ & $<0.00400$ & $<0.00200$ & $<0.00300$ & $<0.00300$ & $<0.00200$ & $<0.00300$ & $<0.00400$ & $<0.00200$ \\
\hline 440402123063900 & $06-13-94$ & $<0.01800$ & $<0.00700$ & $<0.00400$ & $<0.00200$ & $<0.00300$ & $<0.00300$ & $<0.00200$ & $<0.00300$ & $<0.00400$ & $<0.00200$ \\
\hline 441310122095801 & $05-27-94$ & $<0.01800$ & $<0.00700$ & $<0.00400$ & $<0.00200$ & $<0.00300$ & $<0.00300$ & $<0.00200$ & $<0.00300$ & $<0.00400$ & $<0.00200$ \\
\hline 440707123041300 & $05-19-94$ & $<0.01800$ & $<0.00700$ & $<0.00400$ & $<0.00200$ & $<0.00300$ & $<0.00300$ & $<0.00200$ & $<0.00300$ & $<0.00400$ & $<0.00200$ \\
\hline 442223123153703 & $\begin{array}{l}05-19-94 \\
11-01-94\end{array}$ & $\begin{array}{l}<0.01800 \\
\mathrm{E} 0.00600\end{array}$ & $\begin{array}{l}<0.00700 \\
<0.00700\end{array}$ & $\begin{array}{l}<0.00400 \\
<0.00400\end{array}$ & $\begin{array}{l}<0.00200 \\
<0.00200\end{array}$ & $\begin{array}{r}E 2.0000 \\
<0.00300\end{array}$ & $\begin{array}{l}<0.00300 \\
<0.00300\end{array}$ & $\begin{array}{l}0.008000 \\
<0.00200\end{array}$ & $\begin{array}{l}<0.00300 \\
<0.00300\end{array}$ & $\begin{array}{l}<0.00400 \\
<0.00400\end{array}$ & $\begin{array}{l}<0.00200 \\
<0.00200\end{array}$ \\
\hline 442413123122500 & $05-19-94$ & 0.02200 & $<0.00700$ & $<0.00400$ & $<0.00200$ & $\mathrm{E} 0.00600$ & $<0.00300$ & 0.008000 & $<0.00300$ & $<0.00400$ & $<0.00200$ \\
\hline 443138123120901 & $\begin{array}{l}05-26-94 \\
06-03-94 \\
06-20-94 \\
11-02-94 \\
11-06-94 \\
11-09-94\end{array}$ & $\begin{array}{r}0.01300 \\
0.007000 \\
0.005000 \\
\mathrm{E} 0.00800 \\
\mathrm{E} 0.00500 \\
0.03700\end{array}$ & $\begin{array}{l}<0.00700 \\
<0.00700 \\
<0.00700 \\
<0.00700 \\
<0.00700 \\
<0.00700\end{array}$ & $\begin{array}{l}<0.00400 \\
<0.00400 \\
<0.00400 \\
<0.00400 \\
<0.00400 \\
<0.00400\end{array}$ & $\begin{array}{l}<0.00200 \\
<0.00200 \\
<0.00200 \\
<0.00200 \\
<0.00200 \\
<0.00200\end{array}$ & $\begin{array}{l}\mathrm{E} 0.00500 \\
<0.00300 \\
<0.00300 \\
<0.00300 \\
<0.00300 \\
<0.00300\end{array}$ & $\begin{array}{l}<0.00300 \\
<0.00300 \\
<0.00300 \\
<0.00300 \\
<0.00300 \\
<0.00300\end{array}$ & $\begin{array}{l}0.002000 \\
<0.00200 \\
<0.00200 \\
<0.00200 \\
<0.00200 \\
<0.00200\end{array}$ & $\begin{array}{l}<0.00300 \\
<0.00300 \\
<0.00300 \\
<0.00300 \\
<0.00300 \\
<0.00300\end{array}$ & $\begin{array}{l}<0.00400 \\
<0.00400 \\
<0.00400 \\
<0.00400 \\
<0.00400 \\
<0.00400\end{array}$ & $\begin{array}{l}<0.00200 \\
<0.00200 \\
<0.00200 \\
<0.00200 \\
<0.00200 \\
<0.00200\end{array}$ \\
\hline 443045123273000 & $05-18-94$ & $<0.01800$ & $<0.00700$ & $<0.00400$ & $<0.00200$ & $<0.00300$ & $<0.00300$ & $<0.00200$ & $<0.00300$ & $<0.00400$ & $<0.00200$ \\
\hline 443321123155201 & $05-18-94$ & $<0.01800$ & $<0.00700$ & $<0.00400$ & $<0.00200$ & $<0.00300$ & $<0.00300$ & $<0.00200$ & $<0.00300$ & $<0.00400$ & $<0.00200$ \\
\hline 14173500 & $\begin{array}{l}05-26-94 \\
11-01-94\end{array}$ & $\begin{array}{r}<0.01800 \\
0.06500\end{array}$ & $\begin{array}{l}<0.00700 \\
<0.00700\end{array}$ & $\begin{array}{l}<0.00400 \\
<0.00400\end{array}$ & $\begin{array}{l}<0.00200 \\
<0.00200\end{array}$ & $\begin{array}{l}<0.00300 \\
<0.00300\end{array}$ & $\begin{array}{l}<0.00300 \\
<0.00300\end{array}$ & $\begin{array}{l}<0.00200 \\
<0.00200\end{array}$ & $\begin{array}{l}<0.00300 \\
<0.00300\end{array}$ & $\begin{array}{l}<0.00400 \\
<0.00400\end{array}$ & $\begin{array}{l}<0.00200 \\
<0.00200\end{array}$ \\
\hline 444349123094000 & $05-16-94$ & $<0.01800$ & $<0.00700$ & $<0.00400$ & $<0.00200$ & $<0.00300$ & $<0.00300$ & $<0.00200$ & $<0.00300$ & $<0.00400$ & $<0.00200$ \\
\hline 444123122562200 & $05-16-94$ & $<0.01800$ & $<0.00700$ & $<0.00400$ & $<0.00200$ & $<0.00300$ & $<0.00300$ & $<0.00200$ & $<0.00300$ & $<0.00400$ & $<0.00200$ \\
\hline 14189000 & $05-17-94$ & $<0.01800$ & $<0.00700$ & $<0.00400$ & $<0.00200$ & $<0.00300$ & $<0.00300$ & $<0.00200$ & $<0.00300$ & $<0.00400$ & $<0.00200$ \\
\hline
\end{tabular}


Table 36. Concentrations of pesticides in filtered water analyzed by gas chromatography/mass spectroscopy from Phase II of the Willamette River Basin Water Quality Study, Oregon, 1994—Continued

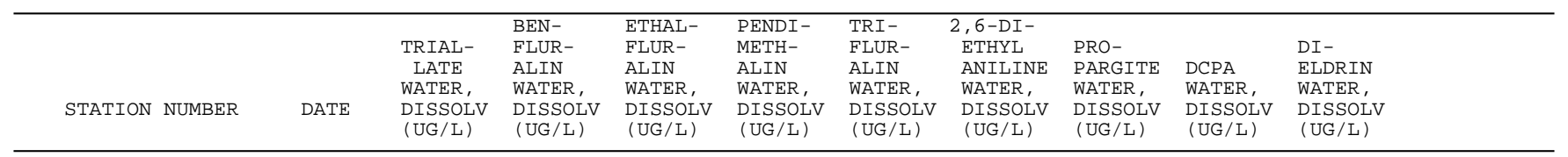

$433548123040600 \quad 05-20-94<0.00100<0.00200<0.00400<0.00400<0.00200<0.00300<0.01300<0.00200<0.00100$

$440045122585600 \quad 05-19-94<0.00100<0.00200<0.00400<0.00400<0.00200<0.00300<0.01300<0.00200<0.00100$

$440402123063900 \quad 06-13-94<0.00100<0.00200<0.00400<0.00400<0.00200<0.00300<0.01300<0.00200<0.00100$

$44131012209580105-27-94<0.00100<0.00200<0.00400<0.00400<0.00200<0.00300<0.01300<0.00200<0.00100$

$440707123041300 \quad 05-19-94<0.00100<0.00200<0.00400<0.00400<0.00200<0.00300<0.01300<0.00200<0.00100$

$44222312315370305-19-94<0.00100<0.00200<0.00400<0.00400<0.00200<0.00300<0.01300<0.00200<0.00100$ $11-01-94<0.00100<0.00200<0.00400<0.00400<0.00200<0.00300<0.01300<0.00200<0.00100$

$442413123122500 \quad 05-19-94<0.00100<0.00200<0.00400<0.00400<0.00200<0.00300<0.01300<0.00200<0.00100$

$44313812312090105-26-94<0.00100<0.00200<0.00400<0.00400<0.00200<0.00300<0.01300<0.00200<0.00100$ $06-03-94<0.00100<0.00200<0.00400<0.00400<0.00200<0.00300<0.01300<0.00200<0.00100$ $06-20-94<0.00100<0.00200<0.00400<0.00400<0.00200<0.00300<0.01300<0.00200<0.00100$ $11-02-94<0.00100<0.00200<0.00400<0.00400<0.00200<0.00300<0.01300<0.00200<0.00100$ $11-06-94<0.00100<0.00200<0.00400<0.00400<0.00200<0.00300<0.01300<0.00200<0.00100$ $11-09-94<0.00100<0.00200<0.00400<0.00400<0.00200<0.00300<0.01300<0.00200<0.00100$

$443045123273000 \quad 05-18-94<0.00100<0.00200<0.00400<0.00400<0.00200<0.00300<0.01300<0.00200<0.00100$

$44332112315520105-18-94<0.00100<0.00200<0.00400<0.00400<0.00200<0.00300<0.01300<0.00200<0.00100$

$14173500 \quad 05-26-94<0.00100<0.00200<0.00400<0.00400<0.00200<0.00300<0.01300<0.00200<0.00100$ $11-01-94<0.00100<0.00200<0.00400<0.00400<0.00200<0.00300<0.01300<0.00200<0.00100$

$444349123094000 \quad 05-16-94<0.00100<0.00200<0.00400<0.00400<0.00200<0.00300<0.01300<0.00200<0.00100$

$444123122562200 \quad 05-16-94<0.00100<0.00200<0.00400<0.00400<0.00200<0.00300<0.01300<0.00200<0.00100$

14189000

$05-17-94<0.00100<0.00200<0.00400<0.00400<0.00200<0.00300<0.01300<0.00200<0.00100$ 
Table 36. Concentrations of pesticides in filtered water analyzed by gas chromatography/mass spectroscopy from Phase II of the Willamette River Basin Water Quality Study, Oregon, 1994—Continued

\begin{tabular}{|c|c|c|c|c|c|c|c|c|c|c|}
\hline STATION NUMBER & DATE & $\begin{array}{l}\text { P, }{ }^{\prime} \\
\text { DDE } \\
\text { WATER, } \\
\text { DISSOLV } \\
(\text { UG/L) }\end{array}$ & $\begin{array}{l}\text { ALPHA } \\
\text { HCH } \\
\text { WATER, } \\
\text { DISSOLV } \\
\text { (UG/L) }\end{array}$ & $\begin{array}{c}\text { LINDANE } \\
\text { WATER, } \\
\text { DISSOL } \\
\text { (UG/L) }\end{array}$ & $\begin{array}{l}\text { CHLOR- } \\
\text { PYRIFO } \\
\text { WATER, } \\
\text { DISSOL } \\
\text { (UG/L) }\end{array}$ & $\begin{array}{c}\text { DI- } \\
\text { AZINON } \\
\text { WATER, } \\
\text { DISSOL } \\
\text { (UG/L) }\end{array}$ & $\begin{array}{ll} & \text { DISUL- } \\
\mathrm{N}, & \text { FOTON } \\
& \text { WATER, } \\
\mathrm{LV} & \text { DISSOLV } \\
)^{\prime} & (\mathrm{UG} / \mathrm{L})\end{array}$ & $\begin{array}{l}\text { ETHO- } \\
\text { PROP } \\
\text { WATER, } \\
\text { DISSOLV } \\
\text { (UG/L) }\end{array}$ & $\begin{array}{l}\text { FONOFOS } \\
\text { WATER, } \\
\text { DISSOLV } \\
\text { (UG/L) }\end{array}$ & $\begin{array}{l}\text { MALA- } \\
\text { THION, } \\
\text { WATER, } \\
\text { DISSOLV } \\
\text { (UG/L) }\end{array}$ \\
\hline 433548123040600 & $05-20-94$ & $<0.00600$ & $<0.00200$ & $<0.00400$ & $<0.00400$ & $<0.00200$ & $<0.01700$ & $<0.00300$ & $<0.00300$ & $<0.00500$ \\
\hline 440045122585600 & $05-19-94$ & $<0.00600$ & $<0.00200$ & $<0.00400$ & $<0.00400$ & $<0.00200$ & $<0.01700$ & $<0.00300$ & $<0.00300$ & $<0.00500$ \\
\hline 440402123063900 & $06-13-94$ & $<0.00600$ & $<0.00200$ & $<0.00400$ & $<0.00400$ & 0.007000 & $<0.01700$ & $<0.00300$ & $<0.00300$ & $<0.00500$ \\
\hline 441310122095801 & $05-27-94$ & $<0.00600$ & $<0.00200$ & $<0.00400$ & $<0.00400$ & $<0.00200$ & $<0.01700$ & $<0.00300$ & $<0.00300$ & $<0.00500$ \\
\hline 440707123041300 & $05-19-94$ & $<0.00600$ & $<0.00200$ & $<0.00400$ & $<0.00400$ & $<0.00200$ & $<0.01700$ & $<0.00300$ & $<0.00300$ & $<0.00500$ \\
\hline 442223123153703 & $\begin{array}{l}05-19-94 \\
11-01-94\end{array}$ & $\begin{array}{l}<0.00600 \\
<0.00600\end{array}$ & $\begin{array}{l}<0.00200 \\
<0.00200\end{array}$ & $\begin{array}{l}<0.00400 \\
<0.00400\end{array}$ & $\begin{array}{l}<0.00400 \\
<0.00400\end{array}$ & $\begin{array}{l}<0.00200 \\
<0.00200\end{array}$ & $\begin{array}{l}<0.01700 \\
<0.01700\end{array}$ & $\begin{array}{l}<0.00300 \\
<0.00300\end{array}$ & $\begin{array}{r}<0.00300 \\
0.01600\end{array}$ & $\begin{array}{l}<0.00500 \\
<0.00500\end{array}$ \\
\hline 442413123122500 & $05-19-94$ & $<0.00600$ & $<0.00200$ & $<0.00400$ & $<0.00400$ & $<0.00200$ & $<0.01700$ & $<0.00300$ & $<0.00300$ & $<0.00500$ \\
\hline 443138123120901 & $\begin{array}{l}05-26-94 \\
06-03-94 \\
06-20-94 \\
11-02-94 \\
11-06-94 \\
11-09-94\end{array}$ & $\begin{array}{l}<0.00600 \\
<0.00600 \\
<0.00600 \\
<0.00600 \\
<0.00600 \\
<0.00600\end{array}$ & $\begin{array}{l}<0.00200 \\
<0.00200 \\
<0.00200 \\
<0.00200 \\
<0.00200 \\
<0.00200\end{array}$ & $\begin{array}{l}<0.00400 \\
<0.00400 \\
<0.00400 \\
<0.00400 \\
<0.00400 \\
<0.00400\end{array}$ & $\begin{array}{l}<0.00400 \\
<0.00400 \\
<0.00400 \\
<0.00400 \\
<0.00400 \\
<0.00400\end{array}$ & $\begin{array}{l}<0.00200 \\
<0.00200 \\
<0.00200 \\
<0.00200 \\
<0.00200 \\
<0.00200\end{array}$ & $\begin{array}{l}<0.01700 \\
<0.01700 \\
<0.01700 \\
<0.01700 \\
<0.01700 \\
<0.01700\end{array}$ & $\begin{array}{l}<0.00300 \\
<0.00300 \\
<0.00300 \\
<0.00300 \\
<0.00300 \\
<0.00300\end{array}$ & $\begin{array}{l}<0.00300 \\
<0.00300 \\
<0.00300 \\
<0.00300 \\
<0.00300 \\
<0.00300\end{array}$ & $\begin{array}{l}<0.00500 \\
<0.00500 \\
<0.00500 \\
<0.00500 \\
<0.00500 \\
<0.00500\end{array}$ \\
\hline 443045123273000 & $05-18-94$ & $<0.00600$ & $<0.00200$ & $<0.00400$ & $<0.00400$ & $<0.00200$ & $<0.01700$ & $<0.00300$ & $<0.00300$ & $<0.00500$ \\
\hline 443321123155201 & $05-18-94$ & $<0.00600$ & $<0.00200$ & $<0.00400$ & $<0.00400$ & $<0.00200$ & $<0.01700$ & $<0.00300$ & $<0.00300$ & $<0.00500$ \\
\hline 14173500 & $\begin{array}{l}05-26-94 \\
11-01-94\end{array}$ & $\begin{array}{l}<0.00600 \\
<0.00600\end{array}$ & $\begin{array}{l}<0.00200 \\
<0.00200\end{array}$ & $\begin{array}{l}<0.00400 \\
<0.00400\end{array}$ & $\begin{array}{l}<0.00400 \\
<0.00400\end{array}$ & $\begin{array}{l}<0.00200 \\
<0.00200\end{array}$ & $\begin{array}{l}<0.01700 \\
<0.01700\end{array}$ & $\begin{array}{l}<0.00300 \\
<0.00300\end{array}$ & $\begin{array}{l}<0.00300 \\
<0.00300\end{array}$ & $\begin{array}{l}<0.00500 \\
<0.00500\end{array}$ \\
\hline 444349123094000 & $05-16-94$ & $<0.00600$ & $<0.00200$ & $<0.00400$ & $<0.00400$ & $<0.00200$ & $<0.01700$ & $<0.00300$ & $<0.00300$ & $<0.00500$ \\
\hline 444123122562200 & $05-16-94$ & $<0.00600$ & $<0.00200$ & $<0.00400$ & $<0.00400$ & $<0.00200$ & $<0.01700$ & $<0.00300$ & $<0.00300$ & $<0.00500$ \\
\hline 14189000 & $05-17-94$ & $<0.00600$ & $<0.00200$ & $<0.00400$ & $<0.00400$ & $<0.00200$ & $<0.01700$ & $<0.00300$ & $<0.00300$ & $<0.00500$ \\
\hline
\end{tabular}


Table 36. Concentrations of pesticides in filtered water analyzed by gas chromatography/mass spectroscopy from Phase II of the Willamette River Basin Water Quality Study, Oregon, 1994-Continued

\begin{tabular}{|c|c|c|c|c|c|c|c|c|c|c|}
\hline STATION NUMBER & DATE & $\begin{array}{l}\text { METHYL } \\
\text { AZIN- } \\
\text { PHOS } \\
\text { WATER, } \\
\text { DISSOLV } \\
(\text { UG/L) }\end{array}$ & $\begin{array}{l}\text { METHYL } \\
\text { PARA- } \\
\text { THION } \\
\text { WATER, } \\
\text { DISSOLV } \\
\text { (UG/L) }\end{array}$ & $\begin{array}{l}\text { PARA- } \\
\text { THION } \\
\text { WATER, } \\
\text { DISSOLV } \\
\text { (UG/L) }\end{array}$ & $\begin{array}{l}\text { PHORATE } \\
\text { WATER, } \\
\text { DISSOLT } \\
\text { (UG/L) }\end{array}$ & $\begin{array}{l}\text { TER- } \\
\text { BUFOS } \\
\text { WATER, } \\
\text { DISSOLV } \\
\text { (UG/L) }\end{array}$ & $\begin{array}{l}\text { PER- } \\
\text { METHRIN } \\
\text { WATER, } \\
\text { DISSOLV } \\
\text { (UG/L) }\end{array}$ & $\begin{array}{l}\text { LIN- } \\
\text { URON } \\
\text { WATER, } \\
\text { DISSOLV } \\
\text { (UG/L) }\end{array}$ & $\begin{array}{l}\text { TEBU- } \\
\text { THIURON } \\
\text { WATER, } \\
\text { DISSOLV } \\
\text { (UG/L) }\end{array}$ & $\begin{array}{l}\text { ATRA- } \\
\text { ZINE } \\
\text { WATER, } \\
\text { DISSOLV } \\
\text { (UG/L) }\end{array}$ \\
\hline 433548123040600 & $05-20-94$ & $<0.00100$ & $<0.00200$ & $<0.00600$ & $<0.00200$ & $<0.00700$ & $<0.00500$ & $<0.00200$ & $<0.01000$ & $<0.00100$ \\
\hline 440045122585600 & $05-19-94$ & $<0.00100$ & $<0.00200$ & $<0.00600$ & $<0.00200$ & $<0.00700$ & $<0.00500$ & $<0.00200$ & $<0.01000$ & 0.01000 \\
\hline 440402123063900 & $06-13-94$ & $<0.00100$ & $<0.00200$ & $<0.00600$ & $<0.00200$ & $<0.00700$ & $<0.00500$ & $<0.00200$ & $<0.01000$ & 0.01600 \\
\hline 441310122095801 & $05-27-94$ & $<0.00100$ & $<0.00200$ & $<0.00600$ & $<0.00200$ & $<0.00700$ & $<0.00500$ & $<0.00200$ & $<0.01000$ & $<0.00100$ \\
\hline 440707123041300 & $05-19-94$ & $<0.00100$ & $<0.00200$ & $<0.00600$ & $<0.00200$ & $<0.00700$ & $<0.00500$ & $<0.00200$ & $<0.01000$ & $<0.00100$ \\
\hline 442223123153703 & $\begin{array}{l}05-19-94 \\
11-01-94\end{array}$ & $\begin{array}{l}<0.00100 \\
<0.00100\end{array}$ & $\begin{array}{l}<0.00200 \\
<0.00200\end{array}$ & $\begin{array}{l}<0.00600 \\
<0.00600\end{array}$ & $\begin{array}{l}<0.00200 \\
<0.00200\end{array}$ & $\begin{array}{l}<0.00700 \\
<0.00700\end{array}$ & $\begin{array}{l}<0.00500 \\
<0.00500\end{array}$ & $\begin{array}{l}<0.00200 \\
<0.00200\end{array}$ & $\begin{array}{l}<0.01000 \\
<0.01000\end{array}$ & $\begin{array}{l}0.05600 \\
0.02600\end{array}$ \\
\hline 442413123122500 & $05-19-94$ & $<0.00100$ & $<0.00200$ & $<0.00600$ & $<0.00200$ & $<0.00700$ & $<0.00500$ & $<0.00200$ & $<0.01000$ & 0.28000 \\
\hline \multirow[t]{2}{*}{443138123120901} & $\begin{array}{l}05-26-94 \\
06-03-94 \\
06-20-94 \\
11-02-94 \\
11-06-94\end{array}$ & $\begin{array}{c}<0.00100 \\
-- \\
<0.00100 \\
<0.00100 \\
<0.00100\end{array}$ & $\begin{array}{l}<0.00200 \\
<0.00200 \\
<0.00200 \\
<0.00200 \\
<0.00200\end{array}$ & $\begin{array}{l}<0.00600 \\
<0.00600 \\
<0.00600 \\
<0.00600 \\
<0.00600\end{array}$ & $\begin{array}{l}<0.00200 \\
<0.00200 \\
<0.00200 \\
<0.00200 \\
<0.00200\end{array}$ & $\begin{array}{l}<0.00700 \\
<0.00700 \\
<0.00700 \\
<0.00700 \\
<0.00700\end{array}$ & $\begin{array}{l}<0.00500 \\
<0.00500 \\
<0.00500 \\
<0.00500 \\
<0.00500\end{array}$ & $\begin{array}{l}<0.00200 \\
<0.00200 \\
<0.00200 \\
<0.00200 \\
<0.00200\end{array}$ & $\begin{array}{l}<0.01000 \\
<0.01000 \\
<0.01000 \\
<0.01000 \\
<0.01000\end{array}$ & $\begin{array}{l}0.43000 \\
0.20000 \\
0.07700 \\
0.88000 \\
0.82000\end{array}$ \\
\hline & $11-09-94$ & $<0.00100$ & $<0.00200$ & $<0.00600$ & $<0.00200$ & $<0.00700$ & $<0.00500$ & $<0.00200$ & $<0.01000$ & 0.58000 \\
\hline 443045123273000 & $05-18-94$ & $<0.00100$ & $<0.00200$ & $<0.00600$ & $<0.00200$ & $<0.00700$ & $<0.00500$ & $<0.00200$ & $<0.01000$ & $<0.00100$ \\
\hline 443321123155201 & $05-18-94$ & $<0.00100$ & $<0.00200$ & $<0.00600$ & $<0.00200$ & $<0.00700$ & $<0.00500$ & $<0.00200$ & $<0.01000$ & 0.03000 \\
\hline 14173500 & $\begin{array}{l}05-26-94 \\
11-01-94\end{array}$ & $\begin{array}{l}<0.00100 \\
<0.00100\end{array}$ & $\begin{array}{l}<0.00200 \\
<0.00200\end{array}$ & $\begin{array}{l}<0.00600 \\
<0.00600\end{array}$ & $\begin{array}{l}<0.00200 \\
<0.00200\end{array}$ & $\begin{array}{l}<0.00700 \\
<0.00700\end{array}$ & $\begin{array}{l}<0.00500 \\
<0.00500\end{array}$ & $\begin{array}{l}<0.00200 \\
<0.00200\end{array}$ & $\begin{array}{l}<0.01000 \\
<0.01000\end{array}$ & $\begin{array}{l}0.02500 \\
0.79000\end{array}$ \\
\hline 444349123094000 & $05-16-94$ & $<0.00100$ & $<0.00200$ & $<0.00600$ & $<0.00200$ & $<0.00700$ & $<0.00500$ & $<0.00200$ & $<0.01000$ & 0.05400 \\
\hline 444123122562200 & $05-16-94$ & $<0.00100$ & $<0.00200$ & $<0.00600$ & $<0.00200$ & $<0.00700$ & $<0.00500$ & $<0.00200$ & $<0.01000$ & 0.02200 \\
\hline 14189000 & $05-17-94$ & $<0.00100$ & $<0.00200$ & $<0.00600$ & $<0.00200$ & $<0.00700$ & $<0.00500$ & $<0.00200$ & $<0.01000$ & $<0.00100$ \\
\hline
\end{tabular}


Table 36. Concentrations of pesticides in filtered water analyzed by gas chromatography/mass spectroscopy from Phase II of the Willamette River Basin Water Quality Study, Oregon, 1994—Continued

\begin{tabular}{|c|c|c|c|c|c|c|c|c|c|c|}
\hline STATION NUMBER & DATE & $\begin{array}{l}\text { CYANA- } \\
\text { ZINE, } \\
\text { WATER, } \\
\text { DISSOLV } \\
(\text { UG/L) }\end{array}$ & $\begin{array}{c}\text { DESETHY } \\
\text { ATRA- } \\
\text { ZINE, } \\
\text { WATER, } \\
\text { DISSOLV } \\
\text { (UG/L) }\end{array}$ & $\begin{array}{ll}\text { YL } & \\
& \\
& \text { METRI- } \\
\text { BUZIN } \\
\text { V } & \text { DISSOLV } \\
& (\text { UG/L) }\end{array}$ & $\begin{array}{l}\text { PRO- } \\
\text { METON, } \\
\text { WATER, } \\
\text { DISSOLV } \\
\text { (UG/L) }\end{array}$ & $\begin{array}{l}\text { SI- } \\
\text { MAZINE, } \\
\text { WATER, } \\
\text { DISSOLV } \\
(\text { UG/L) }\end{array}$ & $\begin{array}{l}\text { TER- } \\
\text { BACIL } \\
\text { WATER, } \\
\text { DISSOLV } \\
(\text { UG/L) }\end{array}$ & $\begin{array}{l}\text { DIAZ- } \\
\text { INON } \\
\text { D10 SRG } \\
\text { WATER, } \\
\text { DISSOLV } \\
\text { PERCENT }\end{array}$ & $\begin{array}{l}\text { TERBUTH } \\
\text { YLAZINE } \\
\text { SURROGT } \\
\text { WATER, } \\
\text { DISSOLV } \\
\text { PERCENT }\end{array}$ & $\begin{array}{c}\text { HCH } \\
\text { ALPHA } \\
\text { D6 SRG } \\
\text { WATER, } \\
\text { DISSOLV } \\
\text { PERCENT }\end{array}$ \\
\hline 433548123040600 & $05-20-94$ & $<0.00400$ & $<0.00200$ & $<0.00400$ & $<0.00300$ & $<0.00500$ & $<0.00700$ & 98.00 & 113.00 & 101.00 \\
\hline 440045122585600 & $05-19-94$ & $<0.00400$ & $<0.00200$ & $<0.00400$ & $<0.00300$ & $<0.00500$ & $<0.00700$ & 103.00 & 122.00 & 106.00 \\
\hline 440402123063900 & $06-13-94$ & $<0.00400$ & $<0.00200$ & $<0.00400$ & 0.01600 & 0.006000 & $<0.00700$ & 113.00 & 108.00 & 118.00 \\
\hline 441310122095801 & $05-27-94$ & $<0.00400$ & $<0.00200$ & $<0.00400$ & $<0.00300$ & $<0.00500$ & $<0.00700$ & 92.00 & 93.00 & 107.00 \\
\hline 440707123041300 & $05-19-94$ & $<0.00400$ & $<0.00200$ & $<0.00400$ & $<0.00300$ & $<0.00500$ & E0.02600 & 97.00 & 117.00 & 98.00 \\
\hline 442223123153703 & $\begin{array}{l}05-19-94 \\
11-01-94\end{array}$ & $\begin{array}{l}<0.00400 \\
<0.00400\end{array}$ & $\begin{array}{l}\text { E0.000600 } \\
\text { E0.00300 }\end{array}$ & $\begin{array}{l}<0.00400 \\
<0.00400\end{array}$ & $\begin{array}{l}<0.00300 \\
\mathrm{E} 0.00500\end{array}$ & $\begin{array}{l}0.01000 \\
0.12000\end{array}$ & $\begin{array}{l}\mathrm{E} 0.07300 \\
\mathrm{E} 0.11000\end{array}$ & $\begin{array}{l}105.00 \\
103.00\end{array}$ & $\begin{array}{l}120.00 \\
110.00\end{array}$ & $\begin{array}{r}104.00 \\
86.00\end{array}$ \\
\hline 442413123122500 & $05-19-94$ & $<0.00400$ & E0.01200 & $<0.00400$ & $<0.00300$ & 0.16000 & E0.29000 & 94.00 & 109.00 & 91.00 \\
\hline 443138123120901 & $\begin{array}{l}05-26-94 \\
06-03-94 \\
06-20-94 \\
11-02-94 \\
11-06-94 \\
11-09-94\end{array}$ & $\begin{array}{l}<0.00400 \\
<0.00400 \\
<0.00400 \\
<0.00400 \\
<0.00400 \\
<0.00400\end{array}$ & $\begin{array}{l}\text { E0.03400 } \\
\text { E0.01900 } \\
\text { E0.01400 } \\
\text { E0.01800 } \\
\text { E0.01500 } \\
\text { E0.01900 }\end{array}$ & $\begin{array}{r}<0.00400 \\
<0.00400 \\
<0.00400 \\
<0.00400 \\
0.11000 \\
0.03800\end{array}$ & $\begin{array}{l}<0.00300 \\
<0.00300 \\
<0.00300 \\
<0.00300 \\
<0.00300 \\
<0.00300\end{array}$ & $\begin{array}{l}0.63000 \\
0.28000 \\
0.47000 \\
1.2000 \\
0.38000 \\
0.19000\end{array}$ & $\begin{array}{c}\text { E0.03300 } \\
-- \\
\text { E0.02900 } \\
\text { E0.05600 } \\
\text { E0.06100 } \\
\text { E0.08700 }\end{array}$ & $\begin{array}{r}78.00 \\
96.00 \\
67.00 \\
106.00 \\
98.00 \\
127.00\end{array}$ & $\begin{array}{r}119.00 \\
101.00 \\
86.00 \\
106.00 \\
108.00 \\
123.00\end{array}$ & $\begin{array}{r}115.00 \\
105.00 \\
85.00 \\
83.00 \\
86.00 \\
116.00\end{array}$ \\
\hline 443045123273000 & $05-18-94$ & $<0.00400$ & $<0.00200$ & $<0.00400$ & $<0.00300$ & $<0.00500$ & $<0.00700$ & 114.00 & 113.00 & 107.00 \\
\hline 443321123155201 & $05-18-94$ & $<0.00400$ & $\mathrm{E} 0.00200$ & $<0.00400$ & $<0.00300$ & 0.007000 & $<0.00700$ & 124.00 & 119.00 & 99.00 \\
\hline 14173500 & $\begin{array}{l}05-26-94 \\
11-01-94\end{array}$ & $\begin{array}{l}<0.00400 \\
<0.00400\end{array}$ & $\begin{array}{l}\text { E0.00300 } \\
\text { E0.01700 }\end{array}$ & $\begin{array}{r}<0.00400 \\
0.06400\end{array}$ & $\begin{array}{l}<0.00300 \\
<0.00300\end{array}$ & $\begin{array}{r}0.006000 \\
0.26000\end{array}$ & $\begin{array}{l}<0.00700 \\
\mathrm{E} 0.00600\end{array}$ & $\begin{array}{r}64.00 \\
108.00\end{array}$ & $\begin{array}{l}110.00 \\
114.00\end{array}$ & $\begin{array}{l}96.00 \\
89.00\end{array}$ \\
\hline 444349123094000 & $05-16-94$ & $<0.00400$ & $<0.00200$ & $<0.00400$ & $<0.00300$ & 0.004000 & $<0.00700$ & 114.00 & 114.00 & 113.00 \\
\hline 444123122562200 & $05-16-94$ & $<0.00400$ & $<0.00200$ & $<0.00400$ & $<0.00300$ & $<0.00500$ & $<0.00700$ & 88.00 & 122.00 & 108.00 \\
\hline 14189000 & $05-17-94$ & $<0.00400$ & $<0.00200$ & $<0.00400$ & $<0.00300$ & $<0.00500$ & $<0.00700$ & 99.00 & 110.00 & 112.00 \\
\hline
\end{tabular}


Table 36. Concentrations of pesticides in filtered water analyzed by gas chromatography/mass spectroscopy from Phase II of the Willamette River Basin Water Quality Study, Oregon, 1994—Continued

\begin{tabular}{|c|c|c|c|c|c|c|}
\hline STATION NUMBER & STATION NAME & DATE & TIME & $\begin{array}{l}\text { ALA- } \\
\text { CHLOR, } \\
\text { WATER } \\
\text { DISSOLV } \\
\text { (UG/L) }\end{array}$ & $\begin{array}{l}\text { METO- } \\
\text { LACHLOR } \\
\text { WATER } \\
\text { DISSOLV } \\
\text { (UG/L) }\end{array}$ & $\begin{array}{l}\text { NAPROP- } \\
\text { AMIDE } \\
\text { WATER } \\
\text { DISSOLV } \\
(\text { UG/L) }\end{array}$ \\
\hline 445547123065400 & RICKREAL CREEK NR MOUTH NR SALEM, OR & $05-26-94$ & 1540 & $<0.00200$ & 0.07100 & $<0.00400$ \\
\hline 14190970 & $\begin{array}{lllllll}\text { PRINGLE } & \text { C AT } & \text { BUSH } & \text { PARK AT SALEM, OR } \\
\text { PRINGLE } & \text { C AT } & \text { BUSH PARK AT SALEM, OR } \\
\text { PRINGLE } & \text { C AT } & \text { BUSH } & \text { PARK AT } & \text { SALEM, OR }\end{array}$ & $\begin{array}{l}11-23-94 \\
11-30-94 \\
11-30-94\end{array}$ & $\begin{array}{l}1200 \\
1530 \\
1710\end{array}$ & $\begin{array}{l}<0.00200 \\
<0.00200 \\
<0.00200\end{array}$ & $\begin{array}{r}0.01100 \\
0.01000 \\
\mathrm{E} 0.00900\end{array}$ & $\begin{array}{l}\mathrm{E} 0.00800 \\
<0.00400 \\
<0.00400\end{array}$ \\
\hline 445037122573800 & $\begin{array}{lllllll}\text { MILL CREEK } & \text { AT } & \text { DELANEY } & \text { ROAD } & \text { NR TURNER, OR } \\
\text { MILL CREEK } & \text { AT } & \text { DELANEY ROAD } & \text { NR TURNER, OR } \\
\text { MILL CREEK } & \text { AT } & \text { DELANEY } & \text { ROAD } & \text { NR TURNER, OR } \\
\text { MILL CREEK } & \text { AT } & \text { DELANEY } & \text { ROAD } & \text { NR TURNER, OR }\end{array}$ & $\begin{array}{l}06-14-94 \\
10-31-94 \\
11-04-94 \\
11-04-94\end{array}$ & $\begin{array}{l}1140 \\
1410 \\
1120 \\
1420\end{array}$ & $\begin{array}{l}<0.00200 \\
<0.00200 \\
<0.00200 \\
<0.00200\end{array}$ & $\begin{array}{l}0.08100 \\
0.28000 \\
0.37000 \\
0.41000\end{array}$ & $\begin{array}{l}<0.00400 \\
<0.00400 \\
<0.00400 \\
<0.00400\end{array}$ \\
\hline 14194150 & $\begin{array}{l}\text { SOUTH YAMHILL RIVER AT MCMINNVILLE, OR } \\
\text { SOUTH YAMHILL RIVER AT MCMINNVILLE, OR }\end{array}$ & $\begin{array}{l}05-17-94 \\
11-02-94\end{array}$ & $\begin{array}{l}1720 \\
1210\end{array}$ & $\begin{array}{l}<0.00200 \\
<0.00200\end{array}$ & $\begin{array}{l}0.09100 \\
0.02600\end{array}$ & $\begin{array}{r}0.16000 \\
<0.00400\end{array}$ \\
\hline 451355123093600 & NORTH YAMHILL RIVER AT HWY. 99E NR MCMINNVILLE, OR & $05-17-94$ & 1220 & $<0.00200$ & $<0.00400$ & $<0.00400$ \\
\hline 451602122564400 & WILLAMETTE RIVER NR NEWBERG, OR & $05-31-94$ & 1400 & $<0.00200$ & 0.005000 & $<0.00400$ \\
\hline 451502122524700 & $\begin{array}{l}\text { CHAMPOEG CREEK BELOW MISSION CR NR BUTTEVILLE, OR } \\
\text { CHAMPOEG CREEK BELOW MISSION CR NR BUTTEVILLE, OR }\end{array}$ & $\begin{array}{l}05-26-94 \\
10-31-94\end{array}$ & $\begin{array}{l}1030 \\
1300\end{array}$ & $\begin{array}{l}<0.00200 \\
<0.00200\end{array}$ & $\begin{array}{r}0.21000 \\
3.3000\end{array}$ & $\begin{array}{r}0.01700 \\
1.6000\end{array}$ \\
\hline 14201300 & $\begin{array}{llll}\text { ZOLLNER CREEK NEAR MT ANGEL, OR } \\
\text { ZOLLNER CREEK NEAR MT ANGEL, OR } \\
\text { ZOLLNER CREEK NEAR MT ANGEL, OR } \\
\text { ZOLLNER CREEK NEAR MT ANGEL, OR }\end{array}$ & $\begin{array}{l}05-25-94 \\
06-13-94 \\
10-28-94 \\
11-28-94\end{array}$ & $\begin{array}{l}1450 \\
1120 \\
1320 \\
1400\end{array}$ & $\begin{array}{r}0.006000 \\
<0.00200 \\
0.01500 \\
\mathrm{E} 0.00700\end{array}$ & $\begin{array}{r}1.4000 \\
0.59000 \\
0.34000 \\
0.10000\end{array}$ & $\begin{array}{r}0.02900 \\
0.08300 \\
\mathrm{E} 0.00800 \\
0.05000\end{array}$ \\
\hline 14202000 & $\begin{array}{lll}\text { PUDDING RIVER AT AURORA, OREG. } \\
\text { PUDDING RIVER AT AURORA, OREG. } \\
\text { PUDDING RIVER AT AURORA, OREG. } \\
\text { PUDDING RIVER AT AURORA, OREG. } \\
\text { PUDDING RIVER AT AURORA, OREG. } \\
\text { PUDDING RIVER AT AURORA, OREG. } \\
\text { PUDDING RIVER AT AURORA, OREG. }\end{array}$ & $\begin{array}{l}04-12-94 \\
05-25-94 \\
06-15-94 \\
10-28-94 \\
10-29-94 \\
11-09-94 \\
11-29-94\end{array}$ & $\begin{array}{l}1110 \\
1100 \\
1145 \\
1710 \\
1230 \\
1300 \\
1110\end{array}$ & $\begin{array}{l}<0.00200 \\
<0.00200 \\
<0.00200 \\
<0.00200 \\
<0.00200 \\
<0.00200 \\
<0.00200\end{array}$ & $\begin{array}{l}0.01400 \\
0.05600 \\
0.01500 \\
0.15000 \\
0.08400 \\
0.08000 \\
0.03600\end{array}$ & $\begin{array}{r}<0.00400 \\
<0.00400 \\
<0.00400 \\
0.13000 \\
0.06600 \\
0.01600 \\
<0.00400\end{array}$ \\
\hline 451603122423301 & MOLALLA R AT KNIGHTS BRIDGE NR CANBY, OR & $05-25-94$ & 1020 & $<0.00200$ & $<0.00400$ & $<0.00400$ \\
\hline 14206200 & $\begin{array}{llllll}\text { DAIRY CREEK AT RTE } 8 & \text { NEAR HILLSBORO, OR } \\
\text { DAIRY CREEK AT RTE } 8 & \text { NEAR HILLSBORO, OR }\end{array}$ & $\begin{array}{l}05-27-94 \\
12-01-94\end{array}$ & $\begin{array}{l}1640 \\
1140\end{array}$ & $\begin{array}{l}<0.00200 \\
<0.00200\end{array}$ & $\begin{array}{r}0.007000 \\
0.03100\end{array}$ & $\begin{array}{r}<0.00400 \\
0.05100\end{array}$ \\
\hline
\end{tabular}


Table 36. Concentrations of pesticides in filtered water analyzed by gas chromatography/mass spectroscopy from Phase II of the Willamette River Basin Water Quality Study, Oregon, 1994-Continued

\begin{tabular}{|c|c|c|c|c|c|c|c|c|c|c|c|}
\hline STATION NUMBER & DATE & $\begin{array}{l}\text { PRON- } \\
\text { AMIDE } \\
\text { WATER, } \\
\text { DISSOLV } \\
(\text { UG/L) }\end{array}$ & $\begin{array}{l}\text { PROPA- } \\
\text { CHLOR } \\
\text { WATER, } \\
\text { DISSOLV } \\
(\text { UG/L) }\end{array}$ & $\begin{array}{l}\text { PRO- } \\
\text { PANIL } \\
\text { WATER, } \\
\text { DISSOLV } \\
(\text { UG/L) }\end{array}$ & $\begin{array}{l}\text { BUTYL- } \\
\text { WATER, } \\
\text { DISSOLV } \\
\text { (UG/L) }\end{array}$ & $\begin{array}{l}\text { CAR- } \\
\text { BARYL } \\
\text { WATER, } \\
\text { DISSOLV } \\
(\text { UG } / L)\end{array}$ & $\begin{array}{l}\text { CARBO- } \\
\text { FURAN } \\
\text { WATER, } \\
\text { DISSOLV } \\
(\text { UG } / L)\end{array}$ & $\begin{array}{l}\text { EPTC } \\
\text { WATER, } \\
\text { DISSOLV } \\
\text { (UG/L) }\end{array}$ & \begin{tabular}{l}
\multicolumn{1}{c}{ MOL- } \\
INATE \\
WATER, \\
DISSOLV \\
$($ UG $/ L)$
\end{tabular} & $\begin{array}{l}\text { PEB- } \\
\text { ULATE } \\
\text { WATER, } \\
\text { DISSOLV } \\
(\text { UG/L) }\end{array}$ & $\begin{array}{l}\text { THIO- } \\
\text { BENCARB } \\
\text { WATER, } \\
\text { DISSOLV } \\
\text { (UG/L) }\end{array}$ \\
\hline
\end{tabular}

$445547123065400 \quad 05-26-94<0.01800<0.00700<0.00400<0.00200 \mathrm{E} 0.00500<0.00300<0.00200<0.00300<0.00400<0.00200$

$14190970 \quad 11-23-94<0.01800<0.00700<0.00400<0.00200<0.00300<0.00300 \quad E 0.00200<0.00300<0.00400<0.00200$ $11-30-94<0.01800<0.00700<0.00400<0.00200<0.00300<0.00300<0.00200<0.00300<0.00400<0.00200$ $11-30-94<0.01800<0.00700<0.00400<0.00200<0.00300<0.00300<0.00200<0.00300<0.00400<0.00200$

$445037122573800 \quad 06-14-94<0.01800<0.00700<0.00400<0.00200<0.00300<0.00300 \quad 0.01900<0.00300<0.00400<0.00200$ $10-31-94<0.01800<0.00700<0.00400<0.00200<0.00300 \mathrm{E} 0.68000<0.00200<0.00300<0.00400<0.00200$ $11-04-94<0.01800<0.00700<0.00400<0.00200<0.00300 \mathrm{E} 0.36000 \quad 0.006000<0.00300<0.00400<0.00200$ $11-04-94<0.01800<0.00700<0.00400<0.00200<0.00300 \mathrm{E} 0.360000 .006000<0.00300<0.00400<0.00200$
$11-04-94<0.01800<0.00700<0.00400<0.00200<0.00300 \mathrm{E} 0.590000 .007000<0.00300<0.00400<0.00200$

$14194150 \quad 05-17-94<0.01800<0.00700<0.00400<0.00200<0.00300<0.00300<0.00200<0.00300<0.00400<0.00200$ $11-02-94<0.01800<0.00700<0.00400<0.00200 \mathrm{E} 0.00800<0.00300<0.00200<0.00300<0.00400<0.00200$

$451355123093600 \quad 05-17-94<0.01800<0.00700<0.00400<0.00200<0.00300<0.00300<0.00200<0.00300<0.00400<0.00200$

$451602122564400 \quad 05-31-94<0.01800<0.00700<0.00400<0.00200<0.00300<0.00300<0.00200<0.00300<0.00400<0.00200$

$451502122524700 \quad 05-26-94 \quad 0.01500<0.00700<0.00400<0.00200<0.00300<0.00300<0.00200<0.00300<0.00400<0.00200$ $10-31-94 \quad 0.02800<0.00700<0.00400<0.00200<0.00300 \mathrm{E} 0.11000<0.00200<0.00300<0.00400<0.00200$

14201300 $05-25-94<0.01800<0.00700<0.00400<0.00200 \mathrm{E} 0.02600 \mathrm{E} 0.38000$ $10-28-94<0.01800<0.00700<0.00400<0.00200 \mathrm{E} 0.01000 \mathrm{E} 0.11000 \quad 0.22000<0.00300<0.00400<0.00200$ $11-28-94<0.01800<0.00700<0.00400<0.00200<0.00300 \mathrm{E} 0.06600 \quad 0.01300<0.00300<0.00400<0.00200$

14202000

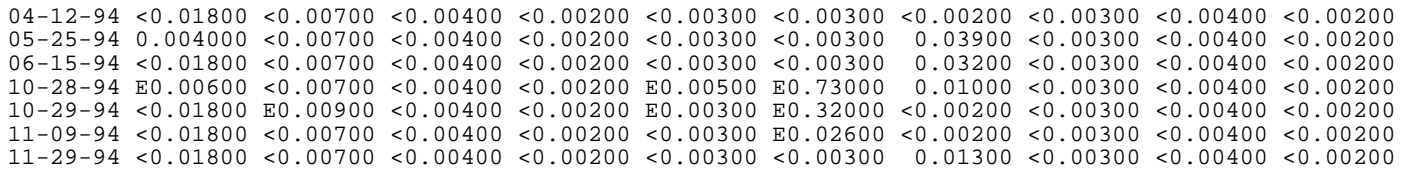

$45160312242330105-25-94<0.01800<0.00700<0.00400<0.00200<0.00300<0.003000 .002000<0.00300<0.00400<0.00200$

14206200

$05-27-94<0.01800<0.00700<0.00400<0.00200<0.00300<0.00300 \quad 0.005000<0.00300<0.00400<0.00200$ $12-01-940.09800<0.00700<0.00400<0.00200<0.00300<0.00300 \mathrm{E} 0.00500<0.00300<0.00400<0.00200$ 
Table 36. Concentrations of pesticides in filtered water analyzed by gas chromatography/mass spectroscopy from Phase II of the Willamette River Basin Water Quality Study, Oregon, 1994—Continued

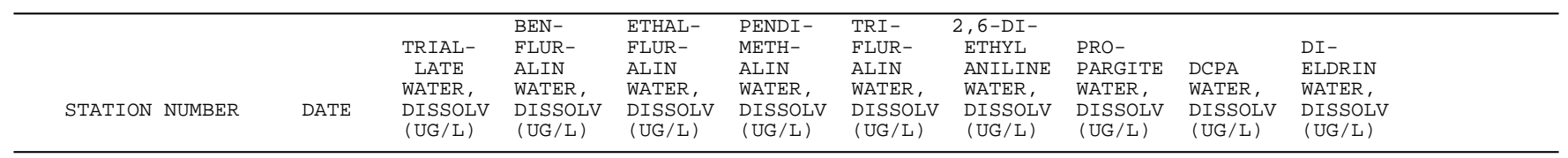

$44554712306540005-26-94<0.00100<0.00200<0.00400<0.00400<0.00200<0.00300<0.01300<0.00200<0.00100$

$14190970 \quad 11-23-94<0.00100<0.00200<0.00400<0.00400<0.00200<0.00300<0.01300<0.00200<0.00100$ $11-30-94<0.00100<0.00200<0.00400<0.00400 \mathrm{E} 0.00600<0.00300<0.01300<0.00200<0.00100$ $11-30-94<0.00100<0.00200<0.00400<0.00400<0.00200<0.00300<0.01300 \mathrm{E} 0.00300<0.00100$

$445037122573800 \quad 06-14-94<0.00100<0.00200<0.00400<0.00400<0.00200<0.00300 \quad 0.003000<0.00200<0.00100$ $10-31-94<0.00100<0.00200<0.00400<0.00400<0.00200<0.003000 .009000<0.00200<0.00100$ $11-04-94<0.00100<0.00200<0.00400<0.00400<0.00200<0.00300<0.01300<0.00200<0.00100$ $11-04-94<0.00100<0.00200<0.00400<0.00400<0.00200<0.003000 .009000<0.00200<0.00100$

$14194150 \quad 05-17-94<0.00100<0.00200<0.00400<0.00400<0.00200<0.00300<0.01300<0.00200<0.00100$ $11-02-94 \quad 0.01000<0.00200<0.00400<0.00400<0.00200<0.00300<0.01300<0.00200<0.00100$

$451355123093600 \quad 05-17-94<0.00100<0.00200<0.00400<0.00400<0.00200<0.00300<0.01300<0.00200<0.00100$

$451602122564400 \quad 05-31-94<0.00100<0.00200<0.00400<0.00400<0.00200<0.00300<0.01300<0.00200<0.00100$

$451502122524700 \quad 05-26-94<0.00100<0.00200<0.00400<0.00400<0.00200<0.00300<0.013000 .001000<0.00100$ $10-31-94<0.00100<0.00200<0.00400<0.00400<0.00200<0.00300<0.01300<0.00200<0.00100$

$14201300 \quad 05-25-94<0.00100<0.00200<0.00400<0.00400 \quad 0.006000<0.00300<0.01300 \quad 0.003000<0.00100$ $06-13-94<0.00100<0.00200<0.00400<0.00400<0.00200<0.00300<0.01300 \quad 0.01000<0.00100$ $10-28-94<0.00100<0.00200<0.00400 \quad 0.05100 \quad 0.01800<0.00300<0.01300 \mathrm{E} 0.00200<0.00100$ $11-28-94<0.00100<0.00200<0.00400<0.00400 \mathrm{E} 0.00600<0.00300<0.01300<0.00200<0.00100$

14202000

$04-12-94<0.00100<0.00200<0.00400<0.00400<0.00200<0.00300<0.01300 \quad 0.02900<0.00100$ $05-25-94<0.00100<0.00200<0.00400<0.00400<0.00200<0.00300<0.013000 .008000<0.00100$ $06-15-94<0.00100<0.00200<0.00400<0.00400<0.00200<0.00300<0.013000 .006000<0.00100$ $10-28-94<0.00100<0.00200<0.00400<0.00400 \mathrm{E} 0.00400<0.00300<0.01300 \quad 0.01100<0.00100$ $10-29-94<0.00100<0.00200<0.00400<0.00400 \mathrm{E} 0.00300<0.00300<0.01300 \quad 0.008000<0.00100$ $11-09-94<0.00100<0.00200<0.00400<0.00400<0.00200<0.00300<0.01300 \quad 0.006000<0.00100$ $11-29-94<0.00100<0.00200<0.00400<0.00400<0.00200<0.00300<0.013000 .005000<0.00100$

$45160312242330105-25-94<0.00100<0.00200<0.00400<0.00400<0.00200<0.00300<0.01300<0.00200<0.00100$

14206200

$05-27-94<0.00100<0.00200<0.00400<0.00400<0.00200<0.00300<0.01300<0.00200<0.00100$ $12-01-940.01100<0.00200<0.00400<0.00400<0.00200<0.00300<0.01300<0.00200<0.00100$ 
Table 36. Concentrations of pesticides in filtered water analyzed by gas chromatography/mass spectroscopy from Phase II of the Willamette River Basin Water Quality Study, Oregon, 1994—Continued

\begin{tabular}{|c|c|c|c|c|c|c|c|c|c|c|}
\hline STATION NUMBER & DATE & $\begin{array}{l}\text { P, } P^{\prime} \\
\text { DDE } \\
\text { WATER, } \\
\text { DISSOLV } \\
(\text { UG/L) }\end{array}$ & $\begin{array}{l}\text { ALPHA } \\
\text { HCH } \\
\text { WATER, } \\
\text { DISSOLV } \\
\text { (UG/L) }\end{array}$ & $\begin{array}{l}\text { LINDANE } \\
\text { WATER, } \\
\text { DISSOI } \\
\text { (UG/L) }\end{array}$ & $\begin{array}{l}\text { CHLOR- } \\
\text { PYRIFO } \\
\text { WATER, } \\
\text { DISSOL } \\
\text { (UG/L) }\end{array}$ & $\begin{array}{l}\text { DI- } \\
\text { AZINON } \\
\text { WATER, } \\
\text { DISSOL } \\
\text { (UG/L) }\end{array}$ & $\begin{array}{ll} & \text { DISUL- } \\
N, & \text { FOTON } \\
\text { WATER, } \\
\text { LV } & \text { DISSOLV } \\
(U G / L)\end{array}$ & $\begin{array}{l}\text { ETHO- } \\
\text { PROP } \\
\text { WATER, } \\
\text { DISSOLV } \\
\text { (UG/L) }\end{array}$ & $\begin{array}{l}\text { FONOFOS } \\
\text { WATER, } \\
\text { DISSOLV } \\
\text { (UG/L) }\end{array}$ & $\begin{array}{l}\text { MALA- } \\
\text { THION, } \\
\text { WATER, } \\
\text { DISSOLV } \\
\text { (UG/L) }\end{array}$ \\
\hline 445547123065400 & $05-26-94$ & $<0.00600$ & $<0.00200$ & $<0.00400$ & $<0.00400$ & 0.005000 & $<0.01700$ & $<0.00300$ & $<0.00300$ & $<0.00500$ \\
\hline 14190970 & $\begin{array}{l}11-23-94 \\
11-30-94 \\
11-30-94\end{array}$ & $\begin{array}{l}<0.00600 \\
<0.00600 \\
<0.00600\end{array}$ & $\begin{array}{l}<0.00200 \\
<0.00200 \\
<0.00200\end{array}$ & $\begin{array}{l}<0.00400 \\
<0.00400 \\
<0.00400\end{array}$ & $\begin{array}{l}<0.00400 \\
<0.02700 \\
<0.00400\end{array}$ & $\begin{array}{r}<0.00200 \\
0.01200 \\
0.02100\end{array}$ & $\begin{array}{l}<0.01700 \\
<0.01700 \\
<0.01700\end{array}$ & $\begin{array}{l}<0.00300 \\
<0.00300 \\
<0.00300\end{array}$ & $\begin{array}{l}<0.00300 \\
<0.00300 \\
<0.00300\end{array}$ & $\begin{array}{l}<0.00500 \\
<0.00500 \\
<0.00500\end{array}$ \\
\hline 445037122573800 & $\begin{array}{l}06-14-94 \\
10-31-94 \\
11-04-94 \\
11-04-94\end{array}$ & $\begin{array}{l}<0.00600 \\
<0.00600 \\
<0.00600 \\
<0.00600\end{array}$ & $\begin{array}{l}<0.00200 \\
<0.00200 \\
<0.00200 \\
<0.00200\end{array}$ & $\begin{array}{l}<0.00400 \\
<0.00400 \\
<0.00400 \\
<0.00400\end{array}$ & $\begin{array}{l}<0.00400 \\
0.008000 \\
<0.00400 \\
0.007000\end{array}$ & $\begin{array}{l}<0.00200 \\
<0.00200 \\
<0.00200 \\
<0.00200\end{array}$ & $\begin{array}{l}<0.01700 \\
<0.01700 \\
<0.01700 \\
<0.01700\end{array}$ & $\begin{array}{r}0.004000 \\
3.1000 \\
1.7000 \\
1.9000\end{array}$ & $\begin{array}{l}0.001000 \\
<0.00300 \\
\text { E0.00400 } \\
\text { E0.00400 }\end{array}$ & $\begin{array}{l}<0.00500 \\
<0.00500 \\
<0.00500 \\
<0.00500\end{array}$ \\
\hline 14194150 & $\begin{array}{l}05-17-94 \\
11-02-94\end{array}$ & $\begin{array}{l}<0.00600 \\
<0.00600\end{array}$ & $\begin{array}{l}<0.00200 \\
<0.00200\end{array}$ & $\begin{array}{l}<0.00400 \\
<0.00400\end{array}$ & $\begin{array}{l}<0.00400 \\
<0.00400\end{array}$ & $\begin{array}{l}<0.00200 \\
<0.00200\end{array}$ & $\begin{array}{l}<0.01700 \\
<0.01700\end{array}$ & $\begin{array}{r}0.14000 \\
<0.00300\end{array}$ & $\begin{array}{l}<0.00300 \\
<0.00300\end{array}$ & $\begin{array}{l}<0.00500 \\
<0.00500\end{array}$ \\
\hline 451355123093600 & $05-17-94$ & $<0.00600$ & $<0.00200$ & $<0.00400$ & $<0.00400$ & $<0.00200$ & $<0.01700$ & $<0.00300$ & $<0.00300$ & $<0.00500$ \\
\hline 451602122564400 & $05-31-94$ & $<0.00600$ & $<0.00200$ & $<0.00400$ & $<0.00400$ & 0.005000 & $<0.01700$ & $<0.00300$ & $<0.00300$ & $<0.00500$ \\
\hline 451502122524700 & $\begin{array}{l}05-26-94 \\
10-31-94\end{array}$ & $\begin{array}{l}<0.00600 \\
\mathrm{E} 0.00200\end{array}$ & $\begin{array}{l}<0.00200 \\
<0.00200\end{array}$ & $\begin{array}{l}0.03800 \\
0.03000\end{array}$ & $\begin{array}{l}<0.00400 \\
<0.00400\end{array}$ & $\begin{array}{l}<0.00200 \\
<0.00200\end{array}$ & $\begin{array}{l}<0.01700 \\
<0.01700\end{array}$ & $\begin{array}{l}0.005000 \\
\mathrm{E} 0.00400\end{array}$ & $\begin{array}{r}<0.00300 \\
0.01500\end{array}$ & $\begin{array}{l}<0.00500 \\
<0.00500\end{array}$ \\
\hline 14201300 & $\begin{array}{l}05-25-94 \\
06-13-94 \\
10-28-94 \\
11-28-94\end{array}$ & $\begin{array}{l}<0.00600 \\
<0.00600 \\
<0.00600 \\
<0.00600\end{array}$ & $\begin{array}{l}<0.00200 \\
<0.00200 \\
<0.00200 \\
<0.00200\end{array}$ & $\begin{array}{l}<0.00400 \\
0.004000 \\
<0.00400 \\
<0.00400\end{array}$ & $\begin{array}{r}0.005000 \\
0.03300 \\
0.01100 \\
0.02500\end{array}$ & $\begin{array}{r}0.12000 \\
0.17000 \\
0.01300 \\
\mathrm{E} 0.00500\end{array}$ & $\begin{array}{l}<0.01700 \\
<0.01700 \\
<0.01700 \\
<0.01700\end{array}$ & $\begin{array}{r}0.01800 \\
0.11000 \\
0.01600 \\
<0.00300\end{array}$ & $\begin{array}{r}0.05100 \\
0.10000 \\
0.04500 \\
0.009000\end{array}$ & $\begin{array}{l}<0.00500 \\
<0.00500 \\
<0.00500 \\
<0.00500\end{array}$ \\
\hline 14202000 & $\begin{array}{l}04-12-94 \\
05-25-94 \\
06-15-94 \\
10-28-94 \\
10-29-94 \\
11-09-94 \\
11-29-94\end{array}$ & $\begin{array}{l}<0.00600 \\
<0.00600 \\
<0.00600 \\
<0.00600 \\
<0.00600 \\
<0.00600 \\
<0.00600\end{array}$ & $\begin{array}{l}<0.00200 \\
<0.00200 \\
<0.00200 \\
<0.00200 \\
<0.00200 \\
<0.00200 \\
<0.00200\end{array}$ & $\begin{array}{l}<0.00400 \\
<0.00400 \\
<0.00400 \\
<0.00400 \\
<0.00400 \\
<0.00400 \\
<0.00400\end{array}$ & $\begin{array}{l}<0.00400 \\
<0.00400 \\
<0.00400 \\
<0.00400 \\
E 0.00500 \\
<0.00400 \\
<0.00400\end{array}$ & $\begin{array}{l}0.005000 \\
0.004000 \\
0.004000 \\
<0.00200 \\
<0.00200 \\
0.009000 \\
\text { E0.00700 }\end{array}$ & $\begin{array}{l}<0.01700 \\
<0.01700 \\
<0.01700 \\
<0.01700 \\
<0.01700 \\
<0.01700 \\
<0.01700\end{array}$ & $\begin{array}{l}<0.00300 \\
<0.00300 \\
0.003000 \\
E 0.00600 \\
E 0.00600 \\
<0.00300 \\
<0.00300\end{array}$ & $\begin{array}{r}<0.00300 \\
<0.00300 \\
0.003000 \\
0.01500 \\
\mathrm{E} 0.00500 \\
<0.00300 \\
<0.00300\end{array}$ & $\begin{array}{l}<0.00500 \\
<0.00500 \\
<0.00500 \\
<0.00500 \\
<0.00500 \\
<0.00500 \\
<0.00500\end{array}$ \\
\hline 451603122423301 & $05-25-94$ & $<0.00600$ & $<0.00200$ & $<0.00400$ & $<0.00400$ & $<0.00200$ & $<0.01700$ & $<0.00300$ & $<0.00300$ & $<0.00500$ \\
\hline 14206200 & $\begin{array}{l}05-27-94 \\
12-01-94\end{array}$ & $\begin{array}{l}<0.00600 \\
<0.00600\end{array}$ & $\begin{array}{l}<0.00200 \\
<0.00200\end{array}$ & $\begin{array}{l}<0.00400 \\
<0.00400\end{array}$ & $\begin{array}{l}<0.00400 \\
0.006000\end{array}$ & $\begin{array}{l}0.003000 \\
<0.00200\end{array}$ & $\begin{array}{l}<0.01700 \\
<0.01700\end{array}$ & $\begin{array}{l}<0.00300 \\
<0.00300\end{array}$ & $\begin{array}{l}<0.00300 \\
<0.00300\end{array}$ & $\begin{array}{l}<0.00500 \\
<0.00500\end{array}$ \\
\hline
\end{tabular}


Table 36. Concentrations of pesticides in filtered water analyzed by gas chromatography/mass spectroscopy from Phase II of the Willamette River Basin Water Quality Study, Oregon, 1994—Continued

\begin{tabular}{|c|c|c|c|c|c|c|c|c|c|c|}
\hline STATION NUMBER & DATE & $\begin{array}{l}\text { METHYL } \\
\text { AZIN- } \\
\text { PHOS } \\
\text { WATER, } \\
\text { DISSOLV } \\
\text { (UG/L) }\end{array}$ & $\begin{array}{l}\text { METHYL } \\
\text { PARA- } \\
\text { THION } \\
\text { WATER, } \\
\text { DISSOLV } \\
\text { (UG/L) }\end{array}$ & $\begin{array}{l}\text { PARA- } \\
\text { THION } \\
\text { WATER, } \\
\text { DISSOLV } \\
\text { (UG/L) }\end{array}$ & $\begin{array}{l}\text { PHORATE } \\
\text { WATER, } \\
\text { DISSOLV } \\
\text { (UG/L) }\end{array}$ & $\begin{array}{l}\text { TER- } \\
\text { BUFOS } \\
\text { WATER, } \\
\text { DISSOLV } \\
(\text { UG/L) }\end{array}$ & $\begin{array}{l}\text { PER- } \\
\text { METHRIN } \\
\text { WATER, } \\
\text { DISSOLV } \\
\text { (UG/L) }\end{array}$ & $\begin{array}{l}\text { LIN- } \\
\text { URON } \\
\text { WATER, } \\
\text { DISSOLV } \\
\text { (UG/L) }\end{array}$ & $\begin{array}{l}\text { TEBU- } \\
\text { THIURON } \\
\text { WATER, } \\
\text { DISSOLV } \\
\text { (UG/L) }\end{array}$ & $\begin{array}{l}\text { ATRA- } \\
\text { ZINE } \\
\text { WATER, } \\
\text { DISSOLV } \\
\text { (UG/L) }\end{array}$ \\
\hline 445547123065400 & $05-26-94$ & $<0.00100$ & $<0.00200$ & $<0.00600$ & $<0.00200$ & $<0.00700$ & $<0.00500$ & $<0.00200$ & $<0.01000$ & 0.04600 \\
\hline 14190970 & $\begin{array}{l}11-23-94 \\
11-30-94 \\
11-30-94\end{array}$ & $\begin{array}{l}<0.00100 \\
<0.00100 \\
<0.00100\end{array}$ & $\begin{array}{l}<0.00200 \\
<0.00200 \\
<0.00200\end{array}$ & $\begin{array}{l}<0.00600 \\
<0.00600 \\
<0.00600\end{array}$ & $\begin{array}{l}<0.00200 \\
<0.00200 \\
<0.00200\end{array}$ & $\begin{array}{l}<0.00700 \\
<0.00700 \\
<0.00700\end{array}$ & $\begin{array}{l}<0.00500 \\
<0.00500 \\
<0.00500\end{array}$ & $\begin{array}{l}<0.00200 \\
<0.00200 \\
<0.00200\end{array}$ & $\begin{array}{l}0.03200 \\
0.05800 \\
0.05100\end{array}$ & $\begin{array}{l}0.04200 \\
0.07600 \\
0.11000\end{array}$ \\
\hline 445037122573800 & $\begin{array}{l}06-14-94 \\
10-31-94 \\
11-04-94 \\
11-04-94\end{array}$ & $\begin{array}{l}<0.00100 \\
<0.00100 \\
<0.00100 \\
<0.00100\end{array}$ & $\begin{array}{l}<0.00200 \\
<0.00200 \\
<0.00200 \\
<0.00200\end{array}$ & $\begin{array}{l}<0.00600 \\
<0.00600 \\
<0.00600 \\
<0.00600\end{array}$ & $\begin{array}{l}<0.00200 \\
<0.00200 \\
<0.00200 \\
<0.00200\end{array}$ & $\begin{array}{l}<0.00700 \\
<0.00700 \\
<0.00700 \\
<0.00700\end{array}$ & $\begin{array}{l}<0.00500 \\
<0.00500 \\
<0.00500 \\
<0.00500\end{array}$ & $\begin{array}{l}<0.00200 \\
<0.00200 \\
<0.00200 \\
<0.00200\end{array}$ & $\begin{array}{l}<0.01000 \\
\mathrm{E} 0.01000 \\
<0.01000 \\
<0.01000\end{array}$ & $\begin{array}{r}0.02700 \\
1.3000 \\
0.50000 \\
0.63000\end{array}$ \\
\hline 14194150 & $\begin{array}{l}05-17-94 \\
11-02-94\end{array}$ & $\begin{array}{l}<0.00100 \\
<0.00100\end{array}$ & $\begin{array}{l}<0.00200 \\
<0.00200\end{array}$ & $\begin{array}{l}<0.00600 \\
<0.00600\end{array}$ & $\begin{array}{l}<0.00200 \\
<0.00200\end{array}$ & $\begin{array}{l}<0.00700 \\
<0.00700\end{array}$ & $\begin{array}{l}<0.00500 \\
<0.00500\end{array}$ & $\begin{array}{l}<0.00200 \\
<0.00200\end{array}$ & $\begin{array}{l}<0.01000 \\
<0.01000\end{array}$ & $\begin{array}{l}0.02600 \\
0.04300\end{array}$ \\
\hline 451355123093600 & $05-17-94$ & $<0.00100$ & $<0.00200$ & $<0.00600$ & $<0.00200$ & $<0.00700$ & $<0.00500$ & $<0.00200$ & $<0.01000$ & 0.01200 \\
\hline 451602122564400 & $05-31-94$ & -- & $<0.00200$ & $<0.00600$ & $<0.00200$ & $<0.00700$ & $<0.00500$ & $<0.00200$ & $<0.01000$ & 0.01200 \\
\hline 451502122524700 & $\begin{array}{l}05-26-94 \\
10-31-94\end{array}$ & $\begin{array}{l}<0.00100 \\
<0.00100\end{array}$ & $\begin{array}{l}<0.00200 \\
<0.00200\end{array}$ & $\begin{array}{l}<0.00600 \\
<0.00600\end{array}$ & $\begin{array}{l}<0.00200 \\
<0.00200\end{array}$ & $\begin{array}{l}<0.00700 \\
<0.00700\end{array}$ & $\begin{array}{l}<0.00500 \\
<0.00500\end{array}$ & $\begin{array}{l}<0.00200 \\
<0.00200\end{array}$ & $\begin{array}{l}<0.01000 \\
<0.01000\end{array}$ & $\begin{array}{l}0.15000 \\
0.12000\end{array}$ \\
\hline 14201300 & $\begin{array}{l}05-25-94 \\
06-13-94 \\
10-28-94 \\
11-28-94\end{array}$ & $\begin{array}{l}<0.00100 \\
<0.00100 \\
<0.00100 \\
<0.00100\end{array}$ & $\begin{array}{l}<0.00200 \\
<0.00200 \\
<0.00200 \\
<0.00200\end{array}$ & $\begin{array}{l}<0.00600 \\
<0.00600 \\
<0.00600 \\
<0.00600\end{array}$ & $\begin{array}{l}<0.00200 \\
<0.00200 \\
<0.00200 \\
<0.00200\end{array}$ & $\begin{array}{l}<0.00700 \\
<0.00700 \\
<0.00700 \\
<0.00700\end{array}$ & $\begin{array}{l}<0.00500 \\
<0.00500 \\
<0.00500 \\
<0.00500\end{array}$ & $\begin{array}{l}<0.00200 \\
<0.00200 \\
<0.00200 \\
<0.00200\end{array}$ & $\begin{array}{l}<0.01000 \\
<0.01000 \\
<0.01000 \\
<0.01000\end{array}$ & $\begin{array}{r}1.8000 \\
2.4000 \\
1.8000 \\
0.19000\end{array}$ \\
\hline 14202000 & $\begin{array}{l}04-12-94 \\
05-25-94 \\
06-15-94 \\
10-28-94 \\
10-29-94 \\
11-09-94 \\
11-29-94\end{array}$ & $\begin{array}{l}<0.00100 \\
<0.00100 \\
<0.00100 \\
<0.00100 \\
<0.00100 \\
<0.00100 \\
<0.00100\end{array}$ & $\begin{array}{l}<0.00200 \\
<0.00200 \\
<0.00200 \\
<0.00200 \\
<0.00200 \\
<0.00200 \\
<0.00200\end{array}$ & $\begin{array}{l}<0.00600 \\
<0.00600 \\
<0.00600 \\
<0.00600 \\
<0.00600 \\
<0.00600 \\
<0.00600\end{array}$ & $\begin{array}{l}<0.00200 \\
<0.00200 \\
<0.00200 \\
<0.00200 \\
<0.00200 \\
<0.00200 \\
<0.00200\end{array}$ & $\begin{array}{l}<0.00700 \\
<0.00700 \\
<0.00700 \\
<0.00700 \\
<0.00700 \\
<0.00700 \\
<0.00700\end{array}$ & $\begin{array}{l}<0.00500 \\
<0.00500 \\
<0.00500 \\
<0.00500 \\
<0.00500 \\
<0.00500 \\
<0.00500\end{array}$ & $\begin{array}{l}<0.00200 \\
<0.00200 \\
<0.00200 \\
<0.00200 \\
<0.00200 \\
<0.00200 \\
<0.00200\end{array}$ & $\begin{array}{l}<0.01000 \\
<0.01000 \\
<0.01000 \\
<0.01000 \\
\mathrm{E} 0.00600 \\
<0.01000 \\
<0.01000\end{array}$ & $\begin{array}{l}0.25000 \\
0.11000 \\
0.06100 \\
0.24000 \\
0.09200 \\
0.16000 \\
0.06500\end{array}$ \\
\hline 451603122423301 & $05-25-94$ & $<0.00100$ & $<0.00200$ & $<0.00600$ & $<0.00200$ & $<0.00700$ & $<0.00500$ & $<0.00200$ & $<0.01000$ & 0.006000 \\
\hline 14206200 & $\begin{array}{l}05-27-94 \\
12-01-94\end{array}$ & $\begin{array}{l}<0.00100 \\
<0.00100\end{array}$ & $\begin{array}{l}<0.00200 \\
<0.00200\end{array}$ & $\begin{array}{l}<0.00600 \\
<0.00600\end{array}$ & $\begin{array}{l}<0.00200 \\
<0.00200\end{array}$ & $\begin{array}{l}<0.00700 \\
<0.00700\end{array}$ & $\begin{array}{l}<0.00500 \\
<0.00500\end{array}$ & $\begin{array}{l}<0.00200 \\
<0.00200\end{array}$ & $\begin{array}{l}<0.01000 \\
<0.01000\end{array}$ & $\begin{array}{l}0.04900 \\
0.75000\end{array}$ \\
\hline
\end{tabular}


Table 36. Concentrations of pesticides in filtered water analyzed by gas chromatography/mass spectroscopy from Phase II of the Willamette River Basin Water Quality Study, Oregon, 1994—Continued

\begin{tabular}{|c|c|c|c|c|c|c|c|c|c|c|}
\hline STATION NUMBER & DATE & $\begin{array}{l}\text { CYANA- } \\
\text { ZINE, } \\
\text { WATER, } \\
\text { DISSOLV } \\
\text { (UG/L) }\end{array}$ & $\begin{array}{l}\text { DESETHY } \\
\text { ATRA- } \\
\text { ZINE, } \\
\text { WATER, } \\
\text { DISSOLV } \\
\text { (UG/L) }\end{array}$ & $\begin{array}{ll}L & \\
\text { METRI- } \\
\text { BUZIN } \\
\text { DISSOLV } \\
(\text { UG/L) }\end{array}$ & $\begin{array}{l}\text { PRO- } \\
\text { METON, } \\
\text { WATER, } \\
\text { DISSOLV } \\
\text { (UG/L) }\end{array}$ & $\begin{array}{l}\text { SI- } \\
\text { MAZINE, } \\
\text { WATER, } \\
\text { DISSOLV } \\
\text { (UG/L) }\end{array}$ & $\begin{array}{l}\text { TER- } \\
\text { BACIL } \\
\text { WATER, } \\
\text { DISSOLV } \\
(\text { UG/L) }\end{array}$ & $\begin{array}{l}\text { DIAZ- } \\
\text { INON } \\
\text { D10 SRG } \\
\text { WATER, } \\
\text { DISSOLV } \\
\text { PERCENT }\end{array}$ & $\begin{array}{l}\text { TERBUTH } \\
\text { YLAZINE } \\
\text { SURROGT } \\
\text { WATER, } \\
\text { DISSOLV } \\
\text { PERCENT }\end{array}$ & $\begin{array}{c}\text { HCH } \\
\text { ALPHA } \\
\text { D } 6 \text { SRG } \\
\text { WATER, } \\
\text { DISSOLV } \\
\text { PERCENT }\end{array}$ \\
\hline 445547123065400 & $05-26-94$ & $<0.00400$ & $<0.00200$ & $<0.00400$ & $<0.00300$ & 0.005000 & $\mathrm{E} 0.20000$ & 58.00 & 101.00 & 90.00 \\
\hline 14190970 & $\begin{array}{l}11-23-94 \\
11-30-94 \\
11-30-94\end{array}$ & $\begin{array}{l}<0.00400 \\
<0.00400 \\
<0.00400\end{array}$ & $\begin{array}{l}\mathrm{E} 0.01100 \\
<0.00200 \\
\mathrm{E} 0.00600\end{array}$ & $\begin{array}{l}<0.00400 \\
<0.00400 \\
<0.00400\end{array}$ & $\begin{array}{l}0.01200 \\
0.02200 \\
0.02600\end{array}$ & $\begin{array}{l}0.05400 \\
0.06800 \\
0.06700\end{array}$ & $\begin{array}{l}<0.00700 \\
<0.00700 \\
<0.00700\end{array}$ & $\begin{array}{l}115.00 \\
126.00 \\
126.00\end{array}$ & $\begin{array}{l}119.00 \\
128.00 \\
126.00\end{array}$ & $\begin{array}{l}106.00 \\
111.00 \\
115.00\end{array}$ \\
\hline 445037122573800 & $\begin{array}{l}06-14-94 \\
10-31-94 \\
11-04-94 \\
11-04-94\end{array}$ & $\begin{array}{l}<0.00400 \\
<0.00400 \\
<0.00400 \\
<0.00400\end{array}$ & $\begin{array}{l}\text { E0.00600 } \\
\text { E0.06100 } \\
\text { E0.03900 } \\
\text { E0.07300 }\end{array}$ & $\begin{array}{r}<0.00400 \\
\mathrm{E} 0.01100 \\
0.08400 \\
0.08700\end{array}$ & $\begin{array}{l}<0.00300 \\
0.009000 \\
<0.00300 \\
\mathrm{E} 0.00600\end{array}$ & $\begin{array}{l}0.01900 \\
0.13000 \\
0.28000 \\
0.40000\end{array}$ & $\begin{array}{r}\text { E0.09100 } \\
\text { E1.0000 } \\
\text { E0.70000 } \\
\text { E0.90000 }\end{array}$ & $\begin{array}{r}110.00 \\
102.00 \\
44.00 \\
48.00\end{array}$ & $\begin{array}{r}100.00 \\
109.00 \\
99.00 \\
98.00\end{array}$ & $\begin{array}{r}111.00 \\
84.00 \\
77.00 \\
78.00\end{array}$ \\
\hline 14194150 & $\begin{array}{l}05-17-94 \\
11-02-94\end{array}$ & $\begin{array}{l}<0.00400 \\
<0.00400\end{array}$ & $\begin{array}{l}\mathrm{E} 0.00200 \\
\mathrm{E} 0.00300\end{array}$ & $\begin{array}{l}<0.00400 \\
<0.00400\end{array}$ & $\begin{array}{l}<0.00300 \\
<0.00300\end{array}$ & $\begin{array}{l}0.02300 \\
0.02700\end{array}$ & $\begin{array}{l}<0.00700 \\
<0.00700\end{array}$ & $\begin{array}{r}99.00 \\
100.00\end{array}$ & $\begin{array}{l}110.00 \\
101.00\end{array}$ & $\begin{array}{r}114.00 \\
85.00\end{array}$ \\
\hline 451355123093600 & $05-17-94$ & $<0.00400$ & $<0.00200$ & $<0.00400$ & $<0.00300$ & $<0.00500$ & $<0.00700$ & 105.00 & 111.00 & 106.00 \\
\hline 451602122564400 & $05-31-94$ & $<0.00400$ & $<0.00200$ & $<0.00400$ & $<0.00300$ & 0.01200 & -- & 124.00 & 124.00 & 129.00 \\
\hline 451502122524700 & $\begin{array}{l}05-26-94 \\
10-31-94\end{array}$ & $\begin{array}{l}<0.00400 \\
<0.00400\end{array}$ & $\begin{array}{l}\mathrm{E} 0.02200 \\
\mathrm{E} 0.00500\end{array}$ & $\begin{array}{l}<0.00400 \\
<0.00400\end{array}$ & $\begin{array}{l}<0.00300 \\
<0.00300\end{array}$ & $\begin{array}{r}0.23000 \\
1.6000\end{array}$ & $\begin{array}{l}<0.00700 \\
<0.00700\end{array}$ & $\begin{array}{r}71.00 \\
102.00\end{array}$ & $\begin{array}{l}116.00 \\
113.00\end{array}$ & $\begin{array}{l}95.00 \\
83.00\end{array}$ \\
\hline 14201300 & $\begin{array}{l}05-25-94 \\
06-13-94 \\
10-28-94 \\
11-28-94\end{array}$ & $\begin{array}{l}<0.00400 \\
<0.00400 \\
<0.00400 \\
<0.02600\end{array}$ & $\begin{array}{l}\text { E0.03500 } \\
\text { E0.06700 } \\
\text { E0.04000 } \\
\text { E0.04800 }\end{array}$ & $\begin{array}{r}<0.00400 \\
<0.00400 \\
<0.00400 \\
0.04700\end{array}$ & $\begin{array}{l}<0.00300 \\
<0.00300 \\
<0.00300 \\
<0.00300\end{array}$ & $\begin{array}{r}3.5000 \\
2.8000 \\
0.94000 \\
0.12000\end{array}$ & $\begin{array}{l}\text { E0.00800 } \\
\text { E0.01100 } \\
\text { E0.03100 } \\
\text { E0.01800 }\end{array}$ & $\begin{array}{r}58.00 \\
80.00 \\
100.00 \\
50.00\end{array}$ & $\begin{array}{l}102.00 \\
102.00 \\
111.00 \\
116.00\end{array}$ & $\begin{array}{r}89.00 \\
83.00 \\
100.00 \\
100.00\end{array}$ \\
\hline 14202000 & $\begin{array}{l}04-12-94 \\
05-25-94 \\
06-15-94 \\
10-28-94 \\
10-29-94 \\
11-09-94 \\
11-29-94\end{array}$ & $\begin{array}{l}<0.00400 \\
<0.00400 \\
<0.00400 \\
<0.00400 \\
<0.00400 \\
<0.00400 \\
<0.02500\end{array}$ & $\begin{array}{l}\text { E0.01100 } \\
\text { E0.00500 } \\
\text { E0.00600 } \\
\text { E0.00700 } \\
\text { E0.00600 } \\
\text { E0.01300 } \\
\text { E0.01600 }\end{array}$ & $\begin{array}{r}<0.00400 \\
<0.00400 \\
<0.00400 \\
<0.00400 \\
<0.00400 \\
0.01100 \\
0.01400\end{array}$ & $\begin{array}{l}<0.00300 \\
<0.00300 \\
<0.00300 \\
<0.00300 \\
<0.00300 \\
<0.00300 \\
<0.00300\end{array}$ & $\begin{array}{l}0.11000 \\
0.09300 \\
0.04500 \\
0.32000 \\
0.21000 \\
0.10000 \\
0.05000\end{array}$ & $\begin{array}{l}<0.00700 \\
\mathrm{E} 0.03700 \\
<0.00700 \\
\mathrm{E} 0.00800 \\
\mathrm{E} 0.01100 \\
\mathrm{E} 0.00700 \\
<0.00700\end{array}$ & $\begin{array}{r}117.00 \\
56.00 \\
87.00 \\
100.00 \\
100.00 \\
116.00 \\
50.00\end{array}$ & $\begin{array}{r}118.00 \\
101.00 \\
96.00 \\
108.00 \\
106.00 \\
123.00 \\
117.00\end{array}$ & $\begin{array}{r}113.00 \\
92.00 \\
95.00 \\
100.00 \\
100.00 \\
111.00 \\
100.00\end{array}$ \\
\hline 451603122423301 & $05-25-94$ & $<0.00400$ & $<0.00200$ & $<0.00400$ & $<0.00300$ & 0.004000 & $<0.00700$ & 53.00 & 98.00 & 90.00 \\
\hline 14206200 & $\begin{array}{l}05-27-94 \\
12-01-94\end{array}$ & $\begin{array}{l}<0.00400 \\
<0.00400\end{array}$ & $\begin{array}{l}<0.00200 \\
\mathrm{E} 0.02800\end{array}$ & $\begin{array}{r}<0.00400 \\
0.01400\end{array}$ & $\begin{array}{l}<0.00300 \\
\mathrm{E} 0.00700\end{array}$ & $\begin{array}{r}<0.00500 \\
0.18000\end{array}$ & $\begin{array}{l}<0.00700 \\
\mathrm{E} 0.01400\end{array}$ & $\begin{array}{l}119.00 \\
104.00\end{array}$ & $\begin{array}{l}110.00 \\
104.00\end{array}$ & $\begin{array}{r}115.00 \\
98.00\end{array}$ \\
\hline
\end{tabular}


Table 36. Concentrations of pesticides in filtered water analyzed by gas chromatography/mass spectroscopy from Phase II of the Willamette River Basin Water Quality Study, Oregon, 1994—Continued

\begin{tabular}{|c|c|c|c|c|c|c|}
\hline STATION NUMBER & STATION NAME & DATE & TIME & $\begin{array}{l}\text { ALA- } \\
\text { CHLOR, } \\
\text { WATER } \\
\text { DISSOLV } \\
(\text { UG/L) }\end{array}$ & $\begin{array}{l}\text { METO- } \\
\text { LACHLOR } \\
\text { WATER } \\
\text { DISSOLV } \\
\text { (UG/L) }\end{array}$ & $\begin{array}{l}\text { NAPROP- } \\
\text { AMIDE } \\
\text { WATER } \\
\text { DISSOLV } \\
\text { (UG/L) }\end{array}$ \\
\hline 14206298 & $\begin{array}{llll}\text { BRONSON CREEK AT } 185 \mathrm{TH} \text { AVE NEAR ALOHA, OR } \\
\text { BRONSON CREEK AT } 185 \mathrm{TH} \text { AVE NEAR ALOHA, OR }\end{array}$ & $\begin{array}{l}05-27-94 \\
11-23-94\end{array}$ & $\begin{array}{l}1100 \\
1030\end{array}$ & $\begin{array}{l}<0.00200 \\
<0.00200\end{array}$ & $\begin{array}{l}<0.00400 \\
\mathrm{E} 0.00400\end{array}$ & $\begin{array}{l}<0.00400 \\
<0.00400\end{array}$ \\
\hline 14206950 & $\begin{array}{lllll}\text { FANNO } & \text { CREEK AT } & \text { DURHAM, OR } \\
\text { FANNO CREEK AT } & \text { DURHAM, OR } \\
\text { FANNO CREEK AT } & \text { DURHAM, OR } \\
\text { FANNO CREEK AT } & \text { DURHAM, OR }\end{array}$ & $\begin{array}{l}05-24-94 \\
06-16-94 \\
10-27-94 \\
11-30-94\end{array}$ & $\begin{array}{l}1420 \\
1040 \\
1620 \\
1010\end{array}$ & $\begin{array}{l}<0.00200 \\
<0.00200 \\
<0.00200 \\
<0.00200\end{array}$ & $\begin{array}{r}0.007000 \\
0.01300 \\
0.01700 \\
\mathrm{E} 0.00800\end{array}$ & $\begin{array}{l}<0.00400 \\
<0.00400 \\
<0.00400 \\
<0.00400\end{array}$ \\
\hline 14207500 & $\begin{array}{llll}\text { TUALATIN RIVER AT WEST LINN, OREG. } \\
\text { TUALATIN RIVER AT WEST LINN, OREG. } \\
\text { TUALATIN RIVER AT WEST LINN, OREG. }\end{array}$ & $\begin{array}{l}05-25-94 \\
10-28-94 \\
12-05-94\end{array}$ & $\begin{array}{l}1540 \\
1610 \\
0910\end{array}$ & $\begin{array}{l}<0.00200 \\
<0.00200 \\
<0.00200\end{array}$ & $\begin{array}{l}0.01500 \\
0.02400 \\
0.07500\end{array}$ & $\begin{array}{r}<0.00400 \\
0.01300 \\
0.01400\end{array}$ \\
\hline 452823122240900 & 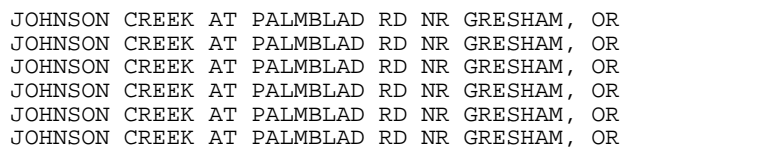 & $\begin{array}{l}05-24-94 \\
10-27-94 \\
10-28-94 \\
11-01-94 \\
11-03-94 \\
11-23-94\end{array}$ & $\begin{array}{l}1440 \\
1640 \\
1135 \\
1100 \\
1330 \\
1410\end{array}$ & $\begin{array}{l}<0.00200 \\
<0.00200 \\
<0.00200 \\
<0.00200 \\
<0.00200 \\
<0.00200\end{array}$ & $\begin{array}{l}0.05800 \\
0.32000 \\
0.22000 \\
0.19000 \\
0.08900 \\
0.05200\end{array}$ & $\begin{array}{l}0.03300 \\
0.48000 \\
0.25000 \\
0.09700 \\
0.03300 \\
0.03000\end{array}$ \\
\hline 453043122402200 & COMMERCIAL/RESIDENTIAL RUNOFF AT HARBOR WAY, PORT, OR & $06-17-94$ & 2150 & $<0.00200$ & 0.02000 & $<0.00400$ \\
\hline 14211720 & $\begin{array}{lll}\text { WILLAMETTE } & \text { RIVER AT PORTLAND, OREG. } \\
\text { WILLAMETTE } & \text { RIVER AT PORTLAND, OREG. } \\
\text { WILLAMETTE } & \text { RIVER AT PORTLAND, OREG. } \\
\text { WILLAMETTE } & \text { RIVER AT PORTLAND, OREG. } \\
\text { WILLAMETTE } & \text { RIVER AT PORTLAND, OREG. } \\
\text { WILLAMETTE RIVER AT PORTLAND, OREG. }\end{array}$ & $\begin{array}{l}05-10-94 \\
06-14-94 \\
10-25-94 \\
10-29-94 \\
11-03-94 \\
12-02-94\end{array}$ & $\begin{array}{l}0939 \\
0949 \\
1300 \\
1230 \\
0956 \\
1040\end{array}$ & $\begin{array}{l}<0.00200 \\
<0.00200 \\
<0.00200 \\
<0.00200 \\
<0.00200 \\
\mathrm{E} 0.00200\end{array}$ & $\begin{array}{r}0.005000 \\
0.004000 \\
\mathrm{E} 0.00600 \\
0.04900 \\
0.11000 \\
0.04800\end{array}$ & $\begin{array}{r}<0.00400 \\
<0.00400 \\
<0.00400 \\
0.02200 \\
0.02900 \\
\mathrm{E} 0.00600\end{array}$ \\
\hline 453154122394200 & 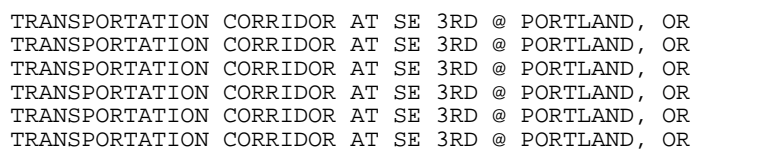 & $\begin{array}{l}05-31-94 \\
11-08-94 \\
11-08-94 \\
11-30-94 \\
11-30-94 \\
12-02-94\end{array}$ & $\begin{array}{l}1800 \\
1800 \\
1950 \\
0840 \\
1020 \\
1020\end{array}$ & $\begin{array}{r}0.06000 \\
<0.00200 \\
<0.00200 \\
<0.00200 \\
<0.00200 \\
<0.00200\end{array}$ & $\begin{array}{r}0.03800 \\
0.02000 \\
0.01900 \\
\text { E0.00900 } \\
\text { E0.00800 } \\
<0.00400\end{array}$ & $\begin{array}{l}<0.00400 \\
<0.00400 \\
<0.00400 \\
<0.00400 \\
<0.00400 \\
<0.00400\end{array}$ \\
\hline 14211805 & WILLAMETTE R AB ST JOHNS BR AT PORTLAND, OREG. & $05-23-94$ & 0950 & $<0.00200$ & 0.005000 & $<0.00400$ \\
\hline
\end{tabular}


Table 36. Concentrations of pesticides in filtered water analyzed by gas chromatography/mass spectroscopy from Phase II of the Willamette River Basin Water Quality Study, Oregon, 1994—Continued

\begin{tabular}{|c|c|c|c|c|c|c|c|c|c|c|c|}
\hline STATION NUMBER & DATE & $\begin{array}{l}\text { PRON- } \\
\text { AMIDE } \\
\text { WATER, } \\
\text { DISSOLV } \\
\text { (UG/L) }\end{array}$ & $\begin{array}{l}\text { PROPA- } \\
\text { CHLOR } \\
\text { WATER, } \\
\text { DISSOLV } \\
\text { (UG/L) }\end{array}$ & $\begin{array}{l}\text { PRO- } \\
\text { PANIL } \\
\text { WATER, } \\
\text { DISSOLV } \\
\text { (UG/L) }\end{array}$ & $\begin{array}{l}\text { BUTYL- } \\
\text { WATER, } \\
\text { DISSOLV } \\
(\text { UG/L) }\end{array}$ & $\begin{array}{l}\text { CAR- } \\
\text { BARYL } \\
\text { WATER, } \\
\text { DISSOLV } \\
\text { (UG/L) }\end{array}$ & $\begin{array}{l}\text { CARBO- } \\
\text { FURAN } \\
\text { WATER, } \\
\text { DISSOLV } \\
\text { (UG/L) }\end{array}$ & $\begin{array}{l}\text { EPTC } \\
\text { WATER, } \\
\text { DISSOLV } \\
(\text { UG/L) }\end{array}$ & \begin{tabular}{l}
\multicolumn{1}{c}{ MOL- } \\
INATE \\
WATER, \\
DISSOLV \\
(UG/L)
\end{tabular} & $\begin{array}{l}\text { PEB- } \\
\text { ULATE } \\
\text { WATER, } \\
\text { DISSOLV } \\
\text { (UG/L) }\end{array}$ & $\begin{array}{c}\text { THIO- } \\
\text { BENCARB } \\
\text { WATER, } \\
\text { DISSOLV } \\
(\text { UG/L) }\end{array}$ \\
\hline 14206298 & $\begin{array}{l}05-27-94 \\
11-23-94\end{array}$ & $\begin{array}{l}<0.01800 \\
<0.01800\end{array}$ & $\begin{array}{l}<0.00700 \\
<0.00700\end{array}$ & $\begin{array}{l}<0.00400 \\
<0.00400\end{array}$ & $\begin{array}{l}<0.00200 \\
<0.00200\end{array}$ & $\begin{array}{l}<0.00300 \\
<0.00300\end{array}$ & $\begin{array}{l}<0.00300 \\
<0.00300\end{array}$ & $\begin{array}{l}0.003000 \\
<0.00200\end{array}$ & $\begin{array}{l}<0.00300 \\
<0.00300\end{array}$ & $\begin{array}{l}<0.00400 \\
<0.00400\end{array}$ & $\begin{array}{l}<0.00200 \\
<0.00200\end{array}$ \\
\hline 14206950 & $\begin{array}{l}05-24-94 \\
06-16-94 \\
10-27-94 \\
11-30-94\end{array}$ & $\begin{array}{l}<0.01800 \\
<0.01800 \\
<0.01800 \\
<0.01800\end{array}$ & $\begin{array}{l}<0.00700 \\
<0.00700 \\
<0.00700 \\
<0.00700\end{array}$ & $\begin{array}{l}<0.00400 \\
<0.00400 \\
<0.00400 \\
<0.00400\end{array}$ & $\begin{array}{l}<0.00200 \\
<0.00200 \\
<0.00200 \\
<0.00200\end{array}$ & $\begin{array}{l}\text { E0.00800 } \\
<0.00300 \\
\text { E0.24000 } \\
<0.00300\end{array}$ & $\begin{array}{l}<0.00300 \\
<0.00300 \\
<0.00300 \\
<0.00300\end{array}$ & $\begin{array}{l}0.003000 \\
0.008000 \\
<0.00200 \\
<0.00200\end{array}$ & $\begin{array}{l}<0.00300 \\
<0.00300 \\
<0.00300 \\
<0.00300\end{array}$ & $\begin{array}{l}<0.00400 \\
<0.00400 \\
<0.00400 \\
<0.00400\end{array}$ & $\begin{array}{l}<0.00200 \\
<0.00200 \\
<0.00200 \\
<0.00200\end{array}$ \\
\hline 14207500 & $\begin{array}{l}05-25-94 \\
10-28-94 \\
12-05-94\end{array}$ & $\begin{array}{r}<0.01800 \\
<0.01800 \\
0.02100\end{array}$ & $\begin{array}{l}<0.00700 \\
<0.00700 \\
<0.00700\end{array}$ & $\begin{array}{l}<0.00400 \\
<0.00400 \\
<0.00400\end{array}$ & $\begin{array}{l}<0.00200 \\
<0.00200 \\
<0.00200\end{array}$ & $\begin{array}{l}\text { E0.01400 } \\
\text { E0.13000 } \\
<0.00300\end{array}$ & $\begin{array}{l}<0.00300 \\
\mathrm{E} 0.03200 \\
<0.00300\end{array}$ & $\begin{array}{r}0.02100 \\
<0.00200 \\
<0.00200\end{array}$ & $\begin{array}{l}<0.00300 \\
<0.00300 \\
<0.00300\end{array}$ & $\begin{array}{l}<0.00400 \\
<0.00400 \\
<0.00400\end{array}$ & $\begin{array}{l}<0.00200 \\
<0.00200 \\
<0.00200\end{array}$ \\
\hline 452823122240900 & $\begin{array}{l}05-24-94 \\
10-27-94 \\
10-28-94 \\
11-01-94 \\
11-03-94 \\
11-23-94\end{array}$ & $\begin{array}{l}0.005000 \\
<0.01800 \\
<0.01800 \\
<0.01800 \\
<0.01800 \\
<0.01800\end{array}$ & $\begin{array}{l}<0.00700 \\
<0.00700 \\
<0.00700 \\
<0.00700 \\
<0.00700 \\
<0.00700\end{array}$ & $\begin{array}{l}<0.00400 \\
<0.00400 \\
<0.00400 \\
<0.00400 \\
<0.00400 \\
<0.00400\end{array}$ & $\begin{array}{l}<0.00200 \\
<0.00200 \\
<0.00200 \\
<0.00200 \\
<0.00200 \\
<0.00200\end{array}$ & $\begin{array}{l}\mathrm{E} 0.01000 \\
<0.00300 \\
<0.00300 \\
<0.00300 \\
<0.00300 \\
<0.00300\end{array}$ & $\begin{array}{l}\mathrm{E} 0.03500 \\
\mathrm{E} 0.00900 \\
<0.00300 \\
<0.00300 \\
<0.00300 \\
\mathrm{E} 0.01200\end{array}$ & $\begin{array}{l}<0.00200 \\
<0.00200 \\
<0.00200 \\
<0.00200 \\
<0.00200 \\
<0.00200\end{array}$ & $\begin{array}{l}<0.00300 \\
<0.00300 \\
<0.00300 \\
<0.00300 \\
<0.00300 \\
<0.00300\end{array}$ & $\begin{array}{l}<0.00400 \\
\mathrm{E} 0.00700 \\
<0.00400 \\
<0.00400 \\
<0.00400 \\
<0.00400\end{array}$ & $\begin{array}{l}<0.00200 \\
<0.00200 \\
<0.00200 \\
<0.00200 \\
<0.00200 \\
<0.00200\end{array}$ \\
\hline 453043122402200 & $06-17-94$ & $<0.01800$ & $<0.00700$ & $<0.00400$ & $<0.00200$ & $<0.00300$ & $<0.00300$ & $<0.00200$ & $<0.00300$ & $<0.00400$ & $<0.00200$ \\
\hline 14211720 & $\begin{array}{l}05-10-94 \\
06-14-94 \\
10-25-94 \\
10-29-94 \\
11-03-94 \\
12-02-94\end{array}$ & $\begin{array}{r}<0.01800 \\
<0.01800 \\
<0.01800 \\
<0.01800 \\
<0.01800 \\
0.01800\end{array}$ & $\begin{array}{l}<0.00700 \\
<0.00700 \\
<0.00700 \\
<0.00700 \\
<0.00700 \\
<0.00700\end{array}$ & $\begin{array}{l}<0.00400 \\
<0.00400 \\
<0.00400 \\
<0.00400 \\
<0.00400 \\
<0.00400\end{array}$ & $\begin{array}{l}<0.00200 \\
<0.00200 \\
<0.00200 \\
<0.00200 \\
<0.00200 \\
<0.00200\end{array}$ & $\begin{array}{l}<0.00300 \\
<0.00300 \\
<0.00300 \\
\mathrm{E} 0.00600 \\
<0.00300 \\
<0.00300\end{array}$ & $\begin{array}{l}<0.00300 \\
<0.00300 \\
<0.00300 \\
\mathrm{E} 0.18000 \\
\mathrm{E} 0.08800 \\
<0.00300\end{array}$ & $\begin{array}{l}0.005000 \\
0.005000 \\
<0.00200 \\
<0.00200 \\
<0.00200 \\
\text { E0.00400 }\end{array}$ & $\begin{array}{l}<0.00300 \\
<0.00300 \\
<0.00300 \\
<0.00300 \\
<0.00300 \\
<0.00300\end{array}$ & $\begin{array}{l}<0.00400 \\
<0.00400 \\
<0.00400 \\
<0.00400 \\
<0.00400 \\
<0.00400\end{array}$ & $\begin{array}{l}<0.00200 \\
<0.00200 \\
<0.00200 \\
<0.00200 \\
<0.00200 \\
<0.00200\end{array}$ \\
\hline 453154122394200 & $\begin{array}{l}05-31-94 \\
11-08-94 \\
11-08-94 \\
11-30-94 \\
11-30-94 \\
12-02-94\end{array}$ & $\begin{array}{l}<0.01800 \\
<0.01800 \\
<0.01800 \\
<0.01800 \\
<0.01800 \\
<0.01800\end{array}$ & $\begin{array}{l}<0.00700 \\
<0.00700 \\
<0.00700 \\
<0.00700 \\
<0.00700 \\
<0.00700\end{array}$ & $\begin{array}{l}<0.00400 \\
<0.00400 \\
<0.00400 \\
<0.00400 \\
<0.00400 \\
<0.00400\end{array}$ & $\begin{array}{l}<0.00200 \\
<0.00200 \\
<0.00200 \\
<0.00200 \\
<0.00200 \\
<0.00200\end{array}$ & $\begin{array}{l}<0.00300 \\
<0.00300 \\
<0.00300 \\
<0.00300 \\
<0.00300 \\
<0.00300\end{array}$ & $\begin{array}{l}<0.00300 \\
<0.00300 \\
<0.00300 \\
<0.00300 \\
<0.00300 \\
<0.00300\end{array}$ & $\begin{array}{l}<0.00200 \\
<0.00200 \\
<0.00200 \\
<0.00200 \\
<0.00200 \\
<0.00200\end{array}$ & $\begin{array}{l}<0.00300 \\
<0.00300 \\
<0.00300 \\
<0.00300 \\
<0.00300 \\
<0.00300\end{array}$ & $\begin{array}{l}<0.00400 \\
<0.00400 \\
<0.00400 \\
<0.00400 \\
<0.00400 \\
<0.00400\end{array}$ & $\begin{array}{l}<0.00200 \\
<0.00200 \\
<0.00200 \\
<0.00200 \\
<0.00200 \\
<0.00200\end{array}$ \\
\hline 14211805 & $05-23-94$ & $<0.01800$ & $<0.00700$ & $<0.00400$ & $<0.00200$ & $<0.00300$ & $<0.00300$ & 0.006000 & $<0.00300$ & $<0.00400$ & $<0.00200$ \\
\hline
\end{tabular}


Table 36. Concentrations of pesticides in filtered water analyzed by gas chromatography/mass spectroscopy from Phase II of the Willamette River Basin Water Quality Study, Oregon, 1994—Continued

\begin{tabular}{|c|c|c|c|c|c|c|c|c|c|c|}
\hline STATION NUMBER & DATE & $\begin{array}{l}\text { TRIAL- } \\
\text { LATE } \\
\text { WATER, } \\
\text { DISSOLV } \\
\text { (UG/L) }\end{array}$ & $\begin{array}{l}\text { BEN- } \\
\text { FLUR- } \\
\text { ALIN } \\
\text { WATER, } \\
\text { DISSOLV } \\
\text { (UG/L) }\end{array}$ & $\begin{array}{l}\text { ETHAL- } \\
\text { FLUR- } \\
\text { ALIN } \\
\text { WATER, } \\
\text { DISSOLV } \\
\text { (UG/L) }\end{array}$ & $\begin{array}{l}\text { PENDI- } \\
\text { METH- } \\
\text { ALIN } \\
\text { WATER, } \\
\text { DISSOLV } \\
(\text { UG/L) }\end{array}$ & $\begin{array}{l}\text { TRI- } \\
\text { FLUR- } \\
\text { ALIN } \\
\text { WATER, } \\
\text { DISSOLV } \\
(\text { UG/L) }\end{array}$ & $\begin{array}{c}2,6-D I- \\
\text { ETHYL } \\
\text { ANILINE } \\
\text { WATER, } \\
\text { DISSOLV } \\
\text { (UG/L) }\end{array}$ & $\begin{array}{l}\text { PRO- } \\
\text { PARGITE } \\
\text { WATER, } \\
\text { DISSOLV } \\
\text { (UG/L) }\end{array}$ & $\begin{array}{l}\text { DCPA } \\
\text { WATER, } \\
\text { DISSOLV } \\
\text { (UG/L) }\end{array}$ & $\begin{array}{l}\text { DI- } \\
\text { ELDRIN } \\
\text { WATER, } \\
\text { DISSOLV } \\
(\mathrm{UG} / \mathrm{L})\end{array}$ \\
\hline 14206298 & $\begin{array}{l}05-27-94 \\
11-23-94\end{array}$ & $\begin{array}{l}<0.00100 \\
<0.00100\end{array}$ & $\begin{array}{l}<0.00200 \\
<0.00200\end{array}$ & $\begin{array}{l}<0.00400 \\
<0.00400\end{array}$ & $\begin{array}{l}<0.00400 \\
<0.00400\end{array}$ & $\begin{array}{l}<0.00200 \\
<0.00200\end{array}$ & $\begin{array}{l}<0.00300 \\
<0.00300\end{array}$ & $\begin{array}{l}<0.01300 \\
<0.01300\end{array}$ & $\begin{array}{l}<0.00200 \\
<0.00200\end{array}$ & $\begin{array}{l}<0.00100 \\
<0.00100\end{array}$ \\
\hline 14206950 & $\begin{array}{l}05-24-94 \\
06-16-94 \\
10-27-94 \\
11-30-94\end{array}$ & $\begin{array}{l}<0.00100 \\
<0.00100 \\
<0.00100 \\
<0.00100\end{array}$ & $\begin{array}{l}<0.00200 \\
<0.00200 \\
<0.00200 \\
<0.00200\end{array}$ & $\begin{array}{l}<0.00400 \\
<0.00400 \\
<0.00400 \\
<0.00400\end{array}$ & $\begin{array}{l}<0.00400 \\
<0.00400 \\
<0.00400 \\
<0.00400\end{array}$ & $\begin{array}{l}<0.00200 \\
<0.00200 \\
<0.00200 \\
<0.00200\end{array}$ & $\begin{array}{l}<0.00300 \\
<0.00300 \\
<0.00300 \\
<0.00300\end{array}$ & $\begin{array}{l}<0.01300 \\
<0.01300 \\
<0.01300 \\
<0.01300\end{array}$ & $\begin{array}{l}0.001000 \\
0.002000 \\
\mathrm{E} 0.00200 \\
\mathrm{E} 0.00300\end{array}$ & $\begin{array}{l}<0.00100 \\
<0.00100 \\
<0.00100 \\
<0.00100\end{array}$ \\
\hline 14207500 & $\begin{array}{l}05-25-94 \\
10-28-94 \\
12-05-94\end{array}$ & $\begin{array}{l}<0.00100 \\
<0.00100 \\
\mathrm{E} 0.00500\end{array}$ & $\begin{array}{l}<0.00200 \\
<0.00200 \\
<0.00200\end{array}$ & $\begin{array}{l}<0.00400 \\
<0.00400 \\
<0.00400\end{array}$ & $\begin{array}{l}<0.00400 \\
\mathrm{E} 0.01100 \\
<0.00400\end{array}$ & $\begin{array}{l}<0.00200 \\
E 0.00500 \\
<0.00200\end{array}$ & $\begin{array}{l}<0.00300 \\
<0.00300 \\
<0.00300\end{array}$ & $\begin{array}{l}<0.01300 \\
<0.01300 \\
<0.01300\end{array}$ & $\begin{array}{l}0.001000 \\
\mathrm{E} 0.00100 \\
<0.00200\end{array}$ & $\begin{array}{l}<0.00100 \\
<0.00100 \\
<0.00100\end{array}$ \\
\hline 452823122240900 & $\begin{array}{l}05-24-94 \\
10-27-94 \\
10-28-94 \\
11-01-94 \\
11-03-94 \\
11-23-94\end{array}$ & $\begin{array}{l}<0.00100 \\
<0.00100 \\
<0.00100 \\
<0.00100 \\
<0.00100 \\
<0.00100\end{array}$ & $\begin{array}{l}<0.00200 \\
<0.00200 \\
<0.00200 \\
<0.00200 \\
<0.00200 \\
<0.00200\end{array}$ & $\begin{array}{l}<0.00400 \\
<0.00400 \\
<0.00400 \\
<0.00400 \\
<0.00400 \\
<0.00400\end{array}$ & $\begin{array}{l}<0.00400 \\
\mathrm{E} 0.01400 \\
<0.00400 \\
<0.00400 \\
<0.00400 \\
<0.00400\end{array}$ & $\begin{array}{r}0.009000 \\
0.01400 \\
\text { E0.00900 } \\
\text { E0.00500 } \\
\text { E0.00300 } \\
\text { E0.01000 }\end{array}$ & $\begin{array}{l}<0.00300 \\
<0.00300 \\
<0.00300 \\
<0.00300 \\
<0.00300 \\
<0.00300\end{array}$ & $\begin{array}{l}<0.01300 \\
<0.01300 \\
<0.01300 \\
<0.01300 \\
<0.01300 \\
<0.01300\end{array}$ & $\begin{array}{l}0.001000 \\
<0.00200 \\
<0.00200 \\
<0.00200 \\
<0.00200 \\
<0.00200\end{array}$ & $\begin{array}{r}0.007000 \\
0.02100 \\
0.01900 \\
0.01600 \\
0.01200 \\
<0.00100\end{array}$ \\
\hline 453043122402200 & $06-17-94$ & $<0.00100$ & $<0.00200$ & $<0.00400$ & $<0.00400$ & $<0.00200$ & $<0.00300$ & $<0.01300$ & $0.004000<$ & $<0.00100$ \\
\hline 14211720 & $\begin{array}{l}05-10-94 \\
06-14-94 \\
10-25-94 \\
10-29-94 \\
11-03-94 \\
12-02-94\end{array}$ & $\begin{array}{l}<0.00100 \\
<0.00100 \\
<0.00100 \\
<0.00100 \\
<0.00100 \\
\mathrm{E} 0.00800\end{array}$ & $\begin{array}{l}<0.00200 \\
<0.00200 \\
<0.00200 \\
<0.00200 \\
<0.00200 \\
<0.00200\end{array}$ & $\begin{array}{l}<0.00400 \\
<0.00400 \\
<0.00400 \\
<0.00400 \\
<0.00400 \\
<0.00400\end{array}$ & $\begin{array}{l}<0.00400 \\
<0.00400 \\
<0.00400 \\
<0.00400 \\
<0.00400 \\
<0.00400\end{array}$ & $\begin{array}{l}<0.00200 \\
<0.00200 \\
<0.00200 \\
<0.00200 \\
<0.00200 \\
<0.00200\end{array}$ & $\begin{array}{l}<0.00300 \\
<0.00300 \\
<0.00300 \\
<0.00300 \\
<0.00300 \\
<0.00300\end{array}$ & $\begin{array}{l}<0.01300 \\
<0.01300 \\
<0.01300 \\
<0.01300 \\
<0.01300 \\
<0.01300\end{array}$ & $\begin{array}{l}<0.00200 \\
<0.00200 \\
<0.00200 \\
0.004000 \\
<0.00200 \\
\mathrm{E} 0.00100\end{array}$ & $\begin{array}{l}<0.00100 \\
<0.00100 \\
<0.00100 \\
<0.00100 \\
<0.00100 \\
<0.00100\end{array}$ \\
\hline 453154122394200 & $\begin{array}{l}05-31-94 \\
11-08-94 \\
11-08-94 \\
11-30-94 \\
11-30-94 \\
12-02-94\end{array}$ & $\begin{array}{l}<0.00100 \\
<0.00100 \\
<0.00100 \\
<0.00100 \\
<0.00100 \\
<0.00100\end{array}$ & $\begin{array}{l}<0.00200 \\
<0.00200 \\
<0.00200 \\
<0.00200 \\
<0.00200 \\
<0.00200\end{array}$ & $\begin{array}{l}<0.00400 \\
<0.00400 \\
<0.00400 \\
<0.00400 \\
<0.00400 \\
<0.00400\end{array}$ & $\begin{array}{l}<0.00400 \\
<0.00400 \\
<0.00400 \\
<0.00400 \\
<0.00400 \\
<0.00400\end{array}$ & $\begin{array}{l}0.006000 \\
<0.00200 \\
<0.00200 \\
<0.00200 \\
<0.00200 \\
<0.00200\end{array}$ & $\begin{array}{l}<0.00300 \\
<0.00300 \\
<0.00300 \\
<0.00300 \\
<0.00300 \\
<0.00300\end{array}$ & $\begin{array}{l}<0.01300 \\
<0.01300 \\
<0.01300 \\
<0.01300 \\
<0.01300 \\
<0.01300\end{array}$ & $\begin{array}{l}0.01200 \\
0.005000 \\
0.004000 \\
\text { E0.00300 } \\
\text { E0.00400 } \\
\text { E0.00100 }\end{array}$ & $\begin{array}{l}<0.00100 \\
<0.00100 \\
<0.00100 \\
<0.00100 \\
<0.00100 \\
<0.00100\end{array}$ \\
\hline 14211805 & $05-23-94$ & $<0.00100$ & $<0.00200$ & $<0.00400$ & $<0.00400$ & $<0.00200$ & $<0.00300$ & $<0.01300$ & $<0.00200<$ & $<0.00100$ \\
\hline
\end{tabular}


Table 36. Concentrations of pesticides in filtered water analyzed by gas chromatography/mass spectroscopy from Phase II of the Willamette River Basin Water Quality Study, Oregon, 1994—Continued

\begin{tabular}{|c|c|c|c|c|c|c|c|c|c|c|}
\hline STATION NUMBER & DATE & $\begin{array}{l}\text { P, } \mathrm{P}^{\prime} \\
\text { DDE } \\
\text { WATER, } \\
\text { DISSOLV } \\
(\mathrm{UG} / \mathrm{L})\end{array}$ & $\begin{array}{l}\text { ALPHA } \\
\text { HCH } \\
\text { WATER, } \\
\text { DISSOLV } \\
\text { (UG/L) }\end{array}$ & $\begin{array}{c}\text { LINDANE } \\
\text { WATER, } \\
\text { DISSOL } \\
\text { (UG/L) }\end{array}$ & $\begin{array}{l}\text { CHLOR- } \\
\text { PYRIFOS } \\
\text { WATER, } \\
\text { DISSOLV } \\
\text { (UG/L) }\end{array}$ & $\begin{array}{l}\text { DI- } \\
\text { AZINON, } \\
\text { WATER, } \\
\text { DISSOLV } \\
(\text { UG/L) }\end{array}$ & $\begin{array}{l}\text { DISUL- } \\
\text { FOTON } \\
\text { WATER, } \\
\text { VISSOLV } \\
\text { DISG/L) } \\
(\mathrm{UG} / \mathrm{L} \text {. }\end{array}$ & $\begin{array}{l}\text { ETHO- } \\
\text { PROP } \\
\text { WATER, } \\
\text { DISSOLV } \\
\text { (UG/L) }\end{array}$ & $\begin{array}{l}\text { FONOFOS } \\
\text { WATER, } \\
\text { DISSOLV } \\
\text { (UG/L) }\end{array}$ & $\begin{array}{l}\text { MALA- } \\
\text { THION, } \\
\text { WATER, } \\
\text { DISSOLV } \\
(\text { UG/L) }\end{array}$ \\
\hline 14206298 & $\begin{array}{l}05-27-94 \\
11-23-94\end{array}$ & $\begin{array}{l}<0.00600 \\
<0.00600\end{array}$ & $\begin{array}{l}<0.00200 \\
<0.00200\end{array}$ & $\begin{array}{l}<0.00400 \\
<0.00400\end{array}$ & $\begin{array}{l}<0.00400 \\
<0.00400<\end{array}$ & $\begin{array}{r}0.01200 \\
<0.00200<\end{array}$ & $\begin{array}{l}<0.01700 \\
<0.01700\end{array}$ & $\begin{array}{l}<0.00300 \\
<0.00300\end{array}$ & $\begin{array}{l}<0.00300 \\
<0.00300\end{array}$ & $\begin{array}{l}<0.00500 \\
<0.00500\end{array}$ \\
\hline 14206950 & $\begin{array}{l}05-24-94 \\
06-16-94 \\
10-27-94 \\
11-30-94\end{array}$ & $\begin{array}{l}<0.00600 \\
<0.00600 \\
<0.00600 \\
<0.00600\end{array}$ & $\begin{array}{l}<0.00200 \\
<0.00200 \\
<0.00200 \\
<0.00200\end{array}$ & $\begin{array}{l}<0.00400 \\
<0.00400 \\
<0.00400 \\
<0.00400\end{array}$ & $\begin{array}{r}0.01000 \\
0.005000 \\
0.01600 \\
0.01400\end{array}$ & $\begin{array}{l}0.02900< \\
0.09400< \\
0.06600< \\
0.01600<\end{array}$ & $\begin{array}{l}<0.01700 \\
<0.01700 \\
<0.01700 \\
<0.01700\end{array}$ & $\begin{array}{l}<0.00300 \\
0.005000 \\
\mathrm{E} 0.00700 \\
<0.00300\end{array}$ & $\begin{array}{l}<0.00300 \\
<0.00300 \\
<0.00300 \\
<0.00300\end{array}$ & $\begin{array}{r}<0.00500 \\
<0.00500 \\
0.01900 \\
<0.00500\end{array}$ \\
\hline 14207500 & $\begin{array}{l}05-25-94 \\
10-28-94 \\
12-05-94\end{array}$ & $\begin{array}{l}<0.00600 \\
<0.00600 \\
<0.00600\end{array}$ & $\begin{array}{l}<0.00200 \\
<0.00200 \\
<0.00200\end{array}$ & $\begin{array}{l}<0.00400 \\
<0.00400 \\
<0.00400\end{array}$ & $\begin{array}{l}<0.00400 \\
0.007000 \\
<0.00400\end{array}$ & $\begin{array}{r}0.02000 \\
0.03100 \\
\mathrm{E} 0.00500\end{array}$ & $\begin{array}{l}<0.01700 \\
<0.01700 \\
<0.01700\end{array}$ & $\begin{array}{r}0.005000 \\
0.01600 \\
<0.00300\end{array}$ & $\begin{array}{l}<0.00300 \\
\mathrm{E} 0.00200 \\
<0.00300\end{array}$ & $\begin{array}{l}<0.00500 \\
\mathrm{E} 0.00900 \\
<0.00500\end{array}$ \\
\hline \multirow[t]{2}{*}{452823122240900} & $\begin{array}{l}05-24-94 \\
10-27-94 \\
10-28-94 \\
11-01-94 \\
11-03-94\end{array}$ & $\begin{array}{l}<0.00600 \\
\text { E0.00400 } \\
\text { E0.00300 } \\
\text { E0.00300 } \\
\text { E0.00100 }\end{array}$ & $\begin{array}{l}<0.00200 \\
<0.00200 \\
<0.00200 \\
<0.00200 \\
<0.00200\end{array}$ & $\begin{array}{l}<0.00400 \\
<0.00400 \\
<0.00400 \\
E 0.00500 \\
<0.00400\end{array}$ & $\begin{array}{l}0.04300 \\
0.01000 \\
0.008000 \\
<0.00400 \\
<0.00400\end{array}$ & $\begin{array}{r}0.01700 \\
0.02400 \\
0.01000 \\
\mathrm{E} 0.00800 \\
<0.00200\end{array}$ & $\begin{array}{l}<0.01700 \\
<0.01700 \\
<0.01700 \\
<0.01700 \\
<0.01700\end{array}$ & $\begin{array}{l}<0.00300 \\
<0.00300 \\
\mathrm{E} 0.00500 \\
<0.00300 \\
<0.00300\end{array}$ & $\begin{array}{l}<0.00300 \\
\mathrm{E} 0.00400 \\
\mathrm{E} 0.00300 \\
<0.00300 \\
<0.00300\end{array}$ & $\begin{array}{l}<0.00500 \\
<0.00500 \\
<0.00500 \\
<0.00500 \\
<0.00500\end{array}$ \\
\hline & $11-23-94$ & E0.00100 & $<0.00200$ & $<0.00400$ & $<0.00400<$ & $<0.00200<$ & $<0.01700<$ & $<0.00300$ & $<0.00300<$ & $<0.00500$ \\
\hline 453043122402200 & $06-17-94$ & $<0.00600$ & $<0.00200$ & $<0.00400$ & $<0.00400$ & $0.02400<$ & $<0.01700<$ & $<0.00300$ & $<0.00300<$ & $<0.00500$ \\
\hline 14211720 & $\begin{array}{l}05-10-94 \\
06-14-94 \\
10-25-94 \\
10-29-94 \\
11-03-94 \\
12-02-94\end{array}$ & $\begin{array}{l}<0.00600 \\
<0.00600 \\
<0.00600 \\
<0.00600 \\
<0.00600 \\
<0.00600\end{array}$ & $\begin{array}{l}<0.00200 \\
<0.00200 \\
<0.00200 \\
<0.00200 \\
<0.00200 \\
<0.00200\end{array}$ & $\begin{array}{l}<0.00400 \\
<0.00400 \\
<0.00400 \\
<0.00400 \\
<0.00400 \\
<0.00400\end{array}$ & $\begin{array}{l}<0.00400 \\
<0.00400 \\
<0.00400 \\
<0.00400 \\
<0.00400 \\
0.006000\end{array}$ & $\begin{array}{l}0.006000 \\
0.009000 \\
<0.00200 \\
\mathrm{E} 0.00600 \\
<0.00200 \\
<0.00200\end{array}$ & $\begin{array}{l}<0.01700 \\
<0.01700 \\
<0.01700 \\
<0.01700 \\
<0.01700 \\
<0.01700\end{array}$ & $\begin{array}{r}<0.00300 \\
<0.00300 \\
<0.00300 \\
0.01500 \\
0.02300 \\
\mathrm{E} 0.00500\end{array}$ & $\begin{array}{l}<0.00300 \\
<0.00300 \\
\mathrm{E} 0.00200 \\
<0.00300 \\
\mathrm{E} 0.00500 \\
\mathrm{E} 0.00500\end{array}$ & $\begin{array}{l}<0.00500 \\
<0.00500 \\
<0.00500 \\
<0.00500 \\
<0.00500 \\
<0.00500\end{array}$ \\
\hline 453154122394200 & $\begin{array}{l}05-31-94 \\
11-08-94 \\
11-08-94 \\
11-30-94 \\
11-30-94 \\
12-02-94\end{array}$ & $\begin{array}{l}<0.00600 \\
<0.00600 \\
<0.00600 \\
<0.00600 \\
<0.00600 \\
<0.00600\end{array}$ & $\begin{array}{l}<0.00200 \\
<0.00200 \\
<0.00200 \\
<0.00200 \\
<0.00200 \\
<0.00200\end{array}$ & $\begin{array}{l}<0.00400 \\
<0.00400 \\
<0.00400 \\
<0.00400 \\
<0.00400 \\
<0.00400\end{array}$ & $\begin{array}{r}<0.00400 \\
<0.00400 \\
<0.00400 \\
<0.16000 \\
<0.15000< \\
0.03700\end{array}$ & $\begin{array}{r}0.07700 \\
0.03500 \\
0.02900 \\
<0.11000 \\
<0.11000 \\
0.03000\end{array}$ & $\begin{array}{l}<0.01700 \\
<0.01700 \\
<0.01700 \\
<0.01700 \\
<0.01700 \\
<0.01700\end{array}$ & $\begin{array}{l}<0.00300 \\
<0.00300 \\
<0.00300 \\
<0.00300 \\
<0.00300 \\
<0.00300\end{array}$ & $\begin{array}{r}<0.00300 \\
0.03000 \\
0.03500 \\
<0.00300 \\
<0.00300 \\
<0.00300\end{array}$ & $\begin{array}{r}0.10000 \\
0.05600 \\
0.07600 \\
<0.00500 \\
<0.00500 \\
<0.00500\end{array}$ \\
\hline 14211805 & $05-23-94$ & $<0.00600$ & $<0.00200$ & $<0.00400$ & $<0.004000$ & $0.003000<$ & $<0.01700$ & 0.002000 & $<0.00300<$ & $<0.00500$ \\
\hline
\end{tabular}


Table 36. Concentrations of pesticides in filtered water analyzed by gas chromatography/mass spectroscopy from Phase II of the Willamette River Basin Water Quality Study, Oregon, 1994—Continued

\begin{tabular}{|c|c|c|c|c|c|c|c|c|c|c|}
\hline STATION NUMBER & DATE & $\begin{array}{l}\text { METHYL } \\
\text { AZIN- } \\
\text { PHOS } \\
\text { WATER, } \\
\text { DISSOLV } \\
\text { (UG/L) }\end{array}$ & $\begin{array}{l}\text { METHYL } \\
\text { PARA- } \\
\text { THION } \\
\text { WATER, } \\
\text { DISSOLV } \\
\text { (UG/L) }\end{array}$ & $\begin{array}{l}\text { PARA- } \\
\text { THION } \\
\text { WATER, } \\
\text { DISSOLV } \\
\text { (UG/L) }\end{array}$ & $\begin{array}{l}\text { PHORATE } \\
\text { WATER, } \\
\text { DISSOLV } \\
(\mathrm{UG} / \mathrm{L})\end{array}$ & $\begin{array}{l}\text { TER- } \\
\text { BUFOS } \\
\text { WATER, } \\
\text { DISSOLV } \\
\text { (UG/L) }\end{array}$ & $\begin{array}{l}\text { PER- } \\
\text { METHRIN } \\
\text { WATER, } \\
\text { DISSOLV } \\
\text { (UG/L) }\end{array}$ & $\begin{array}{l}\text { LIN- } \\
\text { URON } \\
\text { WATER, } \\
\text { DISSOLV } \\
\text { (UG/L) }\end{array}$ & $\begin{array}{l}\text { TEBU- } \\
\text { THIURON } \\
\text { WATER, } \\
\text { DISSOLV } \\
\text { (UG/L) }\end{array}$ & $\begin{array}{l}\text { ATRA- } \\
\text { ZINE } \\
\text { WATER, } \\
\text { DISSOLV } \\
\text { (UG/L) }\end{array}$ \\
\hline 14206298 & $\begin{array}{l}05-27-94 \\
11-23-94\end{array}$ & $\begin{array}{l}<0.00100 \\
<0.00100\end{array}$ & $\begin{array}{l}<0.00200 \\
<0.00200\end{array}$ & $\begin{array}{l}<0.00600 \\
<0.00600\end{array}$ & $\begin{array}{l}<0.00200 \\
<0.00200\end{array}$ & $\begin{array}{l}<0.00700 \\
<0.00700\end{array}$ & $\begin{array}{l}<0.00500 \\
<0.00500\end{array}$ & $\begin{array}{l}<0.00200 \\
<0.00200\end{array}$ & $\begin{array}{l}<0.01000 \\
\mathrm{E} 0.00700\end{array}$ & $\begin{array}{l}0.03000 \\
0.02200\end{array}$ \\
\hline 14206950 & $\begin{array}{l}05-24-94 \\
06-16-94 \\
10-27-94 \\
11-30-94\end{array}$ & $\begin{array}{l}<0.00100 \\
<0.00100 \\
<0.00100 \\
<0.00100\end{array}$ & $\begin{array}{l}<0.00200 \\
<0.00200 \\
<0.00200 \\
<0.00200\end{array}$ & $\begin{array}{l}<0.00600 \\
<0.00600 \\
<0.00600 \\
<0.00600\end{array}$ & $\begin{array}{l}<0.00200 \\
<0.00200 \\
<0.00200 \\
<0.00200\end{array}$ & $\begin{array}{l}<0.00700 \\
<0.00700 \\
<0.00700 \\
<0.00700\end{array}$ & $\begin{array}{l}<0.00500 \\
<0.00500 \\
<0.00500 \\
<0.00500\end{array}$ & $\begin{array}{l}<0.00200 \\
<0.00200 \\
<0.00200 \\
<0.00200\end{array}$ & $\begin{array}{l}0.01700 \\
0.03200 \\
0.12000 \mathrm{E} \\
0.07200 \mathrm{E}\end{array}$ & $\begin{array}{r}0.02000 \\
0.02300 \\
\mathrm{E} 0.01200 \\
\mathrm{E} 0.01300\end{array}$ \\
\hline 14207500 & $\begin{array}{l}05-25-94 \\
10-28-94 \\
12-05-94\end{array}$ & $\begin{array}{l}<0.00100 \\
<0.00100 \\
<0.00100\end{array}$ & $\begin{array}{l}<0.00200 \\
<0.00200 \\
<0.00200\end{array}$ & $\begin{array}{l}<0.00600 \\
<0.00600 \\
<0.00600\end{array}$ & $\begin{array}{l}<0.00200 \\
<0.00200 \\
<0.00200\end{array}$ & $\begin{array}{l}<0.00700 \\
<0.00700 \\
<0.00700\end{array}$ & $\begin{array}{l}<0.00500 \\
<0.00500 \\
<0.00500\end{array}$ & $\begin{array}{l}<0.00200 \\
<0.00200 \\
<0.00200\end{array}$ & $\begin{array}{r}0.05200 \\
0.03700 \\
\mathrm{E} 0.00700\end{array}$ & $\begin{array}{r}0.04800 \\
\mathrm{E} 0.01400 \\
0.16000\end{array}$ \\
\hline 452823122240900 & $\begin{array}{l}05-24-94 \\
10-27-94 \\
10-28-94 \\
11-01-94 \\
11-03-94 \\
11-23-94\end{array}$ & $\begin{array}{l}<0.00100 \\
<0.00100 \\
<0.00100 \\
<0.00100 \\
<0.00100 \\
<0.00100\end{array}$ & $\begin{array}{l}<0.00200 \\
<0.00200 \\
<0.00200 \\
<0.00200 \\
<0.00200 \\
<0.00200\end{array}$ & $\begin{array}{l}<0.00600 \\
<0.00600 \\
<0.00600 \\
<0.00600 \\
<0.00600 \\
<0.00600\end{array}$ & $\begin{array}{l}<0.00200 \\
<0.00200 \\
<0.00200 \\
<0.00200 \\
<0.00200 \\
<0.00200\end{array}$ & $\begin{array}{l}<0.00700 \\
<0.00700 \\
<0.00700 \\
<0.00700 \\
<0.00700 \\
<0.00700\end{array}$ & $\begin{array}{l}<0.00500 \\
<0.00500 \\
<0.00500 \\
<0.00500 \\
<0.00500 \\
<0.00500\end{array}$ & $\begin{array}{l}<0.00200 \\
<0.00200 \\
<0.00200 \\
<0.00200 \\
<0.00200 \\
<0.00200\end{array}$ & $\begin{array}{l}<0.01000 \\
\mathrm{E} 0.00600 \\
<0.01000 \\
<0.01000 \\
<0.01000 \\
<0.01000\end{array}$ & $\begin{array}{l}0.07400 \\
0.17000 \\
0.07200 \\
0.04700 \\
0.02600 \\
0.03800\end{array}$ \\
\hline 453043122402200 & $06-17-94$ & $<0.00100$ & $<0.00200$ & $<0.00600$ & $<0.00200$ & $<0.00700$ & $<0.00500$ & $<0.00200$ & $<0.01000$ & 0.02000 \\
\hline 14211720 & $\begin{array}{l}05-10-94 \\
06-14-94 \\
10-25-94 \\
10-29-94 \\
11-03-94 \\
12-02-94\end{array}$ & $\begin{array}{l}<0.00100 \\
<0.00100 \\
<0.00100 \\
<0.00100 \\
<0.00100 \\
<0.00100\end{array}$ & $\begin{array}{l}<0.00200 \\
<0.00200 \\
<0.00200 \\
<0.00200 \\
<0.00200 \\
<0.00200\end{array}$ & $\begin{array}{l}<0.00600 \\
<0.00600 \\
<0.00600 \\
<0.00600 \\
<0.00600 \\
<0.00600\end{array}$ & $\begin{array}{l}<0.00200 \\
<0.00200 \\
<0.00200 \\
<0.00200 \\
<0.00200 \\
<0.00200\end{array}$ & $\begin{array}{l}<0.00700 \\
<0.00700 \\
<0.00700 \\
<0.00700 \\
<0.00700 \\
<0.00700\end{array}$ & $\begin{array}{l}<0.00500 \\
<0.00500 \\
<0.00500 \\
<0.00500 \\
<0.00500 \\
<0.00500\end{array}$ & $\begin{array}{l}<0.00200 \\
<0.00200 \\
<0.00200 \\
<0.00200 \\
<0.00200 \\
<0.00200\end{array}$ & $\begin{array}{l}<0.01000 \\
<0.01000 \\
<0.01000 \\
\mathrm{E} 0.00700 \\
<0.01000 \\
\mathrm{E} 0.00300\end{array}$ & $\begin{array}{r}0.02000 \\
0.01200 \\
\mathrm{E} 0.00500 \\
0.02900 \\
0.13000 \\
0.18000\end{array}$ \\
\hline 453154122394200 & $\begin{array}{l}05-31-94 \\
11-08-94 \\
11-08-94 \\
11-30-94 \\
11-30-94 \\
12-02-94\end{array}$ & $\begin{array}{c}-- \\
<0.00100 \\
<0.00100 \\
<0.00100 \\
<0.00100 \\
<0.00100\end{array}$ & $\begin{array}{l}<0.00200 \\
<0.00200 \\
<0.00200 \\
<0.00200 \\
<0.00200 \\
<0.00200\end{array}$ & $\begin{array}{l}<0.00600 \\
<0.00600 \\
<0.00600 \\
<0.00600 \\
<0.00600 \\
<0.00600\end{array}$ & $\begin{array}{l}<0.00200 \\
<0.00200 \\
<0.00200 \\
<0.00200 \\
<0.00200 \\
<0.00200\end{array}$ & $\begin{array}{l}<0.00700 \\
<0.00700 \\
<0.00700 \\
<0.00700 \\
<0.00700 \\
<0.00700\end{array}$ & $\begin{array}{l}<0.00500 \\
<0.00500 \\
<0.00500 \\
<0.00500 \\
<0.00500 \\
<0.00500\end{array}$ & $\begin{array}{rl} & 0.78000 \\
< & 0.00200 \\
<0 & 0.00200 \\
< & 0.00200 \\
< & <0.00200 \\
< & <0.00200\end{array}$ & $\begin{array}{l}<0.01000 \\
<0.01000 \\
<0.01000 \\
<0.01000 \\
<0.01000< \\
<0.01000<\end{array}$ & $\begin{array}{r}0.08200 \\
0.02300 \\
0.01800 \\
<0.00100 \\
<0.00100 \\
<0.00100\end{array}$ \\
\hline 14211805 & $05-23-94$ & $<0.00100$ & $<0.00200$ & $<0.00600$ & $<0.00200$ & $<0.00700$ & $<0.00500$ & $<0.00200$ & $<0.01000$ & 0.02400 \\
\hline
\end{tabular}


Table 36. Concentrations of pesticides in filtered water analyzed by gas chromatography/mass spectroscopy from Phase II of the Willamette River Basin Water Quality Study, Oregon, 1994—Continued

\begin{tabular}{|c|c|c|c|c|c|c|c|c|c|c|}
\hline STATION NUMBER & DATE & $\begin{array}{l}\text { CYANA- } \\
\text { ZINE, } \\
\text { WATER, } \\
\text { DISSOLV } \\
(\text { UG/L) }\end{array}$ & $\begin{array}{l}\text { DESETHYI } \\
\text { ATRA- } \\
\text { ZINE, } \\
\text { WATER, } \\
\text { DISSOLV } \\
(\text { UG/L) }\end{array}$ & 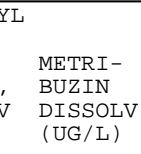 & $\begin{array}{l}\text { PRO- } \\
\text { METON, } \\
\text { WATER, } \\
\text { DISSOLV } \\
(\mathrm{UG} / \mathrm{L})\end{array}$ & $\begin{array}{l}\text { SI- } \\
\text { MAZINE, } \\
\text { WATER, } \\
\text { DISSOLV } \\
\text { (UG/L) }\end{array}$ & $\begin{array}{l}\text { TER- } \\
\text { BACIL } \\
\text { WATER, } \\
\text { DISSOLV } \\
(\text { UG/L) }\end{array}$ & $\begin{array}{l}\text { DIAZ- } \\
\text { INON } \\
\text { D10 SRG } \\
\text { WATER, } \\
\text { DISSOLV } \\
\text { PERCENT }\end{array}$ & $\begin{array}{l}\text { TERBUTH } \\
\text { YLAZINE } \\
\text { SURROGT } \\
\text { WATER, } \\
\text { DISSOLV } \\
\text { PERCENT }\end{array}$ & $\begin{array}{c}\text { HCH } \\
\text { ALPHA } \\
\text { D6 SRG } \\
\text { WATER, } \\
\text { DISSOLV } \\
\text { PERCENT }\end{array}$ \\
\hline 14206298 & $\begin{array}{l}05-27-94 \\
11-23-94\end{array}$ & $\begin{array}{l}<0.00400 \\
<0.00400\end{array}$ & $\begin{array}{l}<0.00200 \\
\mathrm{E} 0.00600\end{array}$ & $\begin{array}{l}<0.00400 \\
<0.00400\end{array}$ & $\begin{array}{l}0.01000 \\
0.01600\end{array}$ & $\begin{array}{l}0.04300 \\
0.89000\end{array}$ & $\begin{array}{l}<0.00700 \\
<0.00700\end{array}$ & $\begin{array}{l}131.00 \\
115.00\end{array}$ & $\begin{array}{l}114.00 \\
119.00\end{array}$ & $\begin{array}{l}120.00 \\
109.00\end{array}$ \\
\hline 14206950 & $\begin{array}{l}05-24-94 \\
06-16-94 \\
10-27-94 \\
11-30-94\end{array}$ & $\begin{array}{l}<0.00400 \\
<0.00400 \\
<0.00400 \\
<0.00400\end{array}$ & $\begin{array}{l}\mathrm{E} 0.00300 \\
\mathrm{E} 0.00600 \\
\mathrm{E} 0.00400 \\
<0.00200\end{array}$ & $\begin{array}{l}<0.00400 \\
<0.00400 \\
<0.00400 \\
<0.00400\end{array}$ & $\begin{array}{l}0.06800 \\
0.05200 \\
0.06500 \\
0.02600\end{array}$ & $\begin{array}{l}0.20000 \\
0.14000 \\
0.18000 \\
0.09600\end{array}$ & $\begin{array}{l}<0.00700 \\
<0.00700 \\
<0.00700 \\
<0.00700\end{array}$ & $\begin{array}{r}59.00 \\
105.00 \\
100.00 \\
100.00\end{array}$ & $\begin{array}{r}125.00 \\
105.00 \\
98.00 \\
130.00\end{array}$ & $\begin{array}{r}92.00 \\
94.00 \\
100.00 \\
100.00\end{array}$ \\
\hline 14207500 & $\begin{array}{l}05-25-94 \\
10-28-94 \\
12-05-94\end{array}$ & $\begin{array}{l}<0.00400 \\
<0.00400 \\
<0.00400\end{array}$ & $\begin{array}{l}\mathrm{E} 0.00400 \\
\mathrm{E} 0.00400 \\
\mathrm{E} 0.00800\end{array}$ & $\begin{array}{l}<0.00400 \\
\mathrm{E} 0.01000 \\
<0.00400\end{array}$ & $\begin{array}{r}<0.00300 \\
0.02400 \\
\mathrm{E} 0.00600\end{array}$ & $\begin{array}{l}0.09600 \\
0.11000 \\
0.18000\end{array}$ & $\begin{array}{l}<0.00700 \\
<0.00700 \\
<0.00700\end{array}$ & $\begin{array}{r}60.00 \\
101.00 \\
60.00\end{array}$ & $\begin{array}{r}105.00 \\
106.00 \\
99.00\end{array}$ & $\begin{array}{r}96.00 \\
102.00 \\
90.00\end{array}$ \\
\hline 452823122240900 & $\begin{array}{l}05-24-94 \\
10-27-94 \\
10-28-94 \\
11-01-94 \\
11-03-94 \\
11-23-94\end{array}$ & $\begin{array}{l}<0.00400 \\
<0.00400 \\
<0.00400 \\
<0.00400 \\
<0.00400 \\
<0.00400\end{array}$ & $\begin{array}{l}\text { E0.00800 } \\
\text { E0.01200 } \\
\text { E0.01100 } \\
\text { E0.00500 } \\
\text { E0.00700 } \\
\text { E0.01400 }\end{array}$ & $\begin{array}{l}<0.00400 \\
<0.00400 \\
<0.00400 \\
<0.00400 \\
<0.00400 \\
<0.00400\end{array}$ & $\begin{array}{r}0.008000 \\
0.01300 \\
0.01000 \\
0.009000 \\
\mathrm{E} 0.00500 \\
\mathrm{E} 0.00800\end{array}$ & $\begin{array}{l}0.20000 \\
0.18000 \\
0.09100 \\
0.07800 \\
0.03200 \\
0.02400\end{array}$ & $\begin{array}{l}<0.00700 \\
\text { E0.01600 } \\
\text { E0.01300 } \\
\text { E0.01000 } \\
\text { E0.00600 } \\
<0.00700\end{array}$ & $\begin{array}{r}59.00 \\
107.00 \\
98.00 \\
97.00 \\
96.00 \\
118.00\end{array}$ & $\begin{array}{r}107.00 \\
103.00 \\
104.00 \\
97.00 \\
104.00 \\
120.00\end{array}$ & $\begin{array}{r}93.00 \\
98.00 \\
100.00 \\
79.00 \\
84.00 \\
111.00\end{array}$ \\
\hline 453043122402200 & $06-17-94$ & $<0.00400$ & $<0.00200$ & $<0.00400$ & $<0.00300$ & 0.06600 & $<0.00700$ & 83.00 & 90.00 & 76.00 \\
\hline 14211720 & $\begin{array}{l}05-10-94 \\
06-14-94 \\
10-25-94 \\
10-29-94 \\
11-03-94 \\
12-02-94\end{array}$ & $\begin{array}{l}<0.00400 \\
<0.00400 \\
<0.00400 \\
<0.00400 \\
<0.00400 \\
<0.00400\end{array}$ & $\begin{array}{l}\mathrm{E} 0.00500 \\
<0.00200 \\
\mathrm{E} 0.00200 \\
\mathrm{E} 0.00400 \\
\mathrm{E} 0.00400 \\
\mathrm{E} 0.00600\end{array}$ & $\begin{array}{r}<0.00400 \\
<0.00400 \\
<0.00400 \\
<0.00400 \\
<0.00400 \\
\quad 0.02000\end{array}$ & $\begin{array}{l}<0.00300 \\
<0.00300 \\
<0.00300 \\
<0.00300 \\
<0.00300 \\
<0.00300\end{array}$ & $\begin{array}{r}0.01000 \\
0.01600 \\
\mathrm{E} 0.00600 \\
0.06600 \\
0.07300 \\
0.04300\end{array}$ & $\begin{array}{l}<0.00700 \\
<0.00700 \\
<0.00700 \\
\text { E0.01700 } \\
\text { E0.02700 } \\
\text { E0.01000 }\end{array}$ & $\begin{array}{r}131.00 \\
96.00 \\
101.00 \\
103.00 \\
50.00 \\
60.00\end{array}$ & $\begin{array}{l}109.00 \\
100.00 \\
103.00 \\
109.00 \\
100.00 \\
106.00\end{array}$ & $\begin{array}{r}97.00 \\
96.00 \\
103.00 \\
99.00 \\
80.00 \\
90.00\end{array}$ \\
\hline 453154122394200 & $\begin{array}{l}05-31-94 \\
11-08-94 \\
11-08-94 \\
11-30-94 \\
11-30-94 \\
12-02-94\end{array}$ & $\begin{array}{l}<0.00400 \\
<0.00400 \\
<0.00400 \\
<0.00400 \\
<0.00400 \\
<0.00400\end{array}$ & $\begin{array}{l}<0.00200 \\
<0.00200 \\
<0.00200 \\
<0.00200 \\
<0.00200 \\
\text { E0.02400 }\end{array}$ & $\begin{aligned} & 0.41000 \\
&<0.00400 \\
&<0.00400 \\
&<0.00400 \\
&<0.00400 \\
&<0.00400\end{aligned}$ & $\begin{array}{l}<0.00300 \\
<0.00300 \\
<0.00300 \\
<0.00300 \\
<0.00300 \\
<0.00300\end{array}$ & $\begin{array}{r}0.12000 \\
<0.00500 \\
<0.00500 \\
<0.00500 \\
<0.00500 \\
0.06700\end{array}$ & $\begin{array}{c}<0 . \\
<0.00700 \\
<0.00700 \\
<0.00700 \\
<0.00700 \\
<0.00700\end{array}$ & $\begin{array}{l}109.00 \\
142.00 \\
149.00 \\
142.00 \\
153.00 \\
114.00\end{array}$ & $\begin{array}{l}-- \\
163.00 \\
142.00 \\
197.00 \\
191.00 \\
129.00\end{array}$ & $\begin{array}{r}98.00 \\
125.00 \\
129.00 \\
113.00 \\
114.00 \\
98.00\end{array}$ \\
\hline 14211805 & $05-23-94$ & $<0.00400$ & $<0.00200$ & $<0.00400$ & $<0.00300$ & 0.02100 & $<0.00700$ & 55.00 & 102.00 & 92.00 \\
\hline
\end{tabular}


Table 37. Concentrations of pesticides in filtered water analyzed by high performance liquid chromatography from Phase I of the Willamette River Basin Water Quality Study, Oregon, 1993

[USGS laboratory schedule 2051; **, not detected. This is considered an experimental analysis; UG/L, micrograms per liter, Examination of native-water data, spiked native-water quality-assurance data, and pesticide use information indicate a possibility of false negative results for a number of analytes. The minimum reporting level for the schedule is listed as $0.05 \mathrm{ug} / \mathrm{L}$ (see Appendix), however the minimum reporting level for each individual analyte may have been variable depending upon sample matrix effects and analytical variability. Chlorothalonil, DNOC, Dichlobenil, Esfenvalerate, and 1-Naphthol are selected for removal from schedule 2051 or for qualitative reporting]

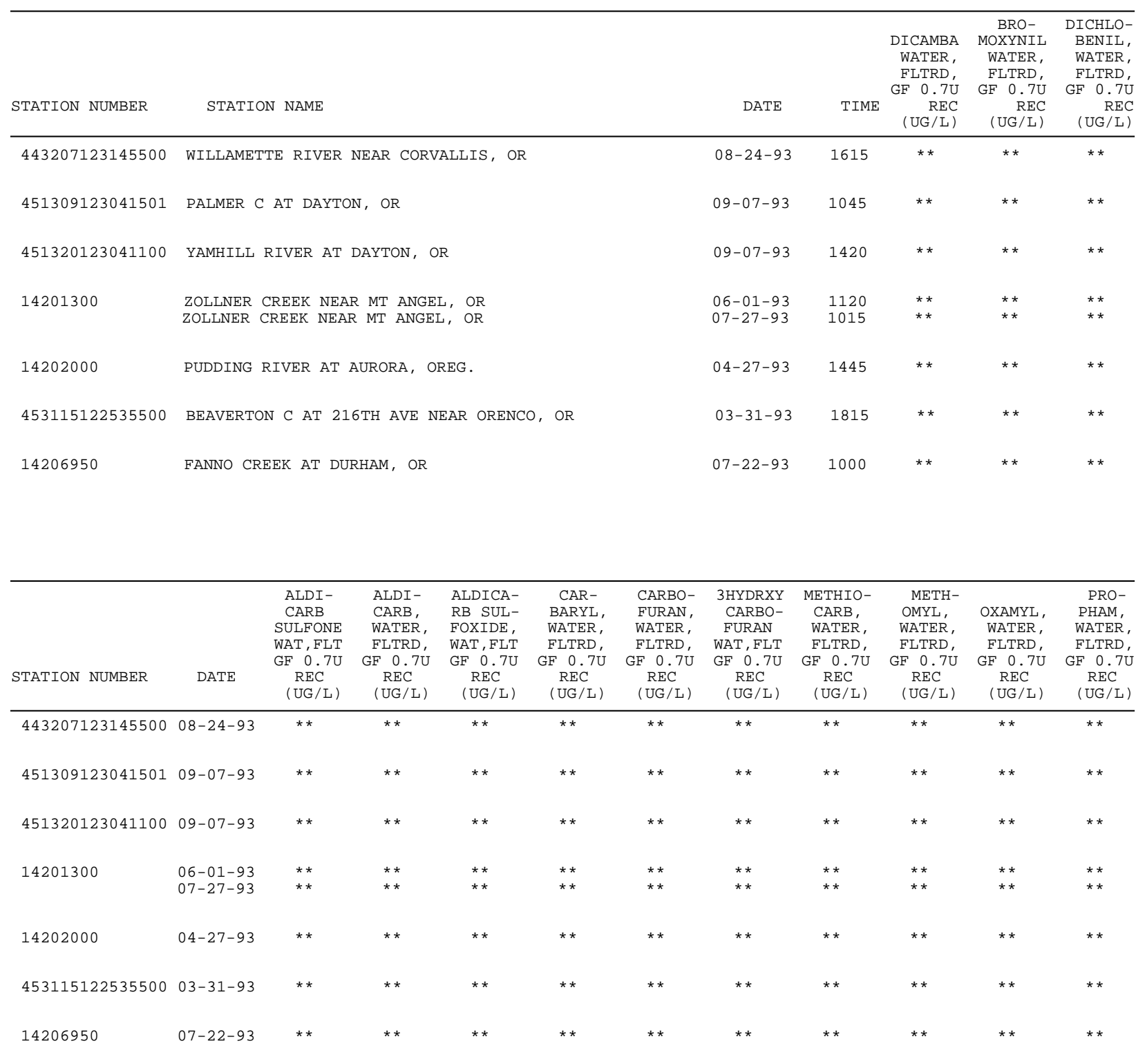


Table 37. Concentrations of pesticides in filtered water analyzed by high performance liquid chromatography from Phase I of the Willamette River Basin Water Quality Study, Oregon, 1993—Continued

\begin{tabular}{|c|c|c|c|c|c|c|c|c|c|c|c|}
\hline STATION NUMBER & DATE & $\begin{array}{c}\text { PRO- } \\
\text { POXUR, } \\
\text { WATER, } \\
\text { FLTRD, } \\
\text { GF } 0.7 \mathrm{U} \\
\text { REC } \\
\text { (UG/L) }\end{array}$ & $\begin{array}{c}\text { BENTA- } \\
\text { ZON, } \\
\text { WATER, } \\
\text { FLTRD, } \\
\text { GF } 0.7 \mathrm{U} \\
\text { REC } \\
\text { (UG/L) }\end{array}$ & $\begin{array}{c}2,4-D, \\
\text { DIS- } \\
\text { SOLVED } \\
(U G / L)\end{array}$ & $\begin{array}{c}\text { 2, 4-DB } \\
\text { WATER, } \\
\text { FLTRD, } \\
\text { GF } 0.7 \mathrm{U} \\
\text { REC } \\
(\mathrm{UG} / \mathrm{L})\end{array}$ & $\begin{array}{c}\text { DACTHAL } \\
\text { MONO- } \\
\text { ACID, } \\
\text { WAT, FLT } \\
\text { GF } 0.7 \mathrm{U} \\
\text { REC } \\
\text { (UG/L) }\end{array}$ & $\begin{array}{l}\text { DICHLOR } \\
\text { PROP, } \\
\text { WATER, } \\
\text { FLTRD, } \\
\text { GF } 0.7 \mathrm{U} \\
\text { REC } \\
\text { (UG/L) }\end{array}$ & $\begin{array}{c}\text { MCPA, } \\
\text { WATER, } \\
\text { FLTRD, } \\
\text { GF 0.7U } \\
\text { REC } \\
(\mathrm{UG} / \mathrm{L})\end{array}$ & $\begin{array}{c}\text { MCPB, } \\
\text { WATER, } \\
\text { FLTRD, } \\
\text { GF 0.7U } \\
\text { REC } \\
(\mathrm{UG} / \mathrm{L})\end{array}$ & $\begin{array}{c}\text { SILVEX, } \\
\text { DIS- } \\
\text { SOLVED } \\
\text { (UG/L) }\end{array}$ & $\begin{array}{c}2,4,5-T \\
\text { DIS- } \\
\text { SOLVED } \\
(\mathrm{UG} / \mathrm{L})\end{array}$ \\
\hline 443207123145500 & $08-24-93$ & $\star \star$ & $\star \star$ & $\star \star$ & ** & $\star \star$ & ** & $\star \star$ & $\star \star$ & ** & $\star \star$ \\
\hline 451309123041501 & $09-07-93$ & $\star \star$ & $\star \star$ & $\star \star$ & *夫 & $\star \star$ & ** & $\star \star$ & $\star \star$ & ** & $\star \star$ \\
\hline 451320123041100 & $09-07-93$ & $\star \star$ & ** & ** & ** & $\star \star$ & ** & $\star \star$ & $\star \star$ & ** & 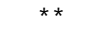 \\
\hline 14201300 & $\begin{array}{l}06-01-93 \\
07-27-93\end{array}$ & $\begin{array}{l}\star \star \\
\star \star\end{array}$ & $\begin{array}{l}\star \star \\
\star \star\end{array}$ & $\begin{array}{c}* * \\
0.79000\end{array}$ & $\begin{array}{l}\star * \\
* *\end{array}$ & $\begin{array}{l}* \star \\
\star \star\end{array}$ & $\begin{array}{l}\star * \\
\star \star\end{array}$ & $\begin{array}{l}\star \star \\
\star \star\end{array}$ & $\begin{array}{l}\star \star \\
\star \star\end{array}$ & $\begin{array}{l}\star \star \\
\star *\end{array}$ & $\begin{array}{l}\star \star \\
\star \star\end{array}$ \\
\hline 14202000 & $04-27-93$ & $\star \star$ & $\star \star$ & $\star \star$ & ** & $\star \star$ & ** & $\star \star$ & $\star \star$ & ** & $\star \star$ \\
\hline 453115122535500 & $03-31-93$ & $\star \star$ & $\star \star$ & $\star \star$ & ** & $\star \star$ & ** & $\star \star$ & $\star \star$ & $\star \star *$ & $\star \star$ \\
\hline 14206950 & $07-22-93$ & $\star \star$ & $\star \star$ & 0.08000 & ** & $\star \star$ & $\star \star$ & $\star \star$ & $\star \star$ & ** & $\star \star$ \\
\hline
\end{tabular}

\begin{tabular}{|c|c|c|c|c|c|c|c|c|c|c|c|}
\hline STATION NUMBER & DATE & $\begin{array}{c}\text { ORY- } \\
\text { ZALIN, } \\
\text { WATER, } \\
\text { FLTRD, } \\
\text { GF } 0.7 \mathrm{U} \\
\text { REC } \\
(\mathrm{UG} / \mathrm{L})\end{array}$ & $\begin{array}{c}\text { DINOSEB } \\
\text { WATER, } \\
\text { FLTRD, } \\
\text { GE 0.7U } \\
\text { REC } \\
(\mathrm{UG} / \mathrm{L})\end{array}$ & $\begin{array}{c}\text { OCRESOL } \\
4,6- \\
\text { DINITRO } \\
\text { WAT, FLT } \\
\text { GF } 0.7 U \\
\text { REC } \\
\text { (UG/L) }\end{array}$ & $\begin{array}{c}\text { ACIFL- } \\
\text { UORFEN } \\
\text { WATER, } \\
\text { FLTRD, } \\
\text { GF } 0.7 \mathrm{U} \\
\text { REC } \\
\text { (UG/L) }\end{array}$ & $\begin{array}{c}\text { 1-NAPH } \\
\text { THOL, } \\
\text { WATER, } \\
\text { FLTRD, } \\
\text { GF } 0.7 \mathrm{U} \\
\text { REC } \\
\text { (UG/L) }\end{array}$ & $\begin{array}{c}\text { AMIBEN, } \\
\text { WATER, } \\
\text { FLTRD, } \\
\text { GF } 0.7 U \\
\text { REC } \\
(\mathrm{UG} / \mathrm{L})\end{array}$ & $\begin{array}{c}\text { DIURON, } \\
\text { WATER, } \\
\text { FLTRD, } \\
\text { GF } 0.7 \mathrm{U} \\
\text { REC } \\
(\mathrm{UG} / \mathrm{L})\end{array}$ & $\begin{array}{c}\text { FEN- } \\
\text { URON, } \\
\text { WATER, } \\
\text { FLTRD, } \\
\text { GF } 0.7 \mathrm{U} \\
\text { REC } \\
\text { (UG/L) }\end{array}$ & $\begin{array}{c}\text { FLUO- } \\
\text { METURON } \\
\text { WATER, } \\
\text { FLTRD, } \\
\text { GF } 0.7 U \\
\text { REC } \\
(U G / L)\end{array}$ & $\begin{array}{c}\text { LINURON } \\
\text { WATER, } \\
\text { FLTRD, } \\
\text { GF } 0.7 \mathrm{U} \\
\text { REC } \\
\text { (UG/L) }\end{array}$ \\
\hline 443207123145500 & $08-24-93$ & $\star \star$ & $\star \star$ & $\star \star$ & $\star \star$ & $\star \star$ & ** & $\star \star$ & ** & $\star \star$ & ** \\
\hline 451309123041501 & $09-07-93$ & $\star *$ & $\star *$ & ** & ** & $\star \star$ & ** & $\star \star$ & ** & $\star \star$ & ** \\
\hline 451320123041100 & $09-07-93$ & $\star *$ & ** & $\star \star$ & $\star \star$ & $\star \star$ & $\star \star$ & $\star \star$ & ** & $\star \star$ & ** \\
\hline 14201300 & $\begin{array}{l}06-01-93 \\
07-27-93\end{array}$ & $\begin{array}{l}* * \\
* *\end{array}$ & $\begin{array}{l}* * \\
\star *\end{array}$ & $\begin{array}{l}* * \\
* *\end{array}$ & $\begin{array}{l}* * \\
\star *\end{array}$ & $\begin{array}{l}* * \\
* *\end{array}$ & $\begin{array}{l}* * \\
\star *\end{array}$ & $\begin{array}{r}0.68000 \\
9.0000\end{array}$ & $\begin{array}{l}* * \\
* *\end{array}$ & $\begin{array}{l}* * \\
\star *\end{array}$ & $\begin{array}{l}* * \\
\star *\end{array}$ \\
\hline 14202000 & $04-27-93$ & $\star \star$ & $\star \star$ & ** & $\star *$ & $\star \star$ & ** & $\star \star$ & $\star \star$ & $\star \star$ & ** \\
\hline 453115122535500 & $03-31-93$ & $\star \star$ & $\star \star$ & ** & $\star \star$ & $\star \star$ & ** & $\star \star$ & ** & $\star \star$ & ** \\
\hline 14206950 & $07-22-93$ & $\star \star$ & $\star \star$ & ** & $\star \star$ & $\star \star$ & ** & 0.06000 & $\star \star$ & $\star \star$ & $\star \star$ \\
\hline
\end{tabular}


Table 37. Concentrations of pesticides in filtered water analyzed by high performance liquid chromatography from Phase I of the Willamette River Basin Water Quality Study, Oregon, 1993—Continued

\begin{tabular}{|c|c|c|c|c|c|c|c|c|c|c|}
\hline STATION NUMBER & DATE & $\begin{array}{c}\text { NEB- } \\
\text { URON, } \\
\text { WATER, } \\
\text { FLTRD, } \\
\text { GF } 0.7 \mathrm{U} \\
\text { REC } \\
\text { (UG/L) }\end{array}$ & $\begin{array}{c}\text { CHLORO- } \\
\text { THALO- } \\
\text { NIL, } \\
\text { WAT, FLT } \\
\text { GF } 0.7 \mathrm{U} \\
\text { REC } \\
\text { (UG/L) }\end{array}$ & $\begin{array}{c}\text { ESFEN- } \\
\text { VAL- } \\
\text { ERATE, } \\
\text { WAT, FLT } \\
\text { GF } 0.7 \mathrm{U} \\
\text { REC } \\
\text { (UG/L) }\end{array}$ & $\begin{array}{c}\text { NORFLUR } \\
\text { AZON, } \\
\text { WATER, } \\
\text { FLTRD, } \\
\text { GF } 0.7 \mathrm{U} \\
\text { REC } \\
\text { (UG/L) }\end{array}$ & $\begin{array}{c}\text { CLOPYR- } \\
\text { ALID, } \\
\text { WATER, } \\
\text { FLTRD, } \\
\text { GF } 0.7 \mathrm{U} \\
\text { REC } \\
\text { (UG/L) }\end{array}$ & $\begin{array}{c}\text { PIC- } \\
\text { LORAM, } \\
\text { WATER, } \\
\text { FLTRD, } \\
\text { GF } 0.7 \mathrm{U} \\
\text { REC } \\
\text { (UG/L) }\end{array}$ & $\begin{array}{c}\text { TRI- } \\
\text { CLOPYR, } \\
\text { WATER, } \\
\text { FLTRD, } \\
\text { GF } 0.7 \mathrm{U} \\
\text { REC } \\
(\mathrm{UG} / \mathrm{L})\end{array}$ & $\begin{array}{l}\text { BRO- } \\
\text { MACIL, } \\
\text { WATER, } \\
\text { DISS, } \\
\text { REC } \\
\text { (UG/L) }\end{array}$ & $\begin{array}{c}\text { BDMC, } \\
\text { SURROG, } \\
\text { WATER, } \\
\text { UNFLTRD } \\
\text { REC } \\
\text { PERCENT }\end{array}$ \\
\hline 443207123145500 & $08-24-93$ & ** & ** & $\star \star$ & ** & $\star \star$ & ** & $\star \star$ & $\star \star$ & 56.000 \\
\hline 451309123041501 & $09-07-93$ & ** & ** & $\star \star$ & ** & $\star \star$ & ** & $\star \star$ & $\star \star$ & 62.000 \\
\hline 451320123041100 & $09-07-93$ & ** & $\star \star$ & $\star \star$ & ** & $\star *$ & ** & $\star \star$ & ** & 50.000 \\
\hline 14201300 & $\begin{array}{l}06-01-93 \\
07-27-93\end{array}$ & ** & $\begin{array}{l}* * \\
* *\end{array}$ & $\begin{array}{l}* * \\
* *\end{array}$ & ** & $\begin{array}{l}* * \\
* *\end{array}$ & $\begin{array}{l}* * \\
\star *\end{array}$ & 0.27000 & $\begin{array}{l}* * \\
* *\end{array}$ & 12.000 \\
\hline 14202000 & $04-27-93$ & ** & $\star \star$ & $\star \star$ & ** & $\star \star$ & ** & $\star \star$ & $\star \star$ & -- \\
\hline 453115122535500 & $03-31-93$ & ** & ** & $\star \star$ & ** & $\star *$ & ** & $\star \star$ & $\star \star$ & 4.0000 \\
\hline 14206950 & $07-22-93$ & ** & ** & $\star \star$ & ** & $\star \star$ & ** & 0.18000 & $\star \star$ & 12.000 \\
\hline
\end{tabular}


Table 38. Concentrations of pesticides in filtered water analyzed by high performance liquid chromatography from Phase II of the Willamette River Basin Water Quality Study, Oregon, 1994

[USGS laboratory schedule 2051; E, estimate; **, not detected; UG/L, micrograms per liter. This is considered an experimental analysis. Examination of native-water data, spiked native-water quality-assurance data, and pesticide use information indicate a possibility of false negative results for a number of analytes. The minimum reporting level for the schedule is listed as $0.05 \mathrm{ug} / \mathrm{L}$ (see Appendix), however the minimum reporting level for each individual analyte may have been variable depending upon sample matrix effects and analytical variability. Chlorothalonil, DNOC, Dichlobenil, Esfenvalerate, and 1-Naphthol are selected for removal from schedule 2051 or for qualitative reporting.]

\begin{tabular}{|c|c|c|c|c|c|c|}
\hline STATION NUMBER & STATION NAME & DATE & TIME & $\begin{array}{r}\text { DICAMBA } \\
\text { WATER, } \\
\text { FLTRD, } \\
\text { GF } 0.7 U \\
\text { REC } \\
(U G / L)\end{array}$ & $\begin{array}{c}\text { BRO- } \\
\text { MOXYNIL } \\
\text { WATER, } \\
\text { FLTRD, } \\
\text { GF } 0.7 U \\
\text { REC } \\
(U G / L)\end{array}$ & $\begin{array}{r}\text { DICHLO- } \\
\text { BENIL, } \\
\text { WATER, } \\
\text { FLTRD, } \\
\text { GF 0.7U } \\
\text { REC } \\
(U G / L)\end{array}$ \\
\hline 433548123040600 & COAST FORK WILLAMETTE R BL BIG RIVER NR LONDON, OR & $05-20-94$ & 1040 & $\star \star$ & $\star \star$ & $\star \star$ \\
\hline 440045122585600 & COAST FORK WILLAM AT SEAVY LOOP RD NR EUGENE, OR & $05-19-94$ & 1020 & $\star \star$ & $\star \star$ & $\star \star$ \\
\hline 440402123063900 & URBAN OUTFALL AT POLK ST. PARK AT EUGENE, OR & $06-13-94$ & 1210 & ** & ** & $\star \star$ \\
\hline 441310122095801 & MACK CREEK NEAR BLUE RIVER, OR & $05-27-94$ & 1030 & $\star \star$ & $\star \star$ & $\star \star$ \\
\hline 440707123041300 & MCKENZIE RIVER NR EUGENE, OR & $05-19-94$ & 1450 & $\star \star$ & $\star \star$ & $\star \star$ \\
\hline 442223123153703 & $\begin{array}{l}\text { LONG TOM R AT BUNDY BRIDGE NR MONROE, OR } \\
\text { LONG TOM R AT BUNDY BRIDGE NR MONROE, OR }\end{array}$ & $\begin{array}{l}05-19-94 \\
11-01-94\end{array}$ & $\begin{array}{l}0950 \\
1720\end{array}$ & $\begin{array}{l}\star * \\
\star *\end{array}$ & $\begin{array}{l}\star \star \\
\star \star\end{array}$ & $\begin{array}{l}\star * \\
\star \star\end{array}$ \\
\hline 442413123122500 & LAKE CAMOUS CR AT PINE GROVE DR NR HARRISBURG, OR & $05-19-94$ & 1720 & $\star *$ & $\star *$ & $\star \star$ \\
\hline 443138123120901 & $\begin{array}{l}\text { MUDDY CREEK NEAR PEORIA, OR } \\
\text { MUDDY CREEK NEAR PEORIA, OR } \\
\text { MUDDY CREEK NEAR PEORIA, OR } \\
\text { MUDDY CREEK NEAR PEORIA, OR } \\
\text { MUDDY CREEK NEAR PEORIA, OR } \\
\text { MUDDY CREEK NEAR PEORIA, OR }\end{array}$ & $\begin{array}{l}05-26-94 \\
06-03-94 \\
06-20-94 \\
11-02-94 \\
11-06-94 \\
11-09-94\end{array}$ & $\begin{array}{l}1730 \\
0930 \\
1050 \\
1010 \\
1200 \\
1430\end{array}$ & $\begin{array}{l}\star \star \\
\star \star \\
\star \star \\
\star \star \\
\star \star \\
\star \star \\
\star\end{array}$ & $\begin{array}{l}\star \star \\
\star \star \\
\star \star \\
\star \star \\
\star \star \\
\star \star \\
\star \star\end{array}$ & $\begin{array}{l}\star \star \\
\star \star \\
\star \star \\
\star \star \\
\star \star \\
\star \star \\
\star \star\end{array}$ \\
\hline 443045123273000 & ROCK CREEK ABOVE GRIFFITH CREEK NR PHILOMATH, OR & $05-18-94$ & 1820 & ** & ** & $\star *$ \\
\hline 443321123155201 & MARYS RIVER AT CORVALLIS, OR & $05-18-94$ & 1350 & ** & ** & $\star \star$ \\
\hline 14173500 & $\begin{array}{l}\text { CALAPOOIA RIVER AT ALBANY, OR } \\
\text { CALAPOOIA RIVER AT ALBANY, OR }\end{array}$ & $\begin{array}{l}05-26-94 \\
11-01-94\end{array}$ & $\begin{array}{l}1220 \\
1230\end{array}$ & $\begin{array}{l}* \star \\
\star *\end{array}$ & $\begin{array}{l}* \star \\
\star *\end{array}$ & 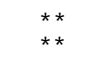 \\
\hline 444349123094000 & LUCKIAMUTE R AT BUENA VISTA RD NR BUENA VISTA, OR & $05-16-94$ & 1720 & $\star \star$ & ** & ** \\
\hline 444123122562200 & THOMAS CREEK AT KELLY RD NR JEFFERSON, OR & $05-16-94$ & 1900 & ** & ** & ** \\
\hline 14189000 & SANTIAM RIVER AT JEFFERSON, OR & $05-17-94$ & 1620 & ** & ** & $\star \star$ \\
\hline
\end{tabular}


Table 38. Concentrations of pesticides in filtered water analyzed by high performance liquid chromatography from Phase II of the Willamette River Basin Water Quality Study, Oregon, 1994-Continued

\begin{tabular}{|c|c|c|c|c|c|c|c|c|c|c|c|}
\hline STATION NUMBER & DATE & $\begin{array}{c}\text { ALDI- } \\
\text { CARB } \\
\text { SULFONE } \\
\text { WAT, FLT } \\
\text { GF } 0.7 \mathrm{U} \\
\text { REC } \\
\text { (UG/L) }\end{array}$ & $\begin{array}{c}\text { ALDI- } \\
\text { CARB, } \\
\text { WATER, } \\
\text { FLTRD, } \\
\text { GF 0.7U } \\
\text { REC } \\
\text { (UG/L) }\end{array}$ & $\begin{array}{c}\text { ALDICA- } \\
\text { RB SUL- } \\
\text { FOXIDE, } \\
\text { WAT, FLT } \\
\text { GF } 0.7 \mathrm{U} \\
\text { REC } \\
\text { (UG/L) }\end{array}$ & $\begin{array}{c}\text { CAR- } \\
\text { BARYL, } \\
\text { WATER, } \\
\text { FLTRD, } \\
\text { GF } 0.7 \mathrm{U} \\
\text { REC } \\
(\mathrm{UG} / \mathrm{L})\end{array}$ & $\begin{array}{c}\text { CARBO- } \\
\text { FURAN, } \\
\text { WATER, } \\
\text { FLTRD, } \\
\text { GF 0.7U } \\
\text { REC } \\
\text { (UG/L) }\end{array}$ & $\begin{array}{c}\text { 3HYDRXY } \\
\text { CARBO- } \\
\text { FURAN } \\
\text { WAT, FLT } \\
\text { GF } 0.7 \mathrm{U} \\
\text { REC } \\
(\mathrm{UG} / \mathrm{L})\end{array}$ & $\begin{array}{c}\text { METHIO- } \\
\text { CARB, } \\
\text { WATER, } \\
\text { FLTRD, } \\
\text { GF 0.7U } \\
\text { REC } \\
\text { (UG/L) }\end{array}$ & $\begin{array}{c}\text { METH- } \\
\text { OMYL, } \\
\text { WATER, } \\
\text { FLTRD, } \\
\text { GF 0.7U } \\
\text { REC } \\
\text { (UG/L) }\end{array}$ & $\begin{array}{c}\text { OXAMYL, } \\
\text { WATER, } \\
\text { FLTRD, } \\
\text { GF } 0.7 U \\
\text { REC } \\
(\mathrm{UG} / \mathrm{L})\end{array}$ & $\begin{array}{c}\text { PRO- } \\
\text { PHAM, } \\
\text { WATER, } \\
\text { FLTRD, } \\
\text { GF 0.7U } \\
\text { REC } \\
(\mathrm{UG} / \mathrm{L})\end{array}$ \\
\hline 433548123040600 & $05-20-94$ & $\star \star$ & $\star \star$ & ** & $\star \star$ & ** & ** & $\star \star$ & ** & ** & $\star \star$ \\
\hline 440045122585600 & $05-19-94$ & $\star \star$ & ** & $\star \star$ & $\star \star$ & $\star \star$ & $\star \star$ & $\star \star$ & $\star \star$ & $\star \star$ & $\star \star$ \\
\hline 440402123063900 & $06-13-94$ & $\star \star$ & ** & $\star \star$ & $\star \star$ & $\star \star$ & $\star \star$ & $\star \star$ & $\star \star$ & $\star \star$ & $\star \star$ \\
\hline 441310122095801 & $05-27-94$ & $\star \star$ & ** & $\star \star$ & $\star \star$ & $\star \star$ & $\star \star$ & $\star \star$ & ** & $\star \star$ & $\star \star$ \\
\hline 440707123041300 & $05-19-94$ & $\star \star$ & $\star *$ & $\star \star$ & $\star \star$ & $\star \star$ & $\star \star$ & $\star \star$ & ** & $\star \star$ & $\star \star$ \\
\hline 442223123153703 & $\begin{array}{l}05-19-94 \\
11-01-94\end{array}$ & $\begin{array}{l}\star * \\
\star *\end{array}$ & $\begin{array}{l}\star * \\
\star *\end{array}$ & $\begin{array}{l}\star * \\
\star *\end{array}$ & $\begin{array}{l}* * \\
\star *\end{array}$ & $\begin{array}{l}\star \star \\
\star *\end{array}$ & $\begin{array}{l}\star \star \\
\star *\end{array}$ & $\begin{array}{l}* \star \\
\star *\end{array}$ & $\begin{array}{l}\star \star \\
\star \star\end{array}$ & $\begin{array}{l}* * \\
\star *\end{array}$ & $\begin{array}{l}\star \star \\
\star \star\end{array}$ \\
\hline 442413123122500 & $05-19-94$ & $\star \star$ & ** & $\star \star$ & ** & $\star \star$ & ** & $\star *$ & ** & $\star \star$ & $\star \star$ \\
\hline 443138123120901 & $\begin{array}{l}05-26-94 \\
06-03-94 \\
06-20-94 \\
11-02-94 \\
11-06-94 \\
11-09-94\end{array}$ & $\begin{array}{l}\star \star \\
\star \star \\
\star \star \\
\star \star \\
\star \star \\
\star \star \\
\star \star\end{array}$ & $\begin{array}{l}\star \star \\
\star \star \\
\star \star \\
\star \star \\
\star \star \\
\star \star \\
\star \star \\
\star \star\end{array}$ & $\begin{array}{l}\star \star \\
\star \star \\
\star \star \\
\star \star \\
\star \star \\
\star \star \\
\star \star\end{array}$ & $\begin{array}{l}\star \star \\
\star \star \\
\star \star \\
\star \star \\
\star \star \\
\star \star \\
\star \star\end{array}$ & $\begin{array}{l}\star \star \\
\star \star \\
\star \star \\
\star \star \\
\star \star \\
\star \star \\
\star \star\end{array}$ & $\begin{array}{l}\star \star \\
\star \star \\
\star \star \\
\star \star \\
\star \star \\
\star \star \\
\star \star\end{array}$ & $\begin{array}{l}\star \star \\
\star \star \\
\star \star \\
\star \star \\
\star \star \\
\star \star \\
\star \star\end{array}$ & $\begin{array}{l}\star \star \\
\star \star \\
\star \star \\
\star \star \\
\star \star \\
\star \star \\
\star \star\end{array}$ & $\begin{array}{l}\star \star \star \\
\star \star \star \\
0.07000 \\
\star \star \\
\star \star \\
\star \star \\
*\end{array}$ & $\begin{array}{l}\star \star \\
\star \star \\
\star \star \\
\star \star \\
\star \star \\
\star \star \\
\star \star\end{array}$ \\
\hline 443045123273000 & $05-18-94$ & $\star *$ & $\star \star$ & $\star \star$ & $\star \star$ & $\star \star$ & $\star \star$ & $\star \star$ & ** & $\star \star$ & $\star \star$ \\
\hline 443321123155201 & $05-18-94$ & $\star \star$ & $\star \star$ & $\star \star$ & $\star \star$ & $\star \star$ & $\star \star$ & $\star \star$ & ** & $\star \star$ & $\star \star$ \\
\hline 14173500 & $\begin{array}{l}05-26-94 \\
11-01-94\end{array}$ & $\begin{array}{l}\star \star \\
\star \star\end{array}$ & $\begin{array}{l}* \star \\
\star *\end{array}$ & $\begin{array}{l}\star * \\
\star *\end{array}$ & $\begin{array}{l}\star \star \\
\star \star\end{array}$ & $\begin{array}{l}\star * \\
\star *\end{array}$ & $\begin{array}{l}\star \star \\
\star *\end{array}$ & $\begin{array}{l}* * \\
\star *\end{array}$ & $\begin{array}{l}* * \\
* *\end{array}$ & $\begin{array}{l}* * \\
\star *\end{array}$ & $\begin{array}{l}\star \star \\
\star *\end{array}$ \\
\hline 444349123094000 & $05-16-94$ & $\star \star$ & $\star \star$ & $\star \star$ & $\star \star$ & $\star \star$ & $\star \star$ & $\star \star$ & ** & $\star \star$ & $\star \star$ \\
\hline 444123122562200 & $05-16-94$ & $\star \star$ & ** & ** & $\star \star$ & $\star \star$ & ** & $\star \star$ & $\star \star$ & $\star \star$ & $\star \star$ \\
\hline 14189000 & $05-17-94$ & $\star \star$ & $\star \star$ & $\star \star$ & ** & $\star \star$ & ** & $\star \star$ & ** & $\star \star$ & $\star \star$ \\
\hline
\end{tabular}


Table 38. Concentrations of pesticides in filtered water analyzed by high performance liquid chromatography from Phase II of the Willamette River Basin Water Quality Study, Oregon, 1994-Continued

\begin{tabular}{|c|c|c|c|c|c|c|c|c|c|c|c|}
\hline STATION NUMBER & DATE & $\begin{array}{c}\text { PRO- } \\
\text { POXUR, } \\
\text { WATER, } \\
\text { FLTRD, } \\
\text { GF } 0.7 \mathrm{U} \\
\text { REC } \\
\text { (UG/L) }\end{array}$ & $\begin{array}{c}\text { BENTA- } \\
\text { ZON, } \\
\text { WATER, } \\
\text { FLTRD, } \\
\text { GF } 0.7 \mathrm{U} \\
\text { REC } \\
\text { (UG/L) }\end{array}$ & $\begin{array}{c}2,4-D, \\
\text { DIS- } \\
\text { SOLVED } \\
(\mathrm{UG} / \mathrm{L})\end{array}$ & $\begin{array}{c}\text { 2, 4-DB } \\
\text { WATER, } \\
\text { FLTRD, } \\
\text { GE } 0.7 \mathrm{U} \\
\text { REC } \\
(\mathrm{UG} / \mathrm{L})\end{array}$ & $\begin{array}{c}\text { DACTHAL } \\
\text { MONO- } \\
\text { ACID, } \\
\text { WAT, FLT } \\
\text { GF } 0.7 \mathrm{U} \\
\text { REC } \\
\text { (UG/L) }\end{array}$ & $\begin{array}{c}\text { DICHLOR } \\
\text { PROP, } \\
\text { WATER, } \\
\text { FLTRD, } \\
\text { GF } 0.7 \mathrm{U} \\
\text { REC } \\
\text { (UG/L) }\end{array}$ & $\begin{array}{l}\text { MCPA, } \\
\text { WATER, } \\
\text { FLTRD, } \\
\text { GF } 0.7 \mathrm{U} \\
\text { REC } \\
(\mathrm{UG} / \mathrm{L})\end{array}$ & $\begin{array}{c}\text { MCPB, } \\
\text { WATER, } \\
\text { FLTRD, } \\
\text { GF } 0.7 \mathrm{U} \\
\text { REC } \\
(\mathrm{UG} / \mathrm{L})\end{array}$ & $\begin{array}{c}\text { SILVEX, } \\
\text { DIS- } \\
\text { SOLVED } \\
\text { (UG/L) }\end{array}$ & $\begin{array}{c}2,4,5-T \\
\text { DIS- } \\
\text { SOLVED } \\
(\mathrm{UG} / \mathrm{L})\end{array}$ \\
\hline 433548123040600 & $05-20-94$ & $\star \star$ & $\star \star$ & ** & $\star \star$ & $\star \star$ & $\star \star$ & ** & $\star \star$ & $\star \star$ & ** \\
\hline 440045122585600 & $05-19-94$ & $\star *$ & $\star \star$ & $\star \star$ & $\star \star$ & $\star \star$ & $\star \star$ & ** & $\star \star$ & $\star \star$ & ** \\
\hline 440402123063900 & $06-13-94$ & $\star \star$ & $\star \star$ & *夫 & $\star \star$ & $\star \star$ & $\star \star$ & ** & $\star \star$ & $\star \star$ & ** \\
\hline 441310122095801 & $05-27-94$ & $\star \star$ & $\star \star$ & ** & $\star \star$ & $\star \star$ & $\star \star$ & ** & $\star \star$ & $\star \star$ & ** \\
\hline 440707123041300 & $05-19-94$ & $\star \star$ & $\star \star$ & ** & $\star \star$ & ** & $\star \star$ & ** & $\star \star$ & $\star \star$ & ** \\
\hline 442223123153703 & $\begin{array}{l}05-19-94 \\
11-01-94\end{array}$ & $\begin{array}{l}\star * \\
\star *\end{array}$ & $\begin{array}{l}\star * \\
* *\end{array}$ & $\begin{array}{l}* * \\
\star *\end{array}$ & $\begin{array}{l}\star \star \\
\star \star\end{array}$ & $\begin{array}{l}\star * \\
\star \star\end{array}$ & $\begin{array}{l}\star \star \\
\star \star\end{array}$ & $\begin{array}{l}* * \\
* *\end{array}$ & $\begin{array}{l}* * \\
* *\end{array}$ & $\begin{array}{l}\star * \\
\star \star\end{array}$ & $\begin{array}{l}* \star \\
\star *\end{array}$ \\
\hline 442413123122500 & $05-19-94$ & $\star \star$ & $\star \star$ & 0.20000 & $\star \star$ & $\star \star$ & $\star \star$ & ** & ** & $\star \star$ & ** \\
\hline 443138123120901 & $\begin{array}{l}05-26-94 \\
06-03-94 \\
06-20-94 \\
11-02-94 \\
11-06-94 \\
11-09-94\end{array}$ & $\begin{array}{l}\star \star \\
\star \star \\
\star \star \\
\star \star \\
\star \star \\
\star \star \\
\star \star\end{array}$ & $\begin{array}{l}\star \star \\
\star \star \\
\star \star \\
\star \star \\
\star \star \\
\star \star \\
\star \star\end{array}$ & $\begin{array}{c}0.21000 \\
\star \star \\
\star \star \\
\star \star \\
\star \star \\
\star \star\end{array}$ & $\begin{array}{l}\star \star \\
\star \star \\
\star \star \\
\star \star \\
\star \star \\
\star \star \\
\star \star\end{array}$ & $\begin{array}{l}\star \star \\
\star * \\
\star * \\
\star \star \\
\star \star \\
\star \star \\
\star \star\end{array}$ & $\begin{array}{l}\star \star \\
\star \star \\
\star \star \\
\star \star \\
\star \star \\
\star \star \\
\star \star\end{array}$ & $\begin{array}{l}\star \star \\
\star * \\
\star \star \\
\star \star \\
\star \star \\
\star \star \\
\star *\end{array}$ & $\begin{array}{l}\star \star \\
\star \star \\
\star \star \\
\star \star \\
\star \star \\
\star \star \\
\star \star\end{array}$ & $\begin{array}{l}\star \star \\
\star \star \\
\star \star \\
\star \star \\
\star \star \\
\star \star \\
\star \star\end{array}$ & $\begin{array}{l}* \star \\
\star * \\
\star \star \\
\star \star \\
\star * \\
\star * \\
* \star\end{array}$ \\
\hline 443045123273000 & $05-18-94$ & $\star *$ & $\star \star$ & $\star \star$ & $\star \star$ & $\star \star$ & $\star \star$ & $\star \star$ & $\star \star$ & $\star \star$ & ** \\
\hline 443321123155201 & $05-18-94$ & $\star *$ & ** & ** & ** & $\star \star$ & $\star \star$ & ** & $\star \star$ & $\star \star$ & ** \\
\hline 14173500 & $\begin{array}{l}05-26-94 \\
11-01-94\end{array}$ & $\begin{array}{l}\star * \\
\star *\end{array}$ & $\begin{array}{l}\star * \\
\star *\end{array}$ & $\begin{array}{l}\star * \\
\star *\end{array}$ & $\begin{array}{l}* * \\
\star *\end{array}$ & $\begin{array}{l}* * \\
* *\end{array}$ & $\begin{array}{l}\star * \\
* *\end{array}$ & $\begin{array}{l}\star * \\
\star *\end{array}$ & $\begin{array}{l}\star * \\
\star *\end{array}$ & $\begin{array}{l}* * \\
\star *\end{array}$ & ** \\
\hline 444349123094000 & $05-16-94$ & $\star \star$ & $\star \star$ & $\star \star$ & ** & $\star \star$ & ** & ** & $\star \star$ & $\star \star$ & ** \\
\hline 444123122562200 & $05-16-94$ & $\star *$ & $\star \star$ & ** & $\star \star$ & $\star \star$ & $\star \star$ & ** & $\star \star$ & $\star \star$ & ** \\
\hline 14189000 & $05-17-94$ & $\star *$ & $\star \star$ & ** & $\star \star$ & ** & $\star \star$ & ** & $\star \star$ & $\star *$ & ** \\
\hline
\end{tabular}


Table 38. Concentrations of pesticides in filtered water analyzed by high performance liquid chromatography from Phase II of the Willamette River Basin Water Quality Study, Oregon, 1994-Continued

\begin{tabular}{|c|c|c|c|c|c|c|c|c|c|c|c|}
\hline STATION NUMBER & DATE & $\begin{array}{c}\text { ORY- } \\
\text { ZALIN, } \\
\text { WATER, } \\
\text { FLTRD, } \\
\text { GF } 0.7 U \\
\text { REC } \\
\text { (UG/L) }\end{array}$ & $\begin{array}{c}\text { DINOSEB } \\
\text { WATER, } \\
\text { FLTRD, } \\
\text { GF } 0.7 U \\
\text { REC } \\
\text { (UG/L) }\end{array}$ & $\begin{array}{c}\text { OCRESOL } \\
4,6- \\
\text { DINITRO } \\
\text { WAT, FLT } \\
\text { GF } 0.7 \mathrm{U} \\
\text { REC } \\
(\mathrm{UG} / \mathrm{L})\end{array}$ & $\begin{array}{c}\text { ACIFL- } \\
\text { UORFEN } \\
\text { WATER, } \\
\text { FLTRD, } \\
\text { GF } 0.7 U \\
\text { REC } \\
\text { (UG/L) }\end{array}$ & $\begin{array}{c}\text { 1-NAPH } \\
\text { THOL, } \\
\text { WATER, } \\
\text { FLTRD, } \\
\text { GF } 0.7 U \\
\text { REC } \\
\text { (UG/L) }\end{array}$ & $\begin{array}{c}\text { AMIBEN, } \\
\text { WATER, } \\
\text { FLTRD, } \\
\text { GF } 0.7 U \\
\text { REC } \\
(U G / L)\end{array}$ & $\begin{array}{c}\text { DIURON, } \\
\text { WATER, } \\
\text { FLTRD, } \\
\text { GF } 0.7 U \\
\text { REC } \\
(U G / L)\end{array}$ & $\begin{array}{c}\text { FEN- } \\
\text { URON, } \\
\text { WATER, } \\
\text { FLTRD, } \\
\text { GF } 0.7 \mathrm{U} \\
\text { REC } \\
(\mathrm{UG} / \mathrm{L})\end{array}$ & $\begin{array}{c}\text { FLUO- } \\
\text { METURON } \\
\text { WATER, } \\
\text { FLTRD, } \\
\text { GF } 0.7 U \\
\text { REC } \\
\text { (UG/L) }\end{array}$ & $\begin{array}{c}\text { LINURON } \\
\text { WATER, } \\
\text { FLTRD, } \\
\text { GF } 0.7 \mathrm{U} \\
\text { REC } \\
\text { (UG/L) }\end{array}$ \\
\hline 433548123040600 & $05-20-94$ & $\star \star$ & $\star \star$ & ** & $\star \star$ & $\star \star$ & $\star *$ & ** & $\star \star$ & $\star \star$ & ** \\
\hline 440045122585600 & $05-19-94$ & $\star \star$ & ** & ** & $\star \star$ & ** & $\star \star$ & ** & $\star \star$ & $\star *$ & ** \\
\hline 440402123063900 & $06-13-94$ & $\star *$ & $\star *$ & ** & $\star \star$ & ** & $\star \star$ & 0.28000 & $\star \star$ & $\star \star$ & ** \\
\hline 441310122095801 & $05-27-94$ & $\star *$ & $\star *$ & $\star \star$ & $\star *$ & $\star \star$ & $\star *$ & ** & $\star \star$ & $\star \star$ & ** \\
\hline 440707123041300 & $05-19-94$ & $\star *$ & $\star \star$ & $\star \star$ & $\star \star$ & $\star \star$ & $\star \star$ & ** & $\star \star$ & $\star \star$ & ** \\
\hline 442223123153703 & $\begin{array}{l}05-19-94 \\
11-01-94\end{array}$ & $\begin{array}{l}\star * \\
\star *\end{array}$ & $\begin{array}{c}* \star \\
0.06000\end{array}$ & $\begin{array}{l}\star \star \\
\star *\end{array}$ & $\begin{array}{l}\star \star \\
\star *\end{array}$ & $\begin{array}{l}* * \\
* *\end{array}$ & $\begin{array}{l}* * \\
* *\end{array}$ & $\begin{array}{c}* * \\
0.12000\end{array}$ & $\begin{array}{l}* \star \\
* *\end{array}$ & $\begin{array}{l}* \star \\
\star *\end{array}$ & $\begin{array}{l}* * \\
* *\end{array}$ \\
\hline 442413123122500 & $05-19-94$ & $\star \star$ & $\star *$ & ** & $\star *$ & ** & $\star \star$ & 0.69000 & $\star \star$ & $\star \star$ & ** \\
\hline 443138123120901 & $\begin{array}{l}05-26-94 \\
06-03-94 \\
06-20-94 \\
11-02-94 \\
11-06-94 \\
11-09-94\end{array}$ & $\begin{array}{l}\star \star \\
\star \star \\
\star \star \\
\star \star \\
\star \star \\
\star \star \\
\star \star\end{array}$ & $\begin{array}{l}\star \star \\
\star \star \\
\star \star \\
\star \star \\
\star \star \\
\star \star \\
\star \star\end{array}$ & $\begin{array}{l}\star \star \\
\star \star \\
\star \star \\
\star \star \\
\star \star \\
\star \star \\
\star \star \\
\star \star\end{array}$ & $\begin{array}{l}\star \star \\
\star \star \\
\star \star \\
\star \star \\
\star \star \\
\star \star \\
\star \star\end{array}$ & $\begin{array}{l}\star \star \\
\star \star \\
\star \star \\
\star \star \\
\star \star \\
\star \star \\
\star \star\end{array}$ & $\begin{array}{l}\star \star \\
\star * \\
\star \star \\
\star \star \\
\star * \\
\star * \\
\star *\end{array}$ & $\begin{array}{l}0.52000 \\
0.10000 \\
0.26000 \\
\mathrm{E} 1.4000 \\
\mathrm{E} 4.2000 \\
\mathrm{E} 2.5000\end{array}$ & $\begin{array}{l}\star \star \\
\star \star \\
\star \star \\
\star \star \\
\star \star \\
\star \star \\
\star \star\end{array}$ & $\begin{array}{l}\star \star \\
\star \star \\
\star \star \\
\star \star \\
\star \star \\
\star \star \\
\star \star\end{array}$ & $\begin{array}{l}\star \star \\
\star \star \\
\star \star \\
\star * \\
\star * \\
\star * \\
\star *\end{array}$ \\
\hline 443045123273000 & $05-18-94$ & $\star *$ & $\star *$ & $\star \star$ & $\star \star$ & $\star \star$ & $\star *$ & ** & $\star \star$ & $\star \star$ & ** \\
\hline 443321123155201 & $05-18-94$ & $\star *$ & $\star \star$ & ** & ** & ** & $\star \star$ & ** & $\star \star *$ & $\star \star$ & ** \\
\hline 14173500 & $\begin{array}{l}05-26-94 \\
11-01-94\end{array}$ & $\begin{array}{l}\star * \\
\star *\end{array}$ & $\begin{array}{l}\star * \\
\star *\end{array}$ & $\begin{array}{l}* * \\
\star *\end{array}$ & $\begin{array}{l}\star * \\
\star *\end{array}$ & $\begin{array}{l}\star * \\
\star *\end{array}$ & $\begin{array}{l}* * \\
* *\end{array}$ & $\begin{array}{c}* * \\
\mathrm{E} 1.9000\end{array}$ & $\begin{array}{l}* * \\
\star *\end{array}$ & $\begin{array}{l}* * \\
* *\end{array}$ & $\begin{array}{l}* * \\
\star *\end{array}$ \\
\hline 444349123094000 & $05-16-94$ & $\star *$ & $\star *$ & ** & $\star *$ & $\star \star$ & ** & $\star *$ & $\star \star$ & $\star *$ & ** \\
\hline 444123122562200 & $05-16-94$ & ** & $\star \star$ & ** & $\star \star$ & $\star \star$ & $\star \star$ & ** & ** & $\star \star$ & ** \\
\hline 14189000 & $05-17-94$ & $\star *$ & ** & ** & $\star \star$ & ** & $\star \star$ & ** & $\star \star$ & $\star \star$ & ** \\
\hline
\end{tabular}


Table 38. Concentrations of pesticides in filtered water analyzed by high performance liquid chromatography from Phase II of the Willamette River Basin Water Quality Study, Oregon, 1994-Continued

\begin{tabular}{|c|c|c|c|c|c|c|c|c|c|c|}
\hline STATION NUMBER & DATE & $\begin{array}{l}\text { NEB- } \\
\text { URON, } \\
\text { WATER, } \\
\text { FLTRD, } \\
\text { GF } 0.7 \mathrm{U} \\
\text { REC } \\
\text { (UG/L) }\end{array}$ & $\begin{array}{c}\text { CHLORO- } \\
\text { THALO- } \\
\text { NIL, } \\
\text { WAT,FLT } \\
\text { GF } 0.7 \mathrm{U} \\
\text { REC } \\
\text { (UG/L) }\end{array}$ & $\begin{array}{l}\text { ESFEN- } \\
\text { VAL- } \\
\text { ERATE, } \\
\text { WAT, FLT } \\
\text { GF } 0.7 \mathrm{U} \\
\text { REC } \\
\text { (UG/L) }\end{array}$ & $\begin{array}{l}\text { NORFLUR } \\
\text { AZON, } \\
\text { WATER, } \\
\text { FLTRD, } \\
\text { GF } 0.7 \mathrm{U} \\
\text { REC } \\
\text { (UG/L) }\end{array}$ & $\begin{array}{l}\text { CLOPYR- } \\
\text { ALID, } \\
\text { WATER, } \\
\text { FLTRD, } \\
\text { GF } 0.7 \mathrm{U} \\
\text { REC } \\
\text { (UG/L) }\end{array}$ & $\begin{array}{l}\text { PIC- } \\
\text { LORAM, } \\
\text { WATER, } \\
\text { FLTRD, } \\
\text { GF } 0.7 \mathrm{U} \\
\text { REC } \\
\text { (UG/L) }\end{array}$ & $\begin{array}{c}\text { TRI- } \\
\text { CLOPYR, } \\
\text { WATER, } \\
\text { FLTRD, } \\
\text { GF } 0.7 \mathrm{U} \\
\text { REC } \\
\text { (UG/L) }\end{array}$ & $\begin{array}{l}\text { BRO- } \\
\text { MACIL, } \\
\text { WATER, } \\
\text { DISS, } \\
\text { REC } \\
\text { (UG/L) }\end{array}$ & $\begin{array}{l}\text { BDMC, } \\
\text { SURROG, } \\
\text { WATER, } \\
\text { UNFLTRD } \\
\text { REC } \\
\text { PERCENT }\end{array}$ \\
\hline 433548123040600 & $05-20-94$ & $\star \star$ & ** & $\star \star$ & $\star \star$ & $\star \star$ & $\star \star$ & $\star \star$ & $\star \star$ & 8.0000 \\
\hline 440045122585600 & $05-19-94$ & $\star \star$ & ** & $\star \star$ & ** & $\star \star$ & $\star \star$ & $\star \star$ & $\star \star$ & 20.000 \\
\hline 440402123063900 & $06-13-94$ & $\star \star$ & ** & $\star \star$ & ** & $\star \star$ & $\star \star$ & $\star \star$ & $\star \star$ & -- \\
\hline 441310122095801 & $05-27-94$ & $\star \star$ & ** & $\star \star$ & ** & $\star \star$ & $\star \star$ & $\star \star$ & $\star \star$ & 90.000 \\
\hline 440707123041300 & $05-19-94$ & $\star \star$ & ** & $\star \star$ & $\star \star$ & $\star \star$ & $\star \star$ & $\star \star$ & $\star \star$ & 12.000 \\
\hline 442223123153703 & $\begin{array}{l}05-19-94 \\
11-01-94\end{array}$ & $\begin{array}{l}* \star \\
\star *\end{array}$ & ** & $\begin{array}{l}\star \star \\
\star \star\end{array}$ & $\begin{array}{l}* \star \\
\star \star\end{array}$ & $\begin{array}{l}* * \\
\star *\end{array}$ & $\begin{array}{l}\star \star \\
\star \star\end{array}$ & 0.29000 & $\begin{array}{l}\star \star \\
\star \star\end{array}$ & $\begin{array}{r}<0.00000 \\
89.000\end{array}$ \\
\hline 442413123122500 & $05-19-94$ & $\star \star$ & ** & $\star \star$ & *夫 & $\star \star$ & ** & $\star \star$ & $\star \star$ & 30.000 \\
\hline 443138123120901 & $\begin{array}{l}05-26-94 \\
06-03-94 \\
06-20-94 \\
11-02-94 \\
11-06-94 \\
11-09-94\end{array}$ & $\begin{array}{l}\star \star \\
\star \star \\
\star \star \\
\star \star \\
\star \star \\
\star \star \\
\star \star\end{array}$ & $\begin{array}{l}\star \star \\
\star \star \\
\star \star \\
\star \star \\
\star \star \\
\star \star \\
\star \star\end{array}$ & $\begin{array}{l}\star \star \\
\star \star \\
\star \star \\
\star \star \\
\star \star \\
\star \star \\
\star \star\end{array}$ & $\begin{array}{l}\star \star \\
\star \star \\
\star \star \\
\star \star \\
\star \star \\
\star \star \\
\star \star\end{array}$ & $\begin{array}{l}\star \star \\
\star \star \\
\star \star \\
\star \star \\
\star \star \\
\star \star \\
\star *\end{array}$ & $\begin{array}{l}\star \star \\
\star \star \\
\star \star \\
\star \star \\
\star \star \\
\star \star \\
\star \star\end{array}$ & $\begin{array}{l}\star \star \\
\star \star \\
\star \star \\
0 . \\
44000 \\
\star \star \\
\star \star\end{array}$ & $\begin{array}{l}0.20000 \\
\star \star \\
\star \star \\
\star \star \\
\star \star \\
\star \star\end{array}$ & $\begin{array}{l}25.000 \\
6.0000 \\
88.000 \\
45.000 \\
66.000 \\
65.000\end{array}$ \\
\hline 443045123273000 & $05-18-94$ & $\star \star$ & ** & $\star \star$ & $\star \star$ & $\star \star$ & $\star \star$ & $\star \star$ & $\star \star$ & 44.000 \\
\hline 443321123155201 & $05-18-94$ & $\star \star$ & ** & $\star \star$ & $\star \star$ & $\star \star$ & $\star \star$ & $\star \star$ & $\star \star$ & 52.000 \\
\hline 14173500 & $\begin{array}{l}05-26-94 \\
11-01-94\end{array}$ & $\begin{array}{l}* \star \\
\star *\end{array}$ & ** & ** & $\begin{array}{l}* \star \\
\star \star\end{array}$ & ** & $\begin{array}{l}* * \\
* *\end{array}$ & 0.72000 & $\begin{array}{l}\star \star \\
\star \star\end{array}$ & $\begin{array}{l}38.000 \\
38.000\end{array}$ \\
\hline 444349123094000 & $05-16-94$ & $\star \star$ & ** & $\star \star$ & $\star \star$ & $\star \star$ & $\star \star$ & $\star \star$ & $\star \star$ & 38.000 \\
\hline 444123122562200 & $05-16-94$ & $\star \star$ & ** & $\star \star$ & $\star \star$ & $\star \star$ & $\star \star$ & $\star \star$ & $\star \star$ & $\mathrm{E} 65.000$ \\
\hline 14189000 & $05-17-94$ & ** & ** & $\star \star$ & ** & $\star \star$ & $\star \star$ & $\star \star$ & $\star \star$ & 89.000 \\
\hline
\end{tabular}


Table 38. Concentrations of pesticides in filtered water analyzed by high performance liquid chromatography from Phase II of the Willamette River Basin Water Quality Study, Oregon, 1994-Continued

\begin{tabular}{|c|c|c|c|c|c|c|}
\hline STATION NUMBER & STATION NAME & DATE & TIME & $\begin{array}{c}\text { DICAMBA } \\
\text { WATER, } \\
\text { FLTRD, } \\
\text { GF } 0.7 U \\
\text { REC } \\
(U G / L)\end{array}$ & $\begin{array}{c}\text { BRO- } \\
\text { MOXYNIL } \\
\text { WATER, } \\
\text { FLTRD, } \\
\text { GF } 0.7 \mathrm{U} \\
\text { REC } \\
\text { (UG/L) }\end{array}$ & $\begin{array}{c}\text { DICHLO- } \\
\text { BENIL, } \\
\text { WATER, } \\
\text { FLTRD, } \\
\text { GF } 0.7 U \\
\text { REC } \\
(U G / L)\end{array}$ \\
\hline 445547123065400 & RICKREAL CREEK NR MOUTH NR SALEM, OR & $05-26-94$ & 1540 & $\star \star$ & $\star \star$ & $\star \star$ \\
\hline 14190970 & $\begin{array}{l}\text { PRINGLE C AT BUSH PARK AT SALEM, OR } \\
\text { PRINGLE C AT BUSH PARK AT SALEM, OR } \\
\text { PRINGLE C AT BUSH PARK AT SALEM, OR }\end{array}$ & $\begin{array}{l}11-23-94 \\
11-30-94 \\
11-30-94\end{array}$ & $\begin{array}{l}1200 \\
1530 \\
1710\end{array}$ & $\begin{array}{l}\star \star \\
\star \star \\
\star \star\end{array}$ & $\begin{array}{l}\star \star \\
\star \star \\
\star \star\end{array}$ & $\begin{array}{l}\star \star \\
\star \star \\
\star \star\end{array}$ \\
\hline 445037122573800 & $\begin{array}{l}\text { MILL CREEK AT DELANEY ROAD NR TURNER, OR } \\
\text { MILL CREEK AT DELANEY ROAD NR TURNER, OR } \\
\text { MILL CREEK AT DELANEY ROAD NR TURNER, OR } \\
\text { MILL CREEK AT DELANEY ROAD NR TURNER, OR }\end{array}$ & $\begin{array}{l}06-14-94 \\
10-31-94 \\
11-04-94 \\
11-04-94\end{array}$ & $\begin{array}{l}1140 \\
1410 \\
1120 \\
1420\end{array}$ & $\begin{array}{l}\star \star \\
\star \star \\
\star \star \\
\star \star\end{array}$ & $\begin{array}{l}\star \star \\
\star \star \\
\star \star \\
\star \star\end{array}$ & $\begin{array}{l}\star \star \\
\star \star \\
\star \star \\
\star \star\end{array}$ \\
\hline 14194150 & $\begin{array}{l}\text { SOUTH YAMHILL RIVER AT MCMINNVILLE, OR } \\
\text { SOUTH YAMHILL RIVER AT MCMINNVILLE, OR }\end{array}$ & $\begin{array}{l}05-17-94 \\
11-02-94\end{array}$ & $\begin{array}{l}1720 \\
1210\end{array}$ & $\begin{array}{l}\star \star \\
\star \star\end{array}$ & ** & 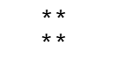 \\
\hline 451355123093600 & NORTH YAMHILL RIVER AT HWY. 99E NR MCMINNVILLE, OR & $05-17-94$ & 1220 & $\star \star$ & $\star \star$ & $\star \star$ \\
\hline 451602122564400 & WILLAMETTE RIVER NR NEWBERG, OR & $05-31-94$ & 1400 & $\star \star$ & ** & $\star \star$ \\
\hline 451502122524700 & $\begin{array}{l}\text { CHAMPOEG CREEK BELOW MISSION CR NR BUTTEVILLE, OR } \\
\text { CHAMPOEG CREEK BELOW MISSION CR NR BUTTEVILLE, OR }\end{array}$ & $\begin{array}{l}05-26-94 \\
10-31-94\end{array}$ & $\begin{array}{l}1030 \\
1300\end{array}$ & ** & ** & ** \\
\hline 14201300 & $\begin{array}{l}\text { ZOLLNER CREEK NEAR MT ANGEL, OR } \\
\text { ZOLLNER CREEK NEAR MT ANGEL, OR } \\
\text { ZOLLNER CREEK NEAR MT ANGEL, OR } \\
\text { ZOLLNER CREEK NEAR MT ANGEL, OR }\end{array}$ & $\begin{array}{l}05-25-94 \\
06-13-94 \\
10-28-94 \\
11-28-94\end{array}$ & $\begin{array}{l}1450 \\
1120 \\
1320 \\
1400\end{array}$ & $\begin{array}{l}0.05000 \\
\star \star \\
\star \star \\
\star \star\end{array}$ & $\begin{array}{l}\star \star \star \\
\star * \star \\
* \star \\
* \star\end{array}$ & $\begin{array}{l}\star \star \\
\star \star \\
\star \star \\
\star \star\end{array}$ \\
\hline 14202000 & $\begin{array}{lll}\text { PUDDING RIVER AT AURORA, OREG. } \\
\text { PUDDING RIVER AT AURORA, OREG. } \\
\text { PUDDING RIVER AT AURORA, OREG. } \\
\text { PUDDING RIVER AT AURORA, OREG. } \\
\text { PUDDING RIVER AT AURORA, OREG. } \\
\text { PUDDING RIVER AT AURORA, OREG. } \\
\text { PUDDING RIVER AT AURORA, OREG. }\end{array}$ & $\begin{array}{l}04-12-94 \\
05-25-94 \\
06-15-94 \\
10-28-94 \\
10-29-94 \\
11-09-94 \\
11-29-94\end{array}$ & $\begin{array}{l}1110 \\
1100 \\
1145 \\
1710 \\
1230 \\
1300 \\
1110\end{array}$ & $\begin{array}{l}\star \star \\
\star \star \\
\star \star \\
\star \star \\
\star \star \\
\star \star \\
\star \star \\
\star \star\end{array}$ & $\begin{array}{l}\star \star \\
\star \star \\
\star \star \\
\star \star \\
\star \star \\
\star \star \\
\star \star \\
\star \star\end{array}$ & $\begin{array}{l}\star \star \\
\star \star \\
\star \star \\
\star \star \\
\star \star \\
\star \star \\
\star \star \\
\star \star\end{array}$ \\
\hline 451603122423301 & MOLALLA R AT KNIGHTS BRIDGE NR CANBY, OR & $05-25-94$ & 1020 & $\star \star$ & $\star \star$ & $\star \star$ \\
\hline 14206200 & $\begin{array}{l}\text { DAIRY CREEK AT RTE } 8 \text { NEAR HILLSBORO, OR } \\
\text { DAIRY CREEK AT RTE } 8 \text { NEAR HILLSBORO, OR }\end{array}$ & $\begin{array}{l}05-27-94 \\
12-01-94\end{array}$ & $\begin{array}{l}1640 \\
1140\end{array}$ & $\begin{array}{l}\star \star \\
\star \star\end{array}$ & $\begin{array}{l}\star \star \\
\star \star\end{array}$ & $\begin{array}{l}\star \star \\
\star \star\end{array}$ \\
\hline
\end{tabular}


Table 38. Concentrations of pesticides in filtered water analyzed by high performance liquid chromatography from Phase II of the Willamette River Basin Water Quality Study, Oregon, 1994-Continued

\begin{tabular}{|c|c|c|c|c|c|c|c|c|c|c|c|}
\hline STATION NUMBER & DATE & $\begin{array}{c}\text { ALDI- } \\
\text { CARB } \\
\text { SULFONE } \\
\text { WAT, FLT } \\
\text { GF } 0.7 \mathrm{U} \\
\text { REC } \\
(\mathrm{UG} / \mathrm{L})\end{array}$ & $\begin{array}{c}\text { ALDI- } \\
\text { CARB, } \\
\text { WATER, } \\
\text { FLTRD, } \\
\text { GF 0.7U } \\
\text { REC } \\
\text { (UG/L) }\end{array}$ & $\begin{array}{c}\text { ALDICA- } \\
\text { RB SUL- } \\
\text { FOXIDE, } \\
\text { WAT, FLT } \\
\text { GF } 0.7 \mathrm{U} \\
\text { REC } \\
\text { (UG/L) }\end{array}$ & $\begin{array}{c}\text { CAR- } \\
\text { BARYL, } \\
\text { WATER, } \\
\text { FLTRD, } \\
\text { GF } 0.7 \mathrm{U} \\
\text { REC } \\
(\mathrm{UG} / \mathrm{L})\end{array}$ & $\begin{array}{c}\text { CARBO- } \\
\text { FURAN, } \\
\text { WATER, } \\
\text { FLTRD, } \\
\text { GF 0.7U } \\
\text { REC } \\
\text { (UG/L) }\end{array}$ & $\begin{array}{c}\text { 3HYDRXY } \\
\text { CARBO- } \\
\text { FURAN } \\
\text { WAT, FLT } \\
\text { GF } 0.7 \mathrm{U} \\
\text { REC } \\
(\mathrm{UG} / \mathrm{L})\end{array}$ & $\begin{array}{c}\text { METHIO- } \\
\text { CARB, } \\
\text { WATER, } \\
\text { FLTRD, } \\
\text { GF 0.7U } \\
\text { REC } \\
\text { (UG/L) }\end{array}$ & $\begin{array}{c}\text { METH- } \\
\text { OMYL, } \\
\text { WATER, } \\
\text { FLTRD, } \\
\text { GF 0.7U } \\
\text { REC } \\
\text { (UG/L) }\end{array}$ & $\begin{array}{c}\text { OXAMYL, } \\
\text { WATER, } \\
\text { FLTRD, } \\
\text { GF } 0.7 U \\
\text { REC } \\
(\mathrm{UG} / \mathrm{L})\end{array}$ & $\begin{array}{c}\text { PRO- } \\
\text { PHAM, } \\
\text { WATER, } \\
\text { FLTRD, } \\
\text { GF 0.7U } \\
\text { REC } \\
(\mathrm{UG} / \mathrm{L})\end{array}$ \\
\hline 445547123065400 & $05-26-94$ & $\star \star$ & $\star \star$ & $\star \star$ & $\star \star$ & $\star \star$ & $\star \star$ & $\star \star$ & ** & $\star \star$ & $\star \star$ \\
\hline 14190970 & $\begin{array}{l}11-23-94 \\
11-30-94 \\
11-30-94\end{array}$ & $\begin{array}{l}\star \star \\
\star \star \\
\star \star\end{array}$ & $\begin{array}{l}\star \star \\
\star * \\
\star *\end{array}$ & $\begin{array}{l}\star \star \\
\star * \\
\star \star\end{array}$ & $\begin{array}{l}\star \star \\
\star \star \\
\star \star\end{array}$ & $\begin{array}{l}\star \star \\
\star \star \\
\star \star\end{array}$ & $\begin{array}{l}\star \star \\
\star \star \\
\star \star\end{array}$ & $\begin{array}{l}\star \star \\
\star \star \\
\star \star\end{array}$ & $\begin{array}{l}\star * \\
\star * \\
\star *\end{array}$ & $\begin{array}{l}\star \star \\
\star * \\
\star \star\end{array}$ & $\begin{array}{l}\star \star \\
\star * \\
\star \star\end{array}$ \\
\hline 445037122573800 & $\begin{array}{l}06-14-94 \\
10-31-94 \\
11-04-94 \\
11-04-94\end{array}$ & $\begin{array}{l}\star \star \\
\star \star \\
\star \star \\
\star \star\end{array}$ & $\begin{array}{l}\star \star \\
\star \star \\
\star \star \\
\star \star\end{array}$ & $\begin{array}{l}\star \star \\
\star \star \\
\star \star \\
\star \star\end{array}$ & $\begin{array}{l}\star \star \\
\star \star \\
\star \star \\
\star \star\end{array}$ & $\begin{array}{c}\star \star \\
\star \star \\
0.30000 \\
0.47000\end{array}$ & $\begin{array}{l}\star \star \\
\star \star \\
\star \star \\
\star \star\end{array}$ & $\begin{array}{l}\star \star \\
\star \star \\
\star \star \\
\star \star\end{array}$ & $\begin{array}{l}\star \star \\
\star \star \\
\star \star \\
\star \star\end{array}$ & $\begin{array}{l}\star \star \\
\star \star \\
\star \star \\
\star \star\end{array}$ & $\begin{array}{l}\star \star \\
\star \star \\
\star \star \\
\star \star\end{array}$ \\
\hline 14194150 & $\begin{array}{l}05-17-94 \\
11-02-94\end{array}$ & $\begin{array}{l}\star \star \\
\star \star\end{array}$ & $\begin{array}{l}\star \star \\
\star \star\end{array}$ & $\begin{array}{l}\star \star \\
\star *\end{array}$ & $\begin{array}{l}\star \star \\
\star *\end{array}$ & $\begin{array}{l}\star \star \\
\star \star\end{array}$ & $\begin{array}{l}\star \star \\
\star *\end{array}$ & $\begin{array}{l}\star \star \\
\star \star\end{array}$ & $\begin{array}{l}\star \star \\
\star *\end{array}$ & $\begin{array}{l}\star \star \\
\star \star\end{array}$ & $\begin{array}{l}\star \star \\
\star \star\end{array}$ \\
\hline 451355123093600 & $05-17-94$ & $\star \star$ & $\star \star$ & $\star \star$ & ** & $\star \star$ & $\star *$ & ** & ** & $\star \star$ & $\star \star$ \\
\hline 451602122564400 & $05-31-94$ & $\star \star$ & $\star \star$ & $\star \star$ & $\star \star$ & $\star \star$ & $\star \star$ & $\star \star$ & ** & $\star \star$ & $\star \star$ \\
\hline 451502122524700 & $\begin{array}{l}05-26-94 \\
10-31-94\end{array}$ & $\begin{array}{l}\star \star \\
\star \star\end{array}$ & $\begin{array}{l}\star * \\
\star *\end{array}$ & $\begin{array}{l}\star \star \\
\star *\end{array}$ & $\begin{array}{l}\star \star \\
\star \star\end{array}$ & $\begin{array}{l}\star \star \\
\star \star\end{array}$ & $\begin{array}{l}\star \star \\
\star \star\end{array}$ & $\begin{array}{l}\star \star \\
\star \star\end{array}$ & $\begin{array}{l}\star \star \\
\star \star\end{array}$ & $\begin{array}{l}\star \star \\
\star *\end{array}$ & $\begin{array}{l}\star \star \\
\star \star\end{array}$ \\
\hline 14201300 & $\begin{array}{l}05-25-94 \\
06-13-94 \\
10-28-94 \\
11-28-94\end{array}$ & $\begin{array}{l}\star \star \\
\star \star \\
\star \star \\
\star \star\end{array}$ & $\begin{array}{l}\star \star \\
\star \star \\
\star \star \\
\star \star\end{array}$ & $\begin{array}{l}\star \star \\
\star \star \\
\star \star \\
\star \star\end{array}$ & $\begin{array}{l}\star \star \\
\star \star \\
\star \star \\
\star \star\end{array}$ & $\begin{array}{c}0.28000 \\
\star \star \\
\star \star \\
\star \star\end{array}$ & $\begin{array}{l}\star \star \\
\star \star \\
\star \star \\
\star \star\end{array}$ & $\begin{array}{l}\star \star \\
\star \star \\
\star \star \\
\star \star\end{array}$ & $\begin{array}{l}\star \star \\
\star \star \\
\star \star \\
\star \star\end{array}$ & $\begin{array}{l}\star \star \\
\star \star \\
\star \star \\
\star \star\end{array}$ & $\begin{array}{l}\star \star \\
\star \star \\
\star \star \\
\star \star\end{array}$ \\
\hline 14202000 & $\begin{array}{l}04-12-94 \\
05-25-94 \\
06-15-94 \\
10-28-94 \\
10-29-94 \\
11-09-94 \\
11-29-94\end{array}$ & $\begin{array}{l}\star * \\
\star * \\
\star * \\
\star * \\
\star * \\
\star * \\
\star * \\
\star *\end{array}$ & $\begin{array}{l}\star \star \\
\star * \\
\star * \\
\star * \\
\star * \\
\star * \\
\star * \\
\star \star\end{array}$ & $\begin{array}{l}\star \star \\
\star * \\
\star * \\
\star * \\
\star * \\
\star * \\
\star * \\
\star \star\end{array}$ & $\begin{array}{l}\star * \\
\star * \\
\star * \\
\star * \\
\star * \\
\star * \\
\star \star\end{array}$ & $\begin{array}{l}\star \star \\
\star \star \\
\star \star \\
\star \star \\
\star \star \\
\star \star \\
\star \star \\
\star \star\end{array}$ & $\begin{array}{l}\star \star \\
\star * \\
\star * \\
\star \star \\
\star \star \\
\star \star \\
\star * \\
\star \star\end{array}$ & $\begin{array}{l}\star * \\
\star * \\
\star * \\
\star * \\
\star * \\
\star * \\
\star *\end{array}$ & 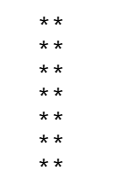 & $\begin{array}{l}* \star \\
\star * \\
\star * \\
\star * \\
\star * \\
\star * \\
\star *\end{array}$ & $\begin{array}{l}* \star \\
\star * \\
\star * \\
\star * \\
\star * \\
\star \star \\
\star \star \\
\star \star\end{array}$ \\
\hline 451603122423301 & $05-25-94$ & $\star \star$ & ** & ** & $\star \star$ & $\star \star$ & $\star \star$ & $\star \star$ & $\star \star$ & $\star \star$ & $\star \star$ \\
\hline 14206200 & $\begin{array}{l}05-27-94 \\
12-01-94\end{array}$ & $\begin{array}{l}\star \star \\
\star \star\end{array}$ & $\begin{array}{l}\star \star \\
\star *\end{array}$ & $\begin{array}{l}\star \star \\
\star \star\end{array}$ & $\begin{array}{l}\star \star \\
\star \star\end{array}$ & $\begin{array}{l}\star \star \\
\star \star\end{array}$ & $\begin{array}{l}\star * \\
\star *\end{array}$ & $\begin{array}{l}\star \star \\
\star \star\end{array}$ & $\begin{array}{l}\star \star \\
\star \star\end{array}$ & $\begin{array}{l}\star * \\
\star *\end{array}$ & $\begin{array}{l}\star \star \\
\star \star\end{array}$ \\
\hline
\end{tabular}


Table 38. Concentrations of pesticides in filtered water analyzed by high performance liquid chromatography from Phase II of the Willamette River Basin Water Quality Study, Oregon, 1994-Continued

\begin{tabular}{|c|c|c|c|c|c|c|c|c|c|c|c|}
\hline STATION NUMBER & DATE & $\begin{array}{c}\text { PRO- } \\
\text { POXUR, } \\
\text { WATER, } \\
\text { FLTRD, } \\
\text { GF 0.7U } \\
\text { REC } \\
\text { (UG/L) }\end{array}$ & $\begin{array}{c}\text { BENTA- } \\
\text { ZON, } \\
\text { WATER, } \\
\text { FLTRD, } \\
\text { GF 0.7U } \\
\text { REC } \\
\text { (UG/L) }\end{array}$ & $\begin{array}{c}2,4-D, \\
\text { DIS- } \\
\text { SOLVED } \\
(\mathrm{UG} / \mathrm{L})\end{array}$ & $\begin{array}{c}\text { 2, 4-DB } \\
\text { WATER, } \\
\text { FLTRD, } \\
\text { GF 0.7U } \\
\text { REC } \\
\text { (UG/L) }\end{array}$ & $\begin{array}{c}\text { DACTHAL } \\
\text { MONO- } \\
\text { ACID, } \\
\text { WAT,FLT } \\
\text { GF } 0.7 \mathrm{U} \\
\text { REC } \\
\text { (UG/L) }\end{array}$ & $\begin{array}{l}\text { DICHLOR } \\
\text { PROP, } \\
\text { WATER, } \\
\text { FLTRD, } \\
\text { GF 0.7U } \\
\text { REC } \\
\text { (UG/L) }\end{array}$ & $\begin{array}{c}\text { MCPA, } \\
\text { WATER, } \\
\text { FLTRD, } \\
\text { GF } 0.7 \mathrm{U} \\
\text { REC } \\
\text { (UG/L) }\end{array}$ & $\begin{array}{c}\text { MCPB, } \\
\text { WATER, } \\
\text { FLTRD, } \\
\text { GF 0.7U } \\
\text { REC } \\
\text { (UG/L) }\end{array}$ & $\begin{array}{c}\text { SILVEX, } \\
\text { DIS- } \\
\text { SOLVED } \\
\text { (UG/L) }\end{array}$ & $\begin{array}{c}2,4,5-T \\
\text { DIS- } \\
\text { SOLVED } \\
(U G / L)\end{array}$ \\
\hline 445547123065400 & $05-26-94$ & $\star \star$ & $\star \star$ & $\star \star$ & $\star \star$ & $\star \star$ & $\star \star$ & $\star \star$ & ** & $\star \star *$ & $\star \star$ \\
\hline 14190970 & $\begin{array}{l}11-23-94 \\
11-30-94 \\
11-30-94\end{array}$ & $\begin{array}{l}* * \\
\star * \\
\star *\end{array}$ & $\begin{array}{l}* * \\
\star * \\
* *\end{array}$ & $\begin{array}{l}* * \\
\star * \\
\star *\end{array}$ & $\begin{array}{l}* * \\
\star * \\
\star *\end{array}$ & $\begin{array}{l}* * \\
\star * \\
\star *\end{array}$ & $\begin{array}{l}\star * \\
\star \star \\
\star *\end{array}$ & $\begin{array}{l}\star * \\
\star \star \\
\star *\end{array}$ & $\begin{array}{l}\star * \\
\star * \\
\star *\end{array}$ & $\begin{array}{l}\star * \\
\star * \\
\star *\end{array}$ & $\begin{array}{l}\star \star \\
\star \star \\
\star \star\end{array}$ \\
\hline 445037122573800 & $\begin{array}{l}06-14-94 \\
10-31-94 \\
11-04-94 \\
11-04-94\end{array}$ & $\begin{array}{l}\star \star \\
\star \star \\
\star \star \\
\star \star\end{array}$ & $\begin{array}{l}\star \star \\
\star \star \\
\star \star \\
\star \star\end{array}$ & $\begin{array}{l}\star \star \\
\star \star \\
\star \star \\
\star \star\end{array}$ & $\begin{array}{l}\star \star \\
\star \star \\
\star \star \\
\star \star\end{array}$ & $\begin{array}{l}\star \star \\
\star \star \\
\star \star \\
\star \star\end{array}$ & $\begin{array}{l}\star \star \\
\star \star \\
\star \star \\
\star \star\end{array}$ & $\begin{array}{l}\star \star \\
\star \star \\
\star \star \\
\star \star\end{array}$ & $\begin{array}{l}\star \star \\
\star \star \\
\star \star \\
\star \star\end{array}$ & $\begin{array}{l}\star \star \\
\star \star \\
\star \star \\
\star \star\end{array}$ & $\begin{array}{l}\star \star \\
\star \star \\
\star \star \\
\star \star\end{array}$ \\
\hline 14194150 & $\begin{array}{l}05-17-94 \\
11-02-94\end{array}$ & $\begin{array}{l}* * \\
\star \star\end{array}$ & $\begin{array}{l}\star * \\
\star *\end{array}$ & $\begin{array}{l}* * \\
\star *\end{array}$ & $\begin{array}{l}* * \\
\star *\end{array}$ & $\begin{array}{l}\star \star \\
\star *\end{array}$ & $\begin{array}{l}\star * \\
\star *\end{array}$ & $\begin{array}{l}\star \star \\
\star *\end{array}$ & $\begin{array}{l}\star \star \\
\star *\end{array}$ & $\begin{array}{l}\star \star \\
\star *\end{array}$ & $\begin{array}{l}\star \star \\
\star *\end{array}$ \\
\hline 451355123093600 & $05-17-94$ & $\star \star$ & $\star \star$ & $\star \star$ & ** & $\star \star$ & $\star \star$ & ** & ** & ** & $\star \star$ \\
\hline 451602122564400 & $05-31-94$ & ** & ** & ** & $\star \star$ & $\star \star$ & $\star \star$ & $\star \star$ & $\star \star$ & ** & $\star \star$ \\
\hline 451502122524700 & $\begin{array}{l}05-26-94 \\
10-31-94\end{array}$ & $\begin{array}{l}\star \star \\
\star \star\end{array}$ & 0.08000 & $\begin{array}{l}* * \\
\star *\end{array}$ & $\begin{array}{l}\star \star \\
\star \star\end{array}$ & $\begin{array}{l}\star * \\
\star *\end{array}$ & $\begin{array}{l}\star * \\
\star *\end{array}$ & $\begin{array}{l}* * \\
\star *\end{array}$ & $\begin{array}{l}\star \star \\
\star \star\end{array}$ & $\begin{array}{l}\star * \\
\star *\end{array}$ & $\begin{array}{l}\star * \\
\star \star\end{array}$ \\
\hline 14201300 & $\begin{array}{l}05-25-94 \\
06-13-94 \\
10-28-94 \\
11-28-94\end{array}$ & $\begin{array}{l}\star \star \\
\star \star \\
\star \star \\
\star \star\end{array}$ & $\begin{array}{c}0.26000 \\
0.41000 \\
\star \star \\
\star \star\end{array}$ & $\begin{array}{l}* \star \\
0.05000 \\
0.27000 \\
* \star\end{array}$ & $\begin{array}{l}\star \star \\
\star \star \\
\star \star \\
\star \star\end{array}$ & $\begin{array}{l}\star \star \\
\star \star \\
\star \star \\
\star \star\end{array}$ & $\begin{array}{l}\star \star \\
\star \star \\
\star \star \\
\star \star\end{array}$ & $\begin{array}{l}\star \star \\
{ }^{\star \star} \\
. \\
* \star \\
\star \star\end{array}$ & $\begin{array}{l}\star \star \\
\star \star \\
\star \star \\
\star \star\end{array}$ & $\begin{array}{l}\star \star \\
\star \star \\
\star \star \\
\star \star\end{array}$ & $\begin{array}{l}\star \star \\
\star \star \\
\star \star \\
\star \star\end{array}$ \\
\hline 14202000 & $\begin{array}{l}04-12-94 \\
05-25-94 \\
06-15-94 \\
10-28-94 \\
10-29-94 \\
11-09-94 \\
11-29-94\end{array}$ & $\begin{array}{l}\star \star \\
\star \star \\
\star \star \\
\star \star \\
\star \star \\
\star \star \\
\star \star \\
\star \star \\
\star\end{array}$ & $\begin{array}{l}\star \star \\
\star \star \\
\star \star \\
\star \star \\
\star \star \\
\star \star \\
\star \star \\
\star \star\end{array}$ & $\begin{array}{l}\star \star \\
\star \star \\
\star \star \\
\star \star \\
\star \star \\
\star \star \\
\star \star \\
\star \star \\
\star \star\end{array}$ & $\begin{array}{l}\star \star \\
\star \star \\
\star \star \\
\star \star \\
\star \star \\
\star \star \\
\star \star \\
\star \star\end{array}$ & $\begin{array}{l}\star \star \\
\star \star \\
\star \star \\
\star \star \\
\star \star \\
\star \star \\
\star \star \\
\star \star\end{array}$ & $\begin{array}{l}\star \star \\
\star \star \\
\star \star \\
\star \star \\
\star \star \\
\star \star \\
\star \star \\
\star \star\end{array}$ & $\begin{array}{l}\star \star \\
\star \star \\
\star \star \\
\star \star \\
\star \star \\
\star \star \\
\star \star \\
\star \star \\
\star \star\end{array}$ & $\begin{array}{l}* \star \\
\star * \\
\star \star \\
\star * \\
\star * \\
\star * \\
\star * \\
\star *\end{array}$ & $\begin{array}{l}* * \\
\star * \\
\star * \\
\star * \\
* * \\
\star * \\
\star * \\
\star *\end{array}$ & $\begin{array}{l}* * \\
\star * \\
\star * \\
\star * \\
\star * \\
\star * \\
\star * \\
\star *\end{array}$ \\
\hline 451603122423301 & $05-25-94$ & $\star \star$ & ** & ** & $\star \star$ & $\star \star$ & $\star \star$ & $\star \star$ & ** & $\star \star$ & $\star *$ \\
\hline 14206200 & $\begin{array}{l}05-27-94 \\
12-01-94\end{array}$ & $\begin{array}{l}-- \\
\star \star\end{array}$ & $\begin{array}{l}\star * \\
\star *\end{array}$ & $\begin{array}{l}\star \star \\
\star \star\end{array}$ & $\begin{array}{l}\star \star \\
\star \star\end{array}$ & $\begin{array}{l}\star \star \\
\star \star\end{array}$ & $\begin{array}{l}\star \star \\
\star \star\end{array}$ & $\begin{array}{l}\star \star \\
\star \star\end{array}$ & $\begin{array}{l}\star \star \\
\star \star\end{array}$ & $\begin{array}{l}* * \\
\star *\end{array}$ & $\begin{array}{l}\star \star \\
\star \star\end{array}$ \\
\hline
\end{tabular}


Table 38. Concentrations of pesticides in filtered water analyzed by high performance liquid chromatography from Phase II of the Willamette River Basin Water Quality Study, Oregon, 1994-Continued

\begin{tabular}{|c|c|c|c|c|c|c|c|c|c|c|c|}
\hline STATION NUMBER & DATE & $\begin{array}{c}\text { ORY- } \\
\text { ZALIN, } \\
\text { WATER, } \\
\text { FLTRD, } \\
\text { GF } 0.7 \mathrm{U} \\
\text { REC } \\
\text { (UG/L) }\end{array}$ & $\begin{array}{c}\text { DINOSEB } \\
\text { WATER, } \\
\text { FLTRD, } \\
\text { GF 0.7U } \\
\text { REC } \\
\text { (UG/L) }\end{array}$ & $\begin{array}{c}\text { OCRESOL } \\
4,6- \\
\text { DINITRO } \\
\text { WAT, FLT } \\
\text { GF } 0.7 U \\
\text { REC } \\
\text { (UG/L) }\end{array}$ & $\begin{array}{c}\text { ACIFL- } \\
\text { UORFEN } \\
\text { WATER, } \\
\text { FLTRD, } \\
\text { GF 0.7U } \\
\text { REC } \\
\text { (UG/L) }\end{array}$ & $\begin{array}{c}\text { 1-NAPH } \\
\text { THOL, } \\
\text { WATER, } \\
\text { FLTRD, } \\
\text { GF 0.7U } \\
\text { REC } \\
\text { (UG/L) }\end{array}$ & $\begin{array}{c}\text { AMIBEN, } \\
\text { WATER, } \\
\text { FLTRD, } \\
\text { GF 0.7U } \\
\text { REC } \\
(\mathrm{UG} / \mathrm{L})\end{array}$ & $\begin{array}{c}\text { DIURON, } \\
\text { WATER, } \\
\text { FLTRD, } \\
\text { GF 0.7U } \\
\text { REC } \\
(\mathrm{UG} / \mathrm{L})\end{array}$ & $\begin{array}{c}\text { FEN- } \\
\text { URON, } \\
\text { WATER, } \\
\text { FLTRD, } \\
\text { GF } 0.7 \mathrm{U} \\
\text { REC } \\
\text { (UG/L) }\end{array}$ & $\begin{array}{c}\text { FLUO- } \\
\text { METURON } \\
\text { WATER, } \\
\text { FLTRD, } \\
\text { GF } 0.7 U \\
\text { REC } \\
\text { (UG/L) }\end{array}$ & $\begin{array}{c}\text { LINURON } \\
\text { WATER, } \\
\text { FLTRD, } \\
\text { GF } 0.7 U \\
\text { REC } \\
(\mathrm{UG} / \mathrm{L})\end{array}$ \\
\hline 445547123065400 & $05-26-94$ & $\star \star$ & $\star \star$ & ** & $\star \star$ & $\star \star$ & ** & $\star \star$ & ** & $\star \star$ & $\star \star$ \\
\hline 14190970 & $\begin{array}{l}11-23-94 \\
11-30-94 \\
11-30-94\end{array}$ & $\begin{array}{l}\star \star \\
\star \star \\
\star \star\end{array}$ & $\begin{array}{l}\star \star \\
\star * \\
\star *\end{array}$ & $\begin{array}{l}\star \star \\
\star \star \\
\star \star\end{array}$ & $\begin{array}{l}\star \star \\
\star \star \\
\star \star\end{array}$ & $\begin{array}{l}\star \star \\
\star \star \\
\star \star\end{array}$ & $\begin{array}{l}\star \star \\
\star \star \\
\star \star\end{array}$ & $\begin{array}{l}\star \star \\
\star \star \\
\star \star\end{array}$ & $\begin{array}{l}\star * \\
\star * \\
\star *\end{array}$ & $\begin{array}{l}\star \star \\
\star * \\
\star \star\end{array}$ & $\begin{array}{l}\star \star \\
\star * \\
\star \star\end{array}$ \\
\hline 445037122573800 & $\begin{array}{l}06-14-94 \\
10-31-94 \\
11-04-94 \\
11-04-94\end{array}$ & $\begin{array}{l}\star \star \\
\star \star \\
\star \star \\
\star \star\end{array}$ & $\begin{array}{l}\star \star \\
\star \star \\
\star \star \\
\star \star\end{array}$ & $\begin{array}{c}* \star \\
\mathrm{E} 0.05000 \\
* \star \\
\star \star\end{array}$ & $\begin{array}{l}\star \star \\
\star \star \\
\star \star \\
\star \star\end{array}$ & $\begin{array}{l}\star \star \\
\star \star \\
\star \star \\
\star \star\end{array}$ & $\begin{array}{l}\star \star \\
\star \star \\
\star \star \\
\star \star\end{array}$ & $\begin{array}{c}\star \star * \\
0.17000 \\
0.45000 \\
0.96000\end{array}$ & $\begin{array}{l}\star \star \\
\star \star \\
\star \star \\
\star \star\end{array}$ & $\begin{array}{l}\star \star \\
\star \star \\
\star \star \\
\star \star\end{array}$ & $\begin{array}{l}\star \star \\
\star \star \\
\star \star \\
\star \star\end{array}$ \\
\hline 14194150 & $\begin{array}{l}05-17-94 \\
11-02-94\end{array}$ & $\begin{array}{l}\star \star \\
\star \star\end{array}$ & $\begin{array}{l}\star * \\
\star *\end{array}$ & $\begin{array}{l}\star \star \\
\star *\end{array}$ & $\begin{array}{l}\star \star \\
\star *\end{array}$ & $\begin{array}{l}\star \star \\
\star *\end{array}$ & $\begin{array}{l}\star \star \\
\star *\end{array}$ & $\begin{array}{c}\star \star \\
0.05000\end{array}$ & $\begin{array}{l}\star \star \\
\star \star\end{array}$ & $\begin{array}{l}\star \star \\
\star \star\end{array}$ & $\begin{array}{l}\star \star \\
\star \star\end{array}$ \\
\hline 451355123093600 & $05-17-94$ & $\star \star$ & $\star \star$ & $\star \star$ & ** & $\star \star$ & $\star *$ & ** & ** & ** & $\star \star$ \\
\hline 451602122564400 & $05-31-94$ & $\star \star$ & $\star \star$ & $\star \star$ & $\star \star$ & $\star \star$ & $\star \star$ & $\star \star$ & ** & $\star \star$ & $\star \star$ \\
\hline 451502122524700 & $\begin{array}{l}05-26-94 \\
10-31-94\end{array}$ & $\begin{array}{l}\star \star \\
\star \star\end{array}$ & $\begin{array}{c}* * \\
0.06000\end{array}$ & $\begin{array}{l}\star \star \\
\star *\end{array}$ & $\begin{array}{l}\star \star \\
\star \star\end{array}$ & $\begin{array}{l}\star \star \\
\star \star\end{array}$ & $\begin{array}{l}\star \star \\
\star \star\end{array}$ & $\begin{array}{l}0.52000 \\
0.58000\end{array}$ & $\begin{array}{l}\star \star \\
\star \star\end{array}$ & $\begin{array}{l}* \star \\
\star *\end{array}$ & $\begin{array}{l}\star \star \\
\star \star\end{array}$ \\
\hline 14201300 & $\begin{array}{l}05-25-94 \\
06-13-94 \\
10-28-94 \\
11-28-94\end{array}$ & $\begin{array}{l}\star \star \\
\star \star \\
\star \star \\
\star \star\end{array}$ & $\begin{array}{c}\star \star \\
\star \star \\
0.23000 \\
0.09000\end{array}$ & $\begin{array}{l}\star \star \\
\star \star \\
\star \star \\
\star \star\end{array}$ & $\begin{array}{l}\star \star \\
\star \star \\
\star \star \\
\star \star\end{array}$ & $\begin{array}{l}\star \star \\
\star \star \\
\star \star \\
\star \star\end{array}$ & $\begin{array}{l}* \star \\
\star * \\
\star \star \\
\star \star\end{array}$ & $\begin{array}{l}\text { E3. } 9000 \\
\text { E3.4000 } \\
\text { E5.8000 } \\
\text { E1.5000 }\end{array}$ & $\begin{array}{l}\star \star \\
\star * \\
\star * \\
\star *\end{array}$ & $\begin{array}{l}\star \star \\
\star \star \\
\star \star \\
\star \star\end{array}$ & $\begin{array}{l}\star \star \\
\star \star \\
\star \star \\
\star \star\end{array}$ \\
\hline 14202000 & $\begin{array}{l}04-12-94 \\
05-25-94 \\
06-15-94 \\
10-28-94 \\
10-29-94 \\
11-09-94 \\
11-29-94\end{array}$ & $\begin{array}{l}\star * \\
\star * \\
\star * \\
\star * \\
\star * \\
\star * \\
\star * \\
\star *\end{array}$ & $\begin{array}{l}\star \star \\
\star * \\
\star * \\
\star * \\
\star \star \\
\star \star \\
\star * \\
\star \star\end{array}$ & $\begin{array}{l}\star \star \\
\star * \\
\star * \\
\star * \\
\star \star \\
\star \star \\
\star * \\
\star \star\end{array}$ & $\begin{array}{l}\star * \\
\star * \\
\star * \\
\star * \\
\star * \\
\star * \\
\star \star\end{array}$ & $\begin{array}{l}\star * \\
\star * \\
\star * \\
\star * \\
\star * \\
\star * \\
\star *\end{array}$ & $\begin{array}{l}\star \star \\
\star * \\
\star * \\
\star * \\
\star \star \\
\star \star \\
\star * \\
\star \star\end{array}$ & $\begin{array}{l}0.06000 \\
0.08000 \\
0.06000 \\
0.87000 \\
0.43000 \\
\star \star \\
0.19000\end{array}$ & $\begin{array}{l}* * \\
\star * \\
\star * \\
\star * \\
\star * \\
\star * \\
* *\end{array}$ & $\begin{array}{l}* \star \\
\star * \\
\star * \\
\star * \\
\star * \\
\star * \\
\star * \\
\star *\end{array}$ & $\begin{array}{l}* \star \\
\star * \\
\star * \\
\star * \\
\star * \\
\star \star \\
\star \star \\
\star \star\end{array}$ \\
\hline 451603122423301 & $05-25-94$ & $\star \star$ & ** & ** & $\star \star$ & $\star \star$ & $\star \star$ & $\star \star$ & $\star \star$ & $\star \star$ & $\star \star$ \\
\hline 14206200 & $\begin{array}{l}05-27-94 \\
12-01-94\end{array}$ & $\begin{array}{l}\star \star \\
\star \star\end{array}$ & $\begin{array}{l}\star \star \\
\star \star\end{array}$ & $\begin{array}{l}\star \star \\
\star \star\end{array}$ & $\begin{array}{l}\star \star \\
\star \star\end{array}$ & $\begin{array}{l}\star \star \\
\star \star\end{array}$ & $\begin{array}{l}\star \star \\
\star \star\end{array}$ & $\begin{array}{l}0.17000 \\
0.94000\end{array}$ & $\begin{array}{l}\star * \\
\star \star\end{array}$ & $\begin{array}{l}\star * \\
\star *\end{array}$ & $\begin{array}{l}\star \star \\
\star \star\end{array}$ \\
\hline
\end{tabular}


Table 38. Concentrations of pesticides in filtered water analyzed by high performance liquid chromatography from Phase II of the Willamette River Basin Water Quality Study, Oregon, 1994-Continued

\begin{tabular}{|c|c|c|c|c|c|c|c|c|c|c|}
\hline STATION NUMBER & DATE & $\begin{array}{c}\text { NEB- } \\
\text { URON, } \\
\text { WATER, } \\
\text { FLTRD, } \\
\text { GF } 0.7 \mathrm{U} \\
\text { REC } \\
\text { (UG/L) }\end{array}$ & $\begin{array}{c}\text { CHLORO- } \\
\text { THALO- } \\
\text { NIL, } \\
\text { WAT, FLT } \\
\text { GF } 0.7 \mathrm{U} \\
\text { REC } \\
\text { (UG/L) }\end{array}$ & $\begin{array}{c}\text { ESFEN- } \\
\text { VAL- } \\
\text { ERATE, } \\
\text { WAT, FLT } \\
\text { GF } 0.7 \mathrm{U} \\
\text { REC } \\
\text { (UG/L) }\end{array}$ & $\begin{array}{c}\text { NORFLUR } \\
\text { AZON, } \\
\text { WATER, } \\
\text { FLTRD, } \\
\text { GF } 0.7 \mathrm{U} \\
\text { REC } \\
\text { (UG/L) }\end{array}$ & $\begin{array}{c}\text { CLOPYR- } \\
\text { ALID, } \\
\text { WATER, } \\
\text { FLTRD, } \\
\text { GF } 0.7 \mathrm{U} \\
\text { REC } \\
\text { (UG/L) }\end{array}$ & $\begin{array}{c}\text { PIC- } \\
\text { LORAM, } \\
\text { WATER, } \\
\text { FLTRD, } \\
\text { GF } 0.7 U \\
\text { REC } \\
\text { (UG/L) }\end{array}$ & $\begin{array}{c}\text { TRI- } \\
\text { CLOPYR, } \\
\text { WATER, } \\
\text { FLTRD, } \\
\text { GF } 0.7 \mathrm{U} \\
\text { REC } \\
(\mathrm{UG} / \mathrm{L})\end{array}$ & $\begin{array}{l}\text { BRO- } \\
\text { MACIL, } \\
\text { WATER, } \\
\text { DISS, } \\
\text { REC } \\
\text { (UG/L) }\end{array}$ & $\begin{array}{c}\text { BDMC, } \\
\text { SURROG, } \\
\text { WATER, } \\
\text { UNFLTRD } \\
\text { REC } \\
\text { PERCENT }\end{array}$ \\
\hline 445547123065400 & $05-26-94$ & ** & $\star \star$ & $\star \star$ & $\star \star$ & $\star \star$ & $\star \star$ & $\star \star$ & $\star \star$ & 40.000 \\
\hline 14190970 & $\begin{array}{l}11-23-94 \\
11-30-94 \\
11-30-94\end{array}$ & $\begin{array}{l}* \star \\
\star * \\
\star *\end{array}$ & $\begin{array}{l}\star \star \\
\star \star \\
\star \star\end{array}$ & $\begin{array}{l}\star \star \\
\star \star \\
\star \star\end{array}$ & $\begin{array}{l}\star \star \\
\star \star \\
\star \star\end{array}$ & $\begin{array}{l}\star \star \\
\star * \\
\star \star\end{array}$ & $\begin{array}{l}\star \star \\
\star \star \\
\star \star\end{array}$ & $\begin{array}{c}0.11000 \\
\star \star \\
\star \star\end{array}$ & $\begin{array}{l}\star \star \\
\star \star \\
\star \star\end{array}$ & $\begin{array}{c}79.000 \\
-- \\
56.000\end{array}$ \\
\hline 445037122573800 & $\begin{array}{l}06-14-94 \\
10-31-94 \\
11-04-94 \\
11-04-94\end{array}$ & $\begin{array}{l}\star \star \\
\star * \\
\star \star \\
\star \star\end{array}$ & $\begin{array}{l}\star \star \\
\star \star \\
\star \star \\
\star \star\end{array}$ & $\begin{array}{l}\star \star \\
\star \star \\
\star \star \\
\star \star\end{array}$ & $\begin{array}{l}\star \star \\
\star * \\
\star * \\
\star \star\end{array}$ & $\begin{array}{l}\star \star \\
\star \star \\
\star \star \\
\star \star\end{array}$ & $\begin{array}{l}\star \star \\
\star \star \\
\star \star \\
\star \star\end{array}$ & $\begin{array}{l}\star \star \star \\
0.14000 \\
\star \star \\
\star \star\end{array}$ & $\begin{array}{l}\star \star \\
\star \star \\
\star \star \\
\star \star\end{array}$ & $\begin{array}{l}35.000 \\
56.000 \\
75.000 \\
85.000\end{array}$ \\
\hline 14194150 & $\begin{array}{l}05-17-94 \\
11-02-94\end{array}$ & $\begin{array}{l}\star * \\
\star *\end{array}$ & $\begin{array}{l}* * \\
* *\end{array}$ & $\begin{array}{l}* * \\
\star *\end{array}$ & $\begin{array}{l}* * \\
* *\end{array}$ & $\begin{array}{l}* * \\
\star *\end{array}$ & ** & $\begin{array}{l}* * \\
* *\end{array}$ & $\begin{array}{l}\star \star \\
\star \star\end{array}$ & $\begin{array}{r}\mathrm{E} 83.000 \\
51.000\end{array}$ \\
\hline 451355123093600 & $05-17-94$ & $\star \star$ & $\star \star$ & $\star \star$ & ** & $\star \star$ & ** & $\star \star$ & $\star \star$ & 113.00 \\
\hline 451602122564400 & $05-31-94$ & $\star \star$ & $\star \star$ & $\star \star$ & ** & $\star \star$ & ** & $\star \star$ & $\star \star$ & 16.000 \\
\hline 451502122524700 & $\begin{array}{l}05-26-94 \\
10-31-94\end{array}$ & ** & $\begin{array}{l}\star \star \\
\star *\end{array}$ & ** & ** & $\begin{array}{l}* * \\
* *\end{array}$ & ** & $\begin{array}{l}\star * \\
\star *\end{array}$ & $\begin{array}{l}* \star \\
* *\end{array}$ & $\begin{array}{l}6.0000 \\
47.000\end{array}$ \\
\hline 14201300 & $\begin{array}{l}05-25-94 \\
06-13-94 \\
10-28-94 \\
11-28-94\end{array}$ & $\begin{array}{l}* \star \\
\star \star \\
\star * \\
\star \star\end{array}$ & $\begin{array}{l}\star \star \\
\star \star \\
\star \star \\
\star \star\end{array}$ & $\begin{array}{l}\star \star \\
\star \star \\
\star \star \\
\star \star\end{array}$ & $\begin{array}{l}\star \star \\
\star \star \\
\star \star \\
\star \star\end{array}$ & $\begin{array}{l}\star \star \\
\star \star \\
\star \star \\
\star \star\end{array}$ & $\begin{array}{l}\star \star \\
\star * \\
\star * \\
\star *\end{array}$ & $\begin{array}{l}\star \star \\
\star \star \\
\star \star \\
\star \star\end{array}$ & $\begin{array}{l}\star \star \\
\star \star \\
\star \star \\
\star \star\end{array}$ & $\begin{array}{l}17.000 \\
30.000 \\
54.000 \\
81.000\end{array}$ \\
\hline 14202000 & $\begin{array}{l}04-12-94 \\
05-25-94 \\
06-15-94 \\
10-28-94 \\
10-29-94 \\
11-09-94 \\
11-29-94\end{array}$ & $\begin{array}{l}\star \star \\
\star \star \\
\star \star \\
\star \star \\
\star \star \\
\star \star \\
\star \star\end{array}$ & $\begin{array}{l}\star \star \\
\star \star \\
\star \star \\
\star \star \\
\star \star \\
\star \star \\
\star \star\end{array}$ & $\begin{array}{l}\star \star \\
\star \star \\
\star \star \\
\star \star \\
\star \star \\
\star \star \\
\star \star \\
\star *\end{array}$ & $\begin{array}{l}\star \star \\
\star \star \\
\star * \\
\star \star \\
\star \star \\
\star \star \\
\star \star\end{array}$ & $\begin{array}{l}\star \star \\
\star \star \\
\star * \\
\star \star \\
\star \star \\
\star \star \\
\star \star\end{array}$ & $\begin{array}{l}\star \star \\
\star \star \\
\star \star \\
\star \star \\
\star \star \\
\star \star \\
\star \star\end{array}$ & $\begin{array}{l}\star * \\
\star * \\
\star \star \\
\star \star \\
\star \star \\
\star \star \\
\star \star\end{array}$ & $\begin{array}{l}\star \star \\
\star \star \\
\star \star \\
\star \star \\
\star \star \\
\star \star \\
\star \star \\
\star \star\end{array}$ & $\begin{array}{l}28.000 \\
42.000 \\
33.000 \\
73.000 \\
77.000 \\
70.000 \\
75.000\end{array}$ \\
\hline 451603122423301 & $05-25-94$ & ** & $\star \star$ & $\star \star$ & ** & $\star \star$ & $\star \star$ & $\star \star$ & $\star \star$ & 90.000 \\
\hline 14206200 & $\begin{array}{l}05-27-94 \\
12-01-94\end{array}$ & $\begin{array}{l}\star * \\
\star \star\end{array}$ & $\begin{array}{l}\star * \\
\star \star\end{array}$ & $\begin{array}{l}\star * \\
\star *\end{array}$ & $\begin{array}{l}* * \\
\star *\end{array}$ & $\begin{array}{l}\star * \\
\star *\end{array}$ & $\begin{array}{l}\star \star \\
\star \star\end{array}$ & $\begin{array}{c}* \star \\
0.14000\end{array}$ & $\begin{array}{l}\star \star \\
\star \star\end{array}$ & $\begin{array}{l}120.00 \\
74.000\end{array}$ \\
\hline
\end{tabular}


Table 38. Concentrations of pesticides in filtered water analyzed by high performance liquid chromatography from Phase II of the Willamette River Basin Water Quality Study, Oregon, 1994-Continued

\begin{tabular}{|c|c|c|c|c|c|}
\hline STATION NUMBER & STATION NAME & TIME & $\begin{array}{r}\text { DICAMBA } \\
\text { WATER, } \\
\text { FLTRD, } \\
\text { GF } 0.7 \mathrm{U} \\
\text { REC } \\
(\mathrm{UG} / \mathrm{L})\end{array}$ & $\begin{array}{l}\text { BRO- } \\
\text { MOXYNIL } \\
\text { WATER, } \\
\text { FLTRD, } \\
\text { GF } 0.7 \mathrm{U} \\
\text { REC } \\
\text { (UG/L) }\end{array}$ & $\begin{array}{l}\text { DICHLO- } \\
\text { BENIL, } \\
\text { WATER, } \\
\text { FLTRD, } \\
\text { GF O.7U } \\
\text { REC } \\
\text { (UG/L) }\end{array}$ \\
\hline 14206298 & $\begin{array}{l}\text { BRONSON CREEK AT 185TH AVE NEAR ALOHA, OR } \\
\text { BRONSON CREEK AT } 185 \mathrm{TH} \text { AVE NEAR ALOHA, OR }\end{array}$ & $\begin{array}{l}1100 \\
1030\end{array}$ & ** & $\star \star *$ & 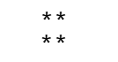 \\
\hline 14206950 & $\begin{array}{llll}\text { FANNO CREEK AT DURHAM, OR } \\
\text { FANNO CREEK AT DURHAM, OR } \\
\text { FANNO CREEK AT DURHAM, OR } \\
\text { FANNO CREEK AT DURHAM, OR }\end{array}$ & $\begin{array}{l}1420 \\
1040 \\
1620 \\
1010\end{array}$ & $\begin{array}{l}\star \star \\
\star \star \\
\star \star \\
\star \star\end{array}$ & $\begin{array}{l}\star \star \\
\star \star \\
\star \star \\
\star \star\end{array}$ & $\begin{array}{l}\star \star \\
\star \star \\
\star \star \\
\star \star\end{array}$ \\
\hline 14207500 & $\begin{array}{l}\text { TUALATIN RIVER AT WEST LINN, OREG. } \\
\text { TUALATIN RIVER AT WEST LINN, OREG. }\end{array}$ & $\begin{array}{l}1540 \\
1610\end{array}$ & 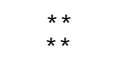 & $\star \star \star ⿱ 乛 ⿻ 上 丨$ & 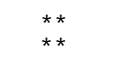 \\
\hline 452823122240900 & $\begin{array}{l}\text { JOHNSON CREEK AT PALMBLAD RD NR GRESHAM, OR } \\
\text { JOHNSON CREEK AT PALMBLAD RD NR GRESHAM, OR } \\
\text { JOHNSON CREEK AT PALMBLAD RD NR GRESHAM, OR } \\
\text { JOHNSON CREEK AT PALMBLAD RD NR GRESHAM, OR } \\
\text { JOHNSON CREEK AT PALMBLAD RD NR GRESHAM, OR } \\
\text { JOHNSON CREEK AT PALMBLAD RD NR GRESHAM, OR }\end{array}$ & $\begin{array}{l}1440 \\
1640 \\
1135 \\
1100 \\
1330 \\
1410\end{array}$ & $\begin{array}{l}\star \star \\
\star \star \\
\star \star \\
\star \star \\
\star \star \\
\star \star \\
\star \star\end{array}$ & $\begin{array}{l}\star \star \\
\star \star \\
\star \star \\
\star \star \\
\star \star \\
\star \star \\
\star \star\end{array}$ & $\begin{array}{l}0.42000 \\
\star \star \\
\star \star \\
\star \star \\
\star \star \\
\star \star\end{array}$ \\
\hline 453043122402200 & COMM/RESIDENTIAL RUNOFF AT HARBOR WAY AT PORT, OR & 2150 & $\star \star$ & $\star \star$ & $\star \star$ \\
\hline 14211720 & $\begin{array}{l}\text { WILLAMETTE RIVER AT PORTLAND, OREG. } \\
\text { WILLAMETTE RIVER AT PORTLAND, OREG. } \\
\text { WILLAMETTE RIVER AT PORTLAND, OREG. } \\
\text { WILLAMETTE RIVER AT PORTLAND, OREG. }\end{array}$ & $\begin{array}{l}0949 \\
1300 \\
1230 \\
0956\end{array}$ & $\begin{array}{l}\star \star \\
\star \star \\
\star \star \\
\star \star\end{array}$ & $\begin{array}{l}\star \star \\
\star \star \\
\star \star \\
\star \star\end{array}$ & $\begin{array}{l}\star \star \\
\star \star \\
\star \star \\
\star \star\end{array}$ \\
\hline 453154122394200 & $\begin{array}{l}\text { TRANSPORTATION CORRIDOR AT SE 3RD @ PORTLAND, OR } \\
\text { TRANSPORTATION CORRIDOR AT SE 3RD @ PORTLAND, OR } \\
\text { TRANSPORTATION CORRIDOR AT SE 3RD @ PORTLAND, OR } \\
\text { TRANSPORTATION CORRIDOR AT SE 3RD @ PORTLAND, OR } \\
\text { TRANSPORTATION CORRIDOR AT SE 3RD @ PORTLAND, OR } \\
\text { TRANSPORTATION CORRIDOR AT SE 3RD @ PORTLAND, OR }\end{array}$ & $\begin{array}{l}1800 \\
1800 \\
1950 \\
0840 \\
1020 \\
1020\end{array}$ & $\begin{array}{l}\star \star \\
\star \star \\
\star \star \\
\star \star \\
\star \star \\
\star \star \\
\star \star\end{array}$ & $\begin{array}{l}\star \star \\
\star \star \\
\star \star \\
\star \star \\
\star \star \\
\star \star \\
\star \star\end{array}$ & $\begin{array}{l}\star \star \\
\star \star \\
\star \star \\
\star \star \\
\star \star \\
\star \star\end{array}$ \\
\hline 14211805 & WILLAMETTE R AB ST JOHNS BR AT PORTLAND, OREG. & 0950 & $\star \star$ & $\star \star$ & ** \\
\hline
\end{tabular}


Table 38. Concentrations of pesticides in filtered water analyzed by high performance liquid chromatography from Phase II of the Willamette River Basin Water Quality Study, Oregon, 1994-Continued

\begin{tabular}{|c|c|c|c|c|c|c|c|c|c|c|c|}
\hline STATION NUMBER & DATE & $\begin{array}{c}\text { ALDI- } \\
\text { CARB } \\
\text { SULFONE } \\
\text { WAT, FLT } \\
\text { GF } 0.7 \mathrm{U} \\
\text { REC } \\
\text { (UG/L) }\end{array}$ & $\begin{array}{c}\text { ALDI- } \\
\text { CARB, } \\
\text { WATER, } \\
\text { FLTRD, } \\
\text { GF } 0.7 \mathrm{U} \\
\text { REC } \\
(\mathrm{UG} / \mathrm{L})\end{array}$ & $\begin{array}{c}\text { ALDICA- } \\
\text { RB SUL- } \\
\text { FOXIDE, } \\
\text { WAT, FLT } \\
\text { GF } 0.7 \mathrm{U} \\
\text { REC } \\
(\mathrm{UG} / \mathrm{L})\end{array}$ & $\begin{array}{c}\text { CAR- } \\
\text { BARYL, } \\
\text { WATER, } \\
\text { FLTRD, } \\
\text { GF } 0.7 \mathrm{U} \\
\text { REC } \\
\text { (UG/L) }\end{array}$ & $\begin{array}{c}\text { CARBO- } \\
\text { FURAN, } \\
\text { WATER, } \\
\text { FLTRD, } \\
\text { GF } 0.7 \mathrm{U} \\
\text { REC } \\
\text { (UG/L) }\end{array}$ & $\begin{array}{c}\text { 3HYDRXY } \\
\text { CARBO- } \\
\text { FURAN } \\
\text { WAT,FLT } \\
\text { GF } 0.7 \mathrm{U} \\
\text { REC } \\
(\mathrm{UG} / \mathrm{L})\end{array}$ & $\begin{array}{c}\text { METHIO- } \\
\text { CARB, } \\
\text { WATER, } \\
\text { FLTRD, } \\
\text { GF } 0.7 \mathrm{U} \\
\text { REC } \\
\text { (UG/L) }\end{array}$ & $\begin{array}{c}\text { METH- } \\
\text { OMYL, } \\
\text { WATER, } \\
\text { FLTRD, } \\
\text { GF 0.7U } \\
\text { REC } \\
\text { (UG/L) }\end{array}$ & $\begin{array}{c}\text { OXAMYL, } \\
\text { WATER, } \\
\text { FLTRD, } \\
\text { GF } 0.7 \mathrm{U} \\
\text { REC } \\
\text { (UG/L) }\end{array}$ & $\begin{array}{c}\text { PRO- } \\
\text { PHAM, } \\
\text { WATER, } \\
\text { FLTRD, } \\
\text { GF } 0.7 \mathrm{U} \\
\text { REC } \\
(\mathrm{UG} / \mathrm{L})\end{array}$ \\
\hline \multirow[t]{2}{*}{14206298} & $05-27-94$ & $\star \star$ & ** & ** & ** & ** & ** & ** & $\star \star$ & ** & ** \\
\hline & $11-23-94$ & ** & $\star \star$ & $\star \star$ & ** & $\star \star$ & ** & ** & $\star \star$ & $\star \star$ & $\star \star$ \\
\hline \multirow[t]{4}{*}{14206950} & $05-24-94$ & $\star \star$ & $\star \star$ & ** & $\star \star *$ & $\star \star$ & $\star \star \star$ & $\star \star$ & ** & $\star \star$ & ** \\
\hline & $06-16-94$ & ** & ** & ** & $\star \star$ & ** & ** & $\star \star$ & $\star \star *$ & ** & $\star \star *$ \\
\hline & $10-27-94$ & ** & 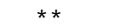 & $\star \star$ & 0.25000 & $\star \star *$ & $\star \star$ & $\star \star$ & $\star \star$ & ** & $\star \star$ \\
\hline & $11-30-94$ & ** & $\star \star$ & $\star \star$ & $\star \star$ & $\star \star$ & ** & $\star \star$ & ** & $\star \star$ & $\star \star$ \\
\hline \multirow{2}{*}{14207500} & $05-25-94$ & ** & $\star *$ & $\star \star *$ & $\star \star$ & $\star \star$ & $\star \star \star$ & $\star \star *$ & ** & $\star \star$ & $\star \star$ \\
\hline & $10-28-94$ & $\star \star$ & $\star \star$ & ** & 0.07000 & ** & ** & $\star \star$ & ** & $\star \star$ & $\star \star$ \\
\hline \multirow[t]{6}{*}{452823122240900} & $05-24-94$ & $\star \star$ & ** & $\star \star \star$ & ** & $\star \star *$ & $\star \star *$ & $\star \star *$ & ** & ** & $\star *$ \\
\hline & $10-27-94$ & ** & ** & ** & ** & ** & $\star *$ & ** & ** & ** & ** \\
\hline & $10-28-94$ & $\star \star$ & ** & $\star \star \star$ & $\star \star *$ & $\star \star$ & $\star \star$ & $\star \star$ & $\star \star$ & $\star \star$ & $\star \star$ \\
\hline & $11-01-94$ & $\star \star$ & 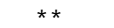 & $\star \star$ & $\star \star$ & $\star \star$ & $\star \star$ & $\star \star$ & $\star \star$ & $\star \star$ & $\star \star$ \\
\hline & $11-03-94$ & $\star \star *$ & ** & ** & ** & $\star \star$ & ** & $\star \star *$ & $\star \star$ & ** & $\star \star$ \\
\hline & $11-23-94$ & $\star \star$ & 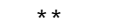 & $\star \star$ & $\star \star *$ & $\star \star$ & $\star \star$ & $\star \star$ & $\star \star$ & $\star \star *$ & $\star \star$ \\
\hline 453043122402200 & $06-17-94$ & $\star \star$ & $\star \star$ & $\star \star$ & $\star \star$ & $\star \star$ & $\star \star$ & $\star \star$ & ** & $\star \star$ & $\star \star$ \\
\hline \multirow[t]{4}{*}{14211720} & $06-14-94$ & ** & $\star \star$ & $\star \star$ & ** & $\star \star$ & $\star \star$ & $\star \star$ & ** & ** & $\star \star$ \\
\hline & $10-25-94$ & $\star \star$ & $\star \star *$ & $\star \star$ & $\star \star *$ & $\star \star$ & $\star \star$ & $\star \star$ & $\star \star \star$ & $\star \star *$ & $\star \star$ \\
\hline & $10-29-94$ & ** & ** & ** & ** & $\star \star$ & ** & $\star \star$ & $\star \star *$ & ** & $\star \star *$ \\
\hline & $11-03-94$ & ** & $\star \star$ & $\star \star$ & $\star \star$ & 0.05000 & $\star \star$ & $\star \star$ & $\star \star$ & $\star \star$ & $\star \star$ \\
\hline \multirow[t]{6}{*}{453154122394200} & $05-31-94$ & ** & $\star \star$ & ** & ** & $\star \star$ & ** & $\star \star$ & ** & $\star \star$ & $\star \star$ \\
\hline & $11-08-94$ & ** & $\star \star$ & ** & ** & $\star \star$ & $\star \star$ & ** & $\star \star$ & ** & $\star \star$ \\
\hline & $11-08-94$ & ** & $\star \star *$ & ** & $\star \star *$ & $\star \star *$ & ** & $\star \star$ & $\star \star *$ & $\star \star *$ & $\star \star$ \\
\hline & $11-30-94$ & $\star \star$ & $\star \star$ & $\star \star$ & $\star \star$ & $\star \star$ & ** & $\star \star$ & $\star *$ & $\star *$ & $\star \star$ \\
\hline & $11-30-94$ & $\star \star$ & $\star \star$ & $\star \star$ & $\star \star$ & $\star \star$ & $\star \star$ & $\star \star$ & $\star \star$ & $\star \star *$ & $\star \star$ \\
\hline & $12-02-94$ & $\star \star$ & $\star \star$ & $\star \star$ & ** & $\star \star$ & $\star \star$ & $\star \star$ & ** & $\star \star$ & $\star \star$ \\
\hline 14211805 & $05-23-94$ & $\star \star$ & $\star \star *$ & $\star \star *$ & ** & $\star \star$ & ** & ** & $\star \star *$ & $\star \star$ & $\star \star$ \\
\hline
\end{tabular}


Table 38. Concentrations of pesticides in filtered water analyzed by high performance liquid chromatography from Phase II of the Willamette River Basin Water Quality Study, Oregon, 1994-Continued

\begin{tabular}{|c|c|c|c|c|c|c|c|c|c|c|c|}
\hline STATION NUMBER & DATE & $\begin{array}{c}\text { PRO- } \\
\text { POXUR, } \\
\text { WATER, } \\
\text { FLTRD, } \\
\text { GF 0.7U } \\
\text { REC } \\
\text { (UG/L) }\end{array}$ & $\begin{array}{c}\text { BENTA- } \\
\text { ZON, } \\
\text { WATER, } \\
\text { FLTRD, } \\
\text { GF 0.7U } \\
\text { REC } \\
\text { (UG/L) }\end{array}$ & $\begin{array}{c}2,4-D, \\
\text { DIS- } \\
\text { SOLVED } \\
(U G / L)\end{array}$ & $\begin{array}{c}\text { 2, 4-DB } \\
\text { WATER, } \\
\text { FLTRD' } \\
\text { GF 0.7U } \\
\text { REC } \\
\text { (UG/L) }\end{array}$ & $\begin{array}{c}\text { DACTHAL } \\
\text { MONO- } \\
\text { ACID, } \\
\text { WAT, FLT } \\
\text { GF } 0.7 \mathrm{U} \\
\text { REC } \\
\text { (UG/L) }\end{array}$ & $\begin{array}{l}\text { DICHLOR } \\
\text { PROP, } \\
\text { WATER, } \\
\text { FLTRD, } \\
\text { GF 0.7U } \\
\text { REC } \\
\text { (UG/L) }\end{array}$ & $\begin{array}{l}\text { MCPA, } \\
\text { WATER, } \\
\text { FLTRD, } \\
\text { GF 0.7U } \\
\text { REC } \\
(\mathrm{UG} / \mathrm{L})\end{array}$ & $\begin{array}{c}\text { MCPB, } \\
\text { WATER, } \\
\text { FLTRD, } \\
\text { GF 0.7U } \\
\text { REC } \\
(\mathrm{UG} / \mathrm{L})\end{array}$ & $\begin{array}{c}\text { SILVEX, } \\
\text { DIS- } \\
\text { SOLVED } \\
\text { (UG/L) }\end{array}$ & $\begin{array}{c}2,4,5-T \\
\text { DIS- } \\
\text { SOLVED } \\
\text { (UG/L) }\end{array}$ \\
\hline \multirow[t]{2}{*}{14206298} & $05-27-94$ & $\star \star$ & $\star \star$ & 0.07000 & $\star \star$ & $\star \star$ & $\star \star$ & $\star \star$ & ** & $\star \star$ & $\star \star$ \\
\hline & $11-23-94$ & $\star \star$ & $\star \star$ & $\star \star$ & $\star \star$ & $\star \star$ & ** & $\star \star$ & ** & $\star \star$ & $\star \star$ \\
\hline \multirow[t]{4}{*}{14206950} & $05-24-94$ & ** & $\star \star$ & $\star \star$ & $\star \star$ & $\star \star$ & $\star \star$ & $\star \star$ & ** & $\star \star$ & $\star \star$ \\
\hline & $06-16-94$ & ** & ** & $\star \star *$ & $\star \star *$ & ** & ** & $\star \star$ & $\star \star *$ & ** & $\star \star *$ \\
\hline & $10-27-94$ & *夫 & $\star \star$ & 0.16000 & ** & $\star \star$ & $\star \star$ & $\star \star$ & ** & $\star \star$ & $\star \star$ \\
\hline & $11-30-94$ & ** & $\star \star$ & $\star \star$ & ** & ** & ** & $\star \star$ & ** & $\star \star$ & $\star \star$ \\
\hline \multirow[t]{2}{*}{14207500} & $05-25-94$ & ** & $\star \star$ & $\star \star$ & $\star \star$ & $\star \star$ & $\star \star$ & $\star \star$ & $\star \star$ & $\star \star$ & $\star \star$ \\
\hline & $10-28-94$ & ** & ** & 0.23000 & $\star \star$ & $\star \star$ & $\star \star$ & $\star \star$ & $\star *$ & $\star \star$ & $\star \star$ \\
\hline \multirow[t]{6}{*}{452823122240900} & $05-24-94$ & ** & ** & $\star \star$ & ** & ** & $\star \star$ & ** & ** & ** & ** \\
\hline & $10-27-94$ & $\star \star$ & $\star \star$ & 0.13000 & $\star \star$ & $\star \star$ & $\star \star$ & $\star \star$ & $\star \star$ & $\star \star$ & $\star \star$ \\
\hline & $10-28-94$ & $\star \star$ & $\star \star *$ & $\star \star$ & ** & $\star \star *$ & $\star \star$ & $\star \star$ & ** & $\star \star *$ & $\star \star$ \\
\hline & $11-01-94$ & $\star \star *$ & ** & ** & ** & $\star *$ & ** & $\star \star *$ & $\star \star *$ & $\star \star *$ & $\star \star *$ \\
\hline & $11-03-94$ & $\star \star$ & ** & ** & ** & $\star \star$ & ** & $\star \star$ & ** & $\star \star$ & $\star \star$ \\
\hline & $11-23-94$ & $\star \star$ & ** & $\star \star$ & $\star \star$ & $\star \star$ & $\star \star$ & $\star \star$ & ** & $\star \star$ & $\star \star$ \\
\hline 453043122402200 & $06-17-94$ & $\star \star$ & ** & $\star \star$ & $\star \star$ & $\star \star$ & $\star \star$ & $\star \star$ & ** & $\star \star$ & $\star \star$ \\
\hline \multirow[t]{4}{*}{14211720} & $06-14-94$ & $\star \star$ & $\star \star$ & $\star \star$ & $\star \star$ & $\star \star$ & $\star \star$ & $\star \star$ & ** & $\star \star$ & $\star \star$ \\
\hline & $10-25-94$ & $\star \star$ & 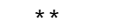 & $\star \star$ & $\star \star$ & $\star \star *$ & $\star \star$ & $\star \star$ & $\star \star$ & 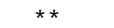 & $\star \star$ \\
\hline & $10-29-94$ & ** & $\star \star$ & ** & $\star \star *$ & ** & $\star \star$ & $\star \star$ & $\star \star$ & $\star \star \star ~$ & $\star \star$ \\
\hline & $11-03-94$ & ** & $\star \star$ & $\star \star$ & ** & ** & $\star \star$ & $\star \star$ & $\star \star$ & $\star \star$ & $\star \star$ \\
\hline \multirow[t]{6}{*}{453154122394200} & $05-31-94$ & $\star \star$ & $\star \star$ & $\star \star$ & ** & $\star \star$ & $\star \star$ & $\star \star$ & $\star \star$ & $\star \star$ & $\star \star$ \\
\hline & $11-08-94$ & $\star \star$ & $\star \star$ & $\star \star$ & $\star \star$ & $\star \star$ & $\star \star$ & ** & ** & ** & ** \\
\hline & $11-08-94$ & $\star \star$ & $\star \star *$ & $\star \star$ & $\star \star$ & $\star \star$ & $\star \star$ & $\star \star$ & $\star \star *$ & $\star \star$ & $\star \star$ \\
\hline & $11-30-94$ & $\star \star$ & $\star \star *$ & ** & $\star \star$ & $\star \star$ & $\star \star$ & $\star \star$ & $\star \star *$ & $\star \star$ & $\star \star *$ \\
\hline & $11-30-94$ & $\star \star$ & ** & ** & ** & $\star *$ & $\star \star$ & $\star \star *$ & $\star \star *$ & $\star \star *$ & $\star \star *$ \\
\hline & $12-02-94$ & $\star \star$ & $\star \star$ & $\star \star$ & $\star \star$ & $\star *$ & $\star \star$ & $\star *$ & $\star \star$ & $\star \star$ & $\star \star$ \\
\hline 14211805 & $05-23-94$ & $\star \star$ & $\star \star$ & $\star \star$ & $\star \star$ & $\star *$ & $\star \star$ & $\star \star$ & $\star \star$ & $\star *$ & $\star \star$ \\
\hline
\end{tabular}


Table 38. Concentrations of pesticides in filtered water analyzed by high performance liquid chromatography from Phase II of the Willamette River Basin Water Quality Study, Oregon, 1994-Continued

\begin{tabular}{|c|c|c|c|c|c|c|c|c|c|c|c|}
\hline STATION NUMBER & DATE & $\begin{array}{c}\text { ORY- } \\
\text { ZALIN, } \\
\text { WATER, } \\
\text { FLTRD, } \\
\text { GF 0.7U } \\
\text { REC } \\
\text { (UG/L) }\end{array}$ & $\begin{array}{c}\text { DINOSEB } \\
\text { WATER, } \\
\text { FLTRD, } \\
\text { GF 0.7U } \\
\text { REC } \\
(\mathrm{UG} / \mathrm{L})\end{array}$ & $\begin{array}{c}\text { OCRESOL } \\
4,6- \\
\text { DINITRO } \\
\text { WAT,FLT } \\
\text { GF } 0.7 \mathrm{U} \\
\text { REC } \\
\text { (UG/L) }\end{array}$ & $\begin{array}{c}\text { ACIFL- } \\
\text { UORFEN } \\
\text { WATER, } \\
\text { FLTRD, } \\
\text { GF 0.7U } \\
\text { REC } \\
\text { (UG/L) }\end{array}$ & $\begin{array}{c}\text { 1-NAPH } \\
\text { THOL, } \\
\text { WATER, } \\
\text { FLTRD, } \\
\text { GF 0.7U } \\
\text { REC } \\
\text { (UG/L) }\end{array}$ & $\begin{array}{c}\text { AMIBEN, } \\
\text { WATER, } \\
\text { FLTRD, } \\
\text { GF } 0.7 U \\
\text { REC } \\
\text { (UG/L) }\end{array}$ & $\begin{array}{c}\text { DIURON, } \\
\text { WATER, } \\
\text { FLTRD, } \\
\text { GF } 0.7 U \\
\text { REC } \\
(U G / L)\end{array}$ & $\begin{array}{c}\text { FEN- } \\
\text { URON, } \\
\text { WATER, } \\
\text { FLTRD, } \\
\text { GF } 0.7 \mathrm{U} \\
\text { REC } \\
\text { (UG/L) }\end{array}$ & $\begin{array}{c}\text { FLUO- } \\
\text { METURON } \\
\text { WATER, } \\
\text { FLTRD, } \\
\text { GF 0.7U } \\
\text { REC } \\
\text { (UG/L) }\end{array}$ & $\begin{array}{c}\text { LINURON } \\
\text { WATER, } \\
\text { FLTRD, } \\
\text { GF 0.7U } \\
\text { REC } \\
\text { (UG/L) }\end{array}$ \\
\hline 14206298 & $\begin{array}{l}05-27-94 \\
11-23-94\end{array}$ & $\begin{array}{l}\star \star \\
\star \star\end{array}$ & $\begin{array}{l}\star \star \\
\star \star\end{array}$ & $\begin{array}{l}\star \star \\
\star \star\end{array}$ & $\begin{array}{l}\star \star \\
\star \star\end{array}$ & $\begin{array}{l}\star \star \\
\star \star\end{array}$ & $\begin{array}{l}* * \\
\star *\end{array}$ & ** & $\begin{array}{l}\star \star \\
\star \star\end{array}$ & $\begin{array}{l}\star \star \\
\star \star\end{array}$ & $\begin{array}{l}\star \star \\
\star \star\end{array}$ \\
\hline 14206950 & $\begin{array}{l}05-24-94 \\
06-16-94 \\
10-27-94 \\
11-30-94\end{array}$ & $\begin{array}{l}\star \star \\
\star \star \\
\star \star \\
\star \star\end{array}$ & $\begin{array}{l}\star \star \\
\star \star \\
\star \star \\
\star \star\end{array}$ & $\begin{array}{l}\star \star \\
\star \star \\
\star \star \\
\star \star\end{array}$ & $\begin{array}{l}\star \star \\
\star \star \\
\star \star \\
\star \star\end{array}$ & $\begin{array}{l}\star \star \\
\star \star \\
\star \star \\
\star \star\end{array}$ & $\begin{array}{l}\star \star \\
\star \star \\
\star * \\
\star *\end{array}$ & $\begin{array}{r}0.27000 \\
0.05000 \\
\mathrm{E} 0.55000 \\
0.19000\end{array}$ & $\begin{array}{l}\star \star \\
\star \star \\
\star \star \\
\star \star\end{array}$ & $\begin{array}{l}\star \star \\
\star \star \\
\star \star \\
\star \star\end{array}$ & $\begin{array}{l}\star \star \\
\star \star \\
\star \star \\
\star \star\end{array}$ \\
\hline 14207500 & $\begin{array}{l}05-25-94 \\
10-28-94\end{array}$ & $\begin{array}{l}\star * \\
\star *\end{array}$ & $\begin{array}{l}* * \\
* *\end{array}$ & $\begin{array}{l}* * \\
* *\end{array}$ & $\begin{array}{l}* \star \\
\star *\end{array}$ & $\begin{array}{l}\star \star \\
\star *\end{array}$ & $\begin{array}{l}\star \star \\
\star *\end{array}$ & $\begin{array}{r}0.05000 \\
\mathrm{E} 0.31000\end{array}$ & $\begin{array}{l}* * \\
\star *\end{array}$ & $\begin{array}{l}\star \star \\
\star *\end{array}$ & $\begin{array}{l}\star * \\
\star *\end{array}$ \\
\hline 452823122240900 & $\begin{array}{l}05-24-94 \\
10-27-94 \\
10-28-94 \\
11-01-94 \\
11-03-94 \\
11-23-94\end{array}$ & $\begin{array}{l}\star \star \star \\
0.23000 \\
\star \star \\
\star \star \\
\star \star \\
\star \star\end{array}$ & 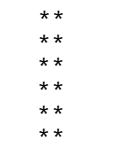 & $\begin{array}{l}* \star \\
\star \star \\
\star \star \\
\star \star \\
\star \star \\
\star \star \\
\star \star \\
\star \star\end{array}$ & $\begin{array}{l}* \star \\
\star \star \\
\star \star \\
\star \star \\
\star \star \\
\star \star \\
\star \star \\
\star \star\end{array}$ & $\begin{array}{l}* \star \\
\star \star \\
\star \star \\
\star \star \\
\star \star \\
\star \star \\
\star \star \\
\star \star\end{array}$ & $\begin{array}{l}\star \star \\
\star \star \\
\star \star \\
\star \star \\
\star \star \\
\star \star \\
\star \star\end{array}$ & $\begin{array}{c}\mathrm{E} 0.33000 \\
* \star \\
* \star \\
\star * \\
0.08000 \\
0.07000\end{array}$ & $\begin{array}{l}\star \star \\
\star \star \\
\star \star \\
\star \star \\
\star \star \\
\star \star \\
\star \star \\
\star \star\end{array}$ & $\begin{array}{l}* \star \\
\star \star \\
\star \star \\
\star \star \\
\star \star \\
\star \star \\
\star \star\end{array}$ & $\begin{array}{l}\star \star \\
\star \star \\
\star \star \\
\star \star \\
\star \star \\
\star \star \\
\star \star\end{array}$ \\
\hline 453043122402200 & $06-17-94$ & $\star \star$ & $\star \star$ & ** & $\star \star$ & ** & $\star \star$ & ** & ** & ** & $\star \star$ \\
\hline 14211720 & $\begin{array}{l}06-14-94 \\
10-25-94 \\
10-29-94 \\
11-03-94\end{array}$ & $\begin{array}{l}\star \star \\
\star \star \\
\star \star \\
\star \star\end{array}$ & $\begin{array}{l}\star \star \\
\star \star \\
\star \star \\
\star \star\end{array}$ & $\begin{array}{l}\star \star \\
\star \star \\
\star \star \\
\star \star\end{array}$ & $\begin{array}{l}\star \star \\
\star \star \\
\star \star \\
\star \star\end{array}$ & $\begin{array}{l}\star \star \\
\star \star \\
\star \star \\
\star \star\end{array}$ & $\begin{array}{l}\star \star \\
\star \star \\
\star \star \\
\star *\end{array}$ & $\begin{array}{c}{ }^{\star *} \\
\mathrm{E} 0.21000 \\
{ }^{\star \star} \\
0.24000\end{array}$ & $\begin{array}{l}\star \star \\
\star \star \\
\star * \\
\star *\end{array}$ & $\begin{array}{l}\star \star \\
\star \star \\
\star \star \\
\star \star\end{array}$ & $\begin{array}{l}\star \star \\
\star \star \\
\star \star \\
\star \star\end{array}$ \\
\hline 453154122394200 & $\begin{array}{l}05-31-94 \\
11-08-94 \\
11-08-94 \\
11-30-94 \\
11-30-94 \\
12-02-94\end{array}$ & $\begin{array}{l}\star \star \\
\star \star \\
\star \star \\
\star \star \\
\star \star \\
\star \star \\
\star \star\end{array}$ & $\begin{array}{l}\star \star \\
\star \star \\
\star \star \\
\star \star \\
\star \star \\
\star \star \\
\star \star \\
\star \star\end{array}$ & $\begin{array}{l}* \star \\
\star \star \\
\star * \\
\star \star \\
\star \star \\
\star \star \\
\star \star \\
\star *\end{array}$ & $\begin{array}{l}\star \star \\
\star \star \\
\star \star \\
\star \star \\
\star \star \\
\star \star \\
\star \star\end{array}$ & $\begin{array}{l}\star \star \\
\star \star \\
\star \star \\
\star \star \\
\star \star \\
\star \star \\
\star \star\end{array}$ & $\begin{array}{l}\star * \\
\star * \\
\star * \\
\star * \\
\star * \\
\star *\end{array}$ & $\begin{array}{c}\mathrm{E} 1.7000 \\
\star * \\
\star \star \\
\star * \\
0.10000 \\
\star *\end{array}$ & $\begin{array}{l}\star * \\
\star * \\
\star * \\
\star * \\
\star * \\
\star *\end{array}$ & $\begin{array}{l}* \star \\
\star \star \\
\star \star \\
\star \star \\
\star \star \\
\star \star \\
\star \star\end{array}$ & $\begin{array}{l}\star \star \\
\star \star \\
\star \star \\
\star \star \\
\star \star \\
\star \star \\
\star \star\end{array}$ \\
\hline 14211805 & $05-23-94$ & $\star \star$ & $\star \star$ & $\star \star$ & ** & $\star *$ & ** & $\star \star$ & $\star \star$ & $\star \star$ & $\star \star$ \\
\hline
\end{tabular}


Table 38. Concentrations of pesticides in filtered water analyzed by high performance liquid chromatography from Phase II of the Willamette River Basin Water Quality Study, Oregon, 1994-Continued

\begin{tabular}{|c|c|c|c|c|c|c|c|c|c|c|}
\hline STATION NUMBER & DATE & $\begin{array}{c}\text { NEB- } \\
\text { URON, } \\
\text { WATER, } \\
\text { FLTRD, } \\
\text { GF } 0.7 \mathrm{U} \\
\text { REC } \\
\text { (UG/L) }\end{array}$ & $\begin{array}{c}\text { CHLORO- } \\
\text { THALO- } \\
\text { NIL, } \\
\text { WAT, FLT } \\
\text { GF } 0.7 \mathrm{U} \\
\text { REC } \\
\text { (UG/L) }\end{array}$ & $\begin{array}{c}\text { ESFEN- } \\
\text { VAL- } \\
\text { ERATE, } \\
\text { WAT, FLT } \\
\text { GF } 0.7 \mathrm{U} \\
\text { REC } \\
(\mathrm{UG} / \mathrm{L})\end{array}$ & $\begin{array}{c}\text { NORFLUR } \\
\text { AZON, } \\
\text { WATER, } \\
\text { FLTRD, } \\
\text { GF } 0.7 \mathrm{U} \\
\text { REC } \\
\text { (UG/L) }\end{array}$ & $\begin{array}{c}\text { CLOPYR- } \\
\text { ALID, } \\
\text { WATER, } \\
\text { FLTRD, } \\
\text { GF } 0.7 \mathrm{U} \\
\text { REC } \\
\text { (UG/L) }\end{array}$ & $\begin{array}{c}\text { PIC- } \\
\text { LORAM, } \\
\text { WATER, } \\
\text { FLTRD, } \\
\text { GF } 0.7 \mathrm{U} \\
\text { REC } \\
\text { (UG/L) }\end{array}$ & $\begin{array}{c}\text { TRI- } \\
\text { CLOPYR, } \\
\text { WATER, } \\
\text { FLTRD, } \\
\text { GF } 0.7 \mathrm{U} \\
\text { REC } \\
\text { (UG/L) }\end{array}$ & $\begin{array}{l}\text { BRO- } \\
\text { MACIL, } \\
\text { WATER, } \\
\text { DISS, } \\
\text { REC } \\
\text { (UG/L) }\end{array}$ & $\begin{array}{c}\text { BDMC, } \\
\text { SURROG, } \\
\text { WATER, } \\
\text { UNFLTRD } \\
\text { REC } \\
\text { PERCENT }\end{array}$ \\
\hline 14206298 & $\begin{array}{l}05-27-94 \\
11-23-94\end{array}$ & $\begin{array}{l}\star * \\
\star *\end{array}$ & $\begin{array}{l}\star * \\
\star *\end{array}$ & $\begin{array}{l}* * \\
* *\end{array}$ & ** & $\begin{array}{l}* * \\
* *\end{array}$ & $\begin{array}{l}* \star \\
\star *\end{array}$ & $\begin{array}{c}* \star \\
0.05000\end{array}$ & $\begin{array}{l}* * \\
\star *\end{array}$ & $\begin{array}{l}106.00 \\
69.000\end{array}$ \\
\hline 14206950 & $\begin{array}{l}05-24-94 \\
06-16-94 \\
10-27-94 \\
11-30-94\end{array}$ & $\begin{array}{l}* * \\
\star * \\
\star \star \\
\star *\end{array}$ & $\begin{array}{l}\star \star \\
\star \star \\
\star \star \\
\star \star\end{array}$ & $\begin{array}{l}\star \star \\
\star \star \\
\star \star \\
\star \star\end{array}$ & $\begin{array}{l}\star \star \\
\star \star \\
\star \star \\
\star \star\end{array}$ & $\begin{array}{l}\star * \\
\star * \\
\star * \\
\star *\end{array}$ & $\begin{array}{l}\star \star \\
\star \star \\
\star \star \\
\star \star\end{array}$ & $\begin{array}{l}\star \star \\
\star \star \\
0.11000 \\
* \star\end{array}$ & $\begin{array}{l}\star \star \\
\star \star \\
\star \star \\
\star \star\end{array}$ & $\begin{array}{c}21.000 \\
-- \\
83.000 \\
70.000\end{array}$ \\
\hline 14207500 & $\begin{array}{l}05-25-94 \\
10-28-94\end{array}$ & ** & $\begin{array}{l}* * \\
* *\end{array}$ & ** & $\begin{array}{l}\star * \\
\star *\end{array}$ & $\begin{array}{l}* * \\
* *\end{array}$ & $\begin{array}{l}\star * \\
\star *\end{array}$ & $\begin{array}{l}* * \\
* *\end{array}$ & $\begin{array}{l}* * \\
\star *\end{array}$ & $\begin{array}{l}26.000 \\
94.000\end{array}$ \\
\hline 452823122240900 & $\begin{array}{l}05-24-94 \\
10-27-94 \\
10-28-94 \\
11-01-94 \\
11-03-94 \\
11-23-94\end{array}$ & $\begin{array}{l}\star * \\
\star * \\
\star * \\
\star \star \\
\star \star \\
\star \star\end{array}$ & $\begin{array}{l}* \star \\
\star \star \\
\star \star \\
\star \star \\
\star \star \\
\star \star \\
\star \star\end{array}$ & $\begin{array}{l}\star \star \\
\star \star \\
\star \star \\
\star \star \\
\star \star \\
\star \star \\
\star \star\end{array}$ & $\begin{array}{l}\star * \\
\star \star \\
\star \star \\
\star \star \\
\star \star \\
\star \star\end{array}$ & $\begin{array}{l}\star \star \\
\star \star \\
\star \star \\
\star \star \\
\star \star \\
\star \star \\
\star \star\end{array}$ & $\begin{array}{l}\star \star \\
\star \star \\
\star \star \\
\star \star \\
\star \star \\
\star \star \\
\star \star\end{array}$ & $\begin{array}{c}\star \star \\
\star \star \\
\star \star \\
0.46000 \\
\star \star \\
\star \star\end{array}$ & $\begin{array}{l}\star \star \\
\star \star \\
\star \star \\
\star \star \\
\star \star \\
\star \star \\
\star \star\end{array}$ & $\begin{array}{l}30.000 \\
70.000 \\
118.00 \\
72.000 \\
104.00 \\
67.000\end{array}$ \\
\hline 453043122402200 & $06-17-94$ & ** & $\star \star$ & $\star \star$ & *夫 & $\star \star$ & ** & $\star \star$ & $\star \star$ & -- \\
\hline 14211720 & $\begin{array}{l}06-14-94 \\
10-25-94 \\
10-29-94 \\
11-03-94\end{array}$ & $\begin{array}{l}\star \star \\
\star \star \\
\star \star \\
\star \star\end{array}$ & $\begin{array}{l}\star \star \\
\star \star \\
\star \star \\
\star \star\end{array}$ & $\begin{array}{l}\star \star \\
\star * \\
\star * \\
\star \star\end{array}$ & $\begin{array}{l}\star \star \\
\star \star \\
\star \star \\
\star \star\end{array}$ & $\begin{array}{l}\star * \\
\star * \\
\star * \\
\star *\end{array}$ & $\begin{array}{l}\star \star \\
\star \star \\
\star \star \\
\star \star\end{array}$ & $\begin{array}{l}\star * \\
\star * \\
\star * \\
\star *\end{array}$ & $\begin{array}{l}* \star \\
\star \star \\
\star \star \\
\star \star\end{array}$ & $\begin{array}{l}93.000 \\
136.00 \\
98.000 \\
58.000\end{array}$ \\
\hline 453154122394200 & $\begin{array}{l}05-31-94 \\
11-08-94 \\
11-08-94 \\
11-30-94 \\
11-30-94 \\
12-02-94\end{array}$ & $\begin{array}{l}\star \star \\
\star * \\
\star * \\
\star * \\
\star \star \\
\star *\end{array}$ & $\begin{array}{l}\star \star \\
\star * \\
\star \star \\
\star \star \\
\star \star \\
\star \star \\
\star \star\end{array}$ & $\begin{array}{l}\star \star \\
\star \star \\
\star \star \\
\star \star \\
\star \star \\
\star \star \\
\star \star\end{array}$ & $\begin{array}{l}\star \star \\
\star * \\
\star * \\
\star * \\
\star \star \\
\star \star\end{array}$ & $\begin{array}{l}\star \star \\
\star \star \\
\star \star \\
\star \star \\
\star \star \\
\star \star \\
\star *\end{array}$ & $\begin{array}{l}\star \star \\
\star \star \\
\star \star \\
\star \star \\
\star \star \\
\star \star\end{array}$ & $\begin{array}{l}\star \star \\
\star * \\
\star * \\
\star \star \\
\star \star \\
\star \star \\
\star *\end{array}$ & $\begin{array}{l}\star \star \\
\star \star \\
\star \star \\
\star \star \\
\star \star \\
\star \star\end{array}$ & $\begin{array}{c}-- \\
62.000 \\
119.00 \\
156.00 \\
56.000 \\
55.000\end{array}$ \\
\hline 14211805 & $05-23-94$ & $\star \star$ & $\star \star$ & $\star \star$ & ** & $\star \star$ & ** & $\star \star$ & $\star \star$ & 56.000 \\
\hline
\end{tabular}


Table 39. Concentrations of triazine herbicides in filtered water from Phase II of the Willamette River Basin Water Quality Study, Oregon, 1994

[USGS laboratory schedule 8015 ; <, less than; ug/L, micrograms per liter]

\begin{tabular}{|c|c|c|c|c|c|c|c|c|}
\hline Station Name & Station number & Date & Time & $\begin{array}{c}\text { Alachlor } \\
\text { (ug/L) }\end{array}$ & $\begin{array}{c}\text { Ametryn } \\
(\mathrm{ug} / \mathrm{L})\end{array}$ & $\begin{array}{c}\text { Atrazine } \\
\text { (ug/L) }\end{array}$ & $\begin{array}{c}\text { Bromacil } \\
\text { (ug/L) }\end{array}$ & $\begin{array}{c}\text { Butachlor } \\
\text { (ug/L) }\end{array}$ \\
\hline Coast Fork Willamette R nr London, OR & 433548123040600 & 940520 & 1050 & $<0.009$ & $<0.008$ & $<0.011$ & $<0.025$ & $<0.010$ \\
\hline Coast Fork Willamette R nr Eugene, OR & 440045122585600 & 940519 & 1030 & $<0.009$ & $<0.008$ & 0.005 & $<0.025$ & $<0.010$ \\
\hline Urban outfall, Eugene, OR & 440402123063900 & 940613 & 1220 & $<0.009$ & $<0.008$ & 0.009 & $<0.025$ & $<0.010$ \\
\hline Mack Cr nr Blue River, OR & 441310122095801 & 940527 & 1040 & $<0.009$ & $<0.008$ & $<0.011$ & $<0.025$ & $<0.010$ \\
\hline McKenzie R nr Eugene, OR & 440707123041300 & 940519 & 1500 & $<0.009$ & $<0.008$ & 0.002 & $<0.025$ & $<0.010$ \\
\hline Long Tom R nr Monroe, OR & 442223123153703 & 941101 & 1730 & $<0.009$ & $<0.008$ & 0.012 & 0.076 & $<0.010$ \\
\hline \multirow[t]{3}{*}{ Muddy Cr nr Peoria, OR } & 443138123120901 & 941102 & 1020 & 0.001 & $<0.008$ & 0.260 & $<0.025$ & $<0.010$ \\
\hline & & 941106 & 1210 & $<0.009$ & $<0.008$ & 0.558 & $<0.025$ & $<0.010$ \\
\hline & & 941109 & 1430 & $<0.009$ & $<0.008$ & 0.209 & $<0.025$ & $<0.010$ \\
\hline Rock Cr nr Philomath, OR & 443045123273000 & 940518 & 1830 & $<0.009$ & $<0.008$ & $<0.011$ & $<0.025$ & $<0.010$ \\
\hline Mary's R at Corvallis, OR & 443321123155201 & 940518 & 1400 & $<0.009$ & $<0.008$ & 0.024 & $<0.025$ & $<0.010$ \\
\hline Calapooia R at Albany, OR & 14173500 & 941101 & 1240 & $<0.009$ & 0.003 & 0.292 & $<0.025$ & $<0.010$ \\
\hline Luckiamute R nr Buena Vista, OR & 444349123094000 & 940516 & 1730 & $<0.009$ & $<0.008$ & 0.031 & $<0.025$ & $<0.010$ \\
\hline Thomas Cr nr Jefferson, OR & 444123122562200 & 940516 & 1910 & $<0.009$ & $<0.008$ & 0.012 & 0.008 & $<0.010$ \\
\hline Santiam R at Jefferson, OR & 14189000 & 940517 & 1630 & $<0.009$ & $<0.008$ & 0.004 & $<0.025$ & $<0.010$ \\
\hline Rickreall Cr nr Salem, OR & 445547123065400 & 940526 & 1550 & $<0.009$ & $<0.008$ & 0.026 & $<0.025$ & $<0.010$ \\
\hline \multirow[t]{3}{*}{ Pringle $\mathrm{Cr}$ at Salem, OR } & 14190970 & 941123 & 1210 & $<0.009$ & $<0.008$ & 0.025 & 0.038 & $<0.010$ \\
\hline & & 941130 & 1540 & $<0.009$ & $<0.008$ & 0.053 & 0.042 & $<0.010$ \\
\hline & & 941130 & 1720 & $<0.009$ & $<0.008$ & 0.066 & 0.049 & $<0.010$ \\
\hline \multirow[t]{4}{*}{ Mill Cr nr Turner, OR } & 445037122573800 & 940614 & 1150 & $<0.009$ & 0.007 & 0.014 & $<0.025$ & $<0.010$ \\
\hline & & 941031 & 1410 & 0.003 & $<0.008$ & 0.657 & $<0.025$ & $<0.010$ \\
\hline & & 941104 & 1130 & 0.003 & $<0.008$ & 0.215 & $<0.025$ & $<0.010$ \\
\hline & & 941104 & 1430 & 0.002 & $<0.008$ & 0.239 & $<0.025$ & $<0.010$ \\
\hline \multirow[t]{2}{*}{ South Yamhill R at McMinneville, OR } & 14194150 & 940517 & 1730 & $<0.009$ & $<0.008$ & 0.015 & $<0.025$ & $<0.010$ \\
\hline & & 941102 & 1210 & $<0.009$ & $<0.008$ & 0.028 & $<0.025$ & $<0.010$ \\
\hline North Yamhill R nr McMinneville, OR & 451355123093600 & 940517 & 1230 & $<0.009$ & $<0.008$ & 0.006 & $<0.025$ & $<0.010$ \\
\hline Willamette R nr Newberg, OR & 451602122564400 & 940531 & 1410 & $<0.009$ & $<0.008$ & 0.011 & $<0.025$ & $<0.010$ \\
\hline \multirow[t]{2}{*}{ Champoeg Cr nr Butteville, OR } & 451502122524700 & 940526 & 1040 & $<0.009$ & 0.002 & 0.076 & $<0.025$ & $<0.010$ \\
\hline & & 941031 & 1310 & $<0.009$ & $<0.008$ & 0.060 & 0.022 & $<0.010$ \\
\hline Zollner Cr nr Mt. Angel, OR & 14201300 & 941028 & 1330 & $<0.009$ & $<0.008$ & 0.566 & $<0.025$ & $<0.010$ \\
\hline \multirow[t]{3}{*}{ Pudding R at Aurora, OR } & 14202000 & 941028 & 1720 & $<0.009$ & $<0.008$ & 0.124 & $<0.025$ & $<0.010$ \\
\hline & & 941029 & 1240 & $<0.009$ & $<0.008$ & 0.041 & 0.010 & $<0.010$ \\
\hline & & 941109 & 1300 & $<0.009$ & $<0.008$ & 0.077 & $<0.025$ & $<0.010$ \\
\hline Molalla R nr Canby, OR & 451603122423301 & 940525 & 1030 & $<0.009$ & $<0.008$ & 0.002 & $<0.025$ & $<0.010$ \\
\hline \multirow[t]{2}{*}{ Dairy Cr nr Hillsboro, OR } & 14206200 & 940527 & 1650 & $<0.009$ & 0.001 & 0.058 & $<0.025$ & $<0.010$ \\
\hline & & 941201 & 1150 & 0.001 & $<0.008$ & 0.454 & 0.063 & $<0.010$ \\
\hline \multirow[t]{2}{*}{ Bronson Cr nr Aloha, OR } & 14206298 & 940527 & 1110 & $<0.009$ & $<0.008$ & 0.033 & $<0.025$ & $<0.010$ \\
\hline & & 941123 & 1040 & $<0.009$ & $<0.008$ & 0.011 & $<0.025$ & $<0.010$ \\
\hline Fanno $\mathrm{Cr}$ at Durham, OR & 14206950 & 941027 & 1630 & $<0.009$ & 0.002 & 0.006 & 0.012 & $<0.010$ \\
\hline
\end{tabular}


Table 39. Concentrations of triazine herbicides in filtered water from Phase II of the Willamette River Basin Water Quality Study, Oregon, 1994-Continued

\begin{tabular}{|c|c|c|c|c|c|c|c|c|}
\hline Station number & Date & $\begin{array}{c}\text { Butylate } \\
\text { (ug/L) }\end{array}$ & $\begin{array}{c}\text { Carboxin } \\
\text { (ug/L) }\end{array}$ & $\begin{array}{l}\text { Cyanazine } \\
\text { (ug/L) }\end{array}$ & $\begin{array}{c}\text { Cycloate } \\
\text { (Estimated) } \\
\text { (ug/L) }\end{array}$ & $\begin{array}{l}\text { Desethyl- } \\
\text { atrazine } \\
\text { (Estimated) } \\
\text { (ug/L) }\end{array}$ & $\begin{array}{l}\text { Desisoproyl- } \\
\text { atrazine } \\
\text { (Estimated) } \\
\text { (ug/L) }\end{array}$ & $\begin{array}{l}\text { Diphenamid } \\
\text { (ug/L) }\end{array}$ \\
\hline 433548123040600 & 940520 & $<0.006$ & $<0.008$ & $<0.008$ & 0.003 & $<0.013$ & $<0.005$ & $<0.010$ \\
\hline 440045122585600 & 940519 & $<0.006$ & $<0.008$ & $<0.008$ & 0.002 & $<0.013$ & $<0.005$ & $<0.010$ \\
\hline 440402123063900 & 940613 & $<0.006$ & $<0.008$ & $<0.008$ & $<0.013$ & 0.003 & 0.011 & $<0.010$ \\
\hline 441310122095801 & 940527 & $<0.006$ & $<0.008$ & $<0.008$ & 0.002 & $<0.013$ & $<0.005$ & $<0.010$ \\
\hline 440707123041300 & 940519 & $<0.006$ & $<0.008$ & $<0.008$ & 0.003 & $<0.013$ & $<0.005$ & $<0.010$ \\
\hline 442223123153703 & 941101 & 0.001 & $<0.008$ & $<0.008$ & 0.001 & 0.002 & 0.004 & $<0.010$ \\
\hline \multirow[t]{3}{*}{443138123120901} & 941102 & $<0.006$ & $<0.008$ & $<0.008$ & $<0.013$ & 0.006 & 0.008 & $<0.010$ \\
\hline & 941106 & $<0.006$ & $<0.008$ & $<0.008$ & $<0.013$ & 0.009 & 0.009 & $<0.010$ \\
\hline & 941109 & $<0.006$ & $<0.008$ & $<0.008$ & $<0.013$ & 0.010 & 0.006 & $<0.010$ \\
\hline 443045123273000 & 940518 & $<0.006$ & $<0.008$ & $<0.008$ & 0.002 & $<0.013$ & $<0.005$ & $<0.010$ \\
\hline 443321123155201 & 940518 & $<0.006$ & $<0.008$ & $<0.008$ & $<0.013$ & 0.005 & $<0.005$ & $<0.010$ \\
\hline 14173500 & 941101 & $<0.006$ & $<0.008$ & $<0.008$ & $<0.013$ & 0.006 & 0.004 & $<0.010$ \\
\hline 444349123094000 & 940516 & $<0.006$ & $<0.008$ & $<0.008$ & 0.003 & $<0.013$ & $<0.005$ & $<0.010$ \\
\hline 444123122562200 & 940516 & $<0.006$ & $<0.008$ & $<0.008$ & 0.002 & $<0.013$ & $<0.005$ & $<0.010$ \\
\hline 14189000 & 940517 & $<0.006$ & $<0.008$ & $<0.008$ & 0.002 & $<0.013$ & $<0.005$ & $<0.010$ \\
\hline 445547123065400 & 940526 & $<0.006$ & $<0.008$ & $<0.008$ & 0.004 & 0.005 & $<0.005$ & $<0.010$ \\
\hline \multirow[t]{3}{*}{14190970} & 941123 & $<0.006$ & $<0.008$ & $<0.008$ & $<0.013$ & 0.003 & 0.013 & $<0.010$ \\
\hline & 941130 & $<0.006$ & $<0.008$ & $<0.008$ & $<0.013$ & 0.003 & $<0.005$ & $<0.010$ \\
\hline & 941130 & $<0.006$ & $<0.008$ & $<0.008$ & $<0.013$ & 0.003 & $<0.005$ & $<0.010$ \\
\hline \multirow[t]{4}{*}{445037122573800} & 940614 & $<0.006$ & $<0.008$ & $<0.008$ & 0.002 & 0.004 & $<0.005$ & $<0.010$ \\
\hline & 941031 & $<0.006$ & $<0.008$ & $<0.008$ & 0.002 & 0.032 & $<0.005$ & $<0.010$ \\
\hline & 941104 & $<0.006$ & $<0.008$ & $<0.008$ & $<0.013$ & 0.012 & 0.003 & $<0.010$ \\
\hline & 941104 & $<0.006$ & $<0.008$ & 0.001 & $<0.013$ & 0.009 & 0.002 & $<0.010$ \\
\hline \multirow[t]{2}{*}{14194150} & 940517 & $<0.006$ & $<0.008$ & $<0.008$ & 0.002 & $<0.013$ & $<0.005$ & $<0.010$ \\
\hline & 941102 & $<0.006$ & $<0.008$ & $<0.008$ & $<0.013$ & 0.002 & 0.002 & $<0.010$ \\
\hline 451355123093600 & 940517 & $<0.006$ & $<0.008$ & $<0.008$ & 0.002 & $<0.013$ & $<0.005$ & $<0.010$ \\
\hline 451602122564400 & 940531 & $<0.006$ & $<0.008$ & $<0.008$ & $<0.013$ & $<0.013$ & $<0.005$ & $<0.010$ \\
\hline \multirow[t]{2}{*}{451502122524700} & 940526 & $<0.006$ & $<0.008$ & $<0.008$ & 0.002 & 0.011 & $<0.005$ & $<0.010$ \\
\hline & 941031 & 0.002 & $<0.008$ & $<0.008$ & 0.002 & 0.003 & 0.012 & 0.001 \\
\hline 14201300 & 941028 & $<0.006$ & $<0.008$ & $<0.008$ & 0.020 & 0.022 & 0.007 & 0.002 \\
\hline \multirow[t]{3}{*}{14202000} & 941028 & $<0.006$ & $<0.008$ & $<0.008$ & 0.010 & 0.003 & 0.003 & 0.001 \\
\hline & 941029 & $<0.006$ & $<0.008$ & $<0.008$ & 0.002 & 0.002 & 0.002 & $<0.010$ \\
\hline & 941109 & $<0.006$ & $<0.008$ & $<0.008$ & 0.001 & 0.006 & 0.002 & $<0.010$ \\
\hline 451603122423301 & 940525 & $<0.006$ & $<0.008$ & $<0.008$ & 0.014 & $<0.013$ & $<0.005$ & $<0.010$ \\
\hline \multirow[t]{2}{*}{14206200} & 940527 & $<0.006$ & $<0.008$ & $<0.008$ & 0.004 & 0.003 & 0.002 & 0.006 \\
\hline & 941201 & $<0.006$ & $<0.008$ & $<0.008$ & $<0.013$ & 0.018 & 0.004 & 0.001 \\
\hline \multirow[t]{2}{*}{14206298} & 940527 & $<0.006$ & $<0.008$ & $<0.008$ & 0.005 & 0.003 & $<0.005$ & $<0.010$ \\
\hline & 941123 & $<0.006$ & $<0.008$ & $<0.008$ & $<0.013$ & 0.002 & 0.007 & $<0.010$ \\
\hline 14206950 & 941027 & $<0.006$ & $<0.008$ & $<0.008$ & $<0.013$ & $<0.013$ & $<0.005$ & $<0.010$ \\
\hline
\end{tabular}


Table 39. Concentrations of triazine herbicides in filtered water from Phase II of the Willamette River Basin Water Quality Study, Oregon, 1994—Continued

\begin{tabular}{|c|c|c|c|c|c|c|c|}
\hline Station number & Date & $\begin{array}{l}\text { Hexazinone } \\
(\mathrm{ug} / \mathrm{L})\end{array}$ & $\begin{array}{c}\text { Metolachlor } \\
\text { (ug/L) }\end{array}$ & $\begin{array}{l}\text { Metribuzin } \\
\text { (ug/L) }\end{array}$ & $\begin{array}{l}\text { Prometon } \\
\text { (ug/L) }\end{array}$ & $\begin{array}{l}\text { Prometryn } \\
\text { (ug/L) }\end{array}$ & $\begin{array}{l}\text { Propachlor } \\
\text { (ug/L) }\end{array}$ \\
\hline 433548123040600 & 940520 & $<0.016$ & $<0.013$ & $<0.006$ & $<0.009$ & $<0.009$ & $<0.010$ \\
\hline 440045122585600 & 940519 & $<0.016$ & $<0.013$ & $<0.006$ & $<0.009$ & $<0.009$ & $<0.010$ \\
\hline 440402123063900 & 940613 & $<0.016$ & 0.004 & 0.050 & 0.008 & 0.002 & $<0.010$ \\
\hline 441310122095801 & 940527 & $<0.016$ & $<0.013$ & $<0.006$ & 0.001 & $<0.009$ & $<0.010$ \\
\hline 440707123041300 & 940519 & $<0.016$ & $<0.013$ & $<0.006$ & $<0.009$ & $<0.009$ & $<0.010$ \\
\hline 442223123153703 & 941101 & 0.001 & 0.008 & 0.002 & 0.003 & $<0.009$ & $<0.010$ \\
\hline \multirow[t]{3}{*}{443138123120901} & 941102 & 0.001 & 0.178 & 0.003 & $<0.009$ & $<0.009$ & $<0.010$ \\
\hline & 941106 & 0.008 & 0.439 & 0.081 & $<0.009$ & $<0.009$ & $<0.010$ \\
\hline & 941109 & 0.002 & 0.147 & 0.028 & 0.001 & $<0.009$ & $<0.010$ \\
\hline 443045123273000 & 940518 & $<0.016$ & $<0.013$ & $<0.006$ & $<0.009$ & $<0.009$ & $<0.010$ \\
\hline 443321123155201 & 940518 & $<0.016$ & $<0.013$ & $<0.006$ & $<0.009$ & $<0.009$ & $<0.010$ \\
\hline 14173500 & 941101 & 0.001 & 0.507 & 0.029 & 0.002 & $<0.009$ & 0.001 \\
\hline 444349123094000 & 940516 & 0.002 & 0.002 & $<0.006$ & $<0.009$ & $<0.009$ & $<0.010$ \\
\hline 444123122562200 & 940516 & 0.003 & 0.001 & $<0.006$ & $<0.009$ & $<0.009$ & $<0.010$ \\
\hline 14189000 & 940517 & $<0.016$ & 0.002 & $<0.006$ & $<0.009$ & $<0.009$ & $<0.010$ \\
\hline 445547123065400 & 940526 & 0.002 & 0.010 & $<0.006$ & 0.006 & $<0.009$ & $<0.010$ \\
\hline \multirow[t]{3}{*}{14190970} & 941123 & $<0.016$ & 0.005 & 0.007 & 0.005 & $<0.009$ & $<0.010$ \\
\hline & 941130 & $<0.016$ & 0.005 & 0.025 & 0.016 & $<0.009$ & $<0.010$ \\
\hline & 941130 & $<0.016$ & 0.005 & 0.025 & 0.016 & $<0.009$ & $<0.010$ \\
\hline \multirow[t]{4}{*}{445037122573800} & 940614 & $<0.016$ & 0.046 & $<0.006$ & 0.001 & $<0.009$ & $<0.010$ \\
\hline & 941031 & 0.003 & 0.131 & 0.009 & 0.006 & $<0.009$ & $<0.010$ \\
\hline & 941104 & 0.005 & 0.132 & 0.024 & 0.002 & $<0.009$ & $<0.010$ \\
\hline & 941104 & 0.005 & 0.130 & 0.017 & 0.003 & $<0.009$ & $<0.010$ \\
\hline \multirow[t]{2}{*}{14194150} & 940517 & $<0.016$ & 0.071 & $<0.006$ & $<0.009$ & $<0.009$ & $<0.010$ \\
\hline & 941102 & 0.001 & 0.014 & 0.001 & $<0.009$ & $<0.009$ & $<0.010$ \\
\hline 451355123093600 & 940517 & $<0.016$ & $<0.013$ & $<0.006$ & $<0.009$ & $<0.009$ & $<0.010$ \\
\hline 451602122564400 & 940531 & $<0.016$ & 0.006 & $<0.006$ & $<0.009$ & $<0.009$ & $<0.010$ \\
\hline \multirow[t]{2}{*}{451502122524700} & 940526 & 0.007 & 0.136 & $<0.006$ & $<0.009$ & $<0.009$ & $<0.010$ \\
\hline & 941031 & 0.001 & 0.842 & 0.006 & $<0.009$ & $<0.009$ & $<0.010$ \\
\hline 14201300 & 941028 & 0.005 & 0.122 & 0.004 & $<0.009$ & $<0.009$ & $<0.010$ \\
\hline \multirow[t]{3}{*}{14202000} & 941028 & 0.004 & 0.086 & 0.002 & $<0.009$ & $<0.009$ & $<0.010$ \\
\hline & 941029 & 0.003 & 0.035 & 0.001 & $<0.009$ & $<0.009$ & 0.003 \\
\hline & 941109 & 0.012 & 0.043 & 0.007 & $<0.009$ & $<0.009$ & $<0.010$ \\
\hline 451603122423301 & 940525 & $<0.016$ & $<0.013$ & $<0.006$ & $<0.009$ & $<0.009$ & $<0.010$ \\
\hline \multirow[t]{2}{*}{14206200} & 940527 & 0.004 & 0.008 & $<0.006$ & 0.003 & $<0.009$ & $<0.010$ \\
\hline & 941201 & 0.040 & 0.019 & 0.012 & 0.003 & $<0.009$ & $<0.010$ \\
\hline \multirow[t]{2}{*}{14206298} & 940527 & 0.003 & $<0.013$ & $<0.006$ & 0.010 & $<0.009$ & $<0.010$ \\
\hline & 941123 & $<0.016$ & 0.002 & 0.004 & 0.007 & $<0.009$ & $<0.010$ \\
\hline 14206950 & 941027 & $<0.016$ & 0.007 & 0.011 & 0.026 & $<0.009$ & 0.001 \\
\hline
\end{tabular}


Table 39. Concentrations of triazine herbicides in filtered water from Phase II of the Willamette River Basin Water Quality Study, Oregon, 1994—Continued

\begin{tabular}{|c|c|c|c|c|c|c|c|c|}
\hline Station number & Date & $\begin{array}{l}\text { Propazine } \\
\text { (ug/L) }\end{array}$ & $\begin{array}{l}\text { Simazine } \\
(\mathrm{ug} / \mathrm{L})\end{array}$ & $\begin{array}{c}\text { Simetryn } \\
\text { (ug/L) }\end{array}$ & $\begin{array}{c}\text { Terbacil } \\
\text { (ug/L) }\end{array}$ & $\begin{array}{c}\text { Trifluralin } \\
\text { (ug/L) }\end{array}$ & $\begin{array}{c}\text { Vernolate } \\
\text { (ug/L) }\end{array}$ & $\begin{array}{c}\text { Terbuthyl- } \\
\text { azine } \\
\text { (surrogate) } \\
\text { (percent) }\end{array}$ \\
\hline 433548123040600 & 940520 & $<0.008$ & $<0.014$ & $<0.006$ & $<0.011$ & $<0.003$ & $<0.011$ & 103.36 \\
\hline 440045122585600 & 940519 & $<0.008$ & $<0.014$ & $<0.006$ & $<0.011$ & $<0.003$ & $<0.011$ & 93.07 \\
\hline 440402123063900 & 940613 & $<0.008$ & 0.013 & $<0.006$ & $<0.011$ & $<0.003$ & $<0.011$ & 56.05 \\
\hline 441310122095801 & 940527 & $<0.008$ & $<0.014$ & $<0.006$ & $<0.011$ & $<0.003$ & $<0.011$ & 104.29 \\
\hline 440707123041300 & 940519 & $<0.008$ & $<0.014$ & $<0.006$ & $<0.011$ & $<0.003$ & $<0.011$ & 89.30 \\
\hline 442223123153703 & 941101 & $<0.008$ & 0.032 & $<0.006$ & 0.081 & 0.002 & 0.002 & 50.71 \\
\hline \multirow[t]{3}{*}{443138123120901} & 941102 & 0.003 & 0.222 & 0.001 & 0.026 & $<0.003$ & $<0.011$ & 57.94 \\
\hline & 941106 & 0.006 & 0.177 & 0.001 & 0.061 & $<0.003$ & $<0.011$ & 87.32 \\
\hline & 941109 & 0.004 & 0.054 & $<0.006$ & 0.069 & $<0.003$ & $<0.011$ & 57.66 \\
\hline 443045123273000 & 940518 & $<0.008$ & $<0.014$ & $<0.006$ & $<0.011$ & $<0.003$ & $<0.011$ & 91.36 \\
\hline 443321123155201 & 940518 & $<0.008$ & 0.004 & $<0.006$ & $<0.011$ & $<0.003$ & $<0.011$ & 105.86 \\
\hline 14173500 & 941101 & 0.004 & 0.064 & $<0.006$ & 0.004 & $<0.003$ & $<0.011$ & 63.41 \\
\hline 444349123094000 & 940516 & $<0.008$ & 0.001 & $<0.006$ & 0.009 & $<0.003$ & $<0.011$ & 98.78 \\
\hline 444123122562200 & 940516 & $<0.008$ & 0.001 & $<0.006$ & $<0.011$ & $<0.003$ & $<0.011$ & 91.98 \\
\hline 14189000 & 940517 & $<0.008$ & $<0.014$ & $<0.006$ & $<0.011$ & $<0.003$ & $<0.011$ & 91.34 \\
\hline 445547123065400 & 940526 & $<0.008$ & 0.038 & $<0.006$ & $<0.011$ & $<0.003$ & $<0.011$ & 90.57 \\
\hline \multirow[t]{3}{*}{14190970} & 941123 & $<0.008$ & 0.033 & 0.001 & $<0.011$ & 0.002 & $<0.011$ & 74.10 \\
\hline & 941130 & $<0.008$ & 0.074 & $<0.006$ & $<0.011$ & 0.002 & $<0.011$ & 98.11 \\
\hline & 941130 & $<0.008$ & 0.076 & $<0.006$ & $<0.011$ & 0.001 & $<0.011$ & 97.29 \\
\hline \multirow[t]{4}{*}{445037122573800} & 940614 & $<0.008$ & 0.025 & $<0.006$ & 0.058 & $<0.003$ & $<0.011$ & 71.13 \\
\hline & 941031 & 0.009 & 0.050 & 0.001 & 0.812 & 0.001 & $<0.011$ & $73 . .77$ \\
\hline & 941104 & 0.004 & 0.070 & $<0.006$ & 0.344 & $<0.003$ & $<0.011$ & 58.45 \\
\hline & 941104 & 0.003 & 0.089 & $<0.006$ & 0.342 & $<0.003$ & $<0.011$ & 53.25 \\
\hline \multirow[t]{2}{*}{14194150} & 940517 & $<0.008$ & 0.009 & $<0.006$ & $<0.011$ & $<0.003$ & $<0.011$ & 76.81 \\
\hline & 941102 & $<0.008$ & 0.010 & $<0.006$ & $<0.011$ & $<0.003$ & $<0.011$ & 70.29 \\
\hline 451355123093600 & 940517 & $<0.008$ & 0.001 & $<0.006$ & 0.007 & $<0.003$ & $<0.011$ & 76.61 \\
\hline 451602122564400 & 940531 & $<0.008$ & $<0.014$ & $<0.006$ & $<0.011$ & $<0.003$ & $<0.011$ & 115.70 \\
\hline \multirow[t]{2}{*}{451502122524700} & 940526 & $<0.008$ & 0.081 & $<0.006$ & $<0.011$ & $<0.003$ & $<0.011$ & 94.17 \\
\hline & 941031 & $<0.008$ & 0.393 & 0.001 & 0.008 & $<0.003$ & $<0.011$ & 62.12 \\
\hline 14201300 & 941028 & 0.008 & 0.242 & $<0.006$ & 0.018 & 0.004 & 0.002 & 59.91 \\
\hline \multirow[t]{3}{*}{14202000} & 941028 & 0.001 & 0.102 & 0.001 & 0.006 & $<0.003$ & $<0.011$ & 67.65 \\
\hline & 941029 & $<0.008$ & 0.056 & $<0.006$ & 0.006 & $<0.003$ & $<0.011$ & 58.98 \\
\hline & 941109 & 0.001 & 0.034 & $<0.006$ & 0.005 & $<0.003$ & $<0.011$ & 68.46 \\
\hline 451603122423301 & 940525 & $<0.008$ & 0.001 & $<0.006$ & $<0.011$ & $<0.003$ & $<0.011$ & 91.42 \\
\hline \multirow[t]{2}{*}{14206200} & 940527 & $<0.008$ & 0.022 & $<0.006$ & 0.004 & $<0.003$ & $<0.011$ & 129.01 \\
\hline & 941201 & 0.005 & 0.072 & $<0.006$ & 0.014 & $<0.003$ & $<0.011$ & 84.69 \\
\hline \multirow[t]{2}{*}{14206298} & 940527 & $<0.008$ & 0.035 & 0.004 & $<0.011$ & $<0.003$ & $<0.011$ & 127.04 \\
\hline & 941123 & $<0.008$ & 0.246 & $<0.006$ & $<0.011$ & $<0.003$ & $<0.011$ & 77.36 \\
\hline 14206950 & 941027 & $<0.008$ & 0.048 & $<0.006$ & $<0.011$ & $<0.003$ & $<0.011$ & 53.52 \\
\hline
\end{tabular}


Table 39. Concentrations of triazine herbicides in filtered water from Phase II of the Willamette River Basin Water Quality Study, Oregon, 1994-Continued

\begin{tabular}{|c|c|c|c|c|c|c|c|c|}
\hline Station Name & Station number & Date & Time & $\begin{array}{l}\text { Alachlor } \\
\text { (ug/L) }\end{array}$ & $\begin{array}{l}\text { Ametryn } \\
(\mathrm{ug} / \mathrm{L})\end{array}$ & $\begin{array}{l}\text { Atrazine } \\
\text { (ug/L) }\end{array}$ & $\begin{array}{c}\text { Bromacil } \\
\text { (ug/L) }\end{array}$ & $\begin{array}{l}\text { Butachlor } \\
\text { (ug/L) }\end{array}$ \\
\hline \multirow[t]{2}{*}{ Tualatin R at West Linn, OR } & \multirow[t]{2}{*}{14207500} & 940525 & 1550 & $<0.009$ & $<0.008$ & 0.022 & $<0.025$ & $<0.010$ \\
\hline & & 941028 & 1620 & $<0.009$ & $<0.008$ & 0.006 & $<0.025$ & $<0.010$ \\
\hline \multirow[t]{6}{*}{ Johnson Cr nr Gresham, OR } & \multirow[t]{6}{*}{452823122240900} & 940524 & 1450 & $<0.009$ & $<0.008$ & 0.020 & $<0.025$ & $<0.010$ \\
\hline & & 941027 & 1650 & $<0.009$ & $<0.008$ & 0.059 & $<0.025$ & $<0.010$ \\
\hline & & 941028 & 1145 & $<0.009$ & $<0.008$ & 0.030 & $<0.025$ & $<0.010$ \\
\hline & & 941101 & 1110 & $<0.009$ & $<0.008$ & 0.019 & $<0.025$ & $<0.010$ \\
\hline & & 941103 & 1340 & $<0.009$ & $<0.008$ & 0.016 & $<0.025$ & $<0.010$ \\
\hline & & 941123 & 1420 & $<0.009$ & $<0.008$ & 0.022 & $<0.025$ & $<0.010$ \\
\hline Urban outfall, Portland, OR & 453043122402200 & 940617 & 2150 & $<0.009$ & $<0.008$ & 0.010 & $<0.025$ & $<0.010$ \\
\hline \multirow[t]{2}{*}{ Willamette R at Portland, OR } & \multirow[t]{2}{*}{14211720} & 941029 & 1240 & $<0.009$ & $<0.008$ & 0.012 & 0.011 & $<0.010$ \\
\hline & & 941103 & 0956 & $<0.009$ & $<0.008$ & 0.051 & $<0.025$ & $<0.010$ \\
\hline \multirow{6}{*}{$\begin{array}{l}\text { Interstate } 84 \text { Transportation Corridor } \\
\text { at Portland, OR }\end{array}$} & \multirow[t]{6}{*}{453154122394200} & 940531 & 1810 & $<0.009$ & $<0.008$ & 0.012 & $<0.025$ & $<0.010$ \\
\hline & & 941108 & 1810 & $<0.009$ & $<0.008$ & 0.013 & $<0.025$ & $<0.010$ \\
\hline & & 941108 & 2000 & $<0.009$ & $<0.008$ & 0.011 & $<0.025$ & $<0.010$ \\
\hline & & 941130 & 0850 & $<0.009$ & $<0.008$ & $<0.011$ & $<0.025$ & $<0.010$ \\
\hline & & 941130 & 1030 & $<0.009$ & $<0.008$ & $<0.011$ & $<0.025$ & $<0.010$ \\
\hline & & 941202 & 1030 & $<0.009$ & $<0.008$ & 0.007 & $<0.025$ & $<0.010$ \\
\hline $\begin{array}{l}\text { Willamette R ab St. John's Bridge, } \\
\text { Portland, OR }\end{array}$ & 14211805 & 940523 & 1000 & $<0.009$ & $<0.008$ & 0.009 & 0.003 & $<0.010$ \\
\hline
\end{tabular}


Table 39. Concentrations of triazine herbicides in filtered water from Phase II of the Willamette River Basin Water Quality Study, Oregon, 1994-Continued

\begin{tabular}{|c|c|c|c|c|c|c|c|c|}
\hline Station number & Date & $\begin{array}{c}\text { Butylate } \\
\text { (ug/L) }\end{array}$ & $\begin{array}{c}\text { Carboxin } \\
\text { (ug/L) }\end{array}$ & $\begin{array}{c}\text { Cyanazine } \\
\text { (ug/L) }\end{array}$ & $\begin{array}{c}\text { Cycloate } \\
\text { (Estimated) } \\
\text { (ug/L) }\end{array}$ & $\begin{array}{l}\text { Desethyl- } \\
\text { atrazine } \\
\text { (Estimated) } \\
\text { (ug/L) }\end{array}$ & $\begin{array}{c}\text { Desisoproyl- } \\
\text { atrazine } \\
\text { (Estimated) } \\
\text { (ug/L) }\end{array}$ & $\begin{array}{c}\text { Diphenamid } \\
(\mathrm{ug} / \mathrm{L})\end{array}$ \\
\hline \multirow[t]{2}{*}{14207500} & 940525 & $<0.006$ & $<0.008$ & $<0.008$ & 0.003 & 0.002 & $<0.005$ & $<0.010$ \\
\hline & 941028 & $<0.006$ & $<0.008$ & $<0.008$ & $<0.013$ & 0.001 & $<0.005$ & 0.001 \\
\hline \multirow[t]{6}{*}{452823122240900} & 940524 & $<0.006$ & $<0.008$ & $<0.008$ & $<0.013$ & 0.005 & $<0.005$ & $<0.010$ \\
\hline & 941027 & $<0.006$ & $<0.008$ & $<0.008$ & $<0.013$ & 0.003 & 0.002 & 0.003 \\
\hline & 941028 & $<0.006$ & $<0.008$ & $<0.008$ & $<0.013$ & 0.004 & 0.002 & 0.002 \\
\hline & 941101 & $<0.006$ & $<0.008$ & $<0.008$ & $<0.013$ & 0.002 & $<0.005$ & 0.001 \\
\hline & 941103 & $<0.006$ & $<0.008$ & $<0.008$ & $<0.013$ & 0.003 & 0.002 & 0.001 \\
\hline & 941123 & $<0.006$ & $<0.008$ & $<0.008$ & $<0.013$ & 0.005 & $<0.005$ & 0.001 \\
\hline 453043122402200 & 940617 & $<0.006$ & $<0.008$ & $<0.008$ & $<0.013$ & $<0.013$ & $<0.005$ & $<0.010$ \\
\hline \multirow[t]{2}{*}{14211720} & 941029 & $<0.006$ & $<0.008$ & $<0.008$ & 0.001 & 0.001 & 0.002 & $<0.010$ \\
\hline & 941103 & $<0.006$ & $<0.008$ & $<0.008$ & $<0.013$ & 0.001 & $<0.005$ & $<0.010$ \\
\hline \multirow[t]{6}{*}{453154122394200} & 940531 & $<0.006$ & $<0.008$ & $<0.008$ & $<0.013$ & $<0.013$ & $<0.005$ & $<0.010$ \\
\hline & 941108 & $<0.006$ & $<0.008$ & $<0.008$ & $<0.013$ & $<0.013$ & $<0.005$ & $<0.010$ \\
\hline & 941108 & $<0.006$ & $<0.008$ & $<0.008$ & $<0.013$ & $<0.013$ & $<0.005$ & $<0.010$ \\
\hline & 941130 & $<0.006$ & $<0.008$ & $<0.008$ & $<0.013$ & $<0.013$ & $<0.005$ & $<0.010$ \\
\hline & 941130 & $<0.006$ & $<0.008$ & $<0.008$ & $<0.013$ & $<0.013$ & $<0.005$ & $<0.010$ \\
\hline & 941202 & $<0.006$ & $<0.008$ & $<0.008$ & 0.002 & 0.018 & 0.023 & $<0.010$ \\
\hline 14211805 & 940523 & $<0.006$ & $<0.008$ & $<0.008$ & 0.003 & $<0.013$ & $<0.005$ & $<0.010$ \\
\hline
\end{tabular}


Table 39. Concentrations of triazine herbicides in filtered water from Phase II of the Willamette River Basin Water Quality Study, Oregon, 1994—Continued

\begin{tabular}{lccccccc}
\hline \multirow{2}{*}{ Station number } & Date & $\begin{array}{c}\text { Hexazinone } \\
\text { (ug/L) }\end{array}$ & $\begin{array}{c}\text { Metolachlor } \\
(\mathrm{ug} / \mathrm{L})\end{array}$ & $\begin{array}{c}\text { Metribuzin } \\
(\mathrm{ug} / \mathrm{L})\end{array}$ & $\begin{array}{c}\text { Prometon } \\
(\mathrm{ug} / \mathrm{L})\end{array}$ & $\begin{array}{c}\text { Prometryn } \\
\text { (ug/L) }\end{array}$ & $\begin{array}{c}\text { Propachlor } \\
(\mathrm{ug} / \mathrm{L})\end{array}$ \\
\hline 14207500 & 940525 & $<0.016$ & 0.046 & $<0.006$ & $<0.009$ & $<0.009$ & $<0.010$ \\
& 941028 & $<0.016$ & 0.010 & $<0.006$ & 0.009 & $<0.009$ & $<0.010$ \\
452823122240900 & 940524 & 0.005 & 0.021 & $<0.006$ & 0.004 & $<0.009$ & $<0.010$ \\
& 941027 & 0.002 & 0.110 & 0.002 & 0.004 & $<0.009$ & $<0.010$ \\
& 941028 & 0.003 & 0.079 & 0.001 & 0.004 & $<0.009$ & $<0.010$ \\
453043122402200 & 940617 & $<0.016$ & 0.017 & 0.147 & $<0.009$ & $<0.009$ & $<0.010$ \\
14211720 & 941101 & 0.006 & 0.067 & $<0.006$ & 0.003 & $<0.009$ & $<0.010$ \\
& 941029 & 0.002 & 0.020 & $<0.006$ & 0.001 & $<0.009$ & $<0.010$ \\
453154122394200 & 941103 & 0.002 & 0.036 & 0.002 & 0.001 & $<0.009$ & $<0.010$ \\
& 940531 & $<0.016$ & 0.005 & 0.158 & $<0.009$ & $<0.009$ & $<0.010$ \\
& 941108 & $<0.016$ & 0.032 & 0.090 & $<0.009$ & $<0.009$ & $<0.010$ \\
& 941108 & $<0.016$ & 0.052 & 0.103 & $<0.009$ & $<0.009$ & $<0.010$ \\
& 941130 & $<0.016$ & 0.040 & 0.135 & $<0.009$ & $<0.009$ & $<0.010$ \\
& 941130 & $<0.016$ & 0.007 & 0.113 & $<0.009$ & $<0.009$ & $<0.010$ \\
& 941202 & $<0.016$ & 0.002 & 0.028 & 0.005 & $<0.009$ & $<0.010$ \\
& 940523 & 0.001 & 0.002 & $<0.006$ & $<0.009$ & $<0.009$ & $<0.010$
\end{tabular}


Table 39. Concentrations of triazine herbicides in filtered water from Phase II of the Willamette River Basin Water Quality Study, Oregon, 1994—Continued

\begin{tabular}{|c|c|c|c|c|c|c|c|c|}
\hline Station number & Date & $\begin{array}{l}\text { Propazine } \\
\text { (ug/L) }\end{array}$ & $\begin{array}{c}\text { Simazine } \\
\text { (ug/L) }\end{array}$ & $\begin{array}{l}\text { Simetryn } \\
\text { (ug/L) }\end{array}$ & $\begin{array}{c}\text { Terbacil } \\
\text { (ug/L) }\end{array}$ & $\begin{array}{l}\text { Trifluralin } \\
\text { (ug/L) }\end{array}$ & $\begin{array}{c}\text { Vernolate } \\
\text { (ug/L) }\end{array}$ & $\begin{array}{c}\text { Terbuthyl- } \\
\text { azine } \\
\text { (surrogate) } \\
\text { (percent) }\end{array}$ \\
\hline \multirow[t]{2}{*}{14207500} & 940525 & $<0.008$ & 0.001 & $<0.006$ & 0.297 & $<0.003$ & $<0.011$ & 87 \\
\hline & 941028 & $<0.008$ & 0.025 & $<0.006$ & $<0.011$ & 0.001 & $<0.011$ & 55 \\
\hline \multirow[t]{6}{*}{452823122240900} & 940524 & $<0.008$ & 0.065 & $<0.006$ & $<0.011$ & 0.004 & $<0.011$ & 52 \\
\hline & 941027 & 0.001 & 0.037 & $<0.006$ & 0.006 & 0.002 & $<0.011$ & 56 \\
\hline & 941028 & $<0.008$ & 0.022 & $<0.006$ & 0.007 & 0.002 & $<0.011$ & 59 \\
\hline & 941101 & $<0.008$ & 0.019 & $<0.006$ & 0.005 & 0.001 & $<0.011$ & 53 \\
\hline & 941103 & $<0.008$ & 0.011 & $<0.006$ & 0.005 & 0.001 & $<0.011$ & 67 \\
\hline & 941123 & $<0.008$ & 0.007 & $<0.006$ & $<0.011$ & 0.003 & $<0.011$ & 83 \\
\hline 453043122402200 & 940617 & $<0.008$ & 0.067 & $<0.006$ & $<0.011$ & $<0.003$ & $<0.011$ & 59 \\
\hline \multirow[t]{2}{*}{14211720} & 941029 & $<0.008$ & 0.018 & $<0.006$ & 0.010 & $<0.003$ & $<0.011$ & 62 \\
\hline & 941103 & $<0.008$ & 0.017 & $<0.006$ & 0.014 & $<0.003$ & $<0.011$ & 52 \\
\hline \multirow[t]{6}{*}{453154122394200} & 940531 & $<0.008$ & 0.014 & $<0.006$ & 0.013 & $<0.003$ & $<0.011$ & 39 \\
\hline & 941108 & $<0.008$ & 0.008 & $<0.006$ & 0.042 & $<0.003$ & $<0.011$ & 56 \\
\hline & 941108 & $<0.008$ & 0.007 & $<0.006$ & 0.036 & 0.001 & $<0.011$ & 78 \\
\hline & 941130 & $<0.008$ & $<0.014$ & $<0.006$ & $<0.011$ & $<0.003$ & $<0.011$ & 92 \\
\hline & 941130 & $<0.008$ & $<0.014$ & $<0.006$ & $<0.011$ & 0.001 & $<0.011$ & 82 \\
\hline & 941202 & $<0.008$ & 0.032 & 0.001 & $<0.011$ & $<0.003$ & $<0.011$ & 100 \\
\hline 14211805 & 940523 & $<0.008$ & 0.007 & $<0.006$ & 0.020 & $<0.003$ & $<0.011$ & 77 \\
\hline
\end{tabular}


Page Intentionally Blank 


\section{QUALITY-ASSURANCE DATA}


Table 40. Concentrations of trace elements in splits of whole water samples from Phase II of the Willamette River Basin Water Quality Study, Oregon, 1994

[Concentrations in micrograms per liter]

\begin{tabular}{lccc}
\hline \begin{tabular}{c} 
Sample type \\
Station \\
\multicolumn{1}{c}{ Date }
\end{tabular} & \multicolumn{3}{c}{$\begin{array}{c}\text { Split } \\
\text { 453154122394200 } \\
94-11-08\end{array}$} \\
\hline \multicolumn{1}{c}{ Analyte } & $\begin{array}{c}\text { Sample } \\
\text { Aample }\end{array}$ & $\begin{array}{c}\text { Relative } \\
\text { percent } \\
\text { difference }\end{array}$ \\
\hline Arsenic & 1 & 2 & 0.0 \\
Cadmium & 1.0 & 1.0 & 0.0 \\
Chromium & 2.0 & 2.0 & 3.4 \\
Copper & 8.6 & 8.9 & 0.0 \\
Lead & 19.0 & 19.0 & 5.3 \\
Nickel & 39.0 & 37.0 & 0.0 \\
Zinc & 8.0 & 8.0 & 0.0 \\
\hline
\end{tabular}

Table 41. Concentrations of trace elements in splits of filtered water samples from Phase II of the Willamette River Basin Water Quality Study, Oregon, 1994

[<, less than; --, not calculated; concentrations in micrograms per liter]

\begin{tabular}{|c|c|c|c|c|c|}
\hline $\begin{array}{l}\text { Sample type } \\
\text { Station } \\
\text { Date }\end{array}$ & & \multicolumn{4}{|c|}{$\begin{array}{c}\text { Split } \\
453154122394200 \\
94-11-08\end{array}$} \\
\hline Analyte & & $\begin{array}{c}\text { Sample } \\
1\end{array}$ & & $\begin{array}{c}\text { Sample } \\
2\end{array}$ & $\begin{array}{l}\text { Relative } \\
\text { percent } \\
\text { difference }\end{array}$ \\
\hline Arsenic & & 2.0 & & 1.0 & 66.7 \\
\hline Aluminum & & 203.0 & & 180.0 & 12.0 \\
\hline Antimony & & 2.0 & & 2.0 & 0.0 \\
\hline Barium & & 52.0 & & 52.0 & 0.0 \\
\hline Beryllium & $<$ & 1.0 & $<$ & 1.0 & -- \\
\hline Cadmium & $<$ & 1.0 & $<$ & 1.0 & -- \\
\hline Chromium & & 2.0 & & 2.0 & 0.0 \\
\hline Cobalt & $<$ & 1.0 & $<$ & 1.0 & -- \\
\hline Copper & & 9.0 & & 7.0 & 25.0 \\
\hline Lead & & 3.0 & & 2.0 & 40.0 \\
\hline Manganese & & 28.0 & & 27.0 & 3.6 \\
\hline Molybdenum & & 2.0 & & 2.0 & 0.0 \\
\hline Nickel & & 4.0 & & 3.0 & 28.6 \\
\hline Selenium & $<$ & 1.0 & $<$ & 1.0 & -- \\
\hline Silver & $<$ & 1.0 & $<$ & 1.0 & -- \\
\hline Uranium & $<$ & 1.0 & $<$ & 1.0 & -- \\
\hline Zinc & & 54.0 & & 55.0 & 1.8 \\
\hline
\end{tabular}


Table 42. Concentrations of organochlorine compounds in splits and field matrix spikes of whole water samples from Phase II of the Willamette River Basin Water Quality Study, Oregon, 1994

[USGS laboratory schedule 1398; < = less than; --, not calculated; concentrations in milligrams per liter; NS, analyte not in spike solution; computed concentration, ((spike volume * spike concentration)/sample volume) + native water concentration; measured concentration, value from laboratory analysis; percent recovery, (measured concentration/computed concentration) $* 100]$

\begin{tabular}{|c|c|c|c|c|c|c|c|c|c|c|c|c|c|c|c|}
\hline $\begin{array}{l}\text { Sample type } \\
\text { Station } \\
\text { Date }\end{array}$ & $\begin{array}{r}\text { Sp } \\
4431381 \\
94-1\end{array}$ & $\begin{array}{l}\text { it } 1 \\
23120901 \\
1-06\end{array}$ & $\begin{array}{r}\mathrm{Sp} \\
4455471 \\
94-\end{array}$ & $\begin{array}{l}\text { lit } 2 \\
23065400 \\
5-26\end{array}$ & $\begin{array}{r}\text { Sp } \\
4450371 \\
94-\end{array}$ & $\begin{array}{l}\text { lit } 3 \\
22573800 \\
10-31\end{array}$ & & $\begin{array}{c}\mathrm{d} \text { matrix sp } \\
3138123120 \\
94-11-06\end{array}$ & $\begin{array}{l}\text { ike } 1 \\
901\end{array}$ & $\begin{array}{r}\text { Field } \\
443\end{array}$ & $\begin{array}{c}\mathrm{d} \text { matrix } \mathrm{sp} \\
3138123120 \\
94-11-06\end{array}$ & $\begin{array}{l}\text { ike } 2 \\
901\end{array}$ & $\begin{array}{r}\text { Fielc } \\
451\end{array}$ & $\begin{array}{c}\text { matrix sp } \\
502122524 \\
94-05-26\end{array}$ & $\begin{array}{l}\text { ike } 3 \\
\text { t701 }\end{array}$ \\
\hline Analyte & Sample 1 & Sample 2 & Sample 1 & Sample 2 & Sample 1 & Sample 2 & $\begin{array}{c}\text { Measured } \\
\text { concen- } \\
\text { tration }\end{array}$ & $\begin{array}{c}\text { Computed } \\
\text { concen- } \\
\text { tration }\end{array}$ & $\begin{array}{l}\text { Percent } \\
\text { recovery }\end{array}$ & $\begin{array}{c}\text { Measured } \\
\text { concen- } \\
\text { tration }\end{array}$ & $\begin{array}{c}\text { Computed } \\
\text { concen- } \\
\text { tration }\end{array}$ & $\begin{array}{l}\text { Percent } \\
\text { recovery }\end{array}$ & $\begin{array}{c}\text { Measured } \\
\text { concen- } \\
\text { tration }\end{array}$ & $\begin{array}{l}\text { Computed } \\
\text { concen- } \\
\text { tration }\end{array}$ & $\begin{array}{l}\text { Percent } \\
\text { recovery }\end{array}$ \\
\hline Perthane & $<0.100$ & $<0.100$ & $<0.100$ & $<0.100$ & $<0.100$ & $<0.100$ & $<0.100$ & NS & -- & $<0.100$ & NS & -- & $<0.100$ & NS & -- \\
\hline Chlordane & $<0.100$ & $<0.100$ & $<0.100$ & $<0.100$ & $<0.100$ & $<0.100$ & $<0.100$ & NS & -- & $<0.100$ & NS & -- & $<0.100$ & NS & -- \\
\hline Toxaphene & $<1.000$ & $<1.000$ & $<1.000$ & $<1.000$ & $<1.000$ & $<1.000$ & $<1.000$ & NS & -- & $<1.000$ & NS & -- & $<1.000$ & NS & -- \\
\hline PCB & $<0.100$ & $<0.100$ & $<0.100$ & $<0.100$ & $<0.100$ & $<0.100$ & $<0.100$ & NS & -- & $<0.100$ & NS & -- & $<0.100$ & NS & -- \\
\hline $\mathrm{PCN}$ & $<0.100$ & $<0.100$ & $<0.100$ & $<0.100$ & $<0.100$ & $<0.100$ & $<0.100$ & NS & -- & $<0.100$ & NS & -- & $<0.100$ & NS & -- \\
\hline Methoxychlor & $<0.010$ & $<0.010$ & $<0.010$ & $<0.010$ & $<0.010$ & $<0.010$ & $<0.010$ & NS & -- & $<0.010$ & NS & -- & $<0.010$ & NS & -- \\
\hline Mirex & $<0.010$ & $<0.010$ & $<0.010$ & $<0.010$ & $<0.010$ & $<0.010$ & $<0.010$ & NS & -- & $<0.010$ & NS & -- & $<0.010$ & NS & -- \\
\hline Endosulfan I & $<0.001$ & $<0.001$ & $<0.001$ & $<0.001$ & $<0.001$ & $<0.001$ & 0.500 & 1.162 & 43.0 & 0.500 & 1.180 & 42.4 & $<0.001$ & NS & -- \\
\hline Aldrin & $<0.001$ & $<0.001$ & $<0.001$ & $<0.001$ & $<0.001$ & $<0.001$ & 0.300 & 0.581 & 51.7 & 0.300 & 0.590 & 50.9 & $<0.001$ & NS & -- \\
\hline DDD & $<0.001$ & $<0.001$ & $<0.001$ & $<0.001$ & $<0.001$ & $<0.001$ & 1.400 & 3.486 & 40.2 & 1.400 & 3.540 & 39.5 & $<0.001$ & NS & -- \\
\hline DDE & $<0.001$ & $<0.001$ & $<0.001$ & $<0.001$ & $<0.001$ & $<0.001$ & 0.500 & 1.162 & 43.0 & 0.500 & 1.180 & 42.4 & 0.070 & 0.112 & 62.6 \\
\hline DDT & $<0.001$ & $<0.001$ & $<0.001$ & $<0.001$ & $<0.001$ & $<0.001$ & 1.000 & 3.486 & 28.7 & 1.000 & 3.540 & 28.2 & $<0.001$ & NS & -- \\
\hline Dieldrin & $<0.001$ & $<0.001$ & $<0.001$ & $<0.001$ & $<0.001$ & $<0.001$ & 0.400 & 1.162 & 34.4 & 0.400 & 1.180 & 33.9 & 0.072 & 0.113 & 63.8 \\
\hline Endrin & $<0.001$ & $<0.001$ & $<0.001$ & $<0.001$ & $<0.001$ & $<0.001$ & 0.700 & 1.162 & 60.2 & 0.700 & 1.180 & 59.3 & $<0.001$ & NS & -- \\
\hline Heptachlor & $<0.001$ & $<0.001$ & $<0.001$ & $<0.001$ & $<0.001$ & $<0.001$ & 0.300 & 0.581 & 51.7 & 0.300 & 0.590 & 50.9 & $<0.001$ & NS & -- \\
\hline Heptachlor epoxide & $<0.001$ & $<0.001$ & $<0.001$ & $<0.001$ & $<0.001$ & $<0.001$ & 0.300 & 0.581 & 51.7 & 0.300 & 0.590 & 50.9 & $<0.001$ & NS & -- \\
\hline Lindane & $<0.001$ & $<0.001$ & $<0.001$ & $<0.001$ & $<0.001$ & $<0.001$ & 0.300 & 0.581 & 51.7 & 0.400 & 0.590 & 67.8 & 0.100 & 0.144 & 69.5 \\
\hline
\end{tabular}


Table 43. Concentrations of pesticides analyzed by gas chromatography/mass spectroscopy in splits and field matrix spikes of filtered water samples from Phase II of the Willamette River Basin Water Quality Study, Oregon, 1994

[USGS laboratory schedule 2010; <, less than method detection limit; E, estimate; --, not calculated; concentrations in micrograms per liter; Breakthrough, concentration passing through first solid-phase extraction cartridge into second cartridge; computed concentration, ((spike volume * spike concentration )/sample volume) + native water concentration; measured concentration, value from laboratory analysis; percent recovery, (measured concentration/computed concentration) $* 100]$

\begin{tabular}{|c|c|c|c|c|c|c|c|c|c|c|c|c|c|c|c|c|}
\hline $\begin{array}{l}\text { Sample type } \\
\text { Station } \\
\text { Date }\end{array}$ & & $\begin{array}{c}\text { Split 1 } \\
554712306 \\
94-05-26\end{array}$ & 5400 & & $\begin{array}{c}\text { Split 2 } \\
282312224 \\
94-11-01\end{array}$ & 0900 & & $\begin{array}{r}\text { Field matr } \\
1419 \\
94-1\end{array}$ & $\begin{array}{l}\text { rix spike } 1 \\
90970 \\
11-23\end{array}$ & & $\begin{array}{r}\text { Fiel } \\
44\end{array}$ & $\begin{array}{c}\mathrm{d} \text { matrix spik } \\
3138123120 \\
94-11-06\end{array}$ & $\begin{array}{l}\text { ike } 2 \\
6901\end{array}$ & & $\begin{array}{c}\text { ld matrix spi } \\
1502122524 \\
94-05-26\end{array}$ & $\begin{array}{l}\text { ike } 3 \\
4701\end{array}$ \\
\hline Analyte & $\begin{array}{c}\text { Sample } \\
1\end{array}$ & $\begin{array}{c}\text { Sample } \\
2\end{array}$ & $\begin{array}{c}\text { Relative } \\
\text { percent } \\
\text { difference }\end{array}$ & $\begin{array}{c}\text { Sample } \\
1\end{array}$ & $\begin{array}{c}\text { Sample } \\
2\end{array}$ & $\begin{array}{c}\text { Relative } \\
\text { percent } \\
\text { difference }\end{array}$ & $\begin{array}{c}\text { Measured } \\
\text { concen- } \\
\text { tration }\end{array}$ & $\begin{array}{c}\text { Computed } \\
\text { concen- } \\
\text { tration }\end{array}$ & $\begin{array}{l}\text { Percent } \\
\text { recovery }\end{array}$ & $\begin{array}{c}\text { Break } \\
\text { through }\end{array}$ & $\begin{array}{c}\text { Measured } \\
\text { concen- } \\
\text { tration }\end{array}$ & $\begin{array}{l}\text { Computed } \\
\text { concen- } \\
\text { tration }\end{array}$ & $\begin{array}{c}\text { Percent } \\
\text { recovery }\end{array}$ & $\begin{array}{c}\text { Measured } \\
\text { concen- } \\
\text { tration }\end{array}$ & $\begin{array}{l}\text { Computed } \\
\text { concen- } \\
\text { tration }\end{array}$ & $\begin{array}{c}\text { Percent } \\
\text { recovery }\end{array}$ \\
\hline Alachlor & $<0.009$ & $<0.009$ & \begin{tabular}{l|l}
-- &
\end{tabular} & $<0.009$ & $<0.009$ & -- & 0.140 & 0.110 & 127.3 & 0.009 & 0.120 & 0.111 & 108.1 & 0.110 & 0.111 & 99.1 \\
\hline Atrazine & 0.046 & 0.047 & 2.2 & 0.047 & 0.047 & 0.0 & 0.160 & 0.152 & 105.3 & $<0.017$ & 1.000 & 0.922 & 108.5 & 0.240 & 0.252 & 95.2 \\
\hline Azinphos methyl & $<0.038$ & $<0.038$ & -- & $<0.038$ & $<0.038$ & -- & E0.120 & 0.110 & 109.1 & $<0.038$ & E0.130 & 0.102 & 127.4 & 0.250 & 0.102 & 245.0 \\
\hline Benflural & $<0.013$ & $<0.013$ & -- & $<0.013$ & $<0.013$ & -- & 0.093 & 0.110 & 84.5 & $<0.013$ & 0.089 & 0.102 & 87.2 & 0.096 & 0.102 & 94.1 \\
\hline Butylate & $<0.008$ & $<0.008$ & -- & $<0.008$ & $<0.008$ & -- & 0.120 & 0.110 & 109.1 & $<0.008$ & 0.100 & 0.102 & 98.0 & 0.100 & 0.102 & 98.0 \\
\hline Carbaryl & 0.005 & 0.007 & 33.3 & $<0.046$ & $<0.046$ & -- & E0.120 & 0.110 & 109.1 & $<0.046$ & E0.120 & 0.102 & 117.6 & 0.160 & 0.102 & 156.8 \\
\hline Carbofuran & $<0.013$ & $<0.013$ & -- & $<0.013$ & $<0.013$ & -- & E0.130 & 0.110 & 118.2 & $<0.013$ & E0.110 & 0.102 & 107.8 & 0.130 & 0.102 & 127.4 \\
\hline Chlorpyrifos & $<0.005$ & $<0.005$ & -- & $<0.005$ & $<0.005$ & -- & 0.130 & 0.110 & 118.2 & $<0.005$ & 0.120 & 0.102 & 117.6 & 0.110 & 0.102 & 107.8 \\
\hline Cyanazine & $<0.013$ & $<0.013$ & -- & $<0.013$ & $<0.013$ & -- & 0.130 & 0.110 & 118.2 & $<0.013$ & 0.100 & 0.102 & 98.0 & 0.099 & 0.102 & 97.0 \\
\hline DCPA & $<0.004$ & $<0.004$ & -- & $<0.004$ & $<0.004$ & -- & 0.130 & 0.110 & 118.2 & $<0.004$ & 0.120 & 0.102 & 117.6 & 0.120 & 0.103 & 116.5 \\
\hline DDE $p, p^{\prime}$ & $<0.010$ & $<0.010$ & -- & E0.003 & E0.002 & 40.0 & 0.066 & 0.110 & 60.0 & E0.007 & 0.073 & 0.102 & 71.5 & 0.072 & 0.102 & 70.6 \\
\hline Desethyl atrazine & $<0.007$ & $<0.007$ & -- & E0.005 & E0.010 & 66.7 & E0.053 & 0.121 & 43.8 & E0.037 & E0.048 & 0.117 & 41.0 & 0.045 & 0.124 & 36.3 \\
\hline Diazinon & 0.005 & 0.006 & 18.2 & E0.008 & E0.007 & 13.3 & 0.100 & 0.110 & 90.9 & $<0.008$ & 0.083 & 0.102 & 81.3 & 0.090 & 0.102 & 88.2 \\
\hline Dieldrin & $<0.008$ & $<0.008$ & -- & 0.016 & 0.015 & 6.5 & 0.120 & 0.110 & 109.1 & $<0.008$ & 0.097 & 0.102 & 95.1 & 0.093 & 0.102 & 91.1 \\
\hline Diethylaniline & $<0.006$ & $<0.006$ & -- & $<0.006$ & $<0.006$ & -- & 0.095 & 0.110 & 86.4 & $<0.006$ & 0.076 & 0.102 & 74.5 & 0.090 & 0.102 & 88.2 \\
\hline Dimethoate & $<0.024$ & $<0.024$ & -- & -- & -- & -- & -- & -- & -- & -- & -- & -- & -- & 0.013 & 0.102 & 12.7 \\
\hline Disulfoton & $<0.008$ & $<0.008$ & -- & $<0.060$ & $<0.060$ & -- & 0.190 & 0.110 & 172.7 & $<0.060$ & 0.099 & 0.102 & 97.0 & 0.230 & 0.102 & 225.4 \\
\hline EPTC & $<0.005$ & $<0.005$ & -- & $<0.005$ & $<0.005$ & -- & 0.110 & 0.112 & 98.2 & $<0.005$ & 0.094 & 0.102 & 92.1 & 0.120 & 0.102 & 117.6 \\
\hline Ethalfluralin & $<0.013$ & $<0.013$ & -- & $<0.013$ & $<0.013$ & -- & 0.130 & 0.110 & 118.2 & $<0.013$ & 0.110 & 0.102 & 107.8 & 0.120 & 0.102 & 117.6 \\
\hline Ethoprop & $<0.012$ & $<0.012$ & -- & $<0.012$ & $<0.012$ & -- & 0.140 & 0.110 & 127.3 & $<0.012$ & 0.120 & 0.102 & 117.6 & 0.110 & 0.107 & 102.8 \\
\hline Fonofos & $<0.008$ & $<0.008$ & -- & $<0.008$ & $<0.008$ & -- & 0.120 & 0.110 & 109.1 & $<0.008$ & 0.100 & 0.102 & 98.0 & 0.100 & 0.102 & 98.0 \\
\hline HCH Alpha & $<0.007$ & $<0.007$ & -- & 0.007 & 0.007 & 0.0 & 0.130 & 0.117 & 111.1 & 0.007 & 0.120 & 0.109 & 110.1 & 0.100 & 0.109 & 91.7 \\
\hline Lindane & $<0.011$ & $<0.011$ & -- & E0.005 & $<0.011$ & -- & 0.130 & 0.110 & 118.2 & $<0.011$ & 0.120 & 0.102 & 117.6 & 0.140 & 0.140 & 100.0 \\
\hline Linuron & $<0.039$ & $<0.039$ & -- & $<0.039$ & $<0.039$ & -- & 0.057 & 0.110 & 51.8 & $<0.039$ & 0.063 & 0.102 & 61.7 & 0.091 & 0.102 & 89.2 \\
\hline Malathion & $<0.010$ & $<0.010$ & -- & $<0.010$ & $<0.010$ & -- & 0.150 & 0.110 & 136.4 & $<0.014$ & 0.140 & 0.102 & 137.2 & 0.140 & 0.102 & 137.2 \\
\hline Methyl parathion & $<0.035$ & $<0.035$ & -- & $<0.035$ & $<0.035$ & -- & 0.130 & 0.110 & 118.2 & $<0.035$ & 0.140 & 0.102 & 137.2 & 0.140 & 0.102 & 137.2 \\
\hline Metolachlor & 0.071 & 0.073 & 2.8 & 0.190 & 0.190 & 0.0 & 0.140 & 0.121 & 115.7 & $<0.009$ & 0.810 & 0.792 & 102.3 & 0.310 & 0.312 & 99.3 \\
\hline Metribuzin & $<0.012$ & $<0.012$ & -- & $<0.012$ & $<0.012$ & -- & 0.110 & 0.110 & 100.0 & 0.017 & 0.200 & 0.212 & 94.3 & 0.120 & 0.102 & 117.6 \\
\hline Molinate & $<0.007$ & $<0.007$ & -- & $<0.007$ & $<0.007$ & -- & 0.120 & 0.110 & 109.1 & $<0.007$ & 0.110 & 0.102 & 107.8 & 0.100 & 0.102 & 98.0 \\
\hline Napropamide & $<0.010$ & $<0.010$ & -- & 0.097 & 0.099 & 2.0 & 0.120 & 0.118 & 101.7 & $<0.010$ & 0.100 & 0.102 & 98.0 & 0.110 & 0.119 & 92.4 \\
\hline Parathion & $<0.022$ & $<0.022$ & -- & $<0.022$ & $<0.022$ & -- & 0.140 & 0.110 & 127.3 & $<0.022$ & 0.140 & 0.102 & 137.2 & 0.130 & 0.102 & 127.4 \\
\hline Pebulate & $<0.009$ & $<0.009$ & -- & $<0.009$ & $<0.009$ & -- & 0.110 & 0.110 & 100.0 & $<0.009$ & 0.096 & 0.102 & 94.1 & 0.096 & 0.102 & 94.1 \\
\hline Pendimethalin & $<0.018$ & $<0.018$ & -- & $<0.018$ & $<0.018$ & -- & 0.100 & 0.110 & 90.9 & $<0.018$ & 0.098 & 0.102 & 96.0 & 0.120 & 0.102 & 117.6 \\
\hline Permethrin & $<0.016$ & $<0.016$ & -- & $<0.016$ & $<0.016$ & -- & E0.014 & 0.110 & 12.7 & $<0.016$ & 0.018 & 0.102 & 17.6 & 0.021 & 0.102 & 20.6 \\
\hline
\end{tabular}


Table 43. Concentrations of pesticides analyzed by gas chromatography/mass spectroscopy in splits and field matrix spikes of filtered water samples from Phase II of the Willamette River Basin Water Quality Study, Oregon, 1994-Continued

\begin{tabular}{|c|c|c|c|c|c|c|c|c|c|c|c|c|c|c|c|c|}
\hline \multirow{2}{*}{$\begin{array}{c}\text { Sample type } \\
\text { Station } \\
\text { Date }\end{array}$} & \multicolumn{3}{|c|}{$\begin{array}{c}\text { Split } 1 \\
445547123065400 \\
94-05-26\end{array}$} & \multicolumn{3}{|c|}{$\begin{array}{c}\text { Split } 2 \\
452823122240900 \\
94-11-01\end{array}$} & \multicolumn{4}{|c|}{$\begin{array}{c}\text { Field matrix spike } 1 \\
14190970 \\
94-11-23\end{array}$} & \multicolumn{3}{|c|}{$\begin{array}{c}\text { Field matrix spike } 2 \\
443138123120901 \\
94-11-06\end{array}$} & \multicolumn{3}{|c|}{$\begin{array}{c}\text { Field matrix spike } 3 \\
451502122524701 \\
94-05-26\end{array}$} \\
\hline & $\begin{array}{c}\text { Sample } \\
1\end{array}$ & $\begin{array}{c}\text { Sample } \\
2\end{array}$ & $\begin{array}{c}\text { Relative } \\
\text { percent } \\
\text { difference }\end{array}$ & $\begin{array}{c}\text { Sample } \\
1\end{array}$ & $\begin{array}{c}\text { Sample } \\
2\end{array}$ & $\begin{array}{c}\text { Relative } \\
\text { percent } \\
\text { difference }\end{array}$ & $\begin{array}{c}\text { Measured } \\
\text { concen- } \\
\text { tration }\end{array}$ & $\begin{array}{l}\text { Computed } \\
\text { concen- } \\
\text { tration }\end{array}$ & $\begin{array}{l}\text { Percent } \\
\text { recovery }\end{array}$ & $\begin{array}{l}\text { Break } \\
\text { through }\end{array}$ & $\begin{array}{l}\text { Measured } \\
\text { concen- } \\
\text { tration }\end{array}$ & $\begin{array}{l}\text { Computed } \\
\text { concen- } \\
\text { tration }\end{array}$ & $\begin{array}{l}\text { Percent } \\
\text { recovery }\end{array}$ & $\begin{array}{c}\text { Measured } \\
\text { concen- } \\
\text { tration }\end{array}$ & $\begin{array}{l}\text { Computed } \\
\text { concen- } \\
\text { tration }\end{array}$ & $\begin{array}{l}\text { Percent } \\
\text { recovery }\end{array}$ \\
\hline Phorate & $<0.011$ & $<0.011$ & -- & $<0.011$ & $<0.011$ & -- & 0.140 & 0.110 & 127.3 & $<0.011$ & 0.068 & 0.102 & 66.6 & 0.089 & 0.102 & 87.2 \\
\hline Prometon & $<0.008$ & $<0.008$ & -- & 0.009 & 0.010 & 10.5 & 0.120 & 0.122 & 98.4 & $<0.008$ & 0.110 & 0.102 & 107.8 & 0.110 & 0.102 & 107.8 \\
\hline Pronamide & $<0.009$ & $<0.009$ & -- & $<0.009$ & $<0.009$ & -- & 0.120 & 0.110 & 109.1 & $<0.009$ & 0.120 & 0.107 & 112.1 & 0.140 & 0.117 & 119.6 \\
\hline Propachlor & $<0.015$ & $<0.015$ & -- & $<0.015$ & $<0.015$ & -- & 0.130 & 0.110 & 118.2 & $<0.015$ & 0.120 & 0.102 & 117.6 & 0.110 & 0.102 & 107.8 \\
\hline Propanil & $<0.016$ & $<0.016$ & -- & $<0.016$ & $<0.016$ & -- & 0.130 & 0.110 & 118.2 & $<0.016$ & 0.120 & 0.102 & 117.6 & 0.120 & 0.102 & 117.6 \\
\hline Propargite & $<0.006$ & $<0.006$ & -- & $<0.006$ & $<0.006$ & -- & 0.120 & 0.110 & 109.1 & $<0.006$ & 0.110 & 0.102 & 107.8 & 0.110 & 0.102 & 107.8 \\
\hline Simazine & 0.005 & 0.005 & 0.0 & 0.078 & 0.085 & 8.6 & 0.170 & 0.164 & 103.7 & $<0.008$ & 0.500 & 0.482 & 103.7 & 0.280 & 0.332 & 84.3 \\
\hline Tebuthiuron & $<0.015$ & $<0.015$ & -- & $<0.015$ & $<0.015$ & -- & 0.130 & 0.142 & 91.5 & $<0.015$ & $<0.015$ & 0.102 & 0.0 & 0.037 & 0.102 & 36.3 \\
\hline Terbacil & 0.200 & 0.210 & 4.9 & E0.010 & E0.013 & 26.1 & E0.110 & 0.110 & 100.0 & E0.019 & E0.130 & 0.163 & 79.7 & 0.100 & 0.102 & 98.0 \\
\hline Terbufos & $<0.012$ & $<0.012$ & -- & $<0.012$ & $<0.012$ & -- & 0.160 & 0.110 & 145.4 & $<0.012$ & 0.110 & 0.102 & 107.8 & 0.130 & 0.102 & 127.4 \\
\hline Thiobencarb & $<0.008$ & $<0.008$ & -- & $<0.008$ & $<0.008$ & -- & 0.130 & 0.110 & 118.2 & $<0.008$ & 0.120 & 0.102 & 117.6 & 0.110 & 0.102 & 107.8 \\
\hline Triallate & $<0.008$ & $<0.008$ & -- & $<0.008$ & $<0.008$ & -- & 0.120 & 0.110 & 109.1 & $<0.008$ & 0.100 & 0.102 & 98.0 & 0.098 & 0.102 & 96.0 \\
\hline Trifluralin & $<0.012$ & $<0.012$ & -- & E0.005 & E0.005 & 0.0 & 0.098 & 0.110 & 89.1 & $<0.012$ & 0.091 & 0.102 & 89.2 & 0.110 & 0.102 & 107.8 \\
\hline
\end{tabular}


Table 44. Concentrations of pesticides analyzed by high performance liquid gas chromatography in splits and field matrix spikes of filtered water samples from Phase II of the Willamette River Basin Water Quality Study, Oregon, 1994

[NS, analyte not in spike solution; <, less than; E, estimate; --, not calculated; concentrations in micrograms per liter; computed concentration, ((spike volume * spike concentration )/sample volume) + native water concentration; measured concentration, value from laboratory analysis; percent recovery, (measured concentration/computed concentration) *100; Chlorothalonil, DNOC, Dichlobenil, Esfenvalerate, and 1-Naphthol are selected for removal from schedule 2051 or for qualitative reporting]

\begin{tabular}{|c|c|c|c|c|c|c|c|c|c|c|}
\hline $\begin{array}{l}\text { Type sample } \\
\text { Station } \\
\text { Date }\end{array}$ & $\begin{array}{r}\mathrm{Spl} \\
44554711 \\
94-0\end{array}$ & $\begin{array}{l}\text { lit } 1 \\
23065400 \\
5-26\end{array}$ & $\begin{array}{r}\mathrm{Sp} \\
4528231 \\
94-1\end{array}$ & $\begin{array}{l}\text { lit } 2 \\
22240900 \\
1-01\end{array}$ & & $\begin{array}{r}\text { Spike 1 } \\
\text { 343812312 } \\
941-06\end{array}$ & 0901 & & $\begin{array}{r}\text { Spike } 2 \\
5150212252 \\
94-05-26\end{array}$ & 4701 \\
\hline Analyte & $\begin{array}{c}\text { Sample } \\
1\end{array}$ & $\begin{array}{c}\text { Sample } \\
2\end{array}$ & $\begin{array}{c}\text { Sample } \\
1\end{array}$ & $\begin{array}{c}\text { Sample } \\
2\end{array}$ & $\begin{array}{c}\text { Measured } \\
\text { concen- } \\
\text { tration }\end{array}$ & $\begin{array}{c}\text { Computed } \\
\text { concen- } \\
\text { tration }\end{array}$ & $\begin{array}{l}\text { Percent } \\
\text { recovery }\end{array}$ & $\begin{array}{c}\text { Measured } \\
\text { concen- } \\
\text { tration }\end{array}$ & $\begin{array}{l}\text { Computed } \\
\text { concen- } \\
\text { tration }\end{array}$ & $\begin{array}{l}\text { Percent } \\
\text { recovery }\end{array}$ \\
\hline Dinoseb & $<0.050$ & $<0.050$ & $<0.050$ & $<0.050$ & 0.720 & 1.154 & 62.4 & 0.560 & 1.125 & 49.8 \\
\hline DNOC & $<0.050$ & $<0.050$ & $<0.050$ & $<0.050$ & 0.800 & 0.868 & 92.2 & 0.310 & 0.847 & 36.6 \\
\hline Dichlobenil & $<0.050$ & $<0.050$ & $<0.050$ & $<0.050$ & $<0.050$ & NS & -- & $<0.050$ & NS & -- \\
\hline 2, 4-DB & $<0.050$ & $<0.050$ & $<0.050$ & $<0.050$ & 0.580 & 1.532 & 37.9 & 0.390 & 1.494 & 26.1 \\
\hline $2,4,5-\mathrm{T}$ & $<0.050$ & $<0.050$ & $<0.050$ & $<0.050$ & 0.520 & 1.021 & 50.9 & 0.650 & 0.996 & 65.3 \\
\hline Aldicarb & $<0.050$ & $<0.050$ & $<0.050$ & $<0.050$ & 0.110 & 0.950 & 11.6 & 0.140 & 0.926 & 15.1 \\
\hline Aldicarb sulfone & $<0.050$ & $<0.050$ & $<0.050$ & $<0.050$ & 0.340 & 1.092 & 31.1 & 0.180 & 1.066 & 16.9 \\
\hline Bromacil & $<0.050$ & $<0.050$ & $<0.050$ & $<0.050$ & -- & 1.001 & -- & 0.290 & 0.976 & 29.7 \\
\hline Carbaryl & $<0.050$ & $<0.050$ & $<0.050$ & $<0.050$ & 0.690 & 0.990 & 69.7 & 0.100 & 0.966 & 10.4 \\
\hline Chloramben & $<0.050$ & $<0.050$ & $<0.050$ & $<0.050$ & $<0.050$ & NS & -- & $<0.050$ & NS & -- \\
\hline Clopyralid & $<0.050$ & $<0.050$ & $<0.050$ & $<0.050$ & $<0.050$ & NS & -- & $<0.050$ & NS & -- \\
\hline Diuron & $<0.050$ & $<0.050$ & $<0.050$ & $<0.050$ & E5.300 & 6.735 & 78.7 & 0.880 & 1.956 & 45.0 \\
\hline Fluometuron & $<0.050$ & $<0.050$ & $<0.050$ & $<0.050$ & 0.730 & 1.041 & 70.1 & 0.590 & 1.016 & 58.1 \\
\hline MCPA & $<0.050$ & $<0.050$ & $<0.050$ & $<0.050$ & 0.680 & 1.123 & 60.5 & 0.390 & 1.096 & 35.6 \\
\hline Methiocarb & $<0.050$ & $<0.050$ & $<0.050$ & $<0.050$ & 0.710 & 1.021 & 69.5 & 0.100 & 0.996 & 10.0 \\
\hline 1-Naphthol & $<0.050$ & $<0.050$ & $<0.050$ & $<0.050$ & $<0.050$ & 1.021 & 0.0 & 0.070 & 0.996 & 7.0 \\
\hline Oryzalin & $<0.050$ & $<0.050$ & $<0.050$ & $<0.050$ & $<0.050$ & NS & -- & $<0.050$ & NS & -- \\
\hline Picloram & $<0.050$ & $<0.050$ & $<0.050$ & $<0.050$ & 0.180 & 1.062 & 17.0 & 0.360 & 1.036 & 34.8 \\
\hline Silvex & $<0.050$ & $<0.050$ & $<0.050$ & $<0.050$ & 0.770 & 1.052 & 73.2 & 0.560 & 1.026 & 54.6 \\
\hline Dacthal, mono-acid- & $<0.050$ & $<0.050$ & $<0.050$ & $<0.050$ & -- & 1.021 & -- & $<0.050$ & NS & -- \\
\hline Propoxur & $<0.050$ & $<0.050$ & $<0.050$ & $<0.050$ & 0.710 & 1.082 & 65.6 & 0.330 & 1.056 & 31.3 \\
\hline Dichlorprop & $<0.050$ & $<0.050$ & $<0.050$ & $<0.050$ & 0.700 & 1.082 & 64.7 & 0.470 & 1.056 & 44.5 \\
\hline Neburon & $<0.050$ & $<0.050$ & $<0.050$ & $<0.050$ & 0.440 & 1.062 & 41.4 & 0.530 & 1.036 & 51.2 \\
\hline Fenuron & $<0.050$ & $<0.050$ & $<0.050$ & $<0.050$ & $\mathrm{E} 1.500$ & 1.123 & 133.6 & E1.100 & 1.096 & 100.4 \\
\hline 2, 4-D & $<0.050$ & $<0.050$ & $<0.050$ & $<0.050$ & 0.660 & 0.980 & 67.3 & 0.440 & 0.956 & 46.0 \\
\hline Acifluorfen & $<0.050$ & $<0.050$ & $<0.050$ & $<0.050$ & $<0.050$ & NS & -- & $<0.050$ & NS & -- \\
\hline Aldicarb sulfoxide & $<0.050$ & $<0.050$ & $<0.050$ & $<0.050$ & 0.650 & 1.021 & 63.6 & 0.530 & 0.996 & 53.2 \\
\hline Bentazon & $<0.050$ & $<0.050$ & $<0.050$ & $<0.050$ & 0.610 & 1.113 & 54.8 & 0.430 & 1.086 & 39.6 \\
\hline Bromoxynil & $<0.050$ & $<0.050$ & $<0.050$ & $<0.050$ & 0.710 & 1.021 & 69.5 & 0.410 & 0.996 & 41.2 \\
\hline Carbofuran & $<0.050$ & $<0.050$ & $<0.050$ & $<0.050$ & 0.700 & 1.021 & 68.5 & 0.440 & 0.996 & 44.2 \\
\hline Chlorothalonil & $<0.050$ & $<0.050$ & $<0.050$ & $<0.050$ & 0.220 & 1.021 & 21.5 & 0.080 & 0.996 & 8.0 \\
\hline Dicamba & $<0.050$ & $<0.050$ & $<0.050$ & $<0.050$ & 0.410 & 1.062 & 38.6 & 0.440 & 1.036 & 42.5 \\
\hline Esfenvalerate & $<0.050$ & $<0.050$ & $<0.050$ & $<0.050$ & $<0.050$ & NS & -- & $<0.050$ & NS & -- \\
\hline Linuron & $<0.050$ & $<0.050$ & $<0.050$ & $<0.050$ & 0.650 & 0.541 & 120.1 & 0.580 & 0.528 & 109.9 \\
\hline МСРВ & $<0.050$ & $<0.050$ & $<0.050$ & $<0.050$ & $<0.050$ & NS & -- & $<0.050$ & NS & -- \\
\hline Methomyl & $<0.050$ & $<0.050$ & $<0.050$ & $<0.050$ & 0.670 & 1.031 & 65.0 & 0.510 & 1.006 & 50.7 \\
\hline Norflurazon & $<0.050$ & $<0.050$ & $<0.050$ & $<0.050$ & $<0.050$ & NS & -- & $<0.050$ & NS & -- \\
\hline Oxamyl & $<0.050$ & $<0.050$ & $<0.050$ & $<0.050$ & E0.540 & 1.031 & 52.4 & E0.060 & 0.996 & 6.0 \\
\hline Propham & $<0.050$ & $<0.050$ & $<0.050$ & $<0.050$ & -- & 0.449 & -- & 0.540 & 0.996 & 54.2 \\
\hline Triclopyr & $<0.050$ & $<0.050$ & 0.460 & 0.380 & $<0.050$ & NS & -- & $<0.050$ & NS & -- \\
\hline Carbofuran, 3-hydroxy- & $<0.050$ & $<0.050$ & $<0.050$ & $<0.050$ & $<0.050$ & NS & -- & $<0.050$ & NS & -- \\
\hline BDMC (surrogate) & 40.00 & 42.00 & 72.00 & 66.00 & 85.00 & 67.02 & 126.8 & 62.00 & 6.996 & 886.2 \\
\hline
\end{tabular}


Table 45. Concentrations of pesticides analyzed by high performance liquid gas chromatography in field matrix spikes of filtered water samples from Phase II of the Willamette River Basin Water Quality Study, Oregon, 1994

[NS, analyte not in spike solution; <, less than; E, estimate; --, not calculated; concentrations in micrograms per liter; computed concentration, ((spike volume * spike concentration )/sample volume) + native water concentration; measured concentration, value from laboratory analysis; percent recovery, (measured concentration/computed concentration) * 100; Breakthrough, concentration passing through solid-Phase extraction cartridge; Chlorothalonil, DNOC, Dichlobenil, Esfenvalerate, and 1-Naphthol are selected for removal from schedule 2051 or for qualitative reporting]

\begin{tabular}{|c|c|c|c|c|c|c|c|c|}
\hline $\begin{array}{l}\text { Type sample } \\
\text { Station } \\
\text { Date }\end{array}$ & \multicolumn{4}{|c|}{$\begin{array}{c}\text { Field matrix spike } 3 \\
14190970 \\
94-11-23\end{array}$} & \multicolumn{4}{|c|}{$\begin{array}{c}\text { Field matrix spike } 4 \\
14190970 \\
94-11-23\end{array}$} \\
\hline Analyte & $\begin{array}{l}\text { Measured } \\
\text { concen- } \\
\text { tration }\end{array}$ & $\begin{array}{c}\text { Computed } \\
\text { concen- } \\
\text { tration }\end{array}$ & $\begin{array}{l}\text { Percent } \\
\text { recovery }\end{array}$ & $\begin{array}{l}\text { Break- } \\
\text { through }\end{array}$ & $\begin{array}{l}\text { Measured } \\
\text { concen- } \\
\text { tration }\end{array}$ & $\begin{array}{l}\text { Computed } \\
\text { concen- } \\
\text { tration }\end{array}$ & $\begin{array}{l}\text { Percent } \\
\text { recovery }\end{array}$ & $\begin{array}{l}\text { Break- } \\
\text { through }\end{array}$ \\
\hline$\overline{\text { Dinoseb }}$ & 0.560 & 1.162 & 48.2 & $<0.050$ & 0.740 & 1.201 & 61.6 & $<0.050$ \\
\hline DNOC & 0.550 & 0.874 & 62.9 & $<0.050$ & 0.780 & 0.904 & 86.3 & $<0.050$ \\
\hline Dichlobenil & $<0.050$ & NS & -- & $<0.050$ & $<0.050$ & NS & -- & $<0.050$ \\
\hline $2,4-\mathrm{DB}$ & 0.530 & 1.542 & 34.4 & -- & 0.770 & 1.595 & 48.3 & $<0.050$ \\
\hline $2,4,5-\mathrm{T}$ & 0.990 & 1.028 & 96.3 & $<0.050$ & 0.670 & 1.063 & 63.0 & $<0.050$ \\
\hline Aldicarb & -- & 0.956 & -- & 0.070 & 0.260 & 0.989 & 26.3 & $<0.050$ \\
\hline Aldicarb sulfone & 0.410 & 1.100 & 37.3 & 0.470 & 0.600 & 1.137 & 52.8 & 0.350 \\
\hline Bromacil & 0.960 & 1.007 & 95.3 & 0.050 & 0.880 & 1.042 & 84.5 & $<0.050$ \\
\hline Carbaryl & 0.560 & 0.997 & 56.2 & $<0.050$ & 0.770 & 1.031 & 74.7 & $<0.050$ \\
\hline Chloramben & $<0.050$ & NS & -- & $<0.050$ & $<0.050$ & NS & -- & $<0.050$ \\
\hline Clopyralid & $<0.050$ & NS & -- & $<0.050$ & $<0.050$ & NS & -- & $<0.050$ \\
\hline Diuron & 0.700 & 1.326 & 52.8 & $<0.050$ & 0.590 & 1.371 & 43.0 & $<0.050$ \\
\hline Fluometuron & 0.750 & 1.049 & 71.5 & $<0.050$ & 0.640 & 1.084 & 59.0 & $<0.050$ \\
\hline MCPA & 0.550 & 1.131 & 48.6 & $<0.050$ & 0.700 & 1.169 & 59.9 & $<0.050$ \\
\hline Methiocarb & 0.500 & 1.028 & 48.7 & $<0.050$ & 0.400 & 1.063 & 37.6 & $<0.050$ \\
\hline 1-Naphthol & $<0.050$ & 1.028 & 0.0 & $<0.050$ & $<0.050$ & 1.063 & 0.0 & $<0.050$ \\
\hline Oryzalin & $<0.050$ & NS & -- & $<0.050$ & $<0.050$ & NS & -- & $<0.050$ \\
\hline Picloram & 0.570 & 1.069 & 53.3 & 0.120 & 0.050 & 1.106 & 4.5 & $<0.050$ \\
\hline Silvex & 0.590 & 1.059 & 55.7 & $<0.050$ & 0.740 & 1.095 & 67.6 & $<0.050$ \\
\hline Dacthal, mono-acid- & $<0.050$ & NS & -- & $<0.050$ & $<0.050$ & NS & -- & $<0.050$ \\
\hline Propoxur & 0.740 & 1.090 & 67.9 & 0.070 & 0.770 & 1.127 & 68.3 & $<0.050$ \\
\hline Dichlorprop & 0.500 & 1.090 & 45.9 & $<0.050$ & 0.640 & 1.127 & 56.8 & $<0.050$ \\
\hline Neburon & 0.540 & 1.069 & 50.5 & $<0.050$ & 0.410 & 1.106 & 37.1 & $<0.050$ \\
\hline Fenuron & 0.930 & 1.131 & 82.2 & 0.050 & 0.910 & 1.169 & 77.8 & $<0.050$ \\
\hline 2, 4-D & 0.620 & 0.987 & 62.8 & $<0.050$ & 0.810 & 1.021 & 79.4 & $<0.050$ \\
\hline Acifluorfen & $<0.050$ & NS & -- & $<0.050$ & $<0.050$ & NS & -- & $<0.050$ \\
\hline Aldicarb sulfoxide & 0.640 & 1.028 & 62.3 & 0.390 & 1.200 & 1.063 & 112.9 & 0.070 \\
\hline Bentazon & 0.520 & 1.121 & 46.4 & 0.160 & 0.820 & 1.159 & 70.8 & $<0.050$ \\
\hline Bromoxynil & 0.570 & 1.028 & 55.5 & $<0.050$ & 0.730 & 1.063 & 68.7 & $<0.050$ \\
\hline Carbofuran & 0.780 & 1.028 & 75.9 & $<0.050$ & 0.770 & 1.063 & 72.5 & $<0.050$ \\
\hline Chlorothalonil & 0.140 & 1.028 & 13.6 & $<0.050$ & 0.050 & 1.063 & 4.7 & $<0.050$ \\
\hline Dicamba & 0.390 & 1.069 & 36.5 & 0.280 & 0.370 & 1.106 & 33.5 & $<0.050$ \\
\hline Esfenvalerate & $<0.050$ & NS & -- & $<0.050$ & $<0.050$ & NS & -- & $<0.050$ \\
\hline Linuron & 0.680 & 0.545 & 124.8 & $<0.050$ & 0.530 & 0.563 & 94.1 & $<0.050$ \\
\hline МСРВ & $<0.050$ & NS & -- & $<0.050$ & $<0.050$ & NS & -- & $<0.050$ \\
\hline Methomyl & 0.730 & 1.038 & 70.3 & $<0.050$ & 0.760 & 1.074 & 70.8 & $<0.050$ \\
\hline Norflurazon & $<0.050$ & NS & -- & $<0.050$ & $<0.050$ & NS & -- & $<0.050$ \\
\hline Oxamyl & 0.420 & 1.038 & 40.5 & 0.070 & 0.500 & 1.074 & 46.6 & $<0.050$ \\
\hline Propham & E0.220 & 0.452 & 48.6 & -- & E0.220 & 0.468 & 47.0 & $<0.050$ \\
\hline Triclopyr & $<0.050$ & NS & -- & $<0.050$ & $<0.050$ & NS & -- & $<0.050$ \\
\hline Carbofuran, 3-hydroxy- & $<0.050$ & NS & -- & $<0.050$ & $<0.050$ & NS & -- & $<0.050$ \\
\hline BDMC (surrogate) & 94.00 & 80.03 & 117.5 & 5.000 & 101.0 & 80.06 & 126.2 & -- \\
\hline
\end{tabular}


Table 46. Concentrations of pesticides analyzed by high performance liquid gas chromatography in field matrix spikes of filtered water samples from Phase II of the Willamette River Basin Water Quality Study, Oregon, 1994

[NS, analyte not in spike solution; <, less than; E, estimate; --, not calculated; concentrations in micrograms per liter; computed concentration, ((spike volume * spike concentration )/sample volume) + native water concentration; measured concentration, value from laboratory analysis; percent recovery, (measured concentration/computed concentration) * 100; Chlorothalonil, DNOC, Dichlobenil, Esfenvalerate, and 1-Naphthol are selected for removal from schedule 2051 or for qualitative reporting]

\begin{tabular}{|c|c|c|c|c|c|c|c|c|c|c|c|c|c|c|c|}
\hline $\begin{array}{l}\text { Type sample } \\
\text { Station } \\
\text { Date }\end{array}$ & \multicolumn{3}{|c|}{$\begin{array}{c}\text { Field matrix spike } 5 \\
14211720 \\
94-10-25\end{array}$} & \multicolumn{3}{|c|}{$\begin{array}{c}\text { Field matrix spike } 6 \\
14211720 \\
94-10-25\end{array}$} & \multicolumn{3}{|c|}{$\begin{array}{c}\text { Field matrix spike } 7 \\
14211720 \\
94-10-25\end{array}$} & \multicolumn{3}{|c|}{$\begin{array}{c}\text { Field matrix spike } 8 \\
14211720 \\
94-10-25\end{array}$} & \multicolumn{3}{|c|}{$\begin{array}{c}\text { Field matrix spike } 9 \\
14211720 \\
94-10-25\end{array}$} \\
\hline Analyte & $\begin{array}{c}\text { Measured } \\
\text { concen- } \\
\text { tration }\end{array}$ & $\begin{array}{l}\text { Computed } \\
\text { concen- } \\
\text { tration }\end{array}$ & $\begin{array}{l}\text { Percent } \\
\text { recovery }\end{array}$ & $\begin{array}{c}\text { Measured } \\
\text { concen- } \\
\text { tration }\end{array}$ & $\begin{array}{l}\text { Computed } \\
\text { concen- } \\
\text { tration }\end{array}$ & $\begin{array}{l}\text { Percent } \\
\text { recovery }\end{array}$ & $\begin{array}{c}\text { Measured } \\
\text { concen- } \\
\text { tration }\end{array}$ & $\begin{array}{c}\text { Computed } \\
\text { concen- } \\
\text { tration }\end{array}$ & $\begin{array}{l}\text { Percent } \\
\text { recovery }\end{array}$ & $\begin{array}{c}\text { Measure } \\
\text { concen- } \\
\text { tration }\end{array}$ & $\begin{array}{l}\text { Computed } \\
\text { concen- } \\
\text { tration }\end{array}$ & $\begin{array}{l}\text { Percent } \\
\text { recovery }\end{array}$ & $\begin{array}{c}\text { Measured } \\
\text { concen- } \\
\text { tration }\end{array}$ & $\begin{array}{l}\text { Computed } \\
\text { concen- } \\
\text { tration }\end{array}$ & $\begin{array}{l}\text { Percent } \\
\text { recovery }\end{array}$ \\
\hline Dinoseb & 0.140 & 0.284 & 49.4 & 0.180 & 0.287 & 62.7 & 0.260 & 0.580 & 44.9 & 0.270 & 0.596 & 45.3 & 0.530 & 1.179 & 45.0 \\
\hline DNOC & 0.140 & 0.213 & 65.6 & 0.190 & 0.216 & 88.0 & 0.260 & 0.436 & 59.6 & 0.250 & 0.448 & 55.8 & 0.360 & .887 & 40.6 \\
\hline Dichlobenil & $<0.050$ & NS & -- & $<0.050$ & NS & -- & $<0.050$ & NS & -- & $<0.050$ & NS & -- & $<0.050$ & NS & -- \\
\hline $2,4-\mathrm{DB}$ & 0.200 & 0.377 & 53.1 & 0.230 & 0.381 & 60.4 & 0.280 & 0.770 & 36.4 & 0.300 & 0.791 & 38.0 & 0.520 & 1.565 & 33.2 \\
\hline $2,4,5-\mathrm{T}$ & -- & 0.251 & -- & -- & 0.254 & -- & -- & 0.513 & -- & -- & 0.527 & -- & -- & 1.043 & -- \\
\hline Aldicarb & 0.060 & 0.233 & 25.7 & $<0.050$ & 0.236 & 0.0 & 0.080 & 0.477 & 16.8 & 0.070 & 0.490 & 14.3 & 0.060 & 0.970 & 6.2 \\
\hline Aldicarb sulfone & 0.090 & 0.269 & 33.5 & 0.100 & 0.272 & 36.8 & 0.150 & 0.548 & 27.3 & 0.210 & 0564 & 37.2 & 0.290 & 1.116 & 26.0 \\
\hline Bromacil & 0.260 & 0.246 & 105.7 & 0.220 & 0.249 & 88.4 & 0.390 & 0.503 & 77.6 & 0.420 & 0.516 & 81.3 & 0.850 & 1.022 & 83.2 \\
\hline Carbaryl & 0.160 & 0.243 & 65.7 & 0.170 & 0.246 & 69.0 & 0.320 & 0.498 & 64.3 & 0.320 & 0.511 & 62.6 & 0.690 & 1.012 & 68.2 \\
\hline Chloramben & $<0.050$ & NS & -- & $<0.050$ & NS & -- & $<0.050$ & NS & -- & $<0.050$ & NS & -- & $<0.050$ & NS & -- \\
\hline Clopyralid & $<0.050$ & NS & -- & $<0.050$ & NS & -- & $<0.050$ & NS & -- & $<0.050$ & NS & -- & $<0.050$ & NS & -- \\
\hline Diuron & E0.220 & 0.595 & 37.0 & E0.180 & 0.599 & 30.1 & E0.370 & 0.933 & 39.7 & E0.410 & 0.951 & 43.1 & E0.900 & 1.616 & 55.7 \\
\hline Fluometuron & 0.190 & 0.256 & 74.2 & 0.200 & 0.259 & 77.2 & 0.350 & 0.523 & 66.9 & 0.380 & 0.538 & 70.7 & 0.800 & 1.064 & 75.2 \\
\hline MCPA & 0.170 & 0.276 & 61.6 & 0.210 & 0.279 & 75.2 & 0.320 & 0.564 & 56.7 & 0.310 & 0.580 & 53.5 & 0.600 & 1.147 & 52.3 \\
\hline Methiocarb & 0.150 & 0.251 & 59.8 & 0.130 & 0.254 & 51.3 & 0.300 & 0.513 & 58.5 & 0.290 & 0.527 & 55.0 & 0.700 & 1.043 & 67.1 \\
\hline 1-Naphthol & $<0.050$ & 0.251 & 0.0 & $<0.050$ & 0.254 & 0.0 & $<0.050$ & 0.513 & 0.0 & $<0.050$ & 0.527 & 0.0 & $<0.050$ & 1.043 & 0.0 \\
\hline Oryzalin & $<0.050$ & NS & -- & $<0.050$ & NS & -- & $<0.050$ & NS & -- & $<0.050$ & NS & -- & $<0.050$ & NS & -- \\
\hline Picloram & -- & 0.261 & -- & -- & 0.264 & -- & -- & 0.534 & -- & -- & 0.548 & -- & -- & 1.085 & -- \\
\hline Silvex & 0.170 & 0.259 & 65.8 & 0.190 & 0.262 & 72.6 & 0.310 & 0.528 & 58.7 & 0.300 & 0.543 & 55.3 & 0.600 & 1.074 & 55.9 \\
\hline Dacthal, mono-acid- & -- & NS & -- & -- & NS & -- & -- & NS & -- & -- & NS & -- & -- & NS & -- \\
\hline Propoxur & 0.150 & 0.266 & 56.4 & 0.140 & 0.269 & 52.0 & 0.320 & 0.544 & 58.9 & 0.300 & 0.559 & 53.7 & 0.740 & 1.106 & 66.9 \\
\hline
\end{tabular}


Table 46. Concentrations of pesticides analyzed by high performance liquid gas chromatography in field matrix spikes of filtered water samples from Phase II of the Willamette River Basin Water Quality Study, Oregon, 1994-Continued

\begin{tabular}{|c|c|c|c|c|c|c|c|c|c|c|c|c|c|c|c|}
\hline $\begin{array}{l}\text { Type sample } \\
\text { Station } \\
\text { Date }\end{array}$ & \multicolumn{3}{|c|}{$\begin{array}{c}\text { Field matrix spike } 5 \\
14211720 \\
94-10-25\end{array}$} & \multicolumn{3}{|c|}{$\begin{array}{c}\text { Field matrix spike } 6 \\
14211720 \\
94-10-25\end{array}$} & \multicolumn{3}{|c|}{$\begin{array}{c}\text { Field matrix spike } 7 \\
14211720 \\
94-10-25\end{array}$} & \multicolumn{3}{|c|}{$\begin{array}{c}\text { Field matrix spike } 8 \\
14211720 \\
94-10-25\end{array}$} & \multicolumn{3}{|c|}{$\begin{array}{c}\text { Field matrix spike } 9 \\
14211720 \\
94-10-25\end{array}$} \\
\hline Analyte & $\begin{array}{c}\text { Measured } \\
\text { concen- } \\
\text { tration }\end{array}$ & $\begin{array}{l}\text { Computed } \\
\text { concen- } \\
\text { tration }\end{array}$ & $\begin{array}{l}\text { Percent } \\
\text { recovery }\end{array}$ & $\begin{array}{c}\text { Measured } \\
\text { concen- } \\
\text { tration }\end{array}$ & $\begin{array}{l}\text { Computed } \\
\text { concen- } \\
\text { tration }\end{array}$ & $\begin{array}{l}\text { Percent } \\
\text { recovery }\end{array}$ & $\begin{array}{c}\text { Measured } \\
\text { concen- } \\
\text { tration }\end{array}$ & $\begin{array}{l}\text { Computed } \\
\text { concen- } \\
\text { tration }\end{array}$ & $\begin{array}{l}\text { Percent } \\
\text { recovery }\end{array}$ & $\begin{array}{c}\text { Measure } \\
\text { concen- } \\
\text { tration }\end{array}$ & $\begin{array}{l}\text { Computed } \\
\text { concen- } \\
\text { tration }\end{array}$ & $\begin{array}{l}\text { Percent } \\
\text { recovery }\end{array}$ & $\begin{array}{c}\text { Measured } \\
\text { concen- } \\
\text { tration }\end{array}$ & $\begin{array}{l}\text { Computed } \\
\text { concen- } \\
\text { tration }\end{array}$ & $\begin{array}{l}\text { Percent } \\
\text { recovery }\end{array}$ \\
\hline Dichlorprop & 0.200 & 0.266 & 75.2 & 0.260 & 0.269 & 96.6 & 0.350 & 0.544 & 64.4 & 0.340 & 0.559 & 60.9 & 0.650 & 1.106 & 58.8 \\
\hline Neburon & 0.160 & 0.261 & 61.3 & 0.140 & 0.264 & 53.0 & 0.360 & 0.534 & 67.5 & 0.370 & 0.548 & 67.5 & 0.770 & 1.085 & 71.0 \\
\hline Fenuron & E0.350 & 0.276 & 126.8 & E0.390 & 0.279 & 139.6 & E0.710 & 0.564 & 125.8 & E0.740 & 0.580 & 127.7 & E1.600 & 1.147 & 139.5 \\
\hline 2, 4 -D & 0.170 & 0.241 & 70.6 & 0.170 & 0.244 & 69.7 & 0.310 & 0.492 & 62.9 & 0.290 & 0.506 & 57.3 & 0.590 & 1.001 & 58.9 \\
\hline Acifluorfen & $<0.050$ & NS & -- & $<0.050$ & NS & -- & $<0.050$ & NS & -- & $<0.050$ & NS & -- & $<0.050$ & NS & -- \\
\hline Aldicarb sulfoxide & 0.280 & 0.251 & 111.6 & 0.230 & 0.254 & 90.7 & 0.440 & 0.513 & 85.8 & 0.520 & 0.527 & 98.7 & 0.990 & 1.043 & 94.9 \\
\hline Bentazon & 0.170 & 0.274 & 62.1 & 0.130 & 0.277 & 47.0 & 0.260 & 0.559 & 46.5 & 0.270 & 0.574 & 47.0 & 0.570 & 1.137 & 50.1 \\
\hline Bromoxynil & 0.160 & 0.251 & 63.7 & 0.170 & 0.254 & 67.0 & 0.290 & 0.513 & 56.6 & 0.290 & 0.527 & 55.0 & 0.600 & 1.043 & 57.5 \\
\hline Carbofuran & 0.430 & 0.251 & 171.3 & E0.330 & 0.254 & 130.2 & 0.640 & 0.513 & 124.8 & 0.670 & 0.527 & 127.2 & 1.200 & 1.043 & 115.1 \\
\hline Chlorothalonil & $<0.050$ & NS & -- & $<0.050$ & NS & -- & $<0.050$ & NS & -- & $<0.050$ & NS & -- & $<0.050$ & NS & -- \\
\hline Dicamba & 0.090 & 0.261 & 34.5 & 0.080 & 0.264 & 30.3 & 0.100 & 0.534 & 18.7 & 0.160 & 0.548 & 29.2 & 0.440 & 1.085 & 40.6 \\
\hline Esfenvalerate & $<0.050$ & NS & -- & $<0.050$ & NS & -- & $<0.050$ & NS & -- & $<0.050$ & NS & -- & $<0.050$ & NS & -- \\
\hline Linuron & 0.190 & 0.133 & 142.8 & 0.170 & 0.135 & 126.3 & 0.340 & 0.272 & 125.1 & 0.370 & 0.279 & 132.5 & 0.780 & 0.553 & 141.1 \\
\hline МCPB & $<0.050$ & NS & -- & $<0.050$ & NS & -- & $<0.050$ & NS & -- & $<0.050$ & NS & -- & $<0.050$ & NS & -- \\
\hline Methomyl & 0.160 & 0.254 & 63.1 & 0.170 & 0.257 & 66.3 & 0.320 & 0.518 & 61.8 & 0.350 & 0.532 & 65.8 & 0.740 & 1.053 & 70.2 \\
\hline Norflurazon & $<0.050$ & NS & -- & $<0.050$ & NS & -- & $<0.050$ & NS & -- & $<0.050$ & NS & -- & $<0.050$ & NS & -- \\
\hline Oxamyl & 0.110 & 0.254 & 43.4 & 0.100 & 0.257 & 39.0 & 0.190 & 0.518 & 36.7 & 0.210 & 0.532 & 39.5 & 0.500 & 1.053 & 47.5 \\
\hline Propham & E0.190 & 0.110 & 172.0 & E0.200 & 0.112 & 179.0 & E0.160 & 0.226 & 70.9 & E0.410 & 0.232 & 176.8 & E0.320 & 0.459 & 69.7 \\
\hline Triclopyr & $<0.050$ & NS & -- & $<0.050$ & NS & -- & $<0.050$ & NS & -- & $<0.050$ & NS & -- & $<0.050$ & NS & -- \\
\hline Carbofuran, 3-hydroxy & $<0.050$ & NS & -- & $<0.050$ & NS & -- & $<0.050$ & NS & -- & $<0.050$ & NS & -- & $<0.050$ & NS & -- \\
\hline BDMC (surrogate) & 104.0 & 136.3 & 76.3 & 76.00 & 136.3 & 55.8 & 146.0 & 136.5 & 106.9 & 114.0 & 136.5 & 83.5 & 134.0 & 137.0 & 97.8 \\
\hline
\end{tabular}


Table 47. Concentrations of triazine compounds in split samples from Phase II of the Willamette River Basin Water Quality Study, Oregon, 1994

[USGS laboratory schedule 8015; concentrations in micrograms per liter; ND, no detection;

E, estimate; --, not calculated]

\begin{tabular}{|c|c|c|c|c|c|c|}
\hline $\begin{array}{l}\text { Sample type } \\
\text { Station } \\
\text { Date }\end{array}$ & \multicolumn{2}{|c|}{$\begin{array}{c}\text { Split 1 } \\
14202000 \\
94-10-29\end{array}$} & \multicolumn{2}{|c|}{$\begin{array}{c}\text { Split 2 } \\
444349123094000 \\
94-05-16\end{array}$} & \multicolumn{2}{|c|}{$\begin{array}{c}\text { Split 3 } \\
445547123065400 \\
94-05-26\end{array}$} \\
\hline Analyte & $\begin{array}{c}\text { Sample } \\
1\end{array}$ & $\begin{array}{c}\text { Sample } \\
2\end{array}$ & $\begin{array}{c}\text { Sample } \\
1\end{array}$ & $\begin{array}{c}\text { Sample } \\
2\end{array}$ & $\begin{array}{c}\text { Sample } \\
1\end{array}$ & $\begin{array}{c}\text { Sample } \\
2\end{array}$ \\
\hline Desisopropylatrzine & 0.002 & 0.003 & ND & ND & ND & ND \\
\hline Desethylatrazine & 0.002 & 0.003 & ND & ND & 0.005 & 0.002 \\
\hline Simazine & 0.056 & 0.060 & 0.001 & ND & 0.038 & 0.002 \\
\hline Prometon & ND & 0.001 & ND & ND & 0.007 & ND \\
\hline Atrazine & 0.041 & 0.044 & 0.031 & 0.030 & 0.026 & 0.024 \\
\hline Propazine & ND & ND & ND & ND & ND & ND \\
\hline Metribuzin & 0.001 & 0.002 & ND & ND & ND & ND \\
\hline Ametryn & ND & ND & ND & ND & ND & ND \\
\hline Alachlor & ND & ND & ND & ND & ND & ND \\
\hline Prometryn & ND & ND & ND & ND & ND & ND \\
\hline Metolachlor & 0.035 & 0.039 & 0.002 & 0.001 & 0.010 & 0.053 \\
\hline Cyanazine & ND & ND & ND & ND & ND & ND \\
\hline Butylate & ND & ND & ND & ND & ND & ND \\
\hline Vernolate & ND & ND & ND & ND & ND & ND \\
\hline Propachlor & 0.003 & 0.003 & ND & ND & ND & ND \\
\hline Cycloate & 0.002 & 0.003 & 0.003 & 0.002 & 0.004 & 0.002 \\
\hline Trifluralin & 0.000 & 0.000 & ND & ND & ND & ND \\
\hline Terbacil & 0.006 & 0.007 & 0.009 & 0.007 & ND & 0.269 \\
\hline Simetryn & 0.000 & 0.000 & ND & ND & ND & ND \\
\hline Bromacil & 0.010 & ND & ND & ND & ND & ND \\
\hline Diphenamid & ND & ND & ND & ND & ND & ND \\
\hline Butachlor & ND & ND & ND & ND & ND & ND \\
\hline Carboxin & ND & ND & ND & ND & ND & ND \\
\hline Hexazinone & 0.003 & 0.004 & 0.002 & ND & 0.002 & ND \\
\hline Terbuthylazine (surrogate) & 0.123 & 0.116 & 0.227 & 0.232 & 0.239 & 0.207 \\
\hline
\end{tabular}


Table 48. Concentrations of triazine compounds in field matrix spikes of filtered water samples from Phase II of the Willamette River Basin Water Quality Study, Oregon, 1994

[USGS laboratory schedule 8015; concentrations in micrograms per liter; NS, analyte not in spike solution; ND, no detection; --, not calculated; computed concentration, ((spike volume * spike concentration)/sample volume) + native water concentration; measured concentration, value from laboratory analysis; percent recovery, (measured concentration/computed concentration) * 100]

\begin{tabular}{|c|c|c|c|c|c|c|c|c|c|c|c|c|}
\hline \multirow{2}{*}{$\begin{array}{c}\begin{array}{c}\text { Sample type } \\
\text { Station } \\
\text { Date }\end{array} \\
\text { Analyte }\end{array}$} & \multicolumn{3}{|c|}{$\begin{array}{c}\text { Field matrix spike } 1 \\
14206298 \\
94-11-23\end{array}$} & \multicolumn{3}{|c|}{$\begin{array}{c}\text { Field matrix spike } 2 \\
14206298 \\
94-11-23\end{array}$} & \multicolumn{3}{|c|}{$\begin{array}{c}\text { Field matrix spike } 3 \\
451502122524700 \\
94-05-26\end{array}$} & \multicolumn{3}{|c|}{$\begin{array}{c}\text { Field matrix spike } 4 \\
452823122240900 \\
94-11-03\end{array}$} \\
\hline & $\begin{array}{c}\text { Measured } \\
\text { concen- } \\
\text { tration }\end{array}$ & $\begin{array}{l}\text { Computed } \\
\text { concen- } \\
\text { tration }\end{array}$ & $\begin{array}{c}\text { Percent } \\
\text { recovery }\end{array}$ & $\begin{array}{c}\text { Measured } \\
\text { concen- } \\
\text { tration }\end{array}$ & $\begin{array}{c}\text { Computed } \\
\text { concen- } \\
\text { tration }\end{array}$ & $\begin{array}{l}\text { Percent } \\
\text { recovery }\end{array}$ & $\begin{array}{c}\text { Measured } \\
\text { concen- } \\
\text { tration }\end{array}$ & $\begin{array}{l}\text { Computed } \\
\text { concen- } \\
\text { tration }\end{array}$ & $\begin{array}{c}\text { Percent } \\
\text { recovery }\end{array}$ & $\begin{array}{c}\text { Measured } \\
\text { concen- } \\
\text { tration }\end{array}$ & $\begin{array}{c}\text { Computed } \\
\text { concen- } \\
\text { tration }\end{array}$ & $\begin{array}{c}\text { Percent } \\
\text { recovery }\end{array}$ \\
\hline Desisopropylatrazine & 0.006 & NS & -- & 0.007 & NS & -- & 0.006 & NS & $\overline{--}$ & 0.001 & NS & -- \\
\hline Desethylatrazine & 0.001 & NS & -- & 0.002 & NS & -- & 0.032 & NS & -- & 0.003 & NS & -- \\
\hline Simazine & 0.256 & 0.437 & 59 & 0.207 & 0.466 & 44 & 0.121 & 0.191 & 63 & 0.073 & 0.202 & 36 \\
\hline Prometon & 0.066 & 0.192 & 34 & 0.098 & 0.220 & 45 & 0.083 & 0.108 & 77 & 0.099 & 0.187 & 53 \\
\hline Atrazine & 0.074 & 0.189 & 39 & 0.105 & 0.216 & 48 & 0.130 & 0.179 & 73 & 0.124 & 0.193 & 64 \\
\hline Propazine & 0.065 & 0.146 & 45 & 0.094 & 0.168 & 56 & ND & 0.085 & 0 & 0.097 & 0.146 & 66 \\
\hline Metribuzin & 0.003 & NS & -- & 0.003 & NS & -- & 0.059 & NS & -- & ND & NS & -- \\
\hline Ametryn & 0.079 & 0.181 & 44 & 0.112 & 0.209 & 54 & 0.003 & 0.108 & 3 & 0.117 & 0.181 & 65 \\
\hline Alachlor & ND & NS & -- & ND & NS & -- & 0.077 & NS & -- & ND & NS & -- \\
\hline Prometryn & 0.081 & 0.165 & 49 & 0.117 & 0.191 & 61 & ND & 0.096 & 0 & 0.112 & 0.165 & 68 \\
\hline Metolachlor & 0.001 & NS & -- & 0.001 & NS & -- & 0.222 & NS & -- & 0.055 & NS & -- \\
\hline Cyanazine & ND & NS & -- & ND & NS & -- & 0.068 & NS & -- & 0.001 & NS & -- \\
\hline Butylate & ND & NS & -- & ND & NS & -- & 0.063 & NS & -- & ND & NS & -- \\
\hline Vernolate & ND & NS & -- & ND & NS & -- & ND & NS & -- & ND & NS & -- \\
\hline Propachlor & ND & NS & -- & ND & NS & -- & 0.055 & NS & -- & ND & NS & -- \\
\hline Cycloate & ND & NS & -- & ND & NS & -- & 0.001 & NS & -- & ND & NS & -- \\
\hline Trifluralin & ND & NS & -- & ND & NS & -- & 0.044 & NS & -- & 0.001 & NS & -- \\
\hline Terbacil & ND & NS & -- & ND & NS & -- & 0.077 & NS & -- & 0.005 & NS & -- \\
\hline Simetryn & 0.000 & NS & -- & 0.001 & NS & -- & ND & NS & -- & ND & NS & -- \\
\hline Bromacil & 0.017 & NS & -- & ND & NS & -- & ND & NS & -- & ND & NS & -- \\
\hline Diphenamid & ND & NS & -- & ND & NS & -- & ND & NS & -- & 0.001 & NS & -- \\
\hline Butachlor & ND & NS & -- & ND & NS & -- & ND & NS & -- & ND & NS & -- \\
\hline Carboxin & ND & NS & -- & ND & NS & -- & 0.001 & NS & -- & ND & NS & -- \\
\hline Hexazinone & ND & NS & -- & ND & NS & -- & 0.007 & NS & -- & 0.012 & NS & -- \\
\hline Terbuthylazine & 0.113 & NS & -- & 0.152 & NS & -- & 0.217 & NS & -- & 0.145 & NS & -- \\
\hline
\end{tabular}


Page Intentionally Blank 


\section{APPENDIX}


Appendix - U.S. Environmental Protection Agency STOage and RETrieval (STORET) codes and minimum reporting level (MRL) or method detection limit (MDL) (Schedules 8308, 2010, and 8015 ) for constituents analyzed during Phases I and II of the Willamette River Basin Water Quality Study, Oregon, 1992-94 [Source: Timme, 1994]

\begin{tabular}{|c|c|c|}
\hline $\begin{array}{l}\text { STORE } \\
\text { Code }\end{array}$ & Constituent & $\begin{array}{l}\text { MRL/ } \\
\text { MDL }\end{array}$ \\
\hline \multicolumn{3}{|c|}{ Ancillary data (Phases I and II): } \\
\hline 00010 & Water temperature & 0.5 degrees $\mathrm{C}$ \\
\hline 00025 & Air pressure & $1 \mathrm{~mm}$ of $\mathrm{Hg}$ \\
\hline 00061 & Discharge, instantaneous & $\mathrm{ft}^{3} / \mathrm{sec}$ \\
\hline 80154 & Concentration,suspended sediment & $\mathrm{mg} / \mathrm{L}$ \\
\hline 70331 & 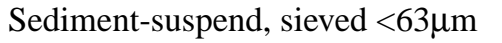 & percent \\
\hline 00095 & Specific conductance & $\begin{array}{l}\mu \mathrm{s} / \mathrm{cm} \text { at } \\
25^{\circ} \mathrm{C}\end{array}$ \\
\hline 00300 & Oxygen, dissolved & $.1 \mathrm{mg} / \mathrm{L}$ \\
\hline 00301 & Oxygen, dissolved & $\begin{array}{l}\text { percent of } \\
\text { saturation }\end{array}$ \\
\hline 00400 & $\mathrm{pH}$, field & $\begin{array}{l}.1 \text { Standard } \\
\text { Units }\end{array}$ \\
\hline 00681 & Carbon, organic, dissolved & $.1 \mathrm{mg} / \mathrm{L}$ as $\mathrm{C}$ \\
\hline 00689 & Carbon, organic, suspended & $.1 \mathrm{mg} / \mathrm{L}$ as $\mathrm{C}$ \\
\hline 00915 & Calcium, dissolved & $.1 \mathrm{mg} / \mathrm{L}$ as $\mathrm{Ca}$ \\
\hline 00925 & Magnesium, dissolved & $.1 \mathrm{mg} / \mathrm{L}$ as $\mathrm{Mg}$ \\
\hline 00900 & Hardness, total & 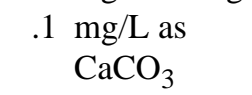 \\
\hline
\end{tabular}

Nutrients [Schedule 2702] (Phases I and II):

00608 Nitrogen, ammonia, dissolved $0.01 \mathrm{mg} / \mathrm{L}$ as N

00613 Nitrogen, nitrite, dissolved $\quad .01 \mathrm{mg} / \mathrm{L}$ as N

00623 Nitrogen, ammonia plus organic, dissolved

00625 Nitrogen, ammonia plus organic, total

00631 Nitrite plus nitrate, dissolved

00665 Phosphorus, total

00666 Phosphorus, dissolved

00671 Phosphorus, ortho-, dissolved

Trace elements in whole water (Phase I):

$\begin{array}{ll}01097 & \text { Antimony, total } \\ 01002 & \text { Arsenic, total } \\ 01012 & \text { Beryllium, total } \\ 01022 & \text { Boron, total } \\ 01027 & \text { Cadmium, total } \\ 01034 & \text { Chromium, total } \\ 01037 & \text { Cobalt, total } \\ 01042 & \text { Copper, total } \\ 01045 & \text { Iron, total } \\ 01051 & \text { Lead, total } \\ 01055 & \text { Manganese, total } \\ 71900 & \text { Mercury, total } \\ 01067 & \text { Nickel, total } \\ 01147 & \text { Selenium, total } \\ 01077 & \text { Silver, total }\end{array}$

$.2 \mathrm{mg} / \mathrm{L}$ as $\mathrm{N}$

$.2 \mathrm{mg} / \mathrm{L}$ as N

$.05 \mathrm{mg} / \mathrm{L}$ as N

$.01 \mathrm{mg} / \mathrm{L}$ as $\mathrm{P}$

$.01 \mathrm{mg} / \mathrm{L}$ as $\mathrm{P}$

$.01 \mathrm{mg} / \mathrm{L}$ as $\mathrm{P}$

\begin{tabular}{ll}
$\begin{array}{l}\text { STORET } \\
\text { Code }\end{array}$ \\
\hline $01092 \quad$ Zinc, total \\
\multicolumn{2}{l}{ Trace elements in wh } \\
01002 Arsenic \\
01027 & Cadmium \\
01034 & Chromium \\
01042 & Copper \\
01051 & Lead \\
71900 & Mercury \\
01067 & Nickel \\
01092 & Zinc
\end{tabular}

MRL/ MDL

Trace elements in filtered water (Phase I):

$\begin{array}{llrl}01095 & \text { Antimony, dissolved } & 1 & \mu \mathrm{g} / \mathrm{L} \text { as } \mathrm{Sb} \\ 01000 & \text { Arsenic, dissolved } & 1 & \mu \mathrm{g} / \mathrm{L} \text { as As } \\ 01010 & \text { Beryllium, dissolved } & 10 & \mu \mathrm{g} / \mathrm{L} \text { as Be } \\ 01020 & \text { Boron, dissolved } & 10 & \mu \mathrm{g} / \mathrm{L} \text { as B } \\ 01025 & \text { Cadmium, dissolved } & .1 & \mu \mathrm{g} / \mathrm{L} \text { as Cd } \\ 01030 & \text { Chromium, dissolved } & .5 & \mu \mathrm{g} / \mathrm{L} \text { as Cr } \\ 01035 & \text { Cobalt, dissolved } & .5 & \mu \mathrm{g} / \mathrm{L} \text { as Co } \\ 01040 & \text { Copper, dissolved } & .5 & \mu \mathrm{g} / \mathrm{L} \text { as Cu } \\ 01046 & \text { Iron, dissolved } & 10 & \mu \mathrm{g} / \mathrm{L} \text { as Fe } \\ 01049 & \text { Lead, dissolved } & .5 & \mu \mathrm{g} / \mathrm{L} \text { as Pb } \\ 01056 & \text { Manganese, dissolved } & .2 & \mu \mathrm{g} / \mathrm{L} \text { as } \mathrm{Mn} \\ 71890 & \text { Mercury, dissolved } & .1 & \mu \mathrm{g} / \mathrm{L} \text { as Hg } \\ 01065 & \text { Nickel, dissolved } & 1 & \mu \mathrm{g} / \mathrm{L} \text { as Ni } \\ 01145 & \text { Selenium, dissolved } & 1 & \mu \mathrm{g} / \mathrm{L} \text { as Se } \\ 01075 & \text { Silver, dissolved } & 1 & \mu \mathrm{g} / \mathrm{L} \text { as Ag } \\ 01090 & \text { Zinc, dissolved } & .5 & \mu \mathrm{g} / \mathrm{L} \text { as Zn }\end{array}$

Trace elements in filtered water [Schedule 2703] (Phase II):

$\begin{array}{llll}01106 & \text { Aluminum } & 1 & \mu \mathrm{g} / \mathrm{L} \text { as Al } \\ 01095 & \text { Antimony } & 1 & \mu \mathrm{g} / \mathrm{L} \text { as Sb } \\ 01000 & \text { Arsenic } & 1 & \mu \mathrm{g} / \mathrm{L} \text { as As } \\ 01005 & \text { Barium } & 1 & \mu \mathrm{g} / \mathrm{L} \text { as Ba } \\ 01010 & \text { Beryllium } & 1 & \mu \mathrm{g} / \mathrm{L} \text { as Be } \\ 01025 & \text { Cadmium } & 1 & \mu \mathrm{g} / \mathrm{L} \text { as } \mathrm{Cd} \\ 01030 & \text { Chromium } & 1 & \mu \mathrm{g} / \mathrm{L} \text { as Cr } \\ 01035 & \text { Cobalt } & 1 & \mu \mathrm{g} / \mathrm{L} \text { as Co } \\ 01040 & \text { Copper } & 1 & \mu \mathrm{g} / \mathrm{L} \text { as Cu } \\ 01049 & \text { Lead } & 1 & \mu \mathrm{g} / \mathrm{L} \text { as } \mathrm{Pb} \\ 01056 & \text { Manganese } & 1 & \mu \mathrm{g} / \mathrm{L} \text { as Mn } \\ 01060 & \text { Molybdenum } & 1 & \mu \mathrm{g} / \mathrm{L} \text { as Mo } \\ 01065 & \text { Nickel } & 1 & \mu \mathrm{g} / \mathrm{L} \text { as Ni } \\ 01145 & \text { Selenium } & 1 & \mu \mathrm{g} / \mathrm{L} \text { as Se } \\ 01075 & \text { Silver } & 1 & \mu \mathrm{g} / \mathrm{L} \text { as Ag } \\ 01090 & \text { Zinc } & 1 & \mu \mathrm{g} / \mathrm{L} \text { as Zn } \\ 22703 & \text { Uranium } & 1 & \mu \mathrm{g} / \mathrm{L} \text { as } \mathrm{U}\end{array}$


Appendix - U.S. Environmental Protection Agency STOage and RETrieval (STORET) codes and minimum reporting level (MRL) or method detection limit (MDL) (Schedules 8308, 2010, and 8015 ) for constituents analyzed during Phases I and II of the Willamette River Basin Water Quality Study, Oregon, 1992-94—Continued

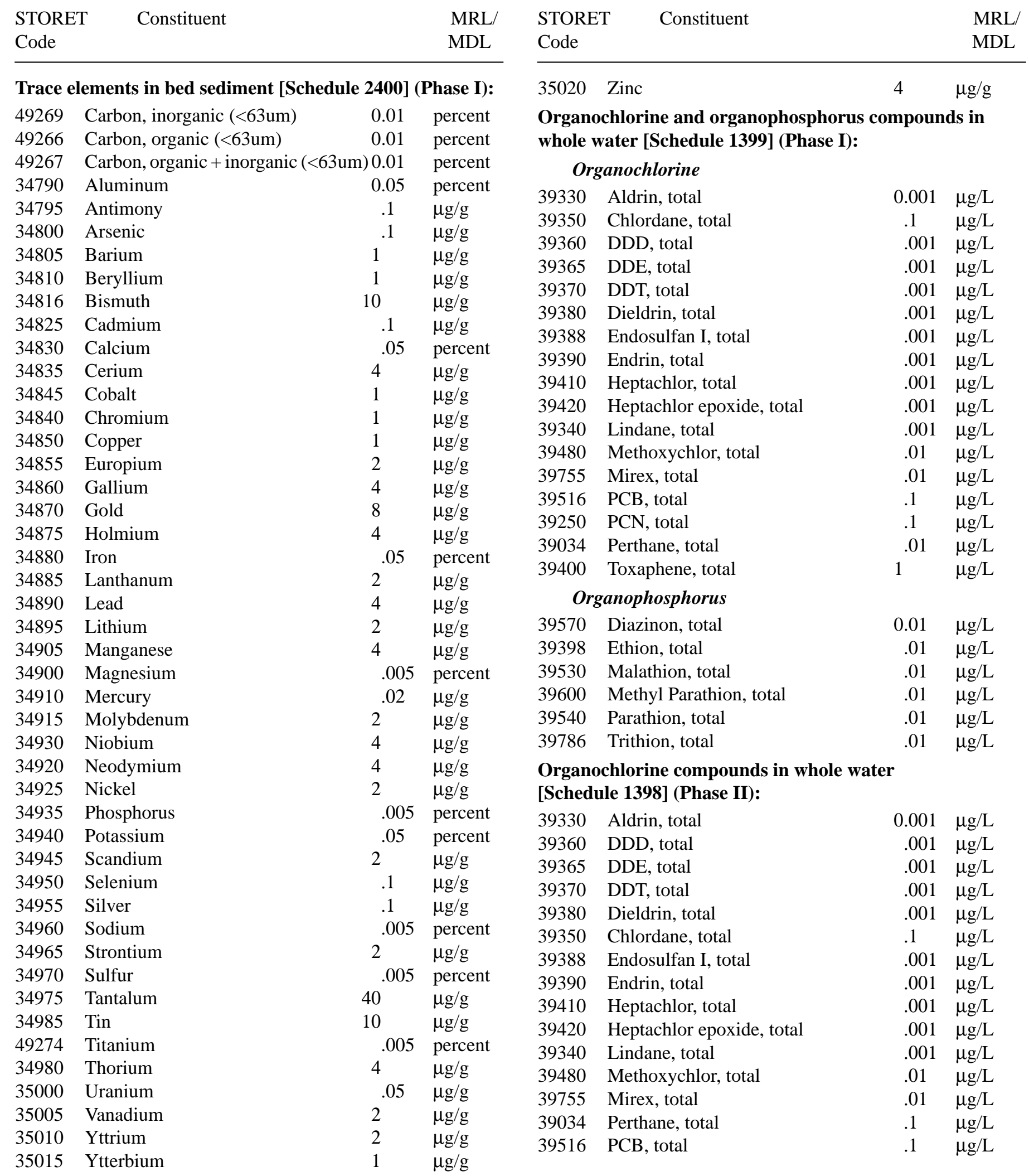


Appendix-U.S. Environmental Protection Agency STOage and RETrieval (STORET) codes and minimum reporting level (MRL) or method detection limit (MDL) (Schedules 8308, 2010, and 8015 ) for constituents analyzed during Phases I and II of the Willamette River Basin Water Quality Study, Oregon, 1992-94—Continued

\begin{tabular}{|c|c|c|c|c|c|c|c|}
\hline $\begin{array}{l}\text { STORE } \\
\text { Code }\end{array}$ & Constituent & & $\begin{array}{l}\text { MRL/ } \\
\text { MDL }\end{array}$ & $\begin{array}{l}\text { STORE' } \\
\text { Code }\end{array}$ & Constituent & & $\begin{array}{l}\text { MRL/ } \\
\text { MDL }\end{array}$ \\
\hline 39250 & PCN, total & 0.1 & $\mu \mathrm{g} / \mathrm{L}$ & 39756 & Mirex, dissolved & 0.01 & $\mu \mathrm{g} / \mathrm{L}$ \\
\hline 39400 & Toxaphene, total & 1 & $\mu \mathrm{g} / \mathrm{L}$ & 39517 & PCB, dissolved & .1 & $\mu \mathrm{g} / \mathrm{L}$ \\
\hline Organo & chlorine and organophosphor & pound & & $\begin{array}{l}82360 \\
82348\end{array}$ & PCN, dissolved. & 1 & $\mu \mathrm{g} / \mathrm{L}$ \\
\hline filtered & water [Schedule 8307] (Phase & & & 82348 & Perthane, dissolved & .1 & $\mu \mathrm{g} / \mathrm{L}$ \\
\hline Or & anochlorine & & & & Toxaphene, dissolved & & \\
\hline 39331 & Aldrin, dissolved & 0.001 & $\mu \mathrm{g} / \mathrm{L}$ & Organo & chlorine and organophosphoru & pounds & \\
\hline 39352 & Chlordane, dissolved & .1 & $\mu \mathrm{g} / \mathrm{L}$ & & ded sediment [Schedule 8308 & & \\
\hline 39361 & DDD, dissolved & .001 & $\mu \mathrm{g} / \mathrm{L}$ & Org & ganochlorine & & \\
\hline 39366 & DDE, dissolved & .001 & $\mu \mathrm{g} / \mathrm{L}$ & 39332 & Aldrin, suspended & 0.001 & $\mu \mathrm{g} / \mathrm{L}$ \\
\hline 39371 & DDT, dissolved & .001 & $\mu \mathrm{g} / \mathrm{L}$ & 39353 & Chlordane, suspended & .1 & $\mu \mathrm{g} / \mathrm{L}$ \\
\hline 39381 & Dieldrin, dissolved & .001 & $\mu \mathrm{g} / \mathrm{L}$ & 39362 & DDD, suspended & .001 & $\mu \mathrm{g} / \mathrm{L}$ \\
\hline 82354 & Endosulfan I, dissolved & .001 & $\mu \mathrm{g} / \mathrm{L}$ & 39367 & DDE, suspended & .001 & $\mu \mathrm{g} / \mathrm{L}$ \\
\hline 39391 & Endrin, dissolved & .001 & $\mu \mathrm{g} / \mathrm{L}$ & 39372 & DDT, suspended & .001 & $\mu \mathrm{g} / \mathrm{L}$ \\
\hline 39411 & Heptachlor, dissolved & .001 & $\mu \mathrm{g} / \mathrm{L}$ & 39382 & Dieldrin, suspended & .001 & $\mu \mathrm{g} / \mathrm{L}$ \\
\hline 39421 & Heptachlor epoxide, dissolved & .001 & $\mu \mathrm{g} / \mathrm{L}$ & 82355 & Endosulfan I, suspended & .001 & $\mu \mathrm{g} / \mathrm{L}$ \\
\hline 39341 & Lindane, dissolved & .001 & $\mu \mathrm{g} / \mathrm{L}$ & 39392 & Endrin, suspended & .001 & $\mu \mathrm{g} / \mathrm{L}$ \\
\hline 82350 & Methoxychlor, dissolved & .01 & $\mu \mathrm{g} / \mathrm{L}$ & 39412 & Heptachlor, suspended & .001 & $\mu \mathrm{g} / \mathrm{L}$ \\
\hline 39756 & Mirex, dissolved & .01 & $\mu \mathrm{g} / \mathrm{L}$ & 39422 & Heptachlor epoxide, suspended & .001 & $\mu \mathrm{g} / \mathrm{L}$ \\
\hline 39517 & PCB, dissolved & .1 & $\mu \mathrm{g} / \mathrm{L}$ & 39342 & Lindane, suspended & .001 & $\mu \mathrm{g} / \mathrm{L}$ \\
\hline 82360 & PCN, dissolved & .1 & $\mu \mathrm{g} / \mathrm{L}$ & 82351 & Methoxychlor, suspended & .01 & $\mu \mathrm{g} / \mathrm{L}$ \\
\hline 82348 & Perthane, dissolved & .1 & $\mu \mathrm{g} / \mathrm{L}$ & 39757 & Mirex, suspended & .01 & $\mu \mathrm{g} / \mathrm{L}$ \\
\hline 39401 & Toxaphene, dissolved & 1 & $\mu \mathrm{g} / \mathrm{L}$ & 39518 & PCB, suspended & .1 & $\mu \mathrm{g} / \mathrm{L}$ \\
\hline Or & anophosphorus & & & 82361 & PCN, suspended & .1 & $\mu \mathrm{g} / \mathrm{L}$ \\
\hline 38933 & Chlorpyrifos, dissolved & 0.01 & $\mu \mathrm{g} / \mathrm{L}$ & 82349 & Perthane, suspended & .1 & $\mu \mathrm{g} / \mathrm{L}$ \\
\hline 39572 & Diazinon, dissolved & $\begin{array}{r}.01 \\
.01\end{array}$ & $\mu \mathrm{g} / \mathrm{L}$ & 39402 & Toxaphene, suspended & 1 & $\mu \mathrm{g} / \mathrm{L}$ \\
\hline 82346 & Ethion, dissolved & .01 & $\mu \mathrm{g} / \mathrm{L}$ & Org & ganophosphorus & & \\
\hline 04095 & Fonofos, dissolved & .01 & $\mu \mathrm{g} / \mathrm{L}$ & 39573 & Diazinon, suspended & 0.01 & $\mu \mathrm{g} / \mathrm{L}$ \\
\hline 39532 & Malathion, dissolved & .01 & $\mu \mathrm{g} / \mathrm{L}$ & 82347 & Ethion, suspended & .01 & $\mu \mathrm{g} / \mathrm{L}$ \\
\hline 39602 & Methyl Parathion, dissolved & .01 & $\mu \mathrm{g} / \mathrm{L}$ & 39533 & Malathion, suspended & .01 & $\mu \mathrm{g} / \mathrm{L}$ \\
\hline 39542 & Parathion, dissolved & .01 & $\mu \mathrm{g} / \mathrm{L}$ & 39603 & Methyl Parathion, suspended & .01 & $\mu \mathrm{g} / \mathrm{L}$ \\
\hline 82342 & Trithion, dissolved & .01 & $\mu \mathrm{g} / \mathrm{L}$ & 39543 & Parathion, suspended & .01 & $\mu \mathrm{g} / \mathrm{L}$ \\
\hline Organo & chlorine compounds in filtere & & & 82343 & Trithion, suspended & .01 & $\mu \mathrm{g} / \mathrm{L}$ \\
\hline
\end{tabular}

\section{[Schedule 8369] (Phase II):}

39331 Aldrin, dissolved

39352 Chlordane, dissolved

39361 DDD, dissolved

39366 DDE, dissolved

39371 DDT, dissolved

39381 Dieldrin, dissolved

82354 Endosulfan I, dissolved

39391 Endrin, dissolved

39411 Heptachlor, dissolved

39421 Heptachlor epoxide, dissolved

39341 Lindane, dissolved

82350 Methoxychlor, dissolved
$0.001 \mu \mathrm{g} / \mathrm{L}$

$.1 \mu \mathrm{g} / \mathrm{L}$

$.001 \mu \mathrm{g} / \mathrm{L}$

$.001 \mu \mathrm{g} / \mathrm{L}$

$.001 \mu \mathrm{g} / \mathrm{L}$

$.001 \mu \mathrm{g} / \mathrm{L}$

$.001 \mu \mathrm{g} / \mathrm{L}$

$.001 \mu \mathrm{g} / \mathrm{L}$

$.001 \mu \mathrm{g} / \mathrm{L}$

$.001 \mu \mathrm{g} / \mathrm{L}$

$.001 \mu \mathrm{g} / \mathrm{L}$

$.01 \mu \mathrm{g} / \mathrm{L}$

Organochlorine compounds in suspended sediment

\section{[Schedule 8370] (Phase II):}

39332 Aldrin, suspended

39353 Chlordane, suspended

39362 DDD, suspended

39367 DDE, suspended

39372 DDT, suspended

39382 Dieldrin, suspended

82355 Endosulfan I, suspended

39392 Endrin, suspended

39412 Heptachlor, suspended

39422 Heptachlor epoxide, suspended

39342 Lindane, suspended
$0.001 \mu \mathrm{g} / \mathrm{L}$

$.1 \mu \mathrm{g} / \mathrm{L}$

$.001 \mu \mathrm{g} / \mathrm{L}$

$.001 \mu \mathrm{g} / \mathrm{L}$

$.001 \mu \mathrm{g} / \mathrm{L}$

$.001 \mu \mathrm{g} / \mathrm{L}$

$.001 \mu \mathrm{g} / \mathrm{L}$

$.001 \mu \mathrm{g} / \mathrm{L}$

$.001 \mu \mathrm{g} / \mathrm{L}$

$.001 \mu \mathrm{g} / \mathrm{L}$

$.001 \mu \mathrm{g} / \mathrm{L}$ 
Appendix - U.S. Environmental Protection Agency STOage and RETrieval (STORET) codes and minimum reporting level (MRL) or method detection limit (MDL) (Schedules 8308, 2010, and 8015 ) for constituents analyzed during Phases I and II of the Willamette River Basin Water Quality Study, Oregon, 1992-94—Continued

\begin{tabular}{|c|c|c|c|c|c|c|c|}
\hline $\begin{array}{l}\text { STORET } \\
\text { Code }\end{array}$ & Constituent & & $\begin{array}{l}\text { MRL/ } \\
\text { MDL }\end{array}$ & $\begin{array}{l}\text { STORET } \\
\text { Code }\end{array}$ & Constituent & & $\begin{array}{l}\text { MRL/ } \\
\text { MDL }\end{array}$ \\
\hline 82351 & Methoxychlor, suspended & .01 & $\mu \mathrm{g} / \mathrm{L}$ & Organic & acids in whole water [ & Phase & e I): \\
\hline 39757 & Mirex, suspended & .01 & $\mu \mathrm{g} / \mathrm{L}$ & 39730 & $2,4-\mathrm{D}$ & .01 & $\mu \mathrm{g} / \mathrm{L}$ \\
\hline 39518 & PCB, suspended & .1 & $\mu \mathrm{g} / \mathrm{L}$ & 39740 & $2,4,5-\mathrm{T}$ & .01 & $\mu \mathrm{g} / \mathrm{L}$ \\
\hline 82361 & PCN, suspended & .1 & $\mu \mathrm{g} / \mathrm{L}$ & 49231 & 3,5-Dinitro Aniline & .01 & $\mu \mathrm{g} / \mathrm{L}$ \\
\hline 82349 & Perthane, suspended & .1 & $\mu \mathrm{g} / \mathrm{L}$ & 82052 & Dicamba & .01 & $\mu \mathrm{g} / \mathrm{L}$ \\
\hline 39402 & Toxaphene, suspended & 1 & $\mu \mathrm{g} / \mathrm{L}$ & 82183 & Dichlorprop (2, 4-DP) & .01 & $\mu \mathrm{g} / \mathrm{L}$ \\
\hline \multirow{2}{*}{\multicolumn{4}{|c|}{$\begin{array}{l}\text { Organochlorine compounds in bed sediment } \\
\text { [Schedule 2501] (Phase I): }\end{array}$}} & 39720 & Picloram & .01 & $\mu \mathrm{g} / \mathrm{L}$ \\
\hline & & & & 39760 & Silvex & .01 & $\mu \mathrm{g} / \mathrm{L}$ \\
\hline
\end{tabular}

$\begin{array}{llll}49271 & \text { Carbon, organic }(<2 \mathrm{~mm}) & 0.1 & \mathrm{~g} / \mathrm{kg} \\ 49270 & \text { Carbon, inorganic }(<2 \mathrm{~mm}) & 0.1 & \mathrm{~g} / \mathrm{kg}\end{array}$

49272 Carbon, organic + inorganic $(<2 \mathrm{~mm}) 0.1 \mathrm{~g} / \mathrm{kg}$

80164 Sediment-bed, sieved $(<2 \mathrm{~mm})$

Aldrin

alpha-HCH

beta-HCH

trans-Chlordane

cis-Chlordane

Chloroneb

cis-Nonachlor

cis-Permethrin

Hexachlorobenzene

DCPA

p,p'-DDD

$o, p^{\prime}-D D D$

o,p'-DDE

p, p'-DDE

p,p'-DDT

o,p'-DDT

Dieldrin

Endosulfan I

Endrin

Heptachlor epoxide

Heptachlor

Isodrin

Lindane (gamma-HCH)

Mirex

o,p'-Methoxychlor

Oxychlordane

PCB

Pentachloroanisole

p,p'-Methoxychlor

Toxaphene

trans-Nonachlor

trans-Permethrin

$\begin{aligned} & \mathrm{percent} \\ 1 & \mu \mathrm{g} / \mathrm{kg} \\ 1 & \mu \mathrm{g} / \mathrm{kg} \\ 1 & \mu \mathrm{g} / \mathrm{kg} \\ 1 & \mu \mathrm{g} / \mathrm{kg} \\ 1 & \mu \mathrm{g} / \mathrm{kg} \\ 5 & \mu \mathrm{g} / \mathrm{kg} \\ 1 & \mu \mathrm{g} / \mathrm{kg} \\ 5 & \mu \mathrm{g} / \mathrm{kg} \\ 1 & \mu \mathrm{g} / \mathrm{kg} \\ 5 & \mu \mathrm{g} / \mathrm{kg} \\ 1 & \mu \mathrm{g} / \mathrm{kg} \\ 1 & \mu \mathrm{g} / \mathrm{kg} \\ 1 & \mu \mathrm{g} / \mathrm{kg} \\ 1 & \mu \mathrm{g} / \mathrm{kg} \\ 2 & \mu \mathrm{g} / \mathrm{kg} \\ 2 & \mu \mathrm{g} / \mathrm{kg} \\ 1 & \mu \mathrm{g} / \mathrm{kg} \\ 1 & \mu \mathrm{g} / \mathrm{kg} \\ 2 & \mu \mathrm{g} / \mathrm{kg} \\ 1 & \mu \mathrm{g} / \mathrm{kg} \\ 1 & \mu \mathrm{g} / \mathrm{kg} \\ 1 & \mu \mathrm{g} / \mathrm{kg} \\ 1 & \mu \mathrm{g} / \mathrm{kg} \\ 1 & \mu \mathrm{g} / \mathrm{kg} \\ 5 & \mu \mathrm{g} / \mathrm{kg} \\ 1 & \mu \mathrm{g} / \mathrm{kg} \\ 50 & \mu \mathrm{g} / \mathrm{kg} \\ 1 & \mu \mathrm{g} / \mathrm{kg} \\ 5 & \mu \mathrm{g} / \mathrm{kg} \\ 200 & \mu \mathrm{g} / \mathrm{kg} \\ 1 & \mu \mathrm{g} / \mathrm{kg} \\ 5 & \mu \mathrm{g} / \mathrm{kg}\end{aligned}$

Semi-volatile compounds in whole water [Schedule 1383] (Phase I):

34205 Acenaphthene, total $5 \mu \mathrm{g} / \mathrm{L}$

34200 Acenaphthylene, total $5 \mu \mathrm{g} / \mathrm{L}$

34220 Anthracene, total $5 \mu \mathrm{g} / \mathrm{L}$

34636 4-Bromophenylphenyether, total $\quad 5 \mu \mathrm{g} / \mathrm{L}$

39120 Benzidine, total $\quad 40 \mu \mathrm{g} / \mathrm{L}$

34526 Benzo[a]anthracene, total $10 \mu \mathrm{g} / \mathrm{L}$

34247 Benzo[a]pyrene, total $\quad 10 \mu \mathrm{g} / \mathrm{L}$

$34230 \quad$ Benzo[b]fluoranthene, total $\quad 10 \mu \mathrm{g} / \mathrm{L}$

34521 Benzo[g,h,i]perylene, total $10 \mu \mathrm{g} / \mathrm{L}$

34242 Benzo[k]fluoranthene, total $\quad 10 \mu \mathrm{g} / \mathrm{L}$

34292 Butyl benzyl phthalate, total $\quad 5 \mu \mathrm{g} / \mathrm{L}$

34278 bis-(2-Chloroethoxy) methane, total $\quad 5 \mu \mathrm{g} / \mathrm{L}$

34273 bis-(2-Chloroethyl) ether, total $\quad 5 \mu \mathrm{g} / \mathrm{L}$

34283 bis-(2-Chlorisopropyl) ether, total $\quad 5 \mu \mathrm{g} / \mathrm{L}$

34581 2-Chloronaphthalene, total $5 \mu \mathrm{g} / \mathrm{L}$

34586 2-Chlorophenol, total $5 \mu \mathrm{g} / \mathrm{L}$

34641 4-Chlorophenylphenyether, total $\quad 5 \mu \mathrm{g} / \mathrm{L}$

34452 4-Chloro-3-methylphenol, total $\quad 30 \mu \mathrm{g} / \mathrm{L}$

34320 Chrysene, total $10 \mu \mathrm{g} / \mathrm{L}$

82626 1,2-Diphenylhydrazine, total $\quad 5 \mu \mathrm{g} / \mathrm{L}$

34536 1,2-Dichlorobenzene, total $\quad 5 \mu \mathrm{g} / \mathrm{L}$

34566 1,3-Dichlorobenzene, total $5 \mu \mathrm{g} / \mathrm{L}$

34571 1,4-Dichlorobenzene, total $5 \mu \mathrm{g} / \mathrm{L}$

34601 2,4-Dichlorophenol, total $5 \mu \mathrm{g} / \mathrm{L}$

34606 2,4-Dimethylphenol, total $5 \mu \mathrm{g} / \mathrm{L}$

34616 2,4-Dinitrophenol, total $\quad 20 \mu \mathrm{g} / \mathrm{L}$

34611 2,4-Dinitrotoluene, total $5 \mu \mathrm{g} / \mathrm{L}$

34626 2,6-Dinitrotoluene, total $5 \mu \mathrm{g} / \mathrm{L}$

34631 3,3-Dichlorobenzidine, total $20 \mu \mathrm{g} / \mathrm{L}$

39110 Di-n-butyl phthalate, total $\quad 5 \mu \mathrm{g} / \mathrm{L}$

34596 Di-n-octyl phthalate, total $\quad 10 \mu \mathrm{g} / \mathrm{L}$

34556 1,2,5,6-Dibenz[a,h]anthracene, total $\quad 10 \mu \mathrm{g} / \mathrm{L}$

34336 Diethyl phthalate, total $\quad 5 \mu \mathrm{g} / \mathrm{L}$

34341 Dimethyl phthalate, total $5 \mu \mathrm{g} / \mathrm{L}$

34657 4,6-Dinitro-2-methylphenol, total $\quad 30 \mu \mathrm{g} / \mathrm{L}$

39100 bis-(2-Ethylhexyl) phthalate, total $\quad 5 \mu \mathrm{g} / \mathrm{L}$ 
Appendix-U.S. Environmental Protection Agency STOage and RETrieval (STORET) codes and minimum reporting level (MRL) or method detection limit (MDL) (Schedules 8308, 2010, and 8015 ) for constituents analyzed during Phases I and II of the Willamette River Basin Water Quality Study, Oregon, 1992-94—Continued

\begin{tabular}{|c|c|c|c|c|c|c|c|}
\hline $\begin{array}{l}\text { STORE } \\
\text { Code }\end{array}$ & Constituent & & $\begin{array}{l}\text { MRL/ } \\
\text { MDL }\end{array}$ & $\begin{array}{l}\text { STORE } \\
\text { Code }\end{array}$ & Constituent & & $\begin{array}{l}\text { MRL/ } \\
\text { MDL }\end{array}$ \\
\hline 34376 & Fluoranthene, total & 5 & $\mu \mathrm{g} / \mathrm{L}$ & 34617 & 2,4-Dinitrophenol, dissolved & 20 & $\mu \mathrm{g} / \mathrm{L}$ \\
\hline 34381 & Fluorene, total & 5 & $\mu \mathrm{g} / \mathrm{L}$ & 34612 & 2,4-Dinitrotoluene, dissolved & 5 & $\mu \mathrm{g} / \mathrm{L}$ \\
\hline 39700 & Hexachlorobenzene, total & 5 & $\mu \mathrm{g} / \mathrm{L}$ & 34627 & 2,6-Dinitrotoluene, dissolved & 5 & $\mu \mathrm{g} / \mathrm{L}$ \\
\hline 39702 & Hexachlorobutadiene, total & 5 & $\mu \mathrm{g} / \mathrm{L}$ & 34632 & 3,3-Dichlorobenzidine, dissolved & 20 & $\mu \mathrm{g} / \mathrm{L}$ \\
\hline 34386 & Hexachlorocyclopentadiene, total & 5 & $\mu \mathrm{g} / \mathrm{L}$ & 34327 & Di-n-butyl phthalate, dissolved & 5 & $\mu \mathrm{g} / \mathrm{L}$ \\
\hline 34396 & Hexachloroethane, total & 5 & $\mu \mathrm{g} / \mathrm{L}$ & 34597 & Di-n-octyl phthalate, dissolved & 10 & $\mu \mathrm{g} / \mathrm{L}$ \\
\hline 34403 & Indeno $(1,2,3-\mathrm{cd})$ pyrene, total & 10 & $\mu \mathrm{g} / \mathrm{L}$ & 34557 & 1,2,5,6-Dibenz[a,h]anthracene, dissolved & 10 & $\mu \mathrm{g} / \mathrm{L}$ \\
\hline 34408 & Isophorone, total & 5 & $\mu \mathrm{g} / \mathrm{L}$ & 34337 & Diethyl phthalate, dissolved & 5 & $\mu \mathrm{g} / \mathrm{L}$ \\
\hline 34428 & n-Nitrosodi-n-propylamine, total & 5 & $\mu \mathrm{g} / \mathrm{L}$ & 34342 & Dimethyl phthalate, dissolved & 5 & $\mu \mathrm{g} / \mathrm{L}$ \\
\hline 34433 & n-Nitrosodiphenylamine, total & 5 & $\mu \mathrm{g} / \mathrm{L}$ & 34607 & 2,4-Dimethyl phenol & 5 & $\mu \mathrm{g} / \mathrm{L}$ \\
\hline 34591 & 2-Nitrophenol, total & 5 & $\mu \mathrm{g} / \mathrm{L}$ & 34658 & 4,6-Dinitro-2-methylphenol, dissolved & 30 & $\mu \mathrm{g} / \mathrm{L}$ \\
\hline 34646 & 4-Nitrophenol, total & 30 & $\mu \mathrm{g} / \mathrm{L}$ & 39103 & bis-(2-Ethylhexyl) phthalate, dissolved & 5 & $\mu \mathrm{g} / \mathrm{L}$ \\
\hline 34696 & Naphthalene, total & 5 & $\mu \mathrm{g} / \mathrm{L}$ & 34377 & Fluoranthene, dissolved & 5 & $\mu \mathrm{g} / \mathrm{L}$ \\
\hline 34447 & Nitrobenzene, total & 5 & $\mu \mathrm{g} / \mathrm{L}$ & 34382 & Fluorene, dissolved & 5 & $\mu \mathrm{g} / \mathrm{L}$ \\
\hline 34438 & n-Nitrosodimethylamine, total & 5 & $\mu \mathrm{g} / \mathrm{L}$ & 34401 & Hexachlorobenzene, dissolved & 5 & $\mu \mathrm{g} / \mathrm{L}$ \\
\hline 39032 & Pentachlorophenol, total & 30 & $\mu \mathrm{g} / \mathrm{L}$ & 34392 & Hexachlorobutadiene, dissolved & 5 & $\mu \mathrm{g} / \mathrm{L}$ \\
\hline 34461 & Phenanthrene, total & 5 & $\mu \mathrm{g} / \mathrm{L}$ & 34387 & Hexachlorocyclopentadiene, dissolved & 5 & $\mu \mathrm{g} / \mathrm{L}$ \\
\hline 34694 & Phenol, total & 5 & $\mu \mathrm{g} / \mathrm{L}$ & 34397 & Hexachloroethane, dissolved & 5 & $\mu \mathrm{g} / \mathrm{L}$ \\
\hline 34469 & Pyrene, total & 5 & $\mu \mathrm{g} / \mathrm{L}$ & 34404 & Indeno $(1,2,3-\mathrm{cd})$ pyrene, dissolved & 10 & $\mu \mathrm{g} / \mathrm{L}$ \\
\hline 34551 & 1,2,4-Trichlorobenzene, total & 5 & $\mu \mathrm{g} / \mathrm{L}$ & 34409 & Isophorone, dissolved & 5 & $\mu \mathrm{g} / \mathrm{L}$ \\
\hline 34621 & 2,4,6-Trichlorophenol, total & 20 & $\mu \mathrm{g} / \mathrm{L}$ & 34429 & n-Nitrosodi-n-propylamine, dissolved & 5 & $\mu \mathrm{g} / \mathrm{L}$ \\
\hline Semi-vo & latile compounds in filtered & 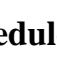 & $8005]$ & 34434 & n-Nitrosodiphenylamine, dissolved & 5 & $\mu \mathrm{g} / \mathrm{L}$ \\
\hline (Phase & & & & 34592 & 2-Nitrophenol, dissolved & 5 & $\mu \mathrm{g} / \mathrm{L}$ \\
\hline 34206 & Acenaphthene, dissolved & 5 & & 34647 & 4-Nitrophenol, dissolved & 30 & $\mu \mathrm{g} / \mathrm{L}$ \\
\hline 34201 & Acenaphthylene, dissolved & 5 & $\mu \mathrm{g} / \mathrm{L}$ & 34443 & Naphthalene, dissolved & 5 & $\mu \mathrm{g} / \mathrm{L}$ \\
\hline 34221 & Anthracene, dissolved & 5 & $\mu \mathrm{g} / \mathrm{L}$ & 34448 & Nitrobenzene, dissolved & 5 & $\mu \mathrm{g} / \mathrm{L}$ \\
\hline 34637 & 4-Bromophenylphenyether, dissolved & 5 & $\mu \mathrm{g} / \mathrm{L}$ & 34439 & n-Nitrosodimethylamine, dissolved & 5 & $\mu \mathrm{g} / \mathrm{L}$ \\
\hline 34239 & Benzidine, dissolved & 40 & $\mu \mathrm{g} / \mathrm{L}$ & 34459 & Pentachlorophenol, dissolved & 30 & $\mu \mathrm{g} / \mathrm{L}$ \\
\hline 34527 & Benzo[a]anthracene, dissolved & 10 & $\mu \mathrm{g} / \mathrm{L}$ & 34462 & Phenanthrene, dissolved & 5 & $\mu \mathrm{g} / \mathrm{L}$ \\
\hline 34248 & Benzo[a]pyrene, dissolved & 10 & $\mu \mathrm{g} / \mathrm{L}$ & 34470 & Pyrene, dissolved & 5 & $\mu \mathrm{g} / \mathrm{L}$ \\
\hline 34231 & Benzo[b]fluoranthene, dissolved & 10 & $\mu \mathrm{g} / \mathrm{L}$ & 34552 & 1,2,4-Trichlorobenzene, dissolved & 5 & $\mu \mathrm{g} / \mathrm{L}$ \\
\hline 34522 & Benzo[g,h,i]perylene, dissolved & 10 & $\mu \mathrm{g} / \mathrm{L}$ & 34622 & 2,4,6-Trichlorophenol, dissolved & 20 & $\mu \mathrm{g} / \mathrm{L}$ \\
\hline
\end{tabular}

34243 Benzo[k]fluoranthene, dissolved $\quad 10 \mu \mathrm{g} / \mathrm{L} \quad$ Semi-volatile compounds in suspended sediment

34293 Butyl benzyl phthalate, dissolved $\quad 5 \mu \mathrm{g} / \mathrm{L} \quad$ [Schedule 8006] (Phase I):

34279 bis-(2-Chloroethoxy) methane, dissolved

34274 bis-(2-Chloroethyl) ether, dissolved

34284 bis-(2-Chlorisopropyl) ether, dissolved

34582 2-Chloronaphthalene, dissolved

34587 2-Chlorophenol, dissolved

34642 4-Chlorophenylphenyether, dissolved

34453 4-Chloro-3-methylphenol, dissolved

34321 Chrysene, dissolved

34347 1,2-Diphenylhydrazine, dissolved

34537 1,2-Dichlorobenzene, dissolved

34567 1,3-Dichlorobenzene, dissolved

34572 1,4-Dichlorobenzene, dissolved

$\mu \mathrm{g} / \mathrm{L}$

$\mu \mathrm{g} / \mathrm{L}$

34207 Acenaphthene, suspended

34202 Acenaphthylene, suspended

34222 Anthracene, suspended

34638 4-Bromophenylphenyether, suspended

34240 Benzidine, suspended

34528 Benzo[a]anthracene, suspended

34249 Benzo[a]pyrene, suspended

34232 Benzo[b]fluoranthene, suspended

34523 Benzo[g,h,i]perylene, suspended

34244 Benzo[k]fluoranthene, suspended

34294 Butyl benzyl phthalate, suspended

34280 bis-(2-Chloroethoxy) methane, suspended

$5 \mu \mathrm{g} / \mathrm{L}$

$5 \mu \mathrm{g} / \mathrm{L}$

$5 \mu \mathrm{g} / \mathrm{L}$

$5 \mu \mathrm{g} / \mathrm{L}$

$30 \mu \mathrm{g} / \mathrm{L}$

$10 \mu \mathrm{g} / \mathrm{L}$

$5 \mu \mathrm{g} / \mathrm{L}$

$5 \mu \mathrm{g} / \mathrm{L}$

$5 \mu \mathrm{g} / \mathrm{L}$

$5 \mu \mathrm{g} / \mathrm{L}$

34602 2,4-Dichlorophenol, dissolved

$5 \mu \mathrm{g} / \mathrm{L} \quad 34275$ bis-(2-Chloroethyl) ether, suspended $\quad 5 \mu \mathrm{g} / \mathrm{L}$

$5 \mu \mathrm{g} / \mathrm{L} \quad 34275$ bis-(2-Chloroethyl) ether, suspended $5 \mu \mathrm{g} / \mathrm{L}$

$10 \mu \mathrm{g} / \mathrm{L}$

$10 \mu \mathrm{g} / \mathrm{L}$

$10 \mu \mathrm{g} / \mathrm{L}$

$10 \mu \mathrm{g} / \mathrm{L}$

$10 \mu \mathrm{g} / \mathrm{L}$

$5 \mu \mathrm{g} / \mathrm{L}$

$5 \mu \mathrm{g} / \mathrm{L}$ 
Appendix - U.S. Environmental Protection Agency STOage and RETrieval (STORET) codes and minimum reporting level (MRL) or method detection limit (MDL) (Schedules 8308, 2010, and 8015 ) for constituents analyzed during Phases I and II of the Willamette River Basin Water Quality Study, Oregon, 1992-94—Continued

\begin{tabular}{|c|c|c|c|c|c|c|c|}
\hline $\begin{array}{l}\text { STORE' } \\
\text { Code }\end{array}$ & Constituent & & $\begin{array}{l}\text { MRL/ } \\
\text { MDL }\end{array}$ & $\begin{array}{l}\text { STORE } \\
\text { Code }\end{array}$ & Constituent & & $\begin{array}{l}\text { MRL } \\
\text { MDL }\end{array}$ \\
\hline 34285 & bis-(2-Chlorisopropyl) ether, suspended & & $\mu \mathrm{g} / \mathrm{L}$ & Semi-v & olatile compounds in bed sediment [S & hedu & le 2502] \\
\hline 34583 & 2-Chloronaphthalene, suspended & & $\mu \mathrm{g} / \mathrm{L}$ & (Phase & & & \\
\hline 34588 & 2-Chlorophenol, suspended & 5 & $\mu \mathrm{g} / \mathrm{L}$ & 49271 & Carbon, organic $(<2 \mathrm{~mm})$ & & $\mathrm{g} / \mathrm{kg}$ \\
\hline 34643 & 4-Chlorophenylphenyether, suspended & 5 & $\mu \mathrm{g} / \mathrm{L}$ & 49270 & Carbon, inorganic $(<2 \mathrm{~mm})$ & & $\mathrm{g} / \mathrm{kg}$ \\
\hline 34454 & 4-Chloro-3-methylphenol, suspended & 30 & $\mu \mathrm{g} / \mathrm{L}$ & 49272 & Carbon, organic + inorganic $(<2 \mathrm{~mm})$ & & $\mathrm{g} / \mathrm{kg}$ \\
\hline 34322 & Chrysene, suspended & 10 & $\mu \mathrm{g} / \mathrm{L}$ & 80164 & Sediment-bed, sieved $(<2 \mathrm{~mm})$ & & percent \\
\hline 34348 & 1,2-Diphenylhydrazine, suspended & 5 & $\mu \mathrm{g} / \mathrm{L}$ & 49429 & Acenaphthene & 50 & $\mu \mathrm{g} / \mathrm{kg}$ \\
\hline 34538 & 1,2-Dichlorobenzene, suspended & 5 & $\mu \mathrm{g} / \mathrm{L}$ & 49428 & Acenaphthylene & 50 & $\mu \mathrm{g} / \mathrm{kg}$ \\
\hline 34568 & 1,3-Dichlorobenzene, suspended & 5 & $\mu \mathrm{g} / \mathrm{L}$ & 49430 & Acridine & 50 & $\mu \mathrm{g} / \mathrm{kg}$ \\
\hline 34573 & 1,4-Dichlorobenzene, suspended & 5 & $\mu \mathrm{g} / \mathrm{L}$ & 49424 & C8-Alkylphenol & 50 & $\mu \mathrm{g} / \mathrm{kg}$ \\
\hline 34603 & 2,4-Dichlorophenol, suspended & 5 & $\mu \mathrm{g} / \mathrm{L}$ & 49434 & Anthracene & 50 & $\mu \mathrm{g} / \mathrm{kg}$ \\
\hline 34618 & 2,4-Dinitrophenol, suspended & 20 & $\mu \mathrm{g} / \mathrm{L}$ & 49437 & Anthraquinone & 50 & $\mu \mathrm{g} / \mathrm{kg}$ \\
\hline 34613 & 2,4-Dinitrotoluene, suspended & 5 & $\mu \mathrm{g} / \mathrm{L}$ & 49443 & Azobenzene & 50 & $\mu \mathrm{g} / \mathrm{kg}$ \\
\hline 34628 & 2,6-Dinitrotoluene, suspended & 5 & $\mu \mathrm{g} / \mathrm{L}$ & 49391 & 2,2’'-Biquinoline & 50 & $\mu \mathrm{g} / \mathrm{kg}$ \\
\hline 34633 & 3,3-Dichlorobenzidine, suspended & 20 & $\mu \mathrm{g} / \mathrm{L}$ & 49401 & bis-(2-Chloroethoxy)methane & 50 & $\mu \mathrm{g} / \mathrm{kg}$ \\
\hline 39114 & Di-n-butyl phthalate, suspended & 5 & $\mu \mathrm{g} / \mathrm{L}$ & 49454 & 4-Bromophenyl-phenylether & 50 & $\mu \mathrm{g} / \mathrm{kg}$ \\
\hline 34598 & Di-n-octyl phthalate, suspended & 10 & $\mu \mathrm{g} / \mathrm{L}$ & 49436 & Benz[a]anthracene & 50 & $\mu \mathrm{g} / \mathrm{kg}$ \\
\hline 34558 & 1,2,5,6-Dibenz[a,h]anthracene, suspended & 10 & $\mu \mathrm{g} / \mathrm{L}$ & 49458 & Benzo[b]fluoranthene & 50 & $\mu \mathrm{g} / \mathrm{kg}$ \\
\hline 34338 & Diethyl phthalate, suspended & 5 & $\mu \mathrm{g} / \mathrm{L}$ & 49408 & Benzo[g,h,i]perylene & 50 & $\mu \mathrm{g} / \mathrm{kg}$ \\
\hline 34343 & Dimethyl phthalate, suspended & 5 & $\mu \mathrm{g} / \mathrm{L}$ & 49397 & Benzo[k]fluoranthene & 50 & $\mu \mathrm{g} / \mathrm{kg}$ \\
\hline 34659 & 4,6-Dinitro-2-methylphenol, suspended & 30 & $\mu \mathrm{g} / \mathrm{L}$ & 49468 & Benzo[c]quinoline & 50 & $\mu \mathrm{g} / \mathrm{kg}$ \\
\hline 39104 & bis-(2-Ethylhexyl) phthalate, suspended & 5 & $\mu \mathrm{g} / \mathrm{L}$ & 49389 & Benzo[a]pyrene & 50 & $\mu \mathrm{g} / \mathrm{kg}$ \\
\hline 34378 & Fluoranthene, suspended & 5 & $\mu \mathrm{g} / \mathrm{L}$ & 49426 & bis-(2-Ethylhexyl)phthalate & 50 & $\mu \mathrm{g} / \mathrm{kg}$ \\
\hline 34383 & Fluorene, suspended & 5 & $\mu \mathrm{g} / \mathrm{L}$ & 49427 & Butylbenzylphthalate & 50 & $\mu \mathrm{g} / \mathrm{kg}$ \\
\hline 34402 & Hexachlorobenzene, suspended & 5 & $\mu \mathrm{g} / \mathrm{L}$ & 49438 & $1,2,4$-Trichlorobenzene & 50 & $\mu \mathrm{g} / \mathrm{kg}$ \\
\hline 34393 & Hexachlorobutadiene, suspended & 5 & $\mu \mathrm{g} / \mathrm{L}$ & 49467 & 2-Chlorophenol & 50 & $\mu \mathrm{g} / \mathrm{kg}$ \\
\hline 34388 & Hexachlorocyclopentadiene, suspended & 5 & $\mu \mathrm{g} / \mathrm{L}$ & 49407 & 2-Chloronaphthalene & 50 & $\mu \mathrm{g} / \mathrm{kg}$ \\
\hline 34398 & Hexachloroethane, suspended & 5 & $\mu \mathrm{g} / \mathrm{L}$ & 49455 & 4-Chlorophenyl-phenylether & 50 & $\mu \mathrm{g} / \mathrm{kg}$ \\
\hline 34405 & Indeno $(1,2,3-\mathrm{cd})$ pyrene, suspended & 10 & $\mu \mathrm{g} / \mathrm{L}$ & 49450 & Chrysene & 50 & $\mu \mathrm{g} / \mathrm{kg}$ \\
\hline 34410 & Isophorone, suspended & 5 & $\mu \mathrm{g} / \mathrm{L}$ & 49446 & Pentachloronitrobenzene & 50 & $\mu \mathrm{g} / \mathrm{kg}$ \\
\hline 34430 & n-Nitrosodi-n-propylamine, suspended & 5 & $\mu \mathrm{g} / \mathrm{L}$ & 49343 & Hexachlorobenzene & 50 & $\mu \mathrm{g} / \mathrm{kg}$ \\
\hline 34435 & n-Nitrosodiphenylamine, suspended & 5 & $\mu \mathrm{g} / \mathrm{L}$ & 49439 & 1,2-Dichlorobenzene & 50 & $\mu \mathrm{g} / \mathrm{kg}$ \\
\hline 34593 & 2-Nitrophenol, suspended & 5 & $\mu \mathrm{g} / \mathrm{L}$ & 49441 & 1,3-Dichlorobenzene & 50 & $\mu \mathrm{g} / \mathrm{kg}$ \\
\hline 34648 & 4-Nitrophenol, suspended & 30 & $\mu \mathrm{g} / \mathrm{L}$ & 49442 & 1,4-Dichlorobenzene & 50 & $\mu \mathrm{g} / \mathrm{kg}$ \\
\hline 34444 & Naphthalene, suspended & 5 & $\mu \mathrm{g} / \mathrm{L}$ & 49395 & 2,4-Dinitrotoluene & 50 & $\mu \mathrm{g} / \mathrm{kg}$ \\
\hline 34449 & Nitrobenzene, suspended & 5 & $\mu \mathrm{g} / \mathrm{L}$ & 49396 & 2,6-Dinitrotoluene & 50 & $\mu \mathrm{g} / \mathrm{kg}$ \\
\hline 34440 & n-Nitrosodimethylamine, suspended & 5 & $\mu \mathrm{g} / \mathrm{L}$ & 49421 & 3,5-Dimethylphenol & 50 & $\mu \mathrm{g} / \mathrm{kg}$ \\
\hline 34460 & Pentachlorophenol, suspended & 30 & $\mu \mathrm{g} / \mathrm{L}$ & 49452 & Dibenzothiophene & 50 & $\mu \mathrm{g} / \mathrm{kg}$ \\
\hline 34463 & Phenanthrene, suspended & 5 & $\mu \mathrm{g} / \mathrm{L}$ & 49381 & Di-n-butylphthalate & 50 & $\mu \mathrm{g} / \mathrm{kg}$ \\
\hline 34467 & Phenol, suspended & 5 & $\mu \mathrm{g} / \mathrm{L}$ & 49461 & Dibenz[a,h]anthracene & 50 & $\mu \mathrm{g} / \mathrm{kg}$ \\
\hline 34471 & Pyrene, suspended & 5 & $\mu \mathrm{g} / \mathrm{L}$ & 49383 & Diethylphthalate & 50 & $\mu \mathrm{g} / \mathrm{kg}$ \\
\hline 34553 & 1,2,4-Trichlorobenzene, suspended & 5 & $\mu \mathrm{g} / \mathrm{L}$ & 49384 & Dimethylphthalate & 50 & $\mu \mathrm{g} / \mathrm{kg}$ \\
\hline 34623 & 2,4,6-Trichlorophenol, suspended & 20 & $\mu \mathrm{g} / \mathrm{L}$ & 49382 & Di-n-octylphthalate & 50 & $\mu \mathrm{g} / \mathrm{kg}$ \\
\hline & & & & 49490 & 2-Ethylnapthalene & 50 & $\mu \mathrm{g} / \mathrm{kg}$ \\
\hline & & & & 49466 & Fluoranthene & 50 & $\mu \mathrm{g} / \mathrm{kg}$ \\
\hline & & & & 49449 & 9H-Carbazole & 50 & $\mu \mathrm{g} / \mathrm{kg}$ \\
\hline & & & & 49399 & 9H-Fluorene & 50 & $\mu \mathrm{g} / \mathrm{kg}$ \\
\hline
\end{tabular}


Appendix-U.S. Environmental Protection Agency STOage and RETrieval (STORET) codes and minimum reporting level (MRL) or method detection limit (MDL) (Schedules 8308, 2010, and 8015 ) for constituents analyzed during Phases I and II of the Willamette River Basin Water Quality Study, Oregon, 1992-94—Continued

\begin{tabular}{|c|c|c|c|c|c|}
\hline \multicolumn{2}{|c|}{$\begin{array}{l}\text { STORET } \\
\text { Code }\end{array}$} & \multirow{2}{*}{$\begin{array}{c}\begin{array}{c}\text { MRL/ } \\
\text { MDL }\end{array} \\
50 \mu \mathrm{g} / \mathrm{kg}\end{array}$} & \multicolumn{2}{|c|}{$\begin{array}{l}\text { STORET } \\
\text { Code }\end{array}$} & \multirow{2}{*}{$\begin{array}{r}\begin{array}{c}\text { MRL/ } \\
\text { MDL }\end{array} \\
0.2 \mu \mathrm{g} / \mathrm{L}\end{array}$} \\
\hline 49390 & Indeno[1,2,3-cd]pyrene & & 34536 & Benzene, 1,2-dichloro- & \\
\hline 49394 & Isoquinoline & $50 \mu \mathrm{g} / \mathrm{kg}$ & 32103 & Ethane, 1,2-dichloro- & $.2 \mu \mathrm{g} / \mathrm{L}$ \\
\hline 49400 & Isophorone & $50 \mu \mathrm{g} / \mathrm{kg}$ & 34541 & Propane, 1,2-dichloro- & $.2 \mu \mathrm{g} / \mathrm{L}$ \\
\hline 49403 & 1,2-Dimethylnaphthalene & $50 \mu \mathrm{g} / \mathrm{kg}$ & 34566 & Benzene, 1,3-dichloro- & $.2 \mu \mathrm{g} / \mathrm{L}$ \\
\hline 49404 & 1,6-Dimethylnaphthalene & $50 \mu \mathrm{g} / \mathrm{kg}$ & 34571 & Benzene, 1,4-dichloro- & $.2 \mu \mathrm{g} / \mathrm{L}$ \\
\hline 49398 & 1-Methyl-9H-fluorene & $50 \mu \mathrm{g} / \mathrm{kg}$ & 77168 & Propene, 1,1-dichloro- & $.2 \mu \mathrm{g} / \mathrm{L}$ \\
\hline 49410 & 1-Methylphenanthrene & $50 \mu \mathrm{g} / \mathrm{kg}$ & 77173 & Propane, 1,3-dichloro- & $.2 \mu \mathrm{g} / \mathrm{L}$ \\
\hline 49388 & 1-Methylpyrene & $50 \mu \mathrm{g} / \mathrm{kg}$ & 77170 & Propane, 2,2-dichloro- & $.2 \mu \mathrm{g} / \mathrm{L}$ \\
\hline 49405 & 2,3,6-Trimethylnaphthalene & $50 \mu \mathrm{g} / \mathrm{kg}$ & 82625 & Propane, 1,2-dibromo-3-chloro- & 1. $\mu \mathrm{g} / \mathrm{L}$ \\
\hline 49406 & 2,6-Dimethylnaphthalene & $50 \mu \mathrm{g} / \mathrm{kg}$ & 30217 & Methane, dibromo- & $.2 \mu \mathrm{g} / \mathrm{L}$ \\
\hline 49435 & 2-Methylanthracene & $50 \mu \mathrm{g} / \mathrm{kg}$ & 32101 & Methane, dichlorobromo- & $.2 \mu \mathrm{g} / \mathrm{L}$ \\
\hline 49411 & 4,5-Methylenephenanthrene & $50 \mu \mathrm{g} / \mathrm{kg}$ & 34668 & Methane, dichlorodifluoro- & $.2 \mu \mathrm{g} / \mathrm{L}$ \\
\hline 49422 & 4-Chloro-3-Methylphenol & $50 \mu \mathrm{g} / \mathrm{kg}$ & 77652 & Ethane, trichlorotrifluoro- (Freon 113) & $.2 \mu \mathrm{g} / \mathrm{L}$ \\
\hline 49433 & $\mathrm{~N}$-nitrosodiphenylamine & $50 \mu \mathrm{g} / \mathrm{kg}$ & 34371 & Benzene, ethyl- & $.2 \mu \mathrm{g} / \mathrm{L}$ \\
\hline 49431 & $\mathrm{~N}$-nitrosodi-n-propylamine & $50 \mu \mathrm{g} / \mathrm{kg}$ & 39702 & Hexachlorobutadiene & $.2 \mu \mathrm{g} / \mathrm{L}$ \\
\hline 49402 & Naphthalene & $50 \mu \mathrm{g} / \mathrm{kg}$ & 77223 & Benzene, isopropyl- & $.2 \mu \mathrm{g} / \mathrm{L}$ \\
\hline 49444 & Nitrobenzene & $50 \mu \mathrm{g} / \mathrm{kg}$ & 34413 & Methane, bromo- (Methyl bromide) & $.2 \mu \mathrm{g} / \mathrm{L}$ \\
\hline 49451 & p-Cresol & $50 \mu \mathrm{g} / \mathrm{kg}$ & 34423 & Methane, dichloro-(Methylene chloride) & $.2 \mu \mathrm{g} / \mathrm{L}$ \\
\hline 49460 & Pentachloroanisole & $50 \mu \mathrm{g} / \mathrm{kg}$ & 78032 & Propene, 2-methoxy-2-methyl -(MTBE) & 1. $\mu \mathrm{g} / \mathrm{L}$ \\
\hline 49409 & Phenanthrene & $50 \mu \mathrm{g} / \mathrm{kg}$ & 77342 & Benzene, n-butyl- & $.2 \mu \mathrm{g} / \mathrm{L}$ \\
\hline 49393 & Phenanthridine & $50 \mu \mathrm{g} / \mathrm{kg}$ & 77224 & Benzene, n-propyl- & $.2 \mu \mathrm{g} / \mathrm{L}$ \\
\hline 49413 & Phenol & $50 \mu \mathrm{g} / \mathrm{kg}$ & 34696 & Naphthalene & $.2 \mu \mathrm{g} / \mathrm{L}$ \\
\hline 49387 & Pyrene & $50 \mu \mathrm{g} / \mathrm{kg}$ & 77356 & Benzene, 1-methyl-4-isopropyl- & $.2 \mu \mathrm{g} / \mathrm{L}$ \\
\hline 49392 & Quinoline & $50 \mu \mathrm{g} / \mathrm{kg}$ & 77350 & Benzene, sec-butyl- & $.2 \mu \mathrm{g} / \mathrm{L}$ \\
\hline \multirow{2}{*}{\multicolumn{3}{|c|}{$\begin{array}{l}\text { Volatile organic compounds in whole water [Schedules } \\
1392 \text { and 1401] (Phase I) and [Schedule 2090] (Phase II): }\end{array}$}} & 77128 & Styrene & $.2 \mu \mathrm{g} / \mathrm{L}$ \\
\hline & & & 34506 & Ethane, 1,1,1-trichloro- & $.2 \mu \mathrm{g} / \mathrm{L}$ \\
\hline 34210 & Acrolein (phase I only) & $20 \mu \mathrm{g} / \mathrm{L}$ & 34516 & Ethane, 1,1,2,2-tetrachloro- & $.2 \mu \mathrm{g} / \mathrm{L}$ \\
\hline 34215 & Acrylonitrile (phase I only) & $20 \mu \mathrm{g} / \mathrm{L}$ & 34511 & Ethane, 1,1,2-trichloro- & $.2 \mu \mathrm{g} / \mathrm{L}$ \\
\hline 34030 & Benzene & $.2 \mu \mathrm{g} / \mathrm{L}$ & 77562 & Ethane, 1,1,1,2-tetrachloro- & $.2 \mu \mathrm{g} / \mathrm{L}$ \\
\hline 81555 & Benzene, bromo- & $.2 \mu \mathrm{g} / \mathrm{L}$ & 77613 & Benzene, 1,2,3-trichloro- & $.2 \mu \mathrm{g} / \mathrm{L}$ \\
\hline 77297 & Methane, bromochloro- & $.2 \mu \mathrm{g} / \mathrm{L}$ & 77443 & Propane, 1,2,3-trichloro- & $.2 \mu \mathrm{g} / \mathrm{L}$ \\
\hline 32104 & Methane, tribromo- & $.2 \mu \mathrm{g} / \mathrm{L}$ & 34551 & Benzene, 1,2,4-trichloro- & $.2 \mu \mathrm{g} / \mathrm{L}$ \\
\hline 77275 & Benzene, 1-chloro-2-methyl- & $.2 \mu \mathrm{g} / \mathrm{L}$ & 77222 & Benzene, 1,2,4-trimethyl- & $.2 \mu \mathrm{g} / \mathrm{L}$ \\
\hline 77277 & Benzene, 1-chloro-4-methyl- & $.2 \mu \mathrm{g} / \mathrm{L}$ & 34546 & Ethylene, trans-1,2-dichloro- & $.2 \mu \mathrm{g} / \mathrm{L}$ \\
\hline 34576 & 2-Chloroethylvinylether (phase I only) & 1. $\mu \mathrm{g} / \mathrm{L}$ & 77226 & Benzene, 1,3,5-trimethyl- & $.2 \mu \mathrm{g} / \mathrm{L}$ \\
\hline \multirow[t]{2}{*}{32102} & Methane, tetrachloro- & & 77353 & Benzene, tert-butyl- & $.2 \mu \mathrm{g} / \mathrm{L}$ \\
\hline & (Carbon tetrachloride) & $.2 \mu \mathrm{g} / \mathrm{L}$ & 34475 & Ethylene, tetrachloro- & $.2 \mu \mathrm{g} / \mathrm{L}$ \\
\hline 34301 & Benzene, chloro- & $.2 \mu \mathrm{g} / \mathrm{L}$ & 34010 & Benzene, methyl- (Toluene) & $.2 \mu \mathrm{g} / \mathrm{L}$ \\
\hline 32105 & Methane, dibromochloro- & $.2 \mu \mathrm{g} / \mathrm{L}$ & 34699 & Propene, trans-1,3-dichloro- & $.2 \mu \mathrm{g} / \mathrm{L}$ \\
\hline 34311 & Ethane, chloro- & $2 \mu \mathrm{g} / \mathrm{L}$ & 39180 & Ethylene, trichloro- & $.2 \mu \mathrm{g} / \mathrm{L}$ \\
\hline 32106 & Methane, trichloro- (Chloroform) & $2 \mu \mathrm{g} / \mathrm{L}$ & 34488 & Methane, trichlorofluoro- & $.2 \mu \mathrm{g} / \mathrm{L}$ \\
\hline 34418 & Methane, chloro- (Methyl chloride) & $.2 \mu \mathrm{g} / \mathrm{L}$ & 39175 & Ethylene, chloro- (Vinyl chloride) & $.2 \mu \mathrm{g} / \mathrm{L}$ \\
\hline 77093 & Ethylene, cis-1,2-dichloro- & $.2 \mu \mathrm{g} / \mathrm{L}$ & 81551 & Benzene, dimethyl- (Xylene) & $.2 \mu \mathrm{g} / \mathrm{L}$ \\
\hline 34704 & Propene, cis-1,3-dichloro- & $.2 \mu \mathrm{g} / \mathrm{L}$ & & & \\
\hline 34501 & Ethylene, 1,1-dichloro- & $.2 \mu \mathrm{g} / \mathrm{L}$ & & & \\
\hline 34496 & Ethane, 1,1-dichloro- & $.2 \mu \mathrm{g} / \mathrm{L}$ & & & \\
\hline 77651 & Ethane, 1,2-dibromo- (EDB) & $.2 \mu \mathrm{g} / \mathrm{L}$ & & & \\
\hline
\end{tabular}


Appendix - U.S. Environmental Protection Agency STOage and RETrieval (STORET) codes and minimum reporting level (MRL) or method detection limit (MDL) (Schedules 8308, 2010, and 8015 ) for constituents analyzed during Phases I and II of the Willamette River Basin Water Quality Study, Oregon, 1992-94—Continued

\begin{tabular}{|c|c|c|c|}
\hline $\begin{array}{l}\text { STORET } \\
\text { Code }\end{array}$ & Constituent & & $\begin{array}{l}\text { MRL } \\
\text { MDL }\end{array}$ \\
\hline \multicolumn{4}{|c|}{$\begin{array}{l}\text { Polychlorinated dibenzo-p-dioxins (PCDD) in suspended } \\
\text { sediment (Phase I): }\end{array}$} \\
\hline \multicolumn{4}{|c|}{ Tetrachlorodibenzo-p-dioxin, total } \\
\hline \multicolumn{4}{|c|}{$\begin{array}{l}\text { (TCDD) } \\
\text { Tetrachlorodibenzo-p-dioxin }\end{array}$} \\
\hline \multicolumn{4}{|c|}{$\begin{array}{l}(2,3,7,8 \text {-TCDD }) \\
\text { Pentachlorodibenzo-p-dioxin, total }\end{array}$} \\
\hline \multicolumn{4}{|c|}{ Pentachlorodibenzo-p-dioxin } \\
\hline & $(1,2,3,7,8-\mathrm{PeCDD})$ & 34 & $\mathrm{pg} / \mathrm{KL}$ \\
\hline \multicolumn{3}{|c|}{ Hexachlorodibenzo-p-dioxin, total } & $\mathrm{pg} / \mathrm{KL}$ \\
\hline \multicolumn{4}{|c|}{ Hexachlorodibenzo-p-dioxin } \\
\hline & $(1,2,3,4,7,8-\mathrm{HxCDD})$ & 21 & $\mathrm{pg} / \mathrm{KL}$ \\
\hline \multicolumn{4}{|c|}{ Hexachlorodibenzo-p-dioxin } \\
\hline \multicolumn{4}{|c|}{ Hexachlorodibenzo-p-dioxin } \\
\hline & $(1,2,3,7,8,9-\mathrm{HxCDD})$ & 20 & $\mathrm{pg} / \mathrm{KL}$ \\
\hline \\
\hline \multicolumn{4}{|c|}{ Heptachlorodibenzo-p-dioxin } \\
\hline & $(1,, 2,3,4,6,7,8-\mathrm{HpCDD})$ & 36 & \\
\hline & Octachlorodibenzo-p-dioxin (OCDD) & 270 & $\mathrm{pg} / \mathrm{Kl}$ \\
\hline
\end{tabular}

Polychlorinated dibenzo-p-dioxins (PCDD) in bed sediment (Phase I):

Tetrachlorodibenzo-p-dioxin, total

(TCDD)

$0.48-2.4 \mathrm{pg} / \mathrm{g}$

Tetrachlorodibenzo-p-dioxin

(2,3,7,8-TCDD)

Pentachlorodibenzo-p-dioxin, total

(PeCDD)

Pentachlorodibenzo-p-dioxin

(1,2,3,7,8-PeCDD)

Hexachlorodibenzo-p-dioxin, total

(HxCDD)

Hexachlorodibenzo-p-dioxin

(1,2,3,4,7,8-HxCDD)

Hexachlorodibenzo-p-dioxin

(1,2,3,6,7,8-HxCDD)

Hexachlorodibenzo-p-dioxin

(1,2,3,7,8,9-HxCDD)

Heptachlorodibenzo-p-dioxin, total

(HpCDD)

Heptachlorodibenzo-p-dioxin

(1,,2,3,4,6,7,8-HpCDD)

$.41-\quad 7.3 \mathrm{pg} / \mathrm{g}$

$.26-\quad 3.9 \mathrm{pg} / \mathrm{g}$
STORET

Constituent

Code

Octachlorodibenzo-p-dioxin

(OCDD)

.93- $2.2 \mathrm{pg} / \mathrm{g}$

\section{Polychlorinated dibenzofurans (PCDF) in suspended} sediment (Phase I):

Tetrachlorodibenzofuran, total

(TCDF)

$12 \mathrm{pg} / \mathrm{KL}$

Tetrachlorodibenzofuran

(2,3,7,8-TCDF)

Pentachlorodibenzofuran, total

(PeCDF)

Pentachlorodibenzofuran

(1,2,3,7,8-PeCDF)

Pentachlorodibenzofuran

(2,3,4,7,8-PeCDF)

Hexachlorodibenzofuran, total

(HxCDF)

Hexachlorodibenzofuran

$(1,2,3,4,7,8-\mathrm{HxCDF})$

Hexachlorodibenzofuran

(1,2,3,6,7,8-HxCDF)

Hexachlorodibenzofuran

(2,3,4,6,7,8-HxCDF)

Hexachlorodibenzofuran

(1,2,3,7,8,9-HxCDF)

Heptachlorodibenzofuran, total

(HpCDF)

Heptachlorodibenzofuran

(1,2,3,4,6,7,8-HpCDF)

Heptachlorodibenzofuran

(1,2,3,4,7,8,9-HpCDF)

Octachlorodibenzofuran (OCDF)

$12 \mathrm{pg} / \mathrm{KL}$

$23 \mathrm{pg} / \mathrm{KL}$

$20 \mathrm{pg} / \mathrm{KL}$

$23 \mathrm{pg} / \mathrm{KL}$

$23 \mathrm{pg} / \mathrm{KL}$

$11 \mathrm{pg} / \mathrm{KL}$

$9 \mathrm{pg} / \mathrm{KL}$

$23 \mathrm{pg} / \mathrm{KL}$

$11 \mathrm{pg} / \mathrm{KL}$

$21 \mathrm{pg} / \mathrm{KL}$

$19 \mathrm{pg} / \mathrm{KL}$

$7.2 \mathrm{pg} / \mathrm{KL}$

$19 \mathrm{pg} / \mathrm{KL}$

Polychlorinated dibenzofurans (PCDF) in bed sediment (Phase I):

Tetrachlorodibenzofuran, total

(TCDF)

Tetrachlorodibenzofuran

(2,3,7,8-TCDF)

Pentachlorodibenzofuran, total

$.39-\quad .51 \mathrm{pg} / \mathrm{g}$

$.31-.51 \mathrm{pg} / \mathrm{g}$
(PeCDF)

Pentachlorodibenzofuran

$(1,2,3,7,8-\mathrm{PeCDF})$

Pentachlorodibenzofuran

$(2,3,4,7,8-\mathrm{PeCDF})$

Hexachlorodibenzofuran, total

(HxCDF)

Hexachlorodibenzofuran
$.22-.72 \mathrm{pg} / \mathrm{g}$

$.13-.79 \mathrm{pg} / \mathrm{g}$

$.34-4.1 \quad \mathrm{pg} / \mathrm{g}$

$.29-7.7 \quad \mathrm{pg} / \mathrm{g}$

$.34-13 \mathrm{pg} / \mathrm{g}$

$.55-4 \mathrm{pg} / \mathrm{g}$ 
Appendix - U.S. Environmental Protection Agency STOage and RETrieval (STORET) codes and minimum reporting level (MRL) or method detection limit (MDL) (Schedules 8308, 2010, and 8015 ) for constituents analyzed during Phases I and II of the Willamette River Basin Water Quality Study, Oregon, 1992-94—Continued

\begin{tabular}{|c|c|c|c|c|c|c|}
\hline $\begin{array}{l}\text { STORET } \\
\text { Code }\end{array}$ & \multicolumn{2}{|l|}{ Constituent } & \multirow{2}{*}{$\begin{array}{l}\begin{array}{l}\text { MRL/ } \\
\text { MDL }\end{array} \\
\mathrm{pg} / \mathrm{g}\end{array}$} & \multicolumn{2}{|c|}{$\begin{array}{l}\text { STORET } \\
\text { Code }\end{array}$} & $\begin{array}{l}\text { MRL/ } \\
\text { MDL }\end{array}$ \\
\hline & $(1,2,3,4,7,8-\mathrm{HxCDF})$ & $.23-9.1$ & & 39381 & Dieldrin & $.001 \mu \mathrm{g} / \mathrm{L}$ \\
\hline & Hexachlorodibenzofuran & & & 34653 & p,p'-DDE & $.006 \mu \mathrm{g} / \mathrm{L}$ \\
\hline & $(1,2,3,6,7,8-\mathrm{HxCDF})$ & $.21-5.5$ & $\mathrm{pg} / \mathrm{g}$ & 34253 & alpha-HCH & $.002 \mu \mathrm{g} / \mathrm{L}$ \\
\hline & Hexachlorodibenzofuran & & & 39341 & Lindane (gamma-HCH) & $.004 \mu \mathrm{g} / \mathrm{L}$ \\
\hline & $(2,3,4,6,7,8-\mathrm{HxCDF})$ & $.55-10$ & $\mathrm{pg} / \mathrm{g}$ & \multicolumn{2}{|c|}{ Organophosphorus } & \\
\hline & Hexachlorodibenzofuran & & & 38933 & Chlorpyrifos & $.004 \mu \mathrm{g} / \mathrm{L}$ \\
\hline & $(1,2,3,7,8,9-\mathrm{HxCDF})$ & $.25-7.7$ & $\mathrm{pg} / \mathrm{g}$ & 39572 & Diazinon & $.002 \mu \mathrm{g} / \mathrm{L}$ \\
\hline & Heptachlorodibenzofuran, total & & & 82677 & Disulfoton & $.017 \mu \mathrm{g} / \mathrm{L}$ \\
\hline & (HpCDF) & $.19-9.6$ & $\mathrm{pg} / \mathrm{g}$ & 82672 & Ethoprop & $.003 \mu \mathrm{g} / \mathrm{L}$ \\
\hline & Heptachlorodibenzofuran & & & 04095 & Fonofos & $.003 \mu \mathrm{g} / \mathrm{L}$ \\
\hline & $(1,2,3,4,6,7,8-\mathrm{HpCDF})$ & $.15-3.3$ & $\mathrm{pg} / \mathrm{g}$ & 39532 & Malathion & $.005 \mu \mathrm{g} / \mathrm{L}$ \\
\hline & Heptachlorodibenzofuran & & & 82686 & Methyl Azinphos & $.001 \mu \mathrm{g} / \mathrm{L}$ \\
\hline & $(1,2,3,4,7,8,9-\mathrm{HpCDF})$ & $.19-10$ & $\mathrm{pg} / \mathrm{g}$ & 82667 & Methyl Parathion & $.002 \mu \mathrm{g} / \mathrm{L}$ \\
\hline & Octachlorodibenzofuran & & & 39542 & Parathion & $.006 \mu \mathrm{g} / \mathrm{L}$ \\
\hline & $(\mathrm{OCDF})$ & $.78-9.9$ & $\mathrm{pg} / \mathrm{g}$ & 82664 & Phorate & $.002 \mu \mathrm{g} / \mathrm{L}$ \\
\hline \multirow{2}{*}{\multicolumn{4}{|c|}{$\begin{array}{l}\text { Pesticides in filtered water by GC/MS [Schedule 2010] } \\
\text { (Phases I and II): }\end{array}$}} & 82675 & Terbufos & $.007 \mu \mathrm{g} / \mathrm{L}$ \\
\hline & & & & \multicolumn{2}{|c|}{ Permethrin } & \\
\hline \multicolumn{2}{|c|}{ Amid } & \multirow{2}{*}{\multicolumn{2}{|c|}{$.002 \mu \mathrm{g} / \mathrm{L}$}} & 82687 & cis-Permethrin & $.005 \mu \mathrm{g} / \mathrm{L}$ \\
\hline 46342 & Alachlor & & & \multicolumn{2}{|c|}{ Phenyl Urea } & \\
\hline 39415 & Metolachlor & \multicolumn{2}{|c|}{$.004 \mu \mathrm{g} / \mathrm{L}$} & & Linuron & $.002 \mu \mathrm{g} / \mathrm{L}$ \\
\hline 82684 & Napropamide & .004 & $\mu \mathrm{g} / \mathrm{L}$ & $\begin{array}{l}82666 \\
82670\end{array}$ & Tebuthiuron & $.01 \mu \mathrm{g} / \mathrm{L}$ \\
\hline 82676 & Pronamide & .018 & $\mu \mathrm{g} / \mathrm{L}$ & \multirow{2}{*}{\multicolumn{2}{|c|}{ Triazine }} & \\
\hline 04024 & Propachlor & .007 & $\mu \mathrm{g} / \mathrm{L}$ & & & \\
\hline 82679 & Propanil & .004 & $\mu \mathrm{g} / \mathrm{L}$ & 39632 & Atrazine & $.001 \mu \mathrm{g} / \mathrm{L}$ \\
\hline \multicolumn{2}{|c|}{ Carbamate } & & & 04041 & Cyanazine & $.004 \mu \mathrm{g} / \mathrm{L}$ \\
\hline 04028 & Butylate & .002 & $\mu \mathrm{g} / \mathrm{L}$ & 04040 & Desethyl Atrazine & $.002 \mu \mathrm{g} / \mathrm{L}$ \\
\hline 82680 & Carbaryl & .003 & $\mu \mathrm{g} / \mathrm{L}$ & 82630 & Metribuzin & $.004 \mu \mathrm{g} / \mathrm{L}$ \\
\hline 82674 & Carbofuran & .003 & $\mu \mathrm{g} / \mathrm{L}$ & 04037 & Prometon & $.003 \mu \mathrm{g} / \mathrm{L}$ \\
\hline 82668 & EPTC & .002 & $\mu \mathrm{g} / \mathrm{L}$ & 04035 & Simazine & $.005 \mu \mathrm{g} / \mathrm{L}$ \\
\hline 82671 & Molinate & .003 & $\mu \mathrm{g} / \mathrm{L}$ & \multicolumn{2}{|c|}{ Uracil } & \\
\hline 82669 & Pebulate & .004 & $\mu \mathrm{g} / \mathrm{L}$ & 82665 & Terbacil & $.007 \mu \mathrm{g} / \mathrm{L}$ \\
\hline 82681 & Thiobencarb & .002 & $\mu \mathrm{g} / \mathrm{L}$ & \multicolumn{2}{|c|}{ Surrogate } & \\
\hline 82678 & Triallate & .001 & $\mu \mathrm{g} / \mathrm{L}$ & 90163 & Diazinon & percent \\
\hline \multicolumn{2}{|c|}{ Dinitroaniline } & & & 90164 & Terbuthylazine & percent \\
\hline 82673 & Benfluralin & .002 & $\mu \mathrm{g} / \mathrm{L}$ & 90165 & alpha D6 HCH & percent \\
\hline 82663 & Ethalfluralin & .004 & $\mu \mathrm{g} / \mathrm{L}$ & \multirow{2}{*}{\multicolumn{3}{|c|}{$\begin{array}{l}\text { Pesticides in filtered water by HPLC [Schedule 2051] } \\
\text { (Phases I and II): }\end{array}$}} \\
\hline 82683 & Pendimethalin & .004 & $\mu \mathrm{g} / \mathrm{L}$ & & & \\
\hline 82661 & Trifluralin & \multirow{3}{*}{\multicolumn{2}{|c|}{$.003 \mu \mathrm{g} / \mathrm{L}$}} & \multicolumn{2}{|c|}{ Benoic Acid } & \\
\hline \multicolumn{2}{|c|}{ Metabolite } & & & 38442 & Dicamba & $.05 \mu \mathrm{g} / \mathrm{L}$ \\
\hline 82660 & 2,6-Diethylaniline & & & \multicolumn{2}{|c|}{ Benzonitrile } & \\
\hline \multicolumn{2}{|c|}{ Miscellaneous } & & & 49311 & Bromoxynil & $.05 \mu \mathrm{g} / \mathrm{L}$ \\
\hline 82685 & Propargite & .013 & $\mu \mathrm{g} / \mathrm{L}$ & 49303 & Dichlobenil & $.05 \mu \mathrm{g} / \mathrm{L}$ \\
\hline Org & anochlorine & & & & rbamate & \\
\hline 82682 & DCPA & .002 & $\mu \mathrm{g} / \mathrm{L}$ & 49312 & Aldicarb & $.05 \mu \mathrm{g} / \mathrm{L}$ \\
\hline
\end{tabular}


Appendix - U.S. Environmental Protection Agency STOage and RETrieval (STORET) codes and minimum reporting level (MRL) or method detection limit (MDL) (Schedules 8308, 2010, and 8015 ) for constituents analyzed during Phases I and II of the Willamette River Basin Water Quality Study, Oregon, 1992-94—Continued

\begin{tabular}{ll}
$\begin{array}{l}\text { STORET } \\
\text { Code }\end{array}$ & \multicolumn{1}{c}{ Constituent } \\
\hline 49313 & Aldicarb Sulfone \\
49314 & Aldicarb Sulfoxide \\
49310 & Carbaryl \\
49309 & Carbofuran \\
49308 & 3-hydroxy-carbofuran \\
38501 & Methiocarb \\
49296 & Methomyl \\
38866 & Oxamyl \\
49236 & Propham (IPC) \\
38538 & Propoxur
\end{tabular}

\section{Chlorophenoxy acid}

$\begin{array}{ll}38711 & \text { Bentazon } \\ 39732 & 2,4-\mathrm{D} \\ 38746 & 2,4-\mathrm{DB} \\ 49304 & \text { Dacthal, mono-acid- } \\ 49302 & \text { Dichlorprop (2,4-DP) } \\ 38482 & \text { MCPA } \\ 38487 & \text { MCPB } \\ 39762 & \text { Silvex (2,4,5-TP) } \\ 39742 & 2,4,5-\mathrm{T}\end{array}$

Dinitroaniline

49292 Oryzalin

Dinitrophenol

49301 Dinoseb (DNBP)

Dinitrophenyl

49299 2,6-Dinitro-o-cresol (DNOC)

\section{Diphenyl ether}

49315 Acifluorfen

\section{Naphthol}

49295 1-Naphthol

\section{Phenoxy acid}

49307 Chloramben (Aimben)

\section{Phenyl urea}

$\begin{array}{ll}49300 & \text { Diuron } \\ 49297 & \text { Fenuron } \\ 38811 & \text { Fluometuron } \\ 38478 & \text { Linuron } \\ 49294 & \text { Neburon }\end{array}$

\section{Phthalimide}

49306 Chlorothalonil

\section{Pyrethroid}

49298 Esfenvalerate

MRL/
MDL
$\mu \mathrm{g} / \mathrm{L}$
$\mu \mathrm{g} / \mathrm{L}$
$\mu \mathrm{g} / \mathrm{L}$
$\mu \mathrm{g} / \mathrm{L}$
$\mu \mathrm{g} / \mathrm{L}$
$\mu \mathrm{g} / \mathrm{L}$
$\mu \mathrm{g} / \mathrm{L}$
$\mu \mathrm{g} / \mathrm{L}$
$\mu \mathrm{g} / \mathrm{L}$
$\mu \mathrm{g} / \mathrm{L}$

STORET
Code

Constituent

MRL/

MDL

\begin{tabular}{|c|c|c|c|}
\hline \multicolumn{4}{|c|}{ Pyridazinone } \\
\hline 49293 & Norflurazon & .05 & $\mu \mathrm{g} / \mathrm{L}$ \\
\hline \multicolumn{4}{|c|}{ Pyridine } \\
\hline 49305 & Clopyralid & .05 & $\mu \mathrm{g} / \mathrm{L}$ \\
\hline 49291 & Picloram & .05 & $\mu \mathrm{g} / \mathrm{L}$ \\
\hline \multicolumn{4}{|c|}{ Pyridyloxyacetic acid } \\
\hline 49235 & Triclopyr & .05 & $\mu \mathrm{g} / \mathrm{L}$ \\
\hline \multicolumn{4}{|c|}{ Uracil } \\
\hline 04029 & Bromacil & .05 & $\mu \mathrm{g} / \mathrm{L}$ \\
\hline \multicolumn{4}{|c|}{ Surrogate } \\
\hline 99835 & BDMC & & percent \\
\hline
\end{tabular}

Triazines, herbicides in filtered water [custom Schedule 8015] (Phase II)):

$\begin{array}{ll}\text { Alachlor } & .007 \mu \mathrm{g} / \mathrm{L} \\ \text { Ametryn } & .006 \mu \mathrm{g} / \mathrm{L} \\ \text { Atrazine } & .009 \mu \mathrm{g} / \mathrm{L} \\ \text { Bromacil } & .02 \mu \mathrm{g} / \mathrm{L} \\ \text { Butachlor } & .008 \mu \mathrm{g} / \mathrm{L} \\ \text { Butylate } & .005 \mu \mathrm{g} / \mathrm{L} \\ \text { Carboxin } & .006 \mu \mathrm{g} / \mathrm{L} \\ \text { Cyanazine } & .006 \mu \mathrm{g} / \mathrm{L} \\ \text { Cycloate } & .01 \mu \mathrm{g} / \mathrm{L} \\ \text { Desethylatrazine } & .01 \mu \mathrm{g} / \mathrm{L} \\ \text { Desisopropylatrazine } & .004 \mu \mathrm{g} / \mathrm{L} \\ \text { Diphenamid } & .008 \mu \mathrm{g} / \mathrm{L} \\ \text { Hexazinone } & .013 \mu \mathrm{g} / \mathrm{L} \\ \text { Metolachlor } & .01 \mu \mathrm{g} / \mathrm{L} \\ \text { Metribuzin } & .005 \mu \mathrm{g} / \mathrm{L} \\ \text { Prometon } & .007 \mu \mathrm{g} / \mathrm{L} \\ \text { Prometryn } & .007 \mu \mathrm{g} / \mathrm{L} \\ \text { Propachlor } & .008 \mu \mathrm{g} / \mathrm{L} \\ \text { Propazine } & .006 \mu \mathrm{g} / \mathrm{L} \\ \text { Trifluralin } & .002 \mu \mathrm{g} / \mathrm{L} \\ \text { Simazine } & .011 \mu \mathrm{g} / \mathrm{L} \\ \text { Simetryn } & .005 \mu \mathrm{g} / \mathrm{L} \\ \text { Terbacil } & .009 \mu \mathrm{g} / \mathrm{L} \\ \text { Terbuthylazine (surrogate) } & \mathrm{percent} \\ \text { Vernolate } & .009 \mu \mathrm{g} / \mathrm{L}\end{array}$

$.05 \mu \mathrm{g} / \mathrm{L}$

$.05 \mu \mathrm{g} / \mathrm{L}$

$.05 \mu \mathrm{g} / \mathrm{L}$

$.05 \mu \mathrm{g} / \mathrm{L}$

$.05 \mu \mathrm{g} / \mathrm{L}$

$.05 \mu \mathrm{g} / \mathrm{L}$

$.05 \mu \mathrm{g} / \mathrm{L}$

$.05 \mu \mathrm{g} / \mathrm{L}$

$.05 \mu \mathrm{g} / \mathrm{L}$

$.05 \mu \mathrm{g} / \mathrm{L}$

$.05 \mu \mathrm{g} / \mathrm{L}$

$.05 \mu \mathrm{g} / \mathrm{L}$

$.05 \mu \mathrm{g} / \mathrm{L}$

$.05 \mu \mathrm{g} / \mathrm{L}$

$.05 \mu \mathrm{g} / \mathrm{L}$

$.05 \mu \mathrm{g} / \mathrm{L}$

$.05 \mu \mathrm{g} / \mathrm{L}$

$.05 \mu \mathrm{g} / \mathrm{L}$

$.05 \mu \mathrm{g} / \mathrm{L}$

$.05 \mu \mathrm{g} / \mathrm{L}$

$.05 \mu \mathrm{g} / \mathrm{L}$ 
Page Intentionally Blank 